\title{
TASK SUMMARY FOR CONE PENETROMETER TESTING SOUNDING AND SOIL AND GROUNDWATER SAMPLING SALMON SITE LAMAR COUNTY, MISSISSIPPI
}

\author{
Prepared for \\ DOE Nevada Operations Office \\ Las Vegas, Nevada \\ Prepared by \\ IT CORPORATION \\ 4330 South Valley View Boulevard, Suite 114 \\ Las Vegas, Nevada
}

Work Performed Under Contract No.

DE-AC08-92NV10972

October 1994

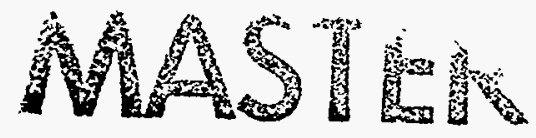




\section{DISCLAIMER}

This report was prepared as an account of work sponsored by an agency of the United States Government. Neither the United States Government nor any agency thereof, nor any of their employees, make any warranty, express or implied, or assumes any legal liability or responsibility for the accuracy, completeness, or usefulness of any information, apparatus, product, or process disclosed, or represents that its use would not infringe privately owned rights. Reference herein to any specific commercial product, process, or service by trade name, trademark, manufacturer, or otherwise does not necessarily constitute or imply its endorsement, recommendation, or favoring by the United States Government or any agency thereof. The views and opinions of authors expressed herein do not necessarily state or reflect those of the United States Government or any agency thereof. 


\section{DISCLAIMER}

Portions of this document may be illegible in electronic image products. Images are produced from the best available original document. 


\section{Table of Contents}

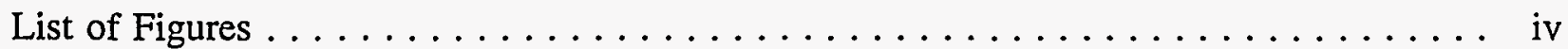

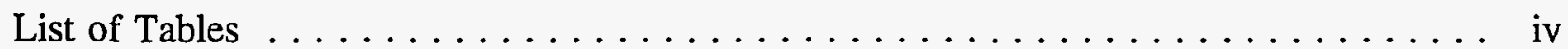

List of Abbreviations and Acronyms $\ldots \ldots \ldots \ldots \ldots \ldots \ldots \ldots \ldots \ldots \ldots \ldots$

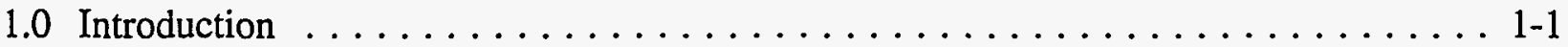

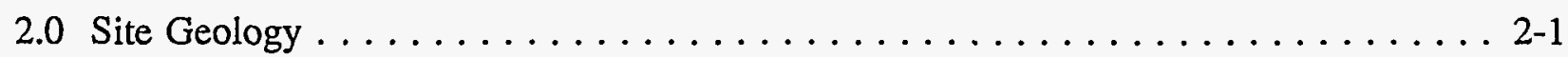

3.0 Cone Penetrometer Testing Soundings and Sampling $\ldots \ldots \ldots \ldots \ldots \ldots$ 3-1

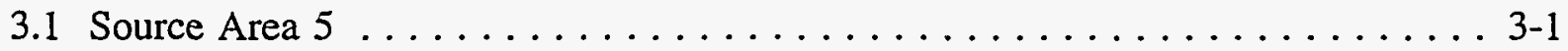

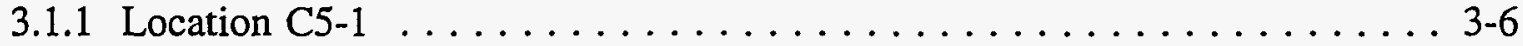

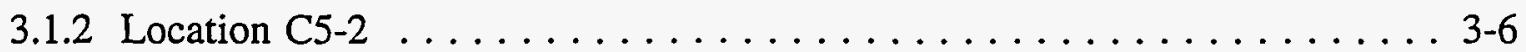

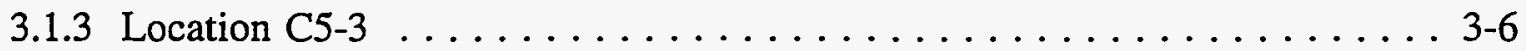

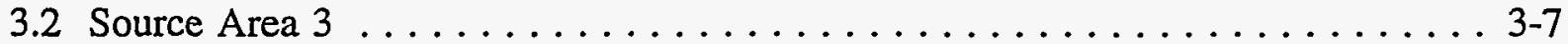

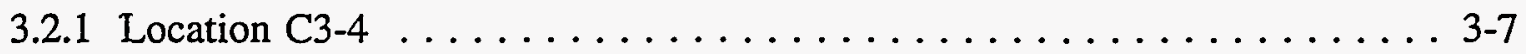

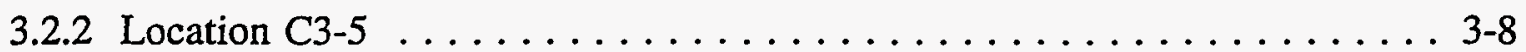

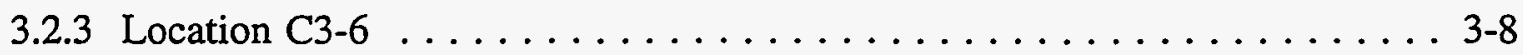

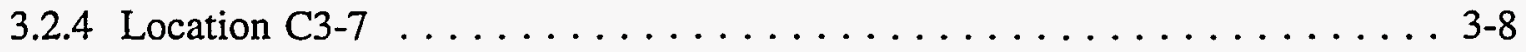

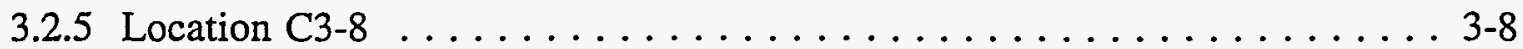

3.2 .6 Location C3-9 . . . . . . . . . . . . . . . . . . . . . 3-9

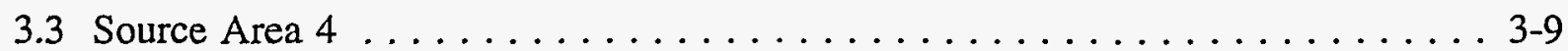

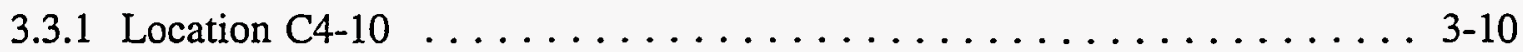

3.3.2 Location C4-11 . . . . . . . . . . . . . . . . . 3-10

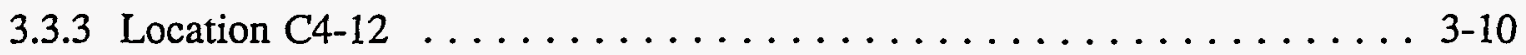

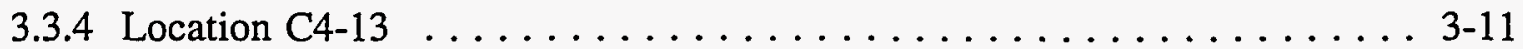

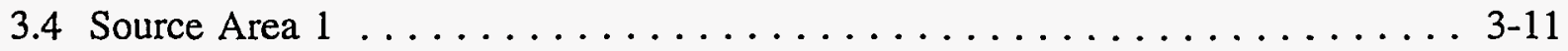

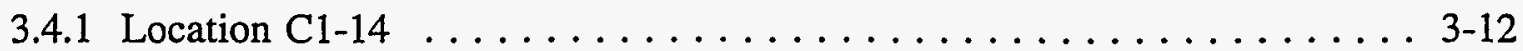

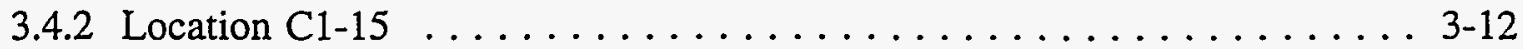

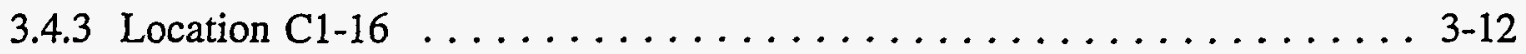

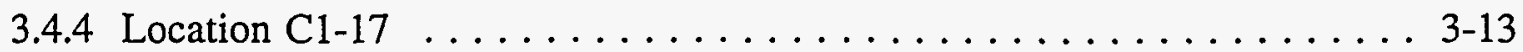

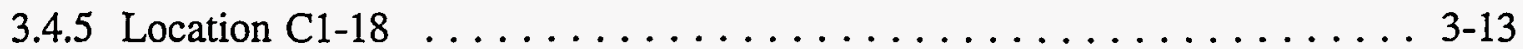

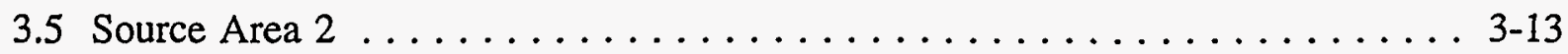

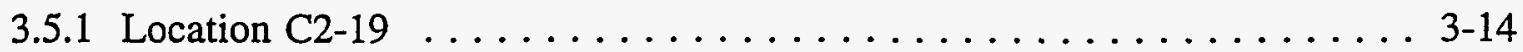

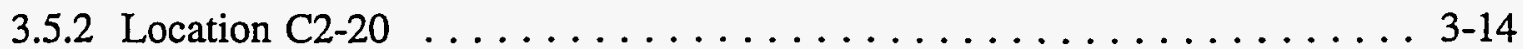




\section{Table of Contents (Continued)}

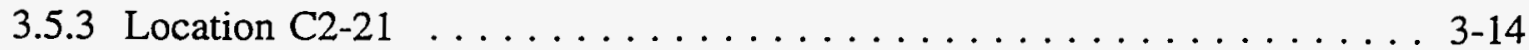

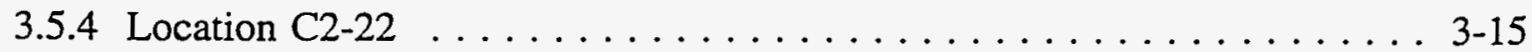

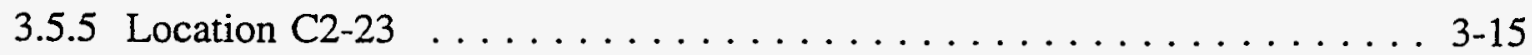

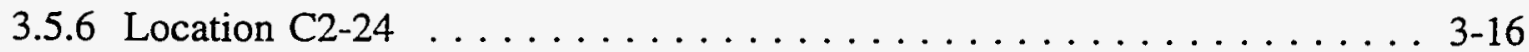

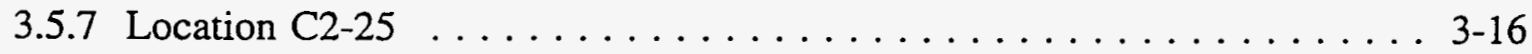

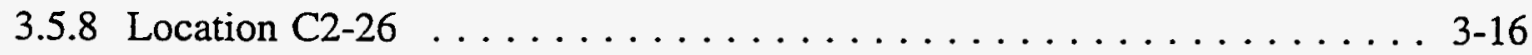

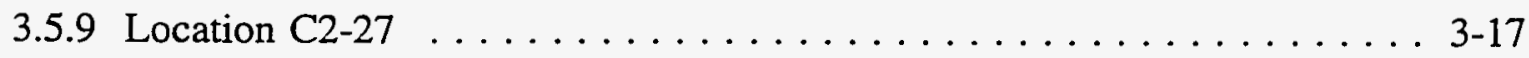

4.0 Analytical Results $\ldots \ldots \ldots \ldots \ldots \ldots \ldots \ldots \ldots \ldots \ldots \ldots \ldots \ldots . \ldots \ldots .4 .1$

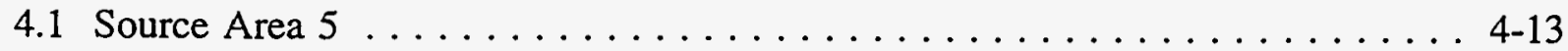

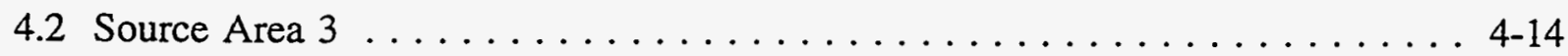

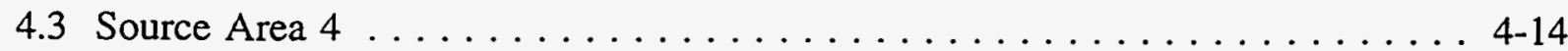

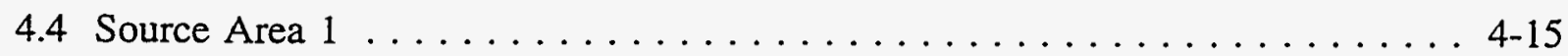

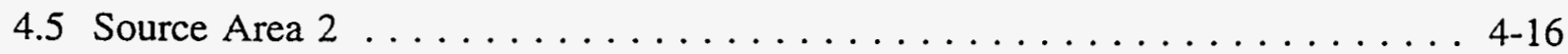

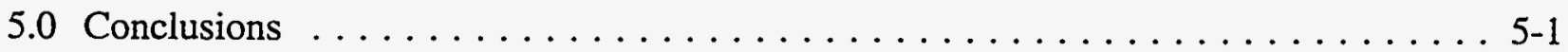

6.0 References $\ldots \ldots \ldots \ldots \ldots \ldots \ldots \ldots \ldots \ldots \ldots \ldots \ldots \ldots \ldots .6 .1$

Appendix A - Cone Penetrometer Testing Logs .................. A-1

Appendix B - Explanation of Terms Used in Data Tables .............. B-1

Appendix C.1 - Results of Volatile Organic Compound Analyses, Soils,

Salmon Site ...................... C.1-1

Appendix C.2 - Results of Volatile Organic Compound Analyses, Water,

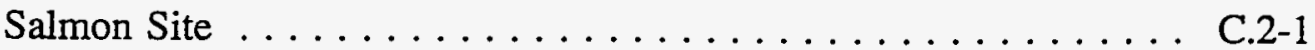

Appendix D.1 - Results of Semivolatile Organic Compound Analyses, Soils,

Salmon Site . . . . . . . . . . . . . . . . . D.1-1

Appendix D.2 - Results of Semivolatile Organic Compound Analyses, Water,

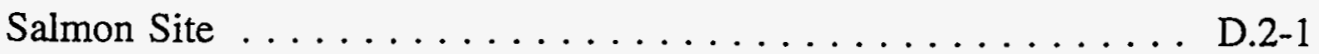

Appendix E.1 - Results of Pesticide and PCB Analyses, Soils, Salmon Site . . . . E. E.1-1

Appendix E.2 - Resuits of Pesticide and PCB Analyses, Water, Salmon Site . . . E E.2-1

Appendix F.1 - Results of Metals Analyses, Soils, Salmon Site ............ F.1-1

Appendix F.2 - Results of Metals Analyses, Water, Salmon Site . . . . . . . . . F.2-1

Appendix G.1 - Results of Gamma Spectroscopy Analyses, Soils, Salmon Site . . . G.1-1

Appendix G.2 - Results of Gamma Spectroscopy Analyses, Water, Salmon Site . . G.2-1 


\section{Table of Contents (Continued)}

Appendix H.1 - Results of Gross Alpha and Beta Analyses, Soils, Salmon Site . . . H.1-1 Appendix H.2 - Results of Gross Alpha and Beta Analyses, Water, Salmon Site . . H.2-1 Appendix I - Results of Tritium Analyses, Water, Salmon Site ........... I-1 


\section{List of Figures}

Number

Title

Page

1-1 General Location Map of the Salmon Site $\ldots \ldots \ldots \ldots \ldots \ldots \ldots \ldots . \ldots \ldots$

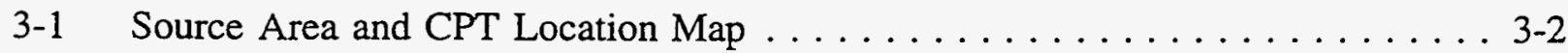

\section{List of Tables}

Number

Title

Page

3-1 Cone Penetrometer Testing Sounding and Soil/Groundwater Sampling

Summary, Salmon Site

3-2 Requested Analytical Parameters for Cone Penetrometer Testing Samples,

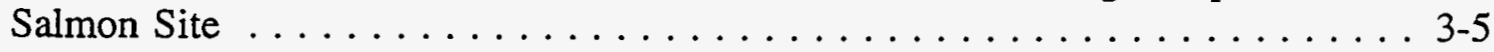

4-1 List of Chemical and Metal Constituents of Concern (CoC), Salmon Site . . . 4 4-2

4-2 List of Radionuclides Included in the Gamma Spectroscopy Analysis . . . . . . 4-3

4-3 Summary of Cone Penetrometer Testing (CPT) Sampling Analytical Data (Preliminary), Salmon Site . . . . . . . . . . . . . . . . . . 4-4

4-4 Radionuclide Activities for Soil Sample REFC-19-SS, Salmon Site . . . . . . 4 4-13 


\section{List of Abbreviations and Acronyms}

\begin{tabular}{|c|c|}
\hline amsl & above mean sea level \\
\hline bgs & below ground surface \\
\hline CERCLA & Comprehensive Environmental Response, Compensation, and Liability Act \\
\hline CLP & Contract Laboratory Program \\
\hline $\mathrm{CoC}$ & constituents of concern \\
\hline CPT & cone penetrometer testing \\
\hline CRDL & Contract Required Detection Limits \\
\hline DOE & U.S. Department of Energy \\
\hline $\mathrm{DOE} / \mathrm{NV}$ & Department of Energy Nevada Operations Office \\
\hline DUP & duplicate \\
\hline EPA & U.S. Environmental Protection Agency \\
\hline $\mathrm{ft}$ & feet \\
\hline $\mathrm{m}$ & meter(s) \\
\hline $\mathrm{m} \ell$ & milliliter \\
\hline MCL & maximum contaminate level \\
\hline MDA & minimum detectable activity \\
\hline MS & matrix spike \\
\hline MSD & matrix spike duplicate \\
\hline NPL & National Priorities List \\
\hline NTS & Nevada Test Sitè \\
\hline PCB & polychlorinated biphenyl \\
\hline PVC & polyvinylchloride \\
\hline REECo & Reynolds Electrical \& Engineering Co., Inc. \\
\hline $\mathrm{RI} / \mathrm{FS}$ & Remedial Investigation/Feasibility Study \\
\hline SARA & Superfund Amendment and Reauthorization Act of 1986 \\
\hline SDWA & Safe Drinking Water Act \\
\hline SS & Salmon Site \\
\hline TDTS & Tatum Dome Test Site \\
\hline VOA & volatile organic analysis \\
\hline
\end{tabular}




\subsection{Introduction}

The Salmon Site (SS), formerly the Tatum Dome Test Site (TDTS), is located in Lamar County, Mississippi, southwest of the city of Hattiesburg and near the communities of Purvis and Baxterville. It was the site of two nuclear and two gas explosion tests conducted deep underground in the Tatum Salt Dome (Figure 1-1). These tests were conducted between 1964 and 1970 as part of the U.S. Atomic Energy Commission's Vela Uniform Program to improve the United States' capability to detect, identify, and locate underground nuclear detonations. The SS is managed by the U.S. Department of Energy, Nevada Operations Office (DOE/NV).

A consequence of these testing activities is that radionuclides generated during the testing were released into the salt dome, where they are presently contained. During reentry drilling and other site activities, incidental liquid and solid wastes that contained radioactivity were generated. These wastes resulted in some soil, groundwater, and equipment contamination at the SS. Most of these wastes and contaminated soil and water were picked up and disposed either in the cavity left by the tests or in an injection well during the 1972 decommissioning of the site. The equipment was decontaminated and transported to the Nevada Test Site for disposal. Nonradioactive wastes were disposed in pits at the site, which were subsequently backfilled with clean soil and graded.

The U.S. Department of Energy (DOE) is conducting a series of investigations as a part of the Remedial Investigation and Feasibility Study (RI/FS). The RI/FS is the methodology under the Comprehensive Environmental Response, Compensation and Liability Act (CERCLA) for evaluating hazardous waste sites on the National Priorities List (NPL). The Salmon Site is not listed on the NPL, but DOE has voluntarily elected to conduct the evaluation of the SS in accordance with CERCLA. This report summarizes the cone penetrometer testing (CPT) and sampling program conducted in the fall of 1993 at the SS. The purpose of this report is to provide a description of the activities and a discussion of the results as a reference document to aid in the overall assessment of the SS.

The objectives of the CPT program were to

- Determine subsurface conditions and stratification

- Determine the depth to the potentiometric surface

- Obtain soil samples from predetermined depths

- Obtain groundwater samples at predetermined depths. 


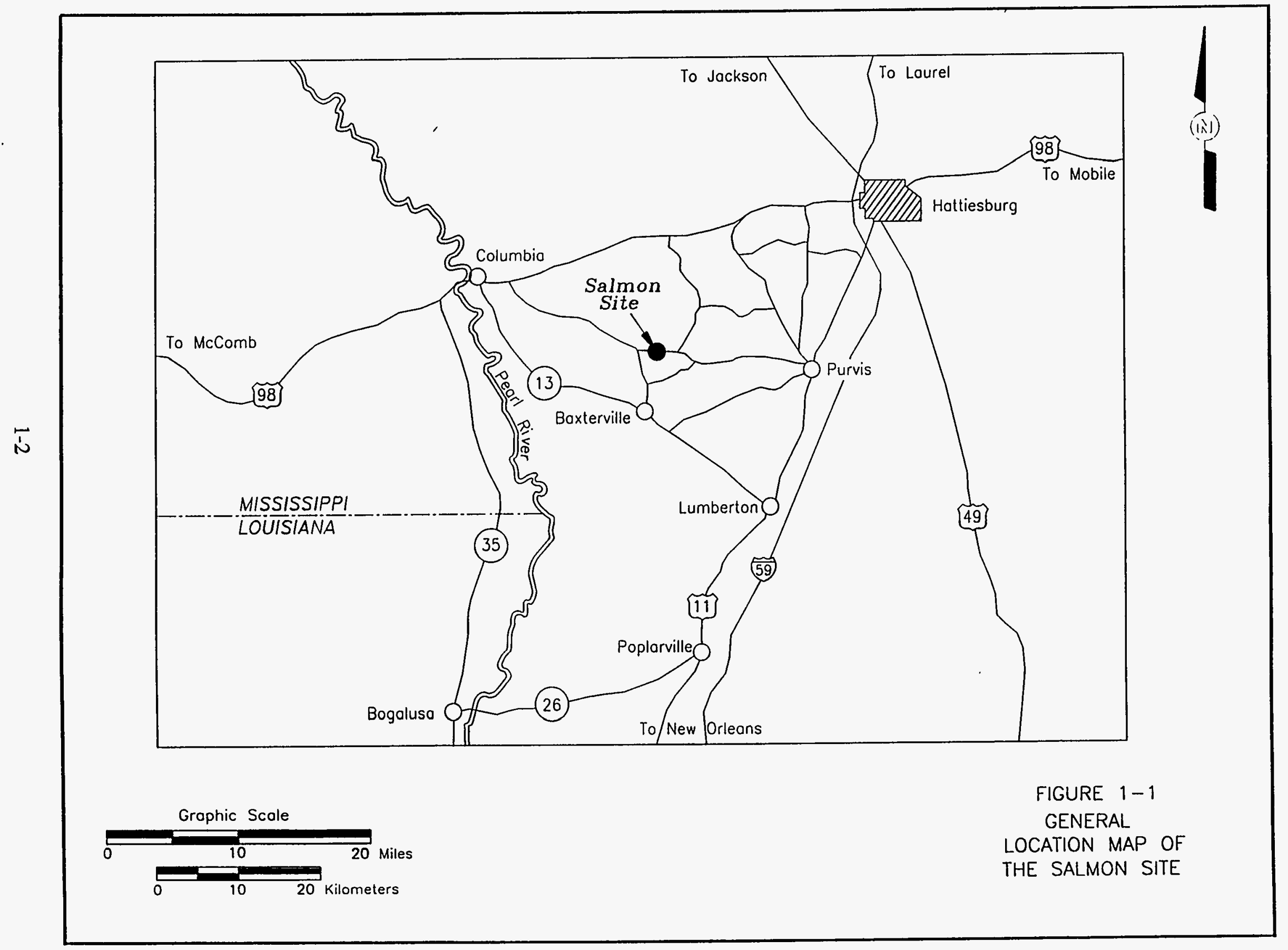


The CPT program was conducted between October 19 and November 11, 1993. Fugro Geoscience, Inc., was contracted to execute the program under the direction of IT Corporation (the Environmental Services Support Contractor to DOE).

The program consisted of conducting stratigraphic tests and collecting soil and groundwater samples at 27 locations. These locations are grouped into five general areas at the SS. Stratigraphy tests were conducted at all locations first, and each location was revisited to collect soil and groundwater samples from intervals selected from interpreted stratigraphic logs.

The stratigraphy tests were pushed to a depth of 30 meters $(\mathrm{m})$ (100 feet [ft]) or until refusal. Stratigraphy tests ranged from 5.5 to $30 \mathrm{~m}(18$ to $100 \mathrm{ft}$ ) in depth. The stratigraphic tool measured cone-tip resistance and sidewall friction in terms of tons per square foot. Penetrated lithologies are interpreted from the ratio of sidewall friction to tip resistance. In general, a sand unit will have a high tip resistance and low sidewall friction, conversely, a clay unit has low tip resistance and a high sidewall friction.

The CPT stratigraphy tool also measured the electrical conductance of the penetrated strata to determine the depth to groundwater. Conductance of the strata is governed by (1) the presence of pore water, (2) the amount of dissolved minerals in the pore water, (3) the geometry of the pores, and (4) the amount of chemically bound water in the strata. The water in the clay strata generally has a higher dissolved mineral content than water in saturated sand units and, therefore, gives a higher conductance measurement than sand units. For this reason, water samples were not always obtainable from the intervals selected from the electrical conductivity measurements. The electrical conductance tool was useful in determining the top of the saturated strata, but this did not always indicate the presence of free water available for sampling. The clayey strata are nearly always saturated with water, yet they will not yield water in sufficient quantities for sampling even when the holes are left open for 24 hours or more.

Soil samples were generally collected from strata interpreted to represent the vadose zone just above the water table. In some locations, water bearing sand could not be retained in the sampler, and the sample had to be collected from finer grained silts and clays just below the sand, which could be retained by the sampler. 
Water samples were collected at 15 of the CPT locations. The other twelve locations either did not have an adequate permeability or the permeable zone did not contain sufficient free moveable water. Most water samples were collected using the CPT groundwater sampling tool described in the work scope document. However, some locations with low-yield waterbearing zones were sampled using a one-inch-diameter polyvinylchloride (PVC) screen and casing. The PVC casing was emplaced by pushing a CPT rod equipped with a sacrificial tip to the desired depth. The PVC screen and casing were then emplaced through the CPT rod. The CPT rod was then extracted leaving the screen and casing in the hole. The hole was then allowed to recharge, ensuring adequate sample volume.

All holes produced by the CPT tool, including stratigraphic sounding holes, soil sampling holes, and water sampling holes, were sealed as prescribed by the state of Mississippi Surface Water and Groundwater Use and Protection Regulations using a volclay, high-solids, bentonite grout. The bentonite grout was mixed according to the manufacturer's specifications and emplaced through a one-inch-diameter PVC tremie line. 


\subsection{Site Geology}

The site geology is based primarily on information collected during the CPT investigation, which was restricted to depths less than $30 \mathrm{~m}$ (100 ft). Geologic data collected during this program are stratigraphic in nature, revealing little about possible geologic structures. The regional structural dip is southerly, but the presence of the Tatum Salt Dome creates local dip variations in the shallow strata.

The Salmon Site is located in southern Mississippi within a broad belt of Miocene Age and younger sediments. Three stratigraphic units were investigated during the CPT program:

- Miocene Age Pascagoula/Hattiesburg Formation

- Pliocene/Pleistocene Age Citronelle Formation

- Holocene Age alluvial sediments associated with local streams (Half Moon Creek).

The Pascagoula/Hattiesburg Formation underlies the entire site. The majority of the Pascagoula/Hattiesburg Formation is composed of stiff, silty clays. Two water-bearing sand units have been identified within this formation, the Local Aquifer and the Upper Aquifer. The top of the Local Aquifer is at depths greater than $61 \mathrm{~m}(200 \mathrm{ft}$ ) below ground surface (bgs) and was not investigated during this CPT program. The Upper Aquifer consists of perched water zones in discontinuous sand lenses present at depths between 9 and $24 \mathrm{~m}$ (30 and $80 \mathrm{ft}$ ) bgs. These sand units probably represent fluvial channel deposits with a meandering linear geometry. Additional soil borings and surveyed surface elevations are needed to accurately determine the distribution of these Upper Aquifer sand units.

The Citronelle Formation overlies the Pascagoula/Hattiesburg Formation with a gradational contact that dips very gently (less than $5^{\circ}$ ) to the south. The Citronelle Formation is generally found at elevations greater than $85 \mathrm{~m}$ ( $280 \mathrm{ft}$ ) above mean sea level (amsl), is fluvial in origin, and is composed primarily of sand and gravel. The thickest section of the

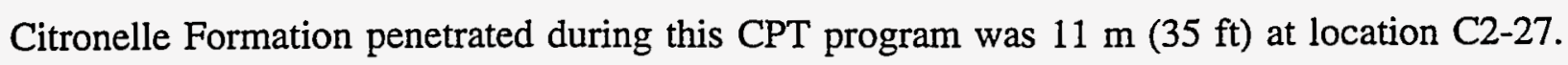
The Citronelle Formation is a very permeable stratum that will yield water during wet seasons. Water infiltrating into the Citronelle Formation tends to drain to the formation contact and then move laterally to hillsides where it forms seeps along the contact between the Citronelle and Pascagoula/Hattiesburg Formations.

The alluvial sediments located in the stream valleys are composed of reworked sediments of the Citronelle Formation and Pascagoula/Hattiesburg Formation. The CPT investigation 
penetrated these sediments in the vicinity of Surface Ground Zero near Half Moon Creek. In

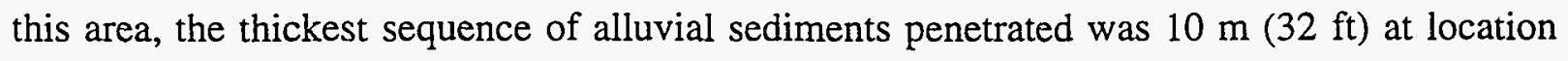
C1-15. The upper 3.7 to $6.1 \mathrm{~m}$ (12 to $20 \mathrm{ft}$ ) of these sediments consist of silty clayey soil, and the deeper sediments consist of silty sand and fine sand with some small gravel. The sandier portions were fully saturated and readily yielded water. 


\subsection{Cone Penetrometer Testing Soundings and Sampling}

The CPT stratigraphy testing (also known as "sounding") and sampling were done at each of the 27 locations. These summaries were developed from the CPT Sounding Logs, Field Activity Daily Logs, and the Sample Collection Logs. Copies of the CPT logs are included in Appendix A. The locations of the Source Areas and the CPT sites are shown on Figure 3-1. Table 3-1 summarizes the CPT sounding, soil, and groundwater sampling program. The term "saturated sediments" in the following sections does not necessarily indicate free or moveable water. In many instances, the clay sediments are moist to wet, but did not yield water even when allowed to recharge for more than 24 hours.

For the purposes of this discussion, the CPT locations are grouped into 5 geographic areas. These areas correspond with the Source Areas established in the work plan for the Salmon Site (DOE, 1992). The locations and samples are designated CX-Y-Z, where $X$ is the source area, $\mathrm{Y}$ is the sequential CPT sounding number, and $\mathrm{Z}$ is the matrix deliminator (e.g., $\mathrm{S}$ - soil, $\mathrm{W}$ - water). Field blanks and rinsate blanks are designated C-FB-XXX-W and C-RB-XXX-W, respectively. The samples were collected and placed in laboratory-certified and preserved plastic or glass sample jars, stored on ice, and shipped to IT Analytical Services, using standard chain-of-custody procedures. A list of the analytical parameters requested for each sample is provided in Table 3-2.

Decontamination of the sounding tool was accomplished through the use of an injection sleeve fitted beneath the CPT truck. The sleeve was connected to a pressurized steam injector during the time that the rods were being pulled from the ground. Rinse water was contained in lined drums. The rods were surveyed for gross radiological contamination as they were pulled from the ground using a Ludlum pancake probe. Soil and water sampling rods and tools were taken to the decontamination pad for cleaning following each sampling event. Equipment rinsate blanks were collected following decontamination at three locations.

Annotated sounding logs with interpreted lithologies for each CPT location are included in Appendix A.

\subsection{Source Area 5}

Source Area 5 is located in the southwestern corner of the SS. The purpose of the CPT in this area was to determine if the alluvial or Citronelle aquifers were contaminated as a result of activities associated with the drilling, operation, and/or decommissioning of Injection Well 


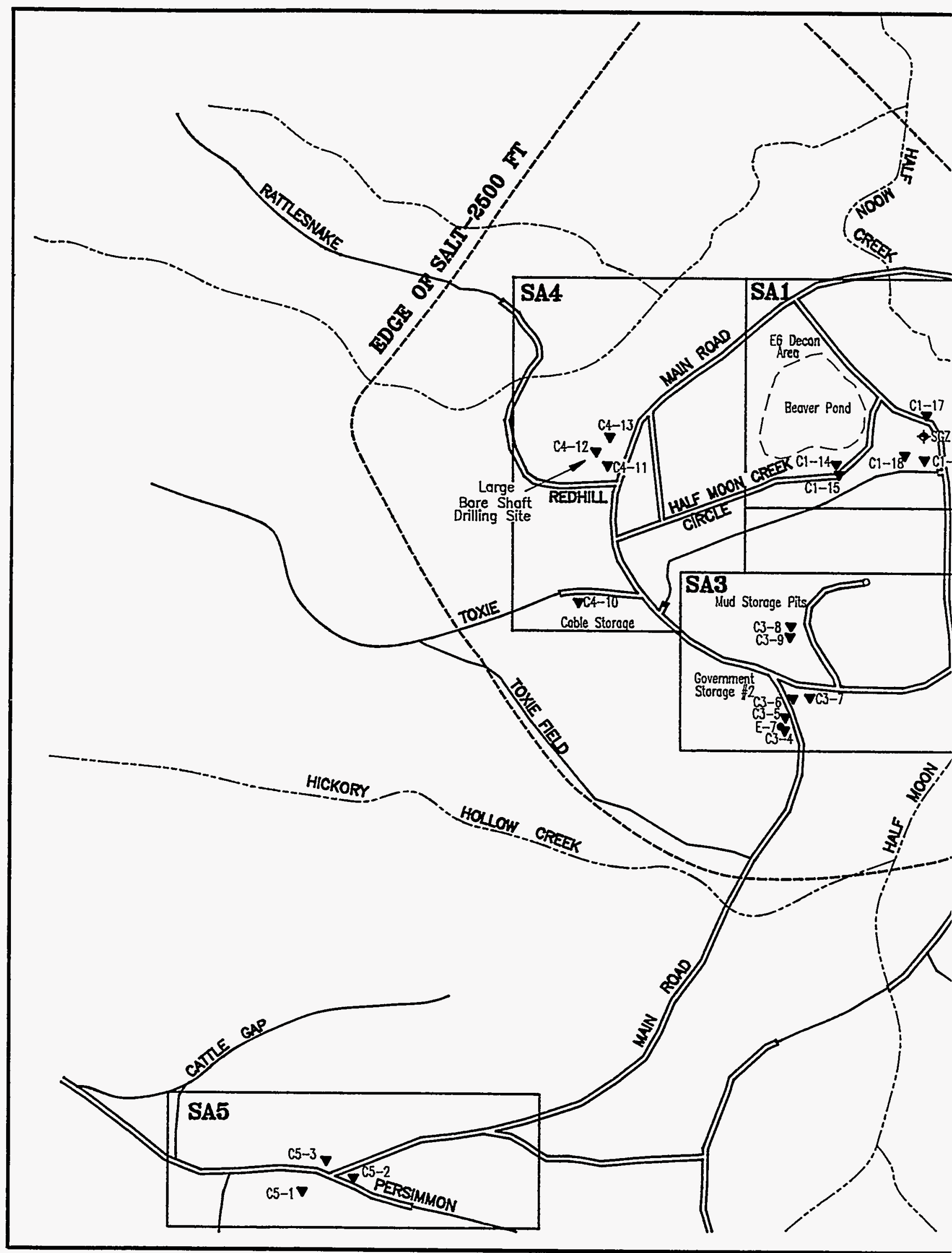


Table 3-1

Cone Penetrometer Testing Sounding and Soil/Groundwater Sampling Summary, Salmon Site

(Page 1 of 2)

\begin{tabular}{|c|c|c|c|c|c|c|c|c|c|c|c|c|c|c|c|c|c|c|c|}
\hline \multirow{3}{*}{$\begin{array}{c}\text { Cone } \\
\text { Pene- } \\
\text { trometer } \\
\text { Testing }\end{array}$} & \multirow{3}{*}{\begin{tabular}{|c|} 
Estimated \\
Surface \\
Elevation \\
ams|" \\
(feet)
\end{tabular}} & \multicolumn{3}{|c|}{ Lithology } & \multicolumn{7}{|c|}{ Soil Sampling } & \multicolumn{7}{|c|}{ Water Sampling } & \multirow[b]{3}{*}{ Notes } \\
\hline & & \multicolumn{2}{|c|}{ Depth } & & \multicolumn{2}{|c|}{ Depth } & \multirow[b]{2}{*}{ Date } & \multirow[b]{2}{*}{ Unit } & \multirow[b]{2}{*}{ MS } & \multirow[b]{2}{*}{ MSD } & \multirow[b]{2}{*}{\begin{tabular}{|l|} 
DUP \\
\end{tabular}} & \multicolumn{2}{|c|}{ Depth } & \multirow[b]{2}{*}{ Date } & \multirow[b]{2}{*}{ Unit } & \multirow[b]{2}{*}{\begin{tabular}{|c|}
$M$ \\
$S$
\end{tabular}} & \multirow[b]{2}{*}{ MSD } & \multirow[b]{2}{*}{ DUP } & \\
\hline & & Feet & Meters & Date & Feet & Meters & & & & & & Feet & Meters & & & & & & \\
\hline$\overline{C 5-1}$ & 290 & 72.0 & 21.9 & 23-Oct & $25.0-27.0$ & 7.6-8.2 & 5-Nov & $\bar{P}$ & & & & 33.5 & 10.2 & 5-Nov & $M$ & $x$ & $\bar{x}$ & $\bar{x}$ & \\
\hline C5-2 & 310 & 45.0 & 13.7 & $22-\mathrm{Oct}$ & $15.5-17.0$ & $4.7-5.2$ & 5-Nov & C & $x$ & $x$ & $x$ & & & & & & & & \\
\hline C5-3 & 315 & 48.0 & 14.6 & $23-\mathrm{Oct}$ & $21.5-23.0$ & $6.5-7.0$ & 5-Nov & C & & & & & & & & & & & \\
\hline C3-6 & 265 & 92.0 & 28.0 & $21-\mathrm{Oct}$ & $40.0-42.0$ & $12.2-12.8$ & 4-Nov & $P$ & & & & & & & & & & & \\
\hline$\overline{C 3-7}$ & 265 & 79.0 & 24.1 & $21-\mathrm{Oct}$ & $24.0-26.0$ & \begin{tabular}{|c|}
$7.3-7.9$ \\
\end{tabular} & 4-Nov & $P$ & & & & & & & & & & & \\
\hline C3-8 & 285 & 78.0 & 23.8 & 23-Oct & $34.0-36.0$ & $10.4-11.0$ & 6-Nov & $P$ & & & & 30.0 & 9.1 & 11-Nov & $P$ & & & & \\
\hline C3-9 & 285 & 82.0 & 25.0 & 23-Oct & $23.0-24.5$ & $7.0-7.5$ & 6-Nov & $P$ & & & & 31.0 & 9.4 & 9-Nov & $P$ & & & & \\
\hline$\overline{C 4-10}$ & 270 & 65.0 & 19.8 & 26-Oct & $30.0-31.5$ & $9.1-9.4$ & 9-Nov & $\mathrm{P}$ & & & & & & & & & & & \\
\hline$\overline{C 1-15}$ & 240 & 73.0 & 22.3 & 24-Oct & $19.0-20.0$ & 5.8-6.1 & 2-Nov & $\bar{A}$ & & & & 22.5 & 6.9 & 3-Nov & A & $\mathrm{x}$ & $\bar{x}$ & $x$ & \\
\hline$\overline{C 1-16}$ & 235 & 78.0 & 23.8 & $24-\mathrm{Oct}$ & $7.5-9.5$ & $2.3-2.9$ & 2-Nov & $\bar{A}$ & & & & 9.0 & 2.7 & 3-Nov & A & & & & \\
\hline C1-17 & 235 & 72.0 & 21.9 & 23-Oct & $12.0-15.5$ & $3.7-4.7$ & 1-Nov & $\bar{A}$ & $x$ & $\bar{x}$ & $x$ & 12.5 & 3.8 & 3-Nov & $A$ & & & & \\
\hline$\overline{C 1-18}$ & 235 & 77.0 & 23.5 & 24-Oct & $6.0-7.5$ & $1.8-2.9$ & 2-Nov & $\bar{A}$ & & & & 9.0 & 2.7 & 2-Nov & $A$ & & & & 3 \\
\hline C2-19 & 255 & 79.7 & 24.3 & 24-Oct & $28.5-30.0$ & $8.7-9.1$ & 6-Nov & $P$ & & & $\bar{x}$ & 38.0 & 11.6 & 8-Nov & $A$ & $x$ & $x$ & $x$ & \\
\hline $\mathrm{C} 2-20$ & 275 & 33.8 & 10.3 & $25-\mathrm{Oct}$ & $7.5-9.0$ & $2.3-2.7$ & 6-Nov & $P$ & & & & & & & & & & & \\
\hline C2-21 & 290 & 69.0 & 21.0 & $25-\mathrm{Oct}$ & $7.0-8.5$ & $2.1-2.6$ & 6-Nov & C & $\bar{x}$ & $\bar{x}$ & $\bar{x}$ & \begin{tabular}{l|}
15.0 \\
\end{tabular} & 4.6 & 10-Nov & $c$ & & & & 4 \\
\hline C2-22 & 295 & 92.0 & 28.0 & $25-\mathrm{Oct}$ & $7.5-9.0$ & $2.3-2.7$ & 6-Nov & C & & & & 15.0 & 4.6 & 11 -Nov & $c$ & & & & 4 \\
\hline C2-23 & 310 & 60.0 & 18.3 & $25-$ Oct & $15.0-17.0$ & $4.6-5.2$ & 6-Nov & $\mathrm{c}$ & & & & & & & & & & & \\
\hline
\end{tabular}


Table 3-1

Cone Penetrometer Testing Sounding and Soil/Groundwater Sampling Summary,

Salmon Site

(Page 2 of 2)

\begin{tabular}{|c|c|c|c|c|c|c|c|c|c|c|c|c|c|c|c|c|c|c|c|}
\hline \multirow{3}{*}{$\begin{array}{c}\text { Cone } \\
\text { Pene- } \\
\text { trometer } \\
\text { Testing }\end{array}$} & \multirow{3}{*}{$\begin{array}{l}\text { Estimated } \\
\text { Surface } \\
\text { Elevation } \\
\text { amsi" } \\
\text { (feet) }\end{array}$} & \multicolumn{3}{|c|}{ Lithology } & \multicolumn{7}{|c|}{ Soil Sampling } & \multicolumn{7}{|c|}{ Water Sampling } & \multirow[b]{3}{*}{ Notes } \\
\hline & & \multicolumn{2}{|c|}{ Depth } & & \multicolumn{2}{|c|}{ Depth } & \multirow[b]{2}{*}{ Date } & \multirow[b]{2}{*}{ Unit } & \multirow[b]{2}{*}{ MS } & \multirow[b]{2}{*}{ MSD } & \multirow[b]{2}{*}{ DUP } & \multicolumn{2}{|c|}{ Depth } & \multirow[b]{2}{*}{ Date } & \multirow[b]{2}{*}{ Unit } & \multirow[b]{2}{*}{\begin{tabular}{|c|} 
\\
$S$
\end{tabular}} & \multirow[b]{2}{*}{ MSD } & \multirow[b]{2}{*}{ DUP } & \\
\hline & & Feet & Meters & Date & Feet & Meters & & & & & & Feet & Meters & & & & & & \\
\hline $\mathrm{C} 2-24$ & 310 & 71.0 & 21.6 & 26-Oct & $53.0-54.5$ & $16.2-16.6$ & 8-Nov & $P$ & & & & 56.0 & 17.1 & 8-Nov & $P$ & & & & 2 \\
\hline C2-25 & 310 & 72.0 & 21.9 & $26 \cdot \mathrm{Oct}$ & $2.0-4.0$ & $.6 \cdot 1.2$ & 7-Nov & $\mathrm{C}$ & & & & & & & & & & & \\
\hline C2-25A & 310 & 72.0 & 21.9 & $26-\mathrm{Oct}$ & $20.0-22.0$ & $6.1-6.7$ & 7-Nov & $P$ & & & & & & & & & & & 1 \\
\hline $\mathrm{C} 2-26$ & 320 & 18.0 & 5.5 & 26-Oct & $15.0-16.5$ & $4.6-5.0$ & $7-\mathrm{Nov}$ & C & & & & & & & & & & & \\
\hline C2.27 & 320 & 65.0 & 19.8 & 26-Oct & $37.0-38.5$ & $11.3-11.7$ & 10-Nov & $\mathrm{C}$ & & & & 34.0 & 10.4 & $10-\mathrm{Nov}$ & $\mathrm{C}$ & & & & \\
\hline
\end{tabular}

aabove mean sea level

MS = Matrix Spike

MSD = Matrix Spike Duplicate

DUP $=$ Duplicate

Remarks: 1 - Two samples collected for soil

2 - Only VOAs collected and approximately 300 milliliters for tritium analysis

3 - Replacement BNA collected 11/10/93

4 - VOAs only

Key to UNITS:

A - Half Moon Creek Alluvium

C - Citronelle Formation

$P$ - Pascagoula/Hattiesburg Formation 
Table 3-2

Requested Analytical Parameters for Cone Penetrometer Testing Samples,

Salmon Site

\begin{tabular}{|c|c|c|c|c|c|c|c|c|c|c|c|c|c|c|c|}
\hline \multicolumn{7}{|c|}{ SOILS } & \multicolumn{9}{|c|}{ WATER } \\
\hline Sample & voc" & $\mathrm{BNA}^{\circ}$ & \begin{tabular}{|l|} 
Pest \\
PCB $^{\circ}$
\end{tabular} & \begin{tabular}{|c|} 
Metals| \\
$\mathrm{CN}^{\circ}$
\end{tabular} & $\begin{array}{l}\text { Alphal } \\
\text { Beta }\end{array}$ & Gamma & Sample & voc" & $\mathrm{BNA}^{\circ}$ & $\begin{array}{l}\text { Pest/ } \\
\text { PCB }^{\circ}\end{array}$ & Metals & \begin{tabular}{|c|} 
Alphal \\
Beta
\end{tabular} & Gamma & Tritium & $\mathrm{CN}^{\circ}$ \\
\hline$C 5-1 \cdot S$ & $\bar{x}$ & $\bar{x}$ & $\bar{x}$ & $\bar{x}$ & $\bar{x}$ & $\bar{x}$ & C5-1-W & $\bar{x}$ & $\bar{x}$ & $\bar{x}$ & $\bar{x}$ & $\bar{x}$ & $\bar{x}$ & $\bar{x}$ & $\bar{x}$ \\
\hline c5.2.S & $x$ & $x$ & $x$ & $x$ & $x$ & $x$ & C5-1-W-MS & $x$ & $x$ & $x$ & $x$ & & & & $x$ \\
\hline C5-2-S-MS & $x$ & $x$ & $x$ & $x$ & & & C5-1-W-MSD & $x$ & $x$ & $x$ & & & & & \\
\hline C5-2-S-MSD & $x$ & $x$ & $x$ & & & & C5-1-W-DUP & & & & $x$ & $\mathrm{x}$ & $x$ & $x$ & $x$ \\
\hline C5.2-S-DUP & & & & $x$ & $x$ & $x$ & C3-8-W & $x$ & $x$ & $x$ & $x$ & $x$ & $x$ & $x$ & $x$ \\
\hline C5-3.S & $x$ & $x$ & $x$ & $x$ & $x$ & $x$ & C3-9.W & $x$ & $x$ & $x$ & $x$ & $x$ & $x$ & $x$ & $x$ \\
\hline C3-4-S & $x$ & $x$ & $x$ & $x$ & $x$ & $x$ & C4-11-W & $x$ & $x$ & $x$ & $x$ & $x$ & $x$ & $x$ & $x$ \\
\hline C3.5.S & $x$ & $x$ & $x$ & $x$ & $x$ & $x$ & C4-13:W & $x$ & $x$ & $x$ & $x$ & $x$ & $x$ & $x$ & $\mathrm{x}$ \\
\hline C3.6-S & $x$ & $x$ & $x$ & $x$ & $x$ & $x$ & C1-14-W & $x$ & $x$ & $x$ & $x$ & $x$ & $x$ & $x$ & $x$ \\
\hline C3.7.S & $x$ & $x$ & $x$ & $x$ & $x$ & $x$ & C1-15-W & $x$ & $x$ & $x$ & $x$ & $x$ & $x$ & $\mathrm{x}$ & $\mathrm{x}$ \\
\hline C3.8.S & $x$ & $x$ & $\mathrm{x}$ & $x$ & $x$ & $x$ & C1-15-W-MS & $x$ & $x$ & $x$ & $x$ & & & & $x$ \\
\hline C3-9.S & $x$ & $x$ & $x$ & $x$ & $x$ & $x$ & C1-15-W-MSD & $x$ & $x$ & $x$ & & & & & \\
\hline C4.10.S & $x$ & $x$ & $x$ & $x$ & $x$ & $x$ & C1-15-W-DUP & & & & $x$ & $x$ & $x$ & $x$ & $x$ \\
\hline C4-11-S & $x$ & $x$ & $x$ & $x$ & $x$ & $x$ & C1-16-W & $x$ & $x$ & $x$ & $x$ & $x$ & $x$ & $x$ & $x$ \\
\hline C4-12-S & $x$ & $x$ & $x$ & $x$ & $x$ & $x$ & C1-17-W & $x$ & $x$ & $x$ & $x$ & $x$ & $x$ & $x$ & $x$ \\
\hline C4-13-S & $x$ & $x$ & $x$ & $x$ & $x$ & $x$ & C1-18-W & $x$ & $x$ & $x$ & $x$ & $x$ & $x$ & $x$ & $x$ \\
\hline$C 1-14-S$ & $x$ & $x$ & $x$ & $x$ & $x$ & $x$ & C2-19-W & $x$ & $x$ & $x$ & $x$ & $x$ & $x$ & $x$ & $x$ \\
\hline C1-15.S & $x$ & $x$ & $\mathrm{x}$ & $x$ & $x$ & $x$ & C2-19-W-MS & $\mathrm{x}$ & $x$ & $x$ & $x$ & & & & $x$ \\
\hline C1-16.S & $x$ & $x$ & $x$ & $x$ & $x$ & $x$ & C2-19-W-MSD & $x$ & $x$ & $x$ & & & & & \\
\hline$c 1-17-s$ & $x$ & $x$ & $x$ & $x$ & $x$ & $x$ & C2-19-W-DUP & & & & $x$ & $x$ & $x$ & $x$ & $x$ \\
\hline C1-17-S-MS & $x$ & $x$ & $x$ & $x$ & & & C2-21-W & $x$ & & & & & & & \\
\hline C1-17-S-MSD & $x$ & $x$ & $x$ & & & & C2-22-W & $x$ & & & & & & & \\
\hline C1-17-S.DUP & & & & $x$ & $x$ & $x$ & C2-24-W & $x$ & & & & & & $x$ & \\
\hline C1-18-S & $x$ & $x$ & $x$ & $x$ & $x$ & $x$ & C2-27-W & $x$ & $x$ & $x$ & $x$ & $x$ & $x$ & $x$ & $x$ \\
\hline |C2-19-S & $x$ & $x$ & $x$ & $x$ & $x$ & $\mathrm{x}$ & C-FB-001-W & $\mathrm{x}$ & $x$ & $x$ & $x$ & $x$ & $x$ & $x$ & $x$ \\
\hline |C2-20-S & $x$ & $x$ & $x$ & $x$ & $\mathrm{x}$ & $x$ & C-FB-002-W & $x$ & $x$ & $x$ & $x$ & $x$ & $x$ & $x$ & $x$ \\
\hline C2.21-S & $\mathrm{x}$ & $x$ & $x$ & $x$ & $x$ & $x$ & C-FB-003-W & $x$ & $x$ & $x$ & $x$ & $x$ & $x$ & $x$ & $x$ \\
\hline C2-21-S-MS & $\mathrm{x}$ & $x$ & $x$ & $x$ & & & C-RB-001-W & $\mathrm{x}$ & $x$ & $x$ & $x$ & $x$ & $x$ & $x$ & $x$ \\
\hline C2-21-S-MSD & $x$ & $x$ & $x$ & & & & C.RB-002-W & $x$ & $x$ & $x$ & $x$ & $x$ & $x$ & $x$ & $x$ \\
\hline C2-21-S.DUP & & & & $x$ & $x$ & $x$ & C-RB-003-W & $x$ & $x$ & $x$ & $x$ & $x$ & $\mathrm{x}$ & $x$ & $\mathrm{x}$ \\
\hline |C2.22.S & $\mathrm{x}$ & $x$ & $x$ & $x$ & $x$ & $x$ & TRIP BLANK & $x$ & & & & & & & \\
\hline |C2-23-S & $x$ & $x$ & $x$ & $x$ & $x$ & $\mathrm{x}$ & TRIP BLANK & $x$ & & & & & & & \\
\hline |C2-24.S & $x$ & $x$ & $x$ & $x$ & $x$ & $x$ & TRIP BLANK & $x$ & & & & & & & \\
\hline |c2.25.S & $x$ & $x$ & $x$ & $x$ & $x$ & $x$ & | TRIP BLANK & $x$ & & & & & & & \\
\hline C2-25a-S & $x$ & $x$ & $x$ & $\mathrm{x}$ & $x$ & $x$ & TRIP BLANK & $\mathrm{x}$ & & & & & & & \\
\hline |C2-26.S & $x$ & $x$ & $x$ & $x$ & $x$ & $x$ & & & & & & & & & \\
\hline C2-27-S & $x$ & $x$ & $x$ & $x$ & $x$ & $x$ & & & & & & & & & \\
\hline
\end{tabular}

Volatile organic compound

"Base/neutralacid extractable (semivolatile organic compounds)

'Pesticide/polychlorinated biphenyls

${ }^{\circ}$ Cyanide 
HT-2 and Monitoring Well HT-2M. Three soundings were conducted in the vicinity of these wells. Three soil samples and one water sample were collected from this area.

\subsubsection{Location C5-1}

The stratigraphic sounding was conducted on October 23,1993, and was pushed to a depth of

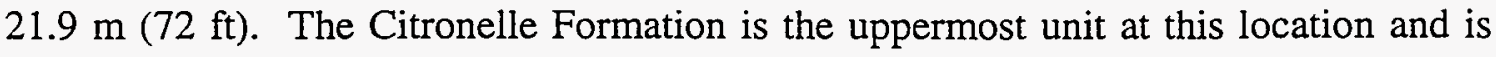
approximately $5.2 \mathrm{~m}(17 \mathrm{ft})$ thick. The top of the saturated sediment is interpreted to be in the Miocene sediments $11 \mathrm{~m}(35 \mathrm{ft})$ bgs.

Soil and water samples were collected on November 5, 1993. One soil sample, a red fine grained sand, was collected from a depth between 7.6 and $8.1 \mathrm{~m} \mathrm{(25}$ and $26.5 \mathrm{ft})$ within the Pascagoula/Hattiesburg Formation section. Water samples were collected from the base of a sandy interval at a depth of $10.2 \mathrm{~m}$ (33.5 ft). Matrix Spike (MS), Matrix Spike Duplicate (MSD), and Duplicate (DUP) water samples were also collected from this interval.

\subsubsection{Location $\mathrm{C5}-2$}

The stratigraphic sounding was conducted on October 22, 1993, and extended to a depth of

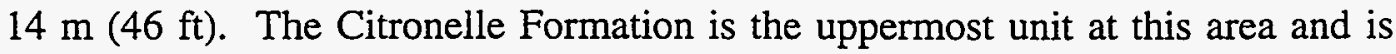
approximately $6.2 \mathrm{~m}(20.5 \mathrm{ft})$ thick. The top of the saturated sediment is interpreted to be at a depth of $6.4 \mathrm{~m}(21 \mathrm{ft})$ bgs, near the contact between the Citronelle and Pascagoula/ Hattiesburg Formations.

A soil sample was collected on November 5, 1993, from a depth between 4.7 and $5.2 \mathrm{~m}$ (15.5 and $17 \mathrm{ft}$ ). The sample consisted of an orange-to-tan, silty, fine-grained sand and a white-to- orange black, fine-grained sand with some small gravel. The soil samples were dry, and no attempt was made to collect a water sample from this location. Matrix Spike, MSD, and DUP soil samples were collected from this location.

\subsubsection{Location C5-3}

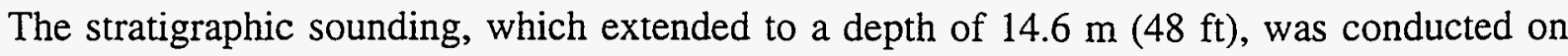
October 23, 1993. The Citronelle Formation, which is the uppermost unit in this area, is

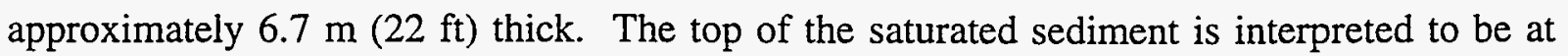
the contact between the Citronelle Formation and the Miocene strata.

On November 5, 1993, a soil sample was collected from 6.6 to $7 \mathrm{~m} \mathrm{(21.5} \mathrm{to} 23 \mathrm{ft}) \mathrm{bgs}$. The sample consisted of a medium- to coarse-grained sand. An attempt to collect a water sample 
was made on November 7, 1993, by setting the CPT water sampling tool between ( 21.5 and $22 \mathrm{ft}$ ) bgs. A water sample was not obtained, and the water sampling tool broke off in the hole after the sampling attempt. The sampler was later retrieved. Another attempt to collect a water sample was made on November 10, 1993, using the PVC screen and casing, but again, no water was present for sampling. A field blank was collected at this location, and an equipment rinsate blank was collected prior to the water sampling (C-FB-002-W and C-RB-002-W).

\subsection{Source Area 3}

Source Area 3 is located in the south central portion of the SS. Several test wells and monitoring wells were drilled in this area which was used for the storage of drilling equipment and drilling fluids (mud). The intent of the CPT in this area was to determine if the drilling, operations, and/or decommissioning of this area resulted in contamination of the subsurface soil and/or the upper water-bearing zones. Six CPT soundings were conducted in this area; six soil and two water samples were collected from this area.

\subsubsection{Location C3-4}

Location C3-4 is in the immediate vicinity of Wells E-2 and E-7, which were drilled to aid in the characterization of the groundwater flow. Immediately to the northwest is an abandoned storage yard (Government Storage \#2) used during operations at the site.

A stratigraphic sounding was completed to a depth of $30.5 \mathrm{~m}(100 \mathrm{ft})$ on October 22, 1993. The Pascagoula/Hattiesburg Formation is the uppermost unit underlying this location. The top of the saturated sediment is interpreted to be approximately $2.7 \mathrm{~m}(9 \mathrm{ft})$ bgs although no free water was encountered.

An attempt to collect a soil sample was made initially on November 9, 1993, in what was interpreted to be a sand at a depth of $17 \mathrm{~m}$ (56 ft). No soil was retrieved on this initial push. The water sampler was then inserted with the screen set between 16.5 and $16.8 \mathrm{~m}$ (54 and $55 \mathrm{ft}$ ) bgs. No water was recovered. The initial sampling hole was abandoned. The CPT truck was moved northeast about $1.5 \mathrm{~m}$ ( $5 \mathrm{ft}$ ), and a soil sample was collected from the interval between 9.4 and $9.9 \mathrm{~m} \mathrm{(31}$ and $32.5 \mathrm{ft}$ ) bgs. The sample was a stiff, green, silty clay. No additional attempts were made to collect a water sample. 


\subsubsection{Location C3-5}

Location C3-5 is immediately north of C3-4. A stratigraphic sounding was completed on October 22, 1993, to a depth of $24.1 \mathrm{~m}$ (79 ft). The sounding was stopped by sidewall friction. The Pascagoula/Hattiesburg Formation is the uppermost unit underlying this location. The top of the saturated sediment is interpreted to be within $3 \mathrm{~m} \mathrm{(10} \mathrm{ft)} \mathrm{of} \mathrm{ground}$ surface.

A soil sample was collected on November 11, 1993, from the interval of 7 to $7.5 \mathrm{~m}$ (23 to $24.5 \mathrm{ft}$ ) bgs. The sample was a stiff, tan clay becoming more silty towards the base. No attempt was made to collect a water sample based on the experience at nearby $\mathrm{C} 3-4$.

\subsubsection{Location C3-6}

Location C3-6 is in a lay-down yard west of Government Storage Yard \#2. Several stratigraphic and hydrologic test wells were drilled in the area. A stratigraphic sounding was completed to a depth of $28 \mathrm{~m}$ ( $92 \mathrm{ft}$ ) on October 21, 1993. The sounding was stopped due to sidewall friction. The Pascagoula/Hattiesburg Formation is the uppermost unit underlying this location. The top of the saturated sediment is interpreted to be about $2 \mathrm{~m}(6.5 \mathrm{ft}) \mathrm{bgs}$.

A soil sample was collected on November 4, 1993. It consisted of a stiff, green, silty clay and was collected between 12.2 and $12.8 \mathrm{~m}$ ( 40 and $42 \mathrm{ft}$ ) bgs. No attempt to collect a water sample was made because no potential water-bearing strata were encountered.

\subsubsection{Location C3-7}

Location C3-7 is also in the lay-down yard west of Government Storage Yard \#2. A stratigraphic sounding was completed to a depth of $24.1 \mathrm{~m}(79 \mathrm{ft})$ on October 21, 1993. The sounding was. stopped due to sidewall friction. The Pascagoula/Hattiesburg Formation is the uppermost unit underlying this location. The top of the saturated sediment is interpreted to be above $1.8 \mathrm{~m}(6 \mathrm{ft})$ bgs.

A soil sample was collected on November 4, 1993, between 7.3 and $7.9 \mathrm{~m} \mathrm{(24}$ and $26 \mathrm{ft}$ ) bgs. The soil sample consisted of a stiff, green, silty clay. No attempt to collect a water sample was made because no potential water bearing strata were encountered.

\subsubsection{Location C3-8}

Location C3-8 is at the Reserve Mud Pits Area. A stratigraphic sounding was completed on October 23,1993 , to a depth of $23.8 \mathrm{~m}$ ( $78 \mathrm{ft}$ ). The sounding was stopped by side-wall 
friction. A thin $1.5-\mathrm{m}(5-\mathrm{ft})$ interval of the Citronelle Formation may be present at this location. The top of the saturated sediment is interpreted to be 1.5 to $2.1 \mathrm{~m}$ ( 5 to $7 \mathrm{ft}$ ) bgs near the Citronelle Formation and Pascagoula/Hattiesburg Formation contact.

A soil sample was collected from the interval of 10.4 to $11 \mathrm{~m} \mathrm{(34} \mathrm{to} 36 \mathrm{ft}$ ) in depth, within the Miocene. strata, on November 6, 1993. This sample was described as a stiff clay. A water sample was collected November 11, 1993, from a sand interval in the Pascagoula/ Hattiesburg Formation. The water sample was retrieved through the PVC screen and casing

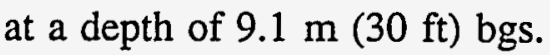

\subsubsection{Location C3-9}

Location C3-9 is also at the Reserve Mud Pits Area. A stratigraphic sounding was completed

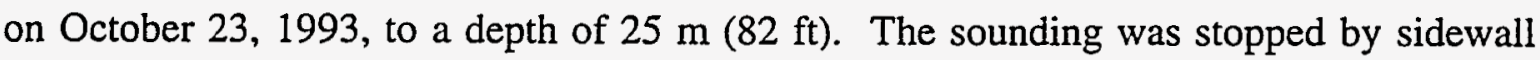
friction. Location C3-9 is on the crest of a low hill and may have actually been pushed through the old mud pit as evidenced by $2 \mathrm{~m}(7 \mathrm{ft})$ of clay at the top of the sounding. The top of the saturated sediment is interpreted to be about $1.5 \mathrm{~m}(5 \mathrm{ft})$ bgs.

On November 5, 1993, a soil sample was collected from the interval of 9 to $9.5 \mathrm{~m}$ ( 29.5 to $31 \mathrm{ft}$ ) bgs; however, not enough soil was recovered for a complete sample suite. A second soil sample was collected from within the Pascagoula/Hattiesburg Formation on November 6, 1993, between 7 and $7.5 \mathrm{~m}$ ( 23 and $23.5 \mathrm{ft}$ ) bgs.

On November 7, 1993, an attempt to collect a water sample was made using the CPT water sampling device. The sampling device broke off in the hole on the first attempt. The sampler was retrieved later that day. The CPT rig was then moved $1.5 \mathrm{~m}(5 \mathrm{ft})$ southwest, and a larger diameter water sampling device was pushed to a depth of $7.9 \mathrm{~m}(26 \mathrm{ft})$. No water was recovered, so the CPT sampler was removed, and the PVC screen and casing were inserted into the hole. The screen section was set between 7.9 and $9.4 \mathrm{~m} \mathrm{(26} \mathrm{and} 31 \mathrm{ft}$ ) bgs. A water sample was eventually collected from the Pascagoula/Hattiesburg Formation on November 9, 1993.

\subsection{Source Area 4}

Source Area 4 is located on the western side of the central portion of the SS. Four CPT soundings, four soil samples, and two water samples were collected from this area. This area was used for construction, storage, and disposal during site operations. Several wells were drilled in this area, including the attempted installation of two large-diameter shafts. Three of 
the CPT tests were conducted to evaluate possible contamination resulting from the construction of the shafts. The other CPT test was conducted in an area where cable was stored and apparently burned.

\subsubsection{Location C4-10}

Location C4-10 is in the Cable Storage Area behind the current decontamination and investigation-derived-waste storage area. The Pascagoula/Hattiesburg Formation is the uppermost unit underlying this location. A stratigraphic sounding was completed to a depth of $19.8 \mathrm{~m}(65 \mathrm{ft})$ on October 26,1993 . The saturated sediments are interpreted to extend to ground surface.

A soil sample was collected on November 9, 1993, from the interval between 9.1 and $9.6 \mathrm{~m}$ (30 and $31.5 \mathrm{ft}$ ) bgs. The soil was a stiff, green, silty clay. No attempt was made to collect a water sample because potential water-bearing sand units were not encountered.

\subsubsection{Location C4-11}

Location C4-11 is near the entrance to the large-bore shaft-drilling site at the intersection of Red Hill Road and the Main Road. A stratigraphic sounding was completed to a depth of $25.5 \mathrm{~m}$ ( $83.8 \mathrm{ft})$ on October 19, 1993. The Citronelle Formation, which is approximately $3 \mathrm{~m}$ $(10 \mathrm{ft})$ thick in this area, is the uppermost unit. The top of the saturated sediment is interpreted to occur at $3.7 \mathrm{~m}$ (12 ft) below contact of the Citronelle Formation and $3 \mathrm{~m}$ $(10 \mathrm{ft})$ of the Pascagoula/Hattiesburg Formation.

A soil sample was collected from the Pascagoula/Hattiesburg Formation at the interval between 8.5 and $9 \mathrm{~m}$ ( 28 and $29.5 \mathrm{ft}$ ) bgs on November 3, 1993. The soil was described as a green-gray, clayey silt becoming sandy towards the base. A water sample was collected on November 10, 1993, using PVC screen and casing. The bottom of the PVC screen was set to a depth of $4 \mathrm{~m}(13 \mathrm{ft})$. A field blank was also collected at this location.

\subsubsection{Location C4-12}

Location $\mathrm{C} 4-12$ is within the large-bore shaft-drilling site. A stratigraphic sounding was completed to a depth of $27.2 \mathrm{~m}(89.2 \mathrm{ft})$ on October 21, 1993. The Citronelle Formation is the uppermost unit in this area and is about $4.9 \mathrm{~m}(16 \mathrm{ft})$ thick. The top of the saturated sediment is interpreted to be just below the Citronelle Formation and the Pascagoula/ Hattiesburg Formation contact. 
On November 4, 1993, a soil sample was collected from the interval between 4.6 and $5.0 \mathrm{~m}$ ( 15 and $16.5 \mathrm{ft}$ ) bgs near the base of the Citronelle Formation. The soil sample was described as a mixed sand and gravel.

Three attempts were made to collect a water sample at C4-12 on November 4, 1994. The first attempt was made with the screen set between 4.6 and $4.7 \mathrm{~m}$ (15 and $15.5 \mathrm{ft}$ ) bgs; the second attempt, with the screen set between 9.4 and $9.6 \mathrm{~m}$ ( 31 and $31.5 \mathrm{ft}$ ) bgs; and the third attempt, with the screen set between 13.6 and $13.7 \mathrm{~m}$ ( 44.5 and $45 \mathrm{ft}$ ) bgs. All attempts were made with the CPT water sampling tool, and the CPT rig was moved for each attempt. No water sample was collected from Location C4-12.

\subsubsection{Location C4-13}

Location C4-13 is also at the large-bore shaft-drilling site. A stratigraphic sounding was completed to a depth of $26.8 \mathrm{~m}(88 \mathrm{ft}$ ) on October 19, 1993.

The Citronelle Formation is the uppermost unit and is 5.5 to $6.1 \mathrm{~m} \mathrm{(18} \mathrm{to} 20 \mathrm{ft})$ thick at this site. The top of the saturated sediments occurs below the interface between the Citronelle Formation and the Pascagoula/Hattiesburg Formation at a depth of about $7.5 \mathrm{~m}(25 \mathrm{ft})$. A soil sample was collected from the base of the Citronelle Formation in the interval between 5.5 and $5.9 \mathrm{~m}$ (18 and $19.5 \mathrm{ft}$ ) bgs on November 3, 1993. The soil sample consisted of mixed sand and gravel.

Water samples were collected on November 4, 1993. The CPT water sampling tool was inserted so the base of the screened interval was at a depth of $5.6 \mathrm{~m}(18.5 \mathrm{ft})$. Because of a bent CPT rod, only a small bailer could get to the water. After filling two sample bottles, the sampling tool was reinserted with the base of the screen set at a depth of $7 \mathrm{~m}(23 \mathrm{ft})$ so that a larger bailer could be used.

\subsection{Source Area 1}

Source Area 1 is located around Surface Ground Zero. Five CPT soundings in two areas were conducted to evaluate potential contamination of the Half Moon Creek Alluvium and the Alluvial Aquifer that may have resulted from construction, operation, and/or decommissioning of the site. Five soil and five water samples were collected from this area. 


\subsubsection{Location C1-14}

Location C1-14 is on Half Moon Creek Road south of the beaver pond. A stratigraphic sounding was completed to a depth of $20.4 \mathrm{~m}(67 \mathrm{ft})$ on October 24, 1993. The Half Moon Creek Alluvium is the uppermost unit underlying this site. The alluvium is believed to be

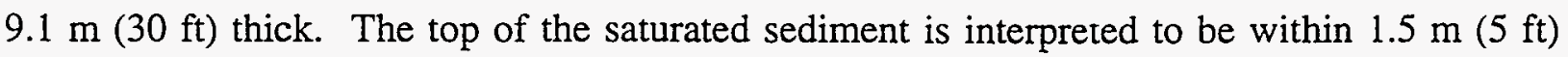
of ground surface.

A soil sample was collected on November 2, 1993, from the interval between 7 and $7.5 \mathrm{~m}$ (23 and $24.5 \mathrm{ft}$ ) bgs, near the base of the alluvium. The sample was described as a fine, silty sand. A water sample was also collected on November 2, 1993, from a depth of $7.8 \mathrm{~m}$ $(25.5 \mathrm{ft})$ using the CPT water sampling tool.

\subsubsection{Location $\mathrm{C1-15}$}

Location C1-15 is on Half Moon Creek Road near the beaver pond. A stratigraphic sounding was completed to a depth of $22.2 \mathrm{~m}$ (73 ft) on October 24, 1993. The sounding was stopped by a hard layer. The Half Moon Creek Alluvium, which is the uppermost unit underlying this location, is believed to be $9.4 \mathrm{~m}(31 \mathrm{ft})$ thick. The top of the saturated sediment is interpreted to be within $1.5 \mathrm{~m}(5 \mathrm{ft})$ of ground surface.

A soil sample was collected on November 2, 1993, from the interval of 5.8 to $6.1 \mathrm{~m}$ (19 to $20 \mathrm{ft}$ ) bgs. The sample was described as a loose, fine-grained sand. A water sample was collected on November 3,1993, using the CPT water sampling tool set at a depth of $6.9 \mathrm{~m}(22.5 \mathrm{ft})$. Matrix spike, MSD, and DUP water samples and field and equipment rinsate blanks were also collected at this location (C-FB-001-W and C-RB-001-W).

\subsubsection{Location C1-16}

Location C1-16 is south of the Surface Ground Zero monument. A stratigraphic sounding was completed to a depth of $23.5 \mathrm{~m}$ (77 ft) on October 24, 1993. The Half Moon Creek Alluvium, the uppermost unit underlying this location, is about $6.7 \mathrm{~m} \mathrm{(22} \mathrm{ft)} \mathrm{thick.} \mathrm{The} \mathrm{top}$ of the saturated sediment is interpreted to be within $1.5 \mathrm{~m}(5 \mathrm{ft})$ of ground surface.

Soil samples were collected on November 2, 1993, from the interval of 2.3 to $2.9 \mathrm{~m}$ ( 7.5 to $9.5 \mathrm{ft}$ ) bgs. The sample was described as a fine- to medium-grained sand with some small gravel. Three attempts were made before enough soil was retained for a sample. Attempts to collect a water sample with the screen set at depths between 2.1 and $2.7 \mathrm{~m}$ ( 7 and $9 \mathrm{ft}$ ) were made on November 2, 1993, with no success. A water sample was 
collected on November 3, 1993, with the CPT water-sampling-tool screen set between 2.6 and $2.9 \mathrm{~m}(8.5$ and $9 \mathrm{ft})$ bgs.

\subsubsection{Location $\mathrm{C1-17}$}

Location C1-17 is north of the Surface Ground Zero monument. A stratigraphic sounding

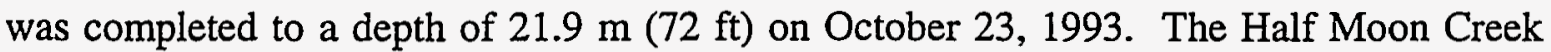

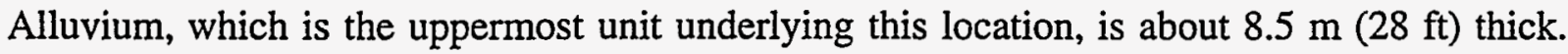
The top of the saturated sediment is interpreted to be within $1.6 \mathrm{~m}(5 \mathrm{ft})$ of ground surface.

A soil sample was collected on November 1, 1993, from the interval between 3.8 and $4.7 \mathrm{~m}$ (12.5 and $15.5 \mathrm{ft}$ ) bgs. The sample was a fine- to medium-grained, white, quartz sand. Matrix spike, MSD, and DUP soil samples were collected from the C1-17 location on November 3, 1993. A water sample was collected on November 3, 1993, with the CPT water-sampling-tool screen set at a depth of $3.8 \mathrm{~m}(12.5 \mathrm{ft})$.

\subsubsection{Location C1-18}

Location C1-18 is south of the Surface Ground Zero monument. A stratigraphic sounding was completed to a depth of $23.5 \mathrm{~m}$ (77 ft) on October 24, 1993. The Half Moon Creek Alluvium, the uppermost unit underlying this location, is about $6.7 \mathrm{~m}(22 \mathrm{ft})$ thick. The top of the saturated sediment is within $1.5 \mathrm{~m}(5 \mathrm{ft})$ of ground surface.

A soil sample was collected on November 2, 1993, from the interval between 1.8 and $2.3 \mathrm{~m}$ ( 6 and $7.5 \mathrm{ft}$ ) bgs. The soil is a gray to black, fine- to medium-grained sand. A water sample was collected on November 2, 1993, with the CPT water-sampling-tool screen set between 2.6 and $2.7 \mathrm{~m}$ ( 8.5 to $9 \mathrm{ft})$. Another water sample was collected on November 10,1993, to replace the semivolatile container that was broken during shipment of the November 2, 1993, samples.

\subsection{Source Area 2}

Source Area 2 is located in the northeastern portion of the site. The area consists of a series of disposal, burn, and borrow pits used in the construction and decommissioning of the site. Collectively these areas are known as the Reynolds Electrical \& Engineering Co., Inc. (REECo) pits. The purpose of the CPT program in this area was to determine if the soil and/or groundwater in the Citronelle and Pascagoula/Hattiesburg Formations was contaminated as a result of the testing and/or clean-up activities conducted at the site. 


\subsubsection{Location C2-19}

Location C2-19 is in the eastern part of Source Area 2. A stratigraphic sounding was completed to a depth of $24.3 \mathrm{~m}$ (79.7 ft) on October 24, 1993. The Pascagoula/Hattiesburg Formation is the uppermost unit underlying this location. The top of the saturated sediment is interpreted to be about $3 \mathrm{~m}(10 \mathrm{ft}) \mathrm{bgs}$.

On November 6, 1993, a soil sample was collected from between 8.7 to $9.1 \mathrm{~m}(28.5$ to $30 \mathrm{ft})$ bgs. The sample was a stiff, orange and gray mottled, sandy clay. A DUP soil sample was also collected during this sampling event.

A water sample was collected on November 8, 1993, using the CPT water sampling tool set

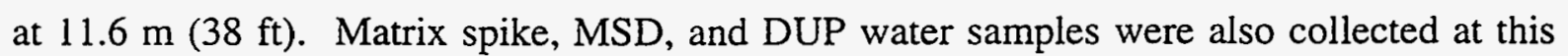
time.

\subsubsection{Location C2-20}

Location C2-20 is on Sand Pit road. On October 25, 1993, a stratigraphic sounding was completed to a depth of $10.3 \mathrm{~m}$ (33.8 ft) before being stopped by a hard layer. The Pascagoula/Hattiesburg Formation is the uppermost unit underlying this location. The top of the saturated sediment is interpreted to be about $3 \mathrm{~m} \mathrm{(10} \mathrm{ft)} \mathrm{bgs.}$

A soil sample was collected from the interval between 2.3 and $2.7 \mathrm{~m}(7.5$ and $9 \mathrm{ft}) \mathrm{bgs}$ on November 6,1993 . The soil was a stiff, orange-to-tan clay with a thin sand interval. A water sample was not collected at this site because no water-bearing sand units were encountered.

\subsubsection{Location C2-21}

Location C2-21 is on Sand Pit road near the REECo disposal pits area. A stratigraphic sounding was completed to a depth of $21 \mathrm{~m}(69 \mathrm{ft})$ on October 25, 1993. The Citronelle Formation, which is the uppermost unit underlying this area, is about $2.1 \mathrm{~m}(7 \mathrm{ft})$ thick. The top of the saturated sediment is at the contact of the Citronelle Formation and the underlying Pascagoula/Hattiesburg Formation.

On November 6, 1993, a soil sample was collected from the interval between 2.1 and $2.6 \mathrm{~m}$ ( 7 and $8.5 \mathrm{ft}$ ) bgs in the Citronelle Formation. Matrix spike, MSD, and DUP soil samples were collected at that location from the interval between 11 and $11.3 \mathrm{~m}$ (36 and $37.5 \mathrm{ft}$ ) bgs in the Pascagoula/Hattiesburg Formation. 
A water sample was collected November 10,1993, using the PVC screen and casing set to a

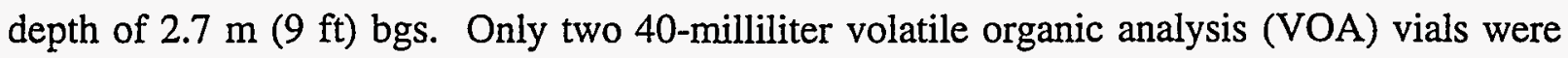
collected due to very low infiltration rates. The sample was red in color and silty. An equipment rinsate blank, C-RB-003-W, was also collected at this location.

\subsubsection{Location C2-22}

Location C2-22 is on Sand Pit road at the REECo Pits. A stratigraphic sounding was completed to a depth of $28 \mathrm{~m}$ (92 ft) on October 25, 1993. The Citronelle Formation, which

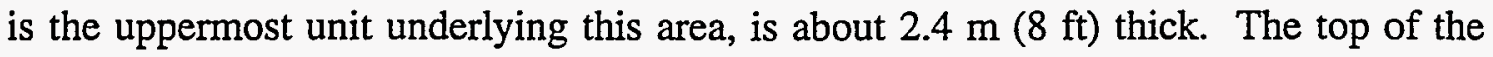
saturated sediment is interpreted to be at the contact of the Citronelle Formation and the Pascagoula/Hattiesburg Formation.

On November 6,1993, a soil sample was collected from the interval between 14.3 and $14.8 \mathrm{~m}$ (47 and $48.5 \mathrm{ft}$ ) bgs. This sample was a stiff clay of the Pascagoula/Hattiesburg Formation. A decision was made to resample the Citronelle Formation from the interval between 2.3 and $2.7 \mathrm{~m}$ ( 7.5 and $9 \mathrm{ft}$ ) bgs as this stratum would more likely be impacted by potential contamination. The Citronelle Formation sample that was submitted for analysis was a red sand and gravel.

On November 11, 1993, a water sample was collected using the PVC screen and casing, set with the bottom of the screen at $4.6 \mathrm{~m}(15 \mathrm{ft})$. The soils only produced enough water for the VOA vials. The water was very muddy and red in color.

\subsubsection{Location C2-23}

Location C2-23 is near the REECo Pits. A stratigraphic sounding was completed to a depth

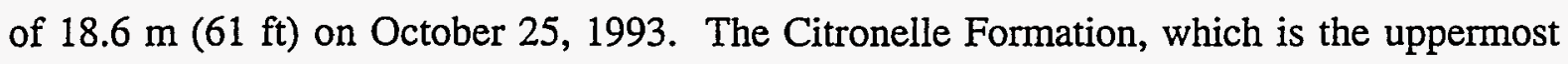

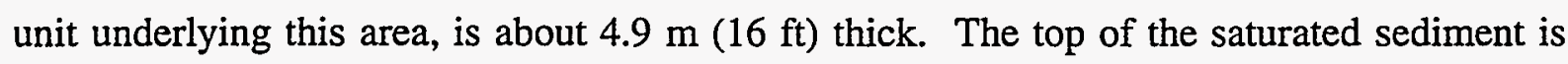

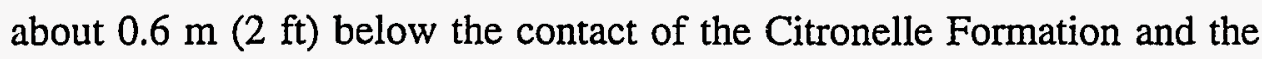
Pascagoula/Hattiesburg Formation.

A soil sample was collected from the interval between 4.6 and $5.2 \mathrm{~m} \mathrm{(15}$ and $17 \mathrm{ft}$ ) bgs on November 6, 1993. The sample was a coarse sand and gravel. An attempt to collect a water sample was made on October 8, 1993, using the CPT water sampling tool. The sampler was placed at various depths between 4.0 and $4.9 \mathrm{~m}$ (13 and $16 \mathrm{ft}$ ) bgs. No sample was recovered. Another attempt to collect a water sample was made on November 10, 1993, 
using the PVC screen and casing set to a depth of $4.9 \mathrm{~m}(16 \mathrm{ft}) \mathrm{bgs}$. Again, no sample was recovered.

\subsubsection{Location C2-24}

Location C2-24 is at the site of an old gas station north of the main road and east of the junction of the main road and Sand Pit road. A stratigraphic sounding was completed to a depth of $21.6 \mathrm{~m}$ (71 ft) on October 26, 1993. The Pascagoula/Hattiesburg Formation is the uppermost unit underlying this location; however, it is covered with about $3 \mathrm{~m}(10 \mathrm{ft})$ of fill material at the surface. The saturated sediments extend to the top of the Pascagoula/ Hattiesburg Formation.

On November 8, 1993, a soil sample was collected from the interval between 16.2 and $16.6 \mathrm{~m} \mathrm{(53}$ and $54.5 \mathrm{ft}$ ) bgs. The soil was a fine green sand with some clayey intervals. The CPT water sampling tool was set with the bottom of the screened interval at a depth of 17.1 $\mathrm{m}(56 \mathrm{ft})$. After two 40-milliliter $(\mathrm{m} l)$ vials were filled, the sampler was pulled up to 17.1, 16.5 , and then $15.8 \mathrm{~m}(56,54$, then $52 \mathrm{ft})$ without producing any more water. The sampler was left in place over night and about a third of a liter was recovered the next morning. No further sampling was conducted at this site.

\subsubsection{Location C2-25}

Location C2-25 is in the REECo Pits. A stratigraphic sounding was completed to a depth of

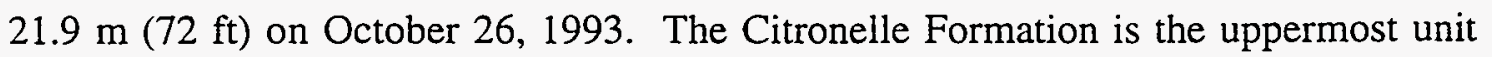
underlying this area; however, it may not be present at the surface due to prior excavation activities. About $1.5 \mathrm{~m}(5 \mathrm{ft})$ of disturbed Citronelle Formation or fill material exist at the

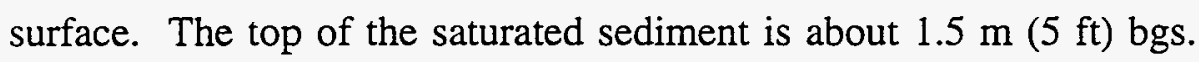

Soil samples were collected from the intervals between 0.6 and $1.2 \mathrm{~m} \mathrm{(2}$ and $4 \mathrm{ft})$ and 6.1 and $6.7 \mathrm{~m}$ (20 and $22 \mathrm{ft}$ ) bgs on November 7, 1993. The 0.6- to 1.2-m (2- to 4-ft) sample was a dry, medium-grained, clayey sand. The 6.1 - to $6.7-\mathrm{m}$ (20- to $22-\mathrm{ft}$ ) sample was a stiff, green and brown mottled, silty clay. An attempt to collect a water sample was not made because no water-bearing sand units were encountered.

\subsubsection{Location C2-26}

Location C2-26 is on Sand Pit Road at the REECo Pits. A stratigraphic sounding was completed to a depth of $5.5 \mathrm{~m}$ (18 ft) on October 26,1993 . The sounding was stopped when 
a hard layer was encountered. There was no indication of saturated sediments being encountered during the stratigraphic test.

A soil sample was collected on November 7, 1993, from the interval between 4.6 and $5.0 \mathrm{~m}$ (15 and $16.5 \mathrm{ft}$ ) bgs. The sample was a course sand. No attempt was made to collect a water sample.

\subsubsection{Location C2-27}

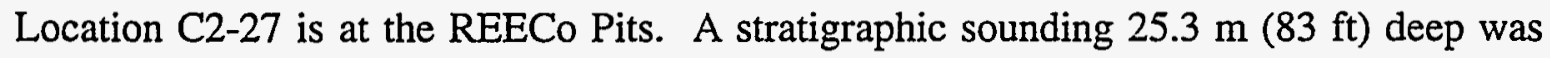
conducted on October 26, 1993. The Citronelle Formation, the uppermost unit underlying this

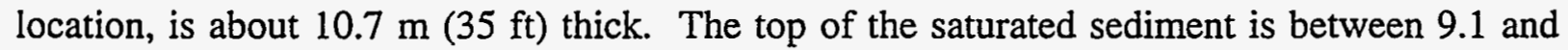
$10.7 \mathrm{~m}$ (30 and $35 \mathrm{ft})$ bgs.

A soil sample was collected on November 10, 1993, from the interval between 11.3 and $11.7 \mathrm{~m}$ (37 and $38.5 \mathrm{ft}$ ) bgs. The soil was a soft, gray clay. A water sample was collected the same day using the PVC screen and casing with the bottom of the screen set to a depth of $11.3 \mathrm{~m}(37 \mathrm{ft})$. A field blank (FB-003-W) was also collected at this location. 


\subsection{Analytical Results}

Fifteen groundwater and 28 soil samples were collected for organic, inorganic, and radiological analyses during the CPT program at the Salmon Site. Table 3-2 lists the sample number and the analyses run for each sample. Because of insufficient sample volume, complete analyses were not possible for several of the water samples. A list of the chemical and metal constituents of concern is given as Table 4-1, and those radionuclides included in the gamma spectroscopy analysis are listed in Table 4-2.

Preliminary results of the analyses from the CPT soil and groundwater samples indicate the presence of chemical, metal, and radiological constituents at levels that exceed the contract required detection limit (CRDL), the Minimum Detectable Activity (MDA) for radiological constituents, and the Maximum Contaminate Level (MCL) under the Safe Drinking Water Act (SDWA). These results are summarized in Table 4-3. Common laboratory contaminates (acetone and methylene chloride) are not included. A complete list of the preliminary results for each sample is provided in Appendices B through $\mathrm{I}$. None of the data contained herein have been validated. Analytical results that exceeded the CRDL or the MDA are highlighted in the appendices by shading.

For comparison purposes, the results of the gamma spectroscopy, gross alpha, and gross beta for the soil samples were compared to those of a reference soil sample collected in conjunction with previous activities at the SS (DOE, 1994). The reference sample was collected from an off-site location near the SS that was not affected by the testing on the site. The values for each radionuclide detected in the reference sample are listed in Table 4-4. The CPT soil samples with radionuclide activities that exceeded those of the reference sample are listed in the "radiological" column of Table 4-3.

The samples were analyzed at the IT Analytical Services laboratory located in Earth City, Missouri. All chemical analyses were conducted according to the U.S. Environmental Protection Agency (EPA) Contract Laboratory Program (CLP) protocols. The requested analytical protocols and the methods used were as follows:

- Volatiles Organic Compounds

- Semivolatiles Organic Compounds

- Pesticides/polychlorinated biphenyls (PCBs)

- Metals
CLP 88

CLP 88

CLP 88

CLP 90 
Table 4-1

List of Chemical and Metal Constituents of Concern (CoC), Salmon Site

\begin{tabular}{|c|c|c|c|c|}
\hline Volatiles EPA CLP & Semivola & les CLP & Pesticides/PCBs & CLP Metals \\
\hline $\begin{array}{l}\text { 1. Chloromethane } \\
\text { 2. Bromomethane } \\
\text { 3. Vinyl Chloride } \\
\text { 4. Chloroethane } \\
\text { 5. Methylene Chloride } \\
\text { 6. Acetone } \\
\text { 7. Carbon Disulfide } \\
\text { 8. 1,1-Dichlorethene } \\
\text { 9. 1,1-Dichlorethane } \\
\text { 10. 1,2-Dichlorethene (total) } \\
\text { 11. Chloroform } \\
\text { 12. 1,2-Dichlorethane } \\
\text { 13. 2--Butanone } \\
\text { 14. 1,1,1-Trichloroethane } \\
\text { 15. Carbon Tetrachloride } \\
\text { 16. Bromodichloromethane } \\
\text { 17. 1,2-Dichloropropane } \\
\text { 18. cis-1,3-Dichloropropene } \\
\text { 19. Trichloroethene } \\
\text { 20. Dibromochloromethane } \\
\text { 21. 1,1,2-Trichloroethane } \\
\text { 22. Benzene } \\
\text { 23. trans-1,3-Dichloropropene } \\
\text { 24. Bromoform } \\
\text { 25. 4-Methyl-2-pentanone } \\
\text { 26. 2-Hexanone } \\
\text { 27. Tetrachloroethene } \\
\text { 28. Toluene } \\
\text { 29. 1,1,2,2-Tetrachloroethane } \\
\text { 30. Chlorobenzene } \\
\text { 31. Elyyl Benzene } \\
\text { 32. Styrene } \\
\text { 33. Xylenes (Total) } \\
\text { 34. Vinyl Acetate }\end{array}$ & $\begin{array}{l}\text { 35. Phenol } \\
\text { 36. bis(2-Chloroethyl) ether } \\
\text { 37. 2-Chlorophenol } \\
\text { 38. 1,3-Dichlorobenzene } \\
\text { 39. 1,4-Dichlorobenzene } \\
\text { 40. 1,2-2ichlorobenzene } \\
\text { 41.2-Methlyphenol } \\
\text { 42. 2,2'-oxybis (1-Chloropropane) } \\
\text { 43. 4-Methylphenol } \\
\text { 44. N-Nitroso-di-n-dipropylamine } \\
\text { 45. Hexachloroethane } \\
\text { 46. Nitrobenzene } \\
\text { 47. Isophorone } \\
\text { 48. 2-Nitrophenol } \\
\text { 49. 2,4-Dimethylphenol } \\
\text { 50. bis(2-Chloroethoxy) methane } \\
\text { 51. 2,4-Dichlorophenol } \\
\text { 52. Naphthalene } \\
\text { 53. 4-Chloroaniline } \\
\text { 54. Hexachlorobutadiene } \\
\text { 55. 4-Chloro-3-methylphenol } \\
\text { 56. 2-Methylnaphthalene } \\
\text { 57. Hexachlorocyclopentadiene } \\
\text { 58. 2,4,6-Trichlorophenol } \\
\text { 59. 2,4,5-Trichlorophenol } \\
\text { 60. 2-Chloronaphthalene } \\
\text { 61. 2-Nitroaniline } \\
\text { 62. Dimethylphthalate } \\
\text { 63. Acenaphthylene } \\
\text { 64. 2,6-Dinitrotoluene } \\
\text { 65. 3-Nitroaniline } \\
\text { 66. Acenaphthene } \\
\text { 67. 2,4-Dinitrophenol } \\
\text { 68. 4-Nitrophenol }\end{array}$ & $\begin{array}{l}\text { 69. Dibenzofuran } \\
\text { 70. 2,4-Dinitrotoluene } \\
\text { 71. Diethylphthalate } \\
\text { 72. 4-Chlorophenyl-phenylether } \\
\text { 73. Fluorene } \\
\text { 74. 4-Nitroaniline } \\
\text { 75. 4,6-Dinitro-2-methylphenol } \\
\text { 76. N-nitrosodiphenylamine } \\
\text { 77. 4-Bromophenyl-phenylether } \\
\text { 78. Hexachlorobenzene } \\
\text { 79. Pentachlorophenol } \\
\text { 80. Phenanthrene } \\
\text { 81. Anthracene } \\
\text { 82. Di-n-butylphthalate } \\
\text { 83. Fluoranthene } \\
\text { 84. Pyrene } \\
\text { 85. Butylbenzylphthalate } \\
\text { 86. 3,3'-Dichlorobenzidine } \\
\text { 87. Benzo(a)anthracene } \\
\text { 88. Chrysene } \\
\text { 89. bis(2-Ethylhexyl)phthalate } \\
\text { 90. Di-n-octylphthalate } \\
\text { 91. Benzo(b)fluoranthene } \\
\text { 92. Benzo(k)fluoranthene } \\
\text { 93. Benzo(a)pyrene } \\
\text { 94. Indeno(1,2,3-cd)pyrene } \\
\text { 95. Dibenz(a,h)anthracene } \\
\text { 96. Benzo(g,hi)perylene } \\
\text { 97. Benzyl Alcohol } \\
\text { 98. Benzoic Acid }\end{array}$ & $\begin{array}{l}\text { 99. alpha-BHC } \\
\text { 100. beta-BHC } \\
\text { 101. delta-BHM } \\
\text { 102. gamma-BHC (lindane) } \\
\text { 103. Heptachlor } \\
\text { 104. Aldrin } \\
\text { 105. Heptachlor epoxide } \\
\text { 106. Endosulfan I } \\
\text { 107. Dieldrin } \\
\text { 108. 4,4'-DDE } \\
\text { 109. Endrin } \\
\text { 110. Endosulfan II } \\
\text { 111. 4,4'-DDT } \\
\text { 112. 4,4'-DDD } \\
\text { 113. Endosulfan Sulfate } \\
\text { 114. Methoxychlor } \\
\text { 115. Endrin ketone } \\
\text { 116. Endrin aldehyde } \\
\text { 117. alpha-Chlordane } \\
\text { 118. gamma-Chlordane } \\
\text { 119. Toxaphene } \\
\text { 120. Aroclor-1016 } \\
\text { 121. Aroclor-1221 } \\
\text { 122. Aroclor-1232 } \\
\text { 123. Aroclor-1242 } \\
\text { 124. Aroclor-1248 } \\
\text { 125. Aroclor-1254 } \\
\text { 126. Aroclor-1260 }\end{array}$ & $\begin{array}{l}\text { 127. Aluminum } \\
\text { 128. Antimony } \\
\text { 12. Arsenic } \\
\text { 130. Barium } \\
\text { 131. Beryllium } \\
\text { 132. Cadmium } \\
\text { 133. Calcium } \\
\text { 134. Chromium } \\
\text { 135. Cobalt } \\
\text { 136. Copper } \\
\text { 137. Iron } \\
\text { 138. Lead } \\
\text { 139. Magnesium } \\
\text { 140. Manganese } \\
\text { 141. Mercury } \\
\text { 142. Nickel } \\
\text { 143. Potassium } \\
\text { 144. Selenium } \\
\text { 145. Silver } \\
\text { 146. Sodium } \\
\text { 147. Thallium } \\
\text { 148. Vanadium } \\
\text { 149. Zinc } \\
\text { 150. Cyanide }\end{array}$ \\
\hline
\end{tabular}

(All 1988 Organics and 1990 Metals) 
Table 4-2

List of Radionuclides Included in the Gamma Spectroscopy Analysis

\begin{tabular}{|c|c|c|c|c|c|c|c|c|}
\hline Radionuclide & Symbol & Mass & Radionuclide & Symbol & Mass & Radionuclide & $\begin{array}{c}\text { Symbo } \\
1\end{array}$ & Mass \\
\hline Beryliium 7 & $\mathrm{Be}-7$ & 7 & Ruthenium 106 & Ru-106 & 106 & Iridium 192 & $\mid r-192$ & 192 \\
\hline Sodium 22 & $\mathrm{Na}-22$ & 22 & Silver $110 \mathrm{M}$ & $\mathrm{Ag}-110 \mathrm{M}$ & 110 & Mercury 203 & $\mathrm{Hg}-203$ & 203 \\
\hline Sodium 24 & $\mathrm{Na}-24$ & 24 & Cadmium 109 & Cd-109 & 109 & Thallium 208 & $T 1-208$ & 208 \\
\hline Potassium 40 & $\mathrm{~K}-40$ & 40 & Tin 113 & Sn-113 & 113 & Lead 210 & $\mathrm{~Pb}-210$ & 210 \\
\hline Scandium 46 & Sc-46 & 46 & Antimony 124 & Sb-124 & 124 & Lead 211 & $\mathrm{~Pb}-211$ & 211 \\
\hline Vanadium 48 & $V-48$ & 48 & Antimony 125 & Sb-125 & 125 & Lead 212 & $\mathrm{~Pb}-212$ & 212 \\
\hline Chromium 51 & Cr-51 & 51 & lodine 129 & $1-129$ & 129 & Bismuth 207 & Bi-207 & 207 \\
\hline Manganese 54 & $M n-54$ & 54 & lodine 131 & $1-131$ & 131 & Bismuth 212 & $8 \mathrm{i}-212$ & 212 \\
\hline Iron 59 & $\mathrm{Fe}-59$ & 59 & lodine 133 & $1-133$ & 133 & Bismuth 214 & $B \mathrm{i}-214$ & $\overline{214}$ \\
\hline Cobalt 56 & $\overline{C o-56}$ & 56 & Cesium 134 & Cs-134 & 134 & Radium 223 & Ra-223 & 223 \\
\hline Cobalt 57 & Co-57 & 57 & Cesium 137 & Cs-137 & 137 & Radium 224 & Ra-224 & 224 \\
\hline Cobalt 58 & Co-58 & 58 & Barium 131 & $\mathrm{Ba}-131$ & 131 & Radium 226 & Ra-226 & 226 \\
\hline Cobalt 60 & Co-60 & 60 & Barium 133 & $\mathrm{Ba}-133$ & 133 & Radium 228 & Ra-228 & 228 \\
\hline Nickel 65 & $\mathrm{Ni}-65$ & 65 & Barium 140 & $\mathrm{Ba}-140$ & 140 & Actinium 227 & Ac-227 & 227 \\
\hline Zinc 65 & $Z n-65$ & 65 & Lanthanum 140 & La-140 & 140 & Thorium 228 & Th-228 & 228 \\
\hline Selenium 75 & Se-75 & 75 & Cerium 139 & Ce-139 & 139 & Thorium 230 & Th-230 & 230 \\
\hline Strontium 85 & Sr-85 & 85 & Cerium 141 & $\mathrm{Ce}-141$ & 141 & Thorium 234 & Th-234 & 234 \\
\hline Yttrium 88 & $\mathrm{Y}-88$ & 88 & Cerium 144 & Ce-144 & 144 & Proactinium 231 & $\mathrm{~Pa}-231$ & 231 \\
\hline Zirconium 95 & Zr-95 & 95 & Europium 152 & Eu-152 & 152 & Proactinium 234 & Pa-234 & 234 \\
\hline Niobium 95 & Nb-95 & 95 & Europium154 & Eu-154 & 154 & Uranium 234 & U-234 & 234 \\
\hline Technetium 99m & TC-99M & 99 & Europium 155 & Eu-155 & 155 & Uranium 235 & U-235 & 234 \\
\hline Ruthenium 103 & Ru-103 & 103 & Gadolinium 153 & Gd-153 & 153 & Americium 241 & Am-241 & 241 \\
\hline
\end{tabular}


Table 4-3

Summary of Cone Penetrometer Testing (CPT)

Sampling Analytical Data (Preliminary), Salmon Site

(Page 1 of 9)

\begin{tabular}{|c|c|c|c|c|}
\hline $\begin{array}{c}\text { CPT } \\
\text { LOCATION }\end{array}$ & $\begin{array}{l}\text { SAMPLE } \\
\text { MATRIX }\end{array}$ & ORGANICS & METALS ${ }^{\mathrm{a}}$ & RADIOLOGICAL \\
\hline \multicolumn{5}{|c|}{ SOURCE AREA 5} \\
\hline C5-1 & SOIL & $\begin{array}{l}\text { Volatiles: } \\
\text { Nondetected (ND) } \\
\text { Semivolatiles: } \\
\text { ND } \\
\text { Pesticides and PCBs: } \\
\text { ND }\end{array}$ & $\begin{array}{l}\text { Chromium }(\mathrm{Cr} \text { ) and lead }(\mathrm{Pb}) \text { exceeded the } \\
\text { Contract Required Detection Limit (CRDL). }\end{array}$ & $\begin{array}{l}\text { Gamma Spectroscopy: } \\
\text { Potassium (K40), Lead } 210 \text { and } 212 \text { (Pb } 210 \text { and } \\
\text { Pb 212) and Radium } 226 \text { (Ra 226) exceeded the } \\
\text { reference sample values. } \\
\text { Gross alpha/beta': } \\
\text { Both exceeded the reference sample values. }\end{array}$ \\
\hline & WATER & $\begin{array}{l}\text { Volatiles: } \\
\text { ND } \\
\text { Semivolatiles: } \\
\text { ND } \\
\text { Pesticides and PCBs: } \\
\text { ND }\end{array}$ & $\mathrm{Cr}$ and $\mathrm{Pb}$ exceeded the CRDL. & $\begin{array}{l}\text { Gamma Spectroscopy: } \\
\text { K40 was detected above the minimum detectable } \\
\text { activity in the DUP. } \\
\text { Gross alpha/beta: } \\
\text { Both exceeded the minimum detectable activity. } \\
\text { Tritium } \\
\text { ND }\end{array}$ \\
\hline$\overline{\mathrm{C} 5-2}$ & SOIL & $\begin{array}{l}\text { Volatiles: } \\
\text { ND } \\
\text { Semivolatiles: } \\
\text { ND } \\
\text { Pesticides and PCBs: } \\
\text { ND }\end{array}$ & Cr and $\mathrm{Pb}$ exceeded the CRDL. & $\begin{array}{l}\text { Gamma Spectroscopy: } \\
\text { Pb212 exceeded the reference sample values. } \\
\text { Gross alpha/beta: } \\
\text { Did not exceed the reference sample values. }\end{array}$ \\
\hline$\overline{\mathrm{C} 5-3}$ & SOIL & $\begin{array}{l}\text { Volatiles: } \\
\text { ND } \\
\text { Semivolatiles: } \\
\text { ND } \\
\text { Pesticides and PCBs: } \\
\text { ND }\end{array}$ & Barium (Ba), $\mathrm{Cr}$, and $\mathrm{Pb}$ exceeded the CRDL. & $\begin{array}{l}\text { Gamma Spectroscopy: } \\
\mathrm{Pb} 212 \text { and Ra226 exceeded the reference } \\
\text { sample values. } \\
\text { Gross alpha/beta: } \\
\text { Did not exceed the reference sample values. }\end{array}$ \\
\hline
\end{tabular}


Table 4-3

Summary of Cone Penetrometer Testing (CPT)

Sampling Analytical Data (Preliminary), Salmon Site

(Page 2 of 9)

\begin{tabular}{|c|c|c|c|c|}
\hline $\begin{array}{c}\text { CPT } \\
\text { LOCATION }\end{array}$ & $\begin{array}{l}\text { SAMPLE } \\
\text { MATRIX }\end{array}$ & ORGANICS & METALS ${ }^{a}$ & RADIOLOGICAL \\
\hline \multicolumn{5}{|c|}{ SOURCE AREA 3} \\
\hline C3-4 & SOIL & $\begin{array}{l}\text { Volatiles: } \\
\text { ND } \\
\text { Semivolatiles: } \\
\text { ND } \\
\text { Pesticides and PCBs: } \\
\text { ND }\end{array}$ & Arsenic (As), $\mathrm{Cr}$, and $\mathrm{Pb}$ exceeded the CRDL. & $\begin{array}{l}\text { Gamma Spectroscopy: } \\
\text { K40, Thallium } 208 \text { (TI208), Pb210, Pb212, Ra226 and } \\
\text { Ra228 exceeded the reference sample values. } \\
\text { Gross alpha/beta: } \\
\text { Did not exceed the reference sample values. }\end{array}$ \\
\hline C3-5 & SOIL & $\begin{array}{l}\text { Volatiles: } \\
\text { ND } \\
\text { Semivolatiles: } \\
\text { ND } \\
\text { Pesticides and PCBs: } \\
\text { ND }\end{array}$ & As and $\mathrm{Pb}$ exceeded the CRDL. & $\begin{array}{l}\text { Gamma Spectroscopy: } \\
\text { Ti 208, Pb212, and Ra226 exceeded the } \\
\text { reference sample values. } \\
\text { Gross alpha/beta: } \\
\text { Both exceeded the reference sample values. }\end{array}$ \\
\hline C3-6 & SOIL & $\begin{array}{l}\text { Volatiles: } \\
\text { ND } \\
\text { Semivolatiles: } \\
\text { ND } \\
\text { Pesticides and PCBs: } \\
\text { ND }\end{array}$ & $\mathrm{As}, \mathrm{Ba}$, and $\mathrm{Pb}$ exceeded the CRDL. & $\begin{array}{l}\text { Gamma Spectroscopy: } \\
\text { TI208, Pb212, Ra228 exceeded the } \\
\text { reference sample values. } \\
\text { Gross alpha/beta: } \\
\text { Both exceeded the reference sample values. }\end{array}$ \\
\hline C3-7 & SOIL & $\begin{array}{l}\text { Volatiles: } \\
\text { ND } \\
\text { Semivolatiles: } \\
\text { ND } \\
\text { Pesticides and PCBs: } \\
\text { ND }\end{array}$ & $\mathrm{As}, \mathrm{Cr}$, and $\mathrm{Pb}$ exceeded the CRDL. & $\begin{array}{l}\text { Gamma Spectroscopy: } \\
\text { K40, TI208, Pb212, Ra228 exceeded the } \\
\text { reference sample values. } \\
\text { Gross alpha/beta: } \\
\text { Both exceeded the reference sample values. }\end{array}$ \\
\hline
\end{tabular}


Table 4-3

Summary of Cone Penetrometer Testing (CPT)

Sampling Analytical Data (Preliminary), Salmon Site

(Page 3 of 9)

\begin{tabular}{|c|c|c|c|c|}
\hline $\begin{array}{c}\text { CPT } \\
\text { LOCATION }\end{array}$ & $\begin{array}{l}\text { SAMPLE } \\
\text { MATRIX }\end{array}$ & ORGANICS & METALS ${ }^{\mathrm{a}}$ & RADIOLOGICAL \\
\hline \multirow[t]{2}{*}{ C3-8 } & SOIL & $\begin{array}{l}\text { Volatiles: } \\
\text { ND } \\
\text { Semivolatiles: } \\
\text { ND } \\
\text { Pesticides and PCBs: } \\
\text { ND }\end{array}$ & $\mathrm{Ba}$ and $\mathrm{Pb}$ exceeded the CRDL & $\begin{array}{l}\text { Gamma Spectroscopy: } \\
\text { K40, T1208, Pb212, Ra226, Ra228 exceeded } \\
\text { the reference sample values. } \\
\text { Gross alpha/beta: } \\
\text { Both exceeded the reference sample values. }\end{array}$ \\
\hline & WATER & \begin{tabular}{|l} 
Volatiles: \\
ND \\
Semivolatiles: \\
ND \\
Pesticides and PCBs: \\
ND
\end{tabular} & $\begin{array}{l}\text { As and } \mathrm{Ni} \text { exceeded the CRDL; } \\
\mathrm{Ba} \text {, } \mathrm{Cr} \text { and } \mathrm{Pb} \text { also exceeded the maximum } \\
\text { contaminant level }{ }^{\circ} \text { (MCL). }\end{array}$ & $\begin{array}{l}\text { Gamma Spectroscopy: } \\
\text { K40 and Pb212 exceeded the MDA. } \\
\text { Gross alpha/beta: } \\
\text { Both exceeded the MDA. } \\
\text { Tritium: } \\
\text { Exceeded the MDA. }\end{array}$ \\
\hline \multirow[t]{2}{*}{ C3-9 } & SOIL & $\begin{array}{l}\text { Volatiles: } \\
\text { ND } \\
\text { Semivolatiles: } \\
\text { ND } \\
\text { Pesticides and PCBs: } \\
\text { ND }\end{array}$ & As and $\mathrm{Pb}$ exceeded the CRDL. & $\begin{array}{l}\text { Gamma Spectroscopy: } \\
\text { Pb212 exceeded the reference sample values. } \\
\text { Gross alpha/beta: } \\
\text { Both exceeded the reference sample values. }\end{array}$ \\
\hline & WATER & $\begin{array}{l}\text { Volatiles: } \\
\text { Toluene exceeded the CRDL } \\
\text { Semivolatiles: } \\
\text { ND } \\
\text { Pesticides and PCBs: } \\
\text { Endosulfan Sulfate exceeded the CRDL }\end{array}$ & $\begin{array}{l}\text { As and } \mathrm{Ni} \text { exceeded the CRDL; } \\
\mathrm{Ba}, \mathrm{Cr} \text { and } \mathrm{Pb} \text { also exceeded the MCL. }\end{array}$ & $\begin{array}{l}\text { Gamma Spectroscopy: } \\
\text { K40, Pb212 and Ra } 226 \text { exceeded the MDA. } \\
\text { Gross alpha/beta: } \\
\text { Both exceeded the MDA. } \\
\text { Tritium: } \\
\text { ND }\end{array}$ \\
\hline \multicolumn{5}{|c|}{ SOURCE AREA 4} \\
\hline$\overline{C 4-10}$ & SOIL & $\begin{array}{l}\text { Volatiles: } \\
\text { ND } \\
\text { Semivolatiles: } \\
\text { ND } \\
\text { Pesticides and PCBs: } \\
\text { ND }\end{array}$ & Pb exceeded the CRDL. & $\begin{array}{l}\text { Gamma Spectroscopy: } \\
\text { K40, Ti208, Pb212 and Ra226 exceeded the } \\
\text { reference sample values. } \\
\text { Gross alpha/beta: } \\
\text { Both exceeded the MDA. }\end{array}$ \\
\hline
\end{tabular}


Table 4-3

Summary of Cone Penetrometer Testing (CPT)

Sampling Analytical Data (Preliminary), Salmon Site

(Page 4 of 9 )

\begin{tabular}{|c|c|c|c|c|}
\hline $\begin{array}{c}\text { CPT } \\
\text { LOCATION }\end{array}$ & $\begin{array}{l}\text { SAMPLE } \\
\text { MATRIX }\end{array}$ & ORGANICS & METALS $^{a}$ & RADIOLOGICAL \\
\hline \multirow[t]{2}{*}{ C4-11 } & SOIL & $\begin{array}{l}\text { Volatiles: } \\
\text { ND } \\
\text { Semivolatiles: } \\
\text { ND } \\
\text { Pesticides and PCBs } \\
\text { ND }\end{array}$ & As, $\mathrm{Pb}$ exceeded the CRDL. & $\begin{array}{l}\text { Gamma Spectroscopy: } \\
\mathrm{K} 40, \mathrm{~T} / 208, \mathrm{~Pb} 210, \mathrm{~Pb} 212 \text { and Ra226 } \\
\text { exceeded the reference sample values. } \\
\text { Gross alpha/beta: } \\
\text { Both exceeded the MDA. }\end{array}$ \\
\hline & WATER & $\begin{array}{l}\text { Volatiles: } \\
\text { ND } \\
\text { Semivolatiles: } \\
\text { bis(2-ethylhexyl)phthalate was detected } \\
\text { below the CRDL } \\
\text { Pesticides and PCBs: } \\
\text { ND }\end{array}$ & $\begin{array}{l}\text { Mercury }(\mathrm{Hg}) \text { and nickel }(\mathrm{Ni}) \text { exceeded the } \\
\mathrm{CRDL} \\
\mathrm{Ba}, \mathrm{Cr} \text {, and } \mathrm{Pb} \text { exceeded the MCL. }\end{array}$ & $\begin{array}{l}\text { Gamma Spectroscopy: } \\
\text { Pb212 and Ra226 exceeded the MDA. } \\
\text { Gross alpha/beta: } \\
\text { Both exceeded the MDA. } \\
\text { Tritium: } \\
\text { ND }\end{array}$ \\
\hline C4-12 & SOIL & \begin{tabular}{|l|} 
Volatiles: \\
ND \\
Semivolatiles: \\
bis(2-ethylhexyl)phthalate was detected at \\
below the CRDL. \\
Pesticides and PCBs: \\
ND
\end{tabular} & $\mathrm{Cr}$ and $\mathrm{Pb}$ exceeded CRDL. & $\begin{array}{l}\text { Gamma Spectroscopy: } \\
\text { ND } \\
\text { Gross alpha/beta: } \\
\text { ND }\end{array}$ \\
\hline \multirow[t]{2}{*}{$\overline{C 4-13}$} & SOIL & $\begin{array}{l}\text { Volatiles: } \\
\text { ND } \\
\text { Semivolatiles: } \\
\text { ND } \\
\text { Pesticides and PCBs } \\
\text { ND }\end{array}$ & $\mathrm{Pb}$ exceeded the CRDL. & $\begin{array}{l}\text { Gamma Spectroscopy: } \\
\mathrm{K} 40, \mathrm{~T} \mid 208, \mathrm{~Pb} 212, \text { Ra226 exceeded reference } \\
\text { sample values. } \\
\text { Gross alpha/beta: } \\
\text { Both exceeded the reference sample }\end{array}$ \\
\hline & WATER & $\begin{array}{l}\text { Volatiles: } \\
\text { ND } \\
\text { Semivolatiles: } \\
\text { ND } \\
\text { Pesticides and PCBs } \\
\text { ND }\end{array}$ & $\begin{array}{l}\mathrm{Ba} \text { exceeded the CRDL; } \\
\mathrm{Cr} \text { and } \mathrm{Pb} \text { exceeded the MCL. }\end{array}$ & $\begin{array}{l}\text { Gamma Spectroscopy: } \\
\text { K40 exceeded reference sample value. } \\
\text { Gross alpha/beta: } \\
\text { Both exceeded the MDA. } \\
\text { Tritium: } \\
\text { Exceeded the MDA. } \\
\end{array}$ \\
\hline
\end{tabular}


Table 4-3

Summary of Cone Penetrometer Testing (CPT)

Sampling Analytical Data (Preliminary), Salmon Site

(Page 5 of 9)

\begin{tabular}{|c|c|c|c|c|}
\hline $\begin{array}{c}\text { CPT } \\
\text { LOCATION }\end{array}$ & $\begin{array}{l}\text { SAMPLE } \\
\text { MATRIX }\end{array}$ & ORGANICS & METALS ${ }^{\mathbf{a}}$ & RADIOLOGICAL \\
\hline \multicolumn{5}{|c|}{ SOURCE AREA 1} \\
\hline \multirow[t]{2}{*}{ C1-14 } & SOIL & \begin{tabular}{|l} 
Volatiles: \\
ND \\
Semivolatiles: \\
ND \\
Pesticides and PCBs \\
ND \\
\end{tabular} & $\mathrm{Cr}$ and $\mathrm{Pb}$ exceeded the CRDL. & $\begin{array}{l}\text { Gamma Spectroscopy: } \\
\text { ND } \\
\text { Gross alpha/beta: } \\
\text { ND }\end{array}$ \\
\hline & WATER & $\begin{array}{l}\text { Volatiles: } \\
\text { ND } \\
\text { Semivolatiles: } \\
\text { ND } \\
\text { Pesticides and PCBs } \\
\text { ND }\end{array}$ & $\begin{array}{l}\mathrm{Hg} \text { and } \mathrm{Ni} \text { exceeded the } \mathrm{CRDL} \\
\mathrm{Ba} \text {, cadmium (Cd), } \mathrm{Cr} \text {, and } \mathrm{Pb} \text { exceeded the } \\
\text { MCL. }\end{array}$ & $\begin{array}{l}\text { Gamma Spectroscopy: } \\
\text { Tl208, Pb212, Bismuth } 214 \text { (Bi214), Ra226, Ra228 } \\
\text { exceeded the MDA. } \\
\text { Gross alpha/beta: } \\
\text { Both exceeded the MDA. } \\
\text { Tritium: } \\
\text { ND } \\
\end{array}$ \\
\hline C1-15 & SOIL & $\begin{array}{l}\text { Volatiles: } \\
\text { Carbon Disulfide exceeded the CRDL } \\
\text { Semivolatiles: } \\
\text { ND } \\
\text { Pesticides and PCBs: } \\
\text { ND } \\
\end{array}$ & $\mathrm{Cr}$ and $\mathrm{Pb}$ exceeded the CRDL. & $\begin{array}{l}\text { Gamma Spectroscopy: } \\
\text { ND } \\
\text { Gross alpha/beta: } \\
\text { ND }\end{array}$ \\
\hline C1-15 & WATER & $\begin{array}{l}\text { Volatiles: } \\
\text { ND } \\
\text { Semivolatiles: } \\
\text { bis(2-ethylhexyl)phthalate was detected } \\
\text { below the CRDL. } \\
\text { Pesticides and PCBs: } \\
\text { ND }\end{array}$ & $\begin{array}{l}\mathrm{As}, \mathrm{Hg} \text {, and } \mathrm{Ni} \text { exceeded the CRDL; } \\
\mathrm{Ba}, \mathrm{Cd}, \mathrm{Cr} \text { and } \mathrm{Pb} \text { exceeded the } \mathrm{MCL} \text {. }\end{array}$ & $\begin{array}{l}\text { Gamma Spectroscopy: } \\
\text { K40, Pb212, Ra226, and Ra228 exceeded the } \\
\text { MDA. } \\
\text { Gross alpha/beta: } \\
\text { Both exceeded the MCL. } \\
\text { Tritium: } \\
\text { ND }\end{array}$ \\
\hline
\end{tabular}


Table 4-3

Summary of Cone Penetrometer Testing (CPT)

Sampling Analytical Data (Preliminary), Salmon Site

(Page 6 of 9)

\begin{tabular}{|c|c|c|c|c|}
\hline $\begin{array}{c}\text { CPT } \\
\text { LOCATION }\end{array}$ & $\begin{array}{l}\text { SAMPLE } \\
\text { MATRIX }\end{array}$ & ORGANICS & METALS & RADIOLOGICAL \\
\hline \multirow[t]{2}{*}{$\overline{C 1-16}$} & $\overline{\text { SOIL }}$ & $\begin{array}{l}\text { Volatiles: } \\
\text { ND } \\
\text { Semivolatiles: } \\
\text { ND } \\
\text { Pesticides and PCBs: } \\
\text { ND }\end{array}$ & Cr and $\mathrm{Pb}$ exceeded the CRDL. & $\begin{array}{l}\text { Gamma Spectroscopy: } \\
\text { K40 exceeded the reference sample value. } \\
\text { Gross alpha/beta: } \\
\text { ND }\end{array}$ \\
\hline & WATER & $\begin{array}{l}\text { Volatiles: } \\
\text { ND } \\
\text { Semivolatiles: } \\
\text { ND } \\
\text { Pesticides and PCBs: } \\
\text { ND }\end{array}$ & $\begin{array}{l}\mathrm{As}, \mathrm{Ba}, \mathrm{Hg} \text { and } \mathrm{Ni} \text { all exceeded the CRDL. } \\
\mathrm{Cr} \text { and } \mathrm{Pb} \text { exceeded the MCL. }\end{array}$ & $\begin{array}{l}\text { Gamma Spectroscopy: } \\
\text { ND } \\
\text { Gross alpha/beta: } \\
\text { Both exceeded the MCL. } \\
\text { Tritium: } \\
\text { ND }\end{array}$ \\
\hline \multirow[t]{2}{*}{ C1-17 } & SOIL & $\begin{array}{l}\text { Volatiles: } \\
\text { Trichlorethane } \\
\text { Semivolatiles: } \\
\text { ND } \\
\text { Pesticides and PCBs: } \\
\text { ND }\end{array}$ & $\begin{array}{l}\mathrm{Pb} \text { exceeded the CRDL; } \\
\mathrm{Cr} \text { was detected at the CRDL. }\end{array}$ & $\begin{array}{l}\text { Gamma Spectroscopy: } \\
\text { Ra226 exceeded the reference sample; Bi214 was } \\
\text { detected in this sample but not in the reference } \\
\text { sample. } \\
\text { Gross alpha/beta: } \\
\text { ND }\end{array}$ \\
\hline & WATER & $\begin{array}{l}\text { Volatiles: } \\
\text { 1,2, Dichlorethane and Trichlorethane } \\
\text { Semivolatiles: } \\
\text { bis(2-ethylhexyl)phthalate exceeded the } \\
\text { CRDL. } \\
\text { Pesticides and PCBs: } \\
\text { ND }\end{array}$ & $\begin{array}{l}\mathrm{As}, \mathrm{Ba}, \mathrm{Hg} \text { and } \mathrm{Ni} \text { exceeded the CRDL; } \\
\mathrm{Cr} \text { and } \mathrm{Pb} \text { exceeded the MCL. }\end{array}$ & $\begin{array}{l}\text { Gamma Spectroscopy: } \\
\text { Pb212 exceeded the MDA. } \\
\text { Gross alpha/beta: } \\
\text { Both exceeded the MCL. } \\
\text { Tritium: } \\
\text { Exceeded the MDA. }\end{array}$ \\
\hline
\end{tabular}


Table 4-3

Summary of Cone Penetrometer Testing (CPT)

Sampling Analytical Data (Preliminary), Salmon Site

(Page 7 of 9 )

\begin{tabular}{|c|c|c|c|c|}
\hline $\begin{array}{c}\text { CPT } \\
\text { LOCATION }\end{array}$ & $\begin{array}{l}\text { SAMPLE } \\
\text { MATRIX }\end{array}$ & ORGANICS & METALS ${ }^{a}$ & RADIOLOGICAL \\
\hline \multirow[t]{2}{*}{ C1-18 } & $\overline{\text { SOIL }}$ & $\begin{array}{l}\text { Volatiles: } \\
\text { ND } \\
\text { Semivolatiles: } \\
\text { ND } \\
\text { Pesticides and PCBs: } \\
\text { ND }\end{array}$ & Cr and $\mathrm{Pb}$ exceeded the CRDL. & $\begin{array}{l}\text { Gamma Spectroscopy: } \\
\text { ND } \\
\text { Gross alpha/beta: } \\
\text { ND }\end{array}$ \\
\hline & WATER & $\begin{array}{l}\text { Oolatiles: } \\
\text { Carbon disulfide exceeded the CRDL; } \\
\text { 1,2,Dichlorethane exceeded the MCL. } \\
\text { Semivolatiles: } \\
\text { ND } \\
\text { Pesticides and PCBs: } \\
\text { ND }\end{array}$ & $\begin{array}{l}\text { As, } \mathrm{Pb}, \mathrm{Hg} \text {, and } \mathrm{Ni} \text { exceeded the CRDL; } \\
\mathrm{Cr} \text { exceeded the MCL. }\end{array}$ & $\begin{array}{l}\text { Gamma Spectroscopy: } \\
\text { ND } \\
\text { Gross alpha/beta: } \\
\text { Both exceeded the MDA and alpha exceeded the } \\
\text { MCL. } \\
\text { Tritium: } \\
\text { ND }\end{array}$ \\
\hline \multirow[t]{2}{*}{ C2-19 } & SOIL & $\begin{array}{l}\text { Volatiles: } \\
\text { Toluene exceeded the CRDL. } \\
\text { Semivolatiles: } \\
\text { ND } \\
\text { Pesticides and PCBs: } \\
\text { ND }\end{array}$ & $\mathrm{Cr}$ and $\mathrm{Pb}$ exceeded the CRDL. & $\begin{array}{l}\text { Gamma Spectroscopy: } \\
\mathrm{K} 40, \mathrm{~Pb} 210, \mathrm{~Pb} 212 \text {, and Ra226 exceeded the } \\
\text { reference sample; Th234 exceeded the MDA but } \\
\text { was not detected in the reference sample. } \\
\text { Gross alpha/beta: } \\
\text { Both exceeded the reference sample. }\end{array}$ \\
\hline & WATER & $\begin{array}{l}\text { Volatiles: } \\
\text { ND } \\
\text { Semivolatiles: } \\
\text { Diethylphthalate } \\
\text { Pesticides and PCBs: } \\
\text { beta-BHC, Aldrin, and Heptachlor epoxide }\end{array}$ & $\begin{array}{l}\text { As, } \mathrm{Cn} \text {, and } \mathrm{Nl} \text { exceeded CRDL; } \\
\mathrm{Cr} \text { and } \mathrm{Pb} \text { exceeded the MCL in the DUP. }\end{array}$ & $\begin{array}{l}\text { Gamma Spectroscopy: } \\
\text { Ra226 and Th234 exceeded the MDA } \\
\text { Gross alpha/beta: } \\
\text { Both exceeded the MDA and alpha exceeded the } \\
\text { MCL. } \\
\text { Tritium: } \\
\text { ND }\end{array}$ \\
\hline C2-20 & SOIL & $\begin{array}{l}\text { Volatiles: } \\
\text { ND } \\
\text { Semivolatiles: } \\
\text { ND } \\
\text { Pesticides and PCBs: } \\
\text { ND }\end{array}$ & Cr exceeded the CRDL. & $\begin{array}{l}\text { Gamma Spectroscopy: } \\
\text { K40, TI208, Pb210, Pb212, Ra226 Ra228 and } \\
\text { Th234 exceeded the reference sample values. } \\
\text { Gross alpha/beta: } \\
\text { Both exceeded the reference sample values. }\end{array}$ \\
\hline
\end{tabular}


Table 4-3

Summary of Cone Penetrometer Testing (CPT)

Sampling Analytical Data (Preliminary), Salmon Site

(Page 8 of 9)

\begin{tabular}{|c|c|c|c|c|}
\hline $\begin{array}{c}\text { CPT } \\
\text { LOCATION }\end{array}$ & $\begin{array}{l}\text { SAMPLE } \\
\text { MATRIX }\end{array}$ & ORGANICS & METALS ${ }^{a}$ & RADIOLOGICAL \\
\hline C2-21 & SOIL & $\begin{array}{l}\text { Volatiles: } \\
\text { ND } \\
\text { Semivolatiles: } \\
\text { ND } \\
\text { Pesticides and PCBs: } \\
\text { ND }\end{array}$ & Pb exceeded the CRDL. & $\begin{array}{l}\text { Gamma Spectroscopy: } \\
\text { ND } \\
\text { Gross alpha/beta: } \\
\text { ND; Alpha exceeded the reference sample } \\
\text { in the DUP. }\end{array}$ \\
\hline$\overline{\mathrm{C} 2-22}$ & $\overline{\text { SOIL }}$ & \begin{tabular}{|l|} 
Volatiles: \\
ND \\
Semivolatiles: \\
ND \\
Pesticides and PCBs: \\
ND
\end{tabular} & $\mathrm{Cr}$ and $\mathrm{Pb}$ exceeded the CRDL. & $\begin{array}{l}\text { Gamma Spectroscopy: } \\
\text { Bi214 exceeded the MDA; ND in reference sample } \\
\text { value. } \\
\text { Gross alpha/beta: } \\
\text { ND }\end{array}$ \\
\hline$\overline{\mathrm{C} 2-23}$ & SOIL & $\begin{array}{l}\text { Volatiles: } \\
\text { ND } \\
\text { Semivolatiles: } \\
\text { ND } \\
\text { Pesticides and PCBs: } \\
\text { ND }\end{array}$ & $\mathrm{Cr}$ and $\mathrm{Pb}$ exceeded the CRDL. & $\begin{array}{l}\text { Gamma Spectroscopy: } \\
\text { Th208, Pb212 and Ra226 exceeded the reference } \\
\text { sample values. } \\
\text { Gross alpha/beta: } \\
\text { Beta ND; Alpha exceeded the reference sample value. }\end{array}$ \\
\hline$\overline{C 2-24}$ & SOIL & $\begin{array}{l}\text { Volatiles: } \\
\text { ND } \\
\text { Semivolatiles: } \\
\text { ND } \\
\text { Pesticides and PCBs: } \\
\text { ND }\end{array}$ & $\mathrm{Ba}$ and $\mathrm{Pb}$ exceeded the CRDL. & $\begin{array}{l}\text { Gamma Spectroscopy: } \\
\text { Pb212 and Ra226 exceeded the reference } \\
\text { sample values. } \\
\text { Gross alpha/beta: } \\
\text { Alpha ND; Beta exceeded the reference sample value. }\end{array}$ \\
\hline C2-24 & WATER & $\begin{array}{l}\text { Volatiles: } \\
\text { ND } \\
\text { Semivolatiles: } \\
\text { Not Sampled. } \\
\text { Pesticides and PCBs: } \\
\text { Not Sampled. }\end{array}$ & Not sampled. & $\begin{array}{l}\text { Gamma Spectroscopy: } \\
\text { Not sampled. } \\
\text { Gross alpha/beta: } \\
\text { Not sampled. } \\
\text { Tritium: } \\
\text { ND }\end{array}$ \\
\hline
\end{tabular}


Table 4-3

Summary of Cone Penetrometer Testing (CPT)

Sampling Analytical Data (Preliminary), Salmon Site

(Page 9 of 9)

\begin{tabular}{|c|c|c|c|c|}
\hline $\begin{array}{c}\text { CPT } \\
\text { LOCATION }\end{array}$ & $\begin{array}{l}\text { SAMPLE } \\
\text { MATRIX }\end{array}$ & ORGANICS & METALS ${ }^{a}$ & RADIOLOGICAL \\
\hline C2-25 & 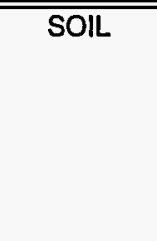 & $\begin{array}{l}\text { Volatiles: } \\
\text { ND } \\
\text { Semivolatiles: } \\
\text { ND } \\
\text { Pesticides and PCBs: } \\
\text { ND }\end{array}$ & $\mathrm{Cr}$ and $\mathrm{Pb}$ exceeded the CRDL. & $\begin{array}{l}\text { Gamma Spectroscopy: } \\
\text { T1208, Pb212, Ra224, and Ra226 exceeded the } \\
\text { reference sample values. } \\
\text { Gross alpha/beta: } \\
\text { ND }\end{array}$ \\
\hline C2-25A & SOIL & $\begin{array}{l}\text { Volatiles: } \\
\text { ND } \\
\text { Semivolatiles: } \\
\text { ND } \\
\text { Pesticides and PCBs: } \\
\text { ND }\end{array}$ & $\mathrm{Cr}$ and $\mathrm{Pb}$ exceeded the CRDL. & $\begin{array}{l}\text { Gamma Spectroscopy: } \\
\text { K40, T1208, Pb212, Ra228,and Bi214 exceeded } \\
\text { the reference sample values. } \\
\text { Gross alpha/beta: } \\
\text { Both exceeded the reference sample values. }\end{array}$ \\
\hline C2-26 & SOIL & \begin{tabular}{|l|} 
Volatiles: \\
ND \\
Semivolatiles: \\
ND \\
Pesticides and PCBs: \\
ND
\end{tabular} & Cr and $\mathrm{Pb}$ exceeded the CRDL. & $\begin{array}{l}\text { Gamma Spectroscopy: } \\
\text { Pb212 exceeded the reference sample values. } \\
\text { Gross alpha/beta: } \\
\text { ND }\end{array}$ \\
\hline C2-27 & SOIL & $\begin{array}{l}\text { Volatiles: } \\
\text { ND } \\
\text { Semivolatiles: } \\
\text { ND } \\
\text { Pesticides and PCBs: } \\
\text { ND }\end{array}$ & $\mathrm{Pb}$ exceeded the CRDL. & $\begin{array}{l}\text { Gamma Spectroscopy: } \\
\text { K40, TI208, Pb210, Pb212, Ra226, Ra228 and } \\
\text { Th234 exceeded the reference sample values. } \\
\text { Gross alpha/beta: } \\
\text { Both exceeded the reference sample values. }\end{array}$ \\
\hline $\mathrm{C2-27}$ & WATER & $\begin{array}{l}\text { Volatiles: } \\
\text { Tetrachlorethane was detected at the } \\
\text { CRDL } \\
\text { Semivolatiles: } \\
\text { ND } \\
\text { Pesticides and PCBs: } \\
\text { ND }\end{array}$ & $\begin{array}{l}\mathrm{Ni} \text { exceeded the CRDL; } \\
\mathrm{Ba}, \mathrm{Cr}, \mathrm{Hg} \text {, and } \mathrm{Pb} \text { exceeded the MCL. }\end{array}$ & $\begin{array}{l}\text { Gamma Spectroscopy: } \\
\text { K40, TI208, Pb210, Pb212, and Ra226, exceeded the } \\
\text { MDA. } \\
\text { Gross alpha/beta: } \\
\text { Both exceeded the MCL. } \\
\text { Tritium: } \\
\text { ND }\end{array}$ \\
\hline
\end{tabular}

a Total metals; samples were unfiltered and unpreserved.

beference soil sample REFC-19-SS: gross alpha activity $8.3 \mathrm{pCi} / \mathrm{g}$ and gross beta activity $12.8 \mathrm{pCi} / \mathrm{g}$ (DOE, 1994)

c From the Safe Drinking Water Act. 
Table 4-4

Radionuclide Activities for Soil Sample REFC-19-SS ${ }^{a}$, Salmon Site

\begin{tabular}{|c|c|c|c|}
\hline Radionuclide & Activity (pCi/g) & Radionuclide & Activity (pCi/g) \\
\hline Cesium 137 & 0.24 & Uranium 234 & nondetect (ND) \\
\hline Potassium 40 & 2.70 & Uranium 235 & ND \\
\hline Lead 212 & 0.31 & Bismuth 214 & ND \\
\hline Radium 226 & 0.46 & Protactinium 234 & ND \\
\hline Radium 228 & 0.47 & Thorium 234 & ND \\
\hline Thallium 208 & 0.13 & Cd-109 & ND \\
\hline Uranium 238 & 0.60 & Strontium 85 & ND \\
\hline Lead 210 & 1.23 & Yttrium 88 & ND \\
\hline Radium 224 & 0.43 & Cerium 144 & ND \\
\hline Radium 223 & 0.16 & Thorium 230 & ND \\
\hline Thorium 228 & ND & Bismuth 212 & 0.32 \\
\hline Protactinium 231 & ND & lodine 131 & ND \\
\hline $\operatorname{Tin} 113$ & ND & Beryllium 7 & ND \\
\hline Mercury 203 & ND & & \\
\hline
\end{tabular}

aSample collected March 1993 (DOE, 1994)

bicocuries per gram

- Cyanide

- Gamma Scan (water)

- Gamma Scan (soil)

- Gross Alpha/Beta (water)

- Gross Alpha/Beta (soil)

- Tritium $\left({ }^{+3} \mathrm{H}\right)$ (water)
- CLP 90

- EPA 901.1

- Health and Safety Laboratory (HSL) 300.0

- EPA 906.0

- SM7110 (soils)

- Eastern Environmental Radiation Facilities (EERF) H.01

\subsection{Source Area 5}

Three soil samples and one water sample were collected from Source Area 5. None of the samples analyzed had organic constituents (volatile, semivolatile, pesticide and PCBs) that exceeded the CRDL. Chromium and lead were identified at levels exceeding the CRDL, but below the MCL in all of the samples in this group. Barium was also identified in sample C5-3-S.

Gamma spectroscopy of the soil samples indicated the presence of radionuclides at levels above those of the reference sample; however, these are all naturally occurring. There were no radionuclides with activities above the MDA in the water sample except potassium 40 , which was detected in the duplicate water sample (C5-1-W-DUP). 
Gross alpha and beta activities in sample C5-1-S exceeded the levels of the reference sample, and the activities in C5-1-W exceeded the MDA.

The tritium level for sample C5-1-W was below the MDA.

\subsection{Source.Area 3}

Six soil and two water samples were collected from the three individual sites within Source Area 3. None of the samples had any volatile or semivolatile organic compounds above the CRDL. A pesticide (Endonsulfan sulfate) was detected at a level that exceeded the CRDL in sample C3-9-W. Arsenic and lead were detected above the CRDL in all but one sample (C3-8-S). Chromium was identified in C3-4-S and C3-7-S at levels above the CRDL. Barium was detected above the CRDL in samples C3-6-S and C3-8-S. Arsenic, barium, chromium, lead and nickel were detected above the CRDL in both water samples. Barium, chromium and lead concentrations also exceeded the MCL set by the SDWA.

Gamma spectroscopy indicated the presence of several naturally occurring radionuclides at activity levels above those for the reference sample. Gross alpha and beta activities for the soil samples exceeded the reference sample results for all but the gross alpha activity in sample C3-9-S. The results of the gross alpha and beta analyses for the water samples indicated activity levels that greatly exceed the MCL. However, because all the radionuclides identified by the gamma spectroscopy are naturally occurring, the alpha and beta activity is also presumed to be naturally occurring.

Tritium was identified in sample C3-8-W at levels above the MDA, but below the MCL.

\subsection{Source Area 4}

Four soil and two water samples were collected from Source Area 4. No volatile organic compounds or pesticide/PCB compounds were detected in any of the samples. Concentrations of bis(2-ethylhexyl)phthalate were detected in C4-11-W and C4-12-S. Because bis(2-ethylhexyl)phthalate is used in laboratory vacuum pumps, it is likely that these detections are the result of laboratory contamination. At location C4-10 only lead exceeded the CRDL for metals in soil. 
All three soil samples taken from the vicinity of the large-bore drilling sites had concentrations of lead, arsenic, and chromium that exceed the CRDL. The water samples indicated the presence of barium, chromium, and lead in both samples at levels that exceeded the CRDL. Barium exceeded the MCL in sample C4-14-W, and chromium and lead exceeded the MCL in both samples. Mercury was detected in C4-11-W above the CRDL, but below the MCL.

Gamma spectroscopy indicated the presence of several naturally occurring radionuclides at activity levels above those for the reference sample. Gross alpha and beta activities for the soil samples exceeded the reference sample results for all but sample C4-12-S. The results of the gross alpha and beta analysis for the water samples indicated activity levels that exceed the MCL. However, because all of the radionuclides identified by the gamma spectroscopy are naturally occurring, the alpha and beta activity is also presumed to be naturally occurring.

Tritium was identified in sample C4-13-W at a level above the MDA, but below the MCL.

\subsection{Source Area 1}

Five soil and five water samples were collected from Source Area 1. Volatile organic compounds were detected in four samples: C1-15-S, C1-17-S, C1-17-W, and C1-18-W. In sample C1-17-W bis(2-ethylhexyl)phthalate was detected above the CRDL. There were no detections of either pesticide or PCB compounds in any of the samples from Source Area 1.

Chromium and lead were detected at or above the CRDL in all of the soil samples in this group. Arsenic, barium, cadmium, chromium, lead, mercury, and nickel exceeded the CRDL in all the water samples. Barium, cadmium, chromium, and lead were detected at levels that exceed the MCL in C1-14-W and C1-15-W. Chromium and lead also exceeded the MCL in sample C1-18-W.

The soil samples analyzed by gamma spectroscopy indicated the presence of radium 226 and bismuth 214 in sample C1-17-S at levels that exceeded those of the reference sample. None

of the other soil samples had gamma spectroscopy or gross alpha and beta activities above the reference sample values. 
Gamma spectroscopy analyses indicated the presence of Thallium-208, Lead-212, Bismuth-214, Radium-226 and Radium-228 at levels that exceed the MDA in Sample C1-14-W. Sample C1-15-W had elevated levels of Potassium-40, Lead-212, Radium-226 and -228. Sample C1-17-W had lead 212 as its sole detectable radionuclide. Samples C1-16-W and C1-18-W had no detectable radionuclide activity. Gross alpha and beta activity exceeded the MCL in all the water samples and is a reflection of the radionuclides in those samples. Samples C1-16-W and C1-18-W had no radionuclides above the MDA, and the alpha and beta activity is unaccounted for. However, one possible explanation is that there are several radionuclides present at levels below the MDA and the sum of their alpha and beta activities is sufficient to produce the high alpha and beta levels in the samples.

Tritium was only detected in Sample C1-17-W at a level that exceeds the MCL.

\subsection{Source Area 2}

Ten soil and three water samples, two complete and one partial suite, were collected from Source Area 2. Volatile organic compounds were detected in soil ample C2-19-S (toluene) and water ample C2-27-W (tetrachlorethane). None of the soil samples had semivolatile organic compounds or pesticide/PCB compounds detected. Diethylphthalate, beta-BHC, aldrin, and heptachlor epoxide were detected in water sample C2-19-W at levels that exceeded the CRDL.

Lead and chromium commonly exceeded the CRDL in the soil samples, and barium was detected in one sample, C2-24-S. Only two water samples were analyzed for metals. Arsenic, chromium, lead, cyanide, and nickel exceeded the CRDL in Sample C2-19-W, and barium, chromium, lead, mercury and nickel exceeded the CRDL in Sample C2-27-W. Chromium and lead exceeded the MCL in both samples, and barium and mercury also exceeded the MCL in C2-27-W.

A variety of radionuclides was detected in the soil samples at levels exceeding the reference sample values. All the radionuclides detected are naturally occurring. The alpha and beta activities generally exceeded those of the reference sample or the MDA, but were of approximately the same magnitude (100 to 500 picocuries per liter) as the other samples collected during this program.

Tritium analyses were conducted on all three water samples, and the results were all below the MDA. 


\subsection{Conclusions}

The objectives of the CPT program were to

- Determine subsoil conditions and stratification

- Determine the depth to the potentiometric surface

- Obtain subsurface soil samples for chemical and radiological analyses from potentially contaminated areas

- Obtain groundwater samples for chemical and radiological analyses from potentially contaminated areas.

The CPT program accomplished these objectives as summarized below:

- Stratigraphic tests were conducted at 27 locations to depths between 5.5 and $30.5 \mathrm{~m}$ (18 and $100 \mathrm{ft}$ ).

- The top of the saturated sediments, not necessarily the potentiometric surface, was determined at each location.

- Soil samples were collected at all 27 locations.

- Water samples were collected at 15 of the locations.

- Chemical and radiological analyses of the soil and groundwater samples identified potentially affected areas.

The following conclusions are derived from the CPT program at the Salmon Site:

- The Upper Aquifer consists of discontinuous sand lenses at elevations between 61 and $79 \mathrm{~m}(200$ and $260 \mathrm{ft})$ amsl.

- The Citronelle Formation consists of sands and gravels and is found on hill tops at elevations greater than $85.3 \mathrm{~m}(280 \mathrm{ft})$ amsl.

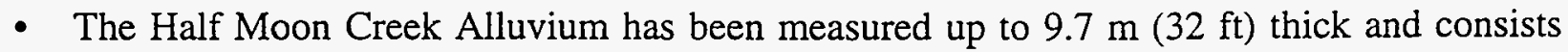
of fine sand with minor small gravels.

- Three water bearing units are present within $30.5 \mathrm{~m}$ (100 ft) of the ground surface:

- Upper Aquifer of the Pascagoula/Hattiesburg Formation

- Pascagoula/Hattiesburg Formation-Citronelle Formation contact

- Half Moon Creek Alluvium associated with that stream.

- A review of the analytical results presented in this report indicates that the Surface Ground Zero area may be affected by volatile organic compounds, metals, and tritium. 


\subsection{References}

U.S. Department of Energy, 1994, draft Preliminary Data Report of Investigations Conducted at the Salmon Site, Lamar County, Mississippi, DOE/NV--369, UC700, Washington, DC. 


\section{Appendix A Cone Penetrometer Testing Logs}


FUGRO GEOSCIENCES, INC.

\section{fukro}

6105 Rookin

Houston, Texes 77074

Tel: (713) 778-5580

Fax: (713) 778-5501

December 10, 1993

Report Number 0301-3144

IT Corporation

4330 South Valley View, Suite 114

Las Vegas, NV 89103-4047

Attention: Mr. Rich Deshler

\section{CONE PENETROMETER TESTING \\ GROUNDWATER AND SOIL SAMPLING RELATED SERVICES \\ TATUM DOME TEST SITE \\ LAMAR COUNTY, MISSISSIPPI \\ IT PROJECT NO: 301965}

Dear Mr. Deshler:

Please find enclosed herewith the resuits of the conductivity cone penetrometer tests conducted at the abovereferenced location. Cone penetrometer tests and sampling programs were carried out under the supervision of IT's field personnel. Also, test holes were grouted using volclay grout.

Fugro Geosciences appreciates the opportunity to be of service to IT Corporation. If you should have any questions, or if we can be of further assistance, please do not hesitate to contact us. We look forward to working with you in the future.

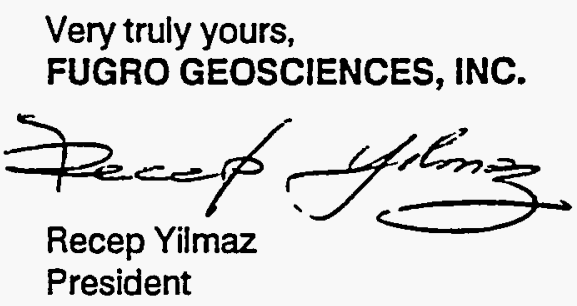

RY/cam 


\section{Key To Soil Classification and Symbols}

\section{SOIL TYPE}

(Shown in Symbol Column)$$
\text { Sand }
$$

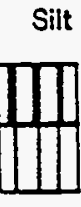

Fill

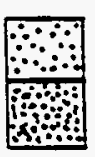

Sandy

Predominant Type Shown Heavy

\section{SAMPLE TYPE}

(Shown in Samples Column)

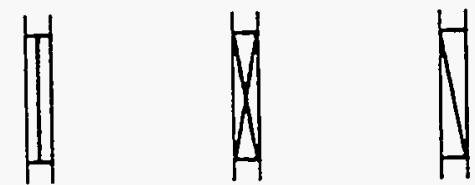

Rock Core Split Spoon No Recovery

\section{TERMS DESCRIBING CONSISTENCY OR CONDITION}

COARSE GRAINED SOILS (Major portion Retained on No. 200 Sieve)

Includes (1) clean gravels and sand described as fine, medium or course, depending on distribution of grain sizes (2) silty or clayey gravels and sands and (3) fine grained low plasticity soils (PI < 10) such as sandy silts. Condition is rated according to relative density, as determined by lab tests or estimated from resistance to sampler penetration.

\begin{tabular}{l} 
Descriptive Term \\
\hline Loose \\
Medium Dense \\
Dense \\
Very Dense
\end{tabular}

\begin{tabular}{c} 
Penetration Resistance* \\
\hline $0-10$ \\
$10-30$ \\
$30-50$ \\
Over 50
\end{tabular}

\section{Relative Density \\ 0 to $40 \%$ \\ 40 to $70 \%$ \\ 70 to $90 \%$ \\ 90 to $100 \%$}

* Blows/Foot, 140* Hammer, 30* Drop

FINE GRAINED SOILS (Majior Portion Passing No. 200 Sieve)

Includes (1) inorganic and organic silts and clays, (2) sandy, gravelly or silty clays, and (3) clayey silts. Consistency is rated according to shearing strength, as indicated by penetrometer readings or by unconfined compression tests for soils with $\mathrm{PI} \geq 10$.

\begin{tabular}{l} 
Descriptive \\
Term \\
\hline Very Soft \\
Soft \\
Firm \\
Stiff \\
Very Stiff \\
Hard
\end{tabular}

\begin{tabular}{l}
$\begin{array}{c}\text { Cohesive Shear Strength } \\
\text { Tons/Square Foot }\end{array}$ \\
\hline Less Than 0.125 \\
0.125 to 0.25 \\
0.25 to 0.50 \\
0.50 to 1.00 \\
1.00 to 2.00 \\
2.00 and Higher
\end{tabular}

Note: Slickensided and fissured clay may have lower unconfined compressive strengths than shown above because of planes of weakness or shrinkage cracks; consistency ratings of such soils ase based on hand penetrometer readings.

\section{TERMS CHARACTERIZING SOIL STRUCTURE}

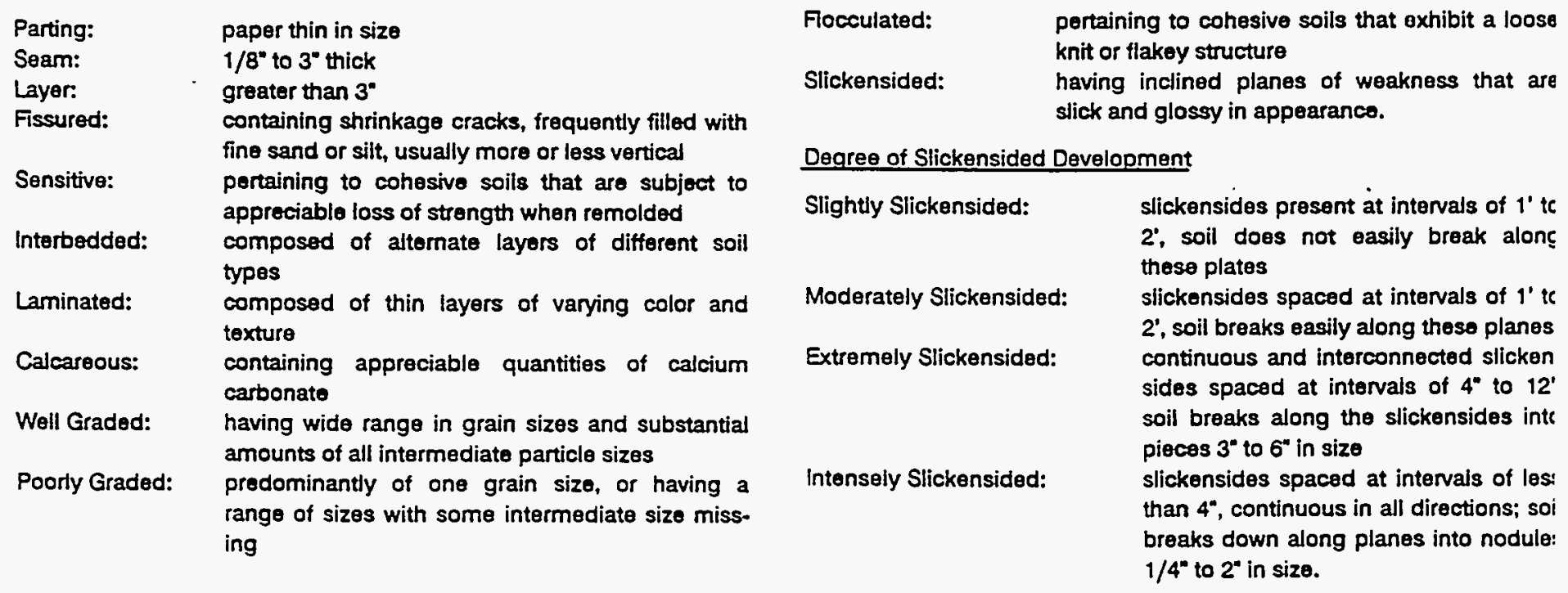




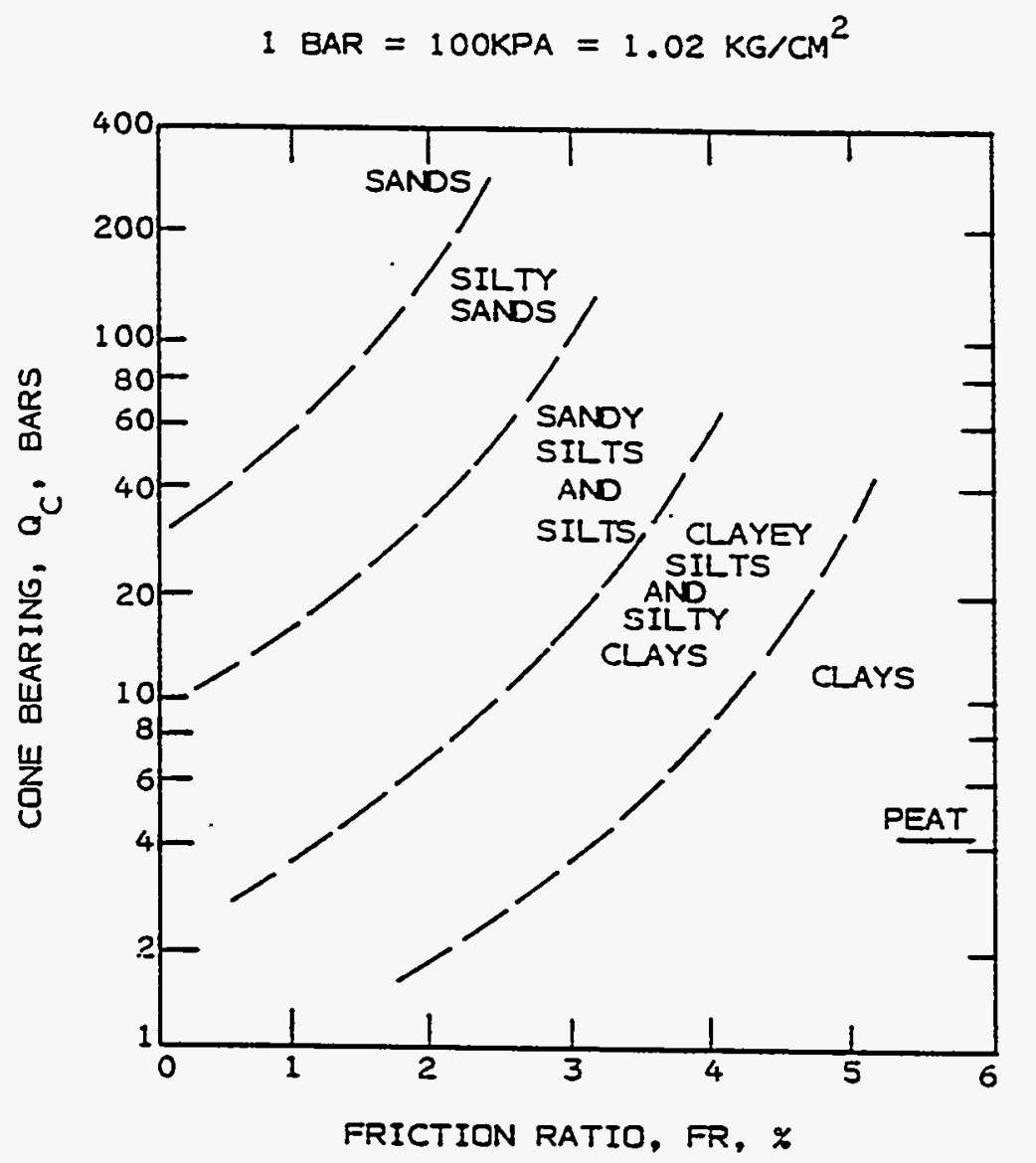

CAMPANELLA AND ROBERTSON CLASSIFICATION CHART 


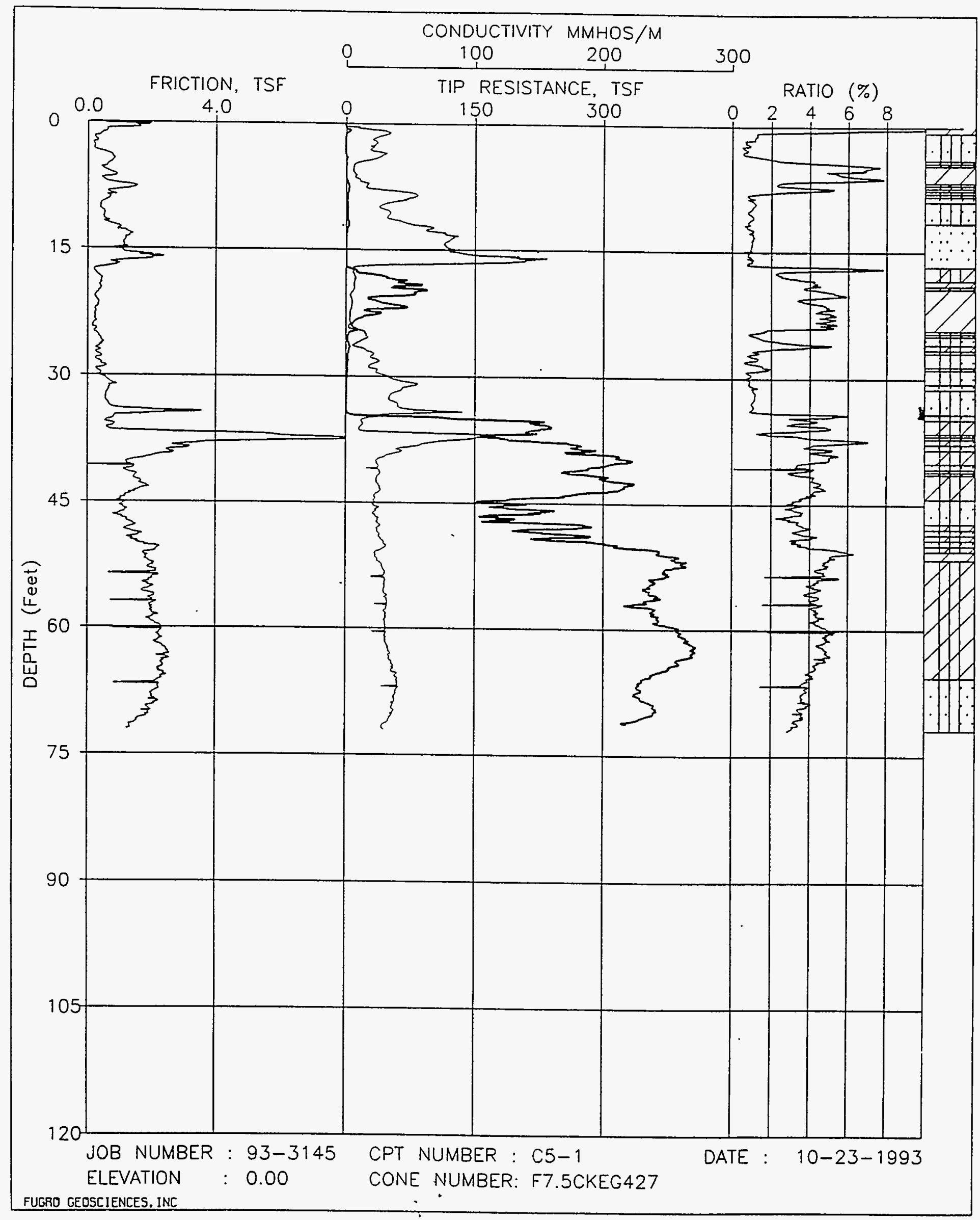




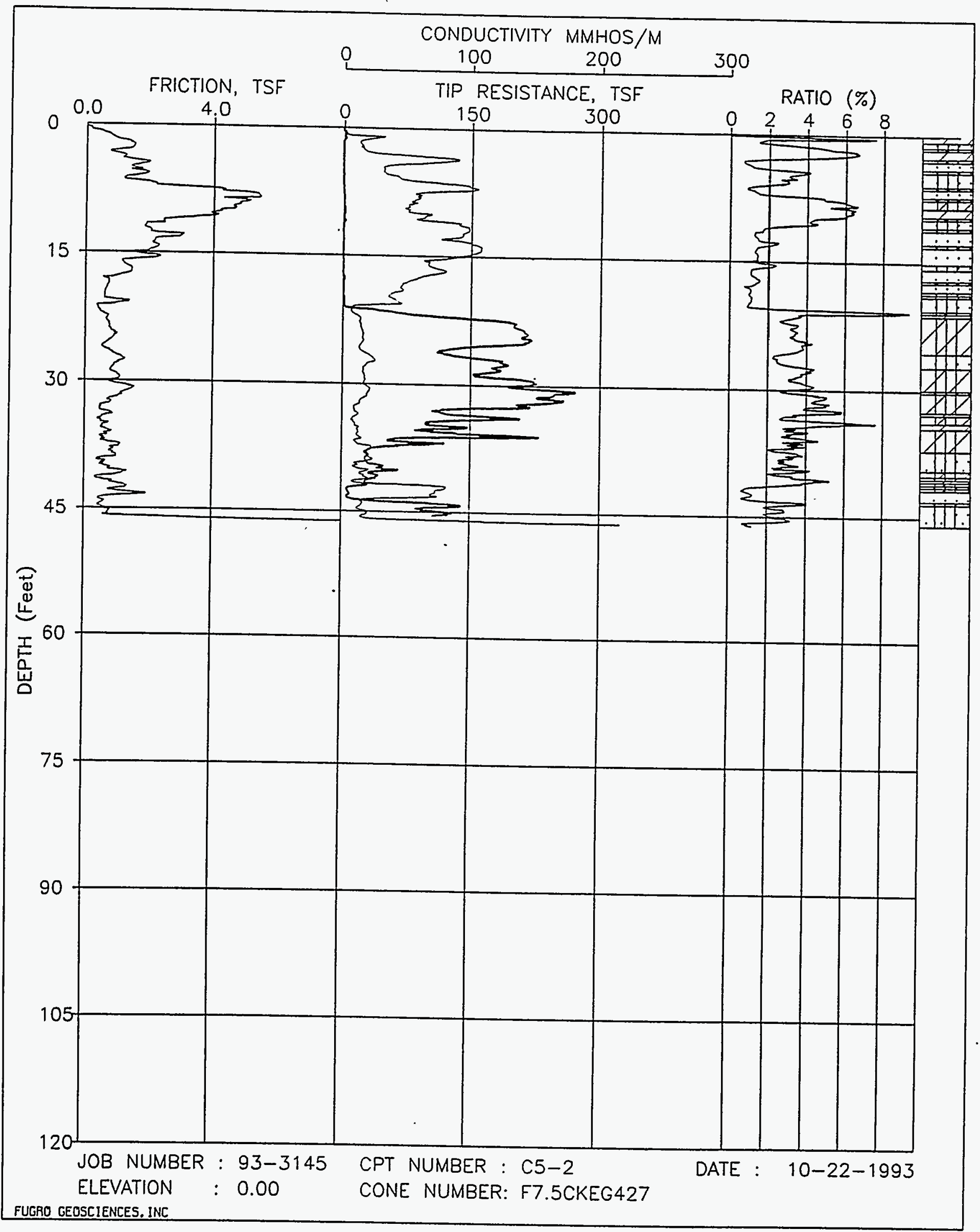




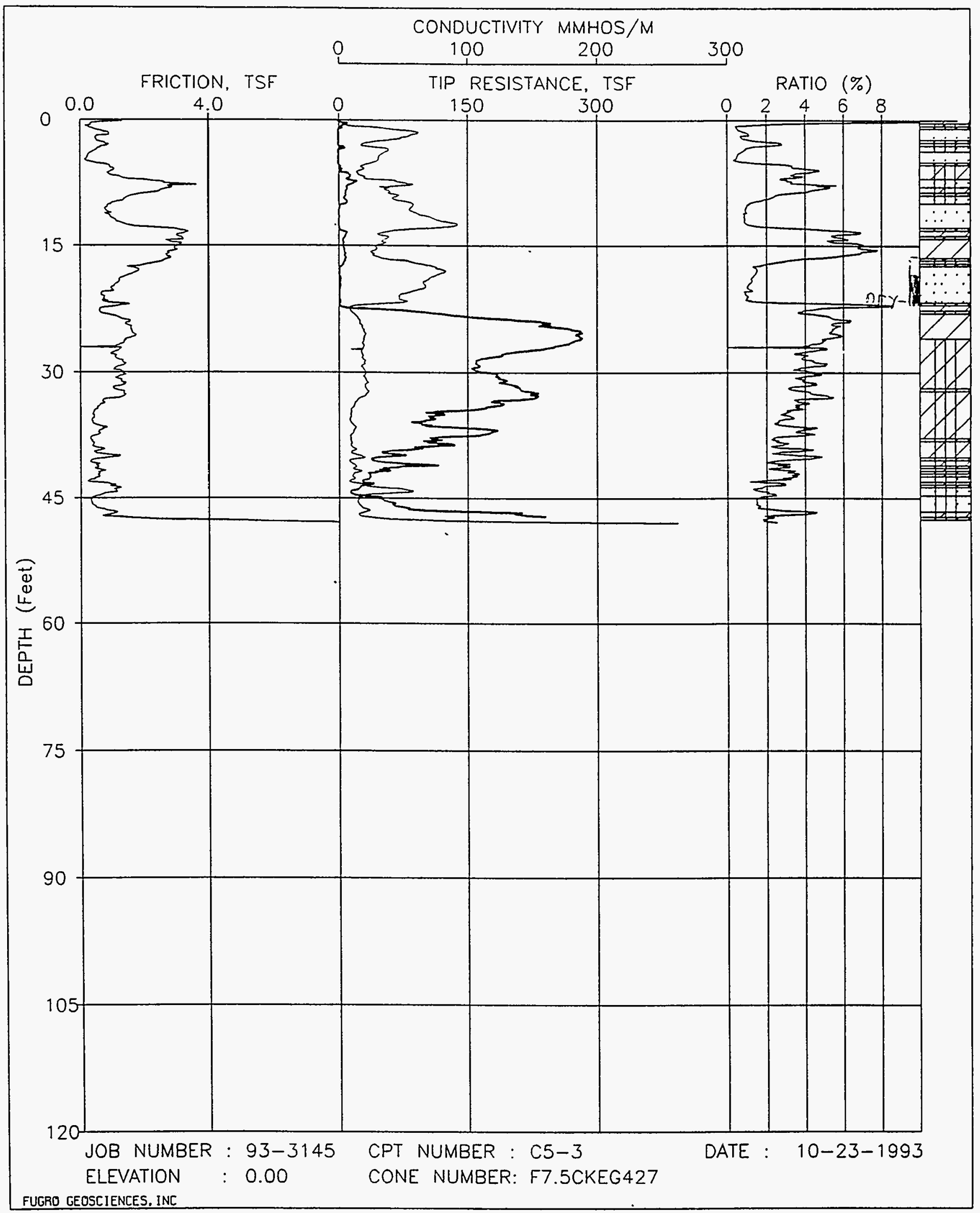




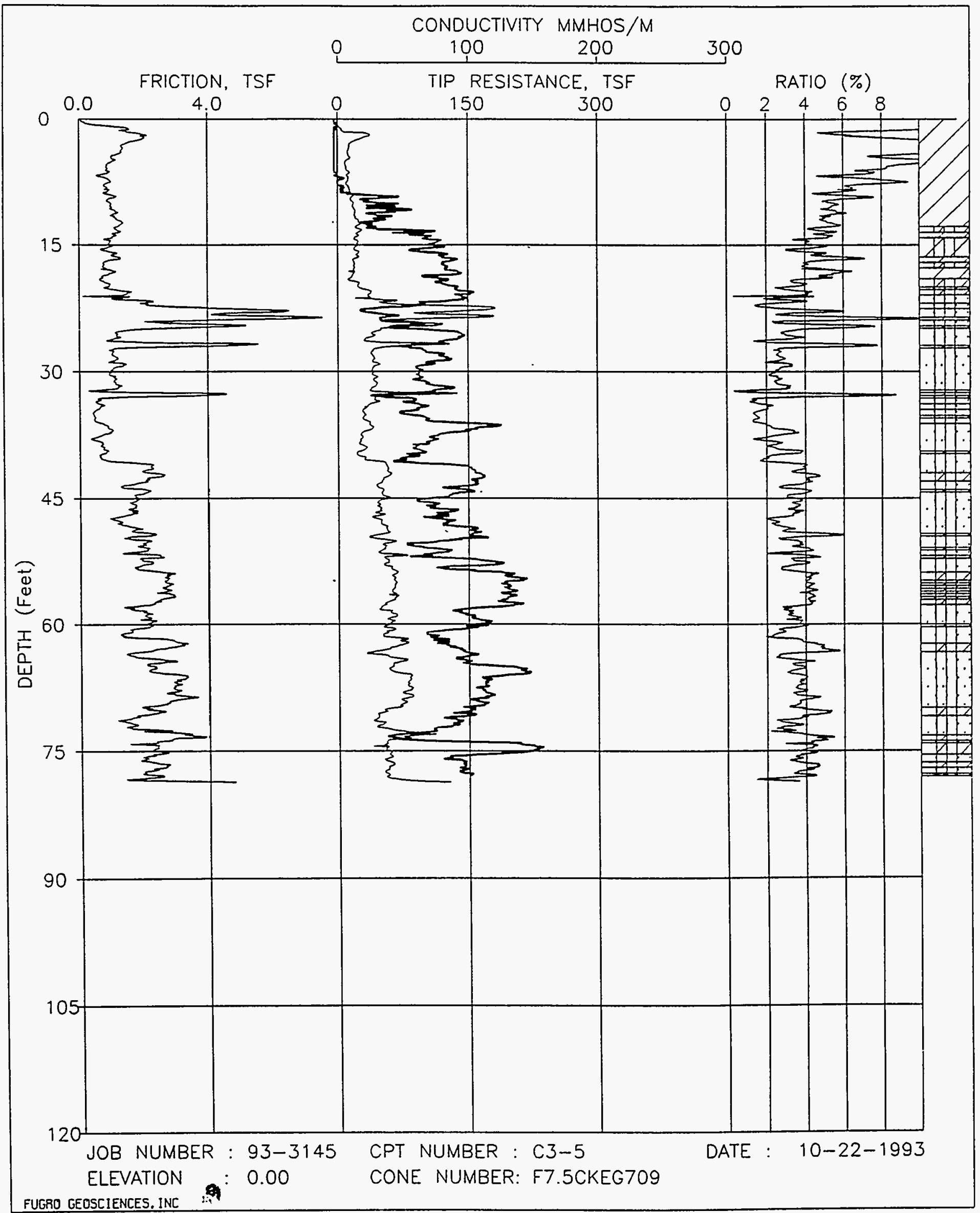




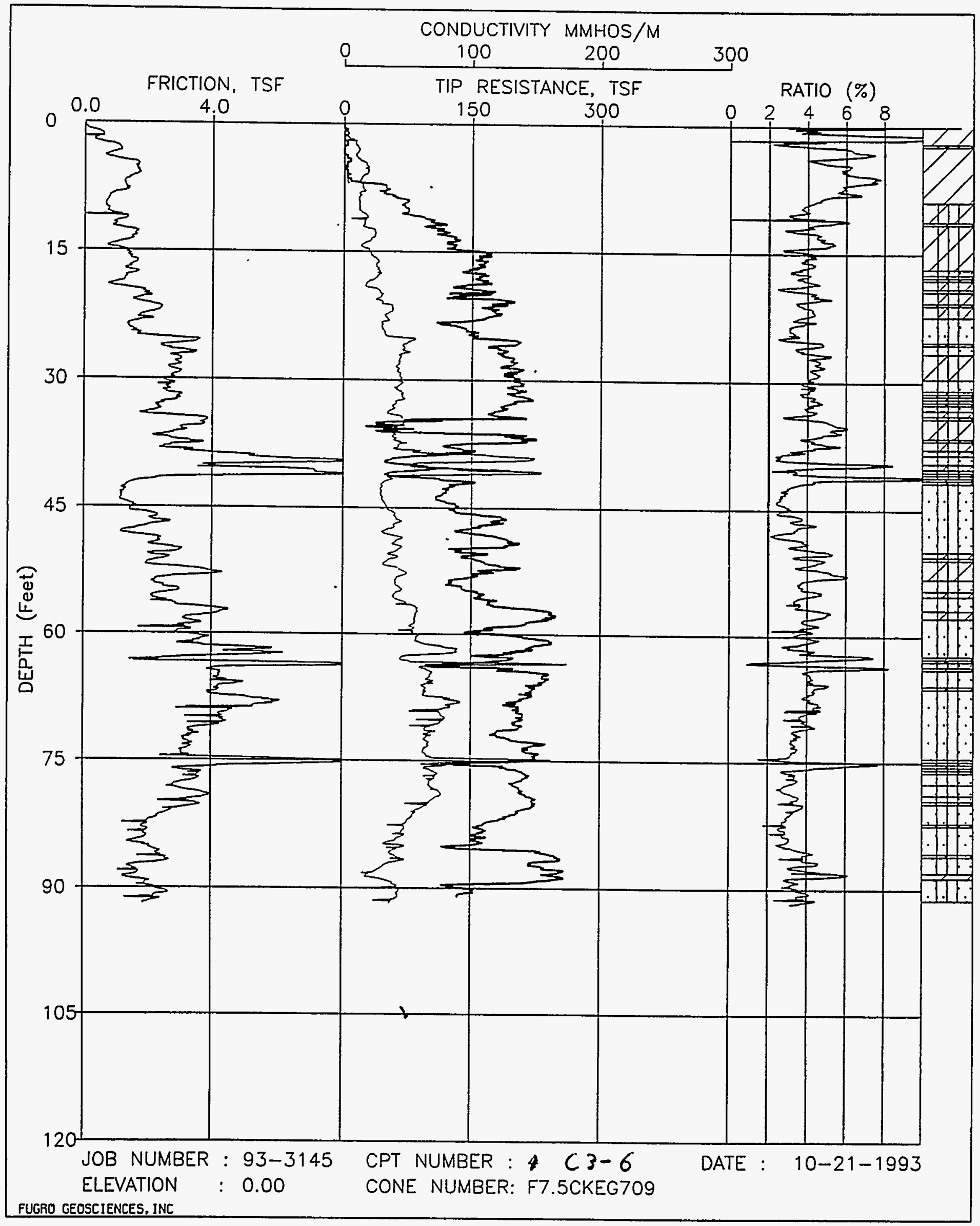




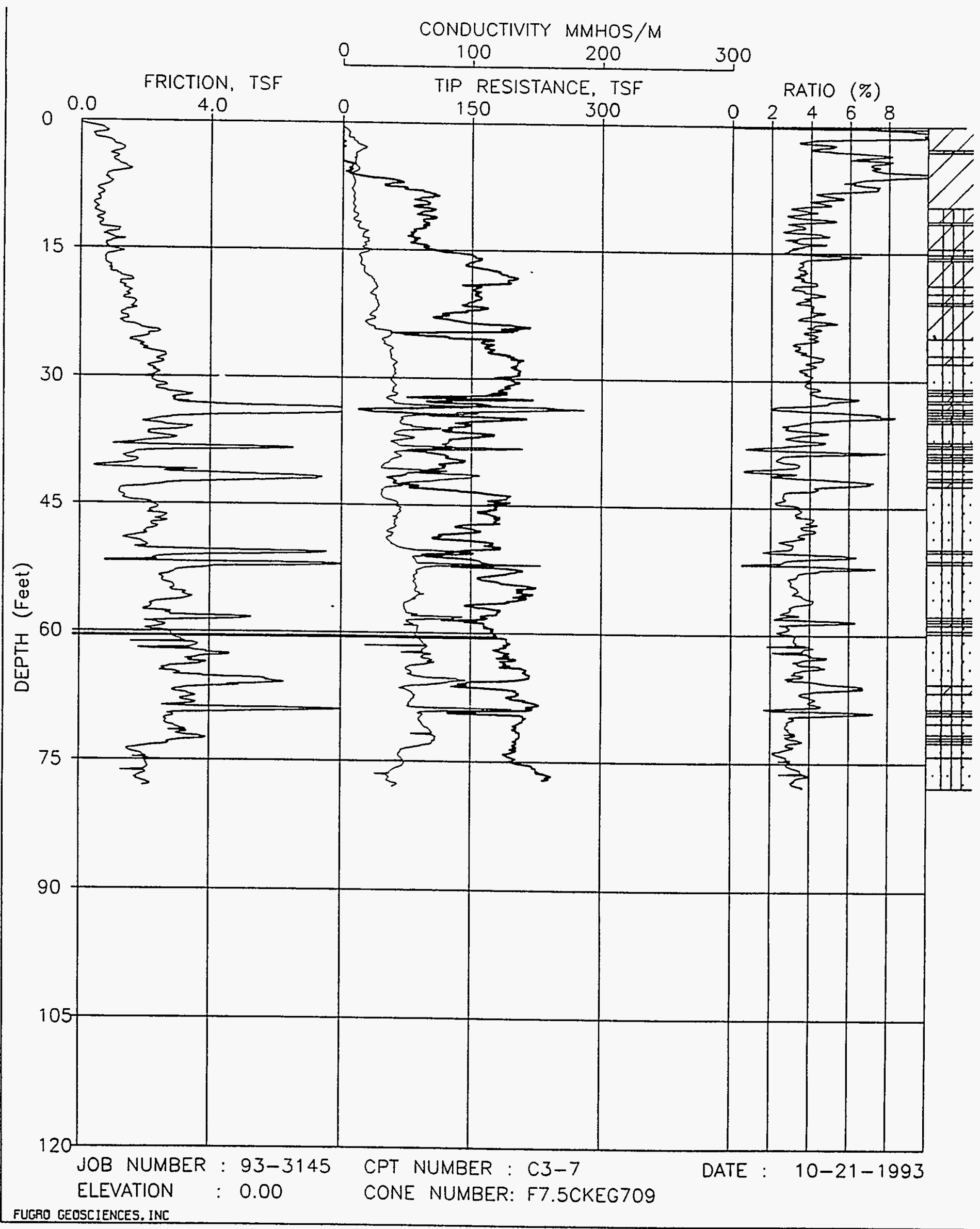




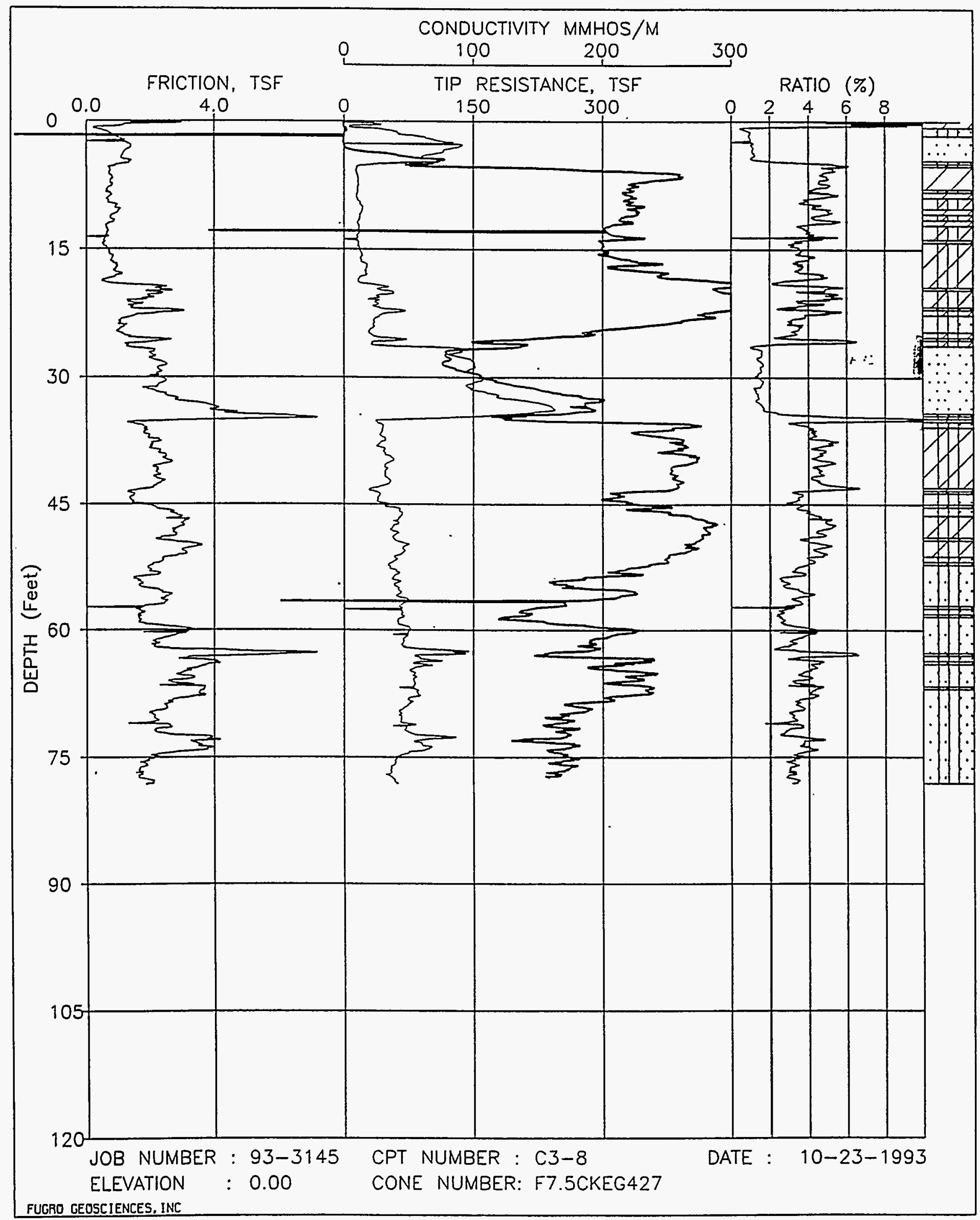




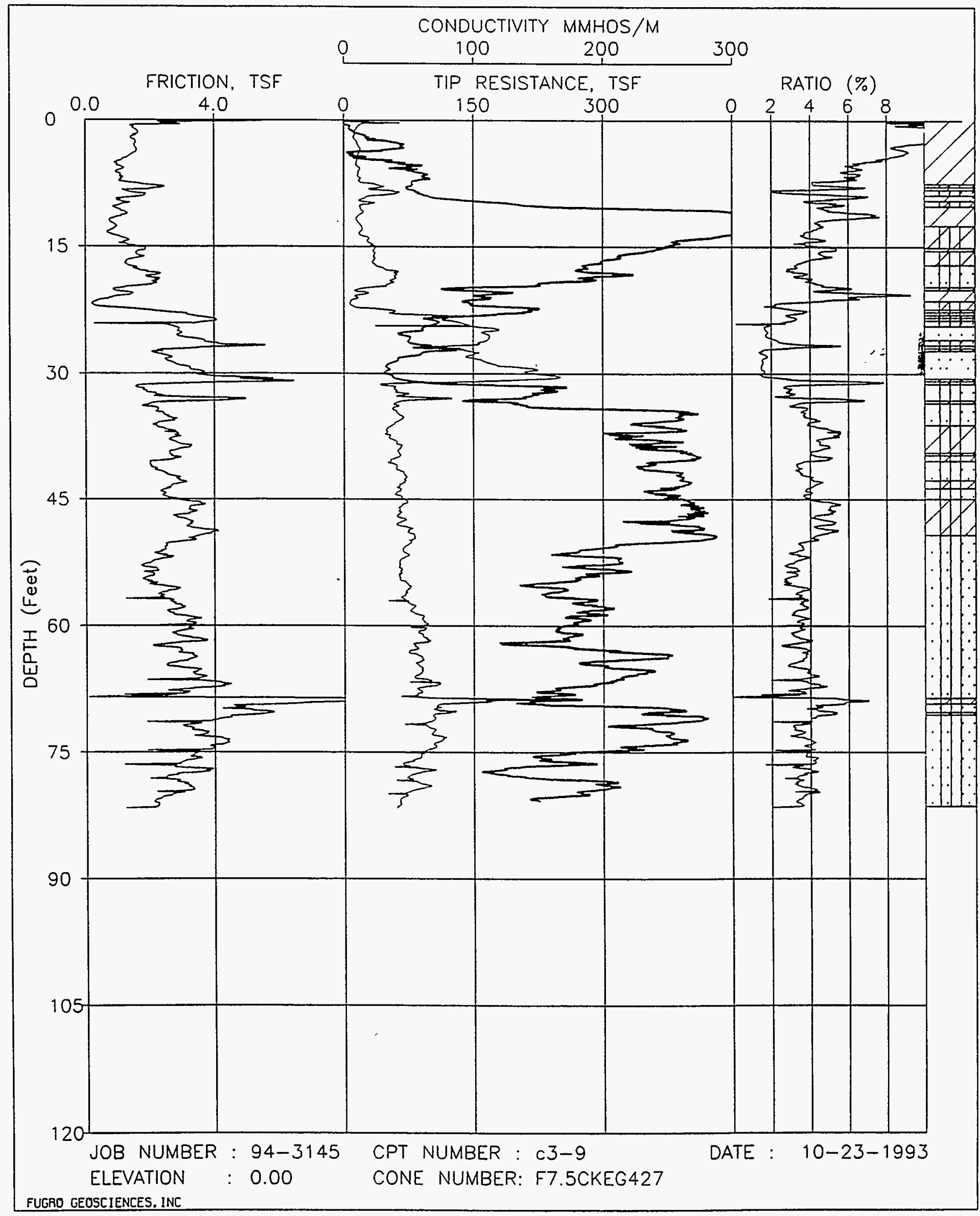




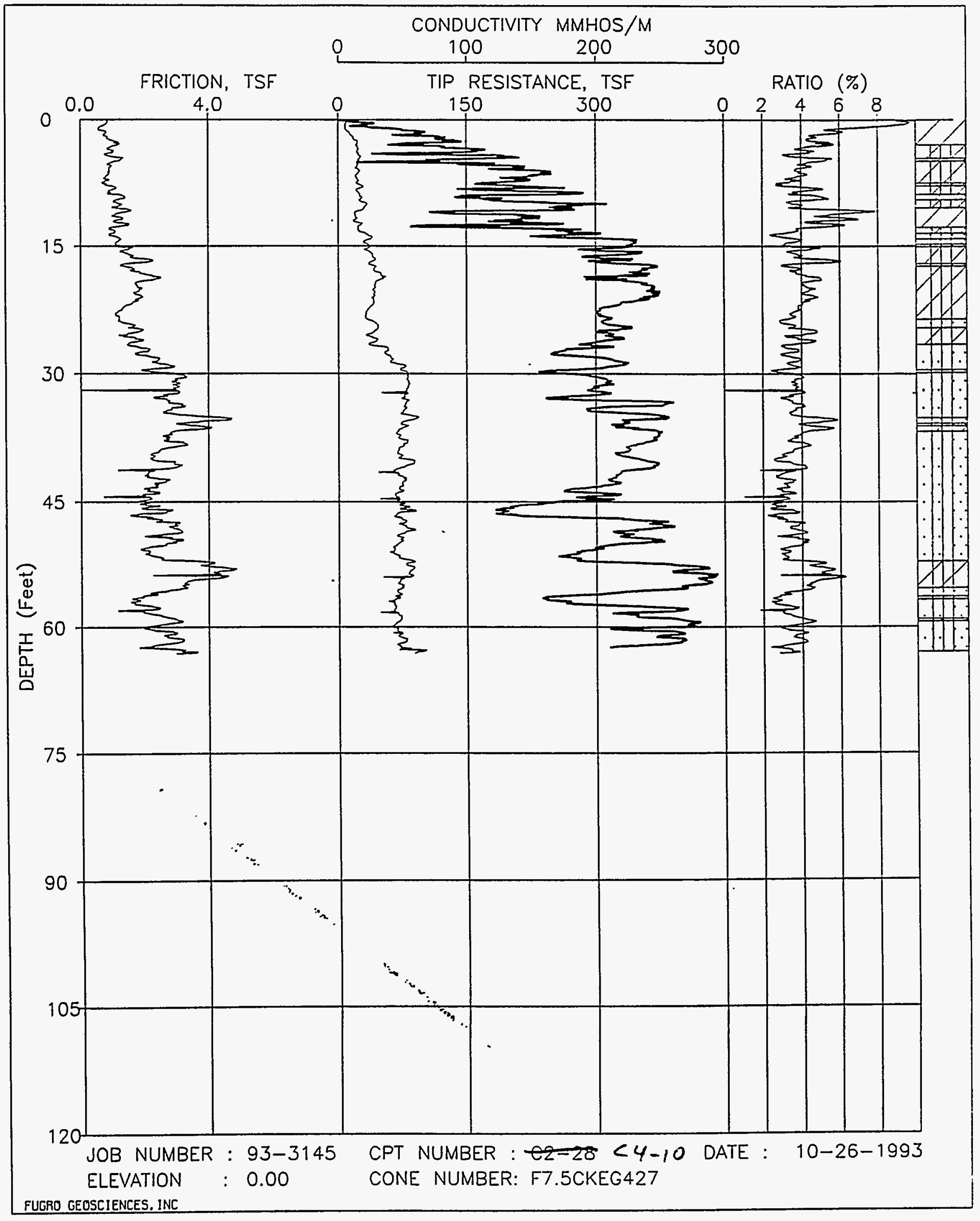




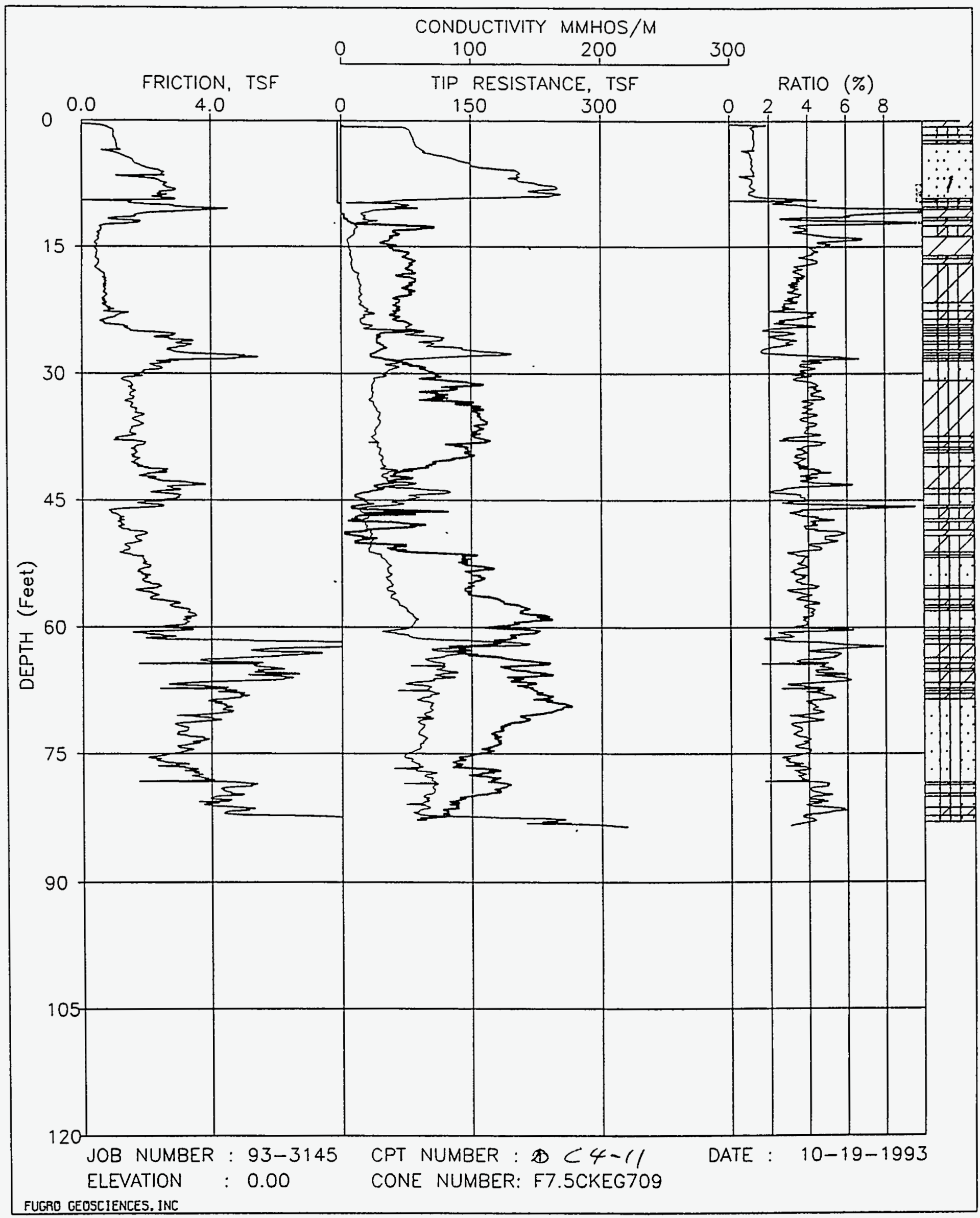




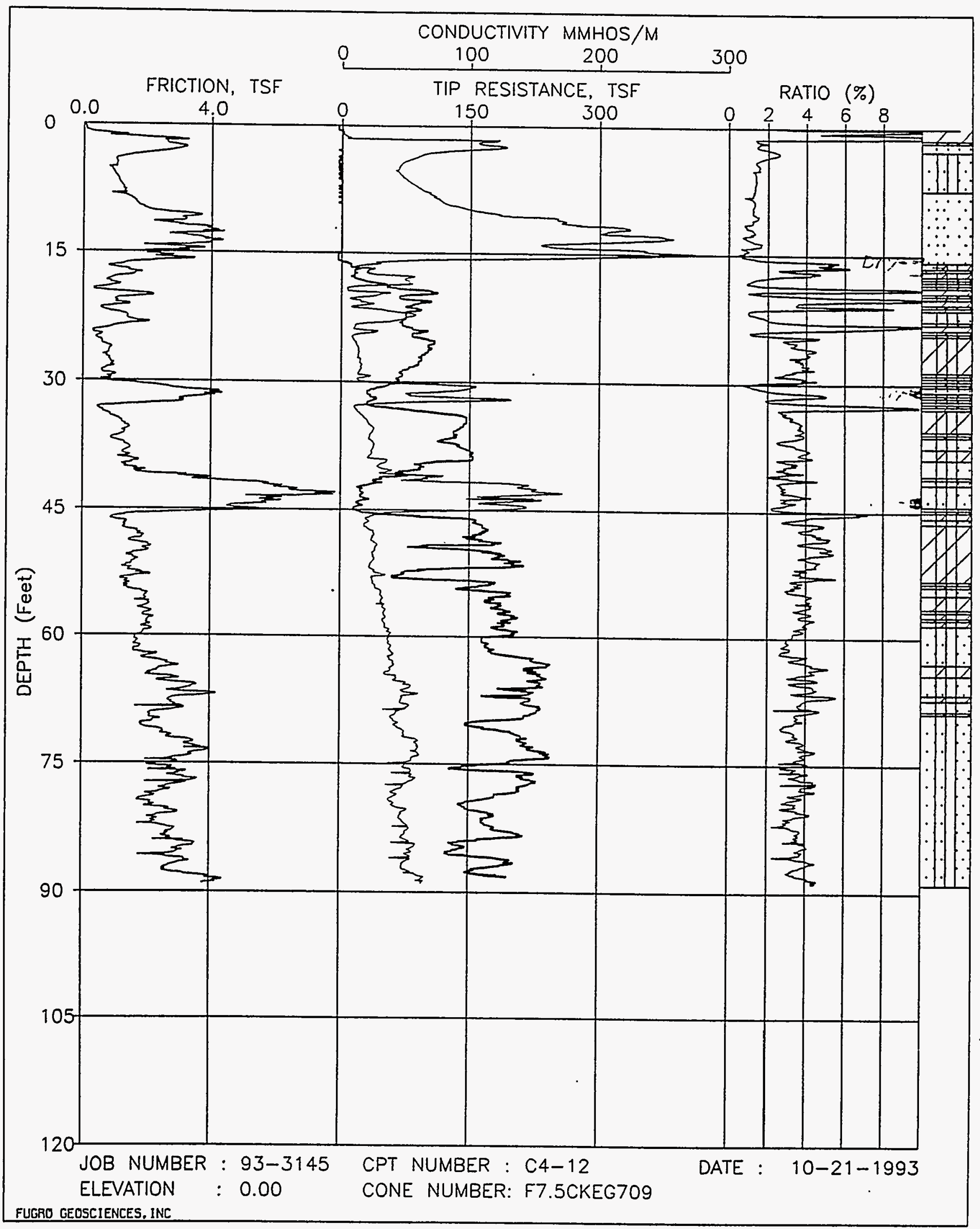




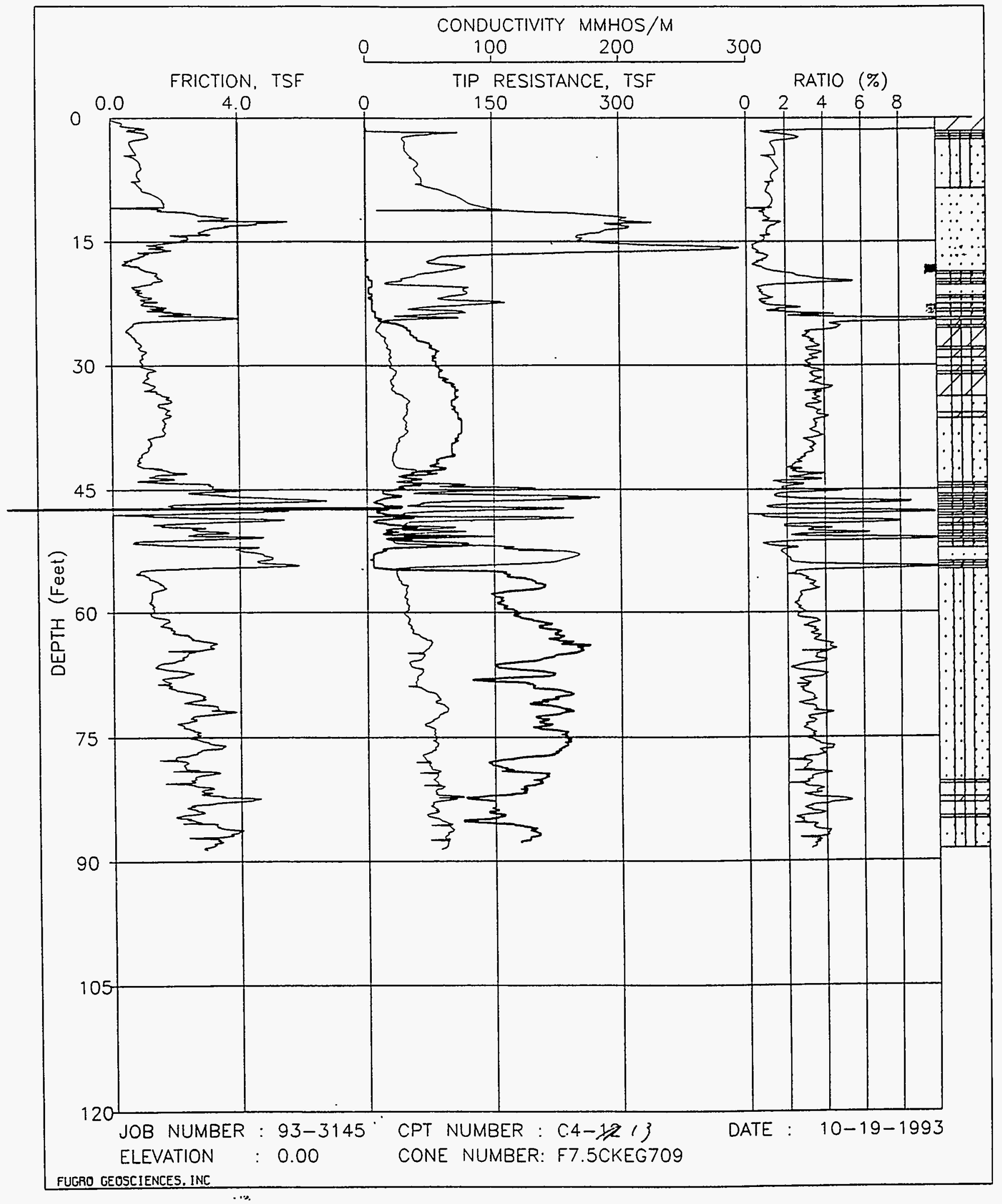




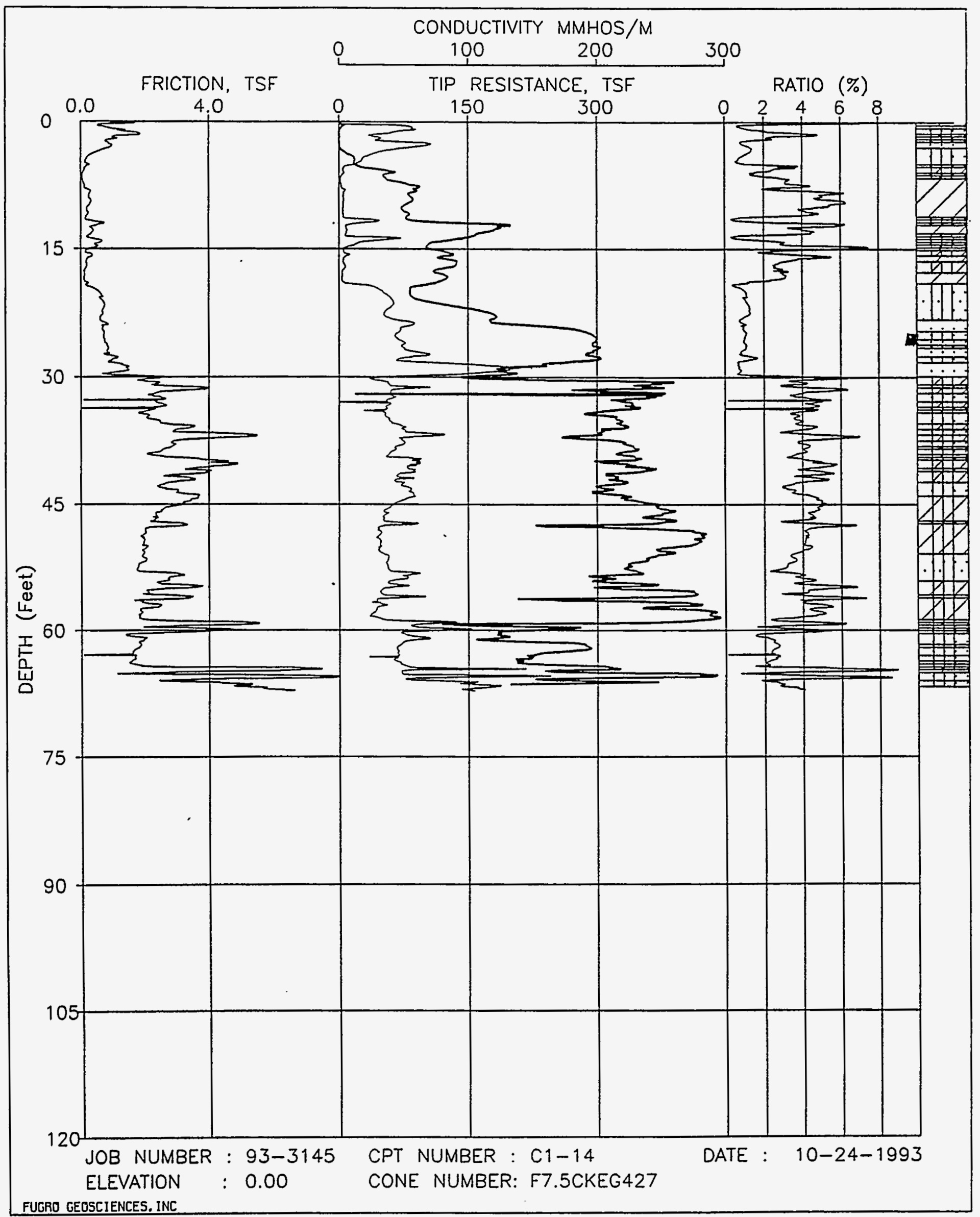




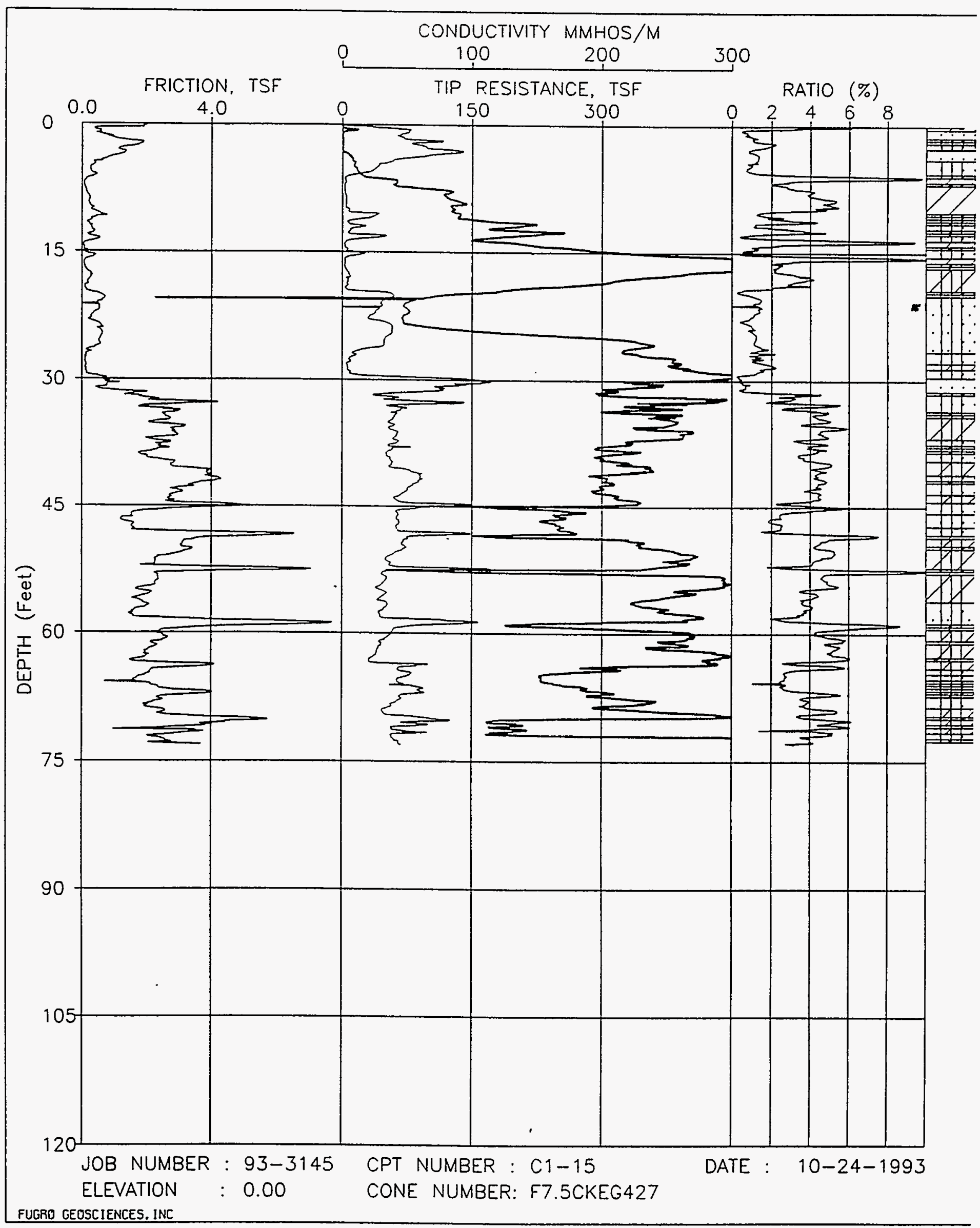




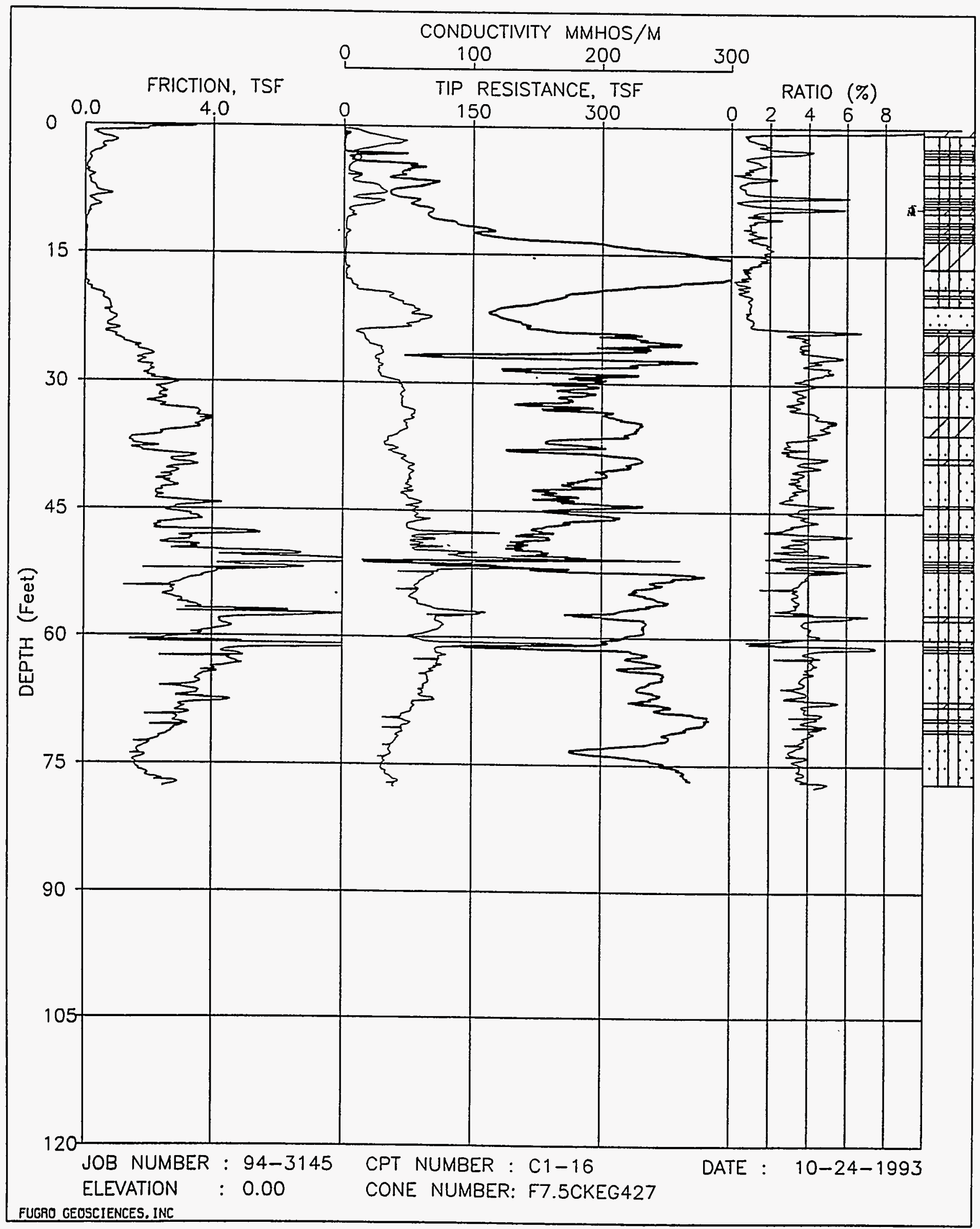




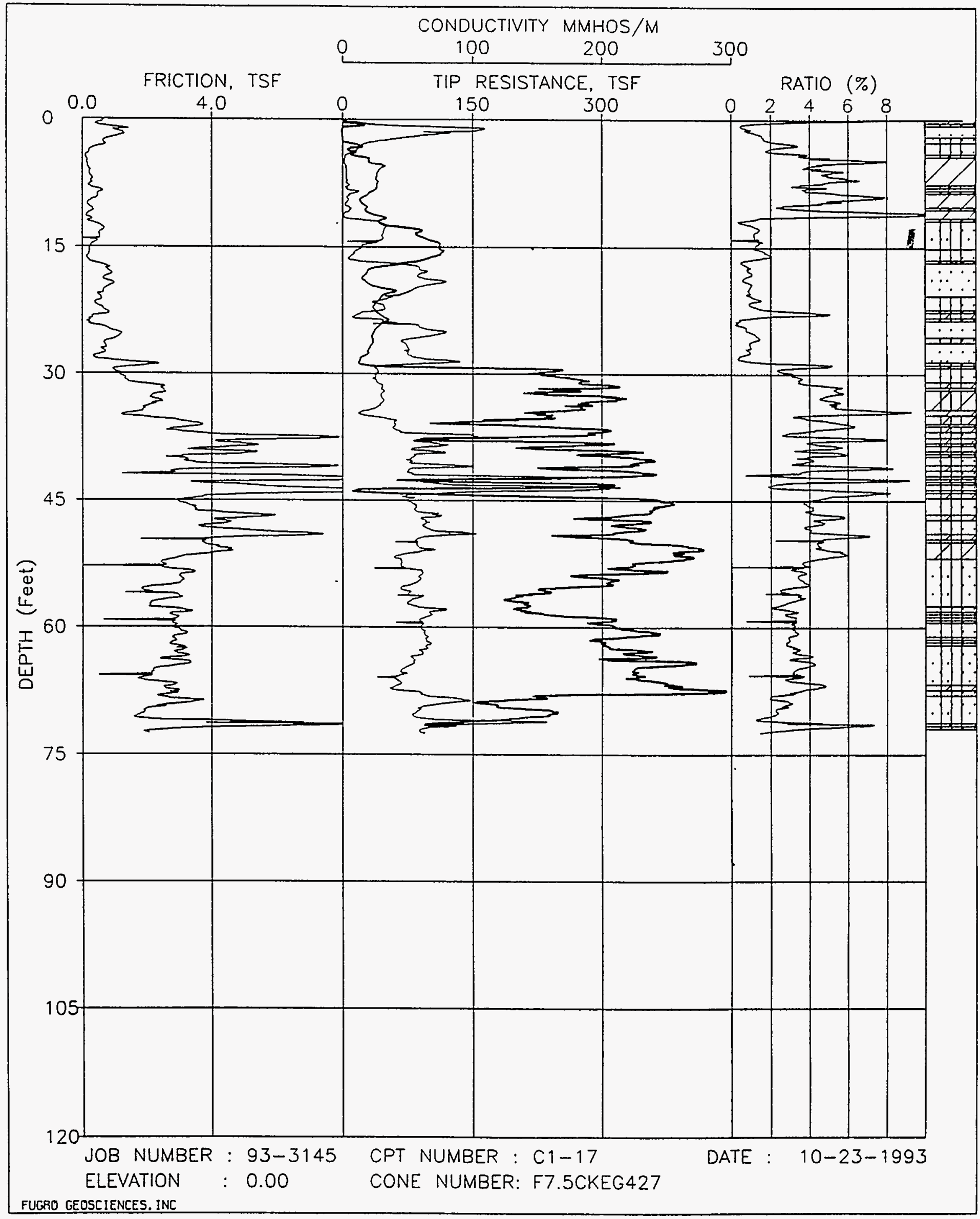




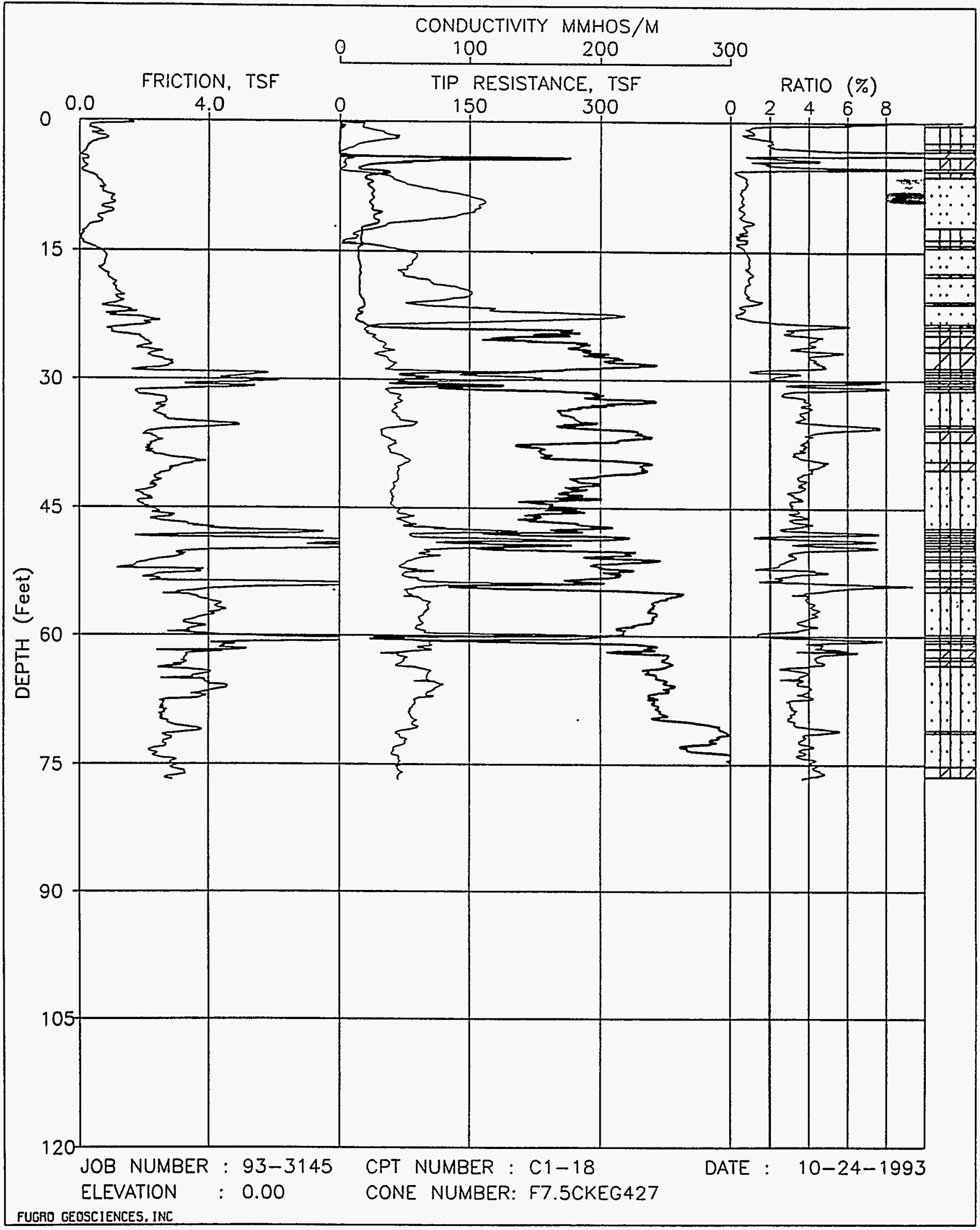




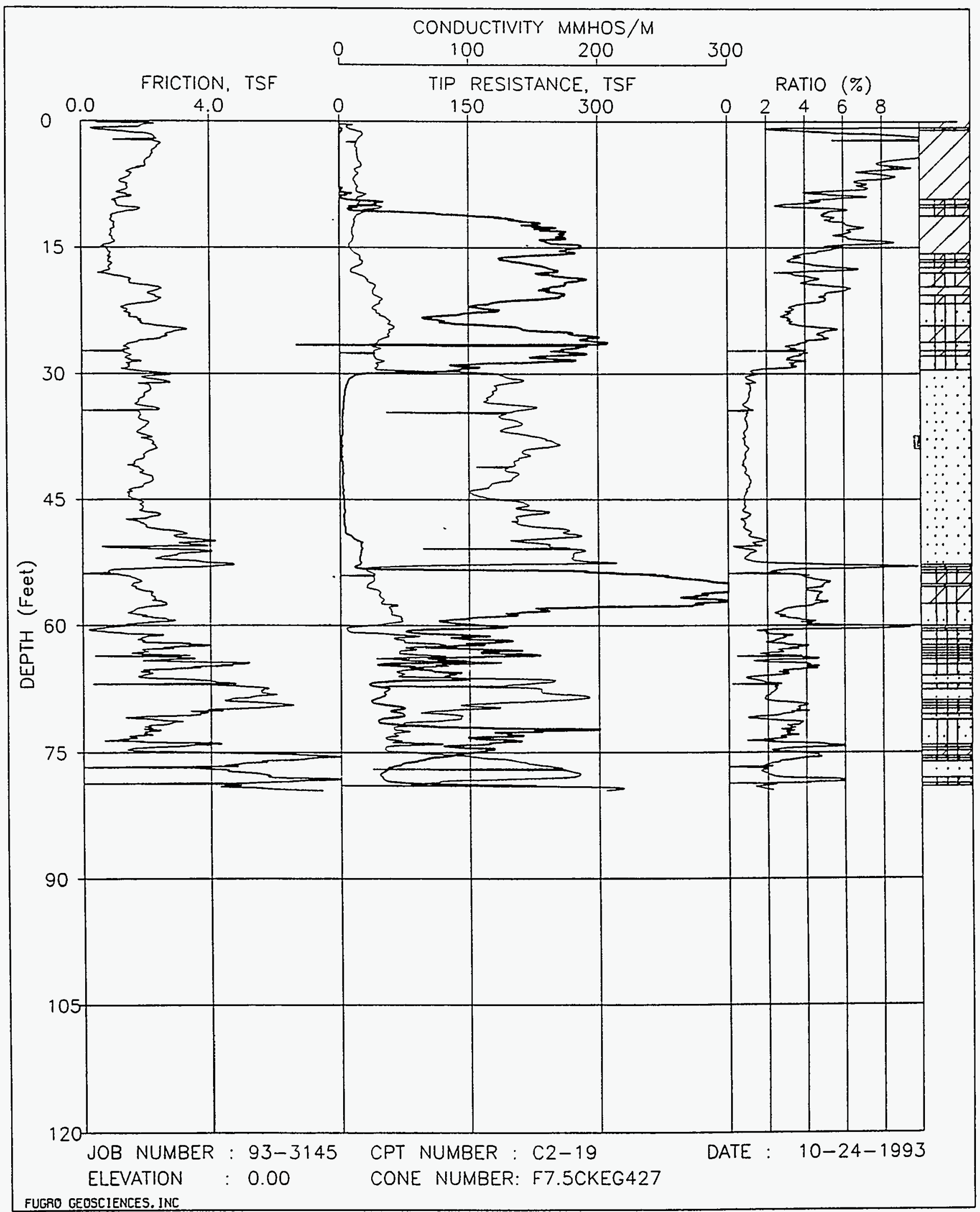




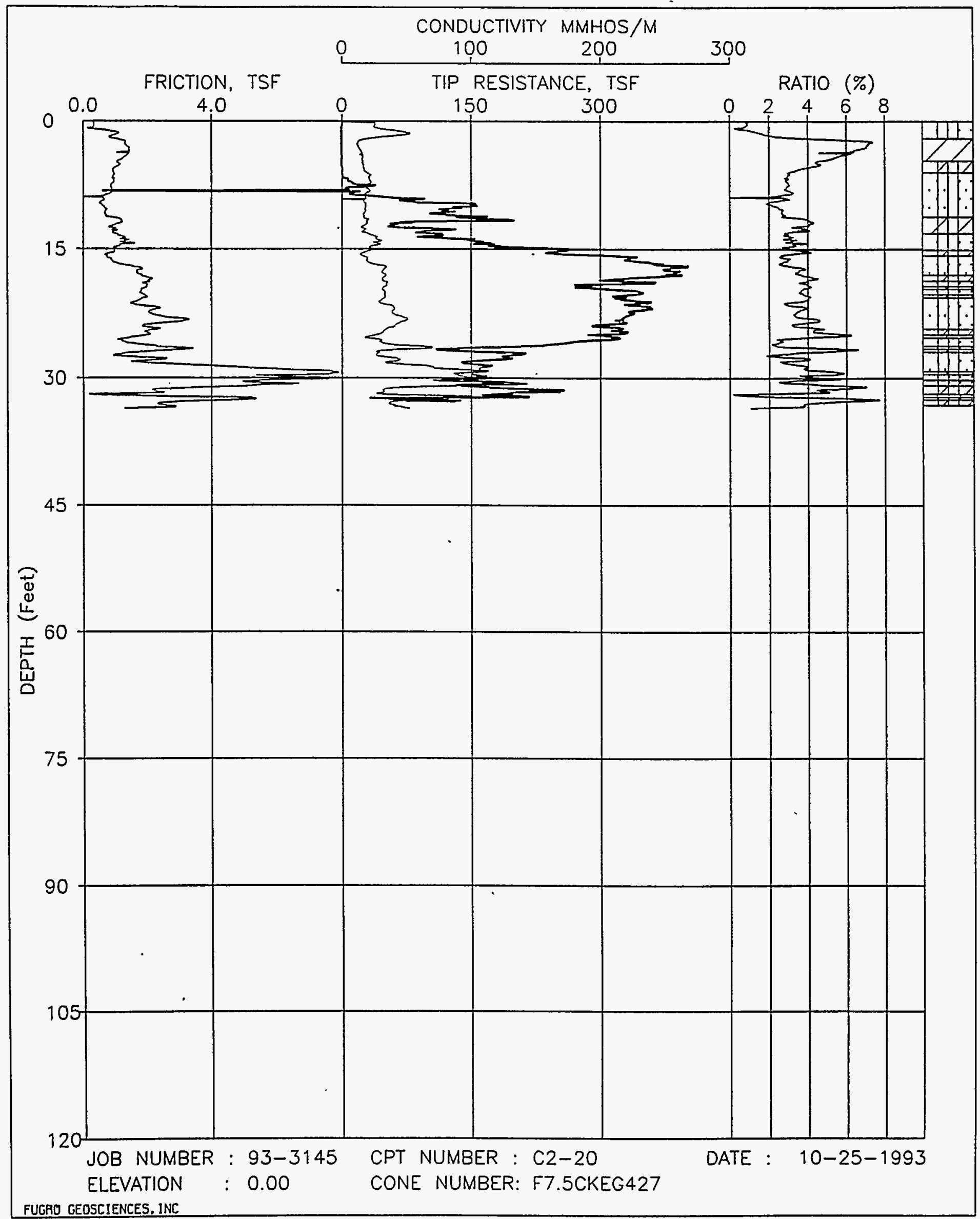




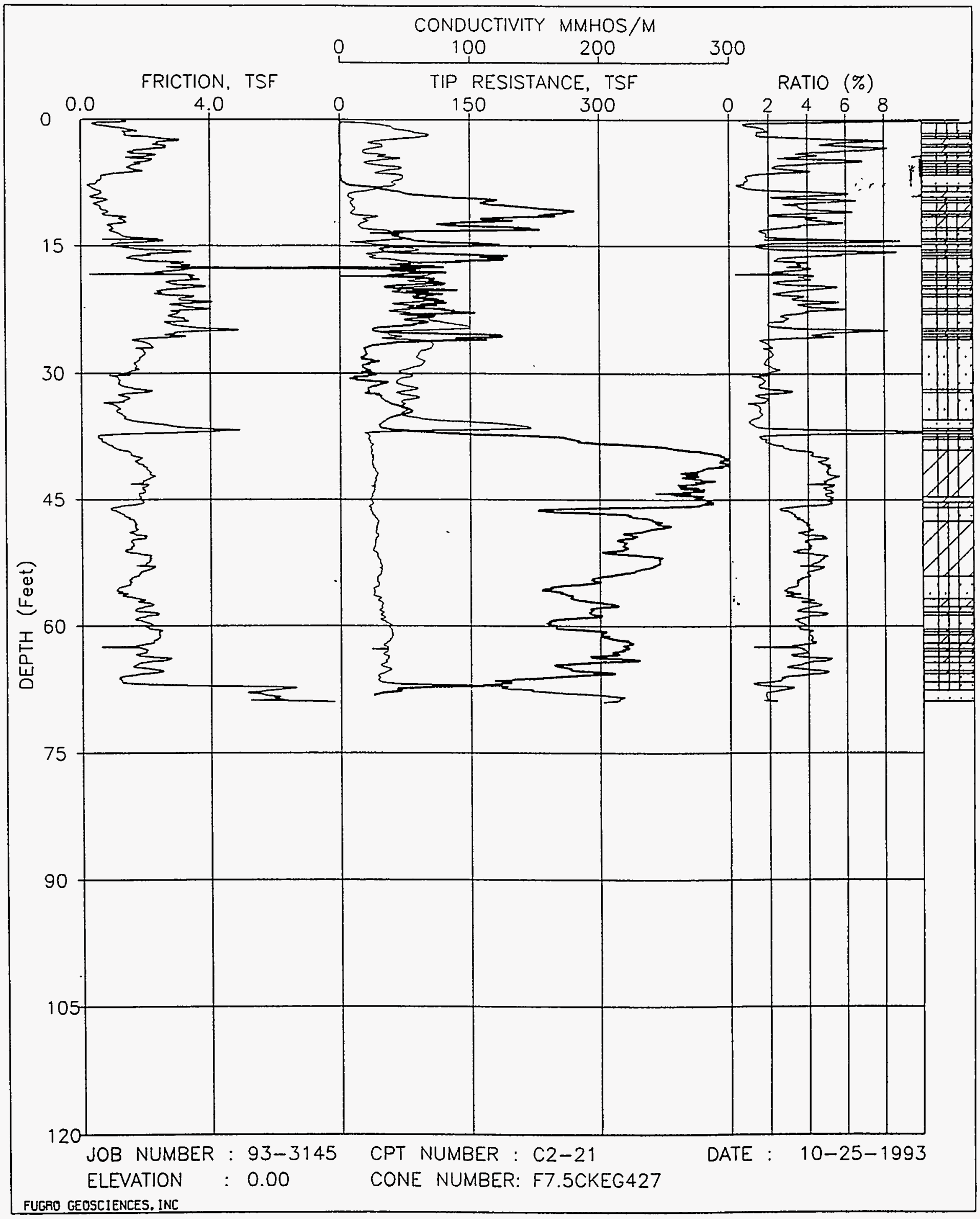




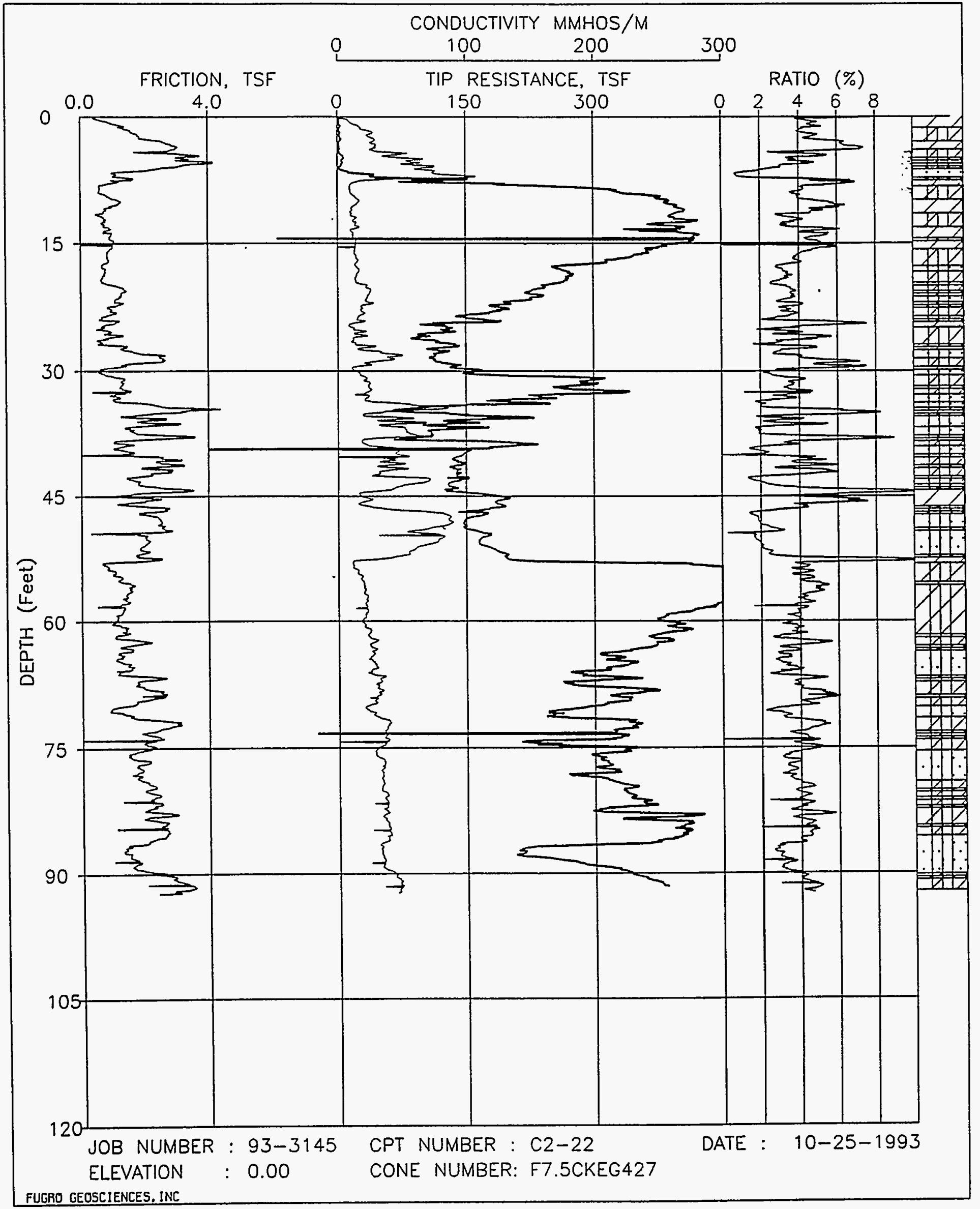




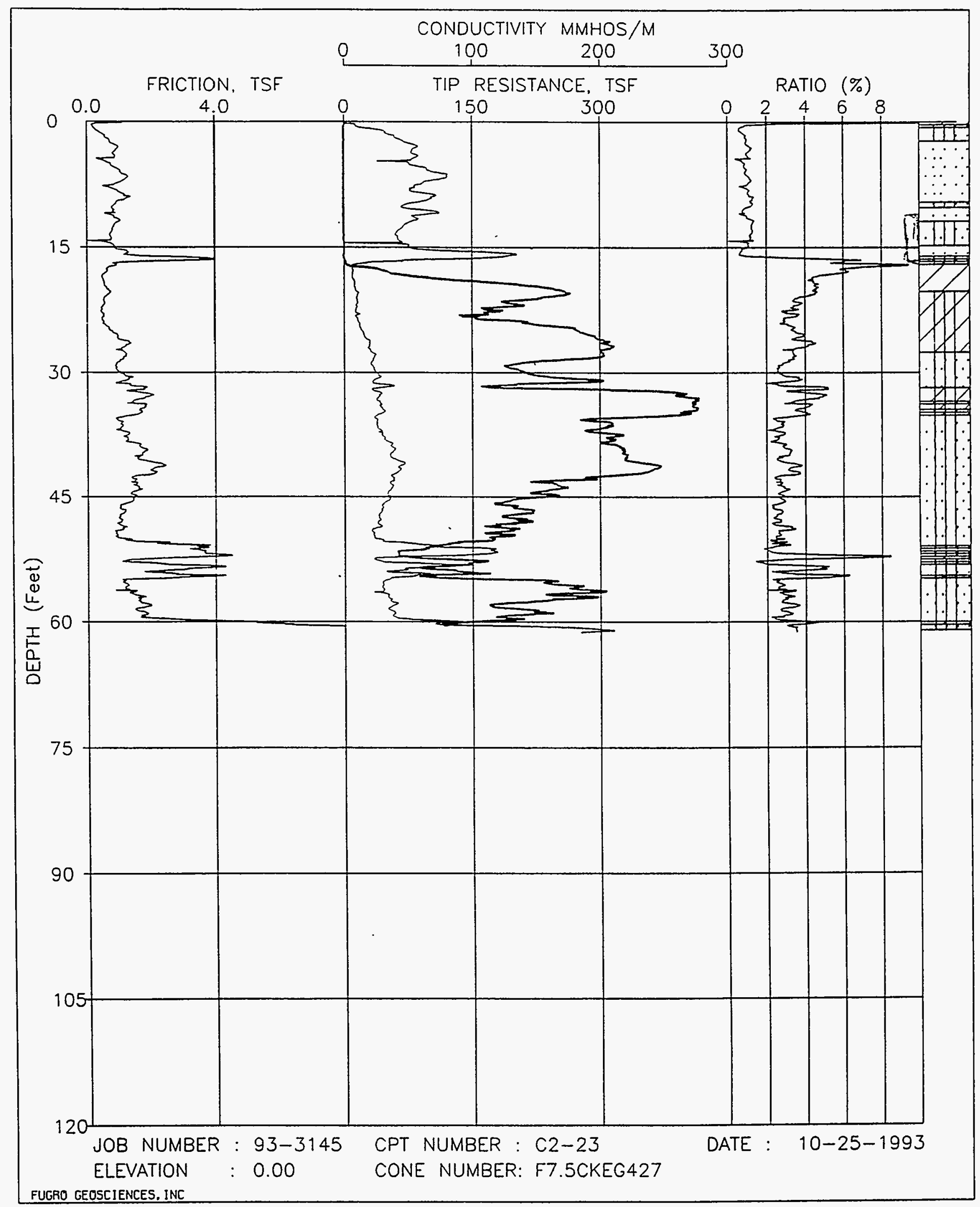




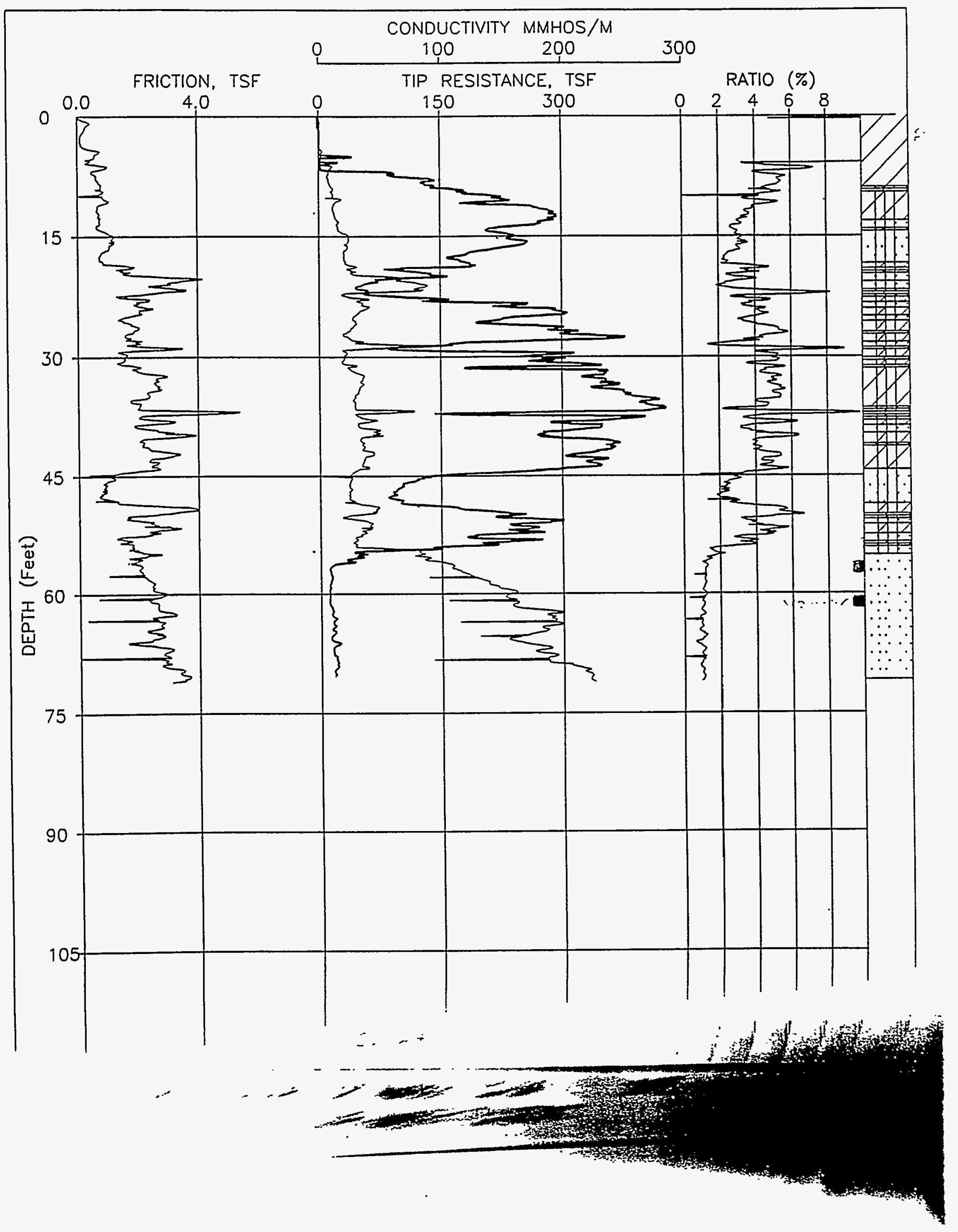




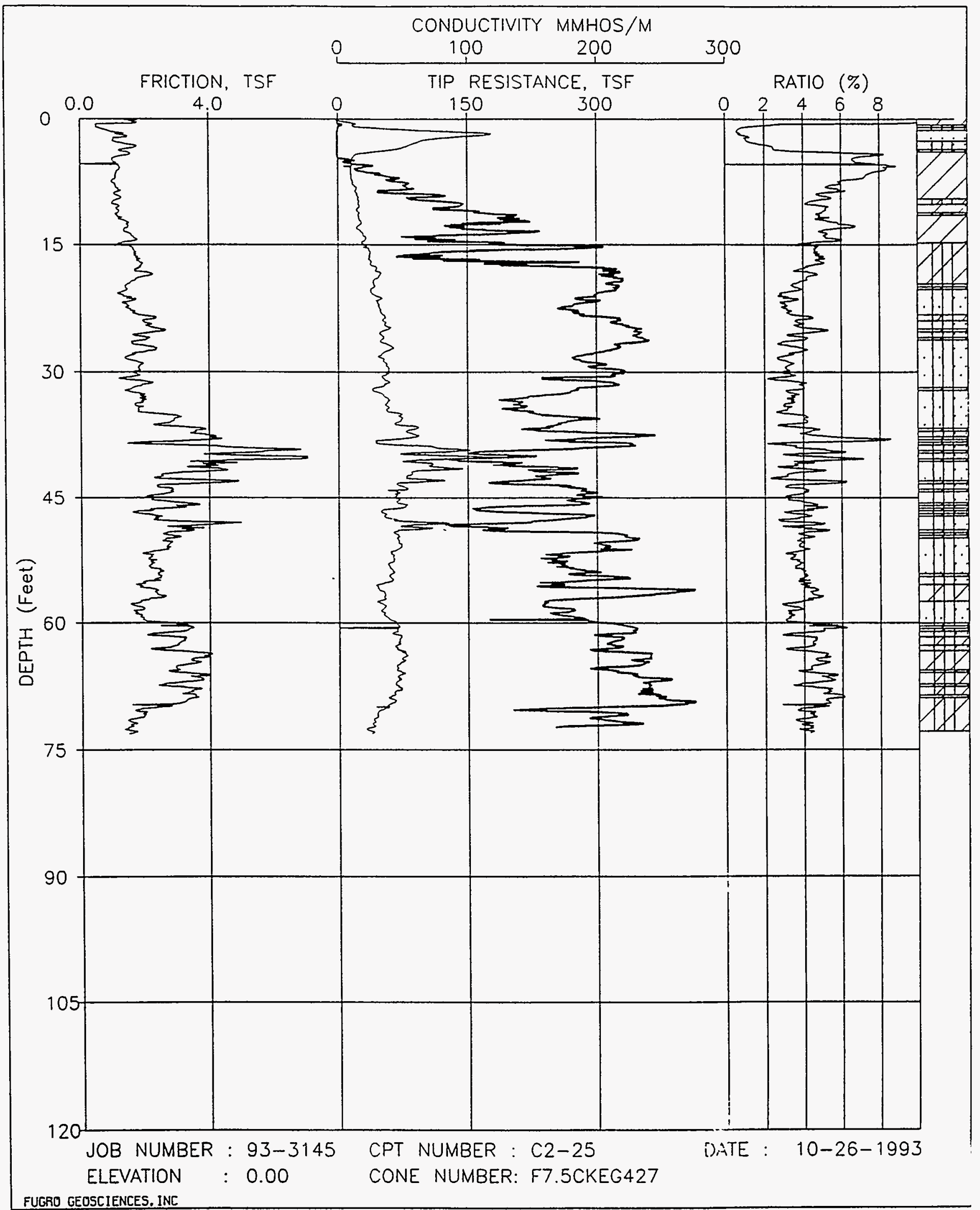




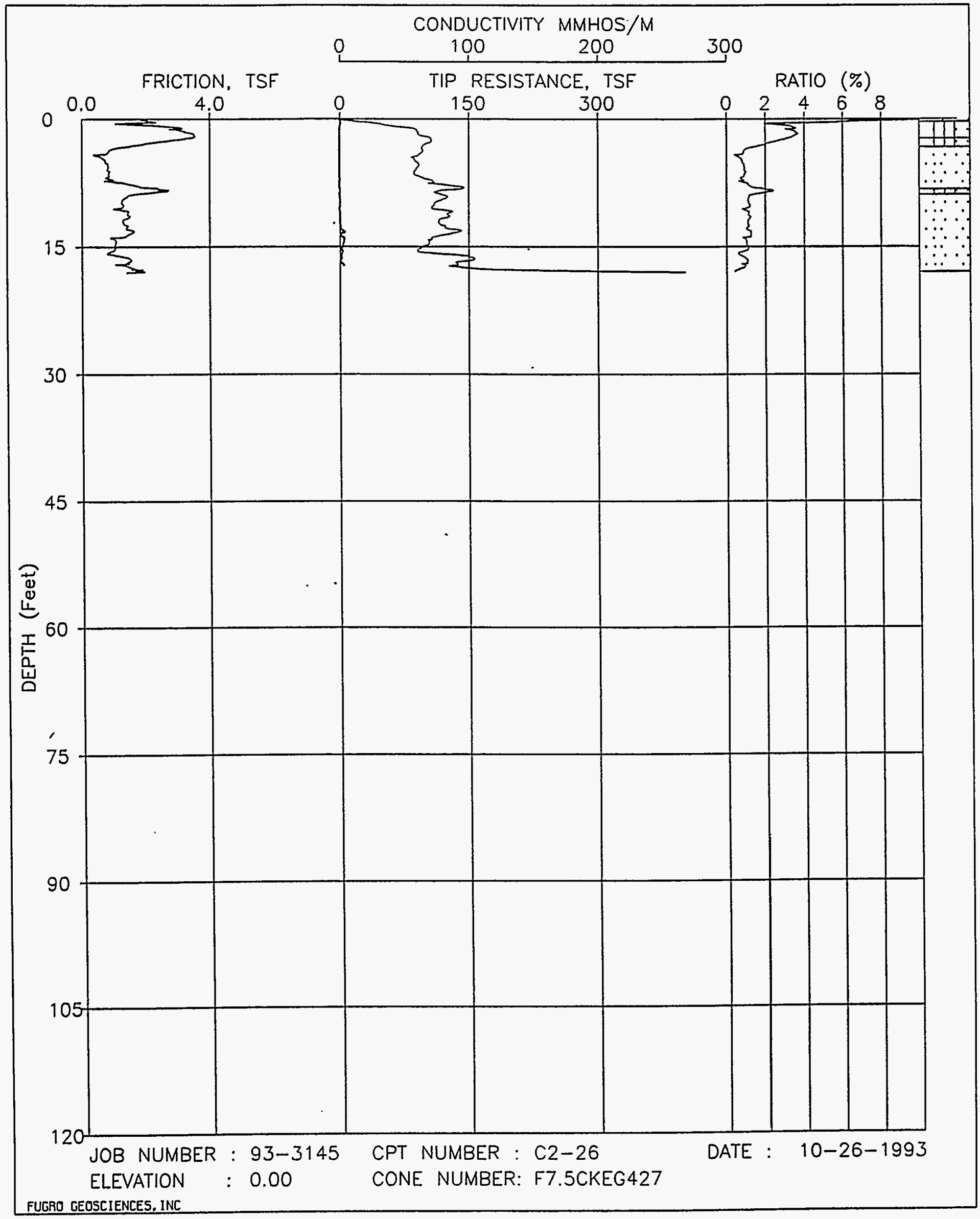


$\mid 1 \cdot-1-=-2=$

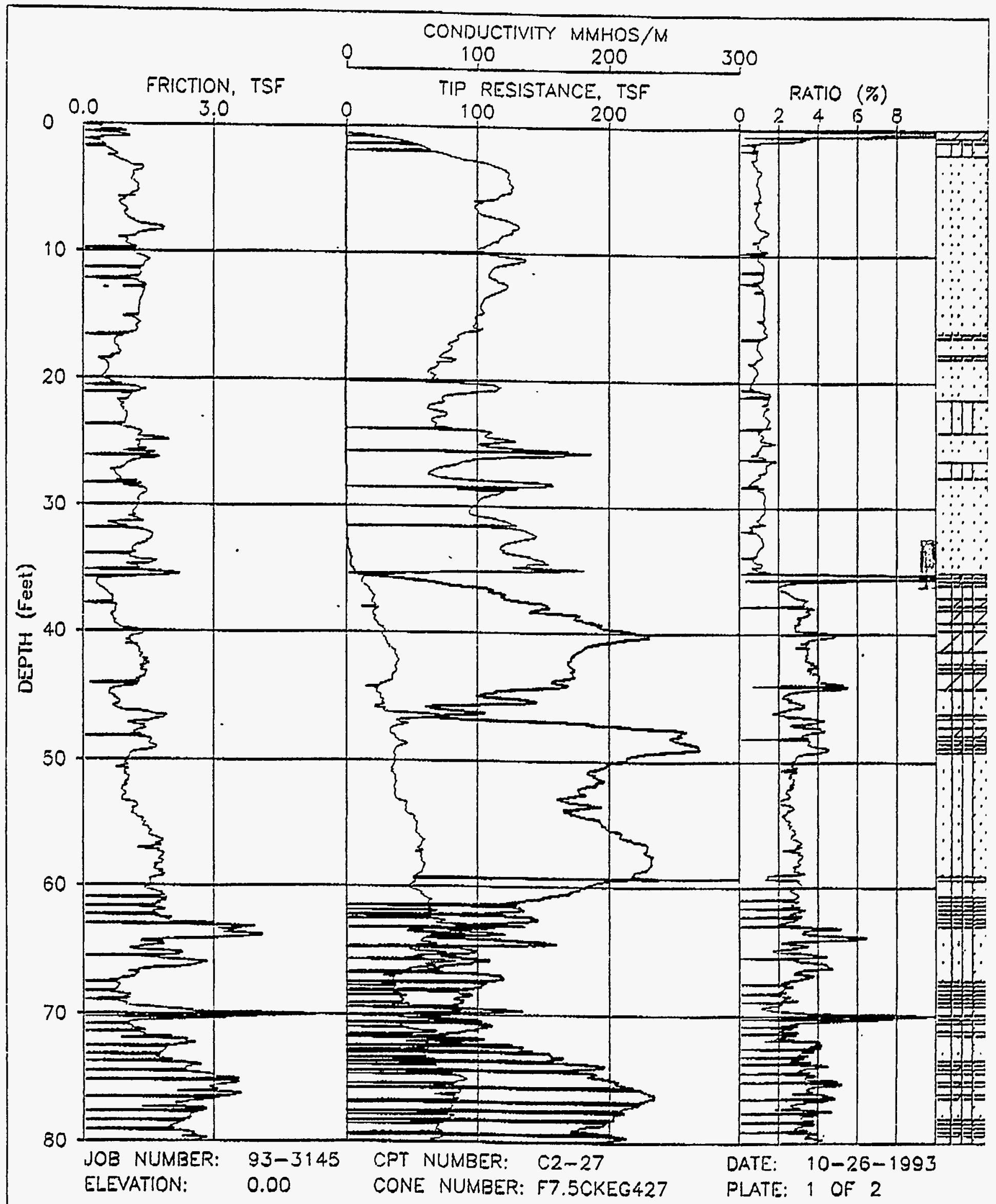




\section{Appendix B \\ Explanation of Terms Used in Data Tables}




\section{APPENDIX B: Explanation of Terms Used in Data Tables}

Terms (letters or symbols) found in the Data Tables are explained below.

\section{Organic Compounds}

B - $\quad$ Analyte was found in associated blank as well as in sample.

CRDL - Contract Required Dectection Limit

D - Indicates sample is a duplicate.

E - Concentrations exceed the calibration range of the Gas Chromatograph/Multispectral Scanner (GC/MS).

$\mathrm{J}$ - Reported result is quantitatively estimated.

NJ - There is presumptive evidence that a Tentatively Identified Compound (TIC) is present at an estimated concentration.

NP - Data Validation was not performed.

$\mathrm{R}$ - Reject, data is qualified as unusable.

RE - Following the sample number indicates a re-extraction

U - Compound was analyzed for but not detected.

UG/G- Micrograms per gram

UG/L- Micrograms per liter

UJ - Reported quantitation limit is qualified as non-detect.

$5 U$ - Example of a number/letter combination in the Data Validation (Data Valid.) column. The $5 \mathrm{x} / 10 \mathrm{x}$ Rule was applied due to blank contamination.

$X$ - Data entered manually.

"----"- Three dashed lines in the DV** column indicate that the data was validated, and there were no changes made to the original data qualifier in the $\mathrm{Q}^{*}$ column. 


\section{APPENDIX B: Explanation of Terms Used in Data Tables}

\section{Inorganic Compounds}

B - The reported value is below the CRDL but above the Instrument Detection Limit (IDL).

CRDL- Contract Required Detection Limit

D - Indicates sample is a duplicate.

E - ICP dilution $\% \mathrm{D}$ is out of control limits.

$\mathrm{J}$ - $\quad$ Reported result is quantitatively estimated.

N - Spiked sample recovery not within control limits.

NJ - There is presumptive evidence that a TIC is present at an estimated concentration.

NP - Data Validation was not performed.

UG/G- Micrograms per gram

UG/L- Micrograms per liter

$\mathrm{R}$ - Reject, data is qualified as unusable.

S - The reported value was determined by Method of Standard Addition.

U - Compound/element was analyzed for but not detected.

UJ - Reported non-detect result is qualified as estimated.

5U - Example of a number/letter combination in the Data Valid. column. Due to blank contamination, the $5 \mathrm{x} / 10 \mathrm{x}$ Rule was applied.

W - Post digestion spike for furnace Atomic Absorption Analysis is out of control limit.

$X$ - Data entered manually.

"---"- Three dashed lines in the Data Valid. column indicate that the data was validated, and there were no changes made to the original data qualifier in the $\mathrm{Q}^{*}$ column. 


\section{APPENDIX B: Explanation of Terms Used in Data Tables}

$\underline{\text { Radiochemical Compounds }}$

D - Indicates sample is a duplicate.

$\mathrm{J}$ - Reported result is quantitatively estimated.

MDA - Minimum Detectable Activity

ND - Recorded as Non-Detect since the result was below the Minimum Detectable Activity (MDA).

"-" - Single dash indicates not applicable.

NP - Data Validation was not performed.

PCi/G- Picocuries per gram

PCi/L- Picocuries per liter

U - Compound was not detected at specified detection limit.

UJ - Reported non-detect result is qualified as estimated.

"---"- Three dashed lines in the Data Validation (Data Valid.) column indicate that the data was validated, and there were no changes made to the original data qualifier in the Data Qualification (Data Qual.)* column. 


\section{Appendix C.1 \\ Results of Volatile Organic Compound Analyses, Soils, Salmon Site}




\section{APPENDIX C.1 Results of Volatile Organic Compound Analyses, Soils, Salmon Site}

\section{DATA DATA}

COMPOUND

\section{C5-1-S}

Chloromethane

Bromomethane

Vinyl Chloride

Chloroethane

Methylene Chloride

Acetone

Carbon Disulfide

1,1-Dichloroethene

1,1-Dichloroethane

1,2-Dichloroethene (total)

Chloroform

1,2-Dichloroethane

2-Butanone

1,1,1-Trichloroethane

Carbon Tetrachloride

Vinyl Acetate

Bromodichloromethane

1,2-Dichloropropane

cis-1,3-Dichloropropene

Trichloroethene

Dibromochloromethane

1,1,2-Trichloroethane

Benzene

trans-1,3-Dichloropropene

Bromoform

4-Methyl-2-Pentanone

2-Hexanone

Tetrachloroethene

1,1,2,2-Tetrachloroethane

Toluene

Chlorobenzene

Ethyl Benzene

Styrene

Xylenes (total)

Unknown

Toluene-d8 (SURR)

Bromofluorobenzene (SURR)

1,2-Dichloroethane-d4 (SURR)

RESULTS UNITS QUAL. VALID.

CRDL

UNITS DILUTION

\begin{tabular}{|c|c|c|c|}
\hline 10 UG/KG & $u$ & NP & $10 \mathrm{UG} / \mathrm{KG}$ \\
\hline 10 UG/KG & $u$ & NP & $10 \mathrm{UG} / \mathrm{KG}$ \\
\hline 10 UG/KG & $U$ & NP & 10 UG/KG \\
\hline 10 UG/KG & $u$ & NP & 10 UG/KG \\
\hline 5 UG/KG & $u$ & NP & 5 UG/KG \\
\hline 35 UG/KG & & NP & 10 UG/KG \\
\hline 5 UG/KG & $u$ & NP & 5 UG/KG \\
\hline 5 UG/KG & $u$ & NP & 5 UG/KG \\
\hline 5 UG/KG & $U$ & NP & 5 UG/KG \\
\hline 5 UG/KG & $u$ & NP & 5 UG/KG \\
\hline 5 UG/KG & $u$ & NP & 5 UG/KG \\
\hline 5 UG/KG & U & NP & 5 UG/KG \\
\hline 10 UG/KG & $u$ & NP & 10 UG/KG \\
\hline 5 UG/KG & $u$ & NP & 5 UG/KG \\
\hline 5 UG/KG & $u$ & NP & 5 UG/KG \\
\hline 10 UG/KG & $u$ & NP & 10 UG/KG \\
\hline 5 UG/KG & $U$ & NP & 5 UG/KG \\
\hline 5 UG/KG & $u$ & NP & 5 UG/KG \\
\hline 5 UG/KG & $u$ & NP & 5 UG/KG \\
\hline 5 UG/KG & $u$ & NP & 5 UG/KG \\
\hline 5 UG/KG & $u$ & NP & 5 UG/KG \\
\hline 5 UG/KG & $u$ & NP & 5 UG/KG \\
\hline 5 UG/KG & $u$ & NP & 5 UG/KG \\
\hline 5 UG/KG & U & NP & 5 UG/KG \\
\hline 5 UG/KG & U & NP & 5 UG/KG \\
\hline 10 UG/KG & $u$ & NP & 10 UG/KG \\
\hline 10 UG/KG & $u$ & NP & 10 UG/KG \\
\hline 5 UG/KG & $u$ & NP & 5 UG/KG \\
\hline 5 UG/KG & $u$ & NP & 5 UG/KG \\
\hline 5 UG/KG & $u$ & NP & 5 UG/KG \\
\hline 5 UG/KG & $u$ & NP & 5 UG/KG \\
\hline 5 UG/KG & U & NP & 5 UG/KG \\
\hline 5 UG/KG & $u$ & NP & 5 UG/KG \\
\hline 5 UG/KG & $u$ & NP & 5 UG/KG \\
\hline 7 UG/KG & $J$ & NP & UG/KG \\
\hline $04 \%$ & & & $\%$ \\
\hline $01 \%$ & & & $\%$ \\
\hline $97 \%$ & & & $\%$ \\
\hline
\end{tabular}


APPENDIX C.1 Results of Volatile Organic Compound Analyses, Soils, Salmon Site

\begin{tabular}{|c|c|c|c|c|c|}
\hline COMPOUND & RESULTS UNITS & $\begin{array}{l}\text { DATA } \\
\text { QUAL. } \\
\end{array}$ & $\begin{array}{l}\text { DATA } \\
\text { VALID. } \\
\end{array}$ & UNITS & DILUTION \\
\hline \multicolumn{6}{|l|}{ C5-2-S } \\
\hline Chloromethane & 10 UG/KG & $u$ & NP & 10 UG/KG & 1 \\
\hline Bromomethane & 10 UG/KG & $u$ & NP & 10 UG/KG & 1 \\
\hline Vinyl Chloride & 10 UG/KG & $u$ & NP & 10 UG/KG & 1 \\
\hline Chloroethane & 10 UG/KG & $u$ & NP & $10 \mathrm{UG} / K G$ & 1 \\
\hline Methylene Chloride & 5 UG/KG & $u$ & NP & 5 UG/KG & 1 \\
\hline Acetone & 20 UG/KG & & NP & $10 \mathrm{UG} / \mathrm{KG}$ & 1 \\
\hline Carbon Disulfide & 5 UG/KG & $u$ & NP & 5 UG/KG & 1 \\
\hline 1,1-Dichloroethene & 5 UG/KG & $u$ & NP & 5 UG/KG & 1 \\
\hline 1,1-Dichloroethane & 5 UG/KG & $u$ & NP & $5 \mathrm{UG} / \mathrm{KG}$ & 1 \\
\hline 1,2-Dichloroethene (total) & 5 UG/KG & $u$ & NP & 5 UG/KG & 1 \\
\hline Chloroform & 5 UG/KG & $u$ & NP & 5 UG/KG & 1 \\
\hline 1,2-Dichloroethane & 5 UG/KG & $u$ & NP & 5 UG/KG & 1 \\
\hline 2-Butanone & 10 UG/KG & $u$ & $\mathrm{NP}$ & $10 \mathrm{UG} / \mathrm{KG}$ & 1 \\
\hline 1,1,1-Trichloroethane & 5 UG/KG & $u$ & NP & 5 UG/KG & 1 \\
\hline Carbon Tetrachloride & 5 UG/KG & $u$ & NP & $5 \mathrm{UG} / \mathrm{KG}$ & 1 \\
\hline Vinyl Acetate & 10 UG/KG & $u$ & NP & $10 \mathrm{UG} / \mathrm{KG}$ & 1 \\
\hline Bromodichloromethane & 5 UG/KG & $u$ & NP & 5 UG/KG & 1 \\
\hline 1,2-Dichloropropane & 5 UG/KG & $u$ & NP & $5 \mathrm{UG} / \mathrm{KG}$ & 1 \\
\hline cis-1,3-Dichloropropene & 5 UG/KG & $u$ & NP & 5 UG/KG & 1 \\
\hline Trichloroethene & 5 UG/KG & $u$ & NP & 5 UG/KG & 1 \\
\hline Dibromochloromethane & 5 UG/KG & $u$ & NP & 5 UG/KG & 1 \\
\hline 1,1,2-Trichloroethane & 5 UG/KG & $u$ & NP & $5 \mathrm{UG} / \mathrm{KG}$ & 1 \\
\hline Benzene & 5 UG/KG & $u$ & nP & 5 UG/KG & 1 \\
\hline trans-1,3-Dichloropropene & 5 UG/KG & $u$ & NP & 5 UG/KG & 1 \\
\hline Bromoform & 5 UG/KG & $u$ & NP & 5 UG/KG & 1 \\
\hline 4-Methyl-2-Pentanone & 10 UG/KG & $u$ & NP & $10 \mathrm{UG} / \mathrm{KG}$ & 1 \\
\hline 2-Hexanone & 10 UG/KG & $u$ & NP & $10 \mathrm{UG} / \mathrm{KG}$ & 1 \\
\hline Tetrachloroethene & 5 UG/KG & $u$ & NP & 5 UG/KG & 1 \\
\hline 1,1,2,2-Tetrachloroethane & 5 UG/KG & $u$ & NP & 5 UG/KG & 1 \\
\hline Toluene & 5 UG/KG & $u$ & NP & 5 UG/KG & 1 \\
\hline Chlorobenzene & 5 UG/KG & $u$ & NP & 5 UG/KG & 1 \\
\hline Ethyl Benzene & 5 UG/KG & $u$ & NP & 5 UG/KG & 1 \\
\hline Styrene & 5 UG/KG & $u$ & NP & 5 UG/KG & 1 \\
\hline Xylenes (total) & 5 UG/KG & $u$ & NP & 5 UG/KG & 1 \\
\hline Toluene-d8 (SURR) & $101 \%$ & & & $\%$ & 1 \\
\hline Bromofluorobenzene (SURR) & $99 \%$ & & & $\%$ & 1 \\
\hline 1,2-Dichloroethane-d4 (SURR) & $98 \%$ & & & $\%$ & 1 \\
\hline
\end{tabular}


APPENDIX C.1 Results of Volatile Organic Compound Analyses, Soils, Salmon Site

\begin{tabular}{|c|c|c|c|c|c|c|}
\hline COMPOUND & RESULTS UNITS & $\begin{array}{l}\text { DATA } \\
\text { QUAL. }\end{array}$ & $\begin{array}{l}\text { DATA } \\
\text { VALID. }\end{array}$ & CRDL & UNITS & DILUTION \\
\hline \multicolumn{7}{|l|}{ C5-2-S-MS } \\
\hline 1,1-Dichloroethene & $91 \%$ & & NP & & $\%$ & 1 \\
\hline Trichloroethene & $105 \%$ & & NP & & $\%$ & 1 \\
\hline Benzene & $106 \%$ & & NP & & $\%$ & 1 \\
\hline Toluene & $103 \%$ & & NP & & $\%$ & 1 \\
\hline Chlorobenzene & $102 \%$ & & NP & & $\%$ & 1 \\
\hline Toluene-d8 (SURR) & $97 \%$ & & NP & & $\%$ & 1 \\
\hline Bromofluorobenzene (SURR) & $99 \%$ & & NP & & $\%$ & 1 \\
\hline 1,2-Dichloroethane-d4 (SURR) & $100 \%$ & & NP & & $\%$ & 1 \\
\hline \multicolumn{7}{|l|}{ C5-2-S-MSD } \\
\hline 1,1-Dichloroethene & $117 \%$ & & NP & & $\%$ & 1 \\
\hline Trichloroethene & $104 \%$ & & NP & & $\%$ & 1 \\
\hline Benzene & $112 \%$ & & NP & & $\%$ & 1 \\
\hline Toluene & $107 \%$ & & NP & & $\%$ & 1 \\
\hline Chlorobenzene & $105 \%$ & & NP & & $\%$ & 1 \\
\hline Toluene-d8 (SURR) & $100 \%$ & & NP & & $\%$ & 1 \\
\hline Bromofluorobenzene (SURR) & $100 \%$ & & NP & & $\%$ & 1 \\
\hline 1,2-Dichloroethane-d4 (SURR) & $105 \%$ & & $\mathrm{NP}$ & & $\%$ & 1 \\
\hline
\end{tabular}


APPENDIX C.1 Results of Volatile Organic Compound Analyses, Soils, Salmon Site

\begin{tabular}{|c|c|c|c|c|c|}
\hline COMPOUND & RESULTS UNITS & $\begin{array}{l}\text { DATA } \\
\text { QUAL. }\end{array}$ & $\begin{array}{l}\text { DATA } \\
\text { VALID. }\end{array}$ & UNITS & DILUTION \\
\hline \multicolumn{6}{|l|}{ C5-3-S } \\
\hline Chloromethane & 10 UG/KG & $u$ & NP & 10 UG/KG & 1 \\
\hline Bromomethane & $10 \mathrm{UG} / \mathrm{KG}$ & $u$ & NP & $10 \mathrm{UG} / \mathrm{KG}$ & 1 \\
\hline Vinyl Chloride & 10 UG/KG & $u$ & NP & 10 UG/KG & 1 \\
\hline Chloroethane & 10 UG/KG & $u$ & NP & $10 \mathrm{UG} / \mathrm{KG}$ & 1 \\
\hline Methylene Chloride & 5 UG/KG & $u$ & NP & 5 UG/KG & 1 \\
\hline Acetone & $10 \mathrm{UG} / \mathrm{KG}$ & $u$ & NP & $10 \mathrm{UG} / \mathrm{KG}$ & 1 \\
\hline Carbon Disulfide & 5 UG/KG & $u$ & NP & 5 UG/KG & 1 \\
\hline 1,1-Dichloroethene & 5 UG/KG & $u$ & NP & 5 UG/KG & 1 \\
\hline 1,1-Dichloroethane & 5 UG/KG & $u$ & NP & 5 UG/KG & 1 \\
\hline 1,2-Dichloroethene (fotal) & 5 UG/KG & $u$ & NP & 5 UG/KG & 1 \\
\hline Chloroform & 5 UG/KG & $u$ & NP & 5 UG/KG & 1 \\
\hline 1,2-Dichloroethane & 5 UG/KG & $u$ & NP & $5 \mathrm{UG} / \mathrm{KG}$ & 1 \\
\hline 2-Butanone & 10 UG/KG & $u$ & NP & 10 UG/KG & 1 \\
\hline 1,1,1-Trichloroethane & 5 UG/KG & $u$ & NP & 5 UG/KG & 1 \\
\hline Carbon Tetrachloride & 5 UG/KG & $u$ & NP & 5 UG/KG & 1 \\
\hline Vinyl Acetate & $10 \mathrm{UG} / \mathrm{KG}$ & $u$ & NP & $10 \mathrm{UG} / \mathrm{KG}$ & 1 \\
\hline Bromodichloromethane & 5 UG/KG & $u$ & NP & 5 UG/KG & 1 \\
\hline 1,2-Dichloropropane & 5 UG/KG & $u$ & NP & 5 UG/KG & 1 \\
\hline cis-1,3-Dichloropropene & 5 UG/KG & $u$ & NP & 5 UG/KG & 1 \\
\hline Trichloroethene & 5 UG/KG & $u$ & NP & 5 UG/KG & 1 \\
\hline Dibromochloromethane & 5 UG/KG & $u$ & NP & 5 UG/KG & 1 \\
\hline 1,1,2-Trichloroethane & 5 UG/KG & $u$ & NP & 5 UG/KG & 1 \\
\hline Benzene & 5 UG/KG & $u$ & NP & 5 UG/KG & 1 \\
\hline trans-1,3-Dichloropropene & 5 UG/KG & $u$ & NP & 5 UG/KG & 1 \\
\hline Bromoform & 5 UG/KG & $u$ & NP & 5 UG/KG & 1 \\
\hline 4-Methyl-2-Pentanone & 10 UG/KG & $u$ & NP & 10 UG/KG & 1 \\
\hline 2-Hexanone & 10 UG/KG & $u$ & NP & 10 UG/KG & 1 \\
\hline Tetrachloroethene & 5 UG/KG & $u$ & NP & 5 UG/KG & 1 \\
\hline $1,1,2,2$-Tetrachloroethane & 5 UG/KG & $u$ & NP & 5 UG/KG & 1 \\
\hline Toluene & 5 UG/KG & $u$ & NP & 5 UGKG & 1 \\
\hline Chlorobenzene & 5 UG/KG & $u$ & NP & 5 UG/KG & 1 \\
\hline Ethyl Benzene & 5 UG/KG & $u$ & NP & 5 UG/KG & 1 \\
\hline Styrene & 5 UG/KG & $u$ & NP & 5 UG/KG & 1 \\
\hline Xylenes (total) & 5 UG/KG & $u$ & NP & 5 UG/KG & 1 \\
\hline Toluene-d8 (SURR) & $99 \%$ & & & $\%$ & 1 \\
\hline Bromofluorobenzene (SURR) & $101 \%$ & & & $\%$ & 1 \\
\hline 1,2-Dichloroethane-d4 (SURR) & $97 \%$ & & & $\%$ & 1 \\
\hline
\end{tabular}




\section{APPENDIX C.1 Results of Volatile Organic Compound Analyses, Soils, Salmon Site}

\begin{tabular}{|c|c|c|c|c|c|}
\hline COMPOUND & RESULTS UNITS & $\begin{array}{l}\text { DATA } \\
\text { QUAL. }\end{array}$ & $\begin{array}{l}\text { DATA } \\
\text { VALID. } \\
\end{array}$ & UNITS & DILUTION \\
\hline \multicolumn{6}{|l|}{ C3-4-S } \\
\hline Chloromethane & 10 UG/KG & $u$ & NP & 10 UG/KG & 1 \\
\hline Bromomethane & 10 UG/KG & $u$ & NP & 10 UG/KG & 1 \\
\hline Vinyl Chloride & 10 UG/KG & $u$ & NP & 10 UG/KG & 1 \\
\hline Chloroethane & 10 UG/KG & $u$ & NP & 10 UG/KG & 1 \\
\hline Methylene Chloride & 5 UG/KG & $u$ & NP & 5 UG/KG & 1 \\
\hline Acetone & 10 UG/KG & $u$ & NP & 10 UG/KG & 1 \\
\hline Carbon Disulfide & 5 UG/KG & $u$ & NP & 5 UG/KG & 1 \\
\hline 1,1-Dichloroethene & 5 UG/KG & $u$ & NP & 5 UG/KG & 1 \\
\hline 1,1-Dichloroethane & 5 UG/KG & $u$ & NP & 5 UG/KG & 1 \\
\hline 1,2-Dichloroethene (total) & 5 UG/KG & $u$ & NP & 5 UG/KG & 1 \\
\hline Chloroform & 5 UG/KG & $u$ & NP & 5 UGKKG & 1 \\
\hline 1,2-Dichloroethane & 5 UG/KG & $u$ & NP & 5 UG/KG & 1 \\
\hline 2-Butanone & 10 UG/KG & $u$ & NP & 10 UG/KG & 1 \\
\hline $1,1,1-$ Trichloroethane & 5 UG/KG & $u$ & NP & 5 UG/KG & 1 \\
\hline Carbon Tetrachloride & 5 UG/KG & $u$ & NP & 5 UG/KG & 1 \\
\hline Vinyl Acetate & 10 UG/KG & $u$ & NP & 10 UG/KG & 1 \\
\hline Bromodichloromethane & 5 UG/KG & $u$ & NP & 5 UG/KG & 1 \\
\hline 1,2-Dichloropropane & 5 UG/KG & $u$ & NP & 5 UG/KG & 1 \\
\hline cis-1,3-Dichloropropene & 5 UG/KG & $u$ & NP & 5 UG/KG & 1 \\
\hline Trichloroethene & 5 UG/KG & $u$ & NP & 5 UG/KG & 1 \\
\hline Dibromochloromethane & 5 UG/KG & $u$ & NP & 5 UG/KG & 1 \\
\hline 1,1,2-Trichloroethane & 5 UG/KG & $u$ & NP & 5 UG/KG & 1 \\
\hline Benzene & 5 UG/KG & $u$ & NP & $5 \mathrm{UG} / \mathrm{KG}$ & 1 \\
\hline trans-1,3-Dichioropropene & 5 UG/KG & $u$ & NP & 5 UG/KG & 1 \\
\hline Bromotorm & 5 UG/KG & $u$ & NP & 5 UG/KG & 1 \\
\hline 4-Methyl-2-Pentanone & 10 UG/KG & $u$ & NP & 10 UG/KG & 1 \\
\hline 2-Hexanone & 10 UG/KG & $u$ & NP & 10 UG/KG & 1 \\
\hline Tetrachloroethene & 5 UG/KG & $u$ & NP & 5 UG/KG & 1 \\
\hline $1,1,2,2-$ Tetrachloroethane & 5 UG/KG & $u$ & NP & 5 UG/KG & 1 \\
\hline Toluene & 5 UG/KG & $u$ & NP & 5 UG/KG & 1 \\
\hline Chlorobenzene & 5 UG/KG & $u$ & NP & 5 UG/KG & 1 \\
\hline Ethyl Benzene & 5 UG/KG & $u$ & NP & 5 UG/KG & 1 \\
\hline Styrene & 5 UG/KG & $u$ & NP & 5 UG/KG & 1 \\
\hline Xylenes (total) & 5 UG/KG & $u$ & $N P$ & 5 UG/KG & 1 \\
\hline Toluene-d8 (SURR) & $100 \%$ & & & $\%$ & 1 \\
\hline Bromofluorobenzene (SURR) & $106 \%$ & & & $\%$ & 1 \\
\hline 1,2-Dichloroethane-d4 (SURR) & $103 \%$ & & & $\%$ & 1 \\
\hline
\end{tabular}


APPENDIX C.1 Results of Volatile Organic Compound Analyses, Soils, Salmon Site

DATA DATA

COMPOUND RESULTS UNITS QUAL. VALID.

\section{C3-5-S}

Chloromethane

Bromomethane

Vinyl Chloride

Chloroethane

Methylene Chloride

Acetone

Carbon Disulfide

1,1-Dichloroethene

1,1-Dichloroethane

1,2-Dichloroethene (total)

Chloroform

1,2-Dichloroethane

2-Butanone

1,1,1-Trichloroethane

Carbon Tetrachloride

Vinyl Acetate

Bromodichloromethane

1,2-Dichloropropane

cis-1,3-Dichloropropene

Trichloroethene

Dibromochloromethane

1,1,2-Trichloroethane

Benzene

trans-1,3-Dichloropropene

Bromoform

4-Methyl-2-Pentanone

2-Hexanone

Tetrachloroethene

1,1,2,2-Tetrachloroethane

Toluene

Chlorobenzene

Ethyl Benzene

Styrene

Xylenes (total)

Toluene-d8 (SURR)

Bromofluorobenzene (SURR)

1,2-Dichloroethane-d4 (SURR)

10 UG/KG
10 UG/KG
10 UG/KG
10 UG/KG
5 UG/KG
10 UG/KG
5 UG/KG
5 UG/KG
5 UG/KG
5 UG/KG
5 UG/KG
5 UG/KG
10 UG/KG
5 UG/KG
5 UG/KG
10 UG/KG
5 UG/KG
5 UG/KG
5 UG/KG
5 UG/KG
5 UG/KG
5 UG/KG
5 UG/KG
5 UG/KG
5 UG/KG
10 UG/KG
$10 ~ U G / K G$
$5 U G / K G$
5 UG/KG
5 UG/KG
5 UG/KG
5 UG/KG
5 UG/KG
5 UG/KG
$100 \%$
$102 \%$
$101 \%$

CRDL UNITS DILUTION

\begin{tabular}{|c|c|c|}
\hline$u$ & NP & 10 UG/KG \\
\hline$u$ & NP & $10 \mathrm{UG} / \mathrm{KG}$ \\
\hline$u$ & NP & 10 UG/KG \\
\hline$u$ & NP & 10 UG/KG \\
\hline$u$ & NP & 5 UG/KG \\
\hline U & NP & 10 UG/KG \\
\hline$u$ & NP & 5 UG/KG \\
\hline U & NP & 5 UG/KG \\
\hline$u$ & NP & 5 UG/KG \\
\hline$U$ & NP & 5 UG/KG \\
\hline$u$ & NP & 5 UG/KG \\
\hline$u$ & NP & 5 UG/KG \\
\hline$U$ & NP & 10 UG/KG \\
\hline$u$ & NP & 5 UG/KG \\
\hline$u$ & NP & 5 UG/KG \\
\hline$u$ & NP & 10 UG/KG \\
\hline$u$ & NP & 5 UG/KG \\
\hline$u$ & NP & 5 UG/KG \\
\hline$u$ & NP & 5 UG/KG \\
\hline$u$ & NP & 5 UG/KG \\
\hline$u$ & NP & 5 UG/KG \\
\hline$u$ & NP & 5 UG/KG \\
\hline$u$ & NP & $5 \mathrm{UG} / \mathrm{KG}$ \\
\hline$u$ & NP & 5 UG/KG \\
\hline$u$ & NP & 5 UG/KG \\
\hline U & NP & 10 UG/KG \\
\hline$u$ & NP & 10 UG/KG \\
\hline$u$ & NP & $5 \mathrm{UG} / \mathrm{KG}$ \\
\hline$u$ & NP & 5 UG/KG \\
\hline$u$ & NP & 5 UG/KG \\
\hline$u$ & NP & 5 UG/KG \\
\hline$U$ & NP & 5 UG/KG \\
\hline$u$ & NP & 5 UG/KG \\
\hline \multirow[t]{4}{*}{$u$} & NP & 5 UG/KG \\
\hline & & $\%$ \\
\hline & & $\%$ \\
\hline & & $\%$ \\
\hline
\end{tabular}


APPENDIX C.1 Results of Volatile Organic Compound Analyses, Soils, Salmon Site

\begin{tabular}{|c|c|c|c|c|c|}
\hline COMPOUND & RESULTS UNITS & $\begin{array}{l}\text { DATA } \\
\text { QUAL. }\end{array}$ & $\begin{array}{l}\text { DATA } \\
\text { VALID. }\end{array}$ & UNITS & DILUTION \\
\hline \multicolumn{6}{|l|}{ C3-6-S } \\
\hline Chloromethane & 10 UG/KG & $u$ & NP & iO UG/KG & 1 \\
\hline Bromomethane & $10 \mathrm{UG} / \mathrm{KG}$ & $u$ & NP & 10 UG/KG & 1 \\
\hline Vinyl Chloride & 10 UG/KG & $u$ & NP & $10 \mathrm{UG} / \mathrm{KG}$ & 1 \\
\hline Chloroethane & 10 UG/KG & $u$ & NP & 10 UG/KG & 1 \\
\hline Methylene Chloride & 5 UG/KG & $u$ & NP & 5 UG/KG & 1 \\
\hline Acetone & 10 UG/KG & $u$ & NP & $10 \mathrm{UG} / \mathrm{KG}$ & 1 \\
\hline Carbon Disulfide & 5 UG/KG & $u$ & NP & 5 UG/KG & 1 \\
\hline 1,1-Dichloroethene & 5 UG/KG & $u$ & NP & 5 UG/KG & 1 \\
\hline 1,1-Dichloroethane & 5 UG/KG & $u$ & NP & 5 UG/KG & 1 \\
\hline 1,2-Dichloroethene (total) & 5 UG/KG & $u$ & NP & 5 UG/KG & 1 \\
\hline Chloroform & 5 UG/KG & $u$ & NP & 5 UG/KG & 1 \\
\hline 1,2-Dichloroethane & 5 UG/KG & $u$ & NP & 5 UG/KG & 1 \\
\hline 2-Butanone & 10 UG/KG & $u$ & NP & 10 UG/KG & 1 \\
\hline $1,1,1$-Trichloroethane & 5 UG/KG & $u$ & NP & 5 UG/KG & 1 \\
\hline Carbon Tetrachloride & 5 UG/KG & $u$ & NP & 5 UG/KG & 1 \\
\hline Vinyl Acetate & 10 UG/KG & $u$ & NP & $10 \mathrm{UG} / \mathrm{KG}$ & 1 \\
\hline Bromodichloromethane & 5 UG/KG & $u$ & NP & 5 UG/KG & 1 \\
\hline 1,2-Dichloropropane & 5 UG/KG & $u$ & NP & 5 UG/KG & 1 \\
\hline cis-1,3-Dichloropropene & 5 UG/KG & $u$ & NP & 5 UG/KG & 1 \\
\hline Trichloroethene & 5 UG/KG & $u$ & NP & 5 UG/KG & 1 \\
\hline Dibromochloromethane & 5 UG/KG & $u$ & NP & 5 UG/KG & 1 \\
\hline 1,1,2-Trichloroethane & 5 UG/KG & $u$ & NP & 5 UG/KG & 1 \\
\hline Benzene & 5 UG/KG & $u$ & NP & 5 UG/KG & 1 \\
\hline trans-1,3-Dichloropropene & 5 UG/KG & $u$ & NP & 5 UG/KG & 1 \\
\hline Bromoform & 5 UG/KG & $u$ & NP & 5 UG/KG & 1 \\
\hline 4-Methyl-2-Pentanone & 10 UG/KG & $u$ & NP & $10 \mathrm{UG} / \mathrm{KG}$ & 1 \\
\hline 2-Hexanone & 10 UG/KG & $u$ & NP & 10 UG/KG & 1 \\
\hline Tetrachloroethene & 5 UG/KG & $u$ & NP & 5 UG/KG & 1 \\
\hline 1,1,2,2-Tetrachloroethane & 5 UG/KG & $u$ & NP & 5 UG/KG & 1 \\
\hline Toluene & 5 UG/KG & $u$ & NP & 5 UG/KG & 1 \\
\hline Chlorobenzene & 5 UG/KG & $u$ & NP & 5 UG/KG & 1 \\
\hline Ethyl Benzene & 5 UG/KG & $u$ & NP & 5 UG/KG & 1 \\
\hline Styrene & 5 UG/KG & $u$ & NP & 5 UG/KG & 1 \\
\hline Xylenes (total) & 5 UG/KG & $u$ & NP & 5 UG/KG & 1 \\
\hline Toluene-d8 (SURR) & $99 \%$ & & & $\%$ & 1 \\
\hline Bromofluorobenzene (SURR) & $102 \%$ & & & $\%$ & 1 \\
\hline 1,2-Dichloroethane-d4 (SURR) & $99 \%$ & & & $\%$ & 1 \\
\hline
\end{tabular}




\section{APPENDIX C.1 Results of Volatile Organic Compound Analyses, Soils,}

Salmon Site

\begin{tabular}{|c|c|c|c|c|c|}
\hline COMPOUND & RESULTS UNITS & $\begin{array}{l}\text { DATA } \\
\text { QUAL. }\end{array}$ & $\begin{array}{l}\text { DATA } \\
\text { VALID. }\end{array}$ & UNITS & DILUTION \\
\hline \multicolumn{6}{|l|}{ C3-7-S } \\
\hline Chloromethane & 10 UG/KG & $u$ & NP & 10 UG/KG & 1 \\
\hline Bromomethane & 10 UG/KG & $u$ & NP & 10 UG/KG & 1 \\
\hline Vinyl Chloride & 10 UG/KG & $u$ & NP & 10 UG/KG & 1 \\
\hline Chloroethane & 10 UG/KG & $u$ & NP & $10 \mathrm{UG} / \mathrm{KG}$ & 1 \\
\hline Methylene Chloride & 5 UG/KG & $u$ & NP & 5 UG/KG & 1 \\
\hline Acetone & $10 \mathrm{UG} / K \mathrm{KG}$ & $u$ & NP & $10 \mathrm{UG} / \mathrm{KG}$ & 1 \\
\hline Carbon Disulfide & 5 UG/KG & $u$ & NP & 5 UG/KG & 1 \\
\hline 1,1-Dichloroethene & 5 UG/KG & $u$ & NP & 5 UG/KG & 1 \\
\hline 1,1-Dichloroethane & $5 \mathrm{UG} / \mathrm{KG}$ & $u$ & NP & 5 UG/KG & 1 \\
\hline 1,2-Dichloroethene (total) & 5 UG/KG & $u$ & NP & 5 UG/KG & 1 \\
\hline Chloroform & 5 UG/KG & $u$ & NP & 5 UG/KG & 1 \\
\hline 1,2-Dichloroethane & 5 UG/KG & $u$ & NP & 5 UG/KG & 1 \\
\hline 2-Butanone & 10 UG/KG & $u$ & NP & $10 \mathrm{UG} / \mathrm{KG}$ & 1 \\
\hline 1,1,1-Trichloroethane & 5 UG/KG & $u$ & NP & 5 UG/KG & 1 \\
\hline Carbon Tetrachloride & 5 UG/KG & $u$ & NP & 5 UG/KG & 1 \\
\hline Vinyl Acetate & $10 \mathrm{UG} / \mathrm{KG}$ & $u$ & NP & 10 UG/KG & 1 \\
\hline Bromodichloromethane & 5 UG/KG & $u$ & NP & 5 UG/KG & 1 \\
\hline 1,2-Dichloropropane & 5 UG/KG & $u$ & NP & 5 UG/KG & 1 \\
\hline cis-1,3-Dichloropropene & 5 UG/KG & $u$ & NP & 5 UG/KG & 1 \\
\hline Trichloroethene & 5 UG/KG & $u$ & NP & 5 UG/KG & 1 \\
\hline Dibromochloromethane & 5 UG/KG & $u$ & NP & 5 UG/KG & 1 \\
\hline 1,1,2-Trichloroethane & 5 UG/KG & $u$ & NP & 5 UG/KG & 1 \\
\hline Benzene & 5 UG/KG & $u$ & NP & 5 UG/KG & 1 \\
\hline trans-1,3-Dichloropropene & 5 UG/KG & $u$ & NP & 5 UG/KG & 1 \\
\hline Bromoform & 5 UG/KG & $u$ & NP & 5 UG/KG & 1 \\
\hline 4-Methyl-2-Pentanone & 10 UG/KG & $u$ & NP & 10 UG/KG & 1 \\
\hline 2-Hexanone & 10 UG/KG & $u$ & NP & 10 UG/KG & 1 \\
\hline Tetrachloroethene & 5 UG/KG & $u$ & NP & 5 UG/KG & 1 \\
\hline 1,1,2,2-Tetrachloroethane & 5 UG/KG & $u$ & NP & 5 UG/KG & 1 \\
\hline Toluene & 5 UG/KG & $u$ & NP & 5 UG/KG & 1 \\
\hline Chlorobenzene & 5 UG/KG & $u$ & NP & 5 UG/KG & 1 \\
\hline Ethyi Benzene & 5 UG/KG & $u$ & NP & 5 UG/KG & 1 \\
\hline Styrene & 5 UG/KG & $u$ & NP & 5 UG/KG & 1 \\
\hline Xylenes (total) & 5 UG/KG & $u$ & NP & 5 UG/KG & 1 \\
\hline Unknown-1 & 5 UG/KG & J & NP & UG/KG & 1 \\
\hline Unknown-2 & 8 UG/KG & $\mathrm{J}$ & NP & UG/KG & 1 \\
\hline Toluene-d8 (SURR) & $98 \%$ & & & $\%$ & 1 \\
\hline Bromofluorobenzene (SURR) & $101 \%$ & & & $\%$ & 1 \\
\hline 1,2-Dichloroethane-d4 (SURR) & $97 \%$ & & & $\%$ & 1 \\
\hline
\end{tabular}




\section{APPENDIX C. 1 Results of Volatile Organic Compound Analyses, Soils, Salmon Site}

\begin{tabular}{|c|c|c|c|c|c|}
\hline COMPOUND & RESULTS UNITS & $\begin{array}{l}\text { DATA } \\
\text { QUAL. }\end{array}$ & $\begin{array}{l}\text { DATA } \\
\text { VALID. }\end{array}$ & UNITS & DILUTIO \\
\hline \multicolumn{6}{|l|}{ C3-7-S -RE } \\
\hline Chloromethane & 10 UG/KG & $u$ & NP & 10 UG/KG & 1 \\
\hline Bromomethane & 10 UG/KG & $u$ & NP & 10 UG/KG & 1 \\
\hline Vinyl Chloride & 10 UG/KG & $u$ & NP & 10 UG/KG & 1 \\
\hline Chloroethane & 10 UG/KG & $u$ & NP & 10 UG/KG & 1 \\
\hline Methylene Chloride & 5 UG/KG & $u$ & NP & 5 UG/KG & 1 \\
\hline Acetone & $10 \mathrm{UG} / \mathrm{KG}$ & $u$ & NP & 10 UG/KG & 1 \\
\hline Carbon Disulfide & 5 UG/KG & $U$ & NP & 5 UGKG & 1 \\
\hline 1,1-Dichloroethene & 5 UG/KG & $u$ & NP & 5 UG/KG & 1 \\
\hline 1,1-Dichloroethane & 5 UG/KG & $u$ & NP & 5 UG/KG & 1 \\
\hline 1,2-Dichloroethene (total) & 5 UG/KG & $u$ & NP & 5 UG/KG & 1 \\
\hline Chloroform & 5 UG/KG & $u$ & NP & 5 UG/KG & 1 \\
\hline 1,2-Dichloroethane & 5 UG/KG & $u$ & NP & 5 UG/KG & 1 \\
\hline 2-Butanone & 10 UG/KG & $u$ & NP & 10 UG/KG & 1 \\
\hline 1,1,1-Trichloroethane & 5 UG/KG & $u$ & NP & 5 UG/KG & 1 \\
\hline Carbon Tetrachloride & 5 UG/KG & $u$ & NP & 5 UG/KG & 1 \\
\hline Vinyl Acetate & 10 UG/KG & $u$ & NP & $10 \mathrm{UG} / \mathrm{KG}$ & 1 \\
\hline Bromodichloromethane & 5 UG/KG & $u$ & NP & 5 UG/KG & 1 \\
\hline 1,2-Dichloropropane & 5 UG/KG & $u$ & NP & 5 UG/KG & 1 \\
\hline cis-1,3-Dichloropropene & 5 UG/KG & $u$ & NP & 5 UG/KG & 1 \\
\hline Trichloroethene & 5 UG/KG & $u$ & NP & 5 UG/KG & 1 \\
\hline Dibromochloromethane & 5 UG/KG & $u$ & NP & 5 UG/KG & 1 \\
\hline 1,1,2-Trichloroethane & 5 UG/KG & $u$ & NP & 5 UG/KG & 1 \\
\hline Benzene & 5 UG/KG & $u$ & NP & 5 UG/KG & 1 \\
\hline trans-1,3-Dichloropropene & 5 UG/KG & $u$ & NP & 5 UG/KG & 1 \\
\hline Bromoform & 5 UG/KG & $u$ & NP & 5 UG/KG & 1 \\
\hline 4-Methyl-2-Pentanone & 10 UG/KG & $u$ & NP & 10 UG/KG & 1 \\
\hline 2-Hexanone & $10 \mathrm{UG} / \mathrm{KG}$ & $u$ & NP & 10 UG/KG & 1 \\
\hline Tetrachloroethene & $5 \mathrm{UG} / \mathrm{KG}$ & $u$ & NP & 5 UG/KG & 1 \\
\hline 1,1,2,2-Tetrachloroethane & 5 UG/KG & $u$ & NP & 5 UG/KG & 1 \\
\hline Toluene & 5 UG/KG & $u$ & NP & 5 UG/KG & 1 \\
\hline Chlorobenzene & 5 UG/KG & $u$ & NP. & 5 UG/KG & 1 \\
\hline Ethyl Benzene & 5 UG/KG & $u$ & NP & 5 UG/KG & 1 \\
\hline Styrene & 5 UG/KG & $u$ & NP & 5 UG/KG & 1 \\
\hline Xylenes (total) & 5 UG/KG & $u$ & NP & 5 UG/KG & 1 \\
\hline Toluene-d8 (SURR) & $99 \%$ & & & $\%$ & 1 \\
\hline Bromofluorobenzene (SURR) & $99 \%$ & & & $\%$ & 1 \\
\hline 1,2-Dichloroethane-d4 (SURR) & $101 \%$ & & & $\%$ & 1 \\
\hline
\end{tabular}


APPENDIX C.1 Results of Volatile Organic Compound Analyses, Soils, Salmon Site

\begin{tabular}{|c|c|c|c|c|c|}
\hline COMPOUND & RESULTS UNITS & $\begin{array}{l}\text { DATA } \\
\text { QUAL. }\end{array}$ & $\begin{array}{l}\text { DATA } \\
\text { VALID. } \\
\end{array}$ & UNITS & DILUTION \\
\hline \multicolumn{6}{|l|}{ C3-8-S } \\
\hline Chloromethane & 10 UG/KG & $u$ & NP & 10 UG/KG & 1 \\
\hline Bromomethane & 10 UG/KG & $u$ & NP & $10 \mathrm{UG} / \mathrm{KG}$ & 1 \\
\hline Vinyl Chloride & 10 UG/KG & $u$ & NP & 10 UG/KG & 1 \\
\hline Chloroethane & 10 UG/KG & $u$ & NP & 10 UG/KG & 1 \\
\hline Methyiene Chloride & 5 UG/KG & $u$ & NP & 5 UG/KG & 1 \\
\hline Acetone & 17 UG/KG & & NP & $10 \mathrm{UG} / \mathrm{KG}$ & 1 \\
\hline Carbon Disulfide & 35 UG/KG & & NP & 5 UG/KG & 1 \\
\hline 1,1-Dichloroethene & 5 UG/KG & $u$ & NP & 5 UG/KG & 1 \\
\hline 1,1-Dichloroethane & 5 UG/KG & $u$ & NP & 5 UG/KG & 1 \\
\hline 1,2-Dichloroethene (total) & 5 UG/KG & $u$ & NP & 5 UG/KG & 1 \\
\hline Chloroform & 5 UG/KG & $u$ & NP & 5 UG/KG & 1 \\
\hline 1,2-Dichloroethane & 5 UG/KG & $u$ & NP & 5 UG/KG & 1 \\
\hline 2-Butanone & 10 UG/KG & $u$ & NP & 10 UG/KG & 1 \\
\hline 1,1,1-Trichloroethane & 5 UG/KG & $u$ & NP & 5 UG/KG & 1 \\
\hline Carbon Tetrachloride & 5 UG/KG & $u$ & NP & 5 UG/KG & 1 \\
\hline Vinyl Acetate & 10 UG/KG & $u$ & NP & 10 UG/KG & 1 \\
\hline Bromodichloromethane & 5 UG/KG & $u$ & NP & 5 UG/KG & 1 \\
\hline 1,2-Dichloropropane & 5 UG/KG & $u$ & NP & 5 UG/KG & 1 \\
\hline cis-1,3-Dichloropropene & 5 UG/KG & $u$ & NP & 5 UG/KG & 1 \\
\hline Trichloroethene & 5 UG/KG & $u$ & NP & 5 UG/KG & 1 \\
\hline Dibromochloromethane & 5 UG/KG & $u$ & NP & 5 UG/KG & 1 \\
\hline 1,1,2-Trichloroethane & 5 UG/KG & $u$ & NP & 5 UG/KG & 1 \\
\hline Benzene & 5 UG/KG & $u$ & NP & 5 UG/KG & 1 \\
\hline trans-1,3-Dichloropropene & $5 \mathrm{UG} / \mathrm{KG}$ & $u$ & NP & 5 UG/KG & 1 \\
\hline Bromoform & 5 UG/KG & $u$ & NP & 5 UG/KG & 1 \\
\hline 4-Methyl-2-Pentanone & $10 \mathrm{UG} / \mathrm{KG}$ & $u$ & NP & 10 UG/KG & 1 \\
\hline 2-Hexanone & $10 \mathrm{UG} / \mathrm{KG}$ & $u$ & NP & $10 \mathrm{UG} / \mathrm{KG}$ & 1 \\
\hline Tetrachloroethene & 5 UG/KG & $u$ & NP & 5 UG/KG & 1 \\
\hline 1,1,2,2-Tetrachloroethane & 5 UG/KG & $u$ & NP & 5 UG/KG & 1 \\
\hline Toluene & 5 UG/KG & $u$ & NP & 5 UG/KG & 1 \\
\hline Chlorobenzene & 5 UG/KG & $u$ & NP & 5 UG/KG & 1 \\
\hline Ethyl Benzene & 5 UG/KG & $u$ & NP & 5 UG/KG & 1 \\
\hline Styrene & 5 UG/KG & $u$ & NP & 5 UG/KG & 1 \\
\hline Xylenes (total) & 5 UG/KG & $u$ & NP & 5 UG/KG & 1 \\
\hline Toluene-d8 (SURR) & $101 \%$ & & & $\%$ & 1 \\
\hline Bromofluorobenzene (SURR) & $101 \%$ & & & $\%$ & 1 \\
\hline 1,2-Dichloroethane-d4 (SURR) & $98 \%$ & & & $\%$ & 1 \\
\hline
\end{tabular}




\section{APPENDIX C.1 Results of Volatile Organic Compound Analyses, Soils, Salmon Site}

\begin{tabular}{|c|c|c|c|c|c|}
\hline COMPOUND & RESULTS UNITS & $\begin{array}{l}\text { DATA } \\
\text { QUAL. }\end{array}$ & $\begin{array}{l}\text { DATA } \\
\text { VALID. }\end{array}$ & UNITS & DILUTION \\
\hline \multicolumn{6}{|l|}{ C3-9-S } \\
\hline Chloromethane & 10 UG/KG & $u$ & NP & $10 \mathrm{UG} / \mathrm{KG}$ & 1 \\
\hline Bromomethane & $10 \mathrm{UG} / \mathrm{KG}$ & $u$ & NP & $10 \mathrm{UG} / \mathrm{KG}$ & 1 \\
\hline Vinyl Chloride & 10 UG/KG & $u$ & NP & 10 UG/KG & 1 \\
\hline Chloroethane & 10 UG/KG & $u$ & NP & 10 UG/KG & 1 \\
\hline Methylene Chloride & 5 UG/KG & $u$ & NP & 5 UG/KG & 1 \\
\hline Acetone & 10 UG/KG & $u$ & NP & 10 UG/KG & 1 \\
\hline Carbon Disulfide & 5 UG/KG & $u$ & NP & 5 UG/KG & 1 \\
\hline 1,1-Dichloroethene & 5 UG/KG & $u$ & NP & 5 UG/KG & 1 \\
\hline 1,1-Dichloroethane & 5 UG/KG & $u$ & NP & 5 UG/KG & 1 \\
\hline 1,2-Dichloroethene (total) & 5 UG/KG & $u$ & NP & 5 UG/KG & 1 \\
\hline Chloroform & 5 UG/KG & $u$ & NP & 5 UG/KG & 1 \\
\hline 1,2-Dichloroethane & 5 UG/KG & $u$ & NP & 5 UGKG & 1 \\
\hline 2-Butanone & 10 UG/KG & $u$ & NP & 10 UG/KG & 1 \\
\hline $1,1,1$-Trichloroethane & 5 UG/KG & $u$ & NP & 5 UG/KG & 1 \\
\hline Carbon Tetrachloride & 5 UG/KG & $u$ & NP & 5 UG/KG & 1 \\
\hline Vinyl Acetate & 10 UG/KG & $u$ & NP & 10 UG/KG & 1 \\
\hline Bromodichloromethane & 5 UG/KG & $u$ & NP & 5 UG/KG & 1 \\
\hline 1,2-Dichloropropane & 5 UG/KG & $u$ & NP & 5 UG/KG & 1 \\
\hline cis-1,3-Dichloropropene & 5 UG/KG & $u$ & NP & 5 UG/KG & 1 \\
\hline Trichloroethene & 5 UG/KG & $u$ & NP & 5 UG/KG & 1 \\
\hline Dibromochloromethane & 5 UG/KG & $u$ & NP & 5 UG/KG & 1 \\
\hline 1,1,2-Trichloroethane & 5 UG/KG & $u$ & NP & 5 UG/KG & 1 \\
\hline Benzene & 5 UG/KG & $u$ & NP & 5 UG/KG & 1 \\
\hline trans-1,3-Dichloropropene & 5 UG/KG & $u$ & NP & 5 UG/KG & 1 \\
\hline Bromoform & 5 UG/KG & $u$ & NP & 5 UG/KG & 1 \\
\hline 4-Methyl-2-Pentanone & 10 UG/KG & $u$ & NP & 10 UG/KG & 1 \\
\hline 2-Hexanone & 10 UG/KG & $u$ & NP & 10 UG/KG & 1 \\
\hline Tetrachloroethene & 5 UG/KG & $u$ & NP & 5 UG/KG & 1 \\
\hline 1,1,2,2-Tetrachloroethane & 5 UG/KG & $u$ & NP & 5 UG/KG & 1 \\
\hline Toluene & 5 UG/KG & $u$ & NP & 5 UG/KG & 1 \\
\hline Chlorobenzene & 5 UG/KG & $u$ & NP & 5 UG/KG & 1 \\
\hline Ethyl Benzene & 5 UG/KG & $u$ & NP & 5 UG/KG & 1 \\
\hline Styrene & 5 UG/KG & $u$ & NP & 5 UG/KG & 1 \\
\hline Xylenes (total) & 5 UG/KG & $u$ & NP & 5 UG/KG & 1 \\
\hline Toluene-d8 (SURR) & $100 \%$ & & & $\%$ & 1 \\
\hline Bromofluorobenzene (SURR) & $103 \%$ & & & $\%$ & 1 \\
\hline 1,2-Dichloroethane-d4 (SURR) & $105 \%$ & & & $\%$ & 1 \\
\hline
\end{tabular}


APPENDIX C.1 Results of Volatile Organic Compound Analyses, Soils, Salmon Site

\begin{tabular}{|c|c|c|c|c|c|}
\hline COMPOUND & RESULTS UNITS & $\begin{array}{l}\text { DATA } \\
\text { QUAL. }\end{array}$ & $\begin{array}{c}\text { DATA } \\
\text { VALID. }\end{array}$ & UNITS & DILUTION \\
\hline \multicolumn{6}{|l|}{ C4-10-S } \\
\hline Chloromethane & 10 UG/KG & $u$ & NP & 10 UG/KG & 1 \\
\hline Bromomethane & 10 UG/KG & $u$ & NP & 10 UG/KG & 1 \\
\hline Vinyl Chloride & 10 UG/KG & $u$ & NP & 10 UG/KG & 1 \\
\hline Chloroethane & 10 UG/KG & $u$ & NP & 10 UG/KG & 1 \\
\hline Methylene Chloride & 5 UG/KG & $u$ & NP & 5 UG/KG & 1 \\
\hline Acetone & $10 \mathrm{UG} / \mathrm{KG}$ & $u$ & NP & 10 UG/KG & 1 \\
\hline Carbon Disulfide & 5 UG/KG & $u$ & NP & 5 UG/KG & 1 \\
\hline 1,1-Dichloroethene & 5 UG/KG & $u$ & NP & 5 UG/KG & 1 \\
\hline 1,1-Dichloroethane & 5 UG/KG & $u$ & NP & 5 UG/KG & 1 \\
\hline 1,2-Dichloroethene (total) & 5 UG/KG & $u$ & NP & 5 UG/KG & 1 \\
\hline Chloroform & 5 UG/KG & $u$ & NP & 5 UG/KG & 1 \\
\hline 1,2-Dichloroethane & 5 UG/KG & $u$ & NP & 5 UG/KG & 1 \\
\hline 2-Butanone & 10 UG/KG & $u$ & NP & 10 UG/KG & 1 \\
\hline 1,1,1-Trichloroethane & 5 UG/KG & $u$ & NP & 5 UG/KG & 1 \\
\hline Carbon Tetrachloride & 5 UG/KG & $u$ & NP & 5 UG/KG & 1 \\
\hline Vinyl Acetate & 10 UG/KG & $u$ & NP & 10 UG/KG & 1 \\
\hline Bromodichloromethane & 5 UG/KG & $u$ & NP & $5 \mathrm{UG} / \mathrm{KG}$ & 1 \\
\hline 1,2-Dichloropropane & 5 UG/KG & $u$ & NP & 5 UG/KG & 1 \\
\hline cis-1,3-Dichloropropene & 5 UG/KG & $u$ & NP & 5 UG/KG & 1 \\
\hline Trichloroethene & 5 UG/KG & $u$ & NP & 5 UG/KG & 1 \\
\hline Dibromochloromethane & 5 UG/KG & $u$ & NP & 5 UG/KG & 1 \\
\hline 1,1,2-Trichloroethane & 5 UG/KG & $u$ & NP & 5 UG/KG & 1 \\
\hline Benzene & 5 UG/KG & $u$ & NP & 5 UG/KG & 1 \\
\hline trans-1,3-Dichloropropene & 5 UG/KG & $u$ & NP & 5 UG/KG & 1 \\
\hline Bromoform & 5 UG/KG & $u$ & NP & 5 UG/KG & 1 \\
\hline 4-Methyl-2-Pentanone & 10 UG/KG & $u$ & NP & 10 UG/KG & 1 \\
\hline 2-Hexanone & 10 UG/KG & $u$ & NP & 10 UG/KG & 1 \\
\hline Tetrachloroethene & 5 UG/KG & $u$ & NP & 5 UG/KG & 1 \\
\hline 1,1,2,2-Tetrachloroethane & 5 UG/KG & $u$ & NP & 5 UG/KG & 1 \\
\hline Toluene & 5 UG/KG & $u$ & NP & 5 UG/KG & 1 \\
\hline Chlorobenzene & 5 UG/KG & $u$ & NP & 5 UG/KG & 1 \\
\hline Ethyl Benzene & 5 UG/KG & $u$ & NP & 5 UG/KG & 1 \\
\hline Styrene & 5 UG/KG & $u$ & NP & 5 UG/KG & 1 \\
\hline Xylenes (total) & 5 UG/KG & $u$ & NP & 5 UG/KG & 1 \\
\hline Toluene-d8 (SURR) & $103 \%$ & & & $\%$ & 1 \\
\hline Bromofluorobenzene (SURR) & $106 \%$ & & & $\%$ & 1 \\
\hline 1,2-Dichloroethane-d4 (SURR) & $105 \%$ & & & $\%$ & 1 \\
\hline
\end{tabular}


APPENDIX C.1 Results of Volatile Organic Compound Analyses, Soils, Salmon Site

\begin{tabular}{|c|c|c|c|c|c|}
\hline COMPOUND & RESULTS UNITS & $\begin{array}{l}\text { DATA } \\
\text { QUAL. }\end{array}$ & $\begin{array}{l}\text { DATA } \\
\text { VALID. }\end{array}$ & CRDL & DILUTION \\
\hline \multicolumn{6}{|l|}{ C4-11-S } \\
\hline Chloromethane & 10 UG/KG & $u$ & NP & 10 UG/KG & 1 \\
\hline Bromomethane & 10 UG/KG & $u$ & NP & 10 UG/KG & 1 \\
\hline Vinyl Chloride & 10 UG/KG & $u$ & NP & 10 UG/KG & 1 \\
\hline Chloroethane & 10 UG/KG & $u$ & NP & 10 UG/KG & 1 \\
\hline Methylene Chloride & 5 UG/KG & $u$ & NP & 5 UG/KG & 1 \\
\hline Acetone & 34 UG/KG & & NP & 10 UG/KG & 1 \\
\hline Carbon Disulfide & 5 UG/KG & $u$ & NP & 5 UG/KG & 1 \\
\hline 1,1-Dichloroethene & 5 UG/KG & $u$ & NP & 5 UG/KG & 1 \\
\hline 1,1-Dichloroethane & 5 UG/KG & $u$ & NP & 5 UG/KG & 1 \\
\hline 1,2-Dichloroethene (total) & 5 UG/KG & $u$ & NP & 5 UG/KG & 1 \\
\hline Chloroform & 5 UG/KG & $u$ & NP & 5 UG/KG & 1 \\
\hline 1,2-Dichloroethane & 5 UG/KG & $u$ & NP & 5 UG/KG & 1 \\
\hline 2-Butanone & 10 UG/KG & $u$ & NP & 10 UG/KG & 1 \\
\hline 1,1,1-Trichloroethane & 5 UG/KG & $u$ & NP & 5 UG/KG & 1 \\
\hline Carbon Tetrachloride & 5 UG/KG & $u$ & NP & 5 UG/KG & 1 \\
\hline Vinyl Acetate & 10 UG/KG & $u$ & NP & $10 \mathrm{UG} / \mathrm{KG}$ & 1 \\
\hline Bromodichloromethane & 5 UG/KG & $u$ & NP & 5 UG/KG & 1 \\
\hline 1,2-Dichloropropane & 5 UG/KG & $u$ & NP & 5 UG/KG & 1 \\
\hline cis-1,3-Dichloropropene & 5 UG/KG & $u$ & NP & 5 UG/KG & 1 \\
\hline Trichloroethene & 5 UG/KG & $u$ & NP & 5 UG/KG & 1 \\
\hline Dibromochloromethane & 5 UG/KG & $u$ & NP & $5 \mathrm{UG} / \mathrm{KG}$ & 1 \\
\hline 1,1,2-Trichloroethane & 5 UG/KG & $u$ & NP & 5 UG/KG & 1 \\
\hline Benzene & 5 UG/KG & $u$ & NP & 5 UG/KG & 1 \\
\hline trans-1,3-Dichloropropene & $5 \mathrm{UG} / \mathrm{KG}$ & $u$ & NP & 5 UG/KG & 1 \\
\hline Bromoform & 5 UG/KG & $u$ & NP & 5 UG/KG & 1 \\
\hline 4-Methyl-2-Pentanone & 10 UG/KG & $u$ & NP & 10 UG/KG & 1 \\
\hline 2-Hexanone & 10 UG/KG & $u$ & NP & 10 UG/KG & 1 \\
\hline Tetrachloroethene & 5 UG/KG & $u$ & NP & 5 UG/KG & 1 \\
\hline 1,1,2,2-Tetrachloroethane & 5 UG/KG & $u$ & NP & 5 UG/KG & 1 \\
\hline Toluene & 5 UG/KG & $u$ & NP & 5 UG/KG & 1 \\
\hline Chlorobenzene & 5 UG/KG & $u$ & NP & 5 UG/KG & 1 \\
\hline Ethyl Benzene & 5 UG/KG & $u$ & NP & 5 UG/KG & 1 \\
\hline Styrene & 5 UG/KG & $u$ & NP & 5 UG/KG & 1 \\
\hline Xylenes (total) & 5 UG/KG & $u$ & NP & 5 UG/KG & 1 \\
\hline Unknown & 6 UG/KG & J & NP & UG/KG & 1 \\
\hline Toluene-d8 (SURR) & $99 \%$ & & & $\%$ & 1 \\
\hline Bromofluorobenzene (SURR) & $100 \%$ & & & $\%$ & 1 \\
\hline 1,2-Dichloroethane-d4 (SURR) & $101 \%$ & & & $\%$ & 1 \\
\hline
\end{tabular}


APPENDIX C.1 Results of Volatile Organic Compound Analyses, Soils, Salmon Site

\begin{tabular}{|c|c|c|c|c|c|}
\hline COMPOUND & RESULTS UNITS & $\begin{array}{l}\text { DATA } \\
\text { QUAL. }\end{array}$ & $\begin{array}{l}\text { DATA } \\
\text { VALID. }\end{array}$ & UNITS & DILUTION \\
\hline \multicolumn{6}{|l|}{$\mathrm{C} 4-12-\mathrm{S}$} \\
\hline Chloromethane & $10 \mathrm{UG} / \mathrm{KG}$ & $u$ & NP & 10 UG/KG & 1 \\
\hline Bromomethane & $10 \mathrm{UG} / \mathrm{KG}$ & $u$ & NP & $10 \mathrm{UG} / \mathrm{KG}$ & 1 \\
\hline Vinyl Chloride & 10 UG/KG & $u$ & NP & $10 \mathrm{UG} / \mathrm{KG}$ & 1 \\
\hline Chloroethane & 10 UG/KG & $u$ & NP & $10 \mathrm{UG} / \mathrm{KG}$ & 1 \\
\hline Methyiene Chloride & 5 UG/KG & $u$ & NP & 5 UG/KG & 1 \\
\hline Acetone & 10 UG/KG & $u$ & NP & 10 UG/KG & 1 \\
\hline Carbon Disulfide & 5 UG/KG & $u$ & NP & 5 UG/KG & 1 \\
\hline 1,1-Dichloroethene & 5 UG/KG & $u$ & NP & 5 UG/KG & 1 \\
\hline 1,1-Dichloroethane & 5 UG/KG & $u$ & NP & 5 UG/KG & 1 \\
\hline 1,2-Dichloroethene (total) & 5 UG/KG & $u$ & NP & 5 UG/KG & 1 \\
\hline Chloroform & 5 UG/KG & $u$ & NP & 5 UG/KG & 1 \\
\hline 1,2-Dichloroethane & 5 UG/KG & $u$ & NP & 5 UG/KG & 1 \\
\hline 2-Butanone & 10 UG/KG & $u$ & NP & 10 UG/KG & 1 \\
\hline 1,1,1-Trichloroethane & 5 UG/KG & $u$ & NP & 5 UG/KG & 1 \\
\hline Carbon Tetrachloride & 5 UG/KG & $u$ & NP & 5 UG/KG & 1 \\
\hline Vinyl Acetate & 10 UG/KG & $u$ & NP & 10 UG/KG & 1 \\
\hline Bromodichloromethane & $5 \mathrm{UG} / \mathrm{KG}$ & $u$ & NP & 5 UG/KG & 1 \\
\hline 1,2-Dichloropropane & 5 UG/KG & $u$ & NP & 5 UG/KG & 1 \\
\hline cis-1,3-Dichloropropene & 5 UG/KG & $u$ & NP & 5 UG/KG & 1 \\
\hline Trichloroethene & 5 UG/KG & $u$ & NP & 5 UG/KG & 1 \\
\hline Dibromochloromethane & 5 UG/KG & $u$ & NP & 5 UG/KG & 1 \\
\hline 1,1,2-Trichloroethane & 5 UG/KG & $u$ & NP & 5 UG/KG & 1 \\
\hline Benzene & 5 UG/KG & $u$ & NP & 5 UG/KG & 1 \\
\hline trans-1,3-Dichloropropene & 5 UG/KG & $u$ & NP & 5 UG/KG & 1 \\
\hline Bromoform & 5 UG/KG & $u$ & NP & 5 UG/KG & 1 \\
\hline 4-Methyl-2-Pentanone & 10 UG/KG & $u$ & NP & 10 UG/KG & 1 \\
\hline 2-Hexanone & 10 UG/KG & $u$ & NP & $10 \mathrm{UG} / \mathrm{KG}$ & 1 \\
\hline Tetrachloroethene & 5 UGKKG & $u$ & NP & 5 UG/KG & 1 \\
\hline 1,1,2,2-Tetrachloroethane & 5 UG/KG & $u$ & NP & 5 UG/KG & 1 \\
\hline Toluene & 5 UG/KG & $u$ & NP & 5 UG/KG & 1 \\
\hline Chlorobenzene & 5 UG/KG & $u$ & NP & 5 UG/KG & 1 \\
\hline Ethyl Benzene & 5 UG/KG & $u$ & NP & 5 UG/KG & 1 \\
\hline Styrene & 5 UG/KG & $u$ & NP & 5 UG/KG & 1 \\
\hline Xylenes (total) & 5 UG/KG & $u$ & NP & 5 UG/KG & 1 \\
\hline Toluene-d8 (SURR) & $99 \%$ & & & $\%$ & 1 \\
\hline Bromofluorobenzene (SURR) & $99 \%$ & & & $\%$ & 1 \\
\hline 1,2-Dichloroethane-d4 (SURR) & $99 \%$ & & & $\%$ & 1 \\
\hline
\end{tabular}




\section{APPENDIX C.1 Results of Volatile Organic Compound Analyses, Soils, Salmon Site}

\begin{tabular}{|c|c|c|c|c|c|}
\hline COMPOUND & RESULTS UNITS & $\begin{array}{l}\text { DATA } \\
\text { QUAL. }\end{array}$ & $\begin{array}{l}\text { DATA } \\
\text { VALID. }\end{array}$ & UNITS & DILUTION \\
\hline \multicolumn{6}{|l|}{ C4-13-s } \\
\hline Chloromethane & 10 UG/KG & $u$ & NP & 10 UG/KG & 1 \\
\hline Bromomethane & 10 UG/KG & $u$ & NP & 10 UG/KG & 1 \\
\hline Vinyl Chloride & 10 UG/KG & $u$ & NP & $10 \mathrm{UG} / \mathrm{KG}$ & 1 \\
\hline Chloroethane & 10 UG/KG & $u$ & $\mathrm{NP}$ & 10 UG/KG & 1 \\
\hline Methylene Chloride & 5 UG/KG & $u$ & NP & 5 UG/KG & 1 \\
\hline Acetone & $10 \mathrm{UG} / \mathrm{KG}$ & $u$ & NP & 10 UG/KG & 1 \\
\hline Carbon Disulfide & 5 UG/KG & $u$ & NP & 5 UG/KG & 1 \\
\hline 1,1-Dichloroethene & 5 UG/KG & $u$ & NP & 5 UG/KG & 1 \\
\hline 1,1-Dichloroethane & 5 UG/KG & $u$ & NP & 5 UG/KG & 1 \\
\hline 1,2-Dichloroethene (total) & 5 UG/KG & $u$ & NP & 5 UG/KG & 1 \\
\hline Chloroform & 5 UG/KG & $u$ & NP & 5 UG/KG & 1 \\
\hline 1,2-Dichloroethane & 5 UG/KG & $\mathrm{u}$ & NP & 5 UG/KG & 1 \\
\hline 2-Butanone & 10 UG/KG & $u$ & NP & $10 \mathrm{UG} / \mathrm{KG}$ & 1 \\
\hline 1,1,1-Trichloroethane & 5 UG/KG & $u$ & NP & 5 UG/KG & 1 \\
\hline Carbon Tetrachloride & 5 UG/KG & $u$ & NP & 5 UG/KG & 1 \\
\hline Vinyl Acetate & 10 UGKG & $u$ & NP & 10 UG/KG & 1 \\
\hline Bromodichloromethane & 5 UG/KG & $u$ & NP & 5 UG/KG & 1 \\
\hline 1,2-Dichloropropane & 5 UG/KG & $u$ & NP & 5 UG/KG & 1 \\
\hline cis-1,3-Dichloropropene & 5 UG/KG & $u$ & NP & 5 UG/KG & 1 \\
\hline Trichloroethene & 5 UG/KG & $u$ & NP & 5 UG/KG & 1 \\
\hline Dibromochloromethane & 5 UG/KG & $u$ & NP & 5 UG/KG & 1 \\
\hline 1,1,2-Trichloroethane & 5 UG/KG & $u$ & NP & 5 UG/KG & 1 \\
\hline Benzene & 5 UG/KG & $u$ & NP & 5 UG/KG & 1 \\
\hline trans-1,3-Dichloropropene & 5 UG/KG & $u$ & NP & 5 UG/KG & 1 \\
\hline Bromoform & 5 UG/KG & $u$ & NP & 5 UG/KG & 1 \\
\hline 4-Methyl-2-Pentanone & 10 UG/KG & $u$ & NP & $10 \mathrm{UG/KG}$ & 1 \\
\hline 2-Hexanone & $10 \mathrm{UG} / \mathrm{KG}$ & $u$ & NP & 10 UG/KG & 1 \\
\hline Tetrachloroethene & 5 UG/KG & $u$ & NP & 5 UG/KG & 1 \\
\hline 1,1,2,2-Tetrachloroethane & 5 UG/KG & $u$ & NP & 5 UG/KG & 1 \\
\hline Toluene & 5 UG/KG & $u$ & NP & 5 UG/KG & 1 \\
\hline Chlorobenzene & 5 UG/KG & $u$ & NP & 5 UG/KG & 1 \\
\hline Ethyl Benzene & 5 UG/KG & $u$ & NP & 5 UG/KG & 1 \\
\hline Styrene & 5 UG/KG & $u$ & NP & 5 UG/KG & 1 \\
\hline Xylenes (total) & 5 UG/KG & $u$ & NP & 5 UG/KG & 1 \\
\hline Toluene-d8 (SURR) & $98 \%$ & & & $\%$ & 1 \\
\hline Bromofluorobenzene (SURR) & $102 \%$ & & & $\%$ & 1 \\
\hline 1,2-Dichloroethane-d4 (SURR) & $95 \%$ & & & $\%$ & 1 \\
\hline
\end{tabular}


APPENDIX C.1 Results of Volatile Organic Compound Analyses, Soils, Salmon Site

\begin{tabular}{|c|c|c|c|c|c|}
\hline COMPOUND & RESULTS UNITS & $\begin{array}{l}\text { DATA } \\
\text { QUAL. }\end{array}$ & $\begin{array}{l}\text { DATA } \\
\text { VALID. }\end{array}$ & UNITS & DILUTION \\
\hline \multicolumn{6}{|l|}{ C1-14-S } \\
\hline Chloromethane & 10 UG/KG & $u$ & NP & $10 \mathrm{UG} / \mathrm{KG}$ & 1 \\
\hline Bromomethane & 10 UG/KG & $u$ & NP & 10 UG/KG & 1 \\
\hline Vinyl Chloride & 10 UG/KG & $u$ & NP & 10 UG/KG & 1 \\
\hline Chloroethane & 10 UG/KG & $u$ & NP & 10 UG/KG & 1 \\
\hline Methylene Chloride & 5 UG/KG & $u$ & NP & 5 UG/KG & 1 \\
\hline Acetone & 10 UG/KG & $u$ & NP & 10 UG/KG & 1 \\
\hline Carbon Disuifide & 5 UG/KG & $u$ & NP & 5 UG/KG & 1 \\
\hline 1,1-Dichloroethene & 5 UG/KG & $u$ & NP & 5 UG/KG & 1 \\
\hline 1,1-Dichloroethane & 5 UG/KG & $u$ & NP & 5 UG/KG & 1 \\
\hline 1,2-Dichloroethene (total) & 5 UG/KG & $u$ & NP & $5 ! 1 G / K G$ & 1 \\
\hline Chloroform & 5 UG/KG & $u$ & NP & 5 UG/KG & 1 \\
\hline 1,2-Dichloroethane & 5 UG/KG & $u$ & NP & 5 UG/KG & 1 \\
\hline 2-Butanone & 10 UG/KG & $u$ & NP & $10 \mathrm{UG} / \mathrm{KG}$ & 1 \\
\hline 1,1,1-Trichloroethane & 5 UG/KG & $u$ & NP & 5 UG/KG & 1 \\
\hline Carbon Tetrachloride & 5 UG/KG & $u$ & NP & 5 UG/KG & 1 \\
\hline Vinyl Acetate & 10 UG/KG & $u$ & NP & 10 UG/KG & 1 \\
\hline Bromodichloromethane & 5 UG/KG & $u$ & NP & 5 UG/KG & 1 \\
\hline 1,2-Dichloropropane & 5 UG/KG & $u$ & NP & 5 UG/KG & 1 \\
\hline cis-1,3-Dichloropropene & 5 UG/KG & $u$ & NP & 5 UG/KG & 1 \\
\hline Trichloroethene & 5 UG/KG & $u$ & NP & 5 UG/KG & 1 \\
\hline Dibromochloromethane & 5 UG/KG & $u$ & NP & 5 UG/KG & 1 \\
\hline $1,1,2-$ Tricloroethane & 5 UG/KG & $u$ & NP & 5 UGKK & 1 \\
\hline Benzene & 5 UG/KG & $u$ & NP & 5 UG/KG & 1 \\
\hline trans-1,3-Dichloropropene & 5 UG/KG & $u$ & NP & 5 UG/KG & 1 \\
\hline Bromoform & 5 UG/KG & $u$ & NP & 5 UG/KG & 1 \\
\hline 4-Methyi-2-Pentanone & 10 UG/KG & $u$ & NP & 10 UG/KG & 1 \\
\hline 2-Hexanone & $10 \mathrm{UG} / \mathrm{KG}$ & $u$ & NP & 10 UG/KG & 1 \\
\hline Tetrachloroethene & 5 UG/KG & $u$ & NP & 5 UG/KG & 1 \\
\hline 1,1,2,2-Tetrachloroethane & 5 UG/KG & $u$ & NP & 5 UG/KG & 1 \\
\hline Toluene & 5 UG/KG & $u$ & NP & 5 UG/KG & 1 \\
\hline Chlorobenzene & 5 UG/KG & $u$ & NP & 5 UG/KG & 1 \\
\hline Ethyl Benzene & 5 UG/KG & $u$ & NP & 5 UG/KG & 1 \\
\hline Styrene & 5 UG/KG & $u$ & NP & 5 UG/KG & 1 \\
\hline Xylenes (total) & 5 UG/KG & $u$ & NP & 5 UG/KG & 1 \\
\hline Toluene-d8 (SURR) & $103 \%$ & & & $\%$ & 1 \\
\hline Bromofluorobenzene (SURR) & $98 \%$ & & & $\%$ & 1 \\
\hline 1,2-Dichloroethane-d4 (SURR) & $100 \%$ & & & $\%$ & 1 \\
\hline
\end{tabular}


APPENDIX C.1 Results of Volatile Organic Compound Analyses, Soils, Salmon Site

DATA DATA

\begin{tabular}{|c|c|c|c|c|c|}
\hline COMPOUND & RESULTS UNITS & $\begin{array}{l}\text { DATA } \\
\text { QUAL. }\end{array}$ & $\begin{array}{l}\text { DATA } \\
\text { VALID. }\end{array}$ & UNITS & DILUTION \\
\hline \multicolumn{6}{|l|}{ C1-15-S } \\
\hline Chloromethane & 10 UG/KG & $u$ & NP & 10 UG/KG & 1 \\
\hline Bromomethane & 10 UG/KG & $u$ & NP & 10 UG/KG & 1 \\
\hline Vinyl Chloride & $10 \mathrm{UG} / \mathrm{KG}$ & $u$ & NP & 10 UG/KG & 1 \\
\hline Chloroethane & 10 UG/KG & $u$ & NP & 10 UG/KG & 1 \\
\hline Methylene Chloride & 5 UG/KG & $u$ & NP & 5 UG/KG & 1 \\
\hline Acetone & 21 UG/KG & & NP & $10 \mathrm{UG} / \mathrm{KG}$ & 1 \\
\hline Carbon Disulfide & 78 UG/KG & & NP & 5 UG/KG & 1 \\
\hline 1,1-Dichloroethene & 5 UG/KG & $u$ & NP & 5 UG/KG & 1 \\
\hline 1,1-Dichloroethane & 5 UG/KG & $u$ & NP & 5 UG/KG & 1 \\
\hline 1,2-Dichloroethene (total) & 5 UG/KG & $u$ & NP & 5 UG/KG & 1 \\
\hline Chloroform & 5 UG/KG & $u$ & NP & 5 UG/KG & 1 \\
\hline 1,2-Dichloroethane & 5 UG/KG & $u$ & NP & 5 UG/KG & 1 \\
\hline 2-Butanone & 10 UG/KG & $u$ & NP & $10 \mathrm{UG} / \mathrm{KG}$ & 1 \\
\hline 1,1,1-Trichloroethane & 5 UG/KG & $u$ & NP & 5 UG/KG & 1 \\
\hline Carbon Tetrachloride & 5 UG/KG & $u$ & NP & 5 UG/KG & 1 \\
\hline Vinyl Acetate & 10 UG/KG & $u$ & NP & 10 UG/KG & 1 \\
\hline Bromodichloromethane & 5 UG/KG & $u$ & NP & 5 UG/KG & 1 \\
\hline 1,2-Dichloropropane & 5 UG/KG & $u$ & NP & 5 UG/KG & 1 \\
\hline cis-1,3-Dichloropropene & 5 UG/KG & $u$ & NP & 5 UG/KG & 1 \\
\hline Trichloroethene & 5 UG/KG & $u$ & NP & 5 UG/KG & 1 \\
\hline Dibromochloromethane & 5 UG/KG & $u$ & NP & 5 UG/KG & 1 \\
\hline 1,1,2-Tricloroethane & 5 UG/KG & $u$ & NP & 5 UG/KG & 1 \\
\hline Benzene & 5 UG/KG & $u$ & NP & 5 UG/KG & 1 \\
\hline trans-1,3-Dichloropropene & 5 UG/KG & $u$ & NP & 5 UG/KG & 1 \\
\hline Bromoform & 5 UG/KG & $u$ & NP & 5 UG/KG & 1 \\
\hline 4-Methyl-2-Pentanone & 10 UG/KG & $u$ & NP & 10 UG/KG & 1 \\
\hline 2-Hexanone & 10 UG/KG & $u$ & NP & 10 UG/KG & 1 \\
\hline Tetrachloroethene & 5 UG/KG & $u$ & NP & $5 \mathrm{UG} / \mathrm{KG}$ & 1 \\
\hline $1,1,2,2-$ Tetrachloroethane & 5 UG/KG & $u$ & NP & 5 UG/KG & 1 \\
\hline Toluene & 5 UG/KG & $u$ & NP & 5 UG/KG & 1 \\
\hline Chlorobenzene & 5 UG/KG & $u$ & NP & 5 UG/KG & 1 \\
\hline Ethyl Benzene & 5 UG/KG & $u$ & NP & $5 \mathrm{UG} / \mathrm{KG}$ & 1 \\
\hline Styrene & 5 UG/KG & $u$ & NP & 5 UG/KG & 1 \\
\hline Xylenes (total) & 5 UG/KG & $u$ & NP & 5 UG/KG & 1 \\
\hline Toluene-d8 (SURR) & $101 \%$ & & & $\%$ & 1 \\
\hline Bromofluorobenzene (SURR) & $99 \%$ & & & $\%$ & 1 \\
\hline 1,2-Dichloroethane-d4 (SURR) & $98 \%$ & & & $\%$ & 1 \\
\hline
\end{tabular}


APPENDIX C.1 Results of Volatile Organic Compound Analyses, Soils, Salmon Site

\begin{tabular}{|c|c|c|c|c|c|}
\hline COMPOUND & RESULTS UNITS & $\begin{array}{l}\text { DATA } \\
\text { QUAL. }\end{array}$ & $\begin{array}{l}\text { DATA } \\
\text { VALID. }\end{array}$ & UNITS & DILUTION \\
\hline \multicolumn{6}{|l|}{ C1-16-S } \\
\hline Chloromethane & 10 UG/KG & $u$ & NP & 10 UG/KG & 1 \\
\hline Bromomethane & 10 UG/KG & $u$ & NP & 10 UG/KG & 1 \\
\hline Vinyl Chloride & $10 \mathrm{UG} / \mathrm{KG}$ & $u$ & NP & 10 UG/KG & 1 \\
\hline Chloroethane & 10 UG/KG & $u$ & NP & 10 UG/KG & 1 \\
\hline Methylene Chloride & 5 UG/KG & $u$ & NP & 5 UG/KG & 1 \\
\hline Acetone & 52 UG/KG & & NP & $10 \mathrm{UG} / \mathrm{KG}$ & 1 \\
\hline Carbon Disulfide & 5 UG/KG & $u$ & NP & 5 UG/KG & 1 \\
\hline 1,1-Dichloroethene & 5 UG/KG & $u$ & NP & 5 UG/KG & 1 \\
\hline 1,1-Dichloroethane & 5 UG/KG & $u$ & NP & 5 UG/KG & 1 \\
\hline 1,2-Dichloroethene (total) & 5 UG/KG & $u$ & NP & 5 UG/KG & 1 \\
\hline Chloroform & 5 UG/KG & $u$ & NP & 5 UG/KG & 1 \\
\hline 1,2-Dichloroethane & 5 UG/KG & $u$ & NP & 5 UG/KG & 1 \\
\hline 2-Butanone & 10 UG/KG & $u$ & NP & 10 UG/KG & 1 \\
\hline 1,1,1-Trichloroethane & 5 UG/KG & $u$ & NP & 5 UG/KG & 1 \\
\hline Carbon Tetrachloride & 5 UG/KG & $u$ & NP & 5 UG/KG & 1 \\
\hline Vinyl Acetate & 10 UG/KG & $u$ & NP & $10 \mathrm{UG} / \mathrm{KG}$ & 1 \\
\hline Bromodichloromethane & 5 UG/KG & $u$ & NP & 5 UG/KG & 1 \\
\hline 1,2-Dichloropropane & 5 UG/KG & $u$ & NP & 5 UG/KG & 1 \\
\hline cis-1,3-Dichloropropene & 5 UG/KG & $u$ & NP & 5 UG/KG & 1 \\
\hline Trichloroethene & 5 UG/KG & $u$ & NP & 5 UG/KG & 1 \\
\hline Dibromochloromethane & 5 UG/KG & $u$ & NP & 5 UG/KG & 1 \\
\hline 1,1,2-Tricloroethane & 5 UG/KG & $u$ & NP & 5 UG/KG & 1 \\
\hline Benzene & 5 UG/KG & $u$ & NP & 5 UG/KG & 1 \\
\hline trans-1,3-Dichloropropene & 5 UG/KG & $u$ & NP & 5 UG/KG & 1 \\
\hline Bromoform & 5 UG/KG & $u$ & NP & 5 UG/KG & 1 \\
\hline 4-Methyl-2-Pentanone & 10 UG/KG & $u$ & NP & 10 UG/KG & 1 \\
\hline 2-Hexanone & $10 \mathrm{UG} / \mathrm{KG}$ & $u$ & NP & 10 UG/KG & 1 \\
\hline Tetrachloroethene & 5 UG/KG & $u$ & NP & 5 UG/KG & 1 \\
\hline 1,1,2,2-Tetrachloroethane & 5 UG/KG & $u$ & NP & 5 UG/KG & 1 \\
\hline Toluene & 5 UG/KG & $u$ & NP & 5 UG/KG & 1 \\
\hline Chlorobenzene & $5 \mathrm{UG} / \mathrm{KG}$ & $u$ & NP & 5 UG/KG & 1 \\
\hline Ethyl Benzene & 5 UG/KG & $u$ & NP & $5 \mathrm{UG} / \mathrm{KG}$ & 1 \\
\hline Styrene & 5 UG/KG & $u$ & NP & 5 UG/KG & 1 \\
\hline Xylenes (total) & 5 UG/KG & $u$ & NP & 5 UG/KG & 1 \\
\hline Toluene-d8 (SURR) & $98 \%$ & & & $\%$ & 1 \\
\hline Bromofluorobenzene (SURR) & $97 \%$ & & & $\%$ & 1 \\
\hline 1,2-Dichloroethane-d4 (SURR) & $100 \%$ & & & $\%$ & 1 \\
\hline
\end{tabular}


APPENDIX C.1 Results of Volatile Organic Compound Analyses, Soils, Salmon Site

\begin{tabular}{|c|c|c|c|c|c|}
\hline COMPOUND & RESULTS UNITS & $\begin{array}{l}\text { DATA } \\
\text { QUAL. }\end{array}$ & $\begin{array}{r}\text { DATA } \\
\text { VALID. } \\
\end{array}$ & UNITS & DILUTION \\
\hline \multicolumn{6}{|l|}{ C1-17-s } \\
\hline Chloromethane & 10 UG/KG & $u$ & NP & 10 UG/KG & 1 \\
\hline Bromomethane & 10 UG/KG & $u$ & NP & $10 \mathrm{UG} / \mathrm{KG}$ & 1 \\
\hline Vinyl Chloride & 10 UG/KG & $u$ & NP & 10 UG/KG & 1 \\
\hline Chloroethane & 10 UG/KG & $u$ & NP & 10 UG/KG & 1 \\
\hline Methylene Chloride & 5 UG/KG & $u$ & NP & 5 UG/KG & 1 \\
\hline Acetone & $10 \mathrm{UG} / \mathrm{KG}$ & $u$ & NP & 10 UG/KG & 1 \\
\hline Carbon Disulfide & 5 UG/KG & $u$ & NP & 5 UG/KG & 1 \\
\hline 1,1-Dichloroethene & 5 UG/KG & $u$ & NP & 5 UG/KG & 1 \\
\hline 1,1-Dichloroethane & 5 UG/KG & $u$ & NP & $5 \mathrm{UG} / \mathrm{KG}$ & 1 \\
\hline 1,2-Dichloroethene (total) & 5 UG/KG & $u$ & NP & 5 UG/KG & 1 \\
\hline Chloroform & 5 UG/KG & $u$ & NP & 5 UG/KG & 1 \\
\hline 1,2-Dichloroethane & 5 UG/KG & $u$ & NP & $5 \mathrm{UG} / \mathrm{KG}$ & 1 \\
\hline 2-Butanone & 10 UG/KG & $u$ & NP & 10 UG/KG & 1 \\
\hline 1,1,1-Trichloroethane & 5 UG/KG & $u$ & NP & 5 UG/KG & 1 \\
\hline Carbon Tetrachloride & 5 UG/KG & $u$ & NP & 5 UG/KG & 1 \\
\hline Vinyl Acetate & 10 UG/KG & $u$ & NP & $10 \mathrm{UG} / \mathrm{KG}$ & 1 \\
\hline Bromodichloromethane & 5 UG/KG & $u$ & NP & 5 UG/KG & 1 \\
\hline 1,2-Dichloropropane & 5 UG/KG & $u$ & NP & 5 UG/KG & 1 \\
\hline cis-1,3-Dichloropropene & 5 UG/KG & $u$ & NP & $5 \mathrm{UG} / \mathrm{KG}$ & 1 \\
\hline Trichloroethene & $6 \mathrm{UG} / \mathrm{KG}$ & & NP & 5 UG/KG & 1 \\
\hline Dibromochloromethane & 5 UG/KG & $u$ & NP & 5 UG/KG & 1 \\
\hline 1,1,2-Tricloroethane & 5 UG/KG & $u$ & NP & 5 UG/KG & 1 \\
\hline Benzene & 5 UG/KG & $u$ & NP & 5 UG/KG & 1 \\
\hline trans-1,3-Dichloropropene & 5 UG/KG & $u$ & NP & 5 UG/KG & 1 \\
\hline Bromoform & 5 UG/KG & $u$ & NP & 5 UG/KG & 1 \\
\hline 4-Methyl-2-Pentanone & 10 UG/KG & $u$ & NP & 10 UG/KG & 1 \\
\hline 2-Hexanone & 10 UG/KG & $u$ & NP & 10 UG/KG & 1 \\
\hline Tetrachloroethene & 5 UG/KG & $u$ & NP & 5 UG/KG & 1 \\
\hline $1,1,2,2-$ Tetrachloroethane & 5 UG/KG & $u$ & NP & 5 UG/KG & 1 \\
\hline Toluene & 5 UG/KG & $u$ & NP & 5 UG/KG & 1 \\
\hline Chlorobenzene & 5 UG/KG & $u$ & NP & 5 UG/KG & 1 \\
\hline Ethyl Benzene & 5 UG/KG & $u$ & NP & 5 UG/KG & 1 \\
\hline Styrene & 5 UG/KG & $u$ & NP & 5 UG/KG & 1 \\
\hline Xylenes (total) & 5 UG/KG & $u$ & NP & 5 UG/KG & 1 \\
\hline Toluene-d8 (SURR) & $105 \%$ & & & $\%$ & 1 \\
\hline Bromofluorobenzene (SURR) & $100 \%$ & & & $\%$ & 1 \\
\hline 1,2-Dichloroethane-d4 (SURR) & $101 \%$ & & & $\%$ & 1 \\
\hline
\end{tabular}


APPENDIX C.1 Results of Volatile Organic Compound Analyses, Soils, Salmon Site

\begin{tabular}{|c|c|c|c|c|c|c|}
\hline COMPOUND & RESULTS UNITS & $\begin{array}{l}\text { DATA } \\
\text { QUAL. }\end{array}$ & $\begin{array}{l}\text { DATA } \\
\text { VALID. }\end{array}$ & CRDL & UNITS & DILUTION \\
\hline \multicolumn{7}{|l|}{ C1-17-S-MS } \\
\hline 1,1-Dichloroethene & $112 \%$ & & NP & & $\%$ & 1 \\
\hline Trichloroethene & $90 \%$ & & NP & & $\%$ & 1 \\
\hline Benzene & $113 \%$ & & NP & & $\%$ & 1 \\
\hline Toluene & $106 \%$ & & NP & & $\%$ & 1 \\
\hline Chlorobenzene & $105 \%$ & & NP & & $\%$ & 1 \\
\hline Toluene-d8 (SURR) & $96 \%$ & & NP & & $\%$ & 1 \\
\hline Bromofluorobenzene (SURR) & $105 \%$ & & NP & & $\%$ & 1 \\
\hline 1,2-Dichloroethane-d4 (SURR) & $114 \%$ & & NP & & $\%$ & 1 \\
\hline \multicolumn{7}{|l|}{ C1-17-S-MSD } \\
\hline 1,1-Dichloroethene & $106 \%$ & & NP & & $\%$ & 1 \\
\hline Trichloroethene & $92 \%$ & & NP & & $\%$ & 1 \\
\hline Benzene & $115 \%$ & & NP & & $\%$ & 1 \\
\hline Toluene & $104 \%$ & & NP & & $\%$ & 1 \\
\hline Chlorobenzene & $107 \%$ & & NP & & $\%$ & 1 \\
\hline Toluene-d8 (SURR) & $95 \%$ & & NP & & $\%$ & 1 \\
\hline Bromofluorobenzene (SURR) & $106 \%$ & & NP & & $\%$ & 1 \\
\hline 1,2-Dichloroethane-d4 (SURR) & $115 \%$ & & NP & & $\%$ & 1 \\
\hline
\end{tabular}


APPENDIX C.1 Results of Volatile Organic Compound Analyses, Soils, Salmon Site

\begin{tabular}{|c|c|c|c|c|c|}
\hline COMPOUND & RESULTS UNITS & $\begin{array}{l}\text { DATA } \\
\text { QUAL. }\end{array}$ & $\begin{array}{l}\text { DATA } \\
\text { VALID. }\end{array}$ & UNITS & DILUTION \\
\hline \multicolumn{6}{|l|}{ C1-18-S } \\
\hline Chloromethane & 10 UG/KG & $u$ & NP & 10 UG/KG & 1 \\
\hline Bromomethane & $10 \mathrm{UG} / \mathrm{KG}$ & $u$ & NP & 10 UG/KG & 1 \\
\hline Vinyl Chloride & 10 UG/KG & $u$ & NP & 10 UG/KG & 1 \\
\hline Chloroethane & 10 UG/KG & $u$ & NP & 10 UG/KG & 1 \\
\hline Methylene Chloride & 5 UG/KG & $U$ & NP & $5 \mathrm{UG} / \mathrm{KG}$ & 1 \\
\hline Acetone & 10 UG/KG & $u$ & NP & 10 UG/KG & 1 \\
\hline Carbon Disulfide & $5 \mathrm{UG} / \mathrm{KG}$ & $u$ & NP & 5 UG/KG & 1 \\
\hline 1,1-Dichloroethene & 5 UG/KG & $u$ & NP & 5 UG/KG & 1 \\
\hline 1,1-Dichloroethane & 5 UG/KG & $u$ & NP & 5 UG/KG & 1 \\
\hline 1,2-Dichloroethene (total) & 5 UG/KG & $u$ & NP & 5 UG/KG & 1 \\
\hline Chloroform & 5 UG/KG & $u$ & NP & 5 UG/KG & 1 \\
\hline 1,2-Dichloroethane & 5 UG/KG & $u$ & NP & 5 UG/KG & 1 \\
\hline 2-Butanone & 10 UG/KG & $u$ & NP & 10 UG/KG & 1 \\
\hline 1,1,1-Trichloroethane & 5 UG/KG & $u$ & NP & 5 UG/KG & 1 \\
\hline Carbon Tetrachloride & 5 UG/KG & $u$ & NP & 5 UG/KG & 1 \\
\hline Vinyl Acetate & 10 UG/KG & $u$ & NP & 10 UG/KG & 1 \\
\hline Bromodichloromethane & 5 UG/KG & $u$ & NP & 5 UG/KG & 1 \\
\hline 1,2-Dichloropropane & 5 UG/KG & $u$ & NP & 5 UG/KG & 1 \\
\hline cis-1,3-Dichloropropene & 5 UG/KG & $u$ & NP & 5 UG/KG & 1 \\
\hline Trichloroethene & 5 UG/KG & $u$ & NP & 5 UG/KG & 1 \\
\hline Dibromochloromethane & 5 UG/KG & $u$ & NP & 5 UG/KG & 1 \\
\hline 1,1,2-Tricloroethane & 5 UG/KG & $u$ & NP & 5 UG/KG & 1 \\
\hline Benzene & 5 UG/KG & $u$ & NP & 5 UG/KG & 1 \\
\hline trans-1,3-Dichloropropene & 5 UGKG & $u$ & NP & 5 UG/KG & 1 \\
\hline Bromoform & 5 UG/KG & $u$ & NP & 5 UG/KG & 1 \\
\hline 4-Methyl-2-Pentanone & 10 UG/KG & $u$ & NP & $10 \mathrm{UG} / \mathrm{KG}$ & 1 \\
\hline 2-Hexanone & $10 \mathrm{UG} / \mathrm{KG}$ & $u$ & NP & $10 \mathrm{UG} / \mathrm{KG}$ & 1 \\
\hline Tetrachloroethene & 5 UG/KG & $u$ & NP & 5 UG/KG & 1 \\
\hline 1,1,2,2-Tetrachloroethane & 5 UG/KG & $u$ & NP & 5 UG/KG & 1 \\
\hline Toluene & 5 UG/KG & $u$ & NP & 5 UG/KG & 1 \\
\hline Chlorobenzene & 5 UG/KG & $u$ & NP & 5 UG/KG & 1 \\
\hline Ethyl Benzene & 5 UG/KG & $u$ & NP & 5 UGKG & 1 \\
\hline Styrene & 5 UG/KG & $u$ & NP & 5 UG/KG & 1 \\
\hline Xylenes (total) & 5 UG/KG & $u$ & NP & 5 UG/KG & 1 \\
\hline Toluene-d8 (SURR) & $101 \%$ & & & $\%$ & 1 \\
\hline Bromofluorobenzene (SURR) & $99 \%$ & & & $\%$ & 1 \\
\hline 1,2-Dichloroethane-d4 (SURR) & $103 \%$ & & & $\%$ & 1 \\
\hline
\end{tabular}


APPENDIX C.1 Results of Volatile Organic Compound Analyses, Soils, Salmon Site

\begin{tabular}{|c|c|c|c|c|c|}
\hline COMPOUND & RESULTS UNITS & $\begin{array}{l}\text { DATA } \\
\text { QUAL. }\end{array}$ & $\begin{array}{l}\text { DATA } \\
\text { VALID. }\end{array}$ & UNITS & DILUTION \\
\hline \multicolumn{6}{|l|}{ C2-19-S } \\
\hline Chloromethane & 10 UG/KG & $u$ & NP & 10 UG/KG & 1 \\
\hline Bromomethane & 10 UG/KG & $u$ & NP & 10 UG/KG & 1 \\
\hline Vinyl Chloride & $10 \mathrm{UG} / \mathrm{KG}$ & $u$ & NP & $10 \mathrm{UG} / \mathrm{KG}$ & 1 \\
\hline Chloroethane & 10 UG/KG & $u$ & NP & 10 UG/KG & 1 \\
\hline Methylene Chloride & 5 UG/KG & $u$ & NP & 5 UG/KG & 1 \\
\hline Acetone & 10 UG/KG & $u$ & NP & 10 UG/KG & 1 \\
\hline Carbon Disulfide & 5 UG/KG & $u$ & NP & 5 UG/KG & 1 \\
\hline 1,1-Dichloroethene & 5 UG/KG & $u$ & NP & 5 UG/KG & 1 \\
\hline 1,1-Dichloroethane & 5 UG/KG & $u$ & NP & 5 UG/KG & 1 \\
\hline 1,2-Dichloroethene (total) & 5 UGKG & $u$ & ND & 5 UG/KG & 1 \\
\hline Chloroform & 5 UG/KG & $u$ & NP & 5 UG/KG & 1 \\
\hline 1,2-Dichloroethane & 5 UG/KG & $u$ & NP & 5 UG/KG & 1 \\
\hline 2-Butanone & $10 \mathrm{UG} / \mathrm{KG}$ & $u$ & NP & 10 UG/KG & 1 \\
\hline 1,1,1-Trichloroethane & 5 UG/KG & $u$ & NP & 5 UG/KG & 1 \\
\hline Carbon Tetrachloride & 5 UG/KG & $u$ & NP & 5 UG/KG & 1 \\
\hline Vinyl Acetate & 10 UG/KG & $u$ & NP & 10 UG/KG & 1 \\
\hline Bromodichloromethane & 5 UG/KG & $u$ & NP & 5 UG/KG & 1 \\
\hline 1,2-Dichloropropane & 5 UG/KG & $u$ & NP & 5 UG/KG & 1 \\
\hline cis-1,3-Dichloropropene & 5 UG/KG & $u$ & NP & 5 UG/KG & 1 \\
\hline Trichloroethene & 5 UG/KG & $u$ & NP & 5 UG/KG & 1 \\
\hline Dibromochloromethane & 5 UG/KG & $u$ & NP & 5 UG/KG & 1 \\
\hline 1,1,2-Trichloroethane & 5 UG/KG & $u$ & NP & 5 UG/KG & 1 \\
\hline Benzene & 5 UG/KG & $u$ & NP & 5 UG/KG & 1 \\
\hline trans-1,3-Dichloropropene & 5 UG/KG & $u$ & NP & 5 UG/KG & 1 \\
\hline Bromoform & 5 UG/KG & $u$ & NP & 5 UG/KG & 1 \\
\hline 4-Methyl-2-Pentanone & 10 UG/KG & $u$ & NP & 10 UG/KG & 1 \\
\hline 2-Hexanone & 10 UG/KG & $u$ & NP & 10 UG/KG & 1 \\
\hline Tetrachloroethene & 5 UG/KG & $u$ & NP & 5 UG/KG & 1 \\
\hline 1,1,2,2-Tetrachloroethane & 5 UG/KG & $u$ & NP & 5 UG/KG & 1 \\
\hline Toluene & $6 \mathrm{UG} / \mathrm{KG}$ & & NP & 5 UG/KG & 1 \\
\hline Chlorobenzene & 5 UG/KG & $u$ & NP & 5 UG/KG & 1 \\
\hline Ethyl Benzene & 5 UG/KG & $u$ & NP & 5 UG/KG & 1 \\
\hline Styrene & 5 UG/KG & $u$ & NP & 5 UG/KG & 1 \\
\hline Xylenes (total) & 5 UG/KG & $u$ & NP & 5 UG/KG & 1 \\
\hline Toluene-d8 (SURR) & $97 \%$ & & & $\%$ & 1 \\
\hline Bromofluorobenzene (SURR) & $101 \%$ & & & $\%$ & 1 \\
\hline 1,2-Dichloroethane-d4 (SURR) & $101 \%$ & & & $\%$ & 1 \\
\hline
\end{tabular}




\section{APPENDIX C.1 Results of Volatile Organic Compound Analyses, Soils, Salmon Site}

\begin{tabular}{|c|c|c|c|c|c|}
\hline COMPOUND & RESULTS UNITS & $\begin{array}{l}\text { DATA } \\
\text { QUAL. }\end{array}$ & $\begin{array}{l}\text { DATA } \\
\text { VALID. } \\
\end{array}$ & UNITS & DILUTION \\
\hline \multicolumn{6}{|l|}{ C2-20-S } \\
\hline Chloromethane & 10 UG/KG & $u$ & $N P$ & $10 \mathrm{UG} / \mathrm{KG}$ & 1 \\
\hline Bromomethane & 10 UG/KG & $u$ & NP & 10 UG/KG & 1 \\
\hline Vinyl Chloride & 10 UG/KG & $u$ & NP & 10 UG/KG & 1 \\
\hline Chloroethane & $10 \mathrm{UG} / \mathrm{KG}$ & $u$ & NP & 10 UG/KG & 1 \\
\hline Methylene Chloride & 5 UG/KG & $u$ & NP & 5 UG/KG & 1 \\
\hline Acetone & 10 UG/KG & $u$ & NP & 10 UG/KG & 1 \\
\hline Carbon Disulfide & 5 UG/KG & $u$ & NP & 5 UG/KG & 1 \\
\hline 1,1-Dichloroethene & 5 UG/KG & $u$ & NP & 5 UG/KG & 1 \\
\hline 1,1-Dichloroethane & 5 UG/KG & $u$ & NP & 5 UG/KG & 1 \\
\hline 1,2-Dichloroethene (total) & 5 UG/KG & $u$ & NP & 5 UG/KG & 1 \\
\hline Chloroform & 5 UG/KG & $u$ & NP & 5 UG/KG & 1 \\
\hline 1,2-Dichloroethane & 5 UG/KG & $u$ & NP & 5 UG/KG & 1 \\
\hline 2-Butanone & 10 UG/KG & $u$ & NP & 10 UG/KG & 1 \\
\hline $1,1,1$-Trichloroethane & 5 UG/KG & $u$ & NP & 5 UG/KG & 1 \\
\hline Carbon Tetrachloride & 5 UG/KG & $u$ & NP & 5 UG/KG & 1 \\
\hline Vinyl Acetate & 10 UG/KG & $u$ & NP & 10 UG/KG & 1 \\
\hline Bromodichloromethane & 5 UG/KG & $u$ & NP & 5 UG/KG & 1 \\
\hline 1,2-Dichloropropane & 5 UG/KG & $u$ & NP & 5 UG/KG & 1 \\
\hline cis-1,3-Dichloropropene & 5 UG/KG & $\mathrm{u}$ & NP & 5 UG/KG & 1 \\
\hline Trichloroethene & 5 UG/KG & $u$ & NP & 5 UG/KG & 1 \\
\hline Dibromochloromethane & 5 UG/KG & $\mathrm{u}$ & $\mathrm{NP}$ & 5 UG/KG & 1 \\
\hline 1,1,2-Trichloroethane & 5 UG/KG & $u$ & NP & $5 \mathrm{UG} / \mathrm{KG}$ & 1 \\
\hline Benzene & 5 UG/KG & $u$ & NP & 5 UG/KG & 1 \\
\hline trans-1,3-Dichloropropene & 5 UG/KG & $u$ & NP & 5 UG/KG & 1 \\
\hline Bromoform & 5 UG/KG & $u$ & NP & 5 UG/KG & 1 \\
\hline 4-Methyl-2-Pentanone & 10 UG/KG & $u$ & NP & 10 UG/KG & 1 \\
\hline 2-Hexanone & 10 UG/KG & $u$ & NP & 10 UG/KG & 1 \\
\hline Tetrachloroethene & 5 UG/KG & $u$ & NP & 5 UG/KG & 1 \\
\hline 1,1,2,2-Tetrachloroethane & 5 UG/KG & $u$ & NP & 5 UG/KG & 1 \\
\hline Toluene & 5 UG/KG & $u$ & NP & 5 UG/KG & 1 \\
\hline Chlorobenzene & 5 UG/KG & $u$ & NP & 5 UG/KG & 1 \\
\hline Ethyl Benzene & 5 UG/KG & $u$ & NP & 5 UG/KG & 1 \\
\hline Styrene & 5 UG/KG & $u$ & NP & 5 UG/KG & 1 \\
\hline Xylenes (total) & 5 UG/KG & $u$ & NP & 5 UG/KG & 1 \\
\hline Toluene-d8 (SURR) & $102 \%$ & & & $\%$ & 1 \\
\hline Bromofluorobenzene (SURR) & $103 \%$ & & & $\%$ & 1 \\
\hline 1,2-Dichloroethane-d4 (SURR) & $103 \%$ & & & $\%$ & 1 \\
\hline
\end{tabular}


APPENDIX C.1 Results of Volatile Organic Compound Analyses, Soils, Salmon Site

\begin{tabular}{|c|c|c|c|c|c|}
\hline COMPOUND & RESULTS UNITS & $\begin{array}{l}\text { DATA } \\
\text { QUAL. }\end{array}$ & $\begin{array}{l}\text { DATA } \\
\text { VALID. }\end{array}$ & UNITS & DILUTION \\
\hline \multicolumn{6}{|l|}{$\mathrm{C} 2-21-\mathrm{S}$} \\
\hline Chloromethane & 10 UG/KG & $u$ & NP & 10 UG/KG & 1 \\
\hline Bromomethane & $10 \mathrm{UG} / \mathrm{KG}$ & $u$ & NP & 10 UG/KG & 1 \\
\hline Vinyl Chloride & $10 \mathrm{UG} / \mathrm{KG}$ & $u$ & NP & 10 UG/KG & 1 \\
\hline Chloroethane & 10 UG/KG & $u$ & NP & $10 \mathrm{UG} / \mathrm{KG}$ & 1 \\
\hline Methylene Chloride & 5 UG/KG & $u$ & NP & 5 UG/KG & 1 \\
\hline Acetone & 10 UG/KG & $u$ & NP & $10 \mathrm{UG} / \mathrm{KG}$ & 1 \\
\hline Carbon Disulfide & 5 UG/KG & $u$ & NP & 5 UG/KG & 1 \\
\hline 1,1-Dichloroethene & 5 UG/KG & $u$ & NP & 5 UG/KG & 1 \\
\hline 1,1-Dichloroethane & 5 UG/KG & $u$ & NP & 5 UG/KG & 1 \\
\hline 1,2-Dichloroethene (total) & 5 UG/KG & $u$ & NP & 5 UG/KG & 1 \\
\hline Chloroform & 5 UG/KG & $u$ & NP & 5 UG/KG & 1 \\
\hline 1,2-Dichloroethane & 5 UG/KG & $u$ & NP & 5 UG/KG & 1 \\
\hline 2-Butanone & 10 UG/KG & $u$ & NP & 10 UG/KG & 1 \\
\hline 1,1,1-Trichloroethane & 5 UG/KG & $u$ & NP & 5 UG/KG & 1 \\
\hline Carbon Tetrachloride & 5 UG/KG & $u$ & NP & 5 UG/KG & 1 \\
\hline Vinyl Acetate & 10 UG/KG & $u$ & NP & 10 UG/KG & 1 \\
\hline Bromodichloromethane & 5 UG/KG & $u$ & NP & 5 UG/KG & 1 \\
\hline 1,2-Dichloropropane & 5 UG/KG & $u$ & NP & 5 UG/KG & 1 \\
\hline cis-1,3-Dichloropropene & 5 UG/KG & $u$ & NP & 5 UG/KG & 1 \\
\hline Trichloroethene & 5 UG/KG & $u$ & NP & 5 UG/KG & 1 \\
\hline Dibromochloromethane & 5 UG/KG & $u$ & NP & 5 UG/KG & 1 \\
\hline 1,1,2-Trichloroethane & 5 UG/KG & $u$ & NP & 5 UG/KG & 1 \\
\hline Benzene & 5 UG/KG & $u$ & NP & 5 UG/KG & 1 \\
\hline trans-1,3-Dichloropropene & 5 UG/KG & $u$ & NP & $5 \mathrm{UG} / \mathrm{KG}$ & 1 \\
\hline Bromoform & 5 UG/KG & $u$ & NP & 5 UG/KG & 1 \\
\hline 4-Methyl-2-Pentanone & 10 UG/KG & $u$ & NP & $10 \cup G / K G$ & 1 \\
\hline 2-Hexanone & 10 UG/KG & $u$ & NP & 10 UG/KG & 1 \\
\hline Tetrachloroethene & 5 UG/KG & $u$ & NP & $5 \mathrm{UG} / \mathrm{KG}$ & 1 \\
\hline 1,1,2,2-Tetrachloroethane & 5 UG/KG & u & NP & 5 UG/KG & 1 \\
\hline Toluene & 5 UG/KG & $u$ & NP & 5 UG/KG & 1 \\
\hline Chlorobenzene & 5 UGKG & $u$ & NP & 5 UG/KG & 1 \\
\hline Ethyl Benzene & 5 UG/KG & $u$ & NP & 5 UG/KG & 1 \\
\hline Styrene & 5 UG/KG & $u$ & NP & 5 UG/KG & 1 \\
\hline Xylenes (total) & 5 UG/KG & $u$ & NP & 5 UG/KG & 1 \\
\hline Unknown & 8 UG/KG & J & NP & UG/KG & 1 \\
\hline Toluene-d8 (SURR) & $97 \%$ & & & $\%$ & 1 \\
\hline Bromofluorobenzene (SURR) & $101 \%$ & & & $\%$ & 1 \\
\hline 1,2-Dichloroethane-d4 (SURR) & $101 \%$ & & & $\%$ & 1 \\
\hline
\end{tabular}


APPENDIX C.1 Results of Volatile Organic Compound Analyses, Soils, Salmon Site

\begin{tabular}{lcccc|}
\hline COMPOUND & RESULTS UNITS & DATA & DATA & \\
QUAL. & VALID. & CRDL & UNITS & DILUTION \\
\hline C2-21-S-MS & & & & \\
1,1-Dichloroethene & $94 \%$ & NP & $\%$ & 1 \\
Trichloroethene & $110 \%$ & NP & $\%$ & 1 \\
Benzene & $112 \%$ & NP & $\%$ & 1 \\
Toluene & $111 \%$ & NP & $\%$ & 1 \\
Chlorobenzene & $116 \%$ & NP & $\%$ & 1 \\
Toluene-d8 (SURR) & $99 \%$ & NP & $\%$ & 1 \\
Bromofluorobenzene (SURR) & $103 \%$ & NP & $\%$ & 1 \\
1,2-Dichloroethane-d4 (SURR) & $108 \%$ & NP & $\%$ & 1 \\
& & & & \\
C2-21-S-MSD & & & & 1 \\
1,1-Dichloroethene & $92 \%$ & NP & $\%$ & 1 \\
Trichloroethene & $102 \%$ & NP & $\%$ & 1 \\
Benzene & $109 \%$ & NP & $\%$ & 1 \\
Toluene & $108 \%$ & NP & $\%$ & 1 \\
Chlorobenzene & $111 \%$ & NP & $\%$ & 1 \\
Toluene-d8 (SURR) & $98 \%$ & NP & $\%$ & 1 \\
Bromofluorobenzene (SURR) & $100 \%$ & NP & $\%$ & 1 \\
1,2-Dichloroethane-d4 (SURR) & $108 \%$ & NP & $\%$ &
\end{tabular}


APPENDIX C.1 Results of Volatile Organic Compound Analyses, Soils, Salmon Site

\begin{tabular}{|c|c|c|c|c|c|}
\hline COMPOUND & RESULTS UNITS & $\begin{array}{l}\text { DATA } \\
\text { QUAL. }\end{array}$ & $\begin{array}{l}\text { DATA } \\
\text { VALID. }\end{array}$ & UNITS & DILUTION \\
\hline \multicolumn{6}{|l|}{ C2-22-S } \\
\hline Chloromethane & 10 UG/KG & $u$ & NP & 10 UG/KG & 1 \\
\hline Bromomethane & 10 UG/KG & $u$ & NP & $10 \mathrm{UG} / \mathrm{KG}$ & 1 \\
\hline Vinyl Chloride & 10 UG/KG & $u$ & NP & $10 \mathrm{UG} / \mathrm{KG}$ & 1 \\
\hline Chloroethane & $10 \mathrm{UG} / \mathrm{KG}$ & $u$ & NP & $10 \mathrm{UG} / \mathrm{KG}$ & 1 \\
\hline Methylene Chloride & 5 UG/KG & $u$ & NP & 5 UG/KG & 1 \\
\hline Acetone & 36 UG/KG & B & NP & $10 \mathrm{UG} / \mathrm{KG}$ & 1 \\
\hline Carbon Disulfide & 5 UG/KG & $u$ & NP & 5 UG/KG & 1 \\
\hline 1,1-Dichloroethene & 5 UG/KG & $u$ & NP & 5 UG/KG & 1 \\
\hline 1,1-Dichloroethane & 5 UG/KG & $u$ & NP & 5 UG/KG & 1 \\
\hline 1,2-Dichloroethene (total) & 5 UG/KG & $u$ & NP & 5 UG/KG & 1 \\
\hline Chloroform & 5 UG/KG & $u$ & NP & 5 UG/KG & 1 \\
\hline 1,2-Dichloroethane & 5 UG/KG & $u$ & NP & 5 UG/KG & 1 \\
\hline 2-Butanone & $10 \mathrm{UG} / \mathrm{KG}$ & $u$ & NP & 10 UG/KG & 1 \\
\hline 1,1,1-Trichloroethane & 5 UG/KG & $u$ & NP & 5 UG/KG & 1 \\
\hline Carbon Tetrachloride & 5 UG/KG & $u$ & NP & 5 UG/KG & 1 \\
\hline Vinyi Acetate & 10 UG/KG & $u$ & NP & $10 \mathrm{UG} / \mathrm{KG}$ & 1 \\
\hline Bromodichloromethane & 5 UG/KG & $u$ & NP & 5 UG/KG & 1 \\
\hline 1,2-Dichloropropane & 5 UG/KG & $u$ & NP & 5 UG/KG & 1 \\
\hline cis-1,3-Dichloropropene & 5 UG/KG & $u$ & NP & 5 UG/KG & 1 \\
\hline Trichloroethene & 5 UG/KG & $u$ & NP & 5 UG/KG & 1 \\
\hline Dibromochloromethane & 5 UG/KG & $u$ & NP & 5 UG/KG & 1 \\
\hline 1,1,2-Trichloroethane & 5 UG/KG & $u$ & NP & 5 UG/KG & 1 \\
\hline Benzene & 5 UG/KG & $u$ & NP & 5 UG/KG & 1 \\
\hline trans-1,3-Dichloropropene & 5 UG/KG & $u$ & NP & 5 UG/KG & 1 \\
\hline Bromoform & 5 UG/KG & $u$ & NP & 5 UG/KG & 1 \\
\hline 4-Methyi-2-Pentanone & 10 UG/KG & $u$ & NP & 10 UG/KG & 1 \\
\hline 2-Hexanone & 10 UG/KG & $u$ & NP & 10 UG/KG & 1 \\
\hline Tetrachloroethene & 5 UG/KG & $u$ & NP & 5 UG/KG & 1 \\
\hline 1,1,2,2-Tetrachloroethane & 5 UG/KG & $u$ & NP & 5 UG/KG & 1 \\
\hline Toluene & 5 UG/KG & $u$ & NP & 5 UG/KG & 1 \\
\hline Chlorobenzene & 5 UG/KG & $u$ & NP & 5 UG/KG & 1 \\
\hline Ethyl Benzene & 5 UG/KG & $u$ & NP & 5 UG/KG & 1 \\
\hline Styrene & 5 UG/KG & $u$ & NP & 5 UG/KG & 1 \\
\hline Xylenes (total) & 5 UG/KG & $u$ & NP & 5 UG/KG & 1 \\
\hline Toluene-d8 (SURR) & $105 \%$ & & & $\%$ & 1 \\
\hline Bromofluorobenzene (SURR) & $109 \%$ & & & $\%$ & 1 \\
\hline 1,2-Dichloroethane-d4 (SURR) & $115 \%$ & & & $\%$ & 1 \\
\hline
\end{tabular}


APPENDIX C.1 Results of Volatile Organic Compound Analyses, Soils, Salmon Site

\begin{tabular}{|c|c|c|c|c|c|}
\hline COMPOUND & RESULTS UNITS & $\begin{array}{l}\text { DATA } \\
\text { QUAL. }\end{array}$ & $\begin{array}{l}\text { DATA } \\
\text { VALID. }\end{array}$ & UNITS & DILUTION \\
\hline \multicolumn{6}{|l|}{ C2-23-S } \\
\hline Chloromethane & 10 UG/KG & $u$ & NP & :0 UG/KG & 1 \\
\hline Bromomethane & $10 \mathrm{UG} / \mathrm{KG}$ & $u$ & NP & 10 UG/KG & 1 \\
\hline Vinyl Chloride & 10 UG/KG & $u$ & NP & 10 UG/KG & 1 \\
\hline Chloroethane & 10 UG/KG & $u$ & NP & :O UG/KG & 1 \\
\hline Methylene Chloride & 5 UG/KG & $u$ & NP & 5 UG/KG & 1 \\
\hline Acetone & 10 UG/KG & $u$ & NP & 10 UG/KG & 1 \\
\hline Carbon Disulfide & 5 UG/KG & $u$ & NP & 5 UG/KG & 1 \\
\hline 1,1-Dichloroethene & 5 UG/KG & $u$ & NP & 5 UG/KG & 1 \\
\hline 1,1-Dichloroethane & 5 UG/KG & $u$ & NP & 5 UG/KG & 1 \\
\hline 1,2-Dichloroethene (total) & 5 UG/KG & $u$ & NP & 5 UG/KG & 1 \\
\hline Chloroform & 5 UG/KG & $u$ & NP & 5 UG/KG & 1 \\
\hline 1,2-Dichloroethane & 5 UG/KG & $u$ & NP & 5 UG/KG & 1 \\
\hline 2-Butanone & $10 \mathrm{UG} / \mathrm{KG}$ & $u$ & NP & 10 UG/KG & 1 \\
\hline 1,1,1-Trichloroethane & 5 UG/KG & $u$ & NP & 5 UG/KG & 1 \\
\hline Carbon Tetrachloride & 5 UG/KG & $u$ & NP & 5 UG/KG & 1 \\
\hline Vinyl Acetate & 10 UG/KG & $u$ & NP & 10 UG/KG & 1 \\
\hline Bromodichloromethane & 5 UG/KG & $u$ & NP & 5 UG/KG & 1 \\
\hline 1,2-Dichloropropane & 5 UG/KG & .u & NP & 5 UG/KG & 1 \\
\hline cis-1,3-Dichloropropene & 5 UG/KG & $u$ & NP & 5 UG/KG & 1 \\
\hline Trichloroethene & 5 UG/KG & $u$ & NP & 5 UG/KG & 1 \\
\hline Dibromochloromethane & 5 UG/KG & $u$ & NP & 5 UG/KG & 1 \\
\hline 1,1,2-Trichloroethane & 5 UG/KG & $u$ & NP & 5 UG/KG & 1 \\
\hline Benzene & 5 UG/KG & $u$ & NP & 5 UG/KG & 1 \\
\hline trans-1,3-Dichloropropene & 5 UG/KG & $u$ & NP & 5 UG/KG & 1 \\
\hline Bromoform & 5 UG/KG & $u$ & NP & 5 UG/KG & 1 \\
\hline 4-Methyl-2-Pentanone & 10 UG/KG & $u$ & NP & 10 UG/KG & 1 \\
\hline 2-Hexanone & $10 \mathrm{UG} / \mathrm{KG}$ & $u$ & NP & 10 UG/KG & 1 \\
\hline Tetrachloroethene & 5 UG/KG & $u$ & NP & 5 UG/KG & 1 \\
\hline $1,1,2,2-$ Tetrachloroethane & 5 UG/KG & $u$ & NP & 5 UG/KG & 1 \\
\hline Toluene & 5 UG/KG & $u$ & NP & 5 UG/KG & 1 \\
\hline Chlorobenzene & 5 UG/KG & $u$ & NP & 5 UG/KG & 1 \\
\hline Ethyl Benzene & $5 \mathrm{UG} / \mathrm{KG}$ & $u$ & NP & 5 UG/KG & 1 \\
\hline Styrene & 5 UG/KG & $u$ & NP & 5 UG/KG & 1 \\
\hline Xylenes (total) & $5 \mathrm{UG} / \mathrm{KG}$ & $u$ & NP & 5 UG/KG & 1 \\
\hline Toluene-d8 (SURR) & $100 \%$ & & & $\%$ & 1 \\
\hline Bromofluorobenzene (SURR) & $112 \%$ & & & $\%$ & 1 \\
\hline 1,2-Dichloroethane-d4 (SURR) & $139 \%$ & - & & $\%$ & 1 \\
\hline
\end{tabular}




\section{APPENDIX C.1 Results of Volatile Organic Compound Analyses, Soils,}

Salmon Site

\begin{tabular}{|c|c|c|c|c|c|}
\hline COMPOUND & RESULTS UNITS & $\begin{array}{l}\text { DATA } \\
\text { QUAL. }\end{array}$ & $\begin{array}{l}\text { DATA } \\
\text { VALID. } \\
\end{array}$ & UNITS & DILUTION \\
\hline \multicolumn{6}{|l|}{ C2-23-S-RE } \\
\hline Chloromethane & 10 UG/KG & $u$ & NP & 10 UG/KG & 1 \\
\hline Bromomethane & $10 \mathrm{UG} / \mathrm{KG}$ & $u$ & NP & 10 UG/KG & 1 \\
\hline Vinyl Chloride & 10 UG/KG & $u$ & NP & 10 UG/KG & 1 \\
\hline Chloroethane & 10 UG/KG & $u$ & NP & 10 UG/KG & 1 \\
\hline Methylene Chloride & 5 UG/KG & $u$ & NP & 5 UG/KG & 1 \\
\hline Acetone & $10 \mathrm{UG} / \mathrm{KG}$ & $u$ & NP & 10 UG/KG & 1 \\
\hline Carbon Disulfide & 5 UG/KG & $u$ & NP & 5 UG/KG & 1 \\
\hline 1,1-Dichloroethene & 5 UG/KG & $u$ & NP & 5 UG/KG & 1 \\
\hline 1,1-Dichloroethane & 5 UG/KG & $u$ & NP & 5 UG/KG & 1 \\
\hline 1,2-Dichloroethene (total) & 5 UG/KG & $u$ & NP & 5 UG/KG & 1 \\
\hline Chloroform & 5 UG/KG & $u$ & NP & 5 UG/KG & 1 \\
\hline 1,2-Dichloroethane & 5 UG/KG & $u$ & NP & 5 UG/KG & 1 \\
\hline 2-Butanone & 10 UG/KG & $u$ & NP & $10 \mathrm{UG} / \mathrm{KG}$ & 1 \\
\hline 1,1,1-Trichloroethane & 5 UG/KG & $u$ & NP & 5 UG/KG & 1 \\
\hline Carbon Tetrachloride & 5 UG/KG & $u$ & NP & 5 UG/KG & 1 \\
\hline Vinyl Acetate & 10 UG/KG & $u$ & NP & 10 UG/KG & 1 \\
\hline Bromodichloromethane & 5 UG/KG & $u$ & NP & 5 UG/KG & 1 \\
\hline 1,2-Dichloropropane & 5 UG/KG & $u$ & NP & 5 UG/KG & 1 \\
\hline cis-1,3-Dichloropropene & 5 UG/KG & $u$ & NP & 5 UG/KG & 1 \\
\hline Trichloroethene & 5 UG/KG & $u$ & NP & 5 UG/KG & 1 \\
\hline Dibromochloromethane & 5 UG/KG & $u$ & NP & 5 UG/KG & 1 \\
\hline 1,1,2-Trichloroethane & 5 UG/KG & $u$ & NP & 5 UG/KG & 1 \\
\hline Benzene & 5 UG/KG & $u$ & NP & 5 UG/KG & 1 \\
\hline trans-1,3-Dichloropropene & 5 UG/KG & $u$ & NP & 5 UG/KG & 1 \\
\hline Bromoform & 5 UG/KG & $u$ & NP & 5 UG/KG & 1 \\
\hline 4-Methyl-2-Pentanone & 10 UG/KG & $u$ & NP & 10 UG/KG & 1 \\
\hline 2-Hexanone & 10 UG/KG & $u$ & NP & 10 UG/KG & 1 \\
\hline Tetrachloroethene & 5 UG/KG & $u$ & NP & 5 UG/KG & 1 \\
\hline 1,1,2,2-Tetrachloroethane & $5 \mathrm{UG} / \mathrm{KG}$ & $u$ & NP & 5 UG/KG & 1 \\
\hline Toluene & 5 UG/KG & $u$ & NP & 5 UG/KG & 1 \\
\hline Chlorobenzene & 5 UG/KG & $u$ & NP & 5 UG/KG & 1 \\
\hline Ethyl Benzene & 5 UG/KG & $u$ & NP & 5 UG/KG & 1 \\
\hline Styrene & 5 UG/KG & $u$ & NP & 5 UG/KG & 1 \\
\hline Xylenes (total) & 5 UG/KG & $u$ & NP & 5 UG/KG & 1 \\
\hline Unknown & 15 UG/KG & J & NP & UG/KG & 1 \\
\hline Toluene-d8 (SURR) & $101 \%$ & & & $\%$ & 1 \\
\hline Bromofluorobenzene (SURR) & $103 \%$ & & & $\%$ & 1 \\
\hline 1,2-Dichloroethane-d4 (SURR) & $109 \%$ & & & $\%$ & 1 \\
\hline
\end{tabular}


APPENDIX C.1 Results of Volatile Organic Compound Analyses, Soils, Salmon Site

\begin{tabular}{|c|c|c|c|c|c|}
\hline COMPOUND & RESULTS UNITS & $\begin{array}{l}\text { DATA } \\
\text { QUAL. }\end{array}$ & $\begin{array}{l}\text { DATA } \\
\text { VALID. }\end{array}$ & UNITS & DILUTIC \\
\hline \multicolumn{6}{|l|}{ C2-24-S } \\
\hline Chloromethane & 10 UG/KG & $u$ & NP & $10 \mathrm{UG} / \mathrm{KG}$ & 1 \\
\hline Bromomethane & 10 UG/KG & $u$ & NP & $10 \mathrm{UG} / \mathrm{KG}$ & 1 \\
\hline Vinyl Chloride & 10 UG/KG & $u$ & NP & 10 UG/KG & 1 \\
\hline Chloroethane & 10 UG/KG & $u$ & NP & 10 UG/KG & 1 \\
\hline Methylene Chloride & 5 UG/KG & $u$ & NP & 5 UG/KG & 1 \\
\hline Acetone & 10 UG/KG & $u$ & NP & 10 UG/KG & 1 \\
\hline Carbon Disulfide & 5 UG/KG & $u$ & NP & 5 UG/KG & 1 \\
\hline 1,1-Dichloroethene & 5 UG/KG & $u$ & NP & 5 UG/KG & 1 \\
\hline 1,1-Dichloroethane & 5 UG/KG & $u$ & NP & 5 UG/KG & 1 \\
\hline 1,2-Dichloroethene (total) & 5 UG/KG & $u$ & NP & 5 UG/KG & 1 \\
\hline Chloroform & 5 UG/KG & $u$ & NP & 5 UG/KG & 1 \\
\hline 1,2-Dichloroethane & 5 UG/KG & $u$ & NP & 5 UG/KG & 1 \\
\hline 2-Butanone & 10 UG/KG & $u$ & NP & 10 UG/KG & 1 \\
\hline 1,1,1-Trichloroethane & 5 UG/KG & $u$ & NP & 5 UG/KG & 1 \\
\hline Carbon Tetrachloride & 5 UG/KG & $u$ & NP & 5 UG/KG & 1 \\
\hline Vinyl Acetate & $10 \mathrm{UG} / \mathrm{KG}$ & $u$ & NP & $10 \mathrm{UG} / \mathrm{KG}$ & 1 \\
\hline Bromodichloromethane & 5 UG/KG & $u$ & NP & 5 UG/KG & 1 \\
\hline 1,2-Dichloropropane & 5 UG/KG & $u$ & NP & 5 UG/KG & 1 \\
\hline cis-1,3-Dichloropropene & 5 UG/KG & $u$ & NP & 5 UG/KG & 1 \\
\hline Trichloroethene & 5 UG/KG & $u$ & NP & 5 UG/KG & 1 \\
\hline Dibromochloromethane & 5 UG/KG & $u$ & NP & 5 UG/KG & 1 \\
\hline 1,1,2-Trichloroethane & 5 UG/KG & $u$ & NP & 5 UG/KG & 1 \\
\hline Benzene & 5 UG/KG & $u$ & NP & 5 UG/KG & 1 \\
\hline trans-1,3-Dichloropropene & 5 UG/KG & $u$ & NP & 5 UG/KG & 1 \\
\hline Bromoform & 5 UG/KG & $u$ & NP & 5 UG/KG & 1 \\
\hline 4-Methyl-2-Pentanone & $10 \mathrm{UG} / \mathrm{KG}$ & $u$ & NP & 10 UG/KG & 1 \\
\hline 2-Hexanone & 10 UG/KG & $u$ & NP & $10 \mathrm{UG/KG}$ & 1 \\
\hline Tetrachloroethene & 5 UG/KG & $u$ & NP & 5 UG/KG & 1 \\
\hline 1,1,2,2-Tetrachloroethane & 5 UG/KG & $u$ & NP & 5 UG/KG & 1 \\
\hline Toluene & 5 UG/KG & $u$ & NP & 5 UG/KG & 1 \\
\hline Chlorobenzene & 5 UG/KG & $u$ & NP & 5 UG/KG & 1 \\
\hline Ethyl Benzene & 5 UG/KG & $u$ & NP & 5 UG/KG & 1 \\
\hline Styrene & 5 UG/KG & $u$ & NP & 5 UG/KG & 1 \\
\hline Xylenes (total) & 5 UG/KG & $u$ & NP & 5 UG/KG & 1 \\
\hline Toluene-d8 (SURR) & $99 \%$ & & & $\%$ & 1 \\
\hline Bromofluorobenzene (SURR) & $105 \%$ & & & $\%$ & 1 \\
\hline 1,2-Dichloroethane-d4 (SURR) & $103 \%$ & & & $\%$ & 1 \\
\hline
\end{tabular}


APPENDIX C.1 Results of Volatile Organic Compound Analyses, Soils, Salmon Site

\begin{tabular}{|c|c|c|c|c|c|}
\hline COMPOUND & RESULTS UNITS & $\begin{array}{l}\text { DATA } \\
\text { QUAL. }\end{array}$ & $\begin{array}{l}\text { DATA } \\
\text { VALID. }\end{array}$ & UNITS & DILUTION \\
\hline \multicolumn{6}{|l|}{ C2-24-S-RE } \\
\hline Chloromethane & $10 \mathrm{UG} / \mathrm{KG}$ & $u$ & NP & 10 UG/KG & 1 \\
\hline Bromomethane & 10 UG/KG & $u$ & NP & 10 UG/KG & 1 \\
\hline Vinyl Chloride & $10 \mathrm{UG} / \mathrm{KG}$ & $u$ & NP & $10 \mathrm{UG} / \mathrm{KG}$ & 1 \\
\hline Chloroethane & 10 UG/KG & $u$ & NP & 10 UG/KG & 1 \\
\hline Methylene Chloride & 5 UG/KG & $u$ & NP & 5 UG/KG & 1 \\
\hline Acetone & $10 \mathrm{UG} / \mathrm{KG}$ & $u$ & NP & 10 UG/KG & 1 \\
\hline Carbon Disulfide & 5 UG/KG & $u$ & NP & 5 UG/KG & 1 \\
\hline 1,1-Dichloroethene & 5 UG/KG & $u$ & NP & 5 UG/KG & 1 \\
\hline 1,1-Dichloroethane & 5 UG/KG & $u$ & NP & 5 UG/KG & 1 \\
\hline 1,2-Dichloroethene (total) & 5 UG/KG & $u$ & NP & 5 UG/KG & 1 \\
\hline Chloroform & 5 UG/KG & $u$ & NP & 5 UG/KG & 1 \\
\hline 1,2-Dichloroethane & 5 UG/KG & $u$ & NP & 5 UG/KG & 1 \\
\hline 2-Butanone & 10 UG/KG & $u$ & NP & $10 \mathrm{UG} / \mathrm{KG}$ & 1 \\
\hline 1,1,1-Trichloroethane & 5 UG/KG & $u$ & NP & 5 UG/KG & 1 \\
\hline Carbon Tetrachloride & 5 UG/KG & $u$ & NP & 5 UG/KG & 1 \\
\hline Vinyl Acetate & 10 UG/KG & $u$ & NP & $10 \mathrm{UG} / \mathrm{KG}$ & 1 \\
\hline Bromodichloromethane & 5 UG/KG & $u$ & NP & 5 UG/KG & 1 \\
\hline 1,2-Dichloropropane & $5 \mathrm{UG} / \mathrm{KG}$ & $u$ & NP & 5 UG/KG & 1 \\
\hline cis-1,3-Dichloropropene & 5 UG/KG & $u$ & NP & 5 UG/KG & 1 \\
\hline Trichloroethene & 5 UG/KG & $u$ & NP & 5 UG/KG & 1 \\
\hline Dibromochloromethane & 5 UG/KG & $u$ & NP & 5 UG/KG & 1 \\
\hline 1,1,2-Trichloroethane & 5 UG/KG & $u$ & NP & 5 UG/KG & 1 \\
\hline Benzene & 5 UG/KG & $u$ & NP & 5 UG/KG & 1 \\
\hline trans-1,3-Dichloropropene & 5 UG/KG & $u$ & NP & 5 UG/KG & 1 \\
\hline Bromoform & 5 UG/KG & $u$ & NP & 5 UG/KG & 1 \\
\hline 4-Methyl-2-Pentanone & 10 UG/KG & $u$ & NP & 10 UG/KG & 1 \\
\hline 2-Hexanone & $10 \mathrm{UG} / \mathrm{KG}$ & $u$ & NP & 10 UG/KG & 1 \\
\hline Tetrachloroethene & 5 UG/KG & $u$ & NP & 5 UG/KG & 1 \\
\hline 1,1,2,2-Tetrachloroethane & 5 UG/KG & $u$ & NP & 5 UG/KG & 1 \\
\hline Toluene & 5 UG/KG & $u$ & NP & 5 UG/KG & 1 \\
\hline Chlorobenzene & 5 UG/KG & $u$ & NP & 5 UG/KG & 1 \\
\hline Ethyl Benzene & $5 \mathrm{UG} / \mathrm{KG}$ & $u$ & NP & 5 UG/KG & 1 \\
\hline Styrene & 5 UG/KG & $u$ & NP & 5 UG/KG & 1 \\
\hline Xylenes (total) & 5 UG/KG & $u$ & NP & 5 UG/KG & 1 \\
\hline Toluene-d8 (SURR) & $95 \%$ & & & $\%$ & 1 \\
\hline Bromofluorobenzene (SURR) & $100 \%$ & & & $\%$ & 1 \\
\hline 1,2-Dichloroethane-d4 (SURR) & $108 \%$ & & & $\%$ & 1 \\
\hline
\end{tabular}




\section{APPENDIX C.1 Results of Volatile Organic Compound Analyses, Soils, Salmon Site}

\begin{tabular}{|c|c|c|c|c|c|}
\hline COMPOUND & RESULTS UNITS & $\begin{array}{l}\text { DATA } \\
\text { QUAL. }\end{array}$ & $\begin{array}{l}\text { DATA } \\
\text { VALID. }\end{array}$ & UNITS & DILUTION \\
\hline \multicolumn{6}{|l|}{ C2-25-S } \\
\hline Chloromethane & 10 UG/KG & $u$ & NP & 10 UG/KG & 1 \\
\hline Bromomethane & 10 UG/KG & $u$ & NP & 10 UG/KG & 1 \\
\hline Vinyl Chloride & 10 UG/KG & $u$ & NP & 10 UG/KG & 1 \\
\hline Chloroethane & 10 UG/KG & $u$ & NP & 10 UG/KG & 1 \\
\hline Methylene Chloride & 5 UG/KG & $u$ & NP & 5 UG/KG & 1 \\
\hline Acetone & 10 UG/KG & $u$ & NP & 10 UG/KG & 1 \\
\hline Carbon Disulfide & 5 UG/KG & $u$ & NP & 5 UG/KG & 1 \\
\hline 1,1-Dichloroethene & 5 UG/KG & $u$ & NP & 5 UG/KG & 1 \\
\hline 1,1-Dichloroethane & 5 UG/KG & $u$ & NP & 5 UG/KG & 1 \\
\hline 1,2-Dichloroethene (total) & 5 UG/KG & $u$ & NP & 5 UG/KG & 1 \\
\hline Chloroform & 5 UG/KG & $u$ & NP & 5 UG/KG & 1 \\
\hline 1,2-Dichloroethane & 5 UG/KG & $u$ & NP & 5 UG/KG & 1 \\
\hline 2-Butanone & $10 \mathrm{UG} / \mathrm{KG}$ & $u$ & NP & 10 UG/KG & 1 \\
\hline $1,1,1-$ Trichloroethane & 5 UG/KG & $u$ & NP & 5 UG/KG & 1 \\
\hline Carbon Tetrachloride & 5 UG/KG & $u$ & NP & 5 UG/KG & 1 \\
\hline Vinyl Acetate & $10 \mathrm{UG} / \mathrm{KG}$ & $u$ & NP & 10 UG/KG & 1 \\
\hline Bromodichloromethane & 5 UG/KG & $u$ & NP & 5 UG/KG & 1 \\
\hline 1,2-Dichloropropane & 5 UG/KG & $u$ & NP & 5 UG/KG & 1 \\
\hline cis-1,3-Dichloropropene & 5 UG/KG & $u$ & NP & 5 UG/KG & 1 \\
\hline Trichloroethene & 5 UG/KG & $u$ & NP & 5 UG/KG & 1 \\
\hline Dibromochloromethane & 5 UG/KG & $u$ & NP & 5 UG/KG & 1 \\
\hline 1,1,2-Trichloroethane & 5 UG/KG & $u$ & NP & 5 UG/KG & 1 \\
\hline Benzene & 5 UG/KG & $u$ & NP & 5 UG/KG & 1 \\
\hline trans-1,3-Dichloropropene & 5 UG/KG & $u$ & NP & 5 UG/KG & 1 \\
\hline Bromoform & 5 UG/KG & $u$ & NP & 5 UG/KG & 1 \\
\hline 4-Methyl-2-Pentanone & 10 UG/KG & $u$ & NP & 10 UG/KG & 1 \\
\hline 2-Hexanone & 10 UG/KG & $u$ & NP & 10 UG/KG & 1 \\
\hline Tetrachloroethene & 5 UG/KG & $u$ & NP & 5 UG/KG & 1 \\
\hline $1,1,2,2$-Tetrachloroethane & 5 UG/KG & $u$ & NP & 5 UG/KG & 1 \\
\hline Toluene & 5 UG/KG & $u$ & NP & 5 UG/KG & 1 \\
\hline Chlorobenzene & 5 UG/KG & $u$ & NP & 5 UG/KG & 1 \\
\hline Ethyl Benzene & 5 UG/KG & $u$ & NP & 5 UG/KG & 1 \\
\hline Styrene & 5 UG/KG & $u$ & NP & 5 UG/KG & 1 \\
\hline Xylenes (total) & 5 UG/KG & $u$ & NP & 5 UG/KG & 1 \\
\hline Toluene-d8 (SURR) & $99 \%$ & & & $\%$ & 1 \\
\hline Bromofluorobenzene (SURR) & $102 \%$ & & & $\%$ & 1 \\
\hline 1,2-Dichloroethane-d4 (SURR) & $107 \%$ & & & $\%$ & 1 \\
\hline
\end{tabular}


APPENDIX C.1 Results of Volatile Organic Compound Analyses, Soils, Salmon Site

\begin{tabular}{|c|c|c|c|c|c|}
\hline COMPOUND & RESULTS UNITS & $\begin{array}{l}\text { DATA } \\
\text { QUAL. }\end{array}$ & $\begin{array}{l}\text { DATA } \\
\text { VALID. }\end{array}$ & UNITS & DILUTION \\
\hline \multicolumn{6}{|l|}{ C2-25A-S } \\
\hline Chloromethane & $10 \mathrm{UG} / \mathrm{KG}$ & $\mathrm{u}$ & NP & 10 UG/KG & 1 \\
\hline Bromomethane & 10 UG/KG & $U$ & NP & 10 UG/KG & 1 \\
\hline Vinyl Chloride & 10 UG/KG & $u$ & NP & $10 \mathrm{UG} / \mathrm{KG}$ & 1 \\
\hline Chloroethane & 10 UG/KG & 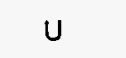 & NP & 10 UG/KG & 1 \\
\hline Methylene Chloride & 5 UG/KG & $u$ & NP & 5 UG/KG & 1 \\
\hline Acetone & 10 UG/KG & $U$ & NP & 10 UG/KG & 1 \\
\hline Carbon Disulfide & $5 \mathrm{UG} / \mathrm{KG}$ & $\mathrm{u}$ & NP & 5 UG/KG & 1 \\
\hline 1,1-Dichloroethene & 5 UG/KG & $u$ & NP & 5 UG/KG & 1 \\
\hline 1,1-Dichloroethane & 5 UG/KG & $u$ & NP & 5 UG/KG & 1 \\
\hline 1,2-Dichloroethene (total) & 5 UG/KG & $u$ & NP & 5 UG/KG & 1 \\
\hline Chloroform & 5 UG/KG & $u$ & NP & 5 UG/KG & 1 \\
\hline 1,2-Dichloroethane & 5 UG/KG & $u$ & NP & 5 UG/KG & 1 \\
\hline 2-Butanone & 10 UG/KG & $u$ & NP & 10 UG/KG & 1 \\
\hline 1,1,1-Trichloroethane & $5 \mathrm{UG} / \mathrm{KG}$ & $\mathrm{U}$ & NP & 5 UG/KG & 1 \\
\hline Carbon Tetrachloride & 5 UG/KG & $u$ & NP & 5 UG/KG & 1 \\
\hline Vinyl Acetate & 10 UG/KG & $u$ & NP & 10 UG/KG & 1 \\
\hline Bromodichloromethane & 5 UG/KG & $u$ & NP & 5 UG/KG & 1 \\
\hline 1,2-Dichloropropane & 5 UG/KG & $U$ & NP & 5 UG/KG & 1 \\
\hline cis-1,3-Dichloropropene & 5 UG/KG & $u$ & NP & 5 UG/KG & 1 \\
\hline Trichloroethene & 5 UG/KG & $\mathrm{U}$ & NP & 5 UG/KG & 1 \\
\hline Dibromochloromethane & 5 UG/KG & $u$ & NP & 5 UG/KG & 1 \\
\hline 1,1,2-Trichloroethane & 5 UG/KG & $u$ & NP & 5 UG/KG & 1 \\
\hline Benzene & 5 UG/KG & $u$ & NP & 5 UG/KG & 1 \\
\hline trans-1,3-Dichloropropene & 5 UG/KG & $u$ & NP & 5 UG/KG & 1 \\
\hline Bromoform & 5 UG/KG & $u$ & NP & 5 UG/KG & 1 \\
\hline 4-Methyl-2-Pentanone & $10 \mathrm{UG} / \mathrm{KG}$ & $\mathrm{U}$ & NP & 10 UG/KG & 1 \\
\hline 2-Hexanone & 10 UG/KG & $u$ & NP & $10 \mathrm{UG} / \mathrm{KG}$ & 1 \\
\hline Tetrachloroethene & 5 UG/KG & $U$ & NP & 5 UG/KG & 1 \\
\hline 1,1,2,2-Tetrachloroethane & 5 UG/KG & $U$ & NP & 5 UG/KG & 1 \\
\hline Toluene & 5 UG/KG & $\mathrm{U}$ & NP & 5 UG/KG & 1 \\
\hline Chlorobenzene & 5 UG/KG & $u$ & NP & 5 UG/KG & 1 \\
\hline Ethyl Benzene & 5 UG/KG & $U$ & NP & 5 UG/KG & 1 \\
\hline Styrene & $5 \mathrm{UG} / \mathrm{KG}$ & U & NP & 5 UG/KG & 1 \\
\hline Xylenes (total) & 5 UG/KG & $\mathrm{u}$ & NP & 5 UG/KG & 1 \\
\hline Toluene-d8 (SURR) & $100 \%$ & & & $\%$ & 1 \\
\hline Bromofluorobenzene (SURR) & $104 \%$ & & & $\%$ & 1 \\
\hline 1,2-Dichloroethane-d4 (SURR) & $102 \%$ & & & $\%$ & 1 \\
\hline
\end{tabular}


APPENDIX C.1 Results of Volatile Organic Compound Analyses, Soils, Salmon Site

\begin{tabular}{|c|c|c|c|c|c|}
\hline COMPOUND & RESULTS UNITS & $\begin{array}{l}\text { DATA } \\
\text { QUAL. }\end{array}$ & $\begin{array}{l}\text { DATA } \\
\text { VALID. }\end{array}$ & UNITS & DILUTIO \\
\hline \multicolumn{6}{|l|}{ C2-26-S } \\
\hline Chloromethane & $10 \mathrm{UG} / \mathrm{KG}$ & $u$ & NP & 10 UG/KG & 1 \\
\hline Bromomethane & 10 UG/KG & $u$ & NP & 10 UG/KG & 1 \\
\hline Vinyl Chloride & 10 UGKG & $u$ & NP & 10 UG/KG & 1 \\
\hline Chloroethane & 10 UG/KG & $u$ & NP & 10 UG/KG & 1 \\
\hline Methylene Chloride & 5 UG/KG & $u$ & NP & 5 UG/KG & 1 \\
\hline Acetone & 10 UG/KG & $u$ & NP & 10 UG/KG & 1 \\
\hline Carbon Disulfide & 5 UG/KG & $u$ & NP & 5 UG/KG & 1 \\
\hline 1,1-Dichloroethene & 5 UG/KG & $u$ & NP & 5 UG/KG & 1 \\
\hline 1,1-Dichloroethane & 5 UG/KG & $u$ & NP & 5 UG/KG & 1 \\
\hline 1,2-Dichloroethene (total) & 5 UG/KG & $u$ & NP & 5 UG/KG & 1 \\
\hline Chloroform & 5 UG/KG & u & NP & 5 UG/KG & 1 \\
\hline 1,2-Dichloroethane & 5 UG/KG & $u$ & NP & 5 UG/KG & 1 \\
\hline 2-Butanone & 10 UG/KG & $u$ & NP & 10 UG/KG & 1 \\
\hline 1,1,1-Trichloroethane & 5 UG/KG & $u$ & NP & 5 UG/KG & 1 \\
\hline Carbon Tetrachloride & 5 UG/KG & $u$ & NP & 5 UG/KG & 1 \\
\hline Vinyl Acetate & 10 UG/KG & $u$ & NP & 10 UG/KG & 1 \\
\hline Bromodichloromethane & 5 UG/KG & $u$ & NP & 5 UG/KG & 1 \\
\hline 1,2-Dichloropropane & 5 UG/KG & $u$ & NP & 5 UG/KG & 1 \\
\hline cis-1,3-Dichloropropene & 5 UG/KG & $u$ & NP & 5 UG/KG & 1 \\
\hline Trichloroethene & 5 UG/KG & $u$ & NP & 5 UG/KG & 1 \\
\hline Dibromochloromethane & 5 UG/KG & $u$ & NP & 5 UG/KG & 1 \\
\hline 1,1,2-Trichloroethane & 5 UG/KG & $u$ & NP & 5 UG/KG & 1 \\
\hline Benzene & 5 UG/KG & $u$ & NP & 5 UG/KG & 1 \\
\hline trans-1,3-Dichloropropene & 5 UG/KG & $u$ & NP & 5 UG/KG & 1 \\
\hline Bromoform & 5 UG/KG & $u$ & NP & 5 UG/KG & 1 \\
\hline 4-Methyl-2-Pentanone & 10 UG/KG & $u$ & NP & 10 UG/KG & 1 \\
\hline 2-Hexanone & 10 UG/KG & $u$ & NP & 10 UG/KG & 1 \\
\hline Tetrachloroethene & 5 UG/KG & $u$ & NP & 5 UG/KG & 1 \\
\hline 1,1,2,2-Tetrachloroethane & 5 UG/KG & $u$ & NP & 5 UG/KG & 1 \\
\hline Toluene & 5 UG/KG & $u$ & NP & 5 UG/KG & 1 \\
\hline Chlorobenzene & 5 UG/KG & $u$ & NP & 5 UG/KG & 1 \\
\hline Ethyl Benzene & 5 UG/KG & $u$ & NP & $5 \mathrm{UG} / \mathrm{KG}$ & 1 \\
\hline Styrene & 5 UG/KG & $u$ & NP & 5 UG/KG & 1 \\
\hline Xylenes (total) & 5 UG/KG & $u$ & NP & 5 UG/KG & 1 \\
\hline Unknown & 6 UG/KG & J & NP & UG/KG & 1 \\
\hline Toluene-d8 (SURR) & $98 \%$ & & & $\%$ & 1 \\
\hline Bromofluorobenzene (SURR) & $103 \%$ & & & $\%$ & 1 \\
\hline 1,2-Dichloroethane-d4 (SURR) & $111 \%$ & & & $\%$ & 1 \\
\hline
\end{tabular}


APPENDIX C.1 Results of Volatile Organic Compound Analyses, Soils, Salmon Site

\begin{tabular}{|c|c|c|c|c|c|}
\hline COMPOUND & RESULTS UNITS & $\begin{array}{l}\text { DATA } \\
\text { QUAL. }\end{array}$ & $\begin{array}{r}\text { DATA } \\
\text { VALID. } \\
\end{array}$ & UNITS & DILUTION \\
\hline \multicolumn{6}{|l|}{ C2-27-S } \\
\hline Chloromethane & $10 \mathrm{UG} / \mathrm{KG}$ & $u$ & NP & 10 UG/KG & 1 \\
\hline Bromomethane & 10 UG/KG & $u$ & NP & $10 \mathrm{UG} / \mathrm{KG}$ & 1 \\
\hline Vinyl Chloride & 10 UG/KG & $u$ & NP & 10 UG/KG & 1 \\
\hline Chloroethane & 10 UG/KG & $u$ & NP & $10 U G / K G$ & 1 \\
\hline Methylene Chloride & 5 UG/KG & $u$ & NP & 5 UG/KG & 1 \\
\hline Acetone & $10 \mathrm{UG} / \mathrm{KG}$ & $u$ & NP & 10 UG/KG & 1 \\
\hline Carbon Disulfide & 5 UG/KG & $u$ & NP & 5 UG/KG & 1 \\
\hline 1,1-Dichloroethene & 5 UG/KG & $u$ & NP & 5 UG/KG & 1 \\
\hline 1,1-Dichloroethane & 5 UG/KG & $u$ & NP & 5 UG/KG & 1 \\
\hline 1,2-Dichloroethene (total) & 5 UG/KG & $u$ & NP & 5 UG/KG & 1 \\
\hline Chloroform & 5 UG/KG & $u$ & NP & 5 UG/KG & 1 \\
\hline 1,2-Dichloroethane & 5 UG/KG & $u$ & NP & 5 UG/KG & 1 \\
\hline 2-Butanone & 10 UG/KG & $u$ & NP & 10 UG/KG & 1 \\
\hline $1,1,1$-Trichloroethane & 5 UG/KG & $u$ & NP & 5 UG/KG & 1 \\
\hline Carbon Tetrachloride & 5 UG/KG & $u$ & NP & 5 UG/KG & 1 \\
\hline Vinyl Acetate & 10 UG/KG & $u$ & NP & 10 UG/KG & 1 \\
\hline Bromodichloromethane & 5 UG/KG & $u$ & NP & 5 UG/KG & 1 \\
\hline 1,2-Dichloropropane & 5 UG/KG & $u$ & NP & 5 UG/KG & 1 \\
\hline cis-1,3-Dichloropropene & 5 UG/KG & $u$ & NP & 5 UG/KG & 1 \\
\hline Trichloroethene & 5 UG/KG & $u$ & NP & 5 UG/KG & 1 \\
\hline Dibromochloromethane & 5 UG/KG & $u$ & NP & 5 UG/KG & 1 \\
\hline 1,1,2-Trichloroethane & .5 UG/KG & $u$ & NP & 5 UG/KG & 1 \\
\hline Benzene & 5 UG/KG & $u$ & NP & 5 UG/KG & 1 \\
\hline trans-1,3-Dichloropropene & 5 UG/KG & $u$ & NP & 5 UG/KG & 1 \\
\hline Bromoform & 5 UG/KG & $u$ & NP & 5 UG/KG & 1 \\
\hline 4-Methyl-2-Pentanone & 10 UG/KG & $u$ & NP & 10 UG/KG & 1 \\
\hline 2-Hexanone & 10 UG/KG & $u$ & NP & 10 UG/KG & 1 \\
\hline Tetrachloroethene & 5 UG/KG & $u$ & NP & 5 UG/KG & 1 \\
\hline 1,1,2,2-Tetrachloroethane & 5 UG/KG & $u$ & NP & 5 UG/KG & 1 \\
\hline Toluene & 5 UG/KG & $u$ & NP & 5 UG/KG & 1 \\
\hline Chlorobenzene & 5 UG/KG & $u$ & NP & 5 UG/KG & 1 \\
\hline Ethyl Benzene & 5 UG/KG & $u$ & NP & 5 UG/KG & 1 \\
\hline Styrene & 5 UG/KG & $u$ & NP & 5 UG/KG & 1 \\
\hline Xylenes (total) & 5 UG/KG & $u$ & NP & 5 UG/KG & 1 \\
\hline Unknown & 7 UG/KG & J & NP & UG/KG & 1 \\
\hline Toluene-d8 (SURR) & $105 \%$ & & & $\%$ & 1 \\
\hline Bromofluorobenzene (SURR) & $104 \%$ & & & $\%$ & 1 \\
\hline 1,2-Dichloroethane-d4 (SURR) & $103 \%$ & & & $\%$ & 1 \\
\hline
\end{tabular}




\section{Appendix C.2 \\ Results of Volatile Organic Compound Analyses, Water, Salmon Site}


APPENDIX C.2 Results of Volatile Organic Analyses, Water, Salmon Site

\begin{tabular}{|c|c|c|c|c|c|}
\hline COMPOUND & RESULTS & $\begin{array}{l}\text { DATA } \\
\text { QUAL. }\end{array}$ & $\begin{array}{l}\text { DATA } \\
\text { VALID. } \\
\end{array}$ & CRDL UNITS & DILUTION \\
\hline \multicolumn{6}{|l|}{ C5-1-W } \\
\hline Chloromethane & 10 UG $\Omega$ & $u$ & NP & 10 UG/ & 1 \\
\hline Bromomethane & 10 UG & $u$ & NP & $10 \mathrm{UG} \Omega$ & 1 \\
\hline Vinyl Chloride & $10 \mathrm{UG} / \mathrm{L}$ & $u$ & NP & 10 UG $/$ & 1 \\
\hline Chloroethane & 10 UG $\Omega$ & $u$ & NP & $10 \mathrm{UG} / \mathrm{L}$ & 1 \\
\hline Methylene Chloride & 2 UG $几$ & BJ & NP & 5 UG/L & 1 \\
\hline Acetone & 10 UG $\Omega$ & $u$ & NP & $10 \mathrm{UG} / \mathrm{L}$ & 1 \\
\hline Carbon Disulfide & 5 UG/ & $u$ & NP & $5 \mathrm{UG} /$ & 1 \\
\hline 1,1-Dichloroethene & 5 UG $\Omega$ & $u$ & NP & 5 UG/ & 1 \\
\hline 1,1-Dichloroethane & 5 UG/ & $u$ & NP & 5 UG $\Omega$ & 1 \\
\hline 1,2-Dichloroethene (total) & 5 UG $\Omega$ & $u$ & NP & 5 UG $\Omega$ & 1 \\
\hline Chloroform & 5 UG/ & $u$ & NP & 5 UG/ & 1 \\
\hline 1,2-Dichloroethane & 5 UG/ & $u$ & NP & $5 \mathrm{UG} \Omega$ & 1 \\
\hline 2-Butanone & $10 \mathrm{UG} / \mathrm{L}$ & $u$ & NP & 10 UG $\Omega$ & 1 \\
\hline 1,1,1-Trichloroethane & 5 UG & $u$ & NP & 5 UG几 & 1 \\
\hline Carbon Tetrachloride & 5 UG/ & $u$ & NP & 5 UG/L & 1 \\
\hline Vinyl Acetate & $10 \mathrm{UG} / \mathrm{L}$ & $u$ & NP & $10 \mathrm{UG} \Omega$ & 1 \\
\hline Bromodichloromethane & 5 UG $\Omega$ & $u$ & NP & $5 \mathrm{UG} / \mathrm{L}$ & 1 \\
\hline 1,2-Dichloropropane & 5 UG/ & $u$ & NP & 5 UG $\Omega$ & 1 \\
\hline cis-1,3-Dichloropropene & 5 UG/ & $u$ & NP & 5 UG $/$ & 1 \\
\hline Trichloroethene & 5 UG $\Omega$ & $u$ & NP & $5 \mathrm{UG} \Omega$ & 1 \\
\hline Dibromochloromethane & 5 UG $\Omega$ & $u$ & NP & 5 UG $\Omega$ & 1 \\
\hline 1,1,2-Trichloroethane & 5 UG几 & $u$ & NP & $5 \mathrm{UG} \Omega$ & 1 \\
\hline Benzene & 5 UG/ & $u$ & NP & $5 \mathrm{UG} /$ & 1 \\
\hline trans-1,3-Dichloropropene & 5 UG/ & $u$ & NP & $5 \mathrm{UG} /$ & 1 \\
\hline Bromoform & 5 UG/ & $u$ & NP & 5 UG $\Omega$ & 1 \\
\hline 4-Methyl-2-Pentanone & 10 UG $\Omega$ & $u$ & NP & 10 UG/ & 1 \\
\hline 2-Hexanone & 10 UG $\Omega$ & $u$ & NP & 10 UG & 1 \\
\hline Tetrachloroethene & 5 UG $\Omega$ & $u$ & NP & 5 UG $\Omega$ & 1 \\
\hline 1,1,2,2-Tetrachloroethane & 5 UG/ & $u$ & NP & 5 UG $\Omega$ & 1 \\
\hline Toluene & 2 UG/L & $J$ & NP & 5 UG & 1 \\
\hline Chlorobenzene & 5 UG/ & $u$ & NP & 5 UG $\Omega$ & 1 \\
\hline Ethyl Benzene & 5 UG/ & $u$ & $\mathrm{NP}$ & $5 \mathrm{UG} \Omega$ & 1 \\
\hline Styrene & 5 UG $/$ & $u$ & NP & 5 UG $\Omega$ & 1 \\
\hline Xylenes (total) & 5 UG $\Omega$ & $u$ & NP & $5 \mathrm{UG} \Omega$ & 1 \\
\hline Unknown-1 & $6 \mathrm{UG} \Omega$ & J & NP & UG/ & 1 \\
\hline Unknown-2 & 12 UG $\Omega$ & J & NP & UG $\Omega$ & 1 \\
\hline Toluene-d8 (SURR) & $100 \%$ & & & $\%$ & 1 \\
\hline Bromofluorobenzene (SURR) & $107 \%$ & & & $\%$ & 1 \\
\hline 1,2-Dichloroethane-d4 (SURR) & $104 \%$ & & & $\%$ & 1 \\
\hline
\end{tabular}


APPENDIX C.2 Results of Volatile Organic Analyses, Water, Salmon Site

\begin{tabular}{lcccc|}
\hline COMPOUND & RESULTS & UNITS & $\begin{array}{c}\text { DATA } \\
\text { QUAL. }\end{array}$ & DATA \\
VALID. & CRDL UNITS & DILUTION \\
\hline C5-1-W-MS & & & & \\
1,1-Dichloroethene & $100 \%$ & NP & $\%$ & 1 \\
Trichloroethene & $111 \%$ & NP & $\%$ & 1 \\
Benzene & $113 \%$ & NP & $\%$ & 1 \\
Toluene & $106 \%$ & NP & $\%$ & 1 \\
Chlorobenzene & $113 \%$ & NP & $\%$ & 1 \\
Toluene-d8 (SURR) & $99 \%$ & NP & $\%$ & 1 \\
Bromofluorobenzene (SURR) & $105 \%$ & NP & $\%$ & 1 \\
1,2-Dichloroethane-d4 (SURR) & $103 \%$ & NP & $\%$ & 1 \\
& & & & \\
C5-1-W-MSD & & & & \\
1,1-Dichloroethene & $101 \%$ & NP & $\%$ & 1 \\
Trichloroethene & $111 \%$ & NP & $\%$ & 1 \\
Benzene & $117 \%$ & NP & $\%$ & 1 \\
Toluene & $109 \%$ & NP & $\%$ & 1 \\
Chlorobenzene & $116 \%$ & NP & $\%$ & 1 \\
Toluene-d8 (SURR) & $98 \%$ & NP & $\%$ & 1 \\
Bromofluorobenzene (SURR) & $105 \%$ & NP & $\%$ & 1 \\
1,2-Dichloroethane-d4 (SURR) & $103 \%$ & NP & $\%$ & 1
\end{tabular}


APPENDIX C.2 Results of Volatile Organic Analyses, Water, Salmon Site

\begin{tabular}{|c|c|c|c|c|c|}
\hline COMPOUND & RESULTS UNITS & $\begin{array}{l}\text { DATA } \\
\text { QUAL. }\end{array}$ & $\begin{array}{c}\text { DATA } \\
\text { VALID. }\end{array}$ & CRDL UNITS & DILUTION \\
\hline \multicolumn{6}{|l|}{ C3-8-W } \\
\hline Chloromethane & $10 \mathrm{UG} / \mathrm{L}$ & $u$ & NP & 10 UG/L & 1 \\
\hline Bromomethane & 10 UG & $u$ & NP & $10 \mathrm{UG} / \mathrm{L}$ & 1 \\
\hline Vinyl Chloride & 10 UG $/$ & $u$ & NP & $10 \mathrm{UG} / \mathrm{L}$ & 1 \\
\hline Chloroethane & $10 \mathrm{UG} / \mathrm{L}$ & $u$ & NP & 10 UG/L & 1 \\
\hline Methylene Chloride & 3 UG/L & BJ & NP & 5 UG/ & 1 \\
\hline Acetone & $10 \mathrm{UG} / \mathrm{L}$ & $u$ & NP & $10 \mathrm{UG} \Omega$ & 1 \\
\hline Carbon Disulfide & 5 UGR & $u$ & NP & $5 \mathrm{UG} / \mathrm{L}$ & 1 \\
\hline 1,1-Dichloroethene & 5 UG/ & $u$ & NP & $5 \mathrm{UG} / \mathrm{L}$ & 1 \\
\hline 1,1-Dichloroethane & 5 UG/L & $u$ & NP & $5 \mathrm{UG} / \mathrm{L}$ & 1 \\
\hline 1,2-Dichloroethene (total) & 5 UG $\Omega$ & $u$ & NP & $5 \mathrm{UG} \Omega$ & 1 \\
\hline Chloroform & 5 UG/ & $u$ & NP & $5 \mathrm{UG} /$ & 1 \\
\hline 1,2-Dichloroethane & 5 UG & $u$ & NP & 5 UG & 1 \\
\hline 2-Butanone & 10 UG $\Omega$ & $u$ & NP & $10 \mathrm{UG} / \mathrm{L}$ & 1 \\
\hline $1,1,1-$ Trichloroethane & 5 UG/L & $u$ & NP & $5 \mathrm{UG} /$ & 1 \\
\hline Carbon Tetrachloride & $5 \mathrm{UG} / \mathrm{L}$ & $u$ & NP & 5 UG $\Omega$ & 1 \\
\hline Vinyl Acetate & $10 \mathrm{UG} \Omega$ & $u$ & NP & 10 UG $\Omega$ & 1 \\
\hline Bromodichloromethane & 5 UG/ & $u$ & NP & 5 UG & 1 \\
\hline 1,2-Dichloropropane & 5 UGL & $u$ & NP & 5 UG几 & 1 \\
\hline cis-1,3-Dichloropropene & 5 UG $\Omega$ & $u$ & NP & 5 UG $\Omega$ & 1 \\
\hline Trichloroethene & 5 UGR & $u$ & NP & 5 UG/ & 1 \\
\hline Dibromochloromethane & 5 UG/L & $u$ & NP & 5 UG $\Omega$ & 1 \\
\hline 1,1,2-Trichloroethane & 5 UGR & $u$ & NP & 5 UG $\Omega$ & 1 \\
\hline Benzene & 5 UG & $u$ & NP & $5 \mathrm{UG} \Omega$ & 1 \\
\hline trans-1,3-Dichloropropene & 5 UG/ & $u$ & NP & 5 UG $\Omega$ & 1 \\
\hline Bromoform & 5 UG/ & $u$ & NP & 5 UG $\Omega$ & 1 \\
\hline 4-Methyl-2-Pentanone & $10 \mathrm{UG} / \mathrm{L}$ & $u$ & NP & 10 UG $\Omega$ & 1 \\
\hline 2-Hexanone & $10 \mathrm{UG} / \mathrm{L}$ & $u$ & NP & 10 UG $\Omega$ & 1 \\
\hline Tetrachloroethene & 5 UG $\Omega$ & $u$ & NP & 5 UG/ & 1 \\
\hline 1,1,2,2-Tetrachloroethane & 5 UG $/$ & $u$ & NP & 5 UG $\Omega$ & 1 \\
\hline Toluene & 5 UG几 & $u$ & NP & 5 UG/ & 1 \\
\hline Chlorobenzene & 5 UG/ & $u$ & NP & $5 \mathrm{UG} \Omega$ & 1 \\
\hline Ethyl Benzene & 5 UGR & $u$ & NP & $5 \mathrm{UG \Omega}$ & 1 \\
\hline Styrene & 5 UG/ & $u$ & NP & 5 UG & 1 \\
\hline Xylenes (total) & 5 UG/ & $u$ & NP & 5 UG $\Omega$ & 1 \\
\hline Unknown & 6 UG & $J$ & NP & UGR & 1 \\
\hline Toluene-d8 (SURR) & $97 \%$ & & & $\%$ & 1 \\
\hline Bromofluorobenzene (SURR) & $103 \%$ & & & $\%$ & 1 \\
\hline 1,2-Dichloroethane-d4 (SURR) & $98 \%$ & & & $\%$ & 1 \\
\hline
\end{tabular}




\section{APPENDIX C.2 Results of Volatile Organic Analyses, Water, Salmon Site}

\begin{tabular}{|c|c|c|c|c|c|}
\hline COMPOUND & RESULTS & $\begin{array}{l}\text { DATA } \\
\text { QUAL. }\end{array}$ & $\begin{array}{l}\text { DATA } \\
\text { VALID. }\end{array}$ & CRDL UNITS & DILUTION \\
\hline \multicolumn{6}{|l|}{ C3-9-W } \\
\hline Chloromethane & 10 UG/L & $u$ & NP & 10 UG/L & 1 \\
\hline Bromomethane & 10 UG $\Omega$ & $u$ & NP & 10 UG/L & 1 \\
\hline Vinyl Chloride & $10 \mathrm{UG} / \mathrm{L}$ & $u$ & $\mathrm{NP}$ & 10 UG $\Omega$ & 1 \\
\hline Chloroethane & $10 \mathrm{UG} / \mathrm{L}$ & $u$ & NP & $10 \mathrm{UG} / \mathrm{L}$ & 1 \\
\hline Methylene Chloride & $3 \mathrm{UG} / \mathrm{L}$ & BJ & NP & $5 \mathrm{UG} /$ & 1 \\
\hline Acetone & $10 \mathrm{UG} \Omega$ & $u$ & NP & $10 \mathrm{UG} / \mathrm{L}$ & 1 \\
\hline Carbon Disulfide & 5 UG/ & $u$ & NP & 5 UG/ & 1 \\
\hline 1,1-Dichloroethene & 5 UG $/$ & $u$ & NP & 5 UG/ & 1 \\
\hline 1,1-Dichloroethane & 5 UG/L & $u$ & NP & 5 UG/L & 1 \\
\hline 1,2-Dichloroethene (total) & 5 UG/L & $u$ & NP & 5 UG/L & 1 \\
\hline Chloroform & 5 UG/L & $u$ & NP & 5 UG/ & 1 \\
\hline 1,2-Dichloroethane & 5 UG/ & $u$ & NP & 5 UG/L & 1 \\
\hline 2-Butanone & 10 UG/L & $u$ & NP & 10 UG/L & 1 \\
\hline 1,1,1-Trichloroethane & 5 UG/ & $u$ & NP & 5 UG/L & 1 \\
\hline Carbon Tetrachloride & 5 UGR & $u$ & NP & 5 UG/ & 1 \\
\hline Vinyl Acetate & 10 UG/L & $u$ & NP & 10 UG/ & 1 \\
\hline Bromodichloromethane & 5 UG几 & $u$ & NP & 5 UG/ & 1 \\
\hline 1,2-Dichloropropane & 5 UG/ & $u$ & NP & 5 UG/ & 1 \\
\hline cis-1,3-Dichloropropene & 5 UG & $u$ & NP & 5 UG/L & 1 \\
\hline Trichloroethene & $5 \mathrm{UG} / \mathrm{L}$ & $u$ & NP & 5 UG/L & 1 \\
\hline Dibromochioromethane & 5 UG/L & $u$ & NP & 5 UG & 1 \\
\hline 1,1,2-Trichloroethane & 5 UGR & $u$ & NP & 5 UG/ & 1 \\
\hline Benzene & 5 UG/ & $u$ & NP & 5 UGR & 1 \\
\hline trans-1,3-Dichloropropene & 5 UG $\Omega$ & $u$ & NP & $5 \mathrm{UG} / \mathrm{L}$ & 1 \\
\hline Bromoform & 5 UG/L & $u$ & NP & 5 UG/ & 1 \\
\hline 4-Methyl-2-Pentanone & 10 UG/ & $u$ & NP & $10 \mathrm{UG} / \mathrm{L}$ & 1 \\
\hline 2-Hexanone & $10 \mathrm{UG} / \mathrm{L}$ & $u$ & NP & $10 \mathrm{UG} / \mathrm{L}$ & 1 \\
\hline Tetrachloroethene & 5 UG/L & $u$ & NP & 5 UG/L & 1 \\
\hline 1,1,2,2-Tetrachloroethane & $5 \mathrm{UG} /$ & $u$ & NP & 5 UGR & 1 \\
\hline Toluene & 10 UG & & NP & 5 UG/ & 1 \\
\hline Chlorobenzene & 5 UG/L & $u$ & NP & 5 UG/ & 1 \\
\hline Ethyl Benzene & 5 UGR & $u$ & NP & 5 UG/L & 1 \\
\hline Styrene & 5 UG/ & $u$ & NP & 5 UG/L & 1 \\
\hline Xylenes (total) & 5 UG/ & $u$ & NP & $5 \mathrm{UG} \Omega$ & 1 \\
\hline Unknown & $6 \mathrm{UG} / \mathrm{L}$ & $J$ & NP & UG/L & 1 \\
\hline Toluene-d8 (SURR) & $99 \%$ & & & $\%$ & 1 \\
\hline Bromofluorobenzene (SURR) & $105 \%$ & & & $\%$ & 1 \\
\hline 1,2-Dichloroethane-d4 (SURR) & $109 \%$ & & & $\%$ & 1 \\
\hline
\end{tabular}


APPENDIX C.2 Results of Volatile Organic Analyses, Water, Salmon Site

\begin{tabular}{|c|c|c|c|c|c|}
\hline COMPOUND & RESULTS & $\begin{array}{l}\text { DATA } \\
\text { QUAL. }\end{array}$ & $\begin{array}{l}\text { DATA } \\
\text { VALID. }\end{array}$ & CRDL UNITS & DILUTION \\
\hline \multicolumn{6}{|l|}{$C 4-11-W$} \\
\hline Chloromethane & $10 \mathrm{UG} / \mathrm{L}$ & $u$ & NP & 10 UG $\Omega$ & 1 \\
\hline Bromomethane & 10 UG $\Omega$ & $u$ & NP & $10 \mathrm{UG} / \mathrm{L}$ & 1 \\
\hline Vinyl Chloride & 10 UG/ & $u$ & NP & $10 \mathrm{UG} / \mathrm{L}$ & 1 \\
\hline Chloroethane & $10 \mathrm{UG} / \mathrm{L}$ & $u$ & NP & 10 UG $\Omega$ & 1 \\
\hline Methylene Chloride & 2 UG/ & BJ & NP & 5 UG & 1 \\
\hline Acetone & 5 UG/L & BJ & NP & $10 \mathrm{UG} \Omega$ & 1 \\
\hline Carbon Disulfide & 5 UG $\Omega$ & $u$ & NP & 5 UG/L & 1 \\
\hline 1,1-Dichloroethene & 5 UGR & $u$ & NP & $5 \mathrm{UG} / \mathrm{L}$ & 1 \\
\hline 1,1-Dichloroethane & 5 UG/ & $u$ & NP & 5 UG/L & 1 \\
\hline 1,2-Dichloroethene (total) & 5 UG/ & $u$ & NP & 5 UGR & 1 \\
\hline Chloroform & 5 UG & $u$ & NP & 5 UG/L & 1 \\
\hline 1,2-Dichloroethane & 5 UG/L & u & NP & 5 UG/ & 1 \\
\hline 2-Butanone & 10 UG/ & $u$ & NP & 10 UG/L & 1 \\
\hline 1,1,1-Trichloroethane & 5 UG & $u$ & NP & 5 UGん & 1 \\
\hline Carbon Tetrachloride & 5 UG/ & $u$ & NP & 5 UG & 1 \\
\hline Vinyl Acetate & $10 \mathrm{UG} / \mathrm{L}$ & $u$ & NP & 10 UG & 1 \\
\hline Bromodichloromethane & 5 UGL & $u$ & NP & 5 UG & 1 \\
\hline 1,2-Dichloropropane & 5 UG/L & $u$ & NP & 5 UG几 & 1 \\
\hline cis-1,3-Dichloropropene & 5 UGR & $u$ & NP & 5 UG/ & 1 \\
\hline Trichloroethene & 5 UGR & $u$ & NP & 5 UG & 1 \\
\hline Dibromochloromethane & 5 UG/ & $u$ & NP & 5 UG几 & 1 \\
\hline 1,1,2-Trichloroethane & $5 \mathrm{UG} / \mathrm{L}$ & $u$ & NP & 5 UGR & 1 \\
\hline Benzene & 5 UG几 & $u$ & NP & 5 UG几 & 1 \\
\hline trans-1,3-Dichloropropene & 5 UG & $u$ & NP & 5 UG $\Omega$ & 1 \\
\hline Bromoform & 5 UGR & $u$ & NP & 5 UGR & 1 \\
\hline 4-Methyl-2-Pentanone & $10 \mathrm{UG} /$ & $u$ & NP & $10 \mathrm{UG} / \mathrm{L}$ & 1 \\
\hline 2-Hexanone & 10 UG/L & $u$ & NP & $10 \mathrm{UG} / \mathrm{L}$ & 1 \\
\hline Tetrachloroethene & 5 UG & $u$ & NP & 5 UG $\Omega$ & 1 \\
\hline 1,1,2,2-Tetrachloroethane & 5 UG/L & $u$ & NP & 5 UG几 & 1 \\
\hline Toluene & 5 UG/L & $u$ & NP & 5 UG & 1 \\
\hline Chlorobenzene & $5 \mathrm{UG} / \mathrm{L}$ & $u$ & NP & 5 UG & 1 \\
\hline Ethyl Benzene & 5 UG/L & $u$ & NP & 5 UG/ & 1 \\
\hline Styrene & 5 UGR & $u$ & NP & 5 UGR & 1 \\
\hline Xylenes (total) & 5 UG/ & $u$ & NP & 5 UGR & 1 \\
\hline Unknown & 6 UG/ & $\mathrm{J}$ & NP & UGR & 1 \\
\hline Toluene-d8 (SURR) & $98 \%$ & & & $\%$ & 1 \\
\hline Bromofluorobenzene (SURR) & $106 \%$ & & & $\%$ & 1 \\
\hline 1,2-Dichloroethane-d4 (SURR) & $105 \%$ & & & $\%$ & 1 \\
\hline
\end{tabular}


APPENDIX C.2 Results of Volatile Organic Analyses, Water, Salmon Site

\begin{tabular}{|c|c|c|c|c|c|}
\hline COMPOUND & RESULTS & $\begin{array}{l}\text { DATA } \\
\text { QUAL. }\end{array}$ & $\begin{array}{l}\text { DATA } \\
\text { VALID. }\end{array}$ & CRDL UNITS & DILUTION \\
\hline \multicolumn{6}{|l|}{$C 4-13-W$} \\
\hline Chloromethane & $10 \mathrm{UG} \Omega$ & $u$ & NP & 10 UG/ & 1 \\
\hline Bromomethane & $10 \mathrm{UG} / \mathrm{L}$ & $u$ & NP & 10 UG $\Omega$ & 1 \\
\hline Vinyl Chloride & 10 UG/L & $u$ & NP & 10 UG & 1 \\
\hline Chloroethane & $10 \mathrm{UG} / \mathrm{L}$ & $u$ & NP & 10 UG/L & 1 \\
\hline Methylene Chloride & $1 \mathrm{UG} / \mathrm{L}$ & BJ & NP & 5 UG/L & 1 \\
\hline Acetone & 6 UG/L & BJ & NP & 10 UG几 & 1 \\
\hline Carbon Disulfide & $5 \mathrm{UG} / \mathrm{L}$ & $u$ & NP & 5 UG几 & 1 \\
\hline 1,1-Dichloroethene & 5 UG/ & $u$ & NP & $5 \mathrm{UG} / \mathrm{L}$ & 1 \\
\hline 1,1-Dichloroethane & 5 UG/ & $u$ & NP & 5 UG/ & 1 \\
\hline 1,2-Dichloroethene (total) & 5 UG & $u$ & NP & 5 UGR & 1 \\
\hline Chloroform & 5 UG/ & $u$ & NP & 5 UG/ & 1 \\
\hline 1,2-Dichloroethane & 5 UG/ & $u$ & NP & 5 UG/ & 1 \\
\hline 2-Butanone & 10 UG/ & $u$ & NP & $10 \mathrm{UG} \Omega$ & 1 \\
\hline 1,1,1-Trichloroethane & 5 UG/. & $u$ & NP & 5 UG $\Omega$ & 1 \\
\hline Carbon Tetrachloride & 5 UG/L & $u$ & NP & 5 UG/ & 1 \\
\hline Vinyl Acetate & $10 \mathrm{UG} / \mathrm{L}$ & $u$ & NP & 10 UG/ & 1 \\
\hline Bromodichloromethane & 5 UG/ & $u$ & NP & 5 UG/L & 1 \\
\hline 1,2-Dichloropropane & 5 UG/L & $u$ & NP & 5 UG $/$ & 1 \\
\hline cis-1,3-Dichloropropene & 5 UG/L & $u$ & NP & 5 UG/L & 1 \\
\hline Trichloroethene & 5 UG/ & $u$ & NP & 5 UG & 1 \\
\hline Dibromochloromethane & 5 UG/ & $u$ & NP & 5 UG/ & 1 \\
\hline 1,1,2-Trichloroethane & 5 UG $\Omega$ & $u$ & NP & 5 UG/ & 1 \\
\hline Benzene & 5 UG/ & $u$ & NP & 5 UG/ & 1 \\
\hline trans-1,3-Dichloropropene & 5 UG $/$ & $u$ & NP & 5 UG/ & 1 \\
\hline Bromotorm & 5 UG/L & $u$ & NP & 5 UG/L & 1 \\
\hline 4-Methyl-2-Pentanone & $10 \mathrm{UG} / \mathrm{L}$ & $u$ & NP & $10 \mathrm{UG} / \mathrm{L}$ & 1 \\
\hline 2-Hexanone & $10 \mathrm{UG} / \mathrm{L}$ & $u$ & NP & 10 UG $\Omega$ & 1 \\
\hline Tetrachloroethene & 5 UG/ & $u$ & NP & 5 UG/ & 1 \\
\hline $1,1,2,2$-Tetrachloroethane & 5 UG/L & $u$ & NP & 5 UG/ & 1 \\
\hline Toluene & 5 UG/ & $u$ & NP & 5 UG/ & 1 \\
\hline Chlorobenzene & 5 UG/L & $u$ & NP & 5 UG/ & 1 \\
\hline Ethyl Benzene & 5 UG/L & $u$ & NP & $5 \mathrm{UG} / \mathrm{L}$ & 1 \\
\hline Styrene & 5 UG/ & $u$ & NP & 5 UG/ & 1 \\
\hline Xylenes (total) & 5 UG $\Omega$ & $u$ & NP & 5 UG & 1 \\
\hline Toluene-d8 (SURR) & $102 \%$ & & & $\%$ & 1 \\
\hline Bromofluorobenzene (SURR) & $103 \%$ & & & $\%$ & 1 \\
\hline 1,2-Dichloroethane-d4 (SURR) & $105 \%$ & & & $\%$ & 1 \\
\hline
\end{tabular}


APPENDIX C.2 Results of Volatile Organic Analyses, Water, Salmon Site

\begin{tabular}{|c|c|c|c|c|c|}
\hline COMPOUND & RESULTS & $\begin{array}{l}\text { DATA } \\
\text { QUAL. }\end{array}$ & $\begin{array}{l}\text { DATA } \\
\text { VALID. }\end{array}$ & CRDL UNITS & DILUTION \\
\hline \multicolumn{6}{|l|}{$C 1-14-W$} \\
\hline Chloromethane & 10 UGR & $u$ & $\cdots$ & 10 UG $\Omega$ & 1 \\
\hline Bromomethane & 10 UG/L & $u$ & $\cdots$ & 10 UG/L & 1 \\
\hline Vinyl Chloride & 10 UG/L & $u$ & - & 10 UG $\Omega$ & 1 \\
\hline Chloroethane & 10 UG $\Omega$ & $u$ & UJ & $10 \mathrm{UG} / \mathrm{L}$ & 1 \\
\hline Methylene Chloride & 5 UGR & $u$ & - & 5 UG/ & 1 \\
\hline Acetone & 10 UG/ & B & us & $10 \mathrm{UG} / \mathrm{L}$ & 1 \\
\hline Carbon Disulfide & 3 UG/L & J & - & 5 UG/ & 1 \\
\hline 1,1-Dichloroethene & 5 UG/ & $u$ & - & 5 UG $\Omega$ & 1 \\
\hline 1,1-Dichloroethane & $5 \mathrm{UG} /$ & $u$ & - & 5 UG & 1 \\
\hline 1,2-Dichloroethene (total) & 5 UG & $u$ & - & 5 UG/ & 1 \\
\hline Chloroform & 5 UG $\Omega$ & $u$ & - & 5 UG/ & 1 \\
\hline 1,2-Dichloroethane & 5 UG/ & $u$ & - & 5 UG/ & 1 \\
\hline 2-Butanone & $10 \mathrm{UG} / \mathrm{L}$ & $u$ & -- & 10 UG $\Omega$ & 1 \\
\hline 1,1,1-Trichloroethane & 5 UG/L & $u$ & -- & 5 UG $/$ & 1 \\
\hline Carbon Tetrachloride & $5 \mathrm{UG} / \mathrm{L}$ & $u$ & $-\cdots$ & 5 UG/ & 1 \\
\hline Vinyl Acetate & $10 \mathrm{UG} / \mathrm{L}$ & $u$ & $\ldots$ & 10 UG/ & 1 \\
\hline Bromodichloromethane & 5 UG几 & $u$ & - & 5 UG $\Omega$ & 1 \\
\hline 1,2-Dichloropropane & 5 UG $\Omega$ & $u$ & $\cdots$ & 5 UG $\Omega$ & 1 \\
\hline cis-1,3-Dichloropropene & 5 UG & $u$ & - & 5 UG/L & 1 \\
\hline Trichloroethene & 5 UGR & $u$ & - & $5 \mathrm{UG} \Omega$ & 1 \\
\hline Dibromochloromethane & 5 UG & $u$ & - & 5 UG $\Omega$ & 1 \\
\hline 1,1,2-Trichloroethane & 5 UGL & $u$ & - & 5 UG几 & 1 \\
\hline Benzene & 5 UG/L & $u$ & - & 5 UG $\Omega$ & 1 \\
\hline trans-1,3-Dichloropropene & 5 UG/ & $u$ & - & 5 UG & 1 \\
\hline Bromoform & 5 UGR & $u$ & - & 5 UG几 & 1 \\
\hline 4-Methyl-2-Pentanone & 10 UG/ & $u$ & - & 10 UG $\Omega$ & 1 \\
\hline 2-Hexanone & 10 UG $/$ & $u$ & - & $10 \mathrm{UG} \Omega$ & 1 \\
\hline Tetrachloroethene & 5 UG/ & $u$ & - & $5 \mathrm{UG} /$ & 1 \\
\hline 1,1,2,2-Tetrachloroethane & 5 UGR & $u$ & - & 5 UG $\Omega$ & 1 \\
\hline Toluene & 5 UG/ & $u$ & - & 5 UG $\Omega$ & 1 \\
\hline Chlorobenzene & $5 \mathrm{UG} /$ & $u$ & - & 5 UG & 1 \\
\hline Ethyl Benzene & 5 UG/L & $u$ & - & 5 UG & 1 \\
\hline Styrene & 5 UG $/$ & $u$ & - & 5 UG/ & 1 \\
\hline Xylenes (total) & 5 UGR & $u$ & - & 5 UGR & 1 \\
\hline Toluene-d8 (SURR) & $95 \%$ & & & $\%$ & 1 \\
\hline Bromofluorobenzene (SURR) & $106 \%$ & & & $\%$ & 1 \\
\hline 1,2-Dichloroethane-d4 (SURR) & $104 \%$ & & & $\%$ & 1 \\
\hline
\end{tabular}


APPENDIX C.2 Results of Volatile Organic Analyses, Water, Salmon Site

\begin{tabular}{|c|c|c|c|c|c|}
\hline COMPOUND & RESULTS UNITS & $\begin{array}{l}\text { DATA } \\
\text { QUAL. }\end{array}$ & $\begin{array}{l}\text { DATA } \\
\text { VALID. }\end{array}$ & CRDL UNITS & DILUTION \\
\hline \multicolumn{6}{|l|}{$C 1-15-W$} \\
\hline Chloromethane & 10 UG/ & $u$ & -- & 10 UG $\Omega$ & 1 \\
\hline Bromomethane & $10 \mathrm{UG} \Omega$ & $u$ & $\ldots$ & $10 \mathrm{UG} / \mathrm{L}$ & 1 \\
\hline Vinyl Chloride & $10 \mathrm{UG} / \mathrm{L}$ & $u$ & --- & $10 \mathrm{UG} \Omega$ & 1 \\
\hline Chloroethane & $10 \mathrm{UG} \Omega$ & $u$ & UJ & 10 UGR & 1 \\
\hline Methylene Chloride & 2 UG/ & BJ & $u$ & 5 UG & 1 \\
\hline Acetone & $10 \mathrm{UG} \Omega$ & $u$ & UJ & $10 \mathrm{UG} / \mathrm{L}$ & 1 \\
\hline Carbon Disulfide & 5 UG/ & $u$ & --- & 5 UG/ & 1 \\
\hline 1,1-Dichloroethene & 5 UGL & $u$ & -- & 5 UG/L & 1 \\
\hline 1,1-Dichloroethane & $5 \mathrm{UG} / \mathrm{L}$ & $u$ & -- & 5 UG/L & 1 \\
\hline 1,2-Dichloroethere (total) & $5 \mathrm{UG} /$ & $u$ & $\ldots$ & 5 UG & 1 \\
\hline Chloroform & 5 UG/ & $u$ & $\ldots$ & 5 UG & 1 \\
\hline 1,2-Dichloroethane & 5 UG $/$ & $u$ & $\ldots$ & 5 UG/L & 1 \\
\hline 2-Butanone & 10 UG/ & $u$ & $\ldots$ & $10 \mathrm{UG} / \mathrm{L}$ & 1 \\
\hline 1,1,1-Trichloroethane & 5 UG & $u$ & -- & 5 UG/ & 1 \\
\hline Carbon Tetrachloride & 5 UG几 & $u$ & $\ldots$ & $5 \mathrm{UG} / \mathrm{L}$ & 1 \\
\hline Vinyl Acetate & $10 \mathrm{UG \Omega}$ & $u$ & -- & 10 UG $\Omega$ & 1 \\
\hline Bromodichloromethane & 5 UG $\Omega$ & $u$ & $\ldots$ & $5 \mathrm{UG} / \mathrm{L}$ & 1 \\
\hline 1,2-Dichloropropane & 5 UG/ & $u$ & - & 5 UG $\Omega$ & 1 \\
\hline cis-1,3-Dichloropropene & 5 UG/ & $u$ & $\ldots$ & 5 UG/ & 1 \\
\hline Trichloroethene & 5 UG/ & $u$ & -- & $5 \mathrm{UG} / \mathrm{L}$ & 1 \\
\hline Dibromochloromethane & 5 UG $\Omega$ & $u$ & $\cdots$ & 5 UG $\Omega$ & 1 \\
\hline 1,1,2-Trichloroethane & 5 UG/ & $u$ & $\ldots$ & $5 \mathrm{UG} /$ & 1 \\
\hline Benzene & $5 \mathrm{UG} /$ & $u$ & $\ldots$ & 5 UG/ & 1 \\
\hline trans-1,3-Dichloropropene & 5 UGת & $u$ & $\ldots$ & 5 UG/ & 1 \\
\hline Bromoform & 5 UGR & $u$ & $\cdots$ & 5 UGん & 1 \\
\hline 4-Methyl-2-Pentanone & 10 UG $\Omega$ & $u$ & $\ldots$ & 10 UG/L & 1 \\
\hline 2-Hexanone & $10 \mathrm{UG} \Omega$ & $u$ & -- & 10 UG $\Omega$ & 1 \\
\hline Tetrachloroethene & 5 UG/ & $u$ & - & 5 UG/L & 1 \\
\hline 1,1,2,2-Tetrachloroethane & 5 UG/ & $u$ & $\cdots$ & 5 UG/ & 1 \\
\hline Toluene & 5 UG/ & $u$ & $\ldots$ & 5 UG/ & 1 \\
\hline Chlorobenzene & 5 UG/ & $u$ & - & 5 UG & 1 \\
\hline Ethyl Benzene & 5 UG/ & $u$ & - & 5 UGR & 1 \\
\hline Styrene & 5 UGR & $u$ & - & 5 UG/ & 1 \\
\hline Xylenes (total) & 5 UG $\Omega$ & $u$ & - & $5 \mathrm{UG} /$ & 1 \\
\hline Toluene-d8 (SURR) & $101 \%$ & & & $\%$ & 1 \\
\hline Bromofluorobenzene (SURR) & $104 \%$ & & & $\%$ & 1 \\
\hline 1,2-Dichloroethane-D4 (SURR) & $100 \%$ & & & $\%$ & 1 \\
\hline
\end{tabular}


APPENDIX C.2 Results of Volatile Organic Analyses, Water, Salmon Site

\begin{tabular}{|c|c|c|c|c|c|c|}
\hline COMPOUND & RESULTS & UNITS & $\begin{array}{l}\text { DATA } \\
\text { QUAL. }\end{array}$ & $\begin{array}{l}\text { DATA } \\
\text { VALID. }\end{array}$ & CRDL UNITS & DILUTION \\
\hline \multicolumn{7}{|l|}{ C1-15-W-MS } \\
\hline 1,1-Dichloroethene & & $8 \%$ & & NP & $\%$ & 1 \\
\hline Trichloroethene & & $1 \%$ & & NP & $\%$ & 1 \\
\hline Benzene & & $2 \%$ & & NP & $\%$ & 1 \\
\hline Toluene & & $7 \%$ & & NP & $\%$ & 1 \\
\hline Chlorobenzene & & $2 \%$ & & NP & $\%$ & 1 \\
\hline Toluene-d8 (SURR) & & $0 \%$ & & NP & $\%$ & 1 \\
\hline Bromofluorobenzene (SURR) & 10 & $5 \%$ & & NP & $\%$ & 1 \\
\hline 1,2-Dichloroethane-D4 (SURR) & 10 & $3 \%$ & & NP & $\%$ & 1 \\
\hline \multicolumn{7}{|l|}{ C1-15-W-MSD } \\
\hline 1,1-Dichloroethene & 10 & $6 \%$ & & NP & $\%$ & 1 \\
\hline Trichloroethene & 11 & & & NP & $\%$ & 1 \\
\hline Benzene & 11 & & & NP & $\%$ & 1 \\
\hline Toluene & 10 & & & NP & $\%$ & 1 \\
\hline Chlorobenzene & 11 & & & NP & $\%$ & 1 \\
\hline Toluene-d8 (SURR) & 10 & & & NP & $\%$ & 1 \\
\hline Bromofluorobenzene (SURR) & 10 & & & NP & $\%$ & 1 \\
\hline 1,2-Dichloroethane-D4 (SURR) & 10 & $4 \%$ & & NP & $\%$ & 1 \\
\hline
\end{tabular}


APPENDIX C.2 Results of Volatile Organic Analyses, Water, Salmon Site

\begin{tabular}{|c|c|c|}
\hline COMPOUND & RESULTS & $\begin{array}{l}\text { DATA } \\
\text { QUAL. }\end{array}$ \\
\hline \multicolumn{3}{|l|}{$C 1-16-W$} \\
\hline Chloromethane & $10 \mathrm{UG} / \mathrm{L}$ & $u$ \\
\hline Bromomethane & $10 \mathrm{UG} / \mathrm{L}$ & $u$ \\
\hline Vinyl Chloride & 10 UG/ & $u$ \\
\hline Chloroethane & 10 UG/L & $u$ \\
\hline Methylene Chloride & 5 UG/ & $u$ \\
\hline Acetone & 10 UG/L & $u$ \\
\hline Carbon Disulfide & 3 UG/ & $J$ \\
\hline 1,1-Dichloroethene & 5 UG/ & $u$ \\
\hline 1,1-Dichloroethane & 5 UG/ & $u$ \\
\hline 1,2-Dichloroethene (total) & 5 UG/ & $u$ \\
\hline Chloroform & 5 UG/ & $u$ \\
\hline 1,2-Dichloroethane & 5 UG/ & $u$ \\
\hline 2-Butanone & $10 \mathrm{UG} /$ & $u$ \\
\hline 1,1,1-Trichloroethane & $5 \mathrm{UG/}$ & $u$ \\
\hline Carbon Tetrachloride & 5 UG/ & $u$ \\
\hline Vinyl Acetate & 10 UG/L & $u$ \\
\hline Bromodichloromethane & 5 UG/ & $u$ \\
\hline 1,2-Dichloropropane & 5 UG/L & $u$ \\
\hline cis-1,3-Dichloropropene & 5 UG & $u$ \\
\hline Trichloroethene & 5 UGR & $u$ \\
\hline Dibromochloromethane & 5 UGR & $u$ \\
\hline 1,1,2-Trichloroethane & 5 UG/ & $u$ \\
\hline Benzene & 5 UG/L & $u$ \\
\hline trans-1,3-Dichloropropene & 5 UG/ & $u$ \\
\hline Bromoform & 5 UG $\Omega$ & $u$ \\
\hline 4-Methyl-2-Pentanone & 10 UG $/$ & $\mathrm{u}$ \\
\hline 2-Hexanone & 10 UG/ & $u$ \\
\hline Tetrachloroethene & 5 UG/L & $u$ \\
\hline 1,1,2,2-Tetrachloroethane & 5 UG/L & $u$ \\
\hline Toluene & 5 UG/ & $u$ \\
\hline Chlorobenzene & 5 UG/L & $u$ \\
\hline Ethyl Benzene & 5 UGR & $u$ \\
\hline Styrene & 5 UG/ & $u$ \\
\hline Xylenes (total) & 5 UG/ & $u$ \\
\hline Unknown & $6 \mathrm{UG} / \mathrm{L}$ & J \\
\hline Toluene-d8 (SURR) & $99 \%$ & \\
\hline Bromofluorobenzene (SURR) & $103 \%$ & \\
\hline 1,2-Dichloroethane-d4 (SURR) & $99 \%$ & \\
\hline
\end{tabular}

\section{CRDL UNITS}

DILUTION

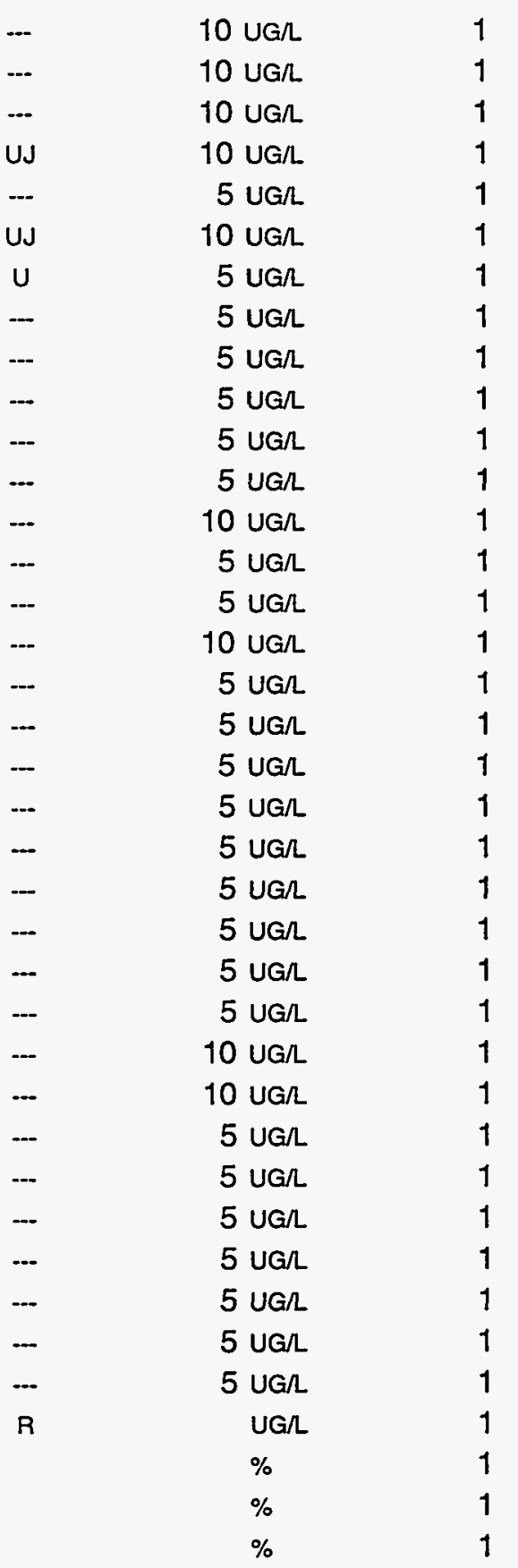


APPENDIX C.2 Results of Volatile Organic Analyses, Water, Salmon Site

\begin{tabular}{|c|c|c|c|c|c|}
\hline COMPOUND & RESULTS & $\begin{array}{l}\text { DATA } \\
\text { QUAL. }\end{array}$ & $\begin{array}{l}\text { DATA } \\
\text { VALID. }\end{array}$ & CRDL UNITS & DILUTION \\
\hline \multicolumn{6}{|l|}{ C1-17-W } \\
\hline Chloromethane & $10 \mathrm{UG} / \mathrm{L}$ & $u$ & $\ldots$ & $10 \mathrm{UG} / \mathrm{L}$ & 1 \\
\hline Bromomethane & 10 UG/L & $u$ & $\ldots$ & 10 UG/L & 1 \\
\hline Vinyl Chloride & 10 UG/L & $u$ & - & 10 UG $\Omega$ & 1 \\
\hline Chloroethane & 10 UG & $u$ & UJ & $10 \mathrm{UG} \Omega$ & 1 \\
\hline Methylene Chloride & $5 \mathrm{UG} /$ & $u$ & $\ldots$ & 5 UG/ & 1 \\
\hline Acetone & $10 \mathrm{UG} / \mathrm{L}$ & $u$ & Us & 10 UGL & 1 \\
\hline Carbon Disulfide & 5 UG $\Omega$ & $u$ & $\ldots$ & 5 UGR & 1 \\
\hline 1,1-Dichloroethene & 5 UG/ & $u$ & - & 5 UG/ & 1 \\
\hline 1,1-Dichloroethane & 5 UG/ & $u$ & $\ldots$ & $5 \mathrm{UG} / \mathrm{L}$ & 1 \\
\hline 1,2-Dichloroethene (total) & 16 UG $\Omega$ & & - & 5 UGR. & 1 \\
\hline Chloroform & $5 \mathrm{UG} /$ & $u$ & $\ldots$ & 5 UG/ & 1 \\
\hline 1,2-Dichloroethane & 5 UG/ & $u$ & ... & 5 UG/ & 1 \\
\hline 2-Butanone & 10 UG/ & $u$ & - & $10 \mathrm{UG} / \mathrm{L}$ & 1 \\
\hline 1,1,1-Trichloroethane & 5 UG/ & $u$ & - & $5 \mathrm{UG} /$ & 1 \\
\hline Carbon Tetrachloride & $5 \mathrm{UG} / \mathrm{L}$ & $u$ & $\cdots$ & $5 \mathrm{UG} / \mathrm{L}$ & 1 \\
\hline Vinyl Acetate & $10 \mathrm{UG} / \mathrm{L}$ & $u$ & - & 10 UGR & 1 \\
\hline Bromodichloromethane & 5 UG $\Omega$ & $u$ & $\ldots$ & $5 \mathrm{UG} / \mathrm{L}$ & 1 \\
\hline 1,2-Dichloropropane & 5 UGR & $u$ & - & 5 UG/ & 1 \\
\hline cis-1,3-Dichloropropene & 5 UG & $u$ & -. & $5 \mathrm{UG} / \mathrm{L}$ & 1 \\
\hline Trichloroethene & 74 UG $\Omega$ & & - & $5 \mathrm{UG} / \mathrm{L}$ & 1 \\
\hline Dibromochloromethane & 5 UG/ & $u$ & - & $5 \mathrm{UG} / \mathrm{L}$ & 1 \\
\hline 1,1,2-Trichloroethane & 5 UGR & $u$ & - & 5 UG $\Omega$ & 1 \\
\hline Benzene & 5 UG/L & $u$ & - & $5 \mathrm{UG} / \mathrm{L}$ & 1 \\
\hline trans-1,3-Dichloropropene & 5 UG/ & $\mathrm{u}$ & - & $5 \mathrm{UG} / \mathrm{L}$ & 1 \\
\hline Bromoform & $5 \mathrm{UG} /$ & $u$ & - & $5 \mathrm{UG} / \mathrm{L}$ & 1 \\
\hline 4-Methyl-2-Pentanone & 10 UG/L & $u$ & - & $10 \mathrm{UG} \Omega$ & 1 \\
\hline 2-Hexanone & 10 UG & $u$ & $\ldots$ & $10 \mathrm{UG} \Omega$ & 1 \\
\hline Tetrachloroethene & 5 UG/ & $u$ & $\ldots$ & $5 \mathrm{UG} / \mathrm{L}$ & 1 \\
\hline $1,1,2,2-$ Tetrachloroethane & 5 UG & $u$ & $\ldots$ & 5 UG $\Omega$ & 1 \\
\hline Toluene & $1 \mathrm{UG} /$ & J & J & 5 UG/L & 1 \\
\hline Chlorobenzene & 5 UG/ & $u$ & - & 5 UG $/$ & 1 \\
\hline Ethyl Benzene & 5 UG/L & $u$ & - & 5 UG $\Omega$ & 1 \\
\hline Styrene & 5 UGR & $u$ & - & $5 \mathrm{UG} \Omega$ & 1 \\
\hline Xylenes (total) & 5 UG/ & $u$ & - & 5 UG $\Omega$ & 1 \\
\hline Unknown-1 & 84 UG/ & J & $\mathbf{R}$ & UG $\Omega$ & 1 \\
\hline Unknown-2 & 63 UG & J & $\mathbf{R}$ & UG $\Omega$ & 1 \\
\hline Unknown-3 & 10 UG $\Omega$ & J & $J$ & UGR & 1 \\
\hline Toluene-d8 (SURR) & $98 \%$ & & & $\%$ & 1 \\
\hline Bromofluorobenzene (SURR) & $107 \%$ & & & $\%$ & 1 \\
\hline 1,2-Dichloroethane-d4 (SURR) & $105 \%$ & & & $\%$ & 1 \\
\hline
\end{tabular}




\begin{tabular}{|c|c|c|c|c|c|}
\hline COMPOUND & RESULTS & $\begin{array}{l}\text { DATA } \\
\text { QUAL. }\end{array}$ & $\begin{array}{c}\text { DATA } \\
\text { VALID. }\end{array}$ & CRDL UNITS & DILUTION \\
\hline \multicolumn{6}{|l|}{$C 1-18-W$} \\
\hline Chloromethane & $10 \mathrm{UG} / \mathrm{L}$ & $u$ & --- & $10 \mathrm{UG} / \mathrm{L}$ & 1 \\
\hline Bromomethane & 10 UG $\Omega$ & $u$ & -- & $10 \mathrm{UG} \Omega$ & 1 \\
\hline Vinyl Chloride & 10 UG & $u$ & $\ldots$ & 10 UG $\Omega$ & 1 \\
\hline Chloroethane & 10 UG/ & $u$ & UJ & $10 \mathrm{UG} / \mathrm{L}$ & 1 \\
\hline Methylene Chloride & $2 U G /$ & BJ & $u$ & 5 UG/L & 1 \\
\hline Acetone & $10 \mathrm{UG} \Omega$ & $u$ & UJ & $10 \mathrm{UG} / \mathrm{L}$ & 1 \\
\hline Carbon Disulfide & 5 UGR & & $u$ & $5 \mathrm{UG} / \mathrm{L}$ & 1 \\
\hline 1,1-Dichloroethene & 5 UG $\Omega$ & $u$ & - & $5 \mathrm{UG} / \mathrm{L}$ & 1 \\
\hline 1,1-Dichloroethane & 5 UG/L & $u$ &.- & 5 UG/ & 1 \\
\hline 1,2-Dichloroethene (total) & 55 UG/ & & $\ldots$ & 5 UG/ & 1 \\
\hline Chloroform & 5 UG/ & $u$ & - & 5 UG/L & 1 \\
\hline 1,2-Dichloroethane & $5 \mathrm{UG/}$ & $u$ & $\ldots$ & $5 \mathrm{UG \Omega}$ & 1 \\
\hline 2-Butanone & 10 UG/L & $u$ & $\ldots$ & 10 UG/ & 1 \\
\hline 1,1,1-Trichloroethane & 5 UGR & $u$ & -.. & 5 UG/ & 1 \\
\hline Carbon Tetrachioride & 5 UG/ & $u$ & $\ldots$ & 5 UG/ & 1 \\
\hline Vinyl Acetate & 10 UG/ & $u$ & -- & $10 \mathrm{UG} /$ & 1 \\
\hline Bromodichloromethane & $5 \mathrm{UGR}$ & $u$ & $\ldots$ & 5 UG $\Omega$ & 1 \\
\hline 1,2-Dichloropropane & 5 UG/ & $u$ & -. & 5 UG/ & 1 \\
\hline cis-1,3-Dichloropropene & 5 UG/ & $u$ & $\ldots$ & 5 UG/ & 1 \\
\hline Trichloroethene & $5 \mathrm{UG} / \mathrm{L}$ & $u$ & $\ldots$ & 5 UG & 1 \\
\hline Dibromochloromethane & $5 \mathrm{UG} / \mathrm{L}$ & $u$ & - & 5 UG/L & 1 \\
\hline 1,1,2-Trichloroethane & 5 UG/ & $u$ & $\ldots$ & 5 UGR & 1 \\
\hline Benzene & $5 \mathrm{UG} / \mathrm{L}$ & $u$ & - & 5 UG/L & 1 \\
\hline trans-1,3-Dichloropropene & 5 UG/L & $u$ & - & 5 UG & 1 \\
\hline Bromoform & 5 UGת & $u$ & $\ldots$ & 5 UG/L & 1 \\
\hline 4-Methyl-2-Pentanone & 10 UG/ & $u$ & - & 10 UGR & 1 \\
\hline 2-Hexanone & 10 Ua/ & $u$ & -- & 10 UG/ & 1 \\
\hline Tetrachloroethene & 5 UG/L & $u$ & -- & 5 UG/L & 1 \\
\hline 1,1,2,2-Tetrachloroethane & 5 UG/L & $u$ & -- & 5 UG/L & 1 \\
\hline Toluene & 5 UG/L & $u$ & -- & 5 UG/ & 1 \\
\hline Chlorobenzene & 5 UGR & $u$ & - & 5 UG/L & 1 \\
\hline Ethyl Benzene & 5 UGR & $u$ & $\ldots$ & $5 \mathrm{UG} /$ & 1 \\
\hline Styrene & 5 UG & $u$ & $\ldots$ & 5 UGR & 1 \\
\hline Xylenes (total) & $5 \mathrm{UG} / \mathrm{L}$ & $u$ & $\ldots$ & 5 UG $\Omega$ & 1 \\
\hline Unknown & $6 \mathrm{UG} / \mathrm{L}$ & J & $\mathrm{R}$ & UG & 1 \\
\hline Toluene-d8 (SURR) & $98 \%$ & & & $\%$ & 1 \\
\hline Bromofluorobenzene (SURR) & $103 \%$ & & & $\%$ & 1 \\
\hline 1,2-Dichloroethane-d4 (SURR) & $103 \%$ & & & $\%$ & 1 \\
\hline
\end{tabular}




\begin{tabular}{|c|c|c|c|c|c|}
\hline COMPOUND & RESULTS & $\begin{array}{l}\text { DATA } \\
\text { QUAL. }\end{array}$ & $\begin{array}{r}\text { DATA } \\
\text { VALID. }\end{array}$ & CRDL UNITS & DILUTION \\
\hline \multicolumn{6}{|l|}{$C 2-19-W$} \\
\hline Chloromethane & $10 \mathrm{UG} / \mathrm{L}$ & $u$ & NP & 10 UG/ & 1 \\
\hline Bromomethane & $10 \mathrm{UG} / \mathrm{L}$ & $u$ & NP & 10 UG $\Omega$ & 1 \\
\hline Vinyl Chloride & $10 \mathrm{UG} \Omega$ & $u$ & NP & 10 UG $\Omega$ & 1 \\
\hline Chloroethane & 10 UG/L & $u$ & NP & 10 Ua $\Omega$ & 1 \\
\hline Methylene Chloride & 2 UG/ & $\mathrm{J}$ & NP & 5 UG/ & 1 \\
\hline Acetone & 10 UG $\Omega$ & $u$ & NP & 10 UG $\Omega$ & 1 \\
\hline Carbon Disulfide & 5 UG $\Omega$ & $u$ & NP & 5 UG $\Omega$ & 1 \\
\hline 1,1-Dichloroethene & 5 UG & $u$ & NP & 5 UG/ & 1 \\
\hline 1,1-Dichloroethane & 5 UGL & $u$ & NP & 5 UG & 1 \\
\hline 1,2-Dichloroethene (total) & 5 UG/L & $u$ & NP & 5 UGR & 1 \\
\hline Chloroform & 5 UG $\Omega$ & $u$ & NP & 5 UG/ & 1 \\
\hline 1,2-Dichloroethane & 5 UGR & $u$ & NP & $5 \mathrm{UG} / \mathrm{L}$ & 1 \\
\hline 2-Butanone & $10 \mathrm{UG} \Omega$ & $u$ & NP & 10 UG $\Omega$ & 1 \\
\hline 1,1,1-Trichloroethane & 5 UG & $u$ & NP & 5 UG & 1 \\
\hline Carbon Tetrachloride & 5 UG & $u$ & NP & $5 \mathrm{UG} \Omega$ & 1 \\
\hline Vinyl Acetate & 10 UG $/$ & $u$ & NP & $10 \mathrm{UG} / \mathrm{L}$ & 1 \\
\hline Bromodichloromethane & 5 UG $\Omega$ & $u$ & NP & 5 UG $\Omega$ & 1 \\
\hline 1,2-Dichloropropane & 5 UG几 & $u$ & NP & 5 UG $\Omega$ & 1 \\
\hline cis-1,3-Dichloropropene & 5 UG & $u$ & NP & 5 UG $\Omega$ & 1 \\
\hline Trichloroethene & 5 UG $\Omega$ & $u$ & NP & $5 \mathrm{UG} /$ & 1 \\
\hline Dibromochloromethane & 5 UG $\Omega$ & $u$ & NP & 5 UG $\Omega$ & 1 \\
\hline 1,1,2-Trichloroethane & 5 UG/ & $u$ & NP & 5 UG $\Omega$ & 1 \\
\hline Benzene & $5 \mathrm{UG} / \mathrm{L}$ & $u$ & NP & 5 UG & 1 \\
\hline trans-1,3-Dichloropropene & 5 UG $\Omega$ & $u$ & NP & 5 UG几 & 1 \\
\hline Bromoform & 5 UG & $u$ & NP & 5 UG几 & 1 \\
\hline 4-Methyl-2-Pentanone & 10 UG/L & $u$ & NP & 10 UG $\Omega$ & 1 \\
\hline 2-Hexanone & $10 \mathrm{UG} / \mathrm{L}$ & $u$ & NP & 10 UG $/$ & 1 \\
\hline Tetrachloroethene & $5 \mathrm{UG} \Omega$ & $u$ & NP & 5 UG/L & 1 \\
\hline 1,1,2,2-Tetrachloroethane & $5 \mathrm{UG} / \mathrm{L}$ & $u$ & NP & 5 UG $\Omega$ & 1 \\
\hline Toluene & 5 UG $\Omega$ & $u$ & NP & 5 UG $\Omega$ & 1 \\
\hline Chlorobenzene & 5 UG & $u$ & NP & 5 UG & 1 \\
\hline Ethyl Benzene & 5 UG/L & $u$ & NP & 5 UG $\Omega$ & 1 \\
\hline Styrene & 5 UG $\Omega$ & $u$ & NP & 5 UG $\Omega$ & 1 \\
\hline Xylenes (total) & $5 \mathrm{UG} \Omega$ & $u$ & NP & 5 UG/L & 1 \\
\hline Toluene-d8 (SURR) & $101 \%$ & & & $\%$ & 1 \\
\hline Bromofluorobenzene (SURR) & $104 \%$ & & & $\%$ & 1 \\
\hline 1,2-Dichloroethane-d4 (SURR) & $94 \%$ & & & $\%$ & 1 \\
\hline
\end{tabular}


APPENDIX C.2 Results of Volatile Organic Analyses, Water, Salmon Site

\begin{tabular}{|llllll}
\hline COMPOUND & DATA DATA & & \\
RESULTS UNITS & QUAL. VALID. & CRDL UNITS & DILUTION \\
\hline
\end{tabular}

C2-19-W-MS

1,1-Dichloroethene

$90 \%$

$106 \%$

Trichloroethene

$112 \%$

Benzene

$111 \%$

Toluene

$117 \%$

Chlorobenzene

$99 \%$

$103 \%$

Bromofluorobenzene (SURR)

1,2-Dichloroethane-d4 (SURR)

$92 \%$

NP

NP

NP

NP

NP

NP

NP

NP

$\begin{array}{ll}\% & 1 \\ \% & 1 \\ \% & 1 \\ \% & 1 \\ \% & 1 \\ \% & 1 \\ \% & 1 \\ \% & 1\end{array}$

\section{C2-19-W-MSD}

1,1-Dichloroethene

$87 \%$

Trichloroethene

$102 \%$

Benzene

$104 \%$

Toluene

$105 \%$

Chlorobenzene

$110 \%$

Toluene-d8 (SURR)

$98 \%$

Bromofluorobenzene (SURR)

$103 \%$

1,2-Dichloroethane-d4 (SURR)

$93 \%$

$N P$
$N P$
$N P$
$N P$
$N P$
$N P$
$N P$
$N P$

1

$\%$

$\%$

1

1

1 1

1

1

1

1 


\begin{tabular}{|c|c|c|c|c|c|}
\hline COMPOUND & RESULTS & $\begin{array}{l}\text { DATA } \\
\text { QUAL. }\end{array}$ & $\begin{array}{l}\text { DATA } \\
\text { VALID. }\end{array}$ & CRDL UNITS & DILUTION \\
\hline \multicolumn{6}{|l|}{$C 2-21-W$} \\
\hline Chloromethane & 10 UG/ & $u$ & NP & $10 \mathrm{UG} / \mathrm{L}$ & 1 \\
\hline Bromomethane & $10 \mathrm{UG} / \mathrm{L}$ & $u$ & NP & 10 UG/L & 1 \\
\hline Vinyl Chloride & $10 \mathrm{UG} / \mathrm{L}$ & $u$ & NP & 10 UG $\Omega$ & 1 \\
\hline Chloroethane & $10 \mathrm{UG} \Omega$ & $u$ & NP & 10 UG $\Omega$ & 1 \\
\hline Methylene Chloride & 3 UG $\Omega$ & BJ & NP & 5 UG $\Omega$ & 1 \\
\hline Acetone & 9 UG $\Omega$ & BJ & NP & $10 \mathrm{UG} / \mathrm{L}$ & 1 \\
\hline Carbon Disulfide & 5 UG/L & $u$ & NP & 5 UG $\Omega$ & 1 \\
\hline 1,1-Dichloroethene & 5 UG $\Omega$ & $u$ & NP & 5 UG/L & 1 \\
\hline 1,1-Dichloroethane & 5 UG/ & $u$ & NP & 5 UG/ & 1 \\
\hline 1,2-Dichloroethene (total) & 5 UG $\Omega$ & $u$ & NP & 5 UG $\Omega$ & 1 \\
\hline Chloroform & 5 UG & $u$ & NP & 5 UG $\Omega$ & 1 \\
\hline 1,2-Dichloroethane & 5 UG $\Omega$ & $u$ & NP & 5 UGR & 1 \\
\hline 2-Butanone & 10 UG $\Omega$ & $u$ & NP & $10 \mathrm{UG} / \mathrm{L}$ & 1 \\
\hline 1,1,1-Trichloroethane & 5 UG $\Omega$ & $u$ & NP & $5 \mathrm{UG} / \mathrm{L}$ & 1 \\
\hline Carbon Tetrachloride & 5 UG & $u$ & NP & 5 UG & 1 \\
\hline Vinyl Acetate & $10 \mathrm{UG} \Omega$ & $u$ & NP & 10 UG & 1 \\
\hline Bromodichloromethane & 5 UGת & $u$ & NP & 5 UGת & 1 \\
\hline 1,2-Dichloropropane & 5 UG & $u$ & NP & 5 UG $\Omega$ & 1 \\
\hline cis-1,3-Dichloropropene & 5 UG/ & $u$ & NP & $5 \mathrm{UG} / \mathrm{L}$ & 1 \\
\hline Trichloroethene & 5 UG & $u$ & NP & 5 UG/ & 1 \\
\hline Dibromochloromethane & 5 UG $\Omega$ & $u$ & NP & 5 UG/L & 1 \\
\hline 1,1,2-Trichloroethane & $5 \mathrm{UG} \Omega$ & $u$ & NP & 5 UG/ & 1 \\
\hline Benzene & 5 UG $\Omega$ & $u$ & NP & 5 UG $\Omega$ & 1 \\
\hline trans-1,3-Dichloropropene & $5 \mathrm{UG} / \mathrm{L}$ & $u$ & $\mathrm{NP}$ & 5 UG & 1 \\
\hline Bromoform & 5 UG/ & $u$ & NP & 5 UG/L & 1 \\
\hline 4-Methyl-2-Pentanone & $10 \mathrm{UG} \Omega$ & $u$ & NP & $10 \mathrm{UG} \Omega$ & 1 \\
\hline 2-Hexanone & 10 UG $\Omega$ & $u$ & NP & 10 UG $\Omega$ & 1 \\
\hline Tetrachloroethene & 5 UG/ & $u$ & NP & 5 UG $\Omega$ & 1 \\
\hline $1,1,2,2-$ Tetrachloroethane & 5 UG/ & $u$ & NP & 5 UG/ & 1 \\
\hline Toluene & $1 \mathrm{UG} / \mathrm{L}$ & J & NP & $5 \mathrm{UG} / \mathrm{L}$ & 1 \\
\hline Chlorobenzene & 5 UG & $u$ & NP & $5 \mathrm{UG} / \mathrm{L}$ & 1 \\
\hline Ethyl Benzene & 5 UG & $u$ & NP & $5 \mathrm{UGR}$ & 1 \\
\hline Styrene & $5 \mathrm{UG} / \mathrm{L}$ & $u$ & NP & $5 \mathrm{UG} / \mathrm{L}$ & 1 \\
\hline Xylenes (total) & $5 \mathrm{UG} / \mathrm{L}$ & $u$ & NP & 5 UG/ & 1 \\
\hline Unknown-1 & 47 UG几 & BJ & NP & UG/ & 1 \\
\hline Unknown-2 & 30 UG $\Omega$ & BJ & NP & UGR & 1 \\
\hline Unknown-3 & $5 \mathrm{UG} /$ & J & NP & UG/ & 1 \\
\hline Toluene-d8 (SURR) & $101 \%$ & & & $\%$ & 1 \\
\hline Bromofluorobenzene (SURR) & $102 \%$ & & & $\%$ & 1 \\
\hline 1,2-Dichloroethane-d4 (SURR) & $104 \%$ & & & $\%$ & 1 \\
\hline
\end{tabular}


APPENDIX C.2 Results of Volatile Organic Analyses, Water, Salmon Site

\begin{tabular}{|c|c|c|c|c|c|}
\hline COMPOUND & RESULTS & $\begin{array}{l}\text { DATA } \\
\text { QUAL. }\end{array}$ & $\begin{array}{l}\text { DATA } \\
\text { VALID. }\end{array}$ & CRDL UNITS & DILUTION \\
\hline \multicolumn{6}{|l|}{ C2-22-W } \\
\hline Chloromethane & 10 UG/L & $u$ & NP & $10 \mathrm{UG} / \mathrm{L}$ & 1 \\
\hline Bromomethane & 10 UG/L & $u$ & NP & 10 UG/ & 1 \\
\hline Vinyl Chloride & $10 \mathrm{UG} / \mathrm{L}$ & $u$ & NP & $10 \mathrm{UG} / \mathrm{L}$ & 1 \\
\hline Chloroethane & $10 \mathrm{UG} / \mathrm{L}$ & $u$ & NP & $10 \mathrm{UG} / \mathrm{L}$ & 1 \\
\hline Methylene Chloride & 3 UG/ & BJ & NP & 5 UG/L & 1 \\
\hline Acetone & 11 UG/ & $B$ & NP & 10 UG & 1 \\
\hline Carbon Disulfide & 5 UG/. & $u$ & NP & 5 UG/ & 1 \\
\hline 1,1-Dichloroethene & 5 UG/ & $u$ & NP & 5 UG $\Omega$ & 1 \\
\hline 1,1-Dichloroethane & 5 UG/ & $u$ & NP & 5 UG/ & 1 \\
\hline 1,2-Dichloroethene (total) & 5 UG/ & $u$ & NP & 5 UG/L & 1 \\
\hline Chloroform & 5 UG/L & $u$ & NP & 5 UG/L & 1 \\
\hline 1,2-Dichloroethane & $5 \mathrm{UG} / \mathrm{L}$ & $u$ & NP & 5 UG $\Omega$ & 1 \\
\hline 2-Butanone & 10 UGR & $u$ & NP & 10 UG/ & 1 \\
\hline 1,1,1-Trichloroethane & 5 UG/ & $u$ & NP & 5 UG/ & 1 \\
\hline Carbon Tetrachloride & 5 UG/ & $u$ & NP & 5 UG/ & 1 \\
\hline Vinyl Acetate & $10 \mathrm{UG} /$ & $u$ & NP & 10 UG/L & 1 \\
\hline Bromodichloromethane & 5 UGR & $u$ & NP & 5 UG/L & 1 \\
\hline 1,2-Dichloropropane & 5 UG & $u$ & NP & 5 UG/ & 1 \\
\hline cis-1,3-Dichloropropene & 5 UG $\Omega$ & $u$ & NP & 5 UG/ & 1 \\
\hline Trichloroethene & 5 UG/ & $u$ & NP & 5 UG/ & 1 \\
\hline Dibromochloromethane & 5 UG/ & $u$ & NP & $5 \mathrm{UG} \Omega$ & 1 \\
\hline 1,1,2-Trichloroethane & 5 UG/ & $u$ & NP & 5 UG/ & 1 \\
\hline Benzene & 5 UG $\Omega$ & $u$ & NP & 5 UG $\Omega$ & 1 \\
\hline trans-1,3-Dichloropropene & 5 UG/ & $u$ & NP & $5 \cup G /$ & 1 \\
\hline Bromoform & 5 UG/ & $u$ & NP & 5 UG/L & 1 \\
\hline 4-Methyl-2-Pentanone & 10 UG/ & $u$ & NP & 10 UG/L & 1 \\
\hline 2-Hexanone & 10 UG $\Omega$ & $u$ & NP & 10 UG/L & 1 \\
\hline Tetrachloroethene & 5 UG/ & $u$ & NP & 5 UG/L & 1 \\
\hline 1,1,2,2-Tetrachloroethane & 5 UG/L & $u$ & NP & 5 UG $\Omega$ & 1 \\
\hline Toluene & 5 UG/ & $u$ & NP & $5 \mathrm{UG} /$ & 1 \\
\hline Chlorobenzene & 5 UG/L & $u$ & NP & 5 UG/ & 1 \\
\hline Ethyl Benzene & 5 UG $\Omega$ & $u$ & NP & 5 UG/L & 1 \\
\hline Styrene & 5 UG & $u$ & NP & $5 \cup G \Omega$ & 1 \\
\hline Xylenes (total) & 5 UG $/$ & $u$ & NP & 5 UG/ & 1 \\
\hline Unknown-1 & 7 UG/L & J & NP & UG几 & 1 \\
\hline Unknown-2 & $6 \mathrm{UG} / \mathrm{L}$ & J & NP & UG $\Omega$ & 1 \\
\hline Toluene-d8 (SURR) & $99 \%$ & & & $\%$ & 1 \\
\hline Bromofluorobenzene (SURR) & $105 \%$ & & & $\%$ & 1 \\
\hline 1,2-Dichloroethane-d4 (SURR) & $103 \%$ & & & $\%$ & 1 \\
\hline
\end{tabular}


APPENDIX C.2 Results of Volatile Organic Analyses, Water, Salmon Site

\begin{tabular}{|c|c|c|c|c|c|}
\hline COMPOUND & RESULTS UNITS & $\begin{array}{l}\text { DATA } \\
\text { QUAL. }\end{array}$ & $\begin{array}{l}\text { DATA } \\
\text { VALID. }\end{array}$ & CRDL UNITS & DILUTION \\
\hline \multicolumn{6}{|l|}{$C 2-24-W$} \\
\hline Chloromethane & $10 \mathrm{UG} /$ & $u$ & NP & 10 UG $\Omega$ & 1 \\
\hline Bromomethane & $10 \mathrm{UG} / \mathrm{L}$ & $u$ & NP & $10 \mathrm{UG} / \mathrm{L}$ & 1 \\
\hline Vinyl Chloride & 10 UG $\Omega$ & $u$ & NP & 10 UG $\Omega$ & 1 \\
\hline Chloroethane & $10 \mathrm{UG} /$ & $u$ & NP & $10 \mathrm{UG} / \mathrm{L}$ & 1 \\
\hline Methylene Chloride & 5 UG几 & $u$ & NP & 5 UG & 1 \\
\hline Acetone & 11 UGR & B & NP & 10 UG/ & 1 \\
\hline Carbon Disulfide & 5 UG/ & $u$ & NP & 5 UG & 1 \\
\hline 1,1-Dichloroethene & 5 UGL & $u$ & NP & 5 UG & 1 \\
\hline 1,1-Dichloroethane & $5 \mathrm{UG} / \mathrm{L}$ & $u$ & NP & 5 UG/ & 1 \\
\hline 1,2-Dichloroethene (total) & 5 UGR & $u$ & NP & 5 UGR & 1 \\
\hline Chloroform & 5 UG/ & $u$ & NP & 5 UG/L & 1 \\
\hline 1,2-Dichloroethane & 5 UGR & $u$ & NP & 5 UGR & 1 \\
\hline 2-Butanone & $10 \mathrm{UG} / \mathrm{L}$ & $u$ & NP & 10 UG $/$ & 1 \\
\hline 1,1,1-Trichloroethane & $5 \mathrm{UG} / \mathrm{L}$ & $u$ & NP & 5 UG & 1 \\
\hline Carbon Tetrachloride & 5 UG & $u$ & NP & 5 UGR & 1 \\
\hline Vinyl Acetate & $10 \mathrm{UG} / \mathrm{L}$ & $u$ & NP & 10 UG $\Omega$ & 1 \\
\hline Bromodichloromethane & 5 UG/ & $u$ & NP & 5 UG/ & 1 \\
\hline 1,2-Dichloropropane & $5 \mathrm{UG} / \mathrm{L}$ & $u$ & NP & 5 UG/ & 1 \\
\hline cis-1,3-Dichloropropene & 5 UG & $u$ & NP & 5 UG/ & 1 \\
\hline Trichloroethene & 5 UG $/$ & $u$ & NP & 5 UGR & 1 \\
\hline Dibromochloromethane & 5 UGR & $u$ & NP & 5 UG/ & 1 \\
\hline 1,1,2-Trichloroethane & 5 UGR & $u$ & NP & $5 \mathrm{UGR}$ & 1 \\
\hline Benzene & 5 UGR & $u$ & NP & $5 \mathrm{UG} /$ & 1 \\
\hline trans-1,3-Dichloropropene & $5 \mathrm{UG} / \mathrm{L}$ & $u$ & NP & 5 UG/ & 1 \\
\hline Bromoform & $5 \mathrm{UG} / \mathrm{L}$ & $u$ & NP & 5 UGR & 1 \\
\hline 4-Methyl-2-Pentanone & $10 \mathrm{UG} \Omega$ & $u$ & NP & 10 UG/ & 1 \\
\hline 2-Hexanone & 10 UG $\Omega$ & $u$ & NP & 10 UG $\Omega$ & 1 \\
\hline Tetrachloroethene & 5 UG/ & $u$ & NP & 5 UG & 1 \\
\hline 1,1,2,2-Tetrachloroethane & 5 UG/ & $u$ & NP & $5 \mathrm{UG} /$ & 1 \\
\hline Toluene & $7 \mathrm{UG} / \mathrm{L}$ & & NP & 5 UG & 1 \\
\hline Chlorobenzene & $5 \mathrm{UG} / \mathrm{L}$ & $u$ & NP & $5 \mathrm{UG} \Omega$ & 1 \\
\hline Ethyl Benzene & 5 UG/ & $u$ & NP & 5 UG $\Omega$ & 1 \\
\hline Styrene & 5 UGR & $u$ & NP & $5 \mathrm{UG} \Omega$ & 1 \\
\hline Xylenes (total) & 5 UG & $u$ & NP & 5 UG & 1 \\
\hline Toluene-d8 (SURR) & $99 \%$ & & & $\%$ & 1 \\
\hline Bromofluorobenzene (SURR) & $105 \%$ & & & $\%$ & 1 \\
\hline 1,2-Dichloroethane-d4 (SURR) & $91 \%$ & & & $\%$ & 1 \\
\hline
\end{tabular}


APPENDIX C.2 Results of Volatile Organic Analyses, Water, Salmon Site

\begin{tabular}{|c|c|c|c|c|c|}
\hline COMPOUND & RESULTS & $\begin{array}{l}\text { DATA } \\
\text { QUAL. }\end{array}$ & $\begin{array}{r}\text { DATA } \\
\text { VALID. } \\
\end{array}$ & CRDL UNITS & DILUTION \\
\hline \multicolumn{6}{|l|}{$C 2-27-W$} \\
\hline Chloromethane & 10 UG/L & $u$ & NP & 10 UG/L & 1 \\
\hline Bromomethane & 10 UG/ & $u$ & NP & $10 \mathrm{UG} / \mathrm{L}$ & 1 \\
\hline Vinyl Chloride & 10 UG & $u$ & NP & 10 UG/ & 1 \\
\hline Chloroethane & 10 UG/L & $u$ & NP & $10 \mathrm{UG} / \mathrm{L}$ & 1 \\
\hline Methylene Chloride & 3 UG/ & BJ & NP & 5 UG/L & 1 \\
\hline Acetone & $8 \mathrm{UG} / \mathrm{L}$ & BJ & NP & $10 \mathrm{UG} / \mathrm{h}$ & 1 \\
\hline Carbon Disulfide & 5 UG/L & $u$ & NP & 5 UG $/$ & 1 \\
\hline 1,1-Dichloroethene & $5 \mathrm{UG} / \mathrm{L}$ & $u$ & NP & 5 UG $\Omega$ & 1 \\
\hline 1,1-Dichloroethane & $5 \mathrm{UG} / \mathrm{L}$ & $u$ & NP & 5 UG/ & 1 \\
\hline 1,2-Dichloroethene (tofal) & 5 UG/L & $u$ & NP & 5 UG/ & 1 \\
\hline Chloroform & 5 UG/ & $u$ & NP & 5 UG/ & 1 \\
\hline 1,2-Dichloroethane & - 5 UG/ & $u$ & NP & 5 UG/L & 1 \\
\hline 2-Butanone & $10 \mathrm{UG} / \mathrm{L}$ & $u$ & NP & 10 UG/ & 1 \\
\hline 1,1,1-Trichloroethane & $5 \mathrm{UG} / \mathrm{L}$ & $u$ & NP & 5 UG几 & 1 \\
\hline Carbon Tetrachloride & 5 UG/L & $u$ & NP & 5 UG & 1 \\
\hline Vinyl Acetate & $10 \mathrm{UG} / \mathrm{L}$ & $u$ & NP & 10 UG $\Omega$ & 1 \\
\hline Bromodichloromethane & 5 UG/L & $u$ & NP & 5 UGR & 1 \\
\hline 1,2-Dichloropropane & 5 UG/ & $u$ & NP & 5 UG $\Omega$ & 1 \\
\hline cis-1,3-Dichloropropene & $5 \mathrm{UG} / \mathrm{L}$ & $u$ & NP & 5 UG/ & 1 \\
\hline Trichloroethene & $1 \cup G \Omega$ & J & NP & 5 UG/ & 1 \\
\hline Dibromochloromethane & 5 UG/ & $u$ & NP & 5 UG/ & 1 \\
\hline 1,1,2-Trichloroethane & 5 UG $\Omega$ & $u$ & NP & $5 \mathrm{UG} /$ & 1 \\
\hline Benzene & 5 UG/ & $u$ & NP & 5 UG $\Omega$ & 1 \\
\hline trans-1,3-Dichloropropene & 5 UG/ & $u$ & $\mathrm{NP}$ & 5 UGת & 1 \\
\hline Bromoform & 5 UG/ & $u$ & NP & 5 UG & 1 \\
\hline 4-Methyl-2-Pentanone & 10 UG $\Omega$ & $u$ & NP & 10 UG $/$ & 1 \\
\hline 2-Hexanone & 10 UG/ & $u$ & NP & 10 UG/ & 1 \\
\hline Tetrachloroethene & 5 UG/ & & NP & 5 UG/ & 1 \\
\hline 1,1,2,2-Tetrachloroethane & 5 UG/L & $u$ & NP & 5 UG/ & 1 \\
\hline Toluene & $5 \mathrm{UG} / \mathrm{L}$ & $u$ & NP & 5 UG/ & 1 \\
\hline Chlorobenzene & 5 UG/ & $u$ & NP & 5 UG $\Omega$ & 1 \\
\hline Ethyl Benzene & 5 UG & $u$ & NP & 5 UG/L & 1 \\
\hline Styrene & 5 UG/ & $u$ & NP & 5 UG/ & 1 \\
\hline Xylenes (total) & 5 UG/ & $u$ & NP & $5 \mathrm{UG} / \mathrm{L}$ & 1 \\
\hline Unknown & $6 \mathrm{UG} / \mathrm{L}$ & J & NP & UG $\Omega$ & 1 \\
\hline Toluene-d8 (SURR) & $99 \%$ & & & $\%$ & 1 \\
\hline Bromofluorobenzene (SURR) & $100 \%$ & & & $\%$ & 1 \\
\hline 1,2-Dichloroethane-d4 (SURR) & $99 \%$ & & & $\%$ & 1 \\
\hline
\end{tabular}




\begin{tabular}{|c|c|c|c|c|c|}
\hline COMPOUND & RESULTS & $\begin{array}{l}\text { DATA } \\
\text { QUAL. }\end{array}$ & $\begin{array}{l}\text { DATA } \\
\text { VALID. }\end{array}$ & CRDL UNITS & DILUTION \\
\hline \multicolumn{6}{|l|}{ C-FB-001-W } \\
\hline Chloromethane & $10 \mathrm{UG} / \mathrm{L}$ & $u$ & - & $10 \mathrm{UG} / \mathrm{L}$ & 1 \\
\hline Bromomethane & $10 \mathrm{UG} / \mathrm{L}$ & $u$ & - & $10 \mathrm{UG} /$ & 1 \\
\hline Vinyl Chloride & $10 \mathrm{UG} / \mathrm{L}$ & $u$ & -. & 10 UG $/ 2$ & 1 \\
\hline Chloroethane & $10 \mathrm{UG} \Omega$ & $u$ & UJ & $10 \mathrm{UG} / \mathrm{L}$ & 1 \\
\hline Methylene Chloride & 2 UG/ & BJ & $u$ & $5 \mathrm{UG} / \mathrm{L}$ & 1 \\
\hline Acetone & $6 \mathrm{UG} / \mathrm{L}$ & BJ & UJ & $10 \mathrm{UG} / \mathrm{L}$ & 1 \\
\hline Carbon Disulfide & 5 UG/L & $u$ & - & 5 UG/ & 1 \\
\hline 1,1-Dichloroethene & 5 UGR & $u$ & - & 5 UG/ & 1 \\
\hline 1,1-Dichloroethane & 5 UG & $u$ & - & 5 UGR & 1 \\
\hline 1,2-Dichloroethene (total) & 5 UG/ & $u$ & $\ldots$ & $5 \mathrm{UG} /$ & 1 \\
\hline Chloroform & 12 UG $\Omega$ & & - & 5 UG/ & 1 \\
\hline 1,2-Dichloroethane & 5 UG $\Omega$ & $u$ & - & 5 UG/ & 1 \\
\hline 2-Butanone & $10 \mathrm{UG} / \mathrm{L}$ & $u$ & - & $10 \mathrm{UG} \Omega$ & 1 \\
\hline $1,1,1$-Trichloroethane & 5 UG/L & $u$ & $\ldots$ & 5 UG & 1 \\
\hline Carbon Tetrachloride & 5 UG & $u$ & - & $5 \mathrm{UG} \Omega$ & 1 \\
\hline Vinyl Acetate & 10 UGR & $u$ & -- & $10 \mathrm{UG} / \mathrm{L}$ & 1 \\
\hline Bromodichloromethane & 5 UGR & $u$ & - & 5 UG $\Omega$ & 1 \\
\hline 1,2-Dichloropropane & 5 UG $\Omega$ & $u$ & - & $5 \mathrm{UG} /$ & 1 \\
\hline cis-1,3-Dichloropropene & 5 UGR & $u$ & - & 5 UGR & 1 \\
\hline Trichloroethene & 5 UG/ & $u$ & - & 5 UG/ & 1 \\
\hline Dibromochloromethane & 5 UGR & $u$ & - & $5 \mathrm{UG} /$ & 1 \\
\hline 1,1,2-Trichloroethane & 5 UG/ & $u$ & - & 5 UG/ & 1 \\
\hline Benzene & 5 UG/L & $u$ & - & $5 \mathrm{UG} / \mathrm{L}$ & 1 \\
\hline trans-1,3-Dichloropropene & 5 UG/L & $u$ & - & 5 UGR & 1 \\
\hline Bromoform & 5 UG/ & $u$ & - & 5 UG & 1 \\
\hline 4-Methyl-2-Pentanone & 10 UG/ & $u$ & - & 10 UGR & 1 \\
\hline 2-Hexanone & $10 \mathrm{UG} / \mathrm{L}$ & $u$ & - & 10 UG $\Omega$ & 1 \\
\hline Tetrachloroethene & 5 UG & $u$ &.- & $5 \mathrm{UG} / \mathrm{L}$ & 1 \\
\hline 1,1,2,2-Tetrachloroethane & 5 UG & $u$ & - & 5 UG & 1 \\
\hline Toluene & 5 UG & $u$ & - & 5 UG & 1 \\
\hline Chlorobenzene & 5 UG $\Omega$ & $u$ & - & 5 UG/ & 1 \\
\hline Ethyl Benzene & 5 UG/ & $u$ & - & 5 UG & 1 \\
\hline Styrene & 5 UGR & $u$ & - & 5 UG $\Omega$ & 1 \\
\hline Xylenes (total) & 5 UG/ & $u$ & - & 5 UG $\Omega$ & 1 \\
\hline Unknown & 6 UG/L & $J$ & J & UG $\Omega$ & 1 \\
\hline Toluene-d8 (SURR) & $100 \%$ & & & $\%$ & 1 \\
\hline Bromofluorobenzene (SURR) & $104 \%$ & & & $\%$ & 1 \\
\hline 1,2-Dichloroethane-d4 (SURR) & $103 \%$ & & & $\%$ & 1 \\
\hline
\end{tabular}




\begin{tabular}{|c|c|c|c|c|c|}
\hline COMPOUND & RESULTS & $\begin{array}{l}\text { DATA } \\
\text { QUAL. }\end{array}$ & $\begin{array}{l}\text { DATA } \\
\text { VALID. }\end{array}$ & CRDL UNITS & DILUTION \\
\hline \multicolumn{6}{|l|}{ C-FB-002-W } \\
\hline Chloromethane & $10 \mathrm{UG} \Omega$ & $u$ & NP & 10 UGR & 1 \\
\hline Bromomethane & 10 UG/ & $u$ & $\mathrm{NP}$ & $10 \mathrm{UG} / \mathrm{L}$ & 1 \\
\hline Vinyl Chloride & 10 UG $/$ & $u$ & NP & 10 UG/ & 1 \\
\hline Chloroethane & 10 UG/L & $u$ & NP & $10 \mathrm{UG} / \mathrm{L}$ & 1 \\
\hline Methylene Chloride & 5 UG/L & $u$ & NP & 5 UG/L & 1 \\
\hline Acetone & $10 \mathrm{UG} / \mathrm{L}$ & $u$ & NP & $10 \mathrm{UG} / \mathrm{L}$ & 1 \\
\hline Carbon Disulfide & 2 UG/L & J & NP & 5 UG/ & 1 \\
\hline 1,1-Dichloroethene & $5 \mathrm{UG} / \mathrm{L}$ & $u$ & NP & 5 UG/ & 1 \\
\hline 1,1-Dichloroethane & 5 UG/ & $u$ & NP & 5 UG/L & 1 \\
\hline 1,2-Dichloroethene (total) & 5 UGR & $u$ & NP & 5 UG & 1 \\
\hline Chloroform & 5 UG几 & $u$ & NP & 5 UG/ & 1 \\
\hline 1,2-Dichloroethane & 5 UG & $u$ & NP & 5 UG/ & 1 \\
\hline 2-Butanone & 10 UGIL & $u$ & NP & $10 \mathrm{UG} \Omega$ & 1 \\
\hline 1,1,1-Trichloroethane & 5 UG & $u$ & NP & 5 UG/ & 1 \\
\hline Carbon Tetrachloride & 5 UG/L & $u$ & NP & 5 UG/ & 1 \\
\hline Vinyl Acetate & $10 \mathrm{UG} / \mathrm{L}$ & $u$ & NP & 10 UG/L & 1 \\
\hline Bromodichloromethane & 5 UG/ & $u$ & NP & 5 UG/ & 1 \\
\hline 1,2-Dichloropropane & 5 UG/L & $u$ & NP & 5 UG/ & 1 \\
\hline cis-1,3-Dichioropropene & $5 \cup G \Omega$ & $u$ & NP & 5 UGR & 1 \\
\hline Trichloroethene & $5 \mathrm{UG} / \mathrm{L}$ & $u$ & NP & 5 UG/ & 1 \\
\hline Dibromochloromethane & 5 UG & $u$ & NP & 5 UG/L & 1 \\
\hline 1,1,2-Trichloroethane & 5 UG $\Omega$ & $u$ & NP & 5 UGR & 1 \\
\hline Benzene & 5 UG & $u$ & NP & $5 \mathrm{UG} / \mathrm{L}$ & 1 \\
\hline trans-1,3-Dichloropropene & 5 UG/ & $u$ & NP & 5 UG/ & 1 \\
\hline Bromoform & $5 \cup G \Omega$ & $u$ & NP & 5 UGR & 1 \\
\hline 4-Methyl-2-Pentanone & $10 \mathrm{UG} / \mathrm{L}$ & $u$ & NP & 10 UG/L & 1 \\
\hline 2-Hexanone & 10 UG/L & $u$ & NP & 10 UG/ & 1 \\
\hline Tetrachioroethene & 5 UGR & $u$ & NP & 5 UGR & 1 \\
\hline 1,1,2,2-Tetrachloroethane & 5 UG几 & $u$ & NP & 5 UG/ & 1 \\
\hline Toluene & 5 UG & $u$ & NP & 5 UG/L & 1 \\
\hline Chlorobenzene & 5 UGR & $u$ & NP & 5 UG/ & 1 \\
\hline Ethyl Benzene & 5 UG/ & $u$ & NP & 5 UG/L & 1 \\
\hline Styrene & 5 UG/ & $u$ & NP & 5 UG/ & 1 \\
\hline Xylenes (total) & 5 UG $\Omega$ & $u$ & NP & 5 UG & 1 \\
\hline Toluene-d8 (SURR) & $97 \%$ & & & $\%$ & 1 \\
\hline Bromofluorobenzene (SURR) & $101 \%$ & & & $\%$ & 1 \\
\hline 1,2-Dichloroethane-d4 (SURR) & $102 \%$ & & & $\%$ & 1 \\
\hline
\end{tabular}


APPENDIX C.2 Results of Volatile Organic Analyses, Water, Salmon Site

\begin{tabular}{|c|c|c|c|c|c|}
\hline COMPOUND & RESULTS UNITS & $\begin{array}{l}\text { DATA } \\
\text { QUAL. }\end{array}$ & $\begin{array}{l}\text { DATA } \\
\text { VALID. }\end{array}$ & CRDL UNITS & DILUTION \\
\hline \multicolumn{6}{|l|}{ C-FB-003-W } \\
\hline Chloromethane & 10 UG $\Omega$ & $u$ & NP & 10 UG $\Omega$ & 1 \\
\hline Bromomethane & 10 UG $\Omega$ & $u$ & NP & 10 UG $\Omega$ & 1 \\
\hline Vinyl Chloride & 10 UG/ & $U$ & NP & $10 \mathrm{UG} \Omega$ & 1 \\
\hline Chloroethane & 10 UG $\Omega$ & $u$ & NP & 10 UG $\Omega$ & 1 \\
\hline Methylene Chloride & 3 UG $\Omega$ & J & NP & 5 UG几 & 1 \\
\hline Acetone & $5 \mathrm{UG} /$ & BJ & NP & $10 \mathrm{UG} / \mathrm{L}$ & 1 \\
\hline Carbon Disulfide & 5 UG $\Omega$ & $u$ & NP & 5 UG & 1 \\
\hline 1,1-Dichloroethene & 5 UG $/$ & $u$ & NP & $5 \mathrm{UG} / \mathrm{L}$ & 1 \\
\hline 1,1-Dichloroethane & 5 UG/L & $u$ & NP & 5 UG $/$ & 1 \\
\hline 1,2-Dichloroethene (total) & 5 UG $\Omega$ & $u$ & NP & 5 UG $\Omega$ & 1 \\
\hline Chloroform & $5 \mathrm{UG} / \mathrm{L}$ & $u$ & NP & 5 UG $\Omega$ & 1 \\
\hline 1,2-Dichloroethane & 5 UG/ & $u$ & NP & $5 \mathrm{UG} /$ & 1 \\
\hline 2-Butanone & 10 UG $/ \mathrm{L}$ & $u$ & NP & $10 \cup G \Omega$ & 1 \\
\hline 1,1,1-Trichloroethane & 5 UG/ & $u$ & NP & 5 UG $\Omega$ & 1 \\
\hline Carbon Tetrachloride & 5 UG $/$ & $u$ & NP & $5 \mathrm{UG} \Omega$ & 1 \\
\hline Vinyl Acetate & $10 \mathrm{UG} / \mathrm{L}$ & $u$ & NP & $10 \mathrm{UG} \Omega$ & 1 \\
\hline Bromodichloromethane & 5 UG & $u$ & NP & 5 UG & 1 \\
\hline 1,2-Dichloropropane & 5 UG $\Omega$ & $u$ & NP & 5 UG $\Omega$ & 1 \\
\hline cis-1,3-Dichloropropene & $5 \mathrm{UG} \Omega$ & $u$ & NP & 5 UG $\Omega$ & 1 \\
\hline Trichloroethene & 5 UG $\Omega$ & $u$ & NP & $5 \cup G \Omega$ & 1 \\
\hline Dibromochloromethane & 5 UG $\Omega$ & $u$ & NP & 5 UG $\Omega$ & 1 \\
\hline 1,1,2-Trichloroethane & 5 UG $\Omega$ & u & NP & 5 UG $\Omega$ & 1 \\
\hline Benzene & 5 UG $\Omega$ & $u$ & NP & $5 \mathrm{UG} / \mathrm{L}$ & 1 \\
\hline trans-1,3-Dichloropropene & 5 UG $\Omega$ & $u$ & NP & 5 UG $\Omega$ & 1 \\
\hline Bromoform & $5 \mathrm{UG} /$ & $u$ & NP & 5 UG $\Omega$ & 1 \\
\hline 4-Methyl-2-Pentanone & $10 \mathrm{UG} / \mathrm{L}$ & $u$ & NP & 10 UG $\Omega$ & 1 \\
\hline 2-Hexanone & 10 UG $\Omega$ & $u$ & $\mathrm{NP}$ & 10 UG $\Omega$ & 1 \\
\hline Tetrachloroethene & 5 UG/ & $u$ & NP & 5 UG $\Omega$ & 1 \\
\hline 1,1,2,2-Tetrachloroethane & $5 \mathrm{UG} / \mathrm{L}$ & $u$ & NP & 5 UG $\Omega$ & 1 \\
\hline Toluene & 5 UG $\Omega$ & $u$ & NP & 5 UG & 1 \\
\hline Chlorobenzene & 5 UG $\Omega$ & $u$ & NP & 5 UG $\Omega$ & 1 \\
\hline Ethyl Benzene & 5 UG $\Omega$ & $u$ & NP & $5 \mathrm{UG \Omega}$ & 1 \\
\hline Styrene & 5 UG/ & $u$ & NP & 5 UG $/$ & 1 \\
\hline Xylenes (total) & 5 UG/ & $u$ & NP & 5 UG & 1 \\
\hline Unknown-1 & 11 UG $\Omega$ & J & NP & UG/L & 1 \\
\hline Unknown-2 & 12 UG $\Omega$ & $\mathrm{J}$ & NP & UG $\Omega$ & 1 \\
\hline Unknown-3 & 23 UG $\Omega$ & J & NP & UG/ & 1 \\
\hline Toluene-d8 (SURR) & $96 \%$ & & & $\%$ & 1 \\
\hline Bromofluorobenzene (SURR) & $104 \%$ & & & $\%$ & 1 \\
\hline 1,2-Dichloroethane-d4 (SURR) & $90 \%$ & & & $\%$ & 1 \\
\hline
\end{tabular}


APPENDIX C.2 Results of Volatile Organic Analyses, Water, Salmon Site

\begin{tabular}{|c|c|c|c|c|c|}
\hline COMPOUND & RESULTS & $\begin{array}{l}\text { DATA } \\
\text { QUAL. }\end{array}$ & $\begin{array}{l}\text { DATA } \\
\text { VALID. }\end{array}$ & CRDL UNITS & DILUTION \\
\hline \multicolumn{6}{|l|}{ C-RB-001-W } \\
\hline Chloromethane & $10 \mathrm{UG} / \mathrm{L}$ & $u$ & $\ldots$ & 10 UG/L & 1 \\
\hline Bromomethane & 10 UG/L & $u$ & -.- & 10 UG $/$ & 1 \\
\hline Vinyl Chloride & $10 \mathrm{UG} / \mathrm{L}$ & $u$ & -- & 10 UG/L & 1 \\
\hline Chloroethane & 10 UG $/$ & $u$ & UJ & $10 \mathrm{UG} / \mathrm{L}$ & 1 \\
\hline Methylene Chloride & 5 UG几 & $u$ & --- & 5 UG/L & 1 \\
\hline Acetone & 10 UG/L & $u$ & us & $10 \mathrm{UG} /$ & 1 \\
\hline Carbon Disulfide & $6 \mathrm{UG} / \mathrm{L}$ & & 6 & 5 UG/ & 1 \\
\hline 1,1-Dichloroethene & 5 UGR & $u$ & $\ldots$ & $5 \mathrm{UG} /$ & 1 \\
\hline 1,1-Dichloroethane & $5 \mathrm{UG} / \mathrm{L}$ & $u$ & $\ldots$ & 5 UG & 1 \\
\hline 1,2-Dichloroethene (total) & 5 UG $\Omega$ & $u$ & -- & $5 \mathrm{UG} /$ & 1 \\
\hline Chloroform & 11 UG/L & & 11 & 5 UG/L & 1 \\
\hline 1,2-Dichloroethane & 5 UG/ & $u$ & $\ldots$ & 5 UG/L & 1 \\
\hline 2-Butanone & 10 UG $\Omega$ & $u$ & -- & 10 UG/L & 1 \\
\hline 1,1,1-Trichloroethane & 5 UG/ & $u$ & $--\cdot$ & 5 UG/ & 1 \\
\hline Carbon Tetrachloride & $5 \mathrm{UG} / \mathrm{L}$ & $u$ & $\cdots$ & 5 UG/L & 1 \\
\hline Vinyl Acetate & $10 \mathrm{UG} / \mathrm{L}$ & $u$ & --- & $10 \mathrm{UG} / \mathrm{L}$ & 1 \\
\hline Bromodichloromethane & $5 \mathrm{UG} / \mathrm{L}$ & $u$ & $\ldots$ & 5 UGR & 1 \\
\hline 1,2-Dichloropropane & 5 UG/ & $u$ & - & 5 UG/ & 1 \\
\hline cis-1,3-Dichloropropene & 5 UGR & $u$ & -- & 5 UG/ & 1 \\
\hline Trichloroethene & 5 UG/ & $u$ & $\cdots$ & 5 UG $\Omega$ & 1 \\
\hline Dibromochloromethane & 5 UG/ & $u$ & $\ldots$ & $5 \mathrm{UG} / \mathrm{L}$ & 1 \\
\hline 1,1,2-Trichloroethane & 5 UGR & $u$ & - & 5 UGR & 1 \\
\hline Benzene & $5 \mathrm{UG} / \mathrm{L}$ & $u$ & $\ldots$ & 5 UG/ & 1 \\
\hline trans-1,3-Dichloropropene & 5 UG/L & $u$ & -- & 5 UG/ & 1 \\
\hline Bromoform & 5 UG/ & $u$ & - & 5 UG/ & 1 \\
\hline 4-Methyl-2-Pentanone & $10 \mathrm{UG} / \mathrm{L}$ & $u$ & -- & $10 \mathrm{UG} / \mathrm{L}$ & 1 \\
\hline 2-Hexanone & $10 \mathrm{UG} / \mathrm{L}$ & $u$ & $\ldots$ & $10 \mathrm{UG} / \mathrm{L}$ & 1 \\
\hline Tetrachloroethene & 5 UG/ & $u$ & -- & $5 \mathrm{UG} /$ & 1 \\
\hline $1,1,2,2-$ Tetrachloroethane & 5 UG/L & $u$ & .-. & 5 UG/L & 1 \\
\hline Toluene & $5 \mathrm{UG} / \mathrm{L}$ & $u$ & $\ldots$ & 5 UG/ & 1 \\
\hline Chlorobenzene & 5 UG/ & $u$ & -.. & 5 UG & 1 \\
\hline Ethyl Benzene & 5 UG & $u$ & $\cdots$ & 5 UG/ & 1 \\
\hline Styrene & 5 UG $/$ & $u$ & $\ldots$ & 5 UG/ & 1 \\
\hline Xylenes (total) & 5 UG/L & $u$ & $\ldots$ & $5 \mathrm{UG} \Omega$ & 1 \\
\hline Unknown-1 & 9 UG/ & J & J & UG $\Omega$ & 1 \\
\hline Unknown-2 & 13 UG/L & J & J & UG $\Omega$ & 1 \\
\hline Toluene-d8 (SURR) & $102 \%$ & & & $\%$ & 1 \\
\hline Bromofluorobenzene (SURR) & $105 \%$ & & & $\%$ & 1 \\
\hline 1,2-Dichloroethane-d4 (SURR) & $104 \%$ & & & $\%$ & 1 \\
\hline
\end{tabular}


APPENDIX C.2 Results of Volatile Organic Analyses, Water, Salmon Site

\begin{tabular}{|c|c|c|c|c|c|}
\hline COMPOUND & RESULTS & $\begin{array}{l}\text { DATA } \\
\text { QUAL. }\end{array}$ & $\begin{array}{l}\text { DATA } \\
\text { VALID. }\end{array}$ & CRDL UNITS & DILUTION \\
\hline \multicolumn{6}{|l|}{ C-RB-002-W } \\
\hline Chloromethane & $10 \mathrm{UG} \Omega$ & $u$ & NP & $10 \mathrm{UG} / \mathrm{L}$ & 1 \\
\hline Bromomethane & 10 UG $\Omega$ & $u$ & NP & $10 \mathrm{UG} \Omega$ & 1 \\
\hline Vinyl Chloride & $10 \mathrm{UG} /$ & $u$ & NP & $10 \mathrm{UG} / \mathrm{L}$ & 1 \\
\hline Chloroethane & 10 UG $\Omega$ & $u$ & NP & 10 UG $\Omega$ & 1 \\
\hline Methylene Chloride & 2 UG/L & BJ & NP & 5 UG $\Omega$ & 1 \\
\hline Acetone & 10 UG $\Omega$ & $u$ & NP & $10 \mathrm{UG} \Omega$ & 1 \\
\hline Carbon Disulfide & 17 UGR & & NP & $5 \mathrm{UG} /$ & 1 \\
\hline 1,1-Dichloroethene & 5 UGR & $u$ & NP & 5 UG $\Omega$ & 1 \\
\hline 1,1-Dichloroethane & $5 \mathrm{UG} / \mathrm{L}$ & $u$ & NP & 5 UG $\Omega$ & 1 \\
\hline 1,2-Dichloroethene (total) & 5 UGR & $u$ & NP & $5 \mathrm{UG} \Omega$ & 1 \\
\hline Chloroform & 5 UG $\Omega$ & $u$ & NP & $5 \mathrm{UG} / \mathrm{L}$ & 1 \\
\hline 1,2-Dichloroethane & 5 UGR & $u$ & NP & 5 UG $\Omega$ & 1 \\
\hline 2-Butanone & 10 UG/ & $u$ & NP & $10 \mathrm{UG} / \mathrm{L}$ & 1 \\
\hline 1,1,1-Trichloroethane & 5 UG几 & $u$ & NP & 5 UGL & 1 \\
\hline Carbon Tetrachloride & 5 UGR & $u$ & NP & 5 UG/ & 1 \\
\hline Vinyl Acetate & $10 \mathrm{UG} / \mathrm{L}$ & $u$ & NP & $10 \mathrm{UG} / \mathrm{L}$ & 1 \\
\hline Bromodichloromethane & $5 \mathrm{UG} / \mathrm{L}$ & $u$ & NP & 5 UG & 1 \\
\hline 1,2-Dichloropropane & 5 UGR & $u$ & NP & 5 UG/ & 1 \\
\hline cis-1,3-Dichloropropene & 5 UGR & $u$ & NP & 5 UG/ & 1 \\
\hline Trichloroethene & 5 UGR & $u$ & NP & 5 UG $\Omega$ & 1 \\
\hline Dibromochloromethane & 5 UG/ & $u$ & NP & 5 UG $\Omega$ & 1 \\
\hline 1,1,2-Trichloroethane & 5 UG几 & $u$ & NP & 5 UG $\Omega$ & 1 \\
\hline Benzene & 5 UGR & $u$ & NP & 5 UG & 1 \\
\hline trans-1,3-Dichloropropene & $5 \mathrm{UG} / \mathrm{L}$ & $u$ & NP & 5 UG & 1 \\
\hline Bromoform & 5 UG/ & $u$ & NP & 5 UGR & 1 \\
\hline 4-Methyl-2-Pentanone & $10 \mathrm{UG} / \mathrm{L}$ & $u$ & NP & 10 UG $\Omega$ & 1 \\
\hline 2-Hexanone & 10 UG $\Omega$ & $u$ & NP & 10 UG/ & 1 \\
\hline Tetrachloroethene & 5 UG & $u$ & NP & 5 UGL & 1 \\
\hline 1,1,2,2-Tetrachloroethane & 5 UGR & $u$ & NP & 5 UG & 1 \\
\hline Toluene & 5 UG & $u$ & NP & 5 UG几 & 1 \\
\hline Chlorobenzene & 5 UG/ & $u$ & NP & 5 UGR & 1 \\
\hline Ethyl Benzene & 5 UG/ & $u$ & NP & 5 UG & 1 \\
\hline Styrene & 5 UGR & $u$ & NP & 5 UG & 1 \\
\hline Xylenes (total) & 5 UGR & $u$ & NP & 5 UG $\Omega$ & 1 \\
\hline Unknown-1 & 70 UG $\Omega$ & J & NP & UGR & 1 \\
\hline Unknown-2 & 82 UG/ & J & NP & UGR & 1 \\
\hline Unknown-3 & 50 UG/ & J & NP & UG/L & 1 \\
\hline Toluene-d8 (SURR) & $97 \%$ & & & $\%$ & 1 \\
\hline Bromofluorobenzene (SURR) & $102 \%$ & & & $\%$ & . \\
\hline 1,2-Dichloroethane-d4 (SURR) & $103 \%$ & & & $\%$ & 1 \\
\hline
\end{tabular}




\begin{tabular}{|c|c|c|c|c|c|}
\hline COMPOUND & RESULTS & $\begin{array}{l}\text { DATA } \\
\text { QUAL. }\end{array}$ & $\begin{array}{l}\text { DATA } \\
\text { VALID. } \\
\end{array}$ & CRDL UNITS & DILUTION \\
\hline \multicolumn{6}{|l|}{ C-RB-003-W } \\
\hline Chloromethane & 10 UG/L & $u$ & NP & 10 UG/L & 1 \\
\hline Bromomethane & $10 \mathrm{UG} / \mathrm{L}$ & $u$ & NP & 10 UG/ & 1 \\
\hline Vinyl Chloride & 10 UG/L & $u$ & NP & 10 UG/ & 1 \\
\hline Chloroethane & 10 UG $\Omega$ & $u$ & NP & 10 UG/ & 1 \\
\hline Methylene Chloride & 2 UG/ & J & NP & 5 UG/ & 1 \\
\hline Acetone & $7 \mathrm{UG} /$ & BJ & NP & $10 \mathrm{UG} / \mathrm{L}$ & 1 \\
\hline Carbon Disulfide & 9 UG $\Omega$ & & NP & 5 UG/ & 1 \\
\hline 1,1-Dichloroethene & 5 UG/ & $u$ & NP & 5 UG/ & 1 \\
\hline 1,1-Dichloroethane & 5 UG/ & $u$ & NP & 5 UG/ & 1 \\
\hline 1,2-Dirshloroethene (total) & 5 UG/ & $u$ & NP & $5 \mathrm{UG} / \mathrm{L}$ & 1 \\
\hline Chloroform & $5 \mathrm{UG} / \mathrm{L}$ & $u$ & NP & $5 \mathrm{UG} /$ & 1 \\
\hline 1,2-Dichloroethane & 5 UG/ & $u$ & NP & $5 \mathrm{UG} / \mathrm{L}$ & 1 \\
\hline 2-Butanone & $10 \mathrm{UG} / \mathrm{L}$ & $u$ & NP & $10 \mathrm{UG} / \mathrm{L}$ & 1 \\
\hline 1,1,1-Trichloroethane & 5 UG $\Omega$ & $u$ & NP & 5 UG $\Omega$ & 1 \\
\hline Carbon Tetrachloride & 5 UG/ & $u$ & NP & 5 UGR & 1 \\
\hline Vinyl Acetate & $10 \mathrm{UG} /$ & $u$ & NP & $10 \mathrm{UG} / \mathrm{L}$ & 1 \\
\hline Bromodichloromethane & 5 UG & $u$ & NP & 5 UG/ & 1 \\
\hline 1,2-Dichloropropane & 5 UG & $u$ & NP & 5 UGR & 1 \\
\hline cis-1,3-Dichloropropene & 5 UG/ & $u$ & NP & 5 UG/L & 1 \\
\hline Trichloroethene & 5 UG $\Omega$ & $u$ & NP & 5 UG/ & 1 \\
\hline Dibromochloromethane & 5 UG/ & $u$ & NP & 5 UG/ & 1 \\
\hline 1,1,2-Trichloroethane & 5 UG几 & $u$ & NP & 5 UG/ & 1 \\
\hline Benzene & 5 UG/ & $u$ & NP & 5 UG & 1 \\
\hline trans-1,3-Dichloropropene & 5 UG几 & $u$ & NP & 5 UGת & 1 \\
\hline Bromoform & 5 UG & $u$ & NP & 5 UG/L & 1 \\
\hline 4-Methyl-2-Pentanone & 10 UG/ & $u$ & NP & 10 UG/ & 1 \\
\hline 2-Hexanone & 10 UG $\Omega$ & $u$ & NP & 10 UG/L & 1 \\
\hline Tetrachloroethene & 5 UG & $u$ & NP & 5 UG/L & 1 \\
\hline $1,1,2,2$-Tetrachloroethane & 5 UG & $u$ & NP & $5 \mathrm{UG} / \mathrm{L}$ & 1 \\
\hline Toluene & 5 UG & $u$ & NP & 5 UG/L & 1 \\
\hline Chlorobenzene & 5 UG几 & $u$ & NP & 5 UG/ & 1 \\
\hline Ethyl Benzene & 5 UG/ & $u$ & NP & $5 \mathrm{UG} / \mathrm{L}$ & 1 \\
\hline Styrene & 5 UG/ & $u$ & NP & 5 UG $\Omega$ & 1 \\
\hline Xylenes (total) & 5 UG/ & $u$ & NP & 5 UG/ & 1 \\
\hline Unknown-1 & $6 \mathrm{UG \Omega}$ & $\mathrm{J}$ & NP & UG/ & 1 \\
\hline Unknown-2 & 17 UG $\Omega$ & $\checkmark$ & NP & $U G / L$ & 1 \\
\hline Toluene-d8 (SURR) & $97 \%$ & & & $\%$ & 1 \\
\hline Bromofluorobenzene (SURR) & $102 \%$ & & & $\%$ & 1 \\
\hline 1,2-Dichloroethane-d4 (SURR) & $90 \%$ & & & $\%$ & 1 \\
\hline
\end{tabular}


APPENDIX C.2 Results of Volatile Organic Analyses, Water, Saimon Site

\begin{tabular}{|c|c|c|c|c|c|}
\hline COMPOUND & RESULTS UNITS & $\begin{array}{l}\text { DATA } \\
\text { QUAL. }\end{array}$ & $\begin{array}{l}\text { DATA } \\
\text { VALID. }\end{array}$ & CRDL UNITS & DILUTIC \\
\hline \multicolumn{6}{|l|}{ TRIP BLANK } \\
\hline Chloromethane & $10 \mathrm{UG} / \mathrm{L}$ & U & - & 10 UG $\Omega$ & 1 \\
\hline Bromomethane & 10 UG/L & U & - & 10 UG $\Omega$ & 1 \\
\hline Vinyl Chloride & $10 \mathrm{UG} / \mathrm{L}$ & $U$ & $\ldots$ & $10 \mathrm{UG} \Omega$ & 1 \\
\hline Chloroethane & 10 UG/L & $U$ & UJ & 10 UG/L & 1 \\
\hline Methylene Chloride & 4 UG/L & BJ & $u$ & 5 UG/ & 1 \\
\hline Acetone & 32 UG/L & B & UJ & 10 UG/ & 1 \\
\hline Carbon Disulfide & 5 UG/L & $U$ & - & 5 UG $\Omega$ & 1 \\
\hline 1,1-Dichloroethene & 5 UG/L & U & $\ldots$ & 5 UG/ & 1 \\
\hline 1,1-Dlchloroethane & $5 U G / L$ & $U$ & - & 5 UG $\Omega$ & 1 \\
\hline 1,2-Dichloroethene (total) & 5 UG/L & $U$ & - & 5 UGת & 1 \\
\hline Chloroform & 5 UG/L & $U$ & - & 5 UG $\Omega$ & 1 \\
\hline 1,2-Dichloroethane & 5 UG/L & $U$ & - & 5 UG $\Omega$ & 1 \\
\hline 2-Butanone & 10 UG/L & U & - & 10 UG $\Omega$ & 1 \\
\hline 1,1,1-Trichloroethane & $5 \cup G / L$ & $U$ & - & 5 UG & 1 \\
\hline Carbon Tetrachloride & $5 \mathrm{UG} / \mathrm{L}$ & $U$ & - & $5 \mathrm{UG} /$ & 1 \\
\hline Vinyl Acetate & $5 \cup G / L$ & U & - & $10 \mathrm{UG} / \mathrm{L}$ & 1 \\
\hline Bromodichloromethane & 5 UG/L & $U$ & - & 5 UG/ & 1 \\
\hline 1,2-Dichloropropane & 5 UG/L & U & - & $5 \mathrm{UG} \Omega$ & 1 \\
\hline cls-1,3-Dlchloropropene & 5 UG/L & U & - & 5 UG & 1 \\
\hline Trichloroethene & 5 UG/L & $\mathrm{U}$ & - & $5 \mathrm{UG} \Omega$ & 1 \\
\hline Dibromochloromethane & 5 UG/L & $U$ & - & 5 UG/ & 1 \\
\hline 1,1,2-Trichloroethane & 5 UG/L & U & - & $5 \mathrm{UG} \Omega$ & 1 \\
\hline Benzene & 5 UG/L & U & 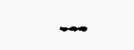 & $5 \mathrm{UG} / \mathrm{L}$ & 1 \\
\hline trans-1,3-Dichloropropene & 5 UG/L & $U$ & - & 5 UG/L & 1 \\
\hline Bromoform & 5 UG/L & $U$ & - & 5 UG $\Omega$ & 1 \\
\hline 4-Methyl-2-Pentanone & 10 UG/L & $U$ & - & $10 \mathrm{UG} / \mathrm{L}$ & 1 \\
\hline 2-Hexanone & 10 UG/L & $U$ & - & 10 UG $\Omega$ & 1 \\
\hline Tetrachloroethene & 5 UG/L & $u$ & - & $5 \cup G \Omega$ & 1 \\
\hline 1,1,2,2-Tetrachloroethane & $5 \mathrm{UG} / \mathrm{L}$ & U & - & 5 UG/ & 1 \\
\hline Toluene & 5 UG/L & $U$ & $\ldots$ & 5 UGR & 1 \\
\hline Chlorobenzene & 5 UG/L & $U$ & - & 5 UG $\Omega$ & 1 \\
\hline Ethyl Benzene & 5 UG/L & $\mathrm{U}$ & - & 5 UG $\Omega$ & 1 \\
\hline Styrene & 5 UG/L & $U$ & - & 5 UG/ & 1 \\
\hline Xylenes (total) & 5 UG/L & $U$ & - & 5 UG/ & 1 \\
\hline Unknown & $16 \mathrm{UG} / \mathrm{L}$ & J & J & $U G \Omega$ & 1 \\
\hline Toluene-d8 (SURR) & $102 \%$ & & & $\%$ & 1 \\
\hline Bromofluorobenzene (SURR) & $98 \%$ & & & $\%$ & 1 \\
\hline 1,2-Dichloroethane-d4 (SURR) & $100 \%$ & & & $\%$ & 1 \\
\hline
\end{tabular}


APPENDIX C.2 Results of Volatile Organic Analyses, Water, Salmon Site

\begin{tabular}{|c|c|c|c|c|c|}
\hline COMPOUND & RESULTS & $\begin{array}{l}\text { DATA } \\
\text { QUAL. }\end{array}$ & $\begin{array}{l}\text { DATA } \\
\text { VALID. }\end{array}$ & CRDL UNITS & DILUTION \\
\hline \multicolumn{6}{|l|}{ TRIP BLANK } \\
\hline Chloromethane & 10 UG/L & $u$ & NP & $10 \mathrm{UG} / \mathrm{L}$ & 1 \\
\hline Bromomethane & 10 UG & $u$ & NP & 10 UG/ & 1 \\
\hline Vinyl Chloride & 10 UG/L & $u$ & NP & 10 UG/ & 1 \\
\hline Chloroethane & 10 UG/L & $u$ & NP & $10 \mathrm{UG} \Omega$ & 1 \\
\hline Methylene Chloride & $6 \mathrm{UG/L}$ & B & NP & $5 \mathrm{UG} \Omega$ & 1 \\
\hline Acetone & 25 UG/L & B & $\mathrm{NP}$ & 10 UG/ & 1 \\
\hline Carbon Disulfide & 2 UG/L & $J$ & NP & 5 UG/L & 1 \\
\hline 1.1-Dichloroethene & 5 UG/L & $\mathrm{u}$ & NP & 5 UG/L & 1 \\
\hline 1,1-Dichloroethane & 5 UG/L & $u$ & NP & 5 UG/L & 1 \\
\hline 1.2-Dichloroethene (total) & 5 UG/L & $u$ & NP & 5 UG/ & 1 \\
\hline Chloroform & 5 UG/L & u & NP & 5 UG/ & 1 \\
\hline 1.2-Dichloroethane & 5 UG/L & $u$ & NP & 5 UG/ & 1 \\
\hline 2-Butanone & 10 UG/L & u & NP & $10 \mathrm{UG} / \mathrm{L}$ & 1 \\
\hline 1,1,1-Trichloroethane & 5 UG/L & $u$ & $\mathrm{NP}$ & $5 \mathrm{UG} \Omega$ & 1 \\
\hline Carbon Tetrachloride & 5 UG/L & $u$ & NP & 5 UG $\Omega$ & 1 \\
\hline Vinyl Acetate & 10 UG/L & $u$ & $\mathrm{NP}$ & $10 \mathrm{UG} \Omega$ & 1 \\
\hline Bromodichloromethane & 5 UG/L & $u$ & NP & 5 UG/ & 1 \\
\hline 1,2-Dichloropropane & 5 UG/L & $u$ & NP & 5 UG $/$ & 1 \\
\hline cis-1,3-Dichloropropene & 5 UG/L & $u$ & NP & 5 UG $\Omega$ & 1 \\
\hline Trichloroethene & 5 UG/L & $u$ & NP & 5 UG/L & 1 \\
\hline Dibromochloromethane & 5 UG/L & $u$ & NP & 5 UG & 1 \\
\hline 1.1.2-Trichloroethane & 5 UG/L & $u$ & $\mathrm{NP}$ & 5 UG/ & 1 \\
\hline Benzene & 5 UG/L & $u$ & NP & 5 UG/ & 1 \\
\hline trans-1,3-Dichloropropene & 5 UG/L & $u$ & NP & 5 UG/L & 1 \\
\hline Bromoform & 5 UG/L & $u$ & NP & 5 UG/ & 1 \\
\hline 4-Methyl-2-Pentanone & 10 UG/L & $u$ & NP & 10 UG/L & 1 \\
\hline 2-Hexanone & 10 UG/L & $u$ & NP & 10 UG/L & 1 \\
\hline Tetrachloroethene & 5 UG/L & $u$ & NP & 5 UG/ & 1 \\
\hline 1,1,2,2-Tetrachloroethane & 5 UG/L & $u$ & NP & 5 UG/ & 1 \\
\hline Toluene & 5 UG/L & $u$ & NP & 5 UG/ & 1 \\
\hline Chlorobenzene & 5 UG/L & $\mathrm{u}$ & NP & 5 UG & 1 \\
\hline Ethyl Benzene & 5 UG/L & $u$ & NP & 5 UG & 1 \\
\hline Styrene & 5 UG/L & $u$ & NP & 5 UG/ & 1 \\
\hline Xylenes (total) & 5 UG/L & U & NP & 5 UG & 1 \\
\hline Unknown-1 & 16 UG/L & $B J$ & NP & UG $\Omega$ & 1 \\
\hline Unknown-2 & 6 UG/L & J & NP & UG $\Omega$ & 1 \\
\hline Toluene-d8 (SURR) & $99 \%$ & & & $\%$ & 1 \\
\hline Bromofluorobenzene (SURR) & $109 \%$ & & & $\%$ & 1 \\
\hline 1.2-Dichloroethane-d4 (SURR) & $106 \%$ & & & $\%$ & 1 \\
\hline
\end{tabular}




\section{Appendix D.1 \\ Results of Semivolatile Organic Compound Analyses, Soils, Salmon Site}


APPENDIX D.1 Results of Semivolatile Organic Compound Analyses, Soils, Salmon Site

\begin{tabular}{|c|c|c|c|c|c|}
\hline COMPOUND & RESULTS & $\begin{array}{l}\text { DATA } \\
\text { QUAL. }\end{array}$ & $\begin{array}{l}\text { DATA } \\
\text { VALID. }\end{array}$ & CRDL UNITS & DILUTION \\
\hline \multicolumn{6}{|l|}{ C5-1-S } \\
\hline Phenol & 330 UG/KG & $u$ & NP & 330 UG/KG & 1 \\
\hline bis(2-Chloroethyl) ether & 330 UG/KG & $u$ & NP & $330 \mathrm{UG} / \mathrm{KG}$ & 1 \\
\hline 2-Chlorophenol & 330 UG/KG & $u$ & NP & 330 UG/KG & 1 \\
\hline 1,3-Dichlorobenzene & 330 UG/KG & $u$ & NP & 330 UG/KG & 1 \\
\hline 1,4-Dichlorobenzene & 330 UG/KG & $u$ & NP & $330 \mathrm{UG} / \mathrm{KG}$ & 1 \\
\hline Benzyl Alcohol & 330 UG/KG & $u$ & NP & $330 \mathrm{UG} / \mathrm{KG}$ & 1 \\
\hline 1,2-Dichlorobenzene & 330 UG/KG & $u$ & NP & 330 UG/KG & 1 \\
\hline 2-Methylphenol & 330 UG/KG & $u$ & NP & 330 UG/KG & 1 \\
\hline bis(2-Chloroisopropyl) ether & 330 UG/KG & $u$ & NP & 330 UG/KG & 1 \\
\hline 4-Methylphenol & 330 UG/KG & $u$ & NP & 330 UG/KG & 1 \\
\hline N-Nitroso-Di-n-dipropylamine & 330 UG/KG & $u$ & NP & 330 UG/KG & 1 \\
\hline Hexachloroethane & 330 UG/KG & $u$ & NP & 330 UG/KG & 1 \\
\hline Nitrobenzene & 330 UG/KG & $u$ & NP & 330 UG/KG & 1 \\
\hline Isophorone & 330 UG/KG & $u$ & NP & 330 UG/KG & 1 \\
\hline 2-Nitrophenol & 330 UG/KG & $u$ & NP & 330 UG/KG & 1 \\
\hline 2,4-Dimethylphenol & 330 UG/KG & $u$ & NP & 330 UG/KG & 1 \\
\hline Benzoic Acid & 1600 UG/KG & $u$ & NP & 1600 UG/KG & 1 \\
\hline bis(2Chloroethoxy)methane & 330 UG/KG & $u$ & NP & 330 UG/KG & 1 \\
\hline 2,4-Dichlorophenol & 330 UG/KG & $u$ & NP & 330 UG/KG & 1 \\
\hline 1,2,4-Trichlorobenzene & 330 UG/KG & $u$ & NP & 330 UG/KG & 1 \\
\hline Naphthalene & 330 UG/KG & $u$ & NP & 330 UG/KG & 1 \\
\hline 4-Chloroaniline & 330 UG/KG & $u$ & NP & 330 UG/KG & 1 \\
\hline Hexachlorobutadiene & 330 UG/KG & $u$ & NP & 330 UG/KG & 1 \\
\hline 4-Chloro-3-Methlyphenol & 330 UG/KG & $u$ & NP & 330 UG/KG & 1 \\
\hline 2-Methylnaphthalene & 330 UG/KG & $u$ & NP & 330 UG/KG & 1 \\
\hline Hexachlorocyclopentadiene & 330 UG/KG & $u$ & NP & 330 UG/KG & 1 \\
\hline 2,4,6-Trichlorophenol & 330 UG/KG & $u$ & NP & 330 UG/KG & 1 \\
\hline 2,4,5-Trichlorophenol & 1600 UG/KG & $u$ & NP & 1600 UG/KG & 1 \\
\hline 2-Chloronaphthalene & 330 UG/KG & $u$ & NP & 330 UG/KG & 1 \\
\hline 2-Nitroaniline & 1600 UG/KG & $u$ & NP & 1600 UG/KG & 1 \\
\hline Dimethyl Phthalate & 330 UG/KG & $u$ & NP & 330 UG/KG & 1 \\
\hline Acenaphthylene & 330 UG/KG & $u$ & NP & 330 UG/KG & 1 \\
\hline 2,6-Dinitrotoluene & 330 UG/KG & $u$ & NP & 330 UG/KG & 1 \\
\hline 3-Nitroaniline & 1600 UG/KG & $u$ & NP & 1600 UG/KG & 1 \\
\hline Acenaphthene & 330 UG/KG & $u$ & NP & 330 UG/KG & 1 \\
\hline 2,4-Dinitrophenol & 1600 UG/KG & $u$ & NP & 1600 UG/KG & 1 \\
\hline 4-Nitrophenol & 1600 UG/KG & $u$ & NP & 1600 UG/KG & 1 \\
\hline Dibenzofuran & 330 UG/KG & $u$ & NP & 330 UG/KG & 1 \\
\hline 2,4-Dinitrotoluene & 330 UG/KG & $u$ & NP & 330 UG/KG & 1 \\
\hline Diethylphthalate & 330 UG/KG & $u$ & NP & 330 UG/KG & 1 \\
\hline 4-Chlorophenyl phenyl ether & 330 UG/KG & $u$ & NP & 330 UG/KG & 1 \\
\hline Fluorene & 330 UG/KG & $u$ & NP & 330 UG/KG & 1 \\
\hline 4-Nitroaniline & 1600 UG/KG & $u$ & NP & 1600 UG/KG & 1 \\
\hline 4,6-Dinitro-2-methylphenol & 1600 UG/KG & $u$ & NP & 1600 UG/KG & 1 \\
\hline N-Nitrosodiphenylamine & 330 UG/KG & $u$ & NP & 330 UG/KG & 1 \\
\hline
\end{tabular}


APPENDIX D.1 Results of Semivolatile Organic Compound Analyses, Soils, Salmon Site

\begin{tabular}{|c|c|c|c|c|c|c|}
\hline COMPOUND & RESULTS & $\begin{array}{l}\text { DATA } \\
\text { QUAL. }\end{array}$ & $\begin{array}{l}\text { DATA } \\
\text { VALID. }\end{array}$ & CRDL & UNITS & DILUTION \\
\hline \multicolumn{7}{|l|}{ C5-1-S (continued) } \\
\hline 4-Bromophenyl phenylether & 330 UG/KG & $u$ & NP & 330 & UG/KG & 1 \\
\hline Hexachlorobenzene & 330 UG/KG & $u$ & NP & 330 & UG/KG & 1 \\
\hline Pentachlorophenol & 1600 UG/KG & $u$ & NP & 1600 & UG/KG & 1 \\
\hline Phenanthrene & 330 UG/KG & $u$ & NP & 330 & UG/KG & 1 \\
\hline Anthracene & 330 UG/KG & $u$ & NP & 330 & UG/KG & 1 \\
\hline Di-N-Butylphthalate & 330 UG/KG & $u$ & NP & 330 & $\mathrm{UG} / \mathrm{KG}$ & 1 \\
\hline Fluoranthene & 330 UG/KG & $u$ & NP & 330 & UG/KG & 1 \\
\hline Pyrene & 330 UG/KG & $u$ & NP & 330 & UG/KG & 1 \\
\hline Butyl Benzyl Phthalate & 330 UG/KG & $u$ & NP & 330 & UG/KG & 1 \\
\hline 3.3'-Dichlorobenzidine & 660 UG/KG & $u$ & NP & 660 & UGKG & 1 \\
\hline Benzo(a)Anthracene & 330 UG/KG & $u$ & NP & 330 & UG/KG & 1 \\
\hline Chrysene & 330 UG/KG & $u$ & $\mathrm{NP}$ & 330 & UG/KG & 1 \\
\hline bis(2-Ethylhexyl)phthalate & 330 UG/KG & $u$ & NP & 330 & UG/KG & 1 \\
\hline di-N-Octyl Phthalate & 330 UG/KG & $u$ & NP & 330 & UG/KG & 1 \\
\hline Benzo(b)Fluoranthene & 330 UG/KG & $u$ & NP & 330 & $U G / K G$ & 1 \\
\hline Benzo(k)Fluoranthene & 330 UG/KG & $u$ & NP & 330 & UG/KG & 1 \\
\hline Benzo(a)Pyrene & 330 UG/KG & $u$ & NP & 330 & UG/KG & 1 \\
\hline Indeno(1,2,3-Cd)pyrene & 330 UG/KG & $u$ & NP & 330 & UG/KG & 1 \\
\hline Dibenzo(a,h)Anthracene & 330 UG/KG & $u$ & NP & 330 & UGKG & 1 \\
\hline Benzo(g,h,i)Perylene & $330 \mathrm{UG} / \mathrm{KG}$ & $u$ & NP & 330 & UG/KG & 1 \\
\hline Aldol Condensation & $1500 \mathrm{UG} / \mathrm{KG}$ & BJ & NP & & UG/KG & 1 \\
\hline Unknown-1 & 76 UG/KG & $J$ & NP & & UG/KG & 1 \\
\hline Unknown-2 & 140 UG/KG & J & NP & & UGKG & 1 \\
\hline Unknown-3 & 87 UG/KG & J & NP & & UG/KG & 1 \\
\hline Unknown-4 & 100 UG/KG & $\mathrm{J}$ & NP & & UGKG & 1 \\
\hline Unknown-5 & 95 UG/KG & J & NP & & UG/KG & 1 \\
\hline Unknown-6 & 71 UG/KG & J & NP & & UG/KG & 1 \\
\hline Unknown-7 & 96 UG/KG & J & NP & & UG/KG & 1 \\
\hline Unknown $\mathrm{C} 18 \mathrm{H} 18$ & 1600 UG/KG & J & NP & & UG/KG & 1 \\
\hline Unknown-8 & 83 UG/KG & $J$ & NP & & UG/KG & 1 \\
\hline Unknown-9 & 150 UG/KG & J & NP & & UG/KG & 1 \\
\hline Unknown-10 & 150 UG/KG & J & NP & & UG/KG & 1 \\
\hline 1-Phenanthrenecarboxylic Acid & 2600 UG/KG & J & NP & & UG/KG & 1 \\
\hline Unknown-11 & 86 UG/KG & J & NP & & UG/KG & 1 \\
\hline Unknown-12 & 83 UG/KG & J & NP & & UG/KG & 1 \\
\hline 1-Phenanthrenecarboxylic Acid & 1700 UG/KG & J & NP & & UG/KG & 1 \\
\hline Unknown-13 & 540 UG/KG & J & NP & & UG/KG & 1 \\
\hline Unknown-14 & 67 UG/KG & J & NP & & UG/KG & 1 \\
\hline Unknown-15 & 73 UG/KG & $\mathrm{J}$ & NP & & UG/KG & 1 \\
\hline Nitrobenzene-d5 & $82 \%$ & & & & $\%$ & 1 \\
\hline 2-Fluorobiphenyl & $72 \%$ & & & & $\%$ & 1 \\
\hline Terphenyl-d14 & $68 \%$ & & & & $\%$ & 1 \\
\hline Phenol-d5 & $89 \%$ & & & & $\%$ & 1 \\
\hline 2-Fluorophenol & $46 \%$ & & & & $\%$ & 1 \\
\hline 2,4,6-Tribromophenol & $69 \%$ & & & & $\%$ & 1 \\
\hline
\end{tabular}


APPENDIX D.1 Results of Semivolatile Organic Compound Analyses, Soils, Salmon Site

\begin{tabular}{|llllll}
\hline COMPOUND & DESULTS & DATA & & \\
\hline
\end{tabular}

\section{C5-2-S}

Phenol

bis(2-Chloroethyl) ether

2-Chlorophenol

1,3-Dichlorobenzene

1,4-Dichlorobenzene

Benzyl Alcohol

1,2-Dichlorobenzene

2-Methyiphenol

bis(2-Chloroisopropyl) ether

4-Methylphenol

N-Nitroso-Di-n-dipropylamine

Hexachloroethane

Nitrobenzene

Isophorone

2-Nitrophenol

2,4-Dimethylphenol

Benzoic Acid

bis(2Chloroethoxy)methane

2,4-Dichlorophenol

1,2,4-Trichlorobenzene

Naphthalene

4-Chloroaniline

Hexachlorobutadiene

4-Chloro-3-Methlyphenol

2-Methylnaphthalene

Hexachlorocyclopentadiene

2,4,6-Trichlorophenol

2,4,5-Trichlorophenol

2-Chloronaphthalene

2-Nitroaniline

Dimethyl Phthalate

Acenaphthylene

2,6-Dinitrotoluene

3-Nitroaniline

Acenaphthene

2,4-Dinitrophenol

4-Nitrophenol

Dibenzofuran

2,4-Dinitrotoluene

Diethylphthalate

4-Chlorophenyl phenyl ether

Fluorene

4-Nitroaniline

4,6-Dinitro-2-methylphenol

$\mathrm{N}$-Nitrosodiphenylamine

330 UG/KG
330 UG/KG
330 UG/KG
330 UG/KG
330 UG/KG
330 UG/KG
330 UG/KG
330 UG/KG
330 UG/KG
330 UG/KG
330 UG/KG
330 UG/KG
330 UG/KG
330 UG/KG
330 UG/KG
330 UG/KG
1600 UG/KG
330 UG/KG
330 UG/KG
330 UG/KG
330 UG/KG
330 UG/KG
330 UG/KG
330 UG/KG
330 UG/KG
330 UG/KG
330 UG/KG
1600 UG/KG
330 UG/KG
1600 UG/KG
330 UG/KG
330 UG/KG
330 UG/KG
1600 UG/KG
330 UG/KG
1600 UG/KG
1600 UG/KG
330 UG/KG
330 UG/KG
330 UG/KG
330 UG/KG
330 UG/KG
1600 UG/KG

$\begin{array}{lll}U & N P & 330 \text { UG/KG } \\ U & N P & 330 \text { UG/KG } \\ U & \text { NP } & 330 \text { UG/KG }\end{array}$

$U \quad N P$

$U \quad N P$

$U \quad N P$

U NP

$U \quad N P$

U NP

$U \quad N P$

$U \quad N P$

U NP

$U \quad N P$

$U \quad N P$

U NP

$U \quad N P$

$U \quad N P$

$U \quad N P$

$U \quad N P$

$U \quad N P$

U NP

U NP

$U \quad N P$

$U \quad N P$

U NP

$U \quad N P$

$U \quad$ NP

$U \quad$ NP

$U \quad$ NP

$U \quad N P$

$U \quad$ NP

U NP

$U \quad N P$

U NP

U NP

$U \quad N P$

$U \quad N P$

$U$ NP

$U$ NP

$U \quad N P$

$U$ NP

$U$ NP

U NP

$U \quad N P$

$U \quad$ NP

33C UG/KG 1

330 UG/KG 1

330 UG/KG 1

$330 \mathrm{UG} / K \mathrm{G}$

330 UG/KG 1

$330 \mathrm{UG} / \mathrm{KG} \quad 1$

330 UG/KG 1

330 UG/KG 1

330 UG/KG 1

330 UG/KG 1

330 UG/KG 1

330 UG/KG 1

330 UG/KG

1600 UG/KG

330 UG/KG

330 UG/KG

330 UG/KG 1

330 UG/KG 1

330 UG/KG 1

330 UG/KG 1

330 UG/KG 1

330 UG/KG 1

330 UG/KG 1

330 UG/KG

1600 UG/KG

330 UG/KG

1600 UG/KG 1

330 UG/KG 1

330 UG/KG 1

330 UG/KG 1

1600 UG/KG 1

330 UG/KG

1600 UG/KG 1

1600 UG/KG 1

330 UG/KG 1

330 UG/KG 1

330 UG/KG 1

330 UG/KG 1

330 UG/KG 1

1600 UG/KG 1

1600 UG/KG

330 UG/KG

19


APPENDIX D.1 Results of Semivolatile Organic Compound Analyses, Soils, Salmon Site

\begin{tabular}{|c|c|c|c|c|c|c|}
\hline COMPOUND & RESULTS & $\begin{array}{l}\text { DATA } \\
\text { QUAL. }\end{array}$ & $\begin{array}{l}\text { DATA } \\
\text { VALID. }\end{array}$ & CRDL & UNITS & DILUTIC \\
\hline \multicolumn{7}{|l|}{ C5-2-S (continued) } \\
\hline 4-Bromophenyl phenyiether & 330 UG/KG & $u$ & NP & 330 & UG/KG & 1 \\
\hline Hexachlorobenzene & 330 UG/KG & $u$ & NP & 330 & $\mathrm{UG} / \mathrm{KG}$ & 1 \\
\hline Pentachlorophenol & 1600 UG/KG & $u$ & NP & 1600 & UG/KG & 1 \\
\hline Phenanthrene & 330 UG/KG & $u$ & NP & 330 & UG/KG & 1 \\
\hline Anthracene & 330 UG/KG & $u$ & NP & 330 & $\mathrm{UG} / \mathrm{KG}$ & 1 \\
\hline Di-N-Butylphthalate & 330 UG/KG & $u$ & NP & 330 & UG/KG & 1 \\
\hline Fiuoranthene & 330 UG/KG & $u$ & NP & 330 & $\mathrm{UG} / \mathrm{KG}$ & 1 \\
\hline Pyrene & 330 UG/KG & $u$ & NP & 330 & $\mathrm{UG} / \mathrm{KG}$ & 1 \\
\hline Butyl Benzyl Phthalate & 330 UG/KG & $u$ & NP & 330 & UG/KG & 1 \\
\hline 3,3'-Dichlorobenzidine & 660 UG/KG & $u$ & NP & 660 & UG/KG & 1 \\
\hline Benzo(a)Anthracene & 330 UG/KG & $u$ & NP & 330 & UG/KG & 1 \\
\hline Chrysene & 330 UG/KG & $u$ & NP & 330 & UG/KG & 1 \\
\hline bis(2-Ethylhexyl)phthalate & 330 UG/KG & $u$ & NP & 330 & UG/KG & 1 \\
\hline di-N-Octyl Phthalate & 330 UG/KG & $u$ & NP & 330 & UG/KG & 1 \\
\hline Benzo(b)Fluoranthene & 330 UG/KG & $u$ & NP & 330 & UG/KG & 1 \\
\hline Benzo(k)Fluoranthene & 330 UG/KG & $u$ & NP & 330 & UG/KG & 1 \\
\hline Benzo(a)Pyrene & 330 UG/KG & $u$ & NP & 330 & UG/KG & 1 \\
\hline Indeno(1,2,3-Cd)pyrene & 330 UG/KG & $u$ & NP & 330 & UG/KG & 1 \\
\hline Dibenzo(a,h)Anthracene & 330 UG/KG & $u$ & NP & 330 & UG/KG & 1 \\
\hline Benzo(g,h,i)Perylene & 330 UG/KG & $u$ & NP & 330 & UG/KG & 1 \\
\hline Aldol Condensation & 1400 UG/KG & BJ & NP & & UG/KG & 1 \\
\hline Unknown-1 & 73 UG/KG & J & NP & & UG/KG & 1 \\
\hline Unknown-2 & 100 UG/KG & BJ & NP & & UG/KG & 1 \\
\hline Nitrobenzene-d5 & $74 \%$ & & & & $\%$ & 1 \\
\hline 2-Fluorobiphenyl & $66 \%$ & & & & $\%$ & 1 \\
\hline Terphenyi-d14 & $58 \%$ & & & & $\%$ & 1 \\
\hline Phenol-d5 & $81 \%$ & & & & $\%$ & 1 \\
\hline 2-Fluorophenol & $46 \%$ & & & & $\%$ & 1 \\
\hline 2,4,6-Tribromophenol & $63 \%$ & & & & $\%$ & 1 \\
\hline
\end{tabular}


APPENDIX D.1 Results of Semivolatile Organic Compound Analyses, Soils, Salmon Site

\begin{tabular}{|c|c|c|c|c|c|c|}
\hline COMPOUND & RESULTS & UNITS & $\begin{array}{l}\text { DATA } \\
\text { QUAL. }\end{array}$ & $\begin{array}{l}\text { DATA } \\
\text { VALID. }\end{array}$ & CRDL UNITS & DILUTION \\
\hline \multicolumn{7}{|l|}{ C5-2-S-MS } \\
\hline Phenol & & $76 \%$ & & NP & $\%$ & 1 \\
\hline 2-Chlorophenol & & $74 \%$ & & NP & $\%$ & 1 \\
\hline 1,4-Dichlorobenzene & & $72 \%$ & & NP & $\%$ & 1 \\
\hline N-Nitroso-Di-n-dipropylamine & & $77 \%$ & & NP & $\%$ & 1 \\
\hline 1,2,4-Trichlorobenzene & & $69 \%$ & & NP & $\%$ & 1 \\
\hline 4-Chloro-3-Methlyphenol & & $83 \%$ & & NP & $\%$ & 1 \\
\hline Acenaphthene & & $62 \%$ & & NP & $\%$ & 1 \\
\hline 4-Nitrophenol & & $75 \%$ & & NP & $\%$ & 1 \\
\hline 2,4-Dinitrotoluene & & $71 \%$ & & NP & $\%$ & 1 \\
\hline Pentachlorophenol & & $85 \%$ & & NP & $\%$ & 1 \\
\hline Pyrene & & $62 \%$ & & NP & $\%$ & 1 \\
\hline Nitrobenzene-d5 & & $79 \%$ & & NP & $\%$ & 1 \\
\hline 2-Fluorobiphenyl & & $73 \%$ & & NP & $\%$ & 1 \\
\hline Terphenyl-d14 & & $64 \%$ & & NP & $\%$ & 1 \\
\hline Phenol-d5 & & $88 \%$ & & NP & $\%$ & 1 \\
\hline 2-Fluorophenol & & $50 \%$ & & NP & $\%$ & 1 \\
\hline 2,4,6-Tribromophenol & & $74 \%$ & & NP & $\%$ & 1 \\
\hline \multicolumn{7}{|l|}{ C5-2-S-MSD } \\
\hline Phenol & & $83 \%$ & & NP & $\%$ & 1 \\
\hline 2-Chlorophenol & & $80 \%$ & & NP & $\%$ & 1 \\
\hline 1,4-Dichlorobenzene & & $79 \%$ & & NP & $\%$ & 1 \\
\hline N-Nitroso-Di-n-dipropylamine & & $84 \%$ & & NP & $\%$ & 1 \\
\hline 1,2,4-Trichiorobenzene & & $75 \%$ & & NP & $\%$ & 1 \\
\hline 4-Chloro-3-Methlyphenol & & $88 \%$ & & NP & $\%$ & 1 \\
\hline Acenaphthene & & $65 \%$ & & NP & $\%$ & 1 \\
\hline 4-Nitrophenol & & $75 \%$ & & NP & $\%$ & 1 \\
\hline 2,4-Dinitrotoluene & & $73 \%$ & & NP & $\%$ & 1 \\
\hline Pentachlorophenol & & $94 \%$ & & NP & $\%$ & 1 \\
\hline Pyrene & & $66 \%$ & & NP & $\%$ & 1 \\
\hline Nitrobenzene-d5 & & $88 \%$ & & NP & $\%$ & 1 \\
\hline 2-Fluorobiphenyl & & $76 \%$ & & NP & $\%$ & 1 \\
\hline Terphenyl-d14 & & $68 \%$ & & NP & $\%$ & 1 \\
\hline Phenol-d5 & & $97 \%$ & & NP & $\%$ & 1 \\
\hline 2-Fluorophenol & & $47 \%$ & & NP & $\%$ & 1 \\
\hline 2,4,6-Tribromophenol & & $76 \%$ & & NP & $\%$ & 1 \\
\hline
\end{tabular}

D.1 - 5 
APPENDIX D.1 Results of Semivolatile Organic Compound Analyses, Soils, Salmon Site

\begin{tabular}{|c|c|c|c|c|c|}
\hline COMPOUND & RESULTS & $\begin{array}{l}\text { DATA } \\
\text { QUAL. }\end{array}$ & $\begin{array}{r}\text { DATA } \\
\text { VALID. }\end{array}$ & CRDL UNITS & DILUTION \\
\hline \multicolumn{6}{|l|}{ C5-3-S } \\
\hline Phenol & 330 UG/KG & $u$ & NP & 330 UG/KG & 1 \\
\hline bis(2-Chloroethyl) ether & 330 UG/KG & $u$ & NP & 330 UG/KG & 1 \\
\hline 2-Chlorophenol & 330 UG/KG & $u$ & NP & 330 UG/KG & 1 \\
\hline 1,3-Dichlorobenzene & 330 UG/KG & $u$ & NP & 330 UG/KG & 1 \\
\hline 1,4-Dichlorobenzene & 330 UG/KG & $u$ & NP & 330 UG/KG & 1 \\
\hline Benzyl Alcohol & 330 UG/KG & $u$ & NP & 330 UG/KG & 1 \\
\hline 1,2-Dichlorobenzene & 330 UG/KG & $u$ & NP & 330 UG/KG & 1 \\
\hline 2-Methylphenol & 330 UG/KG & $u$ & NP & 330 UG/KG & 1 \\
\hline bis(2-Chloroisopropyl) ether & 330 UG/KG & $u$ & NP & 330 UG/KG & 1 \\
\hline 4-Methylphenol & 330 UG/KG & $u$ & NP & 330 UG/KG & 1 \\
\hline N-Nitroso-Di-n-dipropylamine & 330 UG/KG & $u$ & NP & 330 UG/KG & 1 \\
\hline Hexachloroethane & 330 UG/KG & $u$ & NP & 330 UG/KG & 1 \\
\hline Nitrobenzene & 330 UG/KG & $u$ & NP & 330 UG/KG & 1 \\
\hline Isophorone & 330 UG/KG & $u$ & NP & 330 UG/KG & 1 \\
\hline 2-Nitrophenol & 330 UG/KG & $u$ & NP & $330 \mathrm{UG} / \mathrm{KG}$ & 1 \\
\hline 2,4-Dimethylphenol & 330 UG/KG & $u$ & NP & $330 \mathrm{UG} / \mathrm{KG}$ & 1 \\
\hline Benzoic Acid & 1600 UG/KG & $u$ & NP & $1600 \mathrm{UG} / \mathrm{KG}$ & 1 \\
\hline bis(2Chloroethoxy)methane & 330 UG/KG & $u$ & NP & 330 UG/KG & 1 \\
\hline 2,4-Dichlorophenol & 330 UG/KG & $u$ & NP & 330 UG/KG & 1 \\
\hline 1,2,4-Trichlorobenzene & 330 UG/KG & $u$ & NP & 330 UG/KG & 1 \\
\hline Naphthalene & 330 UG/KG & $u$ & NP & 330 UG/KG & 1 \\
\hline 4-Chloroaniline & 330 UG/KG & $u$ & NP & 330 UG/KG & 1 \\
\hline Hexachlorobutadiene & 330 UG/KG & $u$ & NP & 330 UG/KG & 1 \\
\hline 4-Chloro-3-Methlyphenol & 330 UG/KG & $u$ & NP & 330 UG/KG & 1 \\
\hline 2-Methylnaphthalene & 330 UG/KG & $u$ & NP & 330 UG/KG & 1 \\
\hline Hexachiorocyclopentadiene & 330 UG/KG & $u$ & NP & 330 UG/KG & 1 \\
\hline 2,4,6-Trichlorophenol & 330 UG/KG & $u$ & NP & 330 UG/KG & 1 \\
\hline 2,4,5-Trichlorophenol & 1600 UG/KG & $u$ & NP & 1600 UG/KG & 1 \\
\hline 2-Chloronaphthalene & 330 UG/KG & $u$ & NP & 330 UG/KG & 1 \\
\hline 2-Nitroaniline & 1600 UG/KG & $u$ & NP & 1600 UG/KG & 1 \\
\hline Dimethyl Phthalate & 330 UG/KG & $u$ & NP & 330 UG/KG & 1 \\
\hline Acenaphthylene & 330 UG/KG & $u$ & NP & 330 UG/KG & 1 \\
\hline 2,6-Dinitrotoluene & 330 UG/KG & $u$ & NP & 330 UG/KG & 1 \\
\hline 3-Nitroaniline & 1600 UG/KG & $u$ & NP & 1600 UG/KG & 1 \\
\hline Acenaphthene & 330 UG/KG & $u$ & NP & 330 UG/KG & 1 \\
\hline 2,4-Dinitrophenol & 1600 UG/KG & $u$ & NP & 1600 UG/KG & 1 \\
\hline 4-Nitrophenol & 1600 UG/KG & $u$ & NP & 1600 UG/KG & 1 \\
\hline Dibenzofuran & 330 UG/KG & $u$ & NP & 330 UG/KG & 1 \\
\hline 2,4-Dinitrotoluene & $330 \mathrm{UG} / \mathrm{KG}$ & $u$ & NP & 330 UG/KG & 1 \\
\hline Diethylphthalate & 330 UG/KG & $u$ & NP & 330 UG/KG & 1 \\
\hline 4-Chlorophenyl phenyl ether & 330 UG/KG & $u$ & NP & 330 UG/KG & 1 \\
\hline Fluorene & 330 UG/KG & $u$ & NP & 330 UG/KG & 1 \\
\hline 4-Nitroaniline & 1600 UG/KG & $u$ & NP & 1600 UG/KG & 1 \\
\hline 4,6-Dinitro-2-methylphenol & 1600 UG/KG & $u$ & NP & 1600 UG/KG & 1 \\
\hline N-Nitrosodiphenylamine & 330 UG/KG & $u$ & NP & 330 UG/KG & 1 \\
\hline
\end{tabular}


APPENDIX D.1 Results of Semivolatile Organic Compound Analyses, Soils, Salmon Site

\begin{tabular}{|lllllll}
\hline COMPOUND & DESULTS UNA DATA & UNITS & QUAL. VALID. & CRDL UNITS DILUTION \\
\hline
\end{tabular}

C5-3-S (continued)

4-Bromophenyl phenylether

Hexachlorobenzene

Pentachlorophenol

Phenanthrene

Anthracene

Di-N-Butylphthalate

Fluoranthene

Pyrene

Butyl Benzyl Phthalate

3,3'-Dichlorobenzidine

Benzo(a)Anthracene

Chrysene

bis(2-Ethylhexyl)phthalate

di-N-Octyl Phthalate

Benzo(b)Fluoranthene

Benzo(k)Fluoranthene

Benzo(a)Pyrene

Indeno(1,2,3-Cd)pyrene

Dibenzo(a,h)Anthracene

Benzo(g,h,i)Perylene

Aldol Condensation

Unknown-1

Unknown-2

Unknown-3

Nitrobenzene-d5

2-Fluorobiphenyl

Terphenyl-d14

Phenol-d5

2-Fluorophenol

2,4,6-Tribromophenol

330 UG/KG
330 UG/KG
1600 UG/KG
330 UG/KG
330 UG/KG
330 UG/KG
330 UG/KG
330 UG/KG
330 UG/KG
660 UG/KG
330 UG/KG
330 UG/KG
330 UG/KG
330 UG/KG
330 UG/KG
330 UG/KG
330 UG/KG
330 UG/KG
330 UG/KG
330 UG/KG
1200 UG/KG
88 UG/KG
110 UG/KG
90 UG/KG
$76 \%$
$69 \%$
$59 \%$
$75 \%$
$31 \%$
$66 \%$

330 UG/KG

$\begin{array}{llll}U & \text { NP } & 330 \text { UG/KG } & 1\end{array}$

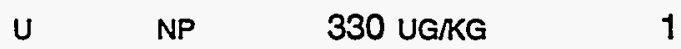

U NP $1600 \mathrm{UG/KG} \quad 1$

U NP $\quad 330$ JGKG

U NP 330 UG/KG 1

$\begin{array}{llll}U & \text { NP } & 330 \mathrm{UG} / \mathrm{KG} & 1\end{array}$

U NP 330 UG/KG 1

$\begin{array}{llll}U & \text { NP } & 330 \text { UG/KG } & 1\end{array}$

$\begin{array}{llll}U & N P & 330 \text { UG/KG } & 1\end{array}$

U NP $\quad 660$ UGKG 1

U NP 330 UG/KG 1

$\begin{array}{llll}U & N P & 330 \mathrm{UG} / K G & 1\end{array}$

U NP 330 UG/KG

$\begin{array}{llll}U & N P & 330 \text { UG/KG } & 1\end{array}$

$\begin{array}{llll}U & N P & 330 \text { UG/KG } & 1\end{array}$

U NP 330 UG/KG 1

U NP 330 UG/KG

$\begin{array}{llll}U & \text { NP } & 330 \text { UG/KG } & 1\end{array}$

$\begin{array}{llll}U & N P & 330 \text { UG/KG } & 1\end{array}$

$\begin{array}{llll}U & N P & 330 \text { UG/KG } & 1\end{array}$

BJ NP

BJ NP

$J \quad N P$

J NP

1
1
1
1
1
1
1
1
1
1
1
1
1
1
1
1
1
1
1
1
1
1
1
1
1
1
1
1
1


APPENDIX D.1 Results of Semivolatile Organic Compound Analyses, Soils, Salmon Site

\begin{tabular}{|c|c|c|c|c|c|}
\hline COMPOUND & RESULTS & $\begin{array}{l}\text { DATA } \\
\text { QUAL. }\end{array}$ & $\begin{array}{l}\text { DATA } \\
\text { VALID. }\end{array}$ & CRDL UNITS & DILUTION \\
\hline \multicolumn{6}{|l|}{ C3-4-S } \\
\hline Phenol & 330 UG/KG & $u$ & NP & $330 \mathrm{UG} / \mathrm{KG}$ & 1 \\
\hline bis(2-Chloroethyl) ether & 330 UG/KG & $u$ & NP & 330 UG/KG & 1 \\
\hline 2-Chlorophenol & 330 UG/KG & $u$ & NP & 330 UG/KG & 1 \\
\hline 1,3-Dichlorobenzene & 330 UG/KG & $u$ & NP & 330 UG/KG & 1 \\
\hline 1,4-Dichlorobenzene & $330 \mathrm{UG} / \mathrm{KG}$ & $u$ & $N P$ & 330 UG/KG & 1 \\
\hline Benzyl Alcohol & 330 UG/KG & $u$ & NP & 330 UG/KG & 1 \\
\hline 1,2-Dichlorobenzene & 330 UG/KG & $u$ & NP & 330 UG/KG & 1 \\
\hline 2-Methylphenol & 330 UG/KG & $u$ & NP & 330 UG/KG & 1 \\
\hline bis(2-Chloroisopropyl) ether & 330 UG/KG & $u$ & NP & 330 UG/KG & 1 \\
\hline 4-Methylphenol & $330 \mathrm{UG} / \mathrm{KG}$ & $u$ & NP & 330 ॥G/KG & 1 \\
\hline N-Nitroso-Di-n-dipropylamine & $330 \mathrm{UG} / \mathrm{KG}$ & $u$ & NP & 330 UG/KG & 1 \\
\hline Hexachloroethane & 330 UG/KG & $u$ & NP & 330 UG/KG & 1 \\
\hline Nitrobenzene & 330 UG/KG & $u$ & NP & 330 UG/KG & 1 \\
\hline Isophorone & 330 UG/KG & $u$ & NP & 330 UG/KG & 1 \\
\hline 2-Nitrophenol & 330 UG/KG & $u$ & NP & 330 UG/KG & 1 \\
\hline 2,4-Dimethylphenol & 330 UG/KG & $u$ & NP & 330 UG/KG & 1 \\
\hline Benzoic Acid & 1600 UG/KG & $u$ & NP & 1600 UG/KG & 1 \\
\hline bis(2Chloroethoxy)methane & $330 \mathrm{UG} / \mathrm{KG}$ & $u$ & NP & 330 UG/KG & 1 \\
\hline 2,4-Dichlorophenol & $330 \mathrm{UG} / \mathrm{KG}$ & $u$ & NP & 330 UG/KG & 1 \\
\hline 1,2,4-Trichlorobenzene & 330 UG/KG & $u$ & NP & 330 UG/KG & 1 \\
\hline Naphthalene & 330 UG/KG & $u$ & NP & 330 UG/KG & 1 \\
\hline 4-Chloroaniline & 330 UG/KG & $u$ & NP & 330 UG/KG & 1 \\
\hline Hexachlorobutadiene & 330 UG/KG & $u$ & NP & 330 UG/KG & 1 \\
\hline 4-Chloro-3-Methlyphenol & 330 UG/KG & $u$ & NP & 330 UG/KG & 1 \\
\hline 2-Methylnaphthalene & 330 UG/KG & $u$ & NP & 330 UG/KG & 1 \\
\hline Hexachlorocyclopentadiene & 330 UG/KG & $u$ & NP & 330 UG/KG & 1 \\
\hline 2,4,6-Trichlorophenol & 330 UG/KG & $u$ & NP & 330 UG/KG & 1 \\
\hline 2,4,5-Trichlorophenol & 1600 UG/KG & $u$ & NP & 1600 UG/KG & 1 \\
\hline 2-Chloronaphthalene & 330 UG/KG & $u$ & NP & 330 UG/KG & 1 \\
\hline 2-Nitroaniline & 1600 UG/KG & $u$ & NP & 1600 UG/KG & 1 \\
\hline Dimethyl Phthalate & 330 UG/KG & $u$ & NP & 330 UG/KG & 1 \\
\hline Acenaphthylene & 330 UG/KG & $u$ & NP & 330 UG/KG & 1 \\
\hline 2,6-Dinitrotoluene & 330 UG/KG & $u$ & NP & $330 \mathrm{UG} / \mathrm{KG}$ & 1 \\
\hline 3-Nitroaniline & 1600 UG/KG & $u$ & NP & 1600 UG/KG & 1 \\
\hline Acenaphthene & 330 UG/KG & $u$ & NP & 330 UG/KG & 1 \\
\hline 2,4-Dinitrophenol & 1600 UG/KG & $u$ & NP & 1600 UG/KG & 1 \\
\hline 4-Nitrophenol & 1600 UG/KG & $u$ & NP & $1600 \mathrm{UG} / \mathrm{KG}$ & 1 \\
\hline Dibenzofuran & 330 UG/KG & $u$ & NP & 330 UG/KG & 1 \\
\hline 2,4-Dinitrotoluene & 330 UG/KG & $u$ & NP & 330 UG/KG & 1 \\
\hline Diethylphthalate & 330 UG/KG & $u$ & NP & 330 UG/KG & 1 \\
\hline 4-Chlorophenyl phenyl ether & 330 UG/KG & $u$ & NP & 330 UG/KG & 1 \\
\hline Fluorene & 330 UG/KG & $u$ & NP & 330 UG/KG & 1 \\
\hline 4-Nitroaniline & 1600 UG/KG & $u$ & NP & 1600 UG/KG & 1 \\
\hline 4,6-Dinitro-2-methylphenol & 1600 UG/KG & $u$ & NP & 1600 UG/KG & 1 \\
\hline N-Nitrosodiphenylamine & 330 UG/KG & $u$ & NP & 330 UG/KG & 1 \\
\hline
\end{tabular}


APPENDIX D.1 Results of Semivolatile Organic Compound Analyses, Soils, Salmon Site

\begin{tabular}{|c|c|c|c|c|c|c|}
\hline COMPOUND & RESULTS & $\begin{array}{l}\text { DATA } \\
\text { QUAL. }\end{array}$ & $\begin{array}{c}\text { DATA } \\
\text { VALID. }\end{array}$ & CRDL & UNITS & DILUTION \\
\hline \multicolumn{7}{|l|}{ C3-4-S (continued) } \\
\hline 4-Bromophenyl phenylether & 330 UG/KG & $u$ & NP & 330 & UG/KG & 1 \\
\hline Hexachlorobenzene & 330 UG/KG & $u$ & NP & 330 & UG/KG & 1 \\
\hline Pentachlorophenol & 1600 UG/KG & $u$ & NP & 1600 & UG/KG & 1 \\
\hline Phenanthrene & 330 UG/KG & $u$ & NP & 330 & UG/KG & 1 \\
\hline Anthracene & 330 UG/KG & $u$ & NP & 330 & UG/KG & 1 \\
\hline Di-N-Butylphthalate & 330 UG/KG & $u$ & NP & 330 & UG/KG & 1 \\
\hline Fluoranthene & 330 UG/KG & $u$ & NP & 330 & UG/KG & 1 \\
\hline Pyrene & 330 UG/KG & $u$ & NP & 330 & UG/KG & 1 \\
\hline Butyl Benzyl Phthalate & 330 UG/KG & $u$ & NP & 330 & UG/KG & 1 \\
\hline 3,3'-Dichlorobenzidine & 660 UG/KG & $u$ & NP & 660 & UG/KG & 1 \\
\hline Benzo(a)Anthracene & 330 UG/KG & $u$ & NP & 330 & UG/KG & 1 \\
\hline Chrysene & 330 UG/KG & $u$ & NP & 330 & UG/KG & 1 \\
\hline bis(2-Ethylhexyl)phthalate & 330 UG/KG & $u$ & NP & 330 & $U G / K G$ & 1 \\
\hline di-N-Octyl Phthalate & 330 UG/KG & $u$ & NP & 330 & UG/KG & 1 \\
\hline Benzo(b)Fluoranthene & 330 UG/KG & $u$ & NP & 330 & UG/KG & 1 \\
\hline Benzo(k)Fluoranthene & 330 UG/KG & $u$ & NP & 330 & UG/KG & 1 \\
\hline Benzo(a)Pyrene & 330 UG/KG & $u$ & NP & 330 & UG/KG & 1 \\
\hline Indeno(1,2,3-Cd)pyrene & 330 UG/KG & $u$ & NP & 330 & UG/KG & 1 \\
\hline Dibenzo(a,h)Anthracene & 330 UG/KG & $u$ & NP & 330 & UG/KG & 1 \\
\hline Benzo(g,h,i)Perylene & 330 UG/KG & $u$ & NP & 330 & UG/KG & 1 \\
\hline Aldol Condensation & $1100 \mathrm{UG} / \mathrm{KG}$ & BS & NP & & UG/KG & 1 \\
\hline Unknown-1 & 99 UG/KG & J & NP & & UG/KG & 1 \\
\hline Unknown-2 & 68 UG/KG & J & NP & & UG/KG & 1 \\
\hline Unknown-3 & $68 \mathrm{UG} / \mathrm{KG}$ & J & NP & & UG/KG & 1 \\
\hline Nitrobenzene-d5 & $71 \%$ & & & & $\%$ & 1 \\
\hline 2-Fluorobiphenyl & $69 \%$ & & & & $\%$ & 1 \\
\hline Terphenyl-d14 & $61 \%$ & & & & $\%$ & 1 \\
\hline Phenol-d5 & $71 \%$ & & & & $\%$ & 1 \\
\hline 2-Fluorophenol & $35 \%$ & & & & $\%$ & 1 \\
\hline 2,4,6-Tribromophenol & $68 \%$ & & & & $\%$ & 1 \\
\hline
\end{tabular}

D. 1 - 9 
APPENDIX D.1 Results of Semivolatile Organic Compound Analyses, Soils, Salmon Site

\begin{tabular}{|llllll}
\hline COMPOUND & DATA DATA & & \\
\hline
\end{tabular}

\section{C3-5-S}

Phenol

bis(2-Chloroethyl) ether

2-Chlorophenol

1,3-Dichlorobenzene

1,4-Dichlorobenzene

Benzyl Alcohol

1,2-Dichlorobenzene

2-Methyiphenol

bis(2-Chloroisopropyl) ether

4-Methylphenol

N-Nitroso-Di-n-dipropylamine

Hexachloroethane

Nitrobenzene

Isophorone

2-Nitrophenol

2,4-Dimethylphenol

Benzoic Acid

bis(2Chloroethoxy)methane

2,4-Dichlorophenol

1,2,4-Trichlorobenzene

Naphthalene

4-Chloroaniline

Hexachlorobutadiene

4-Chloro-3-Methlyphenol

2-Methylnaphthalene

Hexachiorocyclopentadiene

2,4,6-Trichlorophenol

2,4,5-Trichlorophenol

2-Chloronaphthalene

2-Nitroaniline

Dimethyl Phthalate

Acenaphthylene

2,6-Dinitrotoluene

3-Nitroaniline

Acenaphthene

2,4-Dinitrophenol

4-Nitrophenol

Dibenzofuran

2,4-Dinitrotoluene

Diethylphthalate

4-Chlorophenyl phenyl ether

Fluorene

4-Nitroaniline

4,6-Dinitro-2-methylphenol

$\mathrm{N}$-Nitrosodiphenylamine
330 UG/KG

330 UG/KG

330 UG/KG

330 UG/KG

330 UG/KG

330 UG/KG

330 UG/KG

330 UG/KG

330 UG/KG

330 U: $/ K G$

330 UG/KG

330 UG/KG

330 UG/KG

330 UG/KG

330 UG/KG

330 UG/KG

1600 UG/KG

330 UG/KG

330 UG/KG

330 UG/KG

330 UG/KG

330 UG/KG

330 UG/KG

330 UG/KG

330 UG/KG

330 UG/KG

330 UG/KG

1600 UG/KG

330 UG/KG

1600 UG/KG

330 UG/KG

$330 \mathrm{UG} / \mathrm{KG}$

330 UG/KG

1600 UG/KG

330 UG/KG

1600 UG/KG

1600 UG/KG

330 UG/KG

330 UG/KG

330 UG/KG

330 UG/KG

330 UG/KG

1600 UG/KG

1600 UG/KG

330 UG/KG

\begin{tabular}{|c|}
\hline$U$ \\
\hline$u$ \\
\hline U \\
\hline U \\
\hline U \\
\hline$U$ \\
\hline$U$ \\
\hline U \\
\hline$u$ \\
\hline$U$ \\
\hline$U$ \\
\hline u \\
\hline$u$ \\
\hline U \\
\hline U \\
\hline U \\
\hline$u$ \\
\hline$u$ \\
\hline$u$ \\
\hline$u$ \\
\hline$u$ \\
\hline$u$ \\
\hline U \\
\hline$u$ \\
\hline$u$ \\
\hline$u$ \\
\hline$u$ \\
\hline$u$ \\
\hline$u$ \\
\hline$u$ \\
\hline$u$ \\
\hline$u$ \\
\hline$u$ \\
\hline$u$ \\
\hline$u$ \\
\hline$u$ \\
\hline$U$ \\
\hline$u$ \\
\hline$u$ \\
\hline$u$ \\
\hline$u$ \\
\hline$u$ \\
\hline$u$ \\
\hline$u$ \\
\hline$u$ \\
\hline
\end{tabular}

330 UG/KG

330 UG/KG

330 UG/KG

330 UG/KG

330 UG/KG

330 UG/KG

330 UG/KG

330 UG/KG

330 UG/KG

330 UG/KG

330 UG/KG

330 UG/KG

330 UG/KG

330 UG/KG

330 UG/KG

330 UG/KG

1600 UG/KG

330 UG/KG

330 UG/KG

330 UG/KG

330 UG/KG

330 UG/KG

330 UG/KG

330 UG/KG

330 UG/KG

330 UG/KG

330 UG/KG

1600 UG/KG

330 UG/KG

1600 UG/KG

330 UG/KG

330 UG/KG

330 UG/KG

1600 UG/KG

330 UG/KG

1600 UG/KG

1600 UG/KG

330 UG/KG

330 UG/KG

330 UG/KG

330 UG/KG

330 UG/KG

1600 UG/KG

1600 UG/KG

330 UG/KG
1

1

1

1

1

1

1

1


APPENDIX D.1 Results of Semivolatile Organic Compound Analyses, Soils, Salmon Site

\begin{tabular}{|c|c|c|c|c|c|c|}
\hline COMPOUND & RESULTS & $\begin{array}{l}\text { DATA } \\
\text { QUAL. }\end{array}$ & $\begin{array}{l}\text { DATA } \\
\text { VALID. }\end{array}$ & CRDL & UNITS & DILUTION \\
\hline \multicolumn{7}{|l|}{ C3-5-S (continued) } \\
\hline 4-Bromophenyl phenylether & 330 UG/KG & $u$ & NP & 330 & UG/KG & 1 \\
\hline Hexachlorobenzene & 330 UG/KG & $u$ & NP & 330 & UG/KG & 1 \\
\hline Pentachlorophenol & 1600 UG/KG & $u$ & NP & 1600 & UG/KG & 1 \\
\hline Phenanthrene & 330 UG/KG & $u$ & NP & 330 & UG/KG & 1 \\
\hline Anthracene & 330 UG/KG & $u$ & NP & 330 & UG/KG & 1 \\
\hline Di-N-Butylphthalate & 330 UG/KG & $u$ & NP & 330 & UG/KG & 1 \\
\hline Fluoranthene & 330 UG/KG & $u$ & NP & 330 & UG/KG & 1 \\
\hline Pyrene & 330 UG/KG & $u$ & NP & 330 & UG/KG & 1 \\
\hline Butyl Benzyl Phthalate & 330 UG/KG & $u$ & NP & 330 & UG/KG & 1 \\
\hline 3,3'-Dichlorobenzidine & 660 UG/KG & $u$ & NP & 660 & UG/KG & 1 \\
\hline Benzo(a)Anthracene & 330 UG/KG & $u$ & NP & 330 & UG/KG & 1 \\
\hline Chrysene & 330 UG/KG & $u$ & NP & 330 & UG/KG & 1 \\
\hline bis(2-Ethylhexyl)phthalate & 330 UG/KG & $u$ & NP & 330 & UG/KG & 1 \\
\hline di-N-Octyl Phthalate & 330 UG/KG & $u$ & NP & 330 & UG/KG & 1 \\
\hline Benzo(b)Fluoranthene & 330 UG/KG & $u$ & NP & 330 & UG/KG & 1 \\
\hline Benzo(k)Fluoranthene & 330 UG/KG & $u$ & NP & 330 & UG/KG & 1 \\
\hline Benzo(a)Pyrene & 330 UG/KG & $u$ & NP & 330 & UG/KG & 1 \\
\hline Indeno(1,2,3-Cd)pyrene & 330 UG/KG & $u$ & NP & 330 & UG/KG & 1 \\
\hline Dibenzo(a,h)Anthracene & 330 UG/KG & $u$ & NP & 330 & UG/KG & 1 \\
\hline Benzo(g,h,i)Perylene & 330 UG/KG & $u$ & NP & 330 & UG/KG & 1 \\
\hline Aldol Condensation & 1100 UG/KG & BJ & NP & & UG/KG & 1 \\
\hline Unknown-1 & 79 UG/KG & $J$ & NP & & UG/KG & 1 \\
\hline Unknown-2 & 75 UG/KG & J & NP & & UG/KG & 1 \\
\hline Nitrobenzene-d5 & $64 \%$ & & & & $\%$ & 1 \\
\hline 2-Fluorobiphenyl & $60 \%$ & & & & $\%$ & 1 \\
\hline Terphenyl-d14 & $56 \%$ & & & & $\%$ & 1 \\
\hline Phenol-d5 & $63 \%$ & & & & $\%$ & 1 \\
\hline 2-Fluorophenol & $36 \%$ & & & & $\%$ & 1 \\
\hline 2,4,6-Tribromophenol & $64 \%$ & & & & $\%$ & 1 \\
\hline
\end{tabular}


APPENDIX D.1 Results of Semivolatile Organic Compound Analyses, Soils, Salmon Site

\begin{tabular}{|c|c|c|c|c|c|}
\hline COMPOUND & RESULTS & $\begin{array}{l}\text { DATA } \\
\text { QUAL. }\end{array}$ & $\begin{array}{l}\text { DATA } \\
\text { VALID. }\end{array}$ & CRDL UNITS & DILUTION \\
\hline \multicolumn{6}{|l|}{ C3-6-S } \\
\hline Phenol & 330 UG/KG & $u$ & NP & $330 \mathrm{UG} / \mathrm{KG}$ & 1 \\
\hline bis(2-Chloroethyl) ether & 330 UG/KG & $u$ & NP & $330 \mathrm{UG} / \mathrm{KG}$ & 1 \\
\hline 2-Chlorophenol & 330 UG/KG & $u$ & NP & 330 UG/KG & 1 \\
\hline 1,3-Dichlorobenzene & 330 UG/KG & $u$ & NP & 330 UG/KG & 1 \\
\hline 1,4-Dichlorobenzene & $330 \mathrm{UG} / \mathrm{KG}$ & $u$ & NP & 330 UG/KG & 1 \\
\hline Benzyl Alcohol & 330 UG/KG & $u$ & NP & 330 UG/KG & 1 \\
\hline 1,2-Dichlorobenzene & 330 UG/KG & $u$ & NP & 330 UG/KG & 1 \\
\hline 2-Methylphenol & 330 UG/KG & $u$ & NP & 330 UG/KG & 1 \\
\hline bis(2-Chloroisopropyl) ether & 330 UG/KG & $u$ & NP & 330 UG/KG & 1 \\
\hline 4-Methylphenol & 330 ỤG/KG & $u$ & NP & 330 UG/KG & 1 \\
\hline N-Nitroso-Di-n-dipropylamine & 330 UG/KG & $u$ & NP & 330 UG/KG & 1 \\
\hline Hexachloroethane & 330 UG/KG & $u$ & NP & 330 UG/KG & 1 \\
\hline Nitrobenzene & 330 UG/KG & $u$ & NP & 330 UG/KG & 1 \\
\hline Isophorone & 330 UG/KG & $u$ & NP & 330 UG/KG & 1 \\
\hline 2-Nitrophenol & 330 UG/KG & $u$ & NP & 330 UG/KG & 1 \\
\hline 2,4-Dimethylphenol & 330 UG/KG & $u$ & NP & 330 UG/KG & 1 \\
\hline Benzoic Acid & 1600 UG/KG & $u$ & NP & 1600 UG/KG & 1 \\
\hline bis(2Chloroethoxy)methane & 330 UG/KG & $u$ & NP & 330 UG/KG & 1 \\
\hline 2,4-Dichlorophenol & 330 UG/KG & $u$ & NP & 330 UG/KG & 1 \\
\hline 1,2,4-Trichlorobenzene & $330 \mathrm{UG} / \mathrm{KG}$ & $u$ & NP & 330 UG/KG & 1 \\
\hline Naphthalene & 330 UG/KG & $u$ & NP & 330 UG/KG & 1 \\
\hline 4-Chloroaniline & 330 UG/KG & $u$ & NP & 330 UG/KG & 1 \\
\hline Hexachlorobutadiene & 330 UG/KG & $u$ & NP & 330 UG/KG & 1 \\
\hline 4-Chloro-3-Methlyphenol & 330 UG/KG & $u$ & NP & 330 UG/KG & 1 \\
\hline 2-Methylnaphthalene & 330 UG/KG & $u$ & NP & 330 UG/KG & 1 \\
\hline Hexachlorocyclopentadiene & 330 UG/KG & $u$ & NP & 330 UG/KG & 1 \\
\hline 2,4,6-Trichiorophenol & 330 UG/KG & $u$ & NP & 330 UG/KG & 1 \\
\hline 2,4,5-Trichlorophenol & 1600 UG/KG & $u$ & NP & 1600 UG/KG & 1 \\
\hline 2-Chloronaphthalene & 330 UG/KG & $u$ & NP & 330 UG/KG & 1 \\
\hline 2-Nitroaniline & 1600 UG/KG & $u$ & NP & 1600 UG/KG & 1 \\
\hline Dimethyl Phthalate & 330 UG/KG & $u$ & NP & 330 UG/KG & 1 \\
\hline Acenaphthylene & 330 UG/KG & $u$ & NP & 330 UG/KG & 1 \\
\hline 2,6-Dinitrotoluene & 330 UG/KG & $u$ & NP & 330 UG/KG & 1 \\
\hline 3-Nitroaniline & 1600 UG/KG & $u$ & NP & $1600 \mathrm{UG} / \mathrm{KG}$ & 1 \\
\hline Acenaphthene & 330 UG/KG & $u$ & NP & 330 UG/KG & 1 \\
\hline 2,4-Dinitrophenol & 1600 UG/KG & $u$ & NP & 1600 UG/KG & 1 \\
\hline 4-Nitrophenol & 1600 UG/KG & $u$ & NP & 1600 UG/KG & 1 \\
\hline Dibenzofuran & 330 UG/KG & $u$ & NP & 330 UG/KG & 1 \\
\hline 2,4-Dinitrotoluene & 330 UG/KG & $u$ & NP & 330 UG/KG & 1 \\
\hline Diethylphthalate & 330 UG/KG & $u$ & NP & 330 UG/KG & 1 \\
\hline 4-Chlorophenyl phenyl ether & 330 UG/KG & $u$ & NP & 330 UG/KG & 1 \\
\hline Fluorene & 330 UG/KG & $u$ & NP & 330 UG/KG & 1 \\
\hline 4-Nitroaniline & 1600 UG/KG & $u$ & NP & 1600 UG/KG & 1 \\
\hline 4,6-Dinitro-2-methylphenol & 1600 UG/KG & $u$ & NP & 1600 UG/KG & 1 \\
\hline N-Nitrosodiphenylamine & 330 UG/KG & $u$ & NP & 330 UG/KG & 1 \\
\hline
\end{tabular}


APPENDIX D.1 Results of Semivolatile Organic Compound Analyses, Soils, Salmon Site

\begin{tabular}{|c|c|c|c|c|c|c|}
\hline COMPOUND & RESULTS & $\begin{array}{l}\text { DATA } \\
\text { QUAL. }\end{array}$ & $\begin{array}{l}\text { DATA } \\
\text { VALID. }\end{array}$ & CRDL & UNITS & DILUTION \\
\hline \multicolumn{7}{|l|}{ C3-6-S (continued) } \\
\hline 4-Bromophenyl phenylether & 330 UG/KG & $u$ & NP & 330 & UG/KG & 1 \\
\hline Hexachlorobenzene & 330 UG/KG & $u$ & NP & 330 & UG/KG & 1 \\
\hline Pentachlorophenol & 1600 UG/KG & $u$ & NP & 1600 & UG/KG & 1 \\
\hline Phenanthrene & 330 UG/KG & $u$ & NP & 330 & UGKG & 1 \\
\hline Anthracene & 330 UG/KG & $u$ & NP & 330 & UG/KG & 1 \\
\hline Di-N-Butylphthalate & 330 UG/KG & $u$ & NP & 330 & UG/KG & 1 \\
\hline Fluoranthene & 330 UG/KG & $u$ & NP & 330 & UG/KG & 1 \\
\hline Pyrene & 330 UG/KG & $u$ & NP & 330 & UG/KG & 1 \\
\hline Butyl Benzyl Phthalate & 330 UG/KG & $u$ & NP & 330 & UG/KG & 1 \\
\hline 3,3'-Dichlorobenzidine & $660 \mathrm{IJG/KG}$ & $u$ & NP & 660 & UG/KG & 1 \\
\hline Benzo(a)Anthracene & 330 UG/KG & $u$ & NP & 330 & UG/KG & 1 \\
\hline Chrysene & 330 UG/KG & $u$ & NP & 330 & UG/KG & 1 \\
\hline bis(2-Ethylhexyl)phthalate & 330 UG/KG & $u$ & NP & 330 & UG/KG & 1 \\
\hline di-N-Octyl Phthalate & 330 UG/KG & $u$ & NP & 330 & UG/KG & 1 \\
\hline Benzo(b)Fluoranthene & 330 UG/KG & $u$ & NP & 330 & UG/KG & 1 \\
\hline Benzo(k)Fluoranthene & 330 UG/KG & $u$ & NP & 330 & UG/KG & 1 \\
\hline Benzo(a)Pyrene & 330 UG/KG & $u$ & NP & 330 & UG/KG & 1 \\
\hline Indeno(1,2,3-Cd)pyrene & 330 UG/KG & $u$ & NP & 330 & UG/KG & 1 \\
\hline Dibenzo(a,h)Anthracene & 330 UG/KG & $u$ & NP & 330 & UG/KG & 1 \\
\hline Benzo(g,h,i)Perylene & 330 UG/KG & $u$ & NP & 330 & UG/KG & 1 \\
\hline Aldol Condensation & 1600 UG/KG & BJ & NP & & UG/KG & 1 \\
\hline Unknown-1 & 120 UG/KG & BJ & NP & & UG/KG & 1 \\
\hline Unknown-2 & 78 UG/KG & BJ & NP & & UG/KG & 1 \\
\hline Unknown-3 & 76 UG/KG & $J$ & NP & & UG/KG & 1 \\
\hline Nitrobenzene-d5 & $79 \%$ & & & & $\%$ & 1 \\
\hline 2-Fluorobiphenyl & $69 \%$ & & & & $\%$ & 1 \\
\hline Terphenyl-d14 & $61 \%$ & & & & $\%$ & 1 \\
\hline Phenol-d5 & $82 \%$ & & & & $\%$ & 1 \\
\hline 2-Fluorophenol & $44 \%$ & & & & $\%$ & 1 \\
\hline 2,4,6-Tribromophenol & $60 \%$ & & & & $\%$ & 1 \\
\hline
\end{tabular}


APPENDIX D.1 Results of Semivolatile Organic Compound Analyses, Soils, Salmon Site

\begin{tabular}{|c|c|c|c|c|c|}
\hline COMPOUND & RESULTS & $\begin{array}{l}\text { DATA } \\
\text { QUAL. }\end{array}$ & $\begin{array}{l}\text { DATA } \\
\text { VALID. } \\
\end{array}$ & CRDL UNITS & DILUTION \\
\hline \multicolumn{6}{|l|}{ C3-7-S } \\
\hline Phenol & 330 UG/KG & $u$ & NP & 330 UG/KG & 1 \\
\hline bis(2-Chloroethyl) ether & 330 UG/KG & $u$ & NP & 330 UG/KG & 1 \\
\hline 2-Chlorophenol & 330 UG/KG & $u$ & NP & 330 UG/KG & 1 \\
\hline 1,3-Dichlorobenzene & 330 UG/KG & $u$ & NP & 330 UG/KG & 1 \\
\hline 1,4-Dichlorobenzene & 330 UG/KG & $u$ & NP & 330 UG/KG & 1 \\
\hline Benzyl Alcohol & 330 UG/KG & $u$ & NP & 330 UG/KG & 1 \\
\hline 1,2-Dichlorobenzene & 330 UG/KG & $u$ & NP & 330 UG/KG & 1 \\
\hline 2-Methylphenol & 330 UG/KG & $u$ & NP & 330 UG/KG & 1 \\
\hline bis(2-Chloroisopropyl) ether & 330 UG/KG & $u$ & NP & 330 UG/KG & 1 \\
\hline 4-Methylphenol & 330 UG/KG & $u$ & NP & 330 UG/KG & 1 \\
\hline N-Nitroso-Di-n-dipropylamine & 330 UG/KG & $u$ & NP & 330 UG/KG & 1 \\
\hline Hexachloroethane & 330 UG/KG & $u$ & NP & 330 UG/KG & 1 \\
\hline Nitrobenzene & 330 UG/KG & $u$ & NP & 330 UG/KG & 1 \\
\hline Isophorone & 330 UG/KG & $u$ & NP & 330 UG/KG & 1 \\
\hline 2-Nitrophenol & 330 UG/KG & $u$ & NP & 330 UG/KG & 1 \\
\hline 2,4-Dimethylphenol & 330 UG/KG & $u$ & NP & 330 UG/KG & 1 \\
\hline Benzoic Acid & 1600 UG/KG & $u$ & NP & 1600 UG/KG & 1 \\
\hline bis(2Chloroethoxy)methane & 330 UG/KG & $u$ & NP & 330 UG/KG & 1 \\
\hline 2,4-Dichlorophenol & 330 UG/KG & $u$ & NP & 330 UG/KG & 1 \\
\hline 1,2,4-Trichlorobenzene & 330 UG/KG & $u$ & NP & 330 UG/KG & 1 \\
\hline Naphthalene & 330 UG/KG & $u$ & NP & 330 UG/KG & 1 \\
\hline 4-Chloroaniline & 330 UG/KG & $u$ & NP & 330 UG/KG & 1 \\
\hline Hexachlorobutadiene & 330 UG/KG & $u$ & NP & 330 UG/KG & 1 \\
\hline 4-Chloro-3-Methlyphenol & 330 UG/KG & $u$ & NP & 330 UG/KG & 1 \\
\hline 2-Methylnaphthalene & 330 UG/KG & $u$ & NP & 330 UG/KG & 1 \\
\hline Hexachlorocyclopentadiene & 330 UG/KG & $u$ & NP & 330 UG/KG & 1 \\
\hline 2,4,6-Trichlorophenol & 330 UG/KG & $u$ & NP & 330 UG/KG & 1 \\
\hline 2,4,5-Trichlorophenol & 1600 UG/KG & $u$ & NP & 1600 UG/KG & 1 \\
\hline 2-Chloronaphthalene & 330 UG/KG & $u$ & NP & 330 UG/KG & 1 \\
\hline 2-Nitroaniline & 1600 UG/KG & $u$ & NP & 1600 UG/KG & 1 \\
\hline Dimethyl Phthalate & 330 UG/KG & $u$ & NP & 330 UG/KG & 1 \\
\hline Acenaphthylene & 330 UG/KG & $u$ & NP & 330 UG/KG & 1 \\
\hline 2,6-Dinitrotoluene & 330 UG/KG & $u$ & NP & 330 UG/KG & 1 \\
\hline 3-Nitroaniline & 1600 UG/KG & $u$ & NP & 1600 UG/KG & 1 \\
\hline Acenaphthene & 330 UG/KG & $u$ & NP & 330 UG/KG & 1 \\
\hline 2,4-Dinitrophenol & 1600 UG/KG & $u$ & NP & 1600 UG/KG & 1 \\
\hline 4-Nitrophenol & 1600 UG/KG & $u$ & NP & 1600 UG/KG & 1 \\
\hline Dibenzofuran & 330 UG/KG & $u$ & NP & 330 UG/KG & 1 \\
\hline 2,4-Dinitrotoluene & 330 UG/KG & $u$ & NP & 330 UG/KG & 1 \\
\hline Diethylphthaiate & 330 UG/KG & $u$ & NP & 330 UG/KG & 1 \\
\hline 4-Chlorophenyl phenyl ether & 330 UG/KG & $u$ & NP & 330 UG/KG & 1 \\
\hline Fluorene & 330 UG/KG & $u$ & NP & 330 UG/KG & 1 \\
\hline 4-Nitroaniline & 1600 UG/KG & $u$ & NP & 1600 UG/KG & 1 \\
\hline 4,6-Dinitro-2-methylphenol & 1600 UG/KG & $u$ & NP & 1600 UG/KG & 1 \\
\hline N-Nitrosodiphenylamine & 330 UG/KG & $u$ & NP & 330 UG/KG & 1 \\
\hline
\end{tabular}


APPENDIX D.1 Results of Semivolatile Organic Compound Analyses, Soils, Salmon Site

\begin{tabular}{|lllllll}
\hline COMPOUND & DESULTS UNA & DATA & & \\
\hline
\end{tabular}

\section{C3-7-S (continued)}

4-Bromophenyl phenylether

Hexachlorobenzene

Pentachlorophenol

Phenanthrene

Anthracene

Di-N-Butylphthalate

Fluoranthene

Pyrene

Butyl Benzyl Phthalate

3,3'-Dichlorobenzidine

Benzo(a)Anthracene

Chrysene

bis(2-Ethylhexyl)phthalate

di-N-Octyl Phthalate

Benzo(b)Fluoranthene

Benzo(k)Fluoranthene

Benzo(a)Pyrene

Indeno(1,2,3-Cd)pyrene

Dibenzo(a,h)Anthracene

Benzo(g,h,i)Perylene

Aldol Condensation

Unknown-1

Unknown-2

Nitrobenzene-d5

2-Fluorobiphenyl

Terphenyl-d14

Phenol-d5

2-Fluorophenol

2,4,6-Tribromophenol

330 UG/KG
330 UG/KG
1600 UG/KG
330 UG/KG
330 UG/KG
330 UG/KG
330 UG/KG
330 UG/KG
330 UG/KG
660 UG/KG
330 UG/KG
330 UG/KG
330 UG/KG
330 UG/KG
330 UG/KG
330 UG/KG
330 UG/KG
330 UG/KG
330 UG/KG
330 UG/KG
1600 UG/KG
110 UG/KG
76 UG/KG
$78 \%$
$69 \%$
$63 \%$
$80 \%$
$45 \%$
$64 \%$

$\mathrm{NP}$
$\mathrm{NP}$
$\mathrm{NP}$
$\mathrm{NP}$
$\mathrm{NP}$
$\mathrm{NP}$
$\mathrm{NP}$
$\mathrm{NP}$
$\mathrm{NP}$
$\mathrm{NP}$
$\mathrm{NP}$
$\mathrm{NP}$
$\mathrm{NP}$
$\mathrm{NP}$
$\mathrm{NP}$
$\mathrm{NP}$
$\mathrm{NP}$
$\mathrm{NP}$
$\mathrm{NP}$
$\mathrm{NP}$
$\mathrm{NP}$
$\mathrm{NP}$
$\mathrm{NP}$

$\begin{array}{cc}330 \text { UG/KG } & 1 \\ 330 \text { UG/KG } & 1 \\ \text { 1600 UG/KG } & 1 \\ 330 \text { iJG/KG } & 1 \\ 330 \text { UG/KG } & 1 \\ 330 \text { UG/KG } & 1 \\ 330 \text { UG/KG } & 1 \\ 330 \text { UG/KG } & 1 \\ 330 \text { UG/KG } & 1 \\ 660 \text { UG/KG } & 1 \\ 330 \text { UG/KG } & 1 \\ 330 \text { UG/KG } & 1 \\ 330 \text { UG/KG } & 1 \\ 330 \text { UG/KG } & 1 \\ 330 \text { UG/KG } & 1 \\ 330 \text { UG/KG } & 1 \\ 330 \text { UG/KG } & 1 \\ 330 \text { UG/KG } & 1 \\ 330 \text { UG/KG } & 1 \\ 330 \text { UG/KG } & 1 \\ \text { UG/KG } & 1 \\ \text { UG/KG } & 1 \\ \text { UG/KG } & 1 \\ \% & 1 \\ \% & 1 \\ \% & 1 \\ \% & 1 \\ \% & 1 \\ \% & 1\end{array}$

D. 1 - 15 
APPENDIX D.1 Results of Semivolatile Organic Compound Analyses, Soils, Salmon Site

\begin{tabular}{|c|c|c|c|c|c|}
\hline COMPOUND & RESULTS & $\begin{array}{l}\text { DATA } \\
\text { QUAL. }\end{array}$ & $\begin{array}{l}\text { DATA } \\
\text { VALID. }\end{array}$ & CRDL UNITS & DILUTION \\
\hline \multicolumn{6}{|l|}{ C3-8-S } \\
\hline Phenol & 330 UG/KG & $u$ & NP & 330 UG/KG & 1 \\
\hline bis(2-Chloroethyl) ether & 330 UG/KG & $u$ & NP & 330 UG/KG & 1 \\
\hline 2-Chlorophenol & 330 UG/KG & $u$ & NP & 330 UG/KG & 1 \\
\hline 1,3-Dichlorobenzene & 330 UG/KG & $u$ & NP & $330 \mathrm{UG} / \mathrm{KG}$ & 1 \\
\hline 1,4-Dichlorobenzene & 330 UG/KG & $u$ & NP & 330 UG/KG & 1 \\
\hline Benzy! Alcohol & 330 UG/KG & $u$ & NP & 330 UG/KG & 1 \\
\hline 1,2-Dichlorobenzene & 330 UG/KG & $u$ & NP & 330 UG/KG & 1 \\
\hline 2-Methylphenol & 330 UG/KG & $u$ & NP & 330 UG/KG & 1 \\
\hline bis(2-Chloroisopropyi) ether & 330 UG/KG & $u$ & NP & 330 UG/KG & 1 \\
\hline 4-Methylphenol & 330 UG/KG & $u$ & NP & 330 UG/KG & 1 \\
\hline N-Nitroso-Di-n-dipropylamine & $330 \mathrm{UG} / \mathrm{KG}$ & $u$ & NP & 330 UG/KG & 1 \\
\hline Hexachloroethane & 330 UG/KG & $u$ & NP & 330 UG/KG & 1 \\
\hline Nitrobenzene & 330 UG/KG & $u$ & NP & 330 UG/KG & 1 \\
\hline Isophorone & 330 UG/KG & $u$ & NP & 330 UG/KG & 1 \\
\hline 2-Nitrophenol & 330 UG/KG & $u$ & NP & 330 UG/KG & 1 \\
\hline 2,4-Dimethylphenol & 330 UG/KG & $u$ & NP & 330 UG/KG & 1 \\
\hline Benzoic Acid & 1600 UG/KG & $u$ & NP & 1600 UG/KG & 1 \\
\hline bis(2Chloroethoxy)methane & 330 UG/KG & $u$ & NP & 330 UG/KG & 1 \\
\hline 2,4-Dichlorophenol & 330 UG/KG & $u$ & NP & 330 UG/KG & 1 \\
\hline 1,2,4-Trichlorobenzene & 330 UG/KG & $u$ & NP & 330 UG/KG & 1 \\
\hline Naphthalene & 330 UG/KG & $u$ & NP & 330 UG/KG & 1 \\
\hline 4-Chloroaniline & 330 UG/KG & $u$ & NP & 330 UG/KG & 1 \\
\hline Hexachlorobutadiene & 330 UG/KG & $u$ & NP & 330 UG/KG & 1 \\
\hline 4-Chloro-3-Methlyphenol & 330 UG/KG & $u$ & NP & 330 UG/KG & 1 \\
\hline 2-Methylnaphthalene & 330 UG/KG & $u$ & NP & 330 UG/KG & 1 \\
\hline Hexachlorocyclopentadiene & 330 UG/KG & $u$ & NP & 330 UG/KG & 1 \\
\hline 2,4,6-Trichlorophenol & 330 UG/KG & $u$ & NP & 330 UG/KG & 1 \\
\hline 2,4,5-Trichlorophenol & $1600 \mathrm{UG} / \mathrm{KG}$ & $u$ & NP & 1600 UG/KG & 1 \\
\hline 2-Chloronaphthalene & 330 UG/KG & $u$ & NP & 330 UG/KG & 1 \\
\hline 2-Nitroaniline & 1600 UG/KG & $u$ & NP & 1600 UG/KG & 1 \\
\hline Dimethyl Phthalate & 330 UG/KG & $u$ & NP & 330 UG/KG & 1 \\
\hline Acenaphthylene & 330 UG/KG & $u$ & NP & 330 UG/KG & 1 \\
\hline 2,6-Dinitrotoluene & 330 UG/KG & $u$ & NP & 330 UG/KG & 1 \\
\hline 3-Nitroaniline & $1600 \mathrm{UG} / \mathrm{KG}$ & $u$ & NP & 1600 UG/KG & 1 \\
\hline Acenaphthene & 330 UG/KG & $u$ & NP & 330 UG/KG & 1 \\
\hline 2,4-Dinitrophenol & 1600 UG/KG & $u$ & NP & 1600 UG/KG & 1 \\
\hline 4-Nitrophenol & 1600 UG/KG & $u$ & NP & 1600 UG/KG & 1 \\
\hline Dibenzofuran & 330 UG/KG & $u$ & NP & 330 UG/KG & 1 \\
\hline 2,4-Dinitrotoluene & 330 UG/KG & $u$ & NP & 330 UG/KG & 1 \\
\hline Diethylphthalate & 330 UG/KG & $u$ & NP & 330 UG/KG & 1 \\
\hline 4-Chlorophenyl phenyl ether & 330 UG/KG & $u$ & NP & 330 UG/KG & 1 \\
\hline Fluorene & 330 UG/KG & $u$ & NP & $330 \mathrm{UG} / \mathrm{KG}$ & 1 \\
\hline 4-Nitroaniline & 1600 UG/KG & $u$ & NP & 1600 UG/KG & 1 \\
\hline 4,6-Dinitro-2-methylphenol & 1600 UG/KG & $u$ & NP & 1600 UG/KG & 1 \\
\hline N-Nitrosodiphenylamine & 330 UG/KG & $u$ & NP & 330 UG/KG & 1 \\
\hline
\end{tabular}


APPENDIX D.1 Results of Semivolatile Organic Compound Analyses, Soils, Salmon Site

\begin{tabular}{|c|c|c|c|c|c|c|}
\hline COMPOUND & RESULTS & $\begin{array}{l}\text { DATA } \\
\text { QUAL. }\end{array}$ & $\begin{array}{c}\text { DATA } \\
\text { VALID. }\end{array}$ & CRDL & UNITS & DILUTION \\
\hline \multicolumn{7}{|l|}{ C3-8-S (continued) } \\
\hline Hexachlorobenzene & 330 UG/KG & $u$ & NP & 330 & UG/KG & 1 \\
\hline Pentachlorophenol & $1600 \mathrm{UG} / \mathrm{KG}$ & $u$ & NP & 1600 & UG/KG & 1 \\
\hline Phenanthrene & 330 UG/KG & $u$ & NP & 330 & UG/KG & 1 \\
\hline Anthracene & 330 UG/KG & $u$ & NP & 330 & UG/KG & 1 \\
\hline Di-N-Butylphthalate & 330 UG/KG & $u$ & NP & 330 & UG/KG & 1 \\
\hline Fluoranthene & 330 UG/KG & $u$ & NP & 330 & UG/KG & 1 \\
\hline Pyrene & 330 UG/KG & $u$ & NP & 330 & UG/KG & 1 \\
\hline Butyl Benzyl Phthalate & $330 \mathrm{UG} / \mathrm{KG}$ & $u$ & NP & 330 & UG/KG & 1 \\
\hline 3,3'-Dichlorobenzidine & 660 UG/KG & $u$ & NP & 660 & UG/KG & 1 \\
\hline Benzo(a)Anthracene & $330 \mathrm{UG} / \mathrm{KG}$ & $u$ & NP & 330 & UG/KG & 1 \\
\hline Chrysene & 330 UG/KG & $u$ & NP & 330 & UG/KG & 1 \\
\hline bis(2-Ethylhexyl)phthalate & 330 UG/KG & $u$ & NP & 330 & UG/KG & 1 \\
\hline di-N-Octyl Phthalate & 330 UG/KG & $u$ & NP & 330 & UG/KG & 1 \\
\hline Benzo(b)Fluoranthene & 330 UG/KG & $u$ & NP & 330 & UG/KG & 1 \\
\hline Benzo(k)Fluoranthene & 330 UG/KG & $u$ & NP & 330 & UG/KG & 1 \\
\hline Benzo(a)Pyrene & 330 UG/KG & $u$ & NP & 330 & UG/KG & 1 \\
\hline Indeno(1,2,3-Cd)pyrene & 330 UG/KG & $u$ & NP & 330 & UG/KG & 1 \\
\hline Dibenzo(a,h)Anthracene & 330 UG/KG & $u$ & NP & 330 & UG/KG & 1 \\
\hline Benzo $(g, h, i)$ Perylene & 330 UG/KG & $u$ & NP & 330 & UG/KG & 1 \\
\hline Aldol Condensation & 1000 UG/KG & BJ & NP & & UG/KG & 1 \\
\hline Unknown-1 & 110 UG/KG & J & NP & & UG/KG & 1 \\
\hline Unknown-2 & 88 UG/KG & J & NP & & UG/KG & 1 \\
\hline Nitrobenzene-d5 & $68 \%$ & & & & $\%$ & 1 \\
\hline 2-Fluorobiphenyl & $70 \%$ & & & & $\%$ & 1 \\
\hline Terphenyl-d14 & $62 \%$ & & & & $\%$ & 1 \\
\hline Phenol-d5 & $67 \%$ & & & & $\%$ & 1 \\
\hline 2-Fluorophenol & $18 \%$ & - & & & $\%$ & 1 \\
\hline 2,4,6-Tribromophenol & $68 \%$ & & & & $\%$ & 1 \\
\hline
\end{tabular}


APPENDIX D.1 Results of Semivolatile Organic Compound Analyses, Soils, Salmon Site

\begin{tabular}{|c|c|c|c|c|c|c|c|}
\hline COMPOUND & RESULTS & UNITS & $\begin{array}{l}\text { DATA } \\
\text { QUAL. }\end{array}$ & $\begin{array}{l}\text { DATA } \\
\text { VALID. }\end{array}$ & CRDL & UNITS & DILUTION \\
\hline
\end{tabular}

C3-9-S

Phenol

bis(2-Chloroethyl) ether

2-Chlorophenol

1,3-Dichlorobenzene

1,4-Dichlorobenzene

Benzyl Alcohol

1,2-Dichlorobenzene

2-Methylphenol

bis(2-Chloroisopropyl) ether

4-Methylphenol

N-Nitroso-Di-n-dipropylamine

Hexachloroethane

Nitrobenzene

Isophorone

2-Nitrophenol

2,4-Dimethyiphenol

Benzoic Acid

bis(2Chloroethoxy)methane

2,4-Dichlorophenol

1,2,4-Trichiorobenzene

Naphthalene

4-Chloroaniline

Hexachlorobutadiene

4-Chloro-3-Methlyphenol

2-Methyinaphthalene

Hexachlorocyclopentadiene

2,4,6-Trichlorophenol

2,4,5-Trichlorophenol

2-Chloronaphthalene

2-Nitroaniline

Dimethyl Phthalate

Acenaphthylene

2,6-Dinitrotoluene

3-Nitroaniline

Acenaphthene

2,4-Dinitrophenol

4-Nitrophenol

Dibenzofuran

2,4-Dinitrotoluene

Diethylphthalate

4-Chlorophenyl phenyl ether

Fluorene

4-Nitroaniline

4,6-Dinitro-2-methylphenol

$\mathrm{N}$-Nitrosodiphenylamine

330 UG/KG
330 UG/KG
330 UG/KG
330 UG/KG
330 UG/KG
330 UG/KG
330 UG/KG
330 UG/KG
330 UG/KG
330 UG/KG
330 UG/KG
330 UG/KG
330 UG/KG
330 UG/KG
330 UG/KG
330 UG/KG
1600 UG/KG
330 UG/KG
330 UG/KG
330 UG/KG
330 UG/KG
330 UG/KG
330 UG/KG
330 UG/KG
330 UG/KG
330 UG/KG
330 UG/KG
1600 UG/KG
330 UG/KG
1600 UG/KG
330 UG/KG
330 UG/KG
330 UG/KG
1600 UG/KG
330 UG/KG
1600 UG/KG
1600 UG/KG
1600 UG/KG
330 UG/KG
330 UG/KG
330 UG/KG
330 UG/KG

330 UG/KG

u

$U \quad N P$

$U \quad N P$

$U \quad N P$

$U \quad N P$

U NP

U NP

$U \quad N P$

U NP

U NP

U NP

U NP

U NP

U NP

$U$ NP

U NP

U NP

$U$ NP

$U \quad N P$

$U \quad N P$

$U \quad N P$

U NP

U NP

$U \quad N P$

$U \quad N P$

$U \quad N P$

$U$ NP

$U$ NP

$U \quad N P$

U NP

$U \quad N P$

U NP

$U \quad$ NP

$U \quad N P$

$U \quad N P$

$U \quad N P$

$U \quad N P$

$U \quad N P$

$U \quad N P$

$U \quad N P$

$U \quad N P$

U NP

U NP

$U \quad N P$

$U \quad N P$

$U \quad$ NP

\section{UG/KG}

330 UG/KG

330 UG/KG

330 UG/KG

330 UG/KG

330 UG/KG

330 UG/KG

330 UG/KG

330 UG/KG

330 UG/KG

330 UG/KG

330 UG/KG

330 UG/KG

330 UG/KG

330 UG/KG

330 UG/KG

1600 UG/KG

330 UG/KG

330 UG/KG

330 UG/KG

330 UG/KG

330 UG/KG

330 UG/KG

330 UG/KG

330 UG/KG

330 UG/KG

330 UG/KG

1600 UG/KG

330 UG/KG

1600 UG/KG

330 UG/KG

330 UG/KG

330 UG/KG

1600 UG/KG

330 UG/KG

1600 UG/KG

1600 UG/KG

330 UG/KG

330 UG/KG

330 UG/KG

330 UG/KG

330 UG/KG

1600 UG/KG

1600 UG/KG

330 UG/KG
1
1
1
1
1
1
1
1
1
1
1
1
1
1
1
1
1
1
1
1
1
1
1
1
1
1
1
1
1
1
1
1
1
1
1
1
1
1
1
1
1
1
1 
APPENDIX D.1 Results of Semivolatile Organic Compound Analyses, Soils, Salmon Site

\begin{tabular}{|c|c|c|c|c|c|c|}
\hline COMPOUND & RESULTS & $\begin{array}{l}\text { DATA } \\
\text { QUAL. }\end{array}$ & $\begin{array}{l}\text { DATA } \\
\text { VALID. }\end{array}$ & CRDL & UNITS & DILUTIO \\
\hline \multicolumn{7}{|l|}{ C3-9-S (continued) } \\
\hline 4-Bromophenyl phenylether & 330 UG/KG & $u$ & NP & 330 & UG/KG & 1 \\
\hline Hexachlorobenzene & 330 UG/KG & $u$ & NP & 330 & UG/KG & 1 \\
\hline Pentachlorophenol & 1600 UG/KG & $u$ & NP & $16 \mathrm{CO}$ & UG/KG & 1 \\
\hline Phenanthrene & 330 UG/KG & $u$ & NP & 330 & UG/KG & 1 \\
\hline Anthracene & 330 UG/KG & $u$ & NP & 330 & UG/KG & 1 \\
\hline Di-N-Butylphthalate & 330 UG/KG & $u$ & NP & 330 & UG/KG & 1 \\
\hline Fluoranthene & 330 UG/KG & $u$ & NP & 330 & UG/KG & 1 \\
\hline Pyrene & 330 UG/KG & $u$ & NP & 330 & UG/KG & 1 \\
\hline Butyl Benzyl Phthalate & 330 UG/KG & $u$ & NP & 330 & UG/KG & 1 \\
\hline 3,3'-Dichlorobenzidine & 660 UG/KG & $u$ & NP & 660 & UG/KG & 1 \\
\hline Benzo(a)Anthracene & 330 UG/KG & $u$ & NP & 330 & UG/KG & 1 \\
\hline Chrysene & 330 UG/KG & $u$ & NP & 330 & UG/KG & 1 \\
\hline bis(2-Ethylhexyl)phthalate & 330 UG/KG & $u$ & NP & 330 & UG/KG & 1 \\
\hline di-N-Octy! Phthalate & 330 UG/KG & $u$ & NP & 330 & UG/KG & 1 \\
\hline Benzo(b)Fluoranthene & 330 UG/KG & $u$ & NP & 330 & UG/KG & 1 \\
\hline Benzo(k)Fluoranthene & 330 UG/KG & $u$ & NP & 330 & UG/KG & 1 \\
\hline Benzo(a)Pyrene & 330 UG/KG & $u$ & NP & 330 & UG/KG & 1 \\
\hline Indeno(1,2,3-Cd)pyrene & 330 UG/KG & $u$ & NP & 330 & UG/KG & 1 \\
\hline Dibenzo(a,h)Anthracene & 330 UG/KG & $u$ & NP & 330 & UG/KG & 1 \\
\hline Benzo(g,h,i)Perylene & 330 UG/KG & $u$ & NP & 330 & UG/KG & 1 \\
\hline Aldol Condensation & 990 UG/KG & BJ & NP & & UG/KG & 1 \\
\hline Unknown-1 & 84 UG/KG & $\mathrm{J}$ & NP & & UG/KG & 1 \\
\hline Unknown-2 & $130 \mathrm{UG} / \mathrm{KG}$ & J & NP & & UG/KG & 1 \\
\hline Nitrobenzene-d5 & $78 \%$ & & & & $\%$ & 1 \\
\hline 2-Fluorobiphenyl & $80 \%$ & & & & $\%$ & 1 \\
\hline Terphenyl-d14 & $68 \%$ & & & & $\%$ & 1 \\
\hline Phenol-d5 & $77 \%$ & & & & $\%$ & 1 \\
\hline 2-Fluorophenol & $19 \%$ & * & & & $\%$ & 1 \\
\hline 2,4,6-Tribromophenol & $74 \%$ & & & & $\%$ & 1 \\
\hline
\end{tabular}


APPENDIX D.1 Results of Semivolatile Organic Compound Analyses, Soils, Salmon Site

\begin{tabular}{|c|c|c|c|c|c|}
\hline COMPOUND & RESULTS & $\begin{array}{l}\text { DATA } \\
\text { QUAL. }\end{array}$ & $\begin{array}{r}\text { DATA } \\
\text { VALID. } \\
\end{array}$ & CRDL UNITS & DILUTION \\
\hline \multicolumn{6}{|l|}{ C4-10-S } \\
\hline Phenol & 330 UG/KG & $u$ & NP & 330 UG/KG & 1 \\
\hline bis(2-Chloroethyl) ether & 330 UG/KG & $u$ & NP & 330 UG/KG & 1 \\
\hline 2-Chlorophenol & 330 UG/KG & $u$ & NP & 330 UG/KG & 1 \\
\hline 1,3-Dichlorobenzene & 330 UG/KG & $u$ & NP & 330 UG/KG & 1 \\
\hline 1,4-Dichlorobenzene & 330 UG/KG & $u$ & NP & 330 UG/KG & 1 \\
\hline Benzyl Alcohol & 330 UG/KG & $u$ & NP & 330 UG/KG & 1 \\
\hline 1,2-Dichlorobenzene & 330 UG/KG & $u$ & NP & 330 UG/KG & 1 \\
\hline 2-Methylphenol & 330 UG/KG & $u$ & NP & 330 UG/KG & 1 \\
\hline bis(2-Chloroisopropyl) ether & 330 UG/KG & $u$ & NP & 330 UG/KG & 1 \\
\hline 4-Methylphenol & 330 UG/KG & $u$ & NP & 330 UG/KG & 1 \\
\hline N-Nitroso-Di-n-dipropylamine & 330 UG/KG & $u$ & NP & 330 UG/KG & 1 \\
\hline Hexachloroethane & 330 UG/KG & $u$ & NP & 330 UG/KG & 1 \\
\hline Nitrobenzene & 330 UG/KG & $u$ & NP & 330 UG/KG & 1 \\
\hline Isophorone & 330 UG/KG & $u$ & NP & 330 UG/KG & 1 \\
\hline 2-Nitrophenol & 330 UG/KG & $u$ & NP & 330 UG/KG & 1 \\
\hline 2,4-Dimethylphenol & 330 UG/KG & $u$ & NP & 330 UG/KG & 1 \\
\hline Benzoic Acid & 1600 UG/KG & $u$ & NP & 1600 UG/KG & 1 \\
\hline bis(2Chloroethoxy)methane & 330 UG/KG & $u$ & NP & 330 UG/KG & 1 \\
\hline 2,4-Dichlorophenol & $330 \mathrm{UG} / \mathrm{KG}$ & $u$ & NP & 330 UG/KG & 1 \\
\hline 1,2,4-Trichlorobenzene & 330 UG/KG & $u$ & NP & 330 UG/KG & 1 \\
\hline Naphthalene & 330 UG/KG & $u$ & NP & 330 UG/KG & 1 \\
\hline 4-Chloroaniline & 330 UG/KG & $u$ & NP & 330 UG/KG & 1 \\
\hline Hexachlorobutadiene & 330 UG/KG & $u$ & NP & 330 UG/KG & 1 \\
\hline 4-Chloro-3-Methlyphenol & 330 UG/KG & $u$ & NP & 330 UG/KG & 1 \\
\hline 2-Methylnaphthalene & $330 \mathrm{UG} / \mathrm{KG}$ & $u$ & NP & 330 UG/KG & 1 \\
\hline Hexachlorocyclopentadiene & 330 UG/KG & $u$ & NP & 330 UG/KG & 1 \\
\hline 2,4,6-Trichlorophenol & 330 UG/KG & $u$ & NP & 330 UG/KG & 1 \\
\hline 2,4,5-Trichlorophenol & 1600 UG/KG & $u$ & NP & 1600 UG/KG & 1 \\
\hline 2-Chloronaphthalene & 330 UG/KG & $u$ & NP & 330 UG/KG & 1 \\
\hline 2-Nitroaniline & 1600 UG/KG & $u$ & NP & 1600 UG/KG & 1 \\
\hline Dimethyl Phthalate & 330 UG/KG & $u$ & NP & $330 \cup G / K G$ & 1 \\
\hline Acenaphthylene & 330 UG/KG & $u$ & NP & 330 UG/KG & 1 \\
\hline 2,6-Dinitrotoluene & 330 UG/KG & $u$ & NP & 330 UG/KG & 1 \\
\hline 3-Nitroaniline & 1600 UG/KG & $u$ & NP & 1600 UG/KG & 1 \\
\hline Acenaphthene & 330 UG/KG & $u$ & NP & 330 UG/KG & 1 \\
\hline 2,4-Dinitrophenol & 1600 UG/KG & $u$ & NP & 1600 UG/KG & 1 \\
\hline 4-Nitrophenol & 1600 UG/KG & $u$ & NP & 1600 UG/KG & 1 \\
\hline Dibenzofuran & 330 UG/KG & $u$ & NP & $330 \mathrm{UG} / \mathrm{KG}$ & 1 \\
\hline 2,4-Dinitrotoluene & 330 UG/KG & $u$ & NP & 330 UG/KG & 1 \\
\hline Diethylphthalate & 330 UG/KG & $u$ & NP & 330 UG/KG & 1 \\
\hline 4-Chlorophenyl phenyl ether & 330 UG/KG & $u$ & NP & 330 UG/KG & 1 \\
\hline Fluorene & 330 UG/KG & $u$ & NP & 330 UG/KG & 1 \\
\hline 4-Nitroaniline & 1600 UG/KG & $u$ & NP & 1600 UG/KG & 1 \\
\hline 4,6-Dinitro-2-methylphenol & 1600 UG/KG & $u$ & NP & 1600 UG/KG & 1 \\
\hline N-Nitrosodiphenylamine & 330 UG/KG & $u$ & NP & 330 UG/KG & 1 \\
\hline
\end{tabular}


APPENDIX D.1 Results of Semivolatile Organic Compound Analyses, Soils, Salmon Site

\begin{tabular}{|c|c|c|c|c|c|c|}
\hline COMPOUND & RESULTS & $\begin{array}{l}\text { DATA } \\
\text { QUAL. }\end{array}$ & $\begin{array}{l}\text { DATA } \\
\text { VALID. }\end{array}$ & CRDL & UNITS & DILUTION \\
\hline \multicolumn{7}{|l|}{ C4-10-S (continued) } \\
\hline 4-Bromophenyl phenylether & 330 UG/KG & $u$ & NP & 330 & UG/KG & 1 \\
\hline Hexachlorobenzene & 330 UG/KG & $u$ & NP & 330 & UG/KG & 1 \\
\hline Pentachlorophenol & 1600 UG/KG & $u$ & NP & 1600 & UG/KG & 1 \\
\hline Phenanthrene & 330 UG/KG & $u$ & NP & 330 & UG/KG & 1 \\
\hline Anthracene & 330 UG/KG & $u$ & NP & 330 & UG/KG & 1 \\
\hline Di-N-Butylphthalate & $330 \mathrm{UG} / \mathrm{KG}$ & $u$ & NP & 330 & UG/KG & 1 \\
\hline Fluoranthene & 330 UG/KG & $u$ & NP & 330 & UG/KG & 1 \\
\hline Pyrene & 330 UG/KG & $u$ & NP & 330 & UG/KG & 1 \\
\hline Butyl Benzyl Phthalate & 330 UG/KG & $u$ & NP & 330 & UG/KG & 1 \\
\hline 3,3'-Dichlorobenzidine & 660 UG/KG & $u$ & NP & 660 & UG/Ke & 1 \\
\hline Benzo(a)Anthracene & 330 UG/KG & $u$ & NP & 330 & UG/KG & 1 \\
\hline Chrysene & 330 UG/KG & $u$ & NP & 330 & UG/KG & 1 \\
\hline bis(2-Ethylhexyl)phthalate & 330 UG/KG & $u$ & NP & 330 & UG/KG & 1 \\
\hline di-N-Octyl Phthalate & 330 UG/KG & $u$ & NP & 330 & UG/KG & 1 \\
\hline Benzo(b)Fluoranthene & 330 UG/KG & $u$ & NP & 330 & UG/KG & 1 \\
\hline Benzo(k)Fluoranthene & 330 UG/KG & $u$ & NP & 330 & UG/KG & 1 \\
\hline Benzo(a)Pyrene & 330 UG/KG & $u$ & NP & 330 & UG/KG & 1 \\
\hline Indeno(1,2,3-Cd)pyrene & 330 UG/KG & $u$ & NP & 330 & UG/KG & 1 \\
\hline Dibenzo(a,h)Anthracene & 330 UG/KG & $u$ & NP & 330 & UG/KG & 1 \\
\hline Benzo(g,h,i)Perylene & 330 UG/KG & $u$ & NP & 330 & UG/KG & 1 \\
\hline Aldol Condensation & 1100 UG/KG & BJ & NP & & UG/KG & 1 \\
\hline Unknown-1 & 96 UG/KG & $J$ & NP & & UG/KG & 1 \\
\hline Unknown-2 & 150 UG/KG & $J$ & NP & & UG/KG & 1 \\
\hline Unknown-3 & 98 UG/KG & $J$ & NP & & UG/KG & 1 \\
\hline Unknown-4 & 120 UG/KG & J & NP & & UG/KG & 1 \\
\hline Nitrobenzene-d5 & $85 \%$ & & & & $\%$ & 1 \\
\hline 2-Fluorobiphenyl & $79 \%$ & & & & $\%$ & 1 \\
\hline Terphenyl-d14 & $64 \%$ & & & & $\%$ & 1 \\
\hline Phenol-d5 & $78 \%$ & & & & $\%$ & 1 \\
\hline 2-Fluorophenol & $27 \%$ & & & & $\%$ & 1 \\
\hline 2,4,6-Tribromophenol & $75 \%$ & & & & $\%$ & 1 \\
\hline
\end{tabular}


APPENDIX D.1 Results of Semivolatile Organic Compound Analyses, Soils, Salmon Site

\begin{tabular}{|llllll}
\hline COMPOUND & DESULTS UNA DATA & & \\
\hline
\end{tabular}

\section{C4-11-S}

Phenol

bis(2-Chloroethyl) ether

2-Chlorophenol

1,3-Dichlorobenzene

1,4-Dichlorobenzene

Benzyl Alcohol

1,2-Dichlorobenzene

2-Methylphenol

bis(2-Chloroisopropyl) ether

4-Methylphenol

N-Nitroso-Di-n-dipropylamine

Hexachloroethane

Nitrobenzene

Isophorone

2-Nitrophenol

2,4-Dimethylphenol

Benzoic Acid

bis(2Chloroethoxy)methane

2,4-Dichlorophenol

1,2,4-Trichlorobenzene

Naphthalene

4-Chloroaniline

Hexachlorobutadiene

4-Chloro-3-Methlyphenol

2-Methylnaphthalene

Hexachlorocyclopentadiene

2,4,6-Trichlorophenol

2,4,5-Trichlorophenol

2-Chloronaphthalene

2-Nitroaniline

Dimethyl Phthalate

Acenaphthylene

2,6-Dinitrotoluene

3-Nitroaniline

Acenaphthene

2,4-Dinitrophenol

4-Nitrophenol

Dibenzofuran

2,4-Dinitrotoluene

Diethylphthalate

4-Chlorophenyl phenyl ether

Fluorene

4-Nitroaniline

4,6-Dinitro-2-methyiphenoi

$\mathrm{N}$-Nitrosodiphenylamine
330 UG/KG

330 UG/KG

330 UG/KG

330 UG/KG

330 UG/KG

330 UG/KG

330 UG/KG

330 UG/KG

330 UG/KG

330 UG/KG

$330 \mathrm{UG} / \mathrm{KG}$

330 UG/KG

330 UG/KG

$330 \mathrm{UG} / \mathrm{KG}$

330 UG/KG

330 UG/KG

$1600 \mathrm{UG} / \mathrm{KG}$

330 UG/KG

330 UG/KG

330 UG/KG

330 UG/KG

330 UG/KG

330 UG/KG

330 UG/KG

330 UG/KG

330 UG/KG

$330 \mathrm{UG} / K \mathrm{KG}$

1600 UG/KG

330 UG/KG

$1600 \mathrm{UG} / \mathrm{KG}$

$330 \mathrm{UG} / \mathrm{KG}$

330 UG/KG

330 UG/KG

1600 UG/KG

330 UG/KG

1600 UG/KG

1600 UG/KG

330 UG/KG

330 UG/KG

330 UG/KG

330 UG/KG

330 UG/KG

1600 UG/KG

1600 UG/KG

330 UG/KG

\section{u}

U

U

U

U

u

U

u

u

U

u

u

u

u

u

u

u

u

u

u

u

u

U

U

u

u

u

u

u

u

u

U

U

U

U

U

u

u

u

u

u

u

U

U

u
NP

NP

NP

NP

NP

NP

NP

NP

NP

NP

NP

NP

NP

NP

NP

NP

NP

NP

NP

NP

NP

NP

NP

NP

NP

NP

NP

NP

NP

NP

NP

NP

NP

NP

NP

NP

NP

NP

NP

NP

NP

NP

NP

NP

NP
330 UG/KG

330 UG/KG

330 UG/KG

$330 \mathrm{UG} / \mathrm{KG}$

330 UG/KG

330 UG/KG

$330 \mathrm{UG} / \mathrm{KG}$

330 UG/KG

330 UG/KG

330 UG/KG

330 UG/KG

330 UG/KG

330 UG/KG

330 UG/KG

330 UG/KG

330 UG/KG

1600 UG/KG

330 UG/KG

330 UG/KG

330 UG/KG

330 UG/KG

330 UG/KG

330 UG/KG

330 UG/KG

330 UG/KG

330 UG/KG

330 UG/KG

1600 UG/KG

330 UG/KG

1600 UG/KG

330 UG/KG

330 UG/KG

330 UG/KG

1600 UG/KG

330 UG/KG

1600 UG/KG

1600 UG/KG

330 UG/KG

330 UG/KG

330 UG/KG

330 UG/KG

330 UG/KG

1600 UG/KG

1600 UG/KG

330 UG/KG 
APPENDIX D.1 Results of Semivolatile Organic Compound Analyses, Soils, Salmon Site

\begin{tabular}{|llllll}
\hline COMPOUND & DATA DATA & DESULS UNITS & QUAL. VALID. & CRDL UNITS DILUTION \\
\hline
\end{tabular}

\section{C4-11-S (continued)}

4-Bromophenyl phenylether

Hexachlorobenzene

Pentachlorophenol

Phenanthrene

Anthracene

Di-N-Butylphthalate

Fluoranthene

Pyrene

Butyl Benzyl Phthalate

3,3'-Dichlorobenzidine

Benzo(a)Anthracene

Chrysene

bis(2-Ethylhexyl)phthalate

di-N-Octyl Phthalate

Benzo(b)Fluoranthene

Benzo(k)Fluoranthene

Benzo(a)Pyrene

Indeno(1,2,3-Cd)pyrene

Dibenzo(a,h)Anthracene

Benzo(g,h,i)Perylene

Aldol Condensation

Unknown-1

Unknown-2

Nitrobenzene-d5

2-Fluorobiphenyl

Terphenyl-d14

Phenol-d5

2-Fluorophenol

2,4,6-Tribromophenol

330 UG/KG
330 UG/KG
1600 UG/KG
330 UG/KG
330 UG/KG
330 UG/KG
330 UG/KG
330 UG/KG
330 UG/KG
660 UG/KG
330 UG/KG
330 UG/KG
330 UG/KG
330 UG/KG
330 UG/KG
330 UG/KG
330 UG/KG
330 UG/KG
330 UG/KG
330 UG/KG
1500 UG/KG
120 UG/KG
400 UG/KG
$77 \%$
$69 \%$
$63 \%$
$89 \%$
$46 \%$
$69 \%$

330 UG/KG
330 UG/KG
1600 UG/KG
330 UG/KG
330 UG/KG
330 UG/KG
330 UG/KG
330 UG/KG
330 UG/KG
660 UG/KG
330 UG/KG
330 UG/KG
330 UG/KG
330 UG/KG
330 UG/KG
330 UG/KG
330 UG/KG
330 UG/KG
330 UG/KG
330 UG/KG
UG/KG
UG/KG
UG/KG
$\%$
$\%$
$\%$
$\%$
$\%$

1

1

1

1

1

1

1

1

1

1

1

BJ NP

1


APPENDIX D.1 Results of Semivolatile Organic Compound Analyses, Soils, Salmon Site

\begin{tabular}{|c|c|c|c|c|c|}
\hline COMPOUND & RESULTS & $\begin{array}{l}\text { DATA } \\
\text { QUAL. }\end{array}$ & $\begin{array}{l}\text { DATA } \\
\text { VALID. }\end{array}$ & UNITS & DILUTION \\
\hline \multicolumn{6}{|l|}{ C4-12-S } \\
\hline Phenol & $330 \mathrm{UG} / \mathrm{KG}$ & $u$ & NP & 330 UG/KG & 1 \\
\hline bis(2-Chloroethyi) ether & 330 UG/KG & $u$ & NP & 330 UG/KG & 1 \\
\hline 2-Chlorophenoi & 330 UG/KG & $u$ & NP & 330 UG/KG & 1 \\
\hline 1,3-Dichlorobenzene & 330 UG/KG & $u$ & NP & 330 UG/KG & 1 \\
\hline 1,4-Dichlorobenzene & 330 UG/KG & $u$ & NP & 330 UG/KG & 1 \\
\hline Benzyl Alcohol & 330 UG/KG & $u$ & NP & 330 UG/KG & 1 \\
\hline 1,2-Dichlorobenzene & 330 UG/KG & $u$ & NP & 330 UG/KG & 1 \\
\hline 2-Methylphenol & 330 UG/KG & $u$ & NP & $330 \mathrm{UG} / \mathrm{KG}$ & 1 \\
\hline bis(2-Chloroisopropyl) ether & 330 UG/KG & $u$ & NP & 330 UG/KG & 1 \\
\hline 4-Methyiphenol & 330 UG/KG & $u$ & NP & 330 UG/KG & 1 \\
\hline N-Nitroso-Di-n-dipropylamine & 330 UG/KG & $u$ & NP & 330 UG/KG & 1 \\
\hline Hexachloroethane & 330 UG/KG & $U$ & NP & 330 UG/KG & 1 \\
\hline Nitrobenzene & 330 UG/KG & $u$ & NP & 330 UG/KG & 1 \\
\hline Isophorone & 330 UG/KG & $u$ & NP & 330 UG/KG & 1 \\
\hline 2-Nitrophenol & 330 UG/KG & U & NP & $330 \mathrm{UG} / \mathrm{KG}$ & 1 \\
\hline 2,4-Dimethylphenoi & 330 UG/KG & $\mathrm{U}$ & NP & $330 \mathrm{UG} / \mathrm{KG}$ & 1 \\
\hline Benzoic Acid & 1600 UG/KG & $u$ & NP & 1600 UG/KG & 1 \\
\hline bis(2Chloroethoxy)methane & 330 UG/KG & $u$ & NP & 330 UG/KG & 1 \\
\hline 2,4-Dichlorophenol & 330 UG/KG & $u$ & NP & 330 UG/KG & 1 \\
\hline 1,2,4-Trichlorobenzene & 330 UG/KG & $u$ & NP & 330 UG/KG & 1 \\
\hline Naphthalene & 330 UG/KG & $u$ & NP & 330 UG/KG & 1 \\
\hline 4-Chloroaniline & 330 UG/KG & $u$ & NP & $330 \mathrm{UG} / \mathrm{KG}$ & 1 \\
\hline Hexachlorobutadiene & 330 UG/KG & $u$ & NP & $330 \mathrm{UG} / \mathrm{KG}$ & 1 \\
\hline 4-Chloro-3-Methlyphenol & 330 UG/KG & $u$ & NP & $330 \mathrm{UG} / K G$ & 1 \\
\hline 2-Methylnaphthalene & 330 UG/KG & $u$ & NP & $330 \mathrm{UG} / \mathrm{KG}$ & 1 \\
\hline Hexachlorocyclopentadiene & 330 UG/KG & $\mathrm{u}$ & NP & 330 UG/KG & 1 \\
\hline 2,4,6-Trichlorophenol & 330 UG/KG & $u$ & NP & 330 UG/KG & 1 \\
\hline 2,4,5-Trichlorophenol & 1600 UG/KG & $u$ & NP & 1600 UG/KG & 1 \\
\hline 2-Chloronaphthalene & 330 UG/KG & $\mathrm{u}$ & NP & 330 UG/KG & 1 \\
\hline 2-Nitroaniiline & 1600 UG/KG & $\mathrm{U}$ & NP & 1600 UG/KG & 1 \\
\hline Dimethyl Phthalate & 330 UG/KG & $\mathrm{U}$ & NP & 330 UG/KG & 1 \\
\hline Acenaphthylene & 330 UG/KG & $\mathrm{U}$ & NP & 330 UG/KG & 1 \\
\hline 2,6-Dinitrotoluene & 330 UG/KG & $u$ & NP & 330 UG/KG & 1 \\
\hline 3-Nitroaniline & 1600 UG/KG & $u$ & NP & 1600 UG/KG & 1 \\
\hline Acenaphthene & 330 UG/KG & $u$ & NP & 330 UG/KG & 1 \\
\hline 2,4-Dinitrophenol & 1600 UG/KG & $u$ & NP & 1600 UG/KG & 1 \\
\hline 4-Nitrophenol & 1600 UG/KG & $u$ & NP & 1600 UG/KG & 1 \\
\hline Dibenzofuran & 330 UG/KG & $u$ & NP & 330 UG/KG & 1 \\
\hline 2,4-Dinitrotoluene & 330 UG/KG & $U$ & NP & 330 UG/KG & 1 \\
\hline Diethylphthalate & 330 UG/KG & $U$ & NP & 330 UG/KG & 1 \\
\hline 4-Chlorophenyl phenyl ether & 330 UG/KG & $u$ & NP & 330 UG/KG & 1 \\
\hline Fluorene & 330 UG/KG & $u$ & NP & 330 UG/KG & 1 \\
\hline 4-Nitroaniline & 1600 UG/KG & $u$ & NP & 1600 UG/KG & 1 \\
\hline 4,6-Dinitro-2-methylphenol & 1600 UG/KG & $u$ & NP & 1600 UG/KG & 1 \\
\hline N-Nitrosodiphenylamine & 330 UG/KG & $u$ & NP & 330 UG/KG & 1 \\
\hline
\end{tabular}


APPENDIX D.1 Results of Semivolatile Organic Compound Analyses, Soils, Salmon Site

\begin{tabular}{|c|c|c|c|c|c|c|}
\hline COMPOUND & RESULTS & $\begin{array}{l}\text { DATA } \\
\text { QUAL. }\end{array}$ & $\begin{array}{l}\text { DATA } \\
\text { VALID. }\end{array}$ & CRDL & UNITS & DILUTION \\
\hline \multicolumn{7}{|l|}{ C4-12-S (continued) } \\
\hline 4-Bromophenyl phenylether & 330 UG/KG & $u$ & NP & 330 & UG/KG & 1 \\
\hline Hexachlorobenzene & 330 UG/KG & $u$ & NP & 330 & UG/KG & 1 \\
\hline Pentachlorophenol & 1600 UG/KG & $u$ & NP & 1600 & UG/KG & 1 \\
\hline Phenanthrene & 330 UG/KG & $u$ & NP & 330 & UG/KG & 1 \\
\hline Anthracene & 330 UG/KG & $u$ & NP & 330 & UG/KG & 1 \\
\hline Di-N-Butylphthalate & 330 UG/KG & $u$ & NP & 330 & UG/KG & 1 \\
\hline Fluoranthene & 330 UG/KG & $u$ & NP & 330 & UG/KG & 1 \\
\hline Pyrene & 330 UG/KG & $u$ & NP & 330 & UG/KG & 1 \\
\hline Butyl Benzyl Phthalate & 330 UG/KG & $u$ & NP & 330 & UG/KG & 1 \\
\hline 3,3'-Dichlorobenzidine & 660 UG/KG & $u$ & NP & 660 & UG/KG & 1 \\
\hline Benzo(a)Anthracene & 330 UG/KG & $u$ & NP & 330 & UG/KG & 1 \\
\hline Chrysene & 330 UG/KG & $u$ & NP & 330 & UG/KG & 1 \\
\hline bis(2-Ethylhexyl)phthalate & 140 UG/KG & J & NP & 330 & UG/KG & 1 \\
\hline di-N-Octyl Phthalate & 330 UG/KG & $u$ & NP & 330 & UG/KG & 1 \\
\hline Benzo(b)Fluoranthene & 330 UG/KG & $u$ & NP & 330 & UG/KG & 1 \\
\hline Benzo(k)Fluoranthene & 330 UG/KG & $u$ & NP & 330 & UG/KG & 1 \\
\hline Benzo(a)Pyrene & 330 UG/KG & $u$ & NP & 330 & UG/KG & 1 \\
\hline Indeno(1,2,3-Cd)pyrene & 330 UG/KG & $u$ & NP & 330 & UG/KG & 1 \\
\hline Dibenzo(a,h)Anthracene & 330 UG/KG & $u$ & NP & 330 & UG/KG & 1 \\
\hline Benzo(g,h,i)Perylene & 330 UG/KG & $u$ & NP & 330 & UG/KG & 1 \\
\hline Aldol Condensation & 1600 UG/KG & BJ & NP & & UG/KG & 1 \\
\hline Unknown-1 & 98 UG/KG & $\mathrm{J}$ & NP & & UG/KG & 1 \\
\hline Unknown-2 & 130 UG/KG & BJ & NP & & UG/KG & 1 \\
\hline Unknown-3 & 67 UG/KG & J & NP & & UG/KG & 1 \\
\hline Unknown-4 & 120 UG/KG & J & NP & & UG/KG & 1 \\
\hline Octadecanoic Acid & 110 UG/KG & J & NP & & UG/KG & 1 \\
\hline Unknown-5 & 84 UG/KG & J & & & UG/KG & 1 \\
\hline Nitrobenzene-d5 & $82 \%$ & & & & $\%$ & 1 \\
\hline 2-Fluorobiphenyl & $75 \%$ & & & & $\%$ & 1 \\
\hline Terphenyl-d14 & $63 \%$ & & & & $\%$ & 1 \\
\hline Phenol-d5 & $91 \%$ & & & & $\%$ & 1 \\
\hline 2-Fluorophenol & $55 \%$ & & & & $\%$ & 1 \\
\hline 2,4,6-Tribromophenol & $67 \%$ & & & & $\%$ & 1 \\
\hline
\end{tabular}


APPENDIX D.1 Results of Semivolatile Organic Compound Analyses, Soils, Salmon Site

\begin{tabular}{|c|c|c|c|c|c|}
\hline COMPOUND & RESULTS & $\begin{array}{l}\text { DATA } \\
\text { QUAL. }\end{array}$ & $\begin{array}{c}\text { DATA } \\
\text { VALID. }\end{array}$ & CRDL UNITS & DILUTION \\
\hline \multicolumn{6}{|l|}{ C4-13-S } \\
\hline Phenol & 330 UG/KG & $u$ & NP & 330 UG/KG & 1 \\
\hline bis(2-Chloroethyl) ether & 330 UG/KG & $u$ & NP & 330 UG/KG & 1 \\
\hline 2-Chlorophenol & 330 UG/KG & $u$ & NP & 330 UG/KG & 1 \\
\hline 1,3-Dichlorobenzene & 330 UG/KG & $u$ & NP & 330 UG/KG & 1 \\
\hline 1,4-Dichlorobenzene & 330 UG/KG & $u$ & NP & 330 UG/KG & 1 \\
\hline Benzyl Alcohol & 330 UG/KG & $u$ & NP & 330 UG/KG & 1 \\
\hline 1,2-Dichlorobenzene & 330 UG/KG & $u$ & NP & 330 UG/KG & 1 \\
\hline 2-Methylphenol & 330 UG/KG & $u$ & NP & 330 UG/KG & 1 \\
\hline bis(2-Chloroisopropyl) ether & 330 UG/KG & $u$ & NP & 330 UG/KG & 1 \\
\hline 4-Methylphenol & 330 UG/KG & $u$ & NP & 330 UG/KG & 1 \\
\hline N-Nitroso-Di-n-dipropylamine & 330 UG/KG & $u$ & NP & 330 UG/KG & 1 \\
\hline Hexachloroethane & 330 UG/KG & $u$ & NP & 330 UG/KG & 1 \\
\hline Nitrobenzene & 330 UG/KG & $u$ & NP & $330 \mathrm{UG} / \mathrm{KG}$ & 1 \\
\hline Isophorone & 330 UG/KG & $u$ & NP & 330 UG/KG & 1 \\
\hline 2-Nitrophenol & 330 UG/KG & $u$ & NP & 330 UG/KG & 1 \\
\hline 2,4-Dimethylphenol & 330 UG/KG & $u$ & NP & 330 UG/KG & 1 \\
\hline Benzoic Acid & 1600 UG/KG & $u$ & NP & $1600 \mathrm{UG} / \mathrm{KG}$ & 1 \\
\hline bis(2Chloroethoxy)methane & 330 UG/KG & $u$ & NP & 330 UG/KG & 1 \\
\hline 2,4-Dichlorophenol & 330 UG/KG & $u$ & NP & 330 UG/KG & 1 \\
\hline 1,2,4-Trichlorobenzene & 330 UG/KG & $u$ & NP & 330 UG/KG & 1 \\
\hline Naphthalene & 330 UG/KG & $u$ & NP & 330 UG/KG & 1 \\
\hline 4-Chloroaniline & 330 UG/KG & $u$ & NP & 330 UG/KG & 1 \\
\hline Hexachlorobutadiene & 330 UG/KG & $u$ & NP & 330 UG/KG & 1 \\
\hline 4-Chloro-3-Methlyphenol & 330 UG/KG & $u$ & NP & 330 UG/KG & 1 \\
\hline 2-Methyinaphthalene & 330 UG/KG & $u$ & NP & 330 UG/KG & 1 \\
\hline Hexachlorocyclopentadiene & 330 UG/KG & $u$ & NP & 330 UG/KG & 1 \\
\hline 2,4,6-Trichlorophenol & 330 UG/KG & $u$ & NP & 330 UG/KG & 1 \\
\hline 2,4,5-Trichlorophenol & 1600 UG/KG & $u$ & NP & 1600 UG/KG & 1 \\
\hline 2-Chloronaphthalene & 330 UG/KG & $u$ & NP & 330 UG/KG & 1 \\
\hline 2-Nitroaniline & 1600 UG/KG & $u$ & NP & 1600 UG/KG & 1 \\
\hline Dimethyl Phthalate & 330 UG/KG & $u$ & NP & 330 UG/KG & 1 \\
\hline Acenaphthylene & 330 UG/KG & $u$ & NP & 330 UG/KG & 1 \\
\hline 2,6-Dinitrotoluene & 330 UG/KG & $u$ & NP & 330 UG/KG & 1 \\
\hline 3-Nitroaniline & 1600 UG/KG & $u$ & NP & 1600 UG/KG & 1 \\
\hline Acenaphthene & 330 UG/KG & $u$ & NP & 330 UG/KG & 1 \\
\hline 2,4-Dinitrophenol & 1600 UG/KG & $u$ & NP & 1600 UG/KG & 1 \\
\hline 4-Nitrophenol & 1600 UG/KG & $u$ & NP & 1600 UG/KG & 1 \\
\hline Dibenzofuran & 330 UG/KG & $u$ & NP & 330 UG/KG & 1 \\
\hline 2,4-Dinitrotoluene & 330 UG/KG & $u$ & NP & 330 UG/KG & 1 \\
\hline Diethylphthalate & 330 UG/KG & $u$ & NP & 330 UG/KG & 1 \\
\hline 4-Chlorophenyl phenyl ether & $330 \mathrm{UG} / \mathrm{KG}$ & $u$ & NP & 330 UG/KG & 1 \\
\hline Fluorene & 330 UG/KG & $u$ & NP & 330 UG/KG & 1 \\
\hline 4-Nitroaniline & 1600 UG/KG & $u$ & NP & 1600 UG/KG & 1 \\
\hline 4,6-Dinitro-2-methylphenol & 1600 UG/KG & $u$ & NP & 1600 UG/KG & 1 \\
\hline N-Nitrosodiphenylamine & 330 UG/KG & $u$ & NP & 330 UG/KG & 1 \\
\hline
\end{tabular}


APPENDIX D.1 Results of Semivolatile Organic Compound Analyses, Soils, Salmon Site

\begin{tabular}{|c|c|c|c|c|c|c|}
\hline COMPOUND & RESULTS & $\begin{array}{l}\text { DATA } \\
\text { QUAL. }\end{array}$ & $\begin{array}{l}\text { DATA } \\
\text { VALID. }\end{array}$ & CRDL & UNITS & DILUTION \\
\hline \multicolumn{7}{|l|}{ C4-13-S (continued) } \\
\hline 4-Bromophenyl phenylether & 330 UG/KG & $u$ & NP & 330 & $\mathrm{UG} / \mathrm{KG}$ & 1 \\
\hline Hexachlorobenzene & 330 UG/KG & $u$ & NP & 330 & UG/KG & 1 \\
\hline Pentachlorophenol & 1600 UG/KG & $u$ & NP & 1600 & UG/KG & 1 \\
\hline Phenanthrene & 330 UG/KG & $u$ & NP & 330 & UG/KG & 1 \\
\hline Anthracene & 330 UG/KG & $u$ & NP & 330 & UG/KG & 1 \\
\hline Di-N-Butylphthalate & 330 UG/KG & $u$ & NP & 330 & UG/KG & 1 \\
\hline Fluoranthene & 330 UG/KG & $u$ & NP & 330 & UG/KG & 1 \\
\hline Pyrene & 330 UG/KG & $u$ & NP & 330 & UG/KG & 1 \\
\hline Butyl Benzyl Phthalate & 330 UG/KG & $u$ & NP & 330 & UG/KG & 1 \\
\hline 3,3'-Dichlorobenzidine & 660 UG/KG & $u$ & NP & 660 & UG/KG & 1 \\
\hline Benzo(a)Anthracene & 330 UG/KG & $u$ & NP & 330 & UG/KG & 1 \\
\hline Chrysene & 330 UG/KG & $u$ & NP & 330 & UG/KG & 1 \\
\hline bis(2-Ethylhexyl)phthalate & 330 UG/KG & $u$ & NP & 330 & UG/KG & 1 \\
\hline di-N-Octyl Phthalate & 330 UG/KG & $u$ & NP & 330 & $\mathrm{UG} / \mathrm{KG}$ & 1 \\
\hline Benzo(b)Fluoranthene & 330 UG/KG & $u$ & NP & 330 & UG/KG & 1 \\
\hline Benzo(k)Fluoranthene & 330 UG/KG & $u$ & NP & 330 & UG/KG & 1 \\
\hline Benzo(a)Pyrene & 330 UG/KG & $u$ & NP & 330 & UG/KG & 1 \\
\hline Indeno(1,2,3-Cd)pyrene & 330 UG/KG & $u$ & NP & 330 & $\mathrm{UG} / \mathrm{KG}$ & 1 \\
\hline Dibenzo(a,h)Anthracene & 330 UG/KG & $u$ & NP & 330 & UG/KG & 1 \\
\hline Benzo(g,h,i)Perylene & 330 UG/KG & $u$ & NP & 330 & UG/KG & 1 \\
\hline Aldol Condensation & 1600 UG/KG & BJ & NP & & UG/KG & 1 \\
\hline Unknown-1 & 150 UG/KG & BJ & NP & & UG/KG & 1 \\
\hline Unknown-2 & 99 UG/KG & BJ & NP & & UG/KG & 1 \\
\hline Nitrobenzene-d5 & $85 \%$ & & & & $\%$ & 1 \\
\hline 2-Fluorobiphenyl & $78 \%$ & & & & $\%$ & 1 \\
\hline Terphenyl-d14 & $66 \%$ & & & & $\%$ & 1 \\
\hline Phenol-d5 & $90 \%$ & & & & $\%$ & 1 \\
\hline 2-Fluorophenol & $49 \%$ & & & & $\%$ & 1 \\
\hline 2,4,6-Tribromophenol & $68 \%$ & & & & $\%$ & 1 \\
\hline
\end{tabular}


APPENDIX D.1 Results of Semivolatile Organic Compound Analyses, Soils, Salmon Site

\begin{tabular}{|c|c|c|c|c|c|c|}
\hline COMPOUND & RESULTS & $\begin{array}{l}\text { DATA } \\
\text { QUAL. }\end{array}$ & $\begin{array}{l}\text { DATA } \\
\text { VALID. }\end{array}$ & CRDL & UNITS & DILUTION \\
\hline \multicolumn{7}{|l|}{ C1-14-S } \\
\hline Phenol & 330 UG/KG & $u$ & NP & 330 & JG/KG & 1 \\
\hline bis(2-Chloroethyl) ether & 330 UG/KG & $u$ & NP & 330 & JG/KG & 1 \\
\hline 2-Chlorophenol & 330 UG/KG & $u$ & NP & 330 & JG/KG & 1 \\
\hline 1,3-Dichlorobenzene & 330 UG/KG & $u$ & NP & 330 & JG/KG & 1 \\
\hline 1,4-Dichlorobenzene & 330 UG/KG & $u$ & NP & 330 & JG/KG & 1 \\
\hline Benzyl Alcohol & 330 UG/KG & $u$ & NP & 330 & $J G / K G$ & 1 \\
\hline 1,2-Dichlorobenzene & 330 UG/KG & $u$ & NP & 330 & JG/KG & 1 \\
\hline 2-Methylphenol & 330 UG/KG & $u$ & NP & 330 & JG/KG & 1 \\
\hline bis(2-Chloroisopropyl) ether & 330 UG/KG & $u$ & NP & 330 & JG/KG & 1 \\
\hline 4-Methylphenol & 330 UG/KG & $u$ & NP & 330 & JG/KG & 1 \\
\hline N-Nitroso-Di-n-dipropylamine & 330 UG/KG & $u$ & NP & 330 & JG/KG & 1 \\
\hline Hexachloroethane & 330 UG/KG & $u$ & NP & 330 & JG/KG & 1 \\
\hline Nitrobenzene & 330 UG/KG & $u$ & NP & 330 & JG/KG & 1 \\
\hline Isophorone & 330 UG/KG & $u$ & NP & 330 & JG/KG & 1 \\
\hline 2-Nitrophenol & 330 UG/KG & $u$ & $\mathrm{NP}$ & 330 & JG/KG & 1 \\
\hline 2,4-Dimethylphenol & 330 UG/KG & $u$ & NP & 330 & JG/KG & 1 \\
\hline Benzoic Acid & 1600 UG/KG & $u$ & NP & 1600 & JG/KG & 1 \\
\hline bis(2Chloroethoxy)methane & 330 UG/KG & $u$ & NP & 330 & JG/KG & 1 \\
\hline 2,4-Dichlorophenol & 330 UG/KG & $u$ & NP & 330 & JG/KG & 1 \\
\hline 1,2,4-Trichlorobenzene & 330 UG/KG & $u$ & NP & 330 & JG/KG & 1 \\
\hline Naphthalene & 330 UG/KG & $u$ & NP & 330 & JG/KG & 1 \\
\hline 4-Chloroaniline & 330 UG/KG & $u$ & NP & 330 & JG/KG & 1 \\
\hline Hexachlorobutadiene & 330 UG/KG & $u$ & NP & 330 & JG/KG & 1 \\
\hline 4-Chloro-3-Methlyphenol & 330 UG/KG & $u$ & NP & 330 & UG/KG & 1 \\
\hline 2-Methylnaphthalene & $330 \mathrm{UG} / \mathrm{KG}$ & $u$ & NP & 330 & JG/KG & 1 \\
\hline Hexachlorocyclopentadiene & 330 UG/KG & $u$ & NP & 330 & UG/KG & 1 \\
\hline 2,4,6-Trichlorophenol & 330 UG/KG & $u$ & NP & 330 & UG/KG & 1 \\
\hline 2,4,5-Trichlorophenol & 1600 UG/KG & $u$ & NP & 1600 & UG/KG & 1 \\
\hline 2-Chloronaphthalene & 330 UG/KG & $u$ & NP & 330 & UG/KG & 1 \\
\hline 2-Nitroaniline & 1600 UG/KG & $u$ & NP & 1600 & UG/KG & 1 \\
\hline Dimethyl Phthalate & 330 UG/KG & $u$ & NP & 330 & UG/KG & 1 \\
\hline Acenaphthylene & 330 UG/KG & $u$ & NP & 330 & UG/KG & 1 \\
\hline 2,6-Dinitrotoluene & 330 UG/KG & $u$ & NP & 330 & UG/KG & 1 \\
\hline 3-Nitroaniline & 1600 UG/KG & $u$ & NP & 1600 & UG/KG & 1 \\
\hline Acenaphthene & 330 UG/KG & $u$ & NP & 330 & UG/KG & 1 \\
\hline 2,4-Dinitrophenol & 1600 UG/KG & $u$ & NP & 1600 & UG/KG & 1 \\
\hline 4-Nitrophenol & 1600 UG/KG & $u$ & NP & 1600 & UG/KG & 1 \\
\hline Dibenzofuran & 330 UG/KG & $u$ & NP & 330 & UG/KG & 1 \\
\hline 2,4-Dinitrotoluene & 330 UG/KG & $u$ & NP & 330 & UG/KG & 1 \\
\hline Diethylphthalate & 330 UG/KG & $u$ & NP & 330 & UG/KG & 1 \\
\hline 4-Chlorophenyl phenyl ether & 330 UG/KG & $u$ & NP & 330 & UG/KG & 1 \\
\hline Fluorene & 330 UG/KG & $u$ & NP & 330 & UG/KG & 1 \\
\hline 4-Nitroaniline & 1600 UG/KG & $u$ & NP & 1600 & UG/KG & 1 \\
\hline 4,6-Dinitro-2-methylphenol & $1600 \mathrm{UG} / \mathrm{KG}$ & $u$ & NP & 1600 & UG/KG & 1 \\
\hline N-Nitrosodiphenylamine & 330 UG/KG & $u$ & NP & 330 & UG/KG & 1 \\
\hline
\end{tabular}


APPENDIX D.1 Results of Semivolatile Organic Compound Analyses, Soils, Salmon Site

\begin{tabular}{|c|c|c|c|c|c|c|}
\hline COMPOUND & RESULTS & $\begin{array}{l}\text { DATA } \\
\text { QUAL. }\end{array}$ & $\begin{array}{l}\text { DATA } \\
\text { VALID. }\end{array}$ & CRDL & UNITS & DILUTION \\
\hline \multicolumn{7}{|l|}{ C1-14-S (continued) } \\
\hline 4-Bromophenyl phenylether & 330 UG/KG & $u$ & NP & 330 & UG/KG & 1 \\
\hline Hexachlorobenzene & 330 UG/KG & $u$ & NP & 330 & UG/KG & 1 \\
\hline Pentachlorophenol & 1600 UG/KG & $u$ & NP & 1600 & UG/KG & 1 \\
\hline Phenanthrene & 330 UG/KG & $u$ & NP & 330 & UG/KG & 1 \\
\hline Anthracene & 330 UG/KG & $u$ & NP & 330 & UG/KG & 1 \\
\hline Di-N-Butylphthalate & 330 UG/KG & $u$ & NP & 330 & UGKG & 1 \\
\hline Fluoranthene & 330 UG/KG & $u$ & NP & 330 & UG/KG & 1 \\
\hline Pyrene & 330 UG/KG & $u$ & NP & 330 & UG/KG & 1 \\
\hline Butyl Benzyl Phthalate & 330 UG/KG & $u$ & NP & 330 & UG/KG & 1 \\
\hline 3,3'-Dichlorobenzidine & 660 UG/KG & $u$ & NP & 660 & UG/KG & 1 \\
\hline Benzo(a)Anthracene & 330 UG/KG & $u$ & NP & 330 & UG/KG & 1 \\
\hline Chrysene & 330 UG/KG & $u$ & NP & 330 & UG/KG & 1 \\
\hline bis(2-Ethylhexyl)phthalate & 330 UG/KG & $u$ & NP & 330 & UG/KG & 1 \\
\hline di-N-Octyl Phthalate & 330 UG/KG & $u$ & NP & 330 & UG/KG & 1 \\
\hline Benzo(b)Fluoranthene & 330 UG/KG & $u$ & NP & 330 & UG/KG & 1 \\
\hline Benzo(k)Fluoranthene & 330 UG/KG & $u$ & NP & 330 & $U G / K G$ & 1 \\
\hline Benzo(a)Pyrene & 330 UG/KG & $u$ & NP & 330 & UG/KG & 1 \\
\hline Indeno(1,2,3-Cd)pyrene & 330 UG/KG & $u$ & NP & 330 & UG/KG & 1 \\
\hline Dibenzo(a,h)Anthracene & 330 UG/KG & $u$ & NP & 330 & UG/KG & 1 \\
\hline Benzo(g,h,i)Perylene & 330 UG/KG & $u$ & NP & 330 & UG/KG & 1 \\
\hline Aldol Condensation & 1500 UG/KG & BJ & NP & & UG/KG & 1 \\
\hline Unknown-1 & 70 UG/KG & $J$ & NP & & UG/KG & 1 \\
\hline Unknown-2 & 130 UG/KG & BJ & NP & & UG/KG & 1 \\
\hline Unknown-3 & 130 UG/KG & BJ & NP & & UG/KG & 1 \\
\hline Nitrobenzene-d5 & $80 \%$ & & & & $\%$ & 1 \\
\hline 2-Fluorobiphenyl & $73 \%$ & & & & $\%$ & 1 \\
\hline Terphenyl-d14 & $58 \%$ & & & & $\%$ & 1 \\
\hline Phenol-d5 & $86 \%$ & & & & $\%$ & 1 \\
\hline 2-Fluorophenol & $53 \%$ & & & & $\%$ & 1 \\
\hline 2,4,6-Tribromophenol & $68 \%$ & & & & $\%$ & 1 \\
\hline
\end{tabular}


APPENDIX D.1 Results of Semivolatile Organic Compound Analyses, Soils, Salmon Site

\begin{tabular}{|c|c|c|c|c|c|c|}
\hline COMPOUND & RESULTS & $\begin{array}{l}\text { DATA } \\
\text { QUAL. }\end{array}$ & $\begin{array}{l}\text { DATA } \\
\text { VALID. } \\
\end{array}$ & CRDL & UNITS & DILUTION \\
\hline \multicolumn{7}{|l|}{ C1-15-S } \\
\hline Phenol & 330 UG/KG & $u$ & NP & 330 & UG/KG & 1 \\
\hline bis(2-Chloroethyl) ether & 330 UG/KG & $u$ & NP & 330 & UG/KG & 1 \\
\hline 2-Chlorophenol & 330 UG/KG & $u$ & NP & 330 & UG/KG & 1 \\
\hline 1,3-Dichlorobenzene & 330 UG/KG & $u$ & NP & 330 & UG/KG & 1 \\
\hline 1,4-Dichlorobenzene & $330 \mathrm{UG} / \mathrm{KG}$ & $u$ & NP & 330 & UG/KG & 1 \\
\hline Benzyl Alcohol & 330 UG/KG & $u$ & NP & 330 & UG/KG & 1 \\
\hline 1,2-Dichlorobenzene & 330 UG/KG & $u$ & NP & 330 & UG/KG & 1 \\
\hline 2-Methyiphenol & 330 UG/KG & $u$ & NP & 330 & UG/KG & 1 \\
\hline bis(2-Chloroisopropyl) ether & 330 UG/KG & $u$ & NP & 330 & UG/KG & 1 \\
\hline 4-Methylphenol & 330 UG/KG & $u$ & NP & 330 & UG/KG & 1 \\
\hline N-Nitroso-Di-n-dipropylamine & 330 UG/KG & $u$ & NP & 330 & UG/KG & 1 \\
\hline Hexachloroethane & 330 UG/KG & $u$ & NP & 330 & UG/KG & 1 \\
\hline Nitrobenzene & 330 UG/KG & $u$ & NP & 330 & UG/KG & 1 \\
\hline Isophorone & 330 UG/KG & $u$ & NP & 330 & UG/KG & 1 \\
\hline 2-Nitrophenol & 330 UG/KG & $u$ & NP & 330 & UG/KG & 1 \\
\hline 2,4-Dimethylphenol & 330 UG/KG & $u$ & NP & 330 & $U G / K G$ & 1 \\
\hline Benzoic Acid & 1600 UG/KG & $u$ & NP & 1600 & UG/KG & 1 \\
\hline bis(2Chloroethoxy)methane & 330 UG/KG & $u$ & NP & 330 & $U G / K G$ & 1 \\
\hline 2,4-Dichlorophenol & 330 UG/KG & $u$ & NP & 330 & UG/KG & 1 \\
\hline 1,2,4-Trichlorobenzene & 330 UG/KG & $u$ & NP & 330 & UG/KG & 1 \\
\hline Naphthalene & 330 UG/KG & $u$ & NP & 330 & UG/KG & 1 \\
\hline 4-Chloroaniline & 330 UG/KG & $u$ & NP & 330 & UG/KG & 1 \\
\hline Hexachlorobutadiene & 330 UG/KG & $u$ & NP & 330 & UG/KG & 1 \\
\hline 4-Chloro-3-Methlyphenol & 330 UG/KG & $u$ & NP & 330 & UG/KG & 1 \\
\hline 2-Methylnaphthalene & 330 UG/KG & $u$ & NP & 330 & UG/KG & 1 \\
\hline Hexachlorocyclopentadiene & 330 UG/KG & $u$ & NP & 330 & UG/KG & 1 \\
\hline 2,4,6-Trichiorophenol & 330 UG/KG & $u$ & NP & 330 & UG/KG & 1 \\
\hline 2,4,5-Trichlorophenol & 1600 UG/KG & $u$ & NP & 1600 & UG/KG & 1 \\
\hline 2-Chloronaphthalene & 330 UG/KG & $u$ & NP & 330 & UG/KG & 1 \\
\hline 2-Nitroaniline & 1600 UG/KG & $u$ & NP & 1600 & UG/KG & 1 \\
\hline Dimethyl Phthalate & 330 UG/KG & $u$ & NP & 330 & UG/KG & 1 \\
\hline Acenaphthylene & 330 UG/KG & $u$ & NP & 330 & UG/KG & 1 \\
\hline 2,6-Dinitrotoluene & 330 UG/KG & $u$ & NP & 330 & UG/KG & 1 \\
\hline 3-Nitroaniline & 1600 UG/KG & $u$ & $N P$ & 1600 & UG/KG & 1 \\
\hline Acenaphthene & 330 UG/KG & $u$ & NP & 330 & UG/KG & 1 \\
\hline 2,4-Dinitrophenol & 1600 UG/KG & $u$ & NP & 1600 & UG/KG & 1 \\
\hline 4-Nitrophenol & 1600 UG/KG & $u$ & NP & 1600 & UG/KG & 1 \\
\hline Dibenzofuran & 330 UG/KG & $u$ & NP & 330 & UG/KG & 1 \\
\hline 2,4-Dinitrotoluene & 330 UG/KG & $u$ & NP & 330 & UG/KG & 1 \\
\hline Diethylphthalate & 330 UG/KG & $u$ & NP & 330 & UG/KG & 1 \\
\hline 4-Chlorophenyl phenyl ether & 330 UG/KG & $u$ & NP & 330 & UG/KG & 1 \\
\hline Fluorene & 330 UG/KG & $u$ & NP & 330 & UG/KG & 1 \\
\hline 4-Nitroaniline & 1600 UG/KG & $u$ & NP & 1600 & UG/KG & 1 \\
\hline 4,6-Dinitro-2-methylphenol & 1600 UG/KG & $u$ & NP & 1600 & UG/KG & 1 \\
\hline N-Nitrosodiphenylamine & 330 UG/KG & $u$ & NP & 330 & UG/KG & 1 \\
\hline
\end{tabular}


APPENDIX D.1 Results of Semivolatile Organic Compound Analyses, Soils, Salmon Site

\begin{tabular}{|c|c|c|c|c|c|c|}
\hline COMPOUND & RESULTS & $\begin{array}{l}\text { DATA } \\
\text { QUAL. }\end{array}$ & $\begin{array}{l}\text { DATA } \\
\text { VALID. }\end{array}$ & CRDL & UNITS & DILUTIO \\
\hline \multicolumn{7}{|l|}{ C1-15-S (continued) } \\
\hline 4-Bromophenyl phenylether & 330 UG/KG & $u$ & NP & 330 & UG/KG & 1 \\
\hline Hexachlorobenzene & 330 UG/KG & $u$ & NP & 330 & UG/KG & 1 \\
\hline Pentachlorophenol & 1600 UG/KG & $u$ & NP & 1600 & UG/KG & 1 \\
\hline Phenanthrene & 330 UG/KG & $u$ & NP & 330 & UG/KG & 1 \\
\hline Anthracene & 330 UG/KG & $u$ & NP & 330 & UG/KG & 1 \\
\hline Di-N-Butylphthalate & 330 UG/KG & $u$ & NP & 330 & UG/KG & 1 \\
\hline Fluoranthene & 330 UG/KG & $u$ & NP & 330 & UG/KG & 1 \\
\hline Pyrene & 330 UG/KG & $u$ & NP & 330 & UG/KG & 1 \\
\hline Butyl Benzyl Phthalate & 330 UG/KG & $u$ & NP & 330 & UG/KG & 1 \\
\hline 3,3'-Dichlorobenzidine & 660 UG/KG & $u$ & NP & 660 & UG/KG & 1 \\
\hline Benzo(a)Anthracene & 330 UG/KG & $u$ & NP & 330 & UG/KG & 1 \\
\hline Chrysene & 330 UG/KG & $u$ & NP & 330 & UG/KG & 1 \\
\hline bis(2-Ethylhexyl)phthalate & 330 UG/KG & $u$ & NP & 330 & UG/KG & 1 \\
\hline di-N-Octyl Phthalate & 330 UG/KG & $u$ & NP & 330 & UG/KG & 1 \\
\hline Benzo(b)Fluoranthene & 330 UG/KG & $u$ & NP & 330 & UG/KG & 1 \\
\hline Benzo(k)Fluoranthene & 330 UG/KG & $u$ & NP & 330 & UG/KG & 1 \\
\hline Benzo(a)Pyrene & 330 UG/KG & $u$ & NP & 330 & UG/KG & 1 \\
\hline Indeno(1,2,3-Cd)pyrene & 330 UG/KG & $u$ & NP & 330 & UG/KG & 1 \\
\hline Dibenzo(a,h)Anthracene & 330 UG/KG & $u$ & NP & 330 & UG/KG & 1 \\
\hline Benzo(g,h,i)Perylene & 330 UG/KG & $u$ & NP & 330 & UG/KG & 1 \\
\hline Aldol Condensation & 1500 UG/KG & BJ & NP & & UG/KG & 1 \\
\hline Unknown-1 & 130 UG/KG & BJ & NP & & UG/KG & 1 \\
\hline Unknown-2 & 160 UG/KG & BJ & NP & & UG/KG & 1 \\
\hline Nitrobenzene-d5 & $79 \%$ & & & & $\%$ & 1 \\
\hline 2-Fluorobiphenyl & $73 \%$ & & & & $\%$ & 1 \\
\hline Terphenyl-d14 & $60 \%$ & & & & $\%$ & 1 \\
\hline Phenol-d5 & $89 \%$ & & & & $\%$ & 1 \\
\hline 2-Fluorophenol & $53 \%$ & & & & $\%$ & 1 \\
\hline 2,4,6-Tribromophenol & $67 \%$ & & & & $\%$ & 1 \\
\hline
\end{tabular}


APPENDIX D.1 Results of Semivolatile Organic Compound Analyses. Soils, Salmon Site

\begin{tabular}{|c|c|c|c|c|c|}
\hline COMPOUND & RESULTS & $\begin{array}{l}\text { DATA } \\
\text { QUAL. }\end{array}$ & $\begin{array}{l}\text { DATA } \\
\text { VALID. }\end{array}$ & UNITS & DILUTION \\
\hline \multicolumn{6}{|l|}{ C1-16-S } \\
\hline Phenol & 330 UG/KG & $u$ & NP & 330 UG/KG & 1 \\
\hline bis(2-Chloroethyl) ether & 330 UG/KG & $u$ & NP & 330 UG/KG & 1 \\
\hline 2-Chlorophenol & 330 UG/KG & $u$ & NP & $330 \mathrm{UG} / \mathrm{KG}$ & 1 \\
\hline 1,3-Dichlorobenzene & 330 UG/KG & $u$ & NP & 330 UG/KG & 1 \\
\hline 1,4-Dichlorobenzene & 330 UG/KG & $u$ & NP & 330 UG/KG & 1 \\
\hline Benzyl Alcohol & 330 UG/KG & $u$ & NP & 330 UG/KG & 1 \\
\hline 1,2-Dichlorobenzene & 330 UG/KG & $u$ & NP & 330 UG/KG & 1 \\
\hline 2-Methylphenol & 330 UG/KG & $u$ & NP & 330 UG/KG & 1 \\
\hline bis(2-Chloroisopropyl) ether & 330 UG/KG & $u$ & NP & 330 UG/KG & 1 \\
\hline 4-Methylphenol & 330 UG/KG & $u$ & NP & 330 UG/KG & 1 \\
\hline N-Nitroso-Di-n-dipropylamine & 330 UG/KG & $u$ & NP & 330 UG/KG & 1 \\
\hline Hexachloroethane & 330 UG/KG & $u$ & NP & 330 UG/KG & 1 \\
\hline Nitrobenzene & 330 UG/KG & $U$ & NP & 330 UG/KG & 1 \\
\hline Isophorone & 330 UG/KG & $u$ & NP & 330 UG/KG & 1 \\
\hline 2-Nitrophenol & 330 UG/KG & $u$ & NP & 330 UG/KG & 1 \\
\hline 2,4-Dimethylphenol & 330 UG/KG & $u$ & NP & 330 UG/KG & 1 \\
\hline Benzoic Acid & 1600 UG/KG & $u$ & NP & 1600 UG/KG & 1 \\
\hline bis(2Chloroethoxy)methane & 330 UG/KG & $u$ & NP & 330 UG/KG & 1 \\
\hline 2,4-Dichlorophenol & 330 UG/KG & $u$ & NP & 330 UG/KG & 1 \\
\hline 1,2,4-Trichlorobenzene & 330 UG/KG & $\mathrm{u}$ & NP & 330 UG/KG & 1 \\
\hline Naphthalene & 330 UG/KG & $u$ & NP & 330 UG/KG & 1 \\
\hline 4-Chloroaniline & 330 UG/KG & $u$ & NP & 330 UG/KG & 1 \\
\hline Hexachlorobutadiene & 330 UG/KG & $u$ & NP & 330 UG/KG & 1 \\
\hline 4-Chloro-3-Methlyphenol & 330 UG/KG & $u$ & NP & 330 UG/KG & 1 \\
\hline 2-Methylnaphthalene & 330 UG/KG & $u$ & NP & $330 \mathrm{UG} / \mathrm{KG}$ & 1 \\
\hline Hexachlorocyclopentadiene & 330 UG/KG & $u$ & NP & 330 UG/KG & 1 \\
\hline 2,4,6-Trichlorophenol & 330 UG/KG & u & NP & 330 UG/KG & 1 \\
\hline 2,4,5-Trichlorophenol & 1600 UG/KG & u & NP & 1600 UG/KG & 1 \\
\hline 2-Chloronaphthalene & 330 UG/KG & $\mathrm{U}$ & NP & 330 UG/KG & 1 \\
\hline 2-Nitroaniline & 1600 UG/KG & $U$ & NP & 1600 UG/KG & 1 \\
\hline Dimethyl Phthalate & 330 UG/KG & $u$ & NP & $330 \mathrm{UG} / \mathrm{KG}$ & 1 \\
\hline Acenaphthylene & 330 UG/KG & $\mathrm{u}$ & NP & $330 \mathrm{UG} / \mathrm{KG}$ & 1 \\
\hline 2,6-Dinitrotoluene & 330 UG/KG & $\mathrm{U}$ & NP & 330 UG/KG & 1 \\
\hline 3-Nitroaniline & 1600 UG/KG & $\mathrm{U}$ & NP & 1600 UG/KG & 1 \\
\hline Acenaphthene & 330 UG/KG & $u$ & NP & 330 UG/KG & 1 \\
\hline 2,4-Dinitrophenol & 1600 UG/KG & $\mathrm{u}$ & NP & $1600 \mathrm{UG} / K G$ & 1 \\
\hline 4-Nitrophenol & 1600 UG/KG & $U$ & NP & 1600 UG/KG & 1 \\
\hline Dibenzofuran & 330 UG/KG & $u$ & NP & $330 \mathrm{UG} / \mathrm{KG}$ & 1 \\
\hline 2,4-Dinitrotoluene & 330 UG/KG & $\mathrm{u}$ & NP & 330 UG/KG & 1 \\
\hline Diethylphthalate & 330 UG/KG & $u$ & NP & 330 UG/KG & 1 \\
\hline 4-Chlorophenyl phenyl ether & 330 UG/KG & $u$ & NP & 330 UG/KG & 1 \\
\hline Fluorene & 330 UG/KG & $\mathrm{u}$ & NP & 330 UG/KG & 1 \\
\hline 4-Nitroaniline & $1600 \mathrm{UG} / \mathrm{KG}$ & $u$ & NP & 1600 UG/KG & 1 \\
\hline 4,6-Dinitro-2-methylphenol & 1600 UG/KG & $u$ & NP & 1600 UG/KG & 1 \\
\hline N-Nitrosodiphenylamine & 330 UG/KG & $u$ & NP & 330 UG/KG & 1 \\
\hline
\end{tabular}


APPENDIX D.1 Results of Semivolatile Organic Compound Analyses, Soils, Salmon Site

\begin{tabular}{|c|c|c|c|c|c|c|}
\hline COMPOUND & RESULTS & $\begin{array}{l}\text { DATA } \\
\text { QUAL. }\end{array}$ & $\begin{array}{l}\text { DATA } \\
\text { VALID. }\end{array}$ & CRDL & UNITS & DILUTION \\
\hline \multicolumn{7}{|l|}{ C1-16-S (continued) } \\
\hline 4-Bromophenyl phenylether & 330 UG/KG & $u$ & NP & 330 & UG/KG & 1 \\
\hline Hexachlorobenzene & 330 UG/KG & $u$ & NP & 330 & UG/KG & 1 \\
\hline Pentachlorophenol & 1600 UG/KG & $u$ & NP & 1600 & UG/KG & 1 \\
\hline Phenanthrene & 330 UG/KG & $u$ & NP & 330 & UG/KG & 1 \\
\hline Anthracene & 330 UG/KG & $u$ & NP & 330 & UG/KG & 1 \\
\hline Di-N-Butylphthalate & 330 UG/KG & $u$ & NP & 330 & UG/KG & 1 \\
\hline Fluoranthene & 330 UG/KG & $u$ & NP & 330 & UG/KG & 1 \\
\hline Pyrene & 330 UG/KG & $u$ & NP & 330 & UG/KG & 1 \\
\hline Butyl Benzyl Phthalate & 330 UG/KG & $u$ & NP & 330 & UG/KG & 1 \\
\hline 3,3'-Dichlorobenzidine & 660 UG/KG & $u$ & NP & 660 & UG/KG & 1 \\
\hline Benzo(a)Anthracene & 330 UG/KG & $u$ & NP & 330 & UG/KG & 1 \\
\hline Chrysene & 330 UG/KG & $u$ & NP & 330 & UG/KG & 1 \\
\hline bis(2-Ethylhexyl)phthalate & 330 UG/KG & u & NP & 330 & UG/KG & 1 \\
\hline di-N-Octyl Phthalate & 330 UG/KG & $u$ & NP & 330 & UG/KG & 1 \\
\hline Benzo(b)Fluoranthene & 330 UG/KG & $u$ & NP & 330 & UG/KG & 1 \\
\hline Benzo(k)Fluoranthene & 330 UG/KG & $u$ & NP & 330 & UG/KG & 1 \\
\hline Benzo(a)Pyrene & 330 UG/KG & $u$ & NP & 330 & UG/KG & 1 \\
\hline Indeno(1,2,3-Cd)pyrene & 330 UG/KG & $u$ & NP & 330 & UG/KG & 1 \\
\hline Dibenzo(a,h)Anthracene & 330 UG/KG & $u$ & NP & 330 & UG/KG & 1 \\
\hline Benzo(g,h,i)Perylene & 330 UG/KG & $u$ & NP & 330 & UG/KG & 1 \\
\hline Aldol Condensation & 1400 UG/KG & BJ & NP & & UG/KG & 1 \\
\hline Unknown & 98 UG/KG & BJ & NP & & UG/KG & 1 \\
\hline Nitrobenzene-d5 & $76 \%$ & & & & $\%$ & 1 \\
\hline 2-Fluorobiphenyl & $69 \%$ & & & & $\%$ & 1 \\
\hline Terphenyl-d14 & $58 \%$ & & & & $\%$ & 1 \\
\hline Phenol-d5 & $82 \%$ & & & & $\%$ & 1 \\
\hline 2-Fluorophenol & $58 \%$ & & & & $\%$ & 1 \\
\hline 2,4,6-Tribromophenol & $64 \%$ & & & & $\%$ & 1 \\
\hline
\end{tabular}


APPENDIX D.1 Results of Semivolatile Organic Compound Analyses, Soils, Salmon Site

\begin{tabular}{|lllllll}
\hline COMPOUND & DESULTS & UNITS & QUAL. VALA & & \\
\hline
\end{tabular}

\section{C1-17-S}

Phenol

bis(2-Chloroethyl) ether

330 UG/KG

330 UG/KG

NP

330 UG/KG

1

2-Chlorophenol

330 UG/KG

330 UG/KG

1,3-Dichlorobenzene

330 UG/KG

Benzyl Alcohol

330 UG/KG

1,2-Dichlorobenzene

330 UG/KG

2-Methylphenol

330 UG/KG

bis(2-Chloroisopropyl) ether

4-Methyiphenol

N-Nitroso-Di-n-dipropylamine

330 UG/KG

330 UG/KG

330 UG/KG

Hexachloroethane

330 UG/KG

Nitrobenzene

Isophorone

2-Nitrophenol

2,4-Dimethylphenol

Benzoic Acid

bis(2Chloroethoxy)methane

2,4-Dichlorophenol

1,2,4-Trichlorobenzene

Naphthalene

4-Chloroaniline

Hexachlorobutadiene

4-Chloro-3-Methlyphenol

2-Methylnaphthalene

Hexachlorocyclopentadiene

330 UG/KG

330 UG/KG

330 UG/KG

330 UG/KG

1600 UG/KG

330 UG/KG

$330 \mathrm{UG} / \mathrm{KG}$

330 UG/KG

330 UG/KG

330 UG/KG

330 UG/KG

330 UG/KG

330 UG/KG

330 UG/KG

2,4,6-Trichlorophenol

330 UG/KG

2,4,5-Trichlorophenol

2-Chloronaphthalene

2-Nitroaniline

Dimethyl Phthalate

Acenaphthylene

2,6-Dinitrotoluene

3-Nitroaniline

Acenaphthene

2,4-Dinitrophenol

1600 UG/KG

330 UG/KG

1600 UG/KG

330 UG/KG

330 UG/KG

330 UG/KG

1600 UG/KG

330 UG/KG

1600 UG/KG

4-Nitrophenol

Dibenzofuran

2,4-Dinitrotoluene

1600 UG/KG

330 UG/KG

330 UG/KG

330 UG/KG

330 UG/KG

4-Chlorophenyl phenyl ether

330 UG/KG

Fluorene

4-Nitroaniline

4,6-Dinitro-2-methylphenol

$\mathrm{N}$-Nitrosodiphenyiamine

1600 UG/KG

1600 UG/KG

330 UG/KG

330 UG/KG

330 UG/KG

330 UG/KG

330 UG/KG

330 UG/KG

330 UG/KG

330 UG/KG

330 UG/KG

330 UG/KG

330 UG/KG

330 UG/KG

330 UG/KG

330 UG/KG

330 UG/KG

$330 \mathrm{UG} / \mathrm{KG}$

1600 UG/KG

330 UG/KG

330 UG/KG

330 UG/KG

330 UG/KG

330 UG/KG

330 UG/KG

330 UG/KG

330 UG/KG

330 UG/KG

330 UG/KG

1600 UG/KG

330 UG/KG

1600 UG/KG

330 UG/KG

330 UG/KG

330 UG/KG

1600 UG/KG

330 UG/KG

1600 UG/KG

1600 UG/KG

330 UG/KG

330 UG/KG

330 UG/KG

330 UG/KG

330 UG/KG

1600 UG/KG

1600 UG/KG

330 UG/KG

1

1

1

1

1

1

1

1

1

1

1

1

1

1

1

1

1

1 
APPENDIX D.1 Results of Semivolatile Organic Compound Analyses, Soils, Salmon Site

\begin{tabular}{|c|c|c|c|c|c|c|}
\hline COMPOUND & RESULTS & $\begin{array}{l}\text { DATA } \\
\text { QUAL. }\end{array}$ & $\begin{array}{l}\text { DATA } \\
\text { VALID. }\end{array}$ & CRDL & UNITS & DILUTION \\
\hline \multicolumn{7}{|l|}{ C1-17-S (continued) } \\
\hline 4-Bromophenyl phenylether & 330 UG/KG & $u$ & NP & 330 & JG/KG & 1 \\
\hline Hexachlorobenzene & 330 UG/KG & $u$ & NP & 330 & JG/KG & 1 \\
\hline Pentachlorophenol & 1600 UG/KG & $u$ & NP & 1600 & JG/KG & 1 \\
\hline Phenanthrene & 330 UG/KG & $u$ & NP & 330 & JG/KG & 1 \\
\hline Anthracene & 330 UG/KG & $u$ & NP & 330 & JG/KG & 1 \\
\hline Di-N-Butylphthalate & 330 UG/KG & $u$ & NP & 330 & JG/KG & 1 \\
\hline Fluoranthene & 330 UG/KG & $u$ & NP & 330 & JG/KG & 1 \\
\hline Pyrene & 330 UG/KG & $u$ & NP & 330 & JG/KG & 1 \\
\hline Butyl Benzyl Phthalate & 330 UG/KG & $u$ & NP & 330 & JG/KG & 1 \\
\hline 3,3'-Dichlorobenzidine & 660 UG/KG & $u$ & NP & 660 & JG/KG & 1 \\
\hline Benzo(a)Anthracene & 330 UG/KG & $u$ & NP & 330 & JG/KG & 1 \\
\hline Chrysene & 330 UG/KG & $u$ & NP & 330 & JG/KG & 1 \\
\hline bis(2-Ethylhexyl)phthalate & 330 UG/KG & $u$ & NP & 330 & JG/KG & 1 \\
\hline di-N-Octyl Phthalate & 330 UG/KG & $u$ & NP & 330 & JG/KG & 1 \\
\hline Benzo(b)Fluoranthene & 330 UG/KG & $u$ & NP & 330 & JG/KG & 1 \\
\hline Benzo(k)Fluoranthene & 330 UG/KG & $u$ & NP & 330 & JG/KG & 1 \\
\hline Benzo(a)Pyrene & 330 UG/KG & $u$ & NP & 330 & JG/KG & 1 \\
\hline Indeno(1,2,3-Cd)pyrene & 330 UG/KG & $u^{\circ}$ & NP & 330 & $J G / K G$ & 1 \\
\hline Dibenzo(a,h)Anthracene & 330 UG/KG & $u$ & NP & 330 & JG/KG & 1 \\
\hline Benzo(g,h,i)Perylene & 330 UG/KG & $u$ & NP & 330 & JG/KG & 1 \\
\hline Aldol Condensation & 1200 UG/KG & BJ & NP & & JG/KG & 1 \\
\hline Unknown & 92 UG/KG & BJ & NP & & JG/KG & 1 \\
\hline Nitrobenzene-d5 & $66 \%$ & & & & $\%$ & 1 \\
\hline 2-Fluorobiphenyl & $61 \%$ & & & & $\%$ & 1 \\
\hline Terphenyl-d14 & $54 \%$ & & & & $\%$ & 1 \\
\hline Phenol-d5 & $75 \%$ & & & & $\%$ & 1 \\
\hline 2-Fluorophenol & $54 \%$ & & & & $\%$ & 1 \\
\hline 2,4,6-Tribromophenol & $55 \%$ & & & & $\%$ & 1 \\
\hline
\end{tabular}


APPENDIX D.1 Results of Semivolatile Organic Compound Analyses, Soils, Salmon Site

\begin{tabular}{|c|c|c|c|c|c|c|c|}
\hline COMPOUND & RESULTS & UNITS & $\begin{array}{l}\text { DATA } \\
\text { QUAL. }\end{array}$ & $\begin{array}{c}\text { DATA } \\
\text { VALID. }\end{array}$ & CRDL & UNITS & DILUTION \\
\hline \multicolumn{8}{|l|}{ C1-17-S MS } \\
\hline Phenol & & $63 \%$ & & NP & & $\%$ & 1 \\
\hline 2-Chlorophenol & & $61 \%$ & & NP & & $\%$ & 1 \\
\hline 1,4-Dichlorobenzene & & $59 \%$ & & NP & & $\%$ & 1 \\
\hline N-Nitroso-Di-n-dipropylamine & & $63 \%$ & & NP & & $\%$ & 1 \\
\hline 1,2,4-Trichlorobenzene & & $58 \%$ & & NP & & $\%$ & 1 \\
\hline 4-Chloro-3-Methlyphenol & & $68 \%$ & & NP & & $\%$ & 1 \\
\hline Acenaphthene & & $54 \%$ & & NP & & $\%$ & 1 \\
\hline 4-Nitrophenol & & $71 \%$ & & NP & & $\%$ & 1 \\
\hline 2,4-Dinitrotoluene & & $62 \%$ & & NP & & $\%$ & 1 \\
\hline Pentachlorophenol & & $64 \%$ & & $\mathrm{NP}$ & & $\%$ & 1 \\
\hline Pyrene & & $50 \%$ & & NP & & $\%$ & 1 \\
\hline Nitrobenzene-d5 & & $68 \%$ & & NP & & $\%$ & 1 \\
\hline 2-Fluorobiphenyl & & $62 \%$ & & NP & & $\%$ & 1 \\
\hline Terphenyl-d14 & & $54 \%$ & & NP & & $\%$ & 1 \\
\hline Phenol-d5 & & $72 \%$ & & NP & & $\%$ & 1 \\
\hline 2-Fluorophenol & & $51 \%$ & & NP & & $\%$ & 1 \\
\hline 2,4,6-Tribromophenoi & & $61 \%$ & & NP & & $\%$ & 1 \\
\hline \multicolumn{8}{|l|}{ C1-17-S MSD } \\
\hline Phenol & & $71 \%$ & & NP & & $\%$ & 1 \\
\hline 2-Chlorophenol & & $67 \%$ & & NP & & $\%$ & 1 \\
\hline 1,4-Dichlorobenzene & & $67 \%$ & & NP & & $\%$ & 1 \\
\hline N-Nitroso-Di-n-dipropylamine & & $74 \%$ & & NP & & $\%$ & 1 \\
\hline 1,2,4-Trichlorobenzene & & $63 \%$ & & NP & & $\%$ & 1 \\
\hline 4-Chloro-3-Methlyphenol & & $69 \%$ & & NP & & $\%$ & 1 \\
\hline Acenaphthene & & $56 \%$ & & $\mathrm{NP}$ & & $\%$ & 1 \\
\hline 4-Nitrophenol & & $72 \%$ & & NP & & $\%$ & 1 \\
\hline 2,4-Dinitrotoluene & & $63 \%$ & & NP & & $\%$ & 1 \\
\hline Pentachlorophenol & & $62 \%$ & & NP & & $\%$ & 1 \\
\hline Pyrene & & $55 \%$ & & $\mathrm{NP}$ & & $\%$ & 1 \\
\hline Nitrobenzene-d5 & & $73 \%$ & & NP & & $\%$ & 1 \\
\hline 2-Fluorobiphenyl & & $66 \%$ & & NP & & $\%$ & 1 \\
\hline Terphenyl-d14 & & $58 \%$ & & NP & & $\%$ & 1 \\
\hline Phenol-d5 & & $82 \%$ & & $\mathrm{NP}$ & & $\%$ & 1 \\
\hline 2-Fluorophenol & & $52 \%$ & & $\mathrm{NP}$ & & $\%$ & 1 \\
\hline 2,4,6-Tribromophenol & & $60 \%$ & & NP & & $\%$ & 1 \\
\hline
\end{tabular}



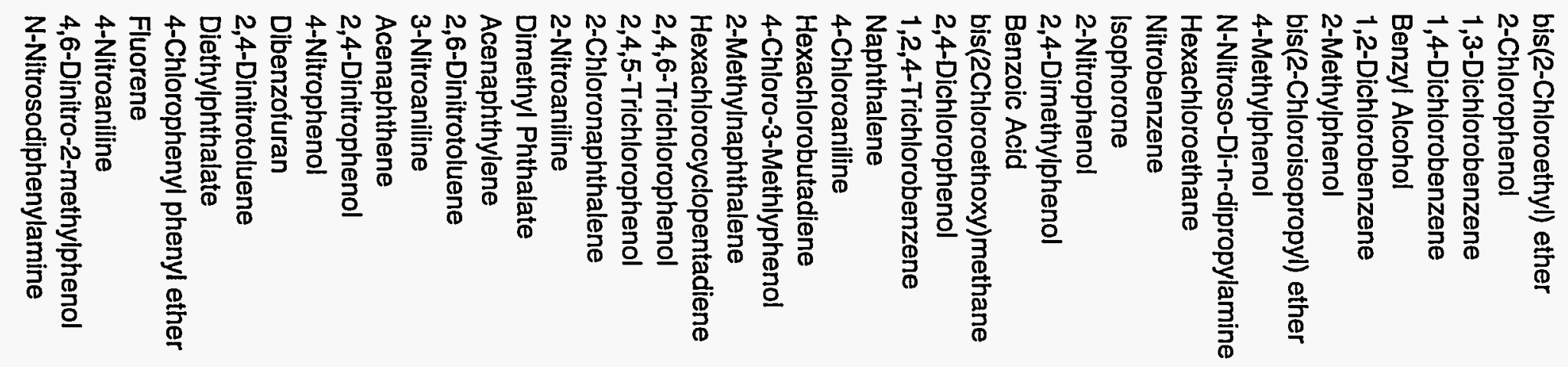

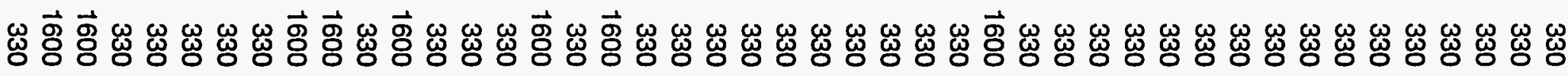

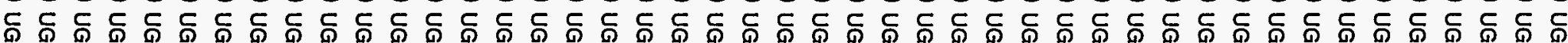

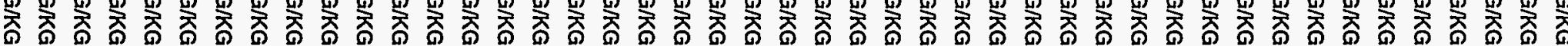

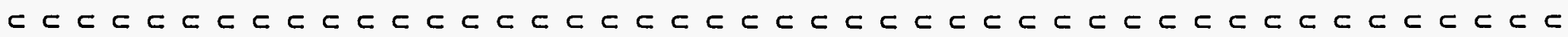

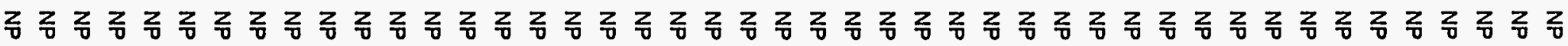

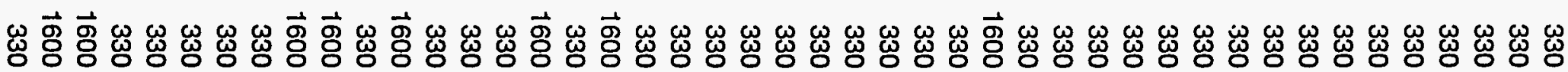

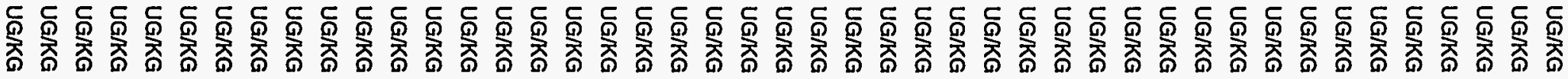


APPENDIX D.1 Results of Semivolatile Organic Compound Analyses, Soils, Salmon Site

\begin{tabular}{|c|c|c|c|c|c|}
\hline COMPOUND & RESULTS & $\begin{array}{l}\text { DATA } \\
\text { QUAL. }\end{array}$ & $\begin{array}{r}\text { DATA } \\
\text { VALID. }\end{array}$ & UNITS & DILUTION \\
\hline \multicolumn{6}{|l|}{ C1-18-S (continued) } \\
\hline 4-Bromophenyl phenylether & 330 UG/KG & $U$ & NP & 330 UG/KG & 1 \\
\hline Hexachlorobenzene & 330 UG/KG & $u$ & NP & 330 UG/KG & 1 \\
\hline Pentachlorophenol & $1600 \mathrm{UG} / \mathrm{KG}$ & $\mathrm{u}$ & NP & 1600 UG/KG & 1 \\
\hline Phenanthrene & 330 UG/KG & $\mathrm{u}$ & NP & 330 UG/KG & 1 \\
\hline Anthracene & 330 UG/KG & $u$ & NP & 330 UG/KG & 1 \\
\hline Di-N-Butylphthalate & $330 \mathrm{UG} / \mathrm{KG}$ & $\mathrm{U}$ & NP & 330 UG/KG & 1 \\
\hline Fluoranthene & 330 UG/KG & $u$ & NP & 330 UG/KG & 1 \\
\hline Pyrene & 330 UG/KG & $u$ & NP & 330 UG/KG & 1 \\
\hline Butyl Benzyl Phthalate & 330 UG/KG & $u$ & NP & 330 UG/KG & 1 \\
\hline 3,3'-Dichlorobenzidine & 660 UG/KG & $u$ & NP & 660 UG/KG & 1 \\
\hline Benzo(a)Anthracene & 330 UG/KG & $\mathrm{u}$ & NP & 330 UG/KG & 1 \\
\hline Chrysene & $330 \mathrm{UG} / \mathrm{KG}$ & $u$ & NP & 330 UG/KG & 1 \\
\hline bis(2-Ethylhexyl)phthalate & 330 UG/KG & $u$ & NP & 330 UG/KG & 1 \\
\hline di-N-Octyl Phthalate & 330 UG/KG & $\mathrm{u}$ & NP & 330 UG/KG & 1 \\
\hline Benzo(b)Fluoranthene & $330 \mathrm{UG} / \mathrm{KG}$ & $u$ & $\mathrm{NP}$ & 330 UG/KG & 1 \\
\hline Benzo(k)Fluoranthene & 330 UG/KG & $u$ & NP & 330 UG/KG & 1 \\
\hline Benzo(a)Pyrene & 330 UG/KG & $u$ & NP & 330 UG/KG & 1 \\
\hline Indeno(1,2,3-Cd)pyrene & $330 \mathrm{UG} / \mathrm{KG}$ & $U$ & NP & $330 \mathrm{UG} / \mathrm{KG}$ & 1 \\
\hline Dibenzo(a,h)Anthracene & 330 UG/KG & $U$ & NP & $330 \mathrm{UG} / \mathrm{KG}$ & 1 \\
\hline Benzo(g,h,i)Perylene & 330 UG/KG & $U$ & NP & 330 UG/KG & 1 \\
\hline Aldol Condensation & 1400 UG/KG & BJ & NP & UG/KG & 1 \\
\hline Unknown-1 & 120 UG/KG & BJ & NP & UG/KG & 1 \\
\hline Unknown-2 & 120 UG/KG & BJ & NP & UG/KG & 1 \\
\hline Sulfur, mol. (S8) & 380 UG/KG & 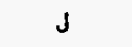 & NP & UG/KG & 1 \\
\hline Nitrobenzene-d5 & $79 \%$ & & & $\%$ & 1 \\
\hline 2-Fluorobiphenyl & $70 \%$ & & & $\%$ & 1 \\
\hline Terphenyl-d14 & $61 \%$ & & & $\%$ & 1 \\
\hline Phenol-d5 & $84 \%$ & & & $\%$ & 1 \\
\hline 2-Fluorophenol & $55 \%$ & & & $\%$ & 1 \\
\hline 2,4,6-Tribromophenol & $64 \%$ & & & $\%$ & 1 \\
\hline
\end{tabular}


APPENDIX D.1 Results of Semivolatile Organic Compound Analyses, Soils, Salmon Site

\begin{tabular}{|c|c|c|c|c|c|c|}
\hline COMPOUND & RESULTS & $\begin{array}{l}\text { DATA } \\
\text { QUAL. }\end{array}$ & $\begin{array}{l}\text { DATA } \\
\text { VALID. }\end{array}$ & CRDL & UNITS & DILUTION \\
\hline \multicolumn{7}{|l|}{ C2-19-S } \\
\hline Phenol & 330 UG/KG & $u$ & NP & 330 & UG/KG & 1 \\
\hline bis(2-Chloroethyl) ether & 330 UG/KG & $u$ & NP & 330 & UG/KG & 1 \\
\hline 2-Chlorophenol & 330 UG/KG & $u$ & NP & 330 & UG/KG & 1 \\
\hline 1,3-Dichlorobenzene & 330 UG/KG & $u$ & NP & 330 & UG/KG & 1 \\
\hline 1,4-Dichlorobenzene & 330 UG/KG & U & NP & 330 & UG/KG & 1 \\
\hline Benzyl Alcohol & 330 UG/KG & $u$ & NP & 330 & UG/KG & 1 \\
\hline 1,2-Dichlorobenzene & 330 UG/KG & $u$ & NP & 330 & UG/KG & 1 \\
\hline 2-Methylphenol & 330 UG/KG & $u$ & NP & 330 & UG/KG & 1 \\
\hline bis(2-Chloroisopropyl) ether & 330 UG/KG & $u$ & NP & 330 & UG/KG & 1 \\
\hline 4-Methylphenol & 330 UG/KG & $u$ & NP & 330 & UG/KG & 1 \\
\hline N-Nitroso-Di-n-dipropylamine & 330 UG/KG & $u$ & NP & 330 & UG/KG & 1 \\
\hline Hexachloroethane & 330 UG/KG & $u$ & NP & 330 & UG/KG & 1 \\
\hline Nitrobenzene & 330 UG/KG & $u$ & NP & 330 & UG/KG & 1 \\
\hline Isophorone & 330 UG/KG & $u$ & NP & 330 & UG/KG & 1 \\
\hline 2-Nitrophenol & 330 UG/KG & $u$ & NP & 330 & UG/KG & 1 \\
\hline 2,4-Dimethylphenol & 330 UG/KG & $u$ & NP & 330 & UG/KG & 1 \\
\hline Benzoic Acid & 1600 UG/KG & $u$ & NP & 1600 & UG/KG & 1 \\
\hline bis(2Chloroethoxy)methane & 330 UG/KG & $u$ & NP & 330 & UG/KG & 1 \\
\hline 2,4-Dichlorophenol & 330 UG/KG & $u$ & NP & 330 & UG/KG & 1 \\
\hline 1,2,4-Trichlorobenzene & 330 UG/KG & $u$ & NP & 330 & UG/KG & 1 \\
\hline Naphthalene & 330 UG/KG & $u$ & NP & 330 & UG/KG & 1 \\
\hline 4-Chloroaniline & 330 UG/KG & $u$ & NP & 330 & UG/KG & 1 \\
\hline Hexachlorobutadiene & 330 UG/KG & $u$ & NP & 330 & UG/KG & 1 \\
\hline 4-Chloro-3-Methlyphenol & 330 UG/KG & $u$ & NP & 330 & UG/KG & 1 \\
\hline 2-Methylnaphthalene & 330 UG/KG & $u$ & NP & 330 & UG/KG & 1 \\
\hline Hexachlorocyclopentadiene & 330 UG/KG & $u$ & NP & 330 & UG/KG & 1 \\
\hline 2,4,6-Trichlorophenol & 330 UG/KG & $u$ & NP & 330 & UG/KG & 1 \\
\hline 2,4,5-Trichlorophenol & 1600 UG/KG & $u$ & NP & 1600 & UG/KG & 1 \\
\hline 2-Chloronaphthalene & 330 UG/KG & $u$ & NP & 330 & UG/KG & 1 \\
\hline 2-Nitroaniline & 1600 UG/KG & $u$ & NP & 1600 & UG/KG & 1 \\
\hline Dimethyl Phthalate & 330 UG/KG & $u$ & NP & 330 & UG/KG & 1 \\
\hline Acenaphthylene & 330 UG/KG & $u$ & NP & 330 & UG/KG & 1 \\
\hline 2,6-Dinitrotoluene & 330 UG/KG & $u$ & NP & 330 & UG/KG & 1 \\
\hline 3-Nitroaniline & 1600 UG/KG & $u$ & NP & 1600 & UG/KG & 1 \\
\hline Acenaphthene & 330 UG/KG & $u$ & NP & 330 & UG/KG & 1 \\
\hline 2,4-Dinitrophenol & 1600 UG/KG & $u$ & NP & 1600 & UG/KG & 1 \\
\hline 4-Nitrophenol & 1600 UG/KG & $u$ & NP & 1600 & UG/KG & 1 \\
\hline Dibenzofuran & 330 UG/KG & $u$ & NP & 330 & UG/KG & 1 \\
\hline 2,4-Dinitrotoluene & 330 UG/KG & $u$ & NP & 330 & UG/KG & 1 \\
\hline Diethylphthalate & 330 UG/KG & $u$ & NP & 330 & UG/KG & 1 \\
\hline 4-Chlorophenyl phenyl ether & 330 UG/KG & $u$ & NP & 330 & UG/KG & 1 \\
\hline Fluorene & 330 UG/KG & $u$ & NP & 330 & UG/KG & 1 \\
\hline 4-Nitroaniline & 1600 UG/KG & $u$ & NP & 1600 & UG/KG & 1 \\
\hline 4,6-Dinitro-2-methylphenol & 1600 UG/KG & $u$ & NP & 1600 & UG/KG & 1 \\
\hline N-Nitrosodiphenylamine & 330 UG/KG & $u$ & NP & 330 & UG/KG & 1 \\
\hline
\end{tabular}


APPENDIX D.1 Results of Semivolatile Organic Compound Analyses, Soils, Salmon Site

\begin{tabular}{|c|c|c|c|c|c|c|}
\hline COMPOUND & RESULTS & $\begin{array}{l}\text { DATA } \\
\text { QUAL. }\end{array}$ & $\begin{array}{l}\text { DATA } \\
\text { VALID. }\end{array}$ & CRDL & UNITS & DILUTION \\
\hline \multicolumn{7}{|l|}{ C2-19-S (continued) } \\
\hline 4-Bromophenyl phenylether & 330 UG/KG & $u$ & NP & 330 & JG/KG & 1 \\
\hline Hexachlorobenzene & 330 UG/KG & $u$ & NP & 330 & JG/KG & 1 \\
\hline Pentachlorophenol & 1600 UG/KG & $u$ & NP & 1600 & JG/KG & 1 \\
\hline Phenanthrene & 330 UG/KG & $u$ & NP & 330 & UG/KG & 1 \\
\hline Anthracene & 330 UG/KG & $u$ & NP & 330 & UG/KG & 1 \\
\hline Di-N-Butylphthalate & 330 UG/KG & $u$ & NP & 330 & UG/KG & 1 \\
\hline Fluoranthene & 330 UG/KG & $u$ & NP & 330 & UG/KG & 1 \\
\hline Pyrene & 330 UG/KG & $u$ & NP & 330 & UG/KG & 1 \\
\hline Butyl Benzyl Phthalate & 330 UG/KG & $u$ & NP & 330 & UG/KG & 1 \\
\hline 3,3'-Dichlorobenzidine & 660 UG/KG & $u$ & NP & 660 & $\mathrm{UG} / \mathrm{KG}$ & 1 \\
\hline Benzo(a)Anthracene & 330 UG/KG & $u$ & NP & 330 & $U G / K G$ & 1 \\
\hline Chrysene & 330 UG/KG & $u$ & NP & 330 & UG/KG & 1 \\
\hline bis(2-Ethylhexyl)phthalate & $330 \mathrm{UG} / \mathrm{KG}$ & $u$ & NP & 330 & UG/KG & 1 \\
\hline di-N-Octyl Phthalate & 330 UG/KG & $u$ & NP & 330 & UG/KG & 1 \\
\hline Benzo(b)Fluoranthene & 330 UG/KG & $u$ & NP & 330 & UG/KG & 1 \\
\hline Benzo(k)Fluoranthene & 330 UG/KG & $u$ & NP & 330 & UG/KG & 1 \\
\hline Benzo(a)Pyrene & 330 UG/KG & $u$ & NP & 330 & UG/KG & 1 \\
\hline Indeno(1,2,3-Cd)pyrene & 330 UG/KG & $u$ & NP & 330 & UG/KG & 1 \\
\hline Dibenzo(a,h)Anthracene & 330 UG/KG & $u$ & NP & 330 & UG/KG & 1 \\
\hline Benzo(g,h,i)Perylene & 330 UG/KG & $u$ & NP & 330 & UG/KG & 1 \\
\hline Aldol Condensation & 1100 UG/KG & BJ & NP & & UG/KG & 1 \\
\hline Unknown-1 & 120 UG/KG & 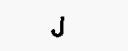 & NP & & UG/KG & 1 \\
\hline Unknown-2 & 94 UG/KG & $\mathrm{J}$ & NP & & UG/KG & 1 \\
\hline Nitrobenzene-d5 & $72 \%$ & & & & $\%$ & 1 \\
\hline 2-Fluorobiphenyi & $76 \%$ & & & & $\%$ & 1 \\
\hline Terphenyi-d14 & $62 \%$ & & & & $\%$ & 1 \\
\hline Phenol-d5 & $74 \%$ & & & & $\%$ & 1 \\
\hline 2-Fluorophenol & $26 \%$ & & & & $\%$ & 1 \\
\hline 2,4,6-Tribromophenol & $71 \%$ & & & & $\%$ & 1 \\
\hline
\end{tabular}


APPENDIX D.1 Results of Semivolatile Organic Compound Analyses, Soils, Salmon Site

\begin{tabular}{|c|c|c|c|c|c|}
\hline COMPOUND & RESULTS & $\begin{array}{l}\text { DATA } \\
\text { QUAL. }\end{array}$ & $\begin{array}{l}\text { DATA } \\
\text { VALID. }\end{array}$ & CRDL UNITS & DILUTION \\
\hline \multicolumn{6}{|l|}{ C2-20-S } \\
\hline Phenol & 330 UG/KG & $u$ & NP & 330 UG/KG & 1 \\
\hline bis(2-Chloroethyl) ether & 330 UG/KG & $u$ & NP & 330 UG/KG & 1 \\
\hline 2-Chlorophenol & 330 UG/KG & $u$ & NP & 330 UG/KG & 1 \\
\hline 1,3-Dichlorobenzene & 330 UG/KG & $u$ & NP & 330 UG/KG & 1 \\
\hline 1,4-Dichlorobenzene & 330 UG/KG & $u$ & NP & 330 UG/KG & 1 \\
\hline Benzyl Alcohol & 330 UG/KG & $u$ & NP & 330 UG/KG & 1 \\
\hline 1,2-Dichlorobenzene & 330 UG/KG & $u$ & NP & 330 UG/KG & 1 \\
\hline 2-Methylphenol & 330 UG/KG & $u$ & NP & 330 UG/KG & 1 \\
\hline bis(2-Chloroisopropyl) ether & 330 UG/KG & $u$ & NP & 330 UG/KG & 1 \\
\hline 4-Methylphenol & 330 UG/KG & $u$ & NP & 330 UG/KG & 1 \\
\hline N-Nitroso-Di-n-dipropylamine & 330 UG/KG & $u$ & NP & 330 UG/KG & 1 \\
\hline Hexachloroethane & 330 UG/KG & $u$ & NP & $330 \mathrm{UG} / \mathrm{KG}$ & 1 \\
\hline Nitrobenzene & 330 UG/KG & $u$ & NP & 330 UG/KG & 1 \\
\hline Isophorone & 330 UG/KG & $u$ & NP & 330 UG/KG & 1 \\
\hline 2-Nitrophenol & 330 UG/KG & $u$ & NP & 330 UG/KG & 1 \\
\hline 2,4-Dimethylphenol & 330 UG/KG & $u$ & NP & 330 UG/KG & 1 \\
\hline Benzoic Acid & 1600 UG/KG & $u$ & NP & 1600 UG/KG & 1 \\
\hline bis(2Chloroethoxy)methane & 330 UG/KG & $u$ & NP & $330 \mathrm{UG} / \mathrm{KG}$ & 1 \\
\hline 2,4-Dichlorophenol & 330 UG/KG & $u$ & NP & 330 UG/KG & 1 \\
\hline 1,2,4-Trichlorobenzene & 330 UG/KG & $u$ & NP & 330 UG/KG & 1 \\
\hline Naphthalene & 330 UG/KG & $u$ & NP & 330 UG/KG & 1 \\
\hline 4-Chloroaniline & 330 UG/KG & $u$ & NP & 330 UG/KG & 1 \\
\hline Hexachlorobutadiene & 330 UG/KG & $u$ & NP & 330 UG/KG & 1 \\
\hline 4-Chloro-3-Methlyphenol & 330 UG/KG & $u$ & NP & 330 UG/KG & 1 \\
\hline 2-Methylnaphthalene & 330 UG/KG & $u$ & NP & 330 UG/KG & 1 \\
\hline Hexachlorocyclopentadiene & 330 UG/KG & $u$ & NP & 330 UG/KG & 1 \\
\hline 2,4,6-Trichlorophenol & 330 UG/KG & $u$ & NP & 330 UG/KG & 1 \\
\hline 2,4,5-Trichlorophenol & 1600 UG/KG & $u$ & NP & 1600 UG/KG & 1 \\
\hline 2-Chloronaphthalene & 330 UG/KG & $u$ & NP & 330 UG/KG & 1 \\
\hline 2-Nitroaniline & 1600 UG/KG & $u$ & NP & 1600 UG/KG & 1 \\
\hline Dimethyl Phthalate & 330 UG/KG & $u$ & NP & 330 UG/KG & 1 \\
\hline Acenaphthylene & 330 UG/KG & $u$ & NP & 330 UG/KG & 1 \\
\hline 2,6-Dinitrotoluene & 330 UG/KG & $u$ & NP & 330 UG/KG & 1 \\
\hline 3-Nitroaniline & 1600 UG/KG & $u$ & NP & 1600 UG/KG & 1 \\
\hline Acenaphthene & 330 UG/KG & $u$ & NP & 330 UG/KG & 1 \\
\hline 2,4-Dinitrophenol & 1600 UG/KG & $u$ & NP & 1600 UG/KG & 1 \\
\hline 4-Nitrophenol & 1600 UG/KG & $u$ & NP & 1600 UG/KG & 1 \\
\hline Dibenzofuran & 330 UG/KG & $u$ & NP & 330 UG/KG & 1 \\
\hline 2,4-Dinitrotoluene & 330 UG/KG & $u$ & NP & 330 UG/KG & 1 \\
\hline Diethylphthalate & $330 \mathrm{UG} / \mathrm{KG}$ & $u$ & NP & 330 UG/KG & 1 \\
\hline 4-Chlorophenyl phenyl ether & 330 UG/KG & $u$ & NP & 330 UG/KG & 1 \\
\hline Fluorene & 330 UG/KG & $u$ & NP & 330 UG/KG & 1 \\
\hline 4-Nitroaniline & 1600 UG̣/KG & $u$ & NP & 1600 UG/KG & 1 \\
\hline 4,6-Dinitro-2-methylphenol & 1600 UG/KG & $u$ & NP & 1600 UG/KG & 1 \\
\hline N-Nitrosodiphenylamine & $330 \mathrm{UG} / \mathrm{KG}$ & $u$ & NP & 330 UG/KG & 1 \\
\hline
\end{tabular}


APPENDIX D.1 Results of Semivolatile Organic Compound Analyses, Soils, Salmon Site

\begin{tabular}{|c|c|c|c|c|c|c|}
\hline COMPOUND & RESULTS & $\begin{array}{l}\text { DATA } \\
\text { QUAL. }\end{array}$ & $\begin{array}{l}\text { DATA } \\
\text { VALID. }\end{array}$ & CRDL & UNITS & DILUTION \\
\hline \multicolumn{7}{|l|}{ C2-20-S (continued) } \\
\hline 4-Bromophenyi phenylether & 330 UG/KG & $u$ & NP & 330 & UG/KG & 1 \\
\hline Hexachlorobenzene & 330 UG/KG & $u$ & NP & 330 & UG/KG & 1 \\
\hline Pentachlorophenol & $1600 \mathrm{UG} / \mathrm{KG}$ & $u$ & NP & 1600 & UG/KG & 1 \\
\hline Phenanthrene & 330 UG/KG & $u$ & NP & 330 & UG/KG & 1 \\
\hline Anthracene & 330 UG/KG & $u$ & NP & 330 & UG/KG & 1 \\
\hline Di-N-Butylphthalate & 330 UG/KG & $u$ & NP & 330 & UG/KG & 1 \\
\hline Fluoranthene & 330 UG/KG & $u$ & NP & 330 & UG/KG & 1 \\
\hline Pyrene & 330 UG/KG & $u$ & NP & 330 & UG/KG & 1 \\
\hline Butyl Benzyl Phthalate & 330 UG/KG & $u$ & NP & 330 & UG/KG & 1 \\
\hline 3,3'-Dichlorobenzidine & 660 UG/KG & $u$ & NP & 660 & UG/KG & 1 \\
\hline Benzo(a)Anthracene & 330 UG/KG & $u$ & NP & 330 & UG/KG & 1 \\
\hline Chrysene & 330 UG/KG & $u$ & NP & 330 & UG/KG & 1 \\
\hline bis(2-Ethylhexyl)phthalate & 330 UG/KG & $u$ & NP & 330 & UG/KG & 1 \\
\hline di-N-Octyl Phthalate & 330 UG/KG & $u$ & NP & 330 & UG/KG & 1 \\
\hline Benzo(b)Fluoranthene & 330 UG/KG & $u$ & $N P$ & 330 & UG/KG & 1 \\
\hline Benzo(k)Fluoranthene & 330 UG/KG & $u$ & NP & 330 & UG/KG & 1 \\
\hline Benzo(a)Pyrene & 330 UG/KG & $u$ & NP & 330 & UG/KG & 1 \\
\hline Indeno(1,2,3-Cd)pyrene & 330 UG/KG & $u$ & NP & 330 & UG/KG & 1 \\
\hline Dibenzo(a,h)Anthracene & 330 UG/KG & $u$ & NP & 330 & UG/KG & 1 \\
\hline Benzo(g,h,i)Perylene & 330 UG/KG & $u$ & NP & 330 & UG/KG & 1 \\
\hline Aldol Condensation & 1100 UG/KG & BJ & NP & & UG/KG & 1 \\
\hline Unknown-1 & 71 UG/KG & $J$ & NP & & UG/KG & 1 \\
\hline Unknown-2 & $96 \mathrm{UG} / \mathrm{KG}$ & J & NP & & UG/KG & 1 \\
\hline Nitrobenzene-d5 & $80 \%$ & & & & $\%$ & 1 \\
\hline 2-Fluorobiphenyl & $80 \%$ & & & & $\%$ & 1 \\
\hline Terphenyl-d14 & $68 \%$ & & & & $\%$ & 1 \\
\hline Phenol-d5 & $80 \%$ & & & & $\%$ & 1 \\
\hline 2-Fluorophenol & $33 \%$ & & & & $\%$ & 1 \\
\hline 2,4,6-Tribromophenol & $74 \%$ & & & & $\%$ & 1 \\
\hline
\end{tabular}


APPENDIX D.1 Results of Semivolatile Organic Compound Analyses, Soils, Salmon Site

\begin{tabular}{|c|c|c|c|c|c|c|}
\hline COMPOUND & RESULTS & $\begin{array}{l}\text { DATA } \\
\text { QUAL. }\end{array}$ & $\begin{array}{l}\text { DATA } \\
\text { VALID. }\end{array}$ & CRDL & UNITS & DILUTION \\
\hline \multicolumn{7}{|l|}{ C2-21-S } \\
\hline Phenol & 330 UG/KG & $u$ & NP & 3301 & UG/KG & 1 \\
\hline bis(2-Chloroethyl) ether & 330 UG/KG & $u$ & NP & 330 & UG/KG & 1 \\
\hline 2-Chlorophenol & 330 UG/KG & $u$ & NP & 3301 & UG/KG & 1 \\
\hline 1,3-Dichlorobenzene & 330 UG/KG & $u$ & NP & 330 & UG/KG & 1 \\
\hline 1,4-Dichlorobenzene & 330 UG/KG & $u$ & NP & 330 & UG/KG & 1 \\
\hline Benzyl Alcohol & 330 UG/KG & $u$ & NP & 330 & UG/KG & 1 \\
\hline 1,2-Dichlorobenzene & 330 UG/KG & $u$ & NP & 330 & UG/KG & 1 \\
\hline 2-Methylphenol & 330 UG/KG & $u$ & NP & 330 & UG/KG & 1 \\
\hline bis(2-Chloroisopropyl) ether & 330 UG/KG & $u$ & NP & 330 & UG/KG & 1 \\
\hline 4-Methylphenol & 330 UG/KG & $u$ & NP & 330 & UG/KG & 1 \\
\hline N-Nitroso-Di-n-dipropylamine & 330 UG/KG & $u$ & NP & 330 & UG/KG & 1 \\
\hline Hexachloroethane & 330 UG/KG & $u$ & NP & 330 & UG/KG & 1 \\
\hline Nitrobenzene & 330 UG/KG & $u$ & NP & 330 & UG/KG & 1 \\
\hline Isophorone & 330 UG/KG & $u$ & NP & 330 & UG/KG & 1 \\
\hline 2-Nitrophenol & 330 UG/KG & $u$ & NP & 330 & UG/KG & 1 \\
\hline 2,4-Dimethylphenol & 330 UG/KG & $u$ & NP & 330 & UG/KG & 1 \\
\hline Benzoic Acid & 1600 UG/KG & $u$ & NP & 1600 & UG/KG & 1 \\
\hline bis(2Chloroethoxy)methane & 330 UG/KG & $u$ & NP & 330 & UG/KG & 1 \\
\hline 2,4-Dichlorophenol & $330 \mathrm{UG} / \mathrm{KG}$ & $u$ & NP & 330 & UG/KG & 1 \\
\hline 1,2,4-Trichlorobenzene & 330 UG/KG & $u$ & NP & 330 & UG/KG & 1 \\
\hline Naphthalene & $330 \mathrm{UG} / \mathrm{KG}$ & $u$ & NP & 330 & UG/KG & 1 \\
\hline 4-Chloroaniline & 330 UG/KG & $u$ & NP & 330 & UG/KG & 1 \\
\hline Hexachlorobutadiene & 330 UG/KG & $u$ & NP & 330 & UG/KG & 1 \\
\hline 4-Chloro-3-Methlyphenol & $330 \mathrm{UG} / \mathrm{KG}$ & $u$ & NP & 330 & UG/KG & 1 \\
\hline 2-Methylnaphthalene & 330 UG/KG & $u$ & NP & 330 & UG/KG & 1 \\
\hline Hexachlorocyclopentadiene & 330 UG/KG & $u$ & NP & 330 & UG/KG & 1 \\
\hline 2,4,6-Trichlorophenol & 330 UG/KG & $u$ & NP & 330 & UG/KG & 1 \\
\hline 2,4,5-Trichlorophenol & 1600 UG/KG & $u$ & NP & 1600 & UG/KG & 1 \\
\hline 2-Chloronaphthalene & 330 UG/KG & $u$ & NP & 330 & UG/KG & 1 \\
\hline 2-Nitroaniline & 1600 UG/KG & $u$ & NP & 1600 & UG/KG & 1 \\
\hline Dimethyl Phthalate & 330 UG/KG & $u$ & NP & 330 & UG/KG & 1 \\
\hline Acenaphthylene & 330 UG/KG & $u$ & NP & 330 & UG/KG & 1 \\
\hline 2,6-Dinitrotoluene & 330 UG/KG & $u$ & NP & 330 & UG/KG & 1 \\
\hline 3-Nitroaniline & 1600 UG/KG & $u$ & NP & 1600 & UG/KG & 1 \\
\hline Acenaphthene & 330 UG/KG & $u$ & NP & 330 & UG/KG & 1 \\
\hline 2,4-Dinitrophenol & 1600 UG/KG & $u$ & NP & 1600 & UG/KG & 1 \\
\hline 4-Nitrophenol & 1600 UG/KG & $u$ & NP & 1600 & UG/KG & 1 \\
\hline Dibenzofuran & 330 UG/KG & $u$ & NP & 330 & UG/KG & 1 \\
\hline 2,4-Dinitrotoluene & 330 UG/KG & $u$ & NP & 330 & UG/KG & 1 \\
\hline Diethylphthalate & 330 UG/KG & $u$ & NP & 330 & UG/KG & 1 \\
\hline 4-Chlorophenyl phenyl ether & 330 UG/KG & $u$ & NP & 330 & UGKG & 1 \\
\hline Fluorene & 330 UG/KG & $u$ & NP & 330 & UG/KG & 1 \\
\hline 4-Nitroaniline & 1600 UG/KG & $u$ & NP & 1600 & UG/KG & 1 \\
\hline 4,6-Dinitro-2-methylphenol & 1600 UG/KG & $u$ & NP & 1600 & UG/KG & 1 \\
\hline N-Nitrosodiphenylamine & 330 UG/KG & $u$ & NP & 330 & UG/KG & 1 \\
\hline
\end{tabular}


APPENDIX D.1 Results of Semivolatile Organic Compound Analyses, Soils, Salmon Site

\begin{tabular}{|c|c|c|c|c|c|c|}
\hline COMPOUND & RESULTS & $\begin{array}{l}\text { DATA } \\
\text { QUAL. }\end{array}$ & $\begin{array}{l}\text { DATA } \\
\text { VALID. }\end{array}$ & CRDL & UNITS & DILUTION \\
\hline \multicolumn{7}{|l|}{ C2-21-S (continued) } \\
\hline 4-Bromophenyl phenylether & 330 UG/KG & $u$ & NP & 330 & JG/KG & 1 \\
\hline Hexachlorobenzene & 330 UG/KG & $u$ & NP & 330 & JG/KG & 1 \\
\hline Pentachlorophenol & 1600 UG/KG & $u$ & NP & 1600 & JG/KG & 1 \\
\hline Phenanthrene & 330 UG/KG & $u$ & NP & 330 & JG/KG & 1 \\
\hline Anthracene & 330 UG/KG & $u$ & NP & 330 & JG/KG & 1 \\
\hline Di-N-Butylphthalate & $330 \mathrm{UG} / \mathrm{KG}$ & $u$ & NP & 330 & JG/KG & 1 \\
\hline Fluoranthene & 330 UG/KG & $u$ & NP & 330 & JG/KG & 1 \\
\hline Pyrene & 330 UG/KG & $\mathrm{u}$ & $\mathrm{NP}$ & 330 & JG/KG & 1 \\
\hline Butyl Benzyl Phthalate & 330 UG/KG & $u$ & NP & 330 & JG/KG & 1 \\
\hline 3,3'-Dichlorobenzidine & 660 UG/KG & $u$ & NP & 660 & IG/KG & 1 \\
\hline Benzo(a)Anthracene & 330 UG/KG & $u$ & NP & 330 & JG/KG & 1 \\
\hline Chrysene & 330 UG/KG & $u$ & NP & 330 & JG/KG & 1 \\
\hline bis(2-Ethylhexyl)phthalate & 330 UG/KG & $u$ & NP & 330 & JG/KG & 1 \\
\hline di-N-Octyl Phthalate & 330 UG/KG & $u$ & NP & 330 & JG/KG & 1 \\
\hline Benzo(b)Fluoranthene & 330 UG/KG & $u$ & NP & 330 & JG/KG & 1 \\
\hline Benzo(k)Fluoranthene & 330 UG/KG & $u$ & NP & 330 & JG/KG & 1 \\
\hline Benzo(a)Pyrene & 330 UG/KG & $u$ & NP & 330 & JG/KG & 1 \\
\hline Indeno(1,2,3-Cd)pyrene & 330 UG/KG & $u$ & NP & 330 & UG/KG & 1 \\
\hline Dibenzo(a,h)Anthracene & 330 UG/KG & $u$ & NP & 330 & JG/KG & 1 \\
\hline Benzo $(g, h, i)$ Perylene & 330 UG/KG & $u$ & NP & 330 & JG/KG & 1 \\
\hline Aldol Condensation & 1200 UG/KG & BJ & NP & & JG/KG & 1 \\
\hline Unknown-1 & 120 UG/KG & J & $\mathrm{NP}$ & & UG/KG & 1 \\
\hline Unknown-2 & 84 UG/KG & J & NP & & UG/KG & 1 \\
\hline Nitrobenzene-d5 & $64 \%$ & & & & $\%$ & 1 \\
\hline 2-Fluorobiphenyl & $67 \%$ & & & & $\%$ & 1 \\
\hline Terphenyl-d14 & $56 \%$ & & & & $\%$ & 1 \\
\hline Phenol-d5 & $72 \%$ & & & & $\%$ & 1 \\
\hline 2-Fluorophenol & $32 \%$ & & & & $\%$ & 1 \\
\hline 2,4,6-Tribromophenol & $61 \%$ & & & & $\%$ & 1 \\
\hline
\end{tabular}


APPENDIX D.1 Results of Semivolatile Organic Compound Analyses, Soils, Salmon Site

\begin{tabular}{|c|c|c|c|c|c|c|c|}
\hline COMPOUND & RESULTS & UNITS & $\begin{array}{l}\text { DATA } \\
\text { QUAL. }\end{array}$ & $\begin{array}{l}\text { DATA } \\
\text { VALID. }\end{array}$ & CRDL & UNITS & DILUTION \\
\hline \multicolumn{8}{|l|}{ C2-21-S-MS } \\
\hline Phenol & & $61 \%$ & & NP & & $\%$ & 1 \\
\hline 2-Chlorophenol & & $58 \%$ & & NP & & $\%$ & 1 \\
\hline 1,4-Dichlorobenzene & & $58 \%$ & & NP & & $\%$ & 1 \\
\hline N-Nitroso-Di-n-dipropylamine & & $74 \%$ & & NP & & $\%$ & 1 \\
\hline 1,2,4-Trichlorobenzene & & $58 \%$ & & NP & & $\%$ & 1 \\
\hline 4-Chloro-3-Methlyphenol & & $67 \%$ & & NP & & $\%$ & 1 \\
\hline Acenaphthene & & $58 \%$ & & NP & & $\%$ & 1 \\
\hline 4-Nitrophenol & & $82 \%$ & & NP & & $\%$ & 1 \\
\hline 2,4-Dinitrotoluene & & $63 \%$ & & NP & & $\%$ & 1 \\
\hline Pentachlorophenol & & $83 \%$ & & NP & & $\%$ & 1 \\
\hline Pyrene & & $51 \%$ & & NP & & $\%$ & 1 \\
\hline Nitrobenzene-d5 & & $66 \%$ & & NP & & $\%$ & 1 \\
\hline 2-Fluorobiphenyl & & $68 \%$ & & NP & & $\%$ & 1 \\
\hline Terphenyl-d14 & & $59 \%$ & & NP & & $\%$ & 1 \\
\hline Phenol-d5 & & $69 \%$ & & NP & & $\%$ & 1 \\
\hline 2-Fluorophenol & & $29 \%$ & & NP & & $\%$ & 1 \\
\hline 2,4,6-Tribromophenol & & $65 \%$ & & NP & & $\%$ & 1 \\
\hline \multicolumn{8}{|l|}{ C2-21-S -MSD } \\
\hline Phenol & & $66 \%$ & & NP & & $\%$ & 1 \\
\hline 2-Chlorophenol & & $60 \%$ & & NP & & $\%$ & 1 \\
\hline 1,4-Dichlorobenzene & & $59 \%$ & & NP & & $\%$ & 1 \\
\hline N-Nitroso-Di-n-dipropylamine & & $76 \%$ & & NP & & $\%$ & 1 \\
\hline 1,2,4-Trichlorobenzene & & $61 \%$ & & NP & & $\%$ & 1 \\
\hline 4-Chloro-3-Methlyphenol & & $70 \%$ & & NP & & $\%$ & 1 \\
\hline Acenaphthene & & $61 \%$ & & NP & & $\%$ & 1 \\
\hline 4-Nitrophenol & & $84 \%$ & & NP & & $\%$ & 1 \\
\hline 2,4-Dinitrotoluene & & $64 \%$ & & NP & & $\%$ & 1 \\
\hline Pentachlorophenol & & $78 \%$ & & NP & & $\%$ & 1 \\
\hline Pyrene & & $52 \%$ & & NP & & $\%$ & 1 \\
\hline Nitrobenzene-d5 & & $66 \%$ & & NP & & $\%$ & 1 \\
\hline 2-Fluorobiphenyl & & $71 \%$ & & NP & & $\%$ & 1 \\
\hline Terphenyl-d14 & & $59 \%$ & & NP & & $\%$ & 1 \\
\hline Phenol-d5 & & $70 \%$ & & NP & & $\%$ & 1 \\
\hline 2-Fluorophenol & & $26 \%$ & & NP & & $\%$ & 1 \\
\hline 2,4,6-Tribromophenol & & $66 \%$ & & NP & & $\%$ & 1 \\
\hline
\end{tabular}


APPENDIX D.1 Results of Semivolatile Organic Compound Analyses, Soils, Salmon Site

\begin{tabular}{|c|c|c|c|c|c|c|}
\hline COMPOUND & RESULTS & $\begin{array}{l}\text { DATA } \\
\text { QUAL. }\end{array}$ & $\begin{array}{l}\text { DATA } \\
\text { VALID. }\end{array}$ & CRDL & UNITS & DILUTION \\
\hline \multicolumn{7}{|l|}{ C2-22-S } \\
\hline Phenol & 330 UG/KG & $u$ & NP & 330 & UG/KG & 1 \\
\hline bis(2-Chloroethyl) ether & 330 UG/KG & $u$ & NP & 330 & $\mathrm{UG} / \mathrm{KG}$ & 1 \\
\hline 2-Chlorophenol & 330 UG/KG & $u$ & NP & 330 & UG/KG & 1 \\
\hline 1,3-Dichlorobenzene & $330 \mathrm{UG} / \mathrm{KG}$ & $u$ & NP & 330 & UG/KG & 1 \\
\hline 1,4-Dichlorobenzene & 330 UG/KG & $u$ & NP & 330 & UG/KG & 1 \\
\hline Benzyl Alcohol & 330 UG/KG & $u$ & NP & 330 & $\mathrm{UG} / \mathrm{KG}$ & 1 \\
\hline 1,2-Dichlorobenzene & 330 UG/KG & $u$ & NP & 330 & UG/KG & 1 \\
\hline 2-Methylphenol & 330 UG/KG & $u$ & NP & 330 & UG/KG & 1 \\
\hline bis(2-Chloroisopropyi) ether & 330 UG/KG & $u$ & NP & 330 & UG/KG & 1 \\
\hline 4-Methylphenol & 330 !IG/KG & $u$ & NP & 330 & UG/KG & 1 \\
\hline N-Nitroso-Di-n-dipropylamine & 330 UG/KG & $u$ & NP & 330 & UG/KG & 1 \\
\hline Hexachloroethane & 330 UG/KG & $u$ & NP & 330 & UG/KG & 1 \\
\hline Nitrobenzene & 330 UG/KG & $u$ & NP & 330 & UG/KG & 1 \\
\hline Isophorone & 330 UG/KG & $u$ & NP & 330 & UG/KG & 1 \\
\hline 2-Nitrophenol & 330 UG/KG & $u$ & NP & 330 & UG/KG & 1 \\
\hline 2,4-Dimethylphenol & 330 UG/KG & $u$ & NP & 330 & UG/KG & 1 \\
\hline Benzoic Acid & 1600 UG/KG & $u$ & NP & 1600 & UG/KG & 1 \\
\hline bis(2Chloroethoxy)methane & 330 UG/KG & $u$ & NP & 330 & UG/KG & 1 \\
\hline 2,4-Dichlorophenol & 330 UG/KG & $u$ & NP & 330 & UG/KG & 1 \\
\hline 1,2,4-Trichlorobenzene & 330 UG/KG & $u$ & NP & 330 & UG/KG & 1 \\
\hline Naphthalene & 330 UG/KG & $u$ & NP & 330 & UG/KG & 1 \\
\hline 4-Chloroaniline & 330 UG/KG & $u$ & NP & 330 & UG/KG & 1 \\
\hline Hexachlorobutadiene & 330 UG/KG & $u$ & NP & 330 & UG/KG & 1 \\
\hline 4-Chloro-3-Methlyphenol & 330 UG/KG & $u$ & NP & 330 & UG/KG & 1 \\
\hline 2-Methylnaphthalene & 330 UG/KG & $u$ & NP & 330 & UG/KG & 1 \\
\hline Hexachlorocyclopentadiene & 330 UG/KG & $u$ & NP & 330 & $\mathrm{UG} / \mathrm{KG}$ & 1 \\
\hline 2,4,6-Tribromophenol & 330 UG/KG & $u$ & NP & 330 & UG/KG & 1 \\
\hline 2,4,5-Trichlorophenol & 1600 UG/KG & $u$ & NP & 1600 & $U G / K G$ & 1 \\
\hline 2-Chloronaphthalene & 330 UG/KG & $u$ & NP & 330 & UG/KG & 1 \\
\hline 2-Nitroaniline & $1600 \mathrm{UG} / \mathrm{KG}$ & $u$ & NP & 1600 & $U G / K G$ & 1 \\
\hline Dimethyl Phthalate & 330 UG/KG & $u$ & NP & 330 & UG/KG & 1 \\
\hline Acenaphthylene & 330 UG/KG & $u$ & NP & 330 & UG/KG & 1 \\
\hline 2,6-Dinitrotoluene & 330 UG/KG & $u$ & NP & 330 & UG/KG & 1 \\
\hline 3-Nitroaniline & 1600 UG/KG & $u$ & NP & 1600 & UG/KG & 1 \\
\hline Acenaphthene & 330 UG/KG & $u$ & NP & 330 & UGKG & 1 \\
\hline 2,4-Dinitrophenol & 1600 UG/KG & $u$ & NP & 1600 & UG/KG & 1 \\
\hline 4-Nitrophenol & 1600 UG/KG & $u$ & NP & 1600 & UG/KG & 1 \\
\hline Dibenzofuran & 330 UG/KG & $u$ & NP & 330 & UG/KG & 1 \\
\hline 2,4-Dinitrotoluene & 330 UG/KG & $u$ & NP & 330 & UG/KG & 1 \\
\hline Diethylphthalate & 330 UG/KG & $u$ & NP & 330 & UG/KG & 1 \\
\hline 4-Chlorophenyi phenyl ether & 330 UG/KG & $u$ & NP & 330 & UG/KG & 1 \\
\hline Fluorene & 330 UG/KG & $u$ & NP & 330 & UG/KG & 1 \\
\hline 4-Nitroaniline & 1600 UG/KG & $u$ & NP & 1600 & UG/KG & 1 \\
\hline 4,6-Dinitro-2-methylphenol & 1600 UG/KG & $u$ & NP & 1600 & UG/KG & 1 \\
\hline N-Nitrosodiphenylamine & 330 UG/KG & $u$ & NP & 330 & UG/KG & 1 \\
\hline
\end{tabular}


APPENDIX D.1 Results of Semivolatile Organic Compound Analyses, Soils, Salmon Site

\begin{tabular}{|c|c|c|c|}
\hline COMPOUND & RESULTS & UNITS & $\begin{array}{l}\text { DATA } \\
\text { QUAL. }\end{array}$ \\
\hline \multicolumn{4}{|l|}{ C2-22-S (continued) } \\
\hline 4-Bromophenyl phenylether & & 330 UG/KG & $u$ \\
\hline Hexachlorobenzene & & 330 UG/KG & $u$ \\
\hline Pentachlorophenol & & 600 UG/KG & $u$ \\
\hline Phenanthrene & & 330 UG/KG & $u$ \\
\hline Anthracene & & 330 UG/KG & $u$ \\
\hline Di-N-Butylphthalate & & 330 UG/KG & $u$ \\
\hline Fluoranthene & & 330 UG/KG & $u$ \\
\hline Pyrene & & 330 UG/KG & $u$ \\
\hline Butyl Benzyl Phthalate & & 330 UG/KG & $u$ \\
\hline 3,3'-Dichlorobenzidine & & 660 UG/KG & $u$ \\
\hline Benzo(a)Anthracene & & 330 UG/KG & $u$ \\
\hline Chrysene & & 330 UG/KG & $u$ \\
\hline bis(2-Ethylhexyl)phthalate & & 330 UG/KG & $u$ \\
\hline di-N-Octyl Phthalate & & 330 UG/KG & $u$ \\
\hline Benzo(b)Fluoranthene & & 330 UG/KG & $u$ \\
\hline Benzo(k)Fluoranthene & & 330 UG/KG & $u$ \\
\hline Benzo(a)Pyrene & & 330 UG/KG & $u$ \\
\hline Indeno(1,2,3-Cd)pyrene & & 330 UG/KG & $u$ \\
\hline Dibenzo(a,h)Anthracene & & 330 UG/KG & $u$ \\
\hline Benzo(g,h,i)Perylene & & 330 UG/KG & $u$ \\
\hline Aldol Condensation & & 100 UG/KG & BJ \\
\hline Unknown-1 & & 82 UG/KG & J \\
\hline Unknown-2 & & 110 UG/KG & $\mathrm{J}$ \\
\hline Nitrobenzene-d5 & & $65 \%$ & \\
\hline 2-Fluorobiphenyl & & $67 \%$ & \\
\hline Terphenyl-d14 & & $56 \%$ & \\
\hline Phenol-d5 & & $70 \%$ & \\
\hline 2-Fluorophenol & & $31 \%$ & \\
\hline 2,4,6-Tribromophenol & & $58 \%$ & \\
\hline
\end{tabular}


APPENDIX D.1 Results of Semivolatile Organic Compound Analyses, Soils, Salmon Site

\begin{tabular}{|c|c|c|c|c|c|}
\hline COMPOUND & RESULTS & $\begin{array}{l}\text { DATA } \\
\text { QUAL. }\end{array}$ & $\begin{array}{r}\text { DATA } \\
\text { VALID. } \\
\end{array}$ & CRDL UNITS & DILUTION \\
\hline \multicolumn{6}{|l|}{ C2-23-S } \\
\hline Phenol & 330 UG/KG & $u$ & NP & 330 UG/KG & 1 \\
\hline bis(2-Chloroethyl) ether & 330 UG/KG & $u$ & NP & 330 UG/KG & 1 \\
\hline 2-Chlorophenol & 330 UG/KG & $u$ & NP & 330 UG/KG & 1 \\
\hline 1,3-Dichlorobenzene & 330 UG/KG & $u$ & NP & 330 UG/KG & 1 \\
\hline 1,4-Dichlorobenzene & 330 UG/KG & $u$ & NP & 330 UG/KG & 1 \\
\hline Benzyl Alcohol & 330 UG/KG & $u$ & NP & 330 UG/KG & 1 \\
\hline 1,2-Dichlorobenzene & 330 UG/KG & $u$ & $N P$ & 330 UG/KG & 1 \\
\hline 2-Methylphenol & 330 UG/KG & $u$ & NP & 330 UG/KG & 1 \\
\hline bis(2-Chloroisopropyl) ether & 330 UG/KG & $u$ & NP & 330 UG/KG & 1 \\
\hline 4-Methylphenol & 330 UG/KG & $u$ & NP & 330 UG/KG & 1 \\
\hline N-Nitroso-Di-n-dipropylamine & 330 UG/KG & $u$ & NP & 330 UG/KG & 1 \\
\hline Hexachloroethane & 330 UG/KG & $u$ & NP & 330 UG/KG & 1 \\
\hline Nitrobenzene & 330 UG/KG & $u$ & NP & 330 UG/KG & 1 \\
\hline Isophorone & 330 UG/KG & $u$ & NP & 330 UG/KG & 1 \\
\hline 2-Nitrophenol & 330 UG/KG & $u$ & NP & 330 UG/KG & 1 \\
\hline 2,4-Dimethyiphenol & 330 UG/KG & $u$ & NP & 330 UG/KG & 1 \\
\hline Benzoic Acid & 1600 UG/KG & $u$ & NP & 1600 UG/KG & 1 \\
\hline bis(2Chloroethoxy)methane & 330 UG/KG & $u$ & NP & 330 UG/KG & 1 \\
\hline 2,4-Dichlorophenol & 330 UG/KG & $u$ & NP & 330 UG/KG & 1 \\
\hline 1,2,4-Trichlorobenzene & 330 UG/KG & $u$ & NP & 330 UG/KG & 1 \\
\hline Naphthalene & 330 UG/KG & $u$ & NP & 330 UG/KG & 1 \\
\hline 4-Chloroaniline & 330 UG/KG & $u$ & NP & 330 UG/KG & 1 \\
\hline Hexachlorobutadiene & 330 UG/KG & $u$ & NP & 330 UG/KG & 1 \\
\hline 4-Chloro-3-Methlyphenol & 330 UG/KG & $u$ & NP & 330 UG/KG & 1 \\
\hline 2-Methylnaphthalene & 330 UG/KG & $u$ & NP & 330 UG/KG & 1 \\
\hline Hexachlorocyclopentadiene & 330 UG/KG & $u$ & NP & 330 UG/KG & 1 \\
\hline 2,4,6-Trichlorophenol & 330 UG/KG & $u$ & NP & 330 UG/KG & 1 \\
\hline 2,4,5-Trichlorophenol & 1600 UG/KG & $u$ & NP & 1600 UG/KG & 1 \\
\hline 2-Chloronaphthalene & 330 UG/KG & $u$ & NP & 330 UG/KG & 1 \\
\hline 2-Nitroaniline & 1600 UG/KG & $u$ & NP & 1600 UG/KG & 1 \\
\hline Dimethyl Phthalate & 330 UG/KG & $u$ & NP & 330 UG/KG & 1 \\
\hline Acenaphthylene & 330 UG/KG & $u$ & NP & 330 UG/KG & 1 \\
\hline 2,6-Dinitrotoluene & 330 UG/KG & $u$ & NP & 330 UG/KG & 1 \\
\hline 3-Nitroaniline & 1600 UG/KG & $u$ & NP & 1600 UG/KG & 1 \\
\hline Acenaphthene & 330 UG/KG & $u$ & NP & 330 UG/KG & 1 \\
\hline 2,4-Dinitrophenol & 1600 UG/KG & $u$ & NP & 1600 UG/KG & 1 \\
\hline 4-Nitrophenoi & 1600 UG/KG & $u$ & NP & 1600 UG/KG & 1 \\
\hline Dibenzofuran & 330 UG/KG & $u$ & NP & 330 UG/KG & 1 \\
\hline 2,4-Dinitrotoluene & 330 UG/KG & $u$ & NP & 330 UG/KG & 1 \\
\hline Diethylphthalate & 330 UG/KG & $u$ & NP & 330 UG/KG & 1 \\
\hline 4-Chlorophenyl phenyl ether & 330 UG/KG & $u$ & NP & 330 UG/KG & 1 \\
\hline Fluorene & 330 UG/KG & $u$ & NP & 330 UG/KG & 1 \\
\hline 4-Nitroaniline & 1600 UG/KG & $u$ & NP & 1600 UG/KG & 1 \\
\hline 4,6-Dinitro-2-methylphenol & 1600 UG/KG & $u$ & NP & 1600 UG/KG & 1 \\
\hline N-Nitrosodiphenylamine & 330 UG/KG & $u$ & NP & 330 UG/KG & 1 \\
\hline
\end{tabular}




\section{APPENDIX D.1 Results of Semivolatile Organic Compound Analyses, Soils, Salmon Site}

\begin{tabular}{|c|c|c|c|c|c|c|}
\hline COMPOUND & RESULTS & $\begin{array}{l}\text { DATA } \\
\text { QUAL. }\end{array}$ & $\begin{array}{l}\text { DATA } \\
\text { VALID. }\end{array}$ & CRDL & UNITS & DILUTION \\
\hline \multicolumn{7}{|l|}{ C2-23-S (continued) } \\
\hline 4-Bromophenyl phenylether & 330 UG/KG & U & $N P$ & 330 & UG/KG & 1 \\
\hline Hexachlorobenzene & 330 UG/KG & $u$ & NP & 330 & UG/KG & 1 \\
\hline Pentachlorophenol & 1600 UG/KG & $u$ & NP & 1600 & UG/KG & 1 \\
\hline Phenanthrene & 330 UG/KG & $u$ & NP & 330 & UG/KG & 1 \\
\hline Anthracene & 330 UG/KG & $u$ & NP & 330 & UG/KG & 1 \\
\hline Di-N-Butylphthalate & 330 UG/KG & $u$ & NP & 3301 & UG/KG & 1 \\
\hline Fluoranthene & 330 UG/KG & $u$ & NP & 3301 & UG/KG & 1 \\
\hline Pyrene & 330 UG/KG & $u$ & NP & 330 & UG/KG & 1 \\
\hline Butyl Benzyl Phthalate & 330 UG/KG & $u$ & NP & 3301 & UG/KG & 1 \\
\hline 3,3'-Dichlorobenzidine & 660 UG/KG & $u$ & NP & 6601 & UG/KG & 1 \\
\hline Benzo(a)Anthracene & 330 UG/KG & $u$ & NP & 330 & UG/KG & 1 \\
\hline Chrysene & 330 UG/KG & $u$ & NP & 3301 & UG/KG & 1 \\
\hline bis(2-Ethylhexyl)phthalate & 330 UG/KG & $u$ & NP & 330 & UG/KG & 1 \\
\hline di-N-Octyl Phthalate & 330 UG/KG & $u$ & NP & 330 & UG/KG & 1 \\
\hline Benzo(b)Fluoranthene & 330 UG/KG & $u$ & NP & 330 & UG/KG & 1 \\
\hline Benzo(k)Fluoranthene & 330 UG/KG & $u$ & NP & 330 & UG/KG & 1 \\
\hline Benzo(a)Pyrene & 330 UG/KG & $u$ & NP & 330 & UG/KG & 1 \\
\hline Indeno(1,2,3-Cd)pyrene & 330 UG/KG & $u$ & NP & 330 & UG/KG & 1 \\
\hline Dibenzo(a,h)Anthracene & 330 UG/KG & $u$ & NP & 330 & UG/KG & 1 \\
\hline Benzo(g,h,i)Perylene & 330 UG/KG & $u$ & NP & 330 & UG/KG & 1 \\
\hline Aldol Condensation & 1100 UG/KG & BJ & NP & & UG/KG & 1 \\
\hline Unknown & 88 UG/KG & 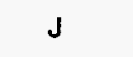 & NP & & UG/KG & 1 \\
\hline Nitrobenzene-d5 & $74 \%$ & & & & $\%$ & 1 \\
\hline 2-Fluorobiphenyl & $76 \%$ & & & & $\%$ & 1 \\
\hline Terphenyl-d14 & $63 \%$ & & & & $\%$ & 1 \\
\hline Phenol-d5 & $75 \%$ & & & & $\%$ & 1 \\
\hline 2-Fluorophenol & $21 \%$ & • & & & $\%$ & 1 \\
\hline 2,4,6-Tribromophenol & $69 \%$ & & & & $\%$ & 1 \\
\hline
\end{tabular}


APPENDIX D.1 Results of Semivolatile Organic Compound Analyses, Soils, Salmon Site

\begin{tabular}{|c|c|c|c|c|c|}
\hline COMPOUND & RESULTS & $\begin{array}{l}\text { DATA } \\
\text { QUAL. }\end{array}$ & $\begin{array}{c}\text { DATA } \\
\text { VALID. }\end{array}$ & CRDL UNITS & DILUTION \\
\hline \multicolumn{6}{|l|}{ C2-24-S } \\
\hline Phenol & 330 UG/KG & $u$ & NP & 330 UGKG & 1 \\
\hline bis(2-Chloroethyl) ether & 330 UG/KG & $u$ & NP & 330 UG/KG & 1 \\
\hline 2-Chlorophenol & 330 UG/KG & $u$ & NP & 330 UG/KG & 1 \\
\hline 1,3-Dichlorobenzene & 330 UG/KG & $u$ & NP & 330 UG/KG & 1 \\
\hline 1,4-Dichlorobenzene & 330 UG/KG & $u$ & NP & 330 UG/KG & 1 \\
\hline Benzyl Alcohol & 330 UG/KG & $u$ & NP & 330 UG/KG & 1 \\
\hline 1,2-Dichlorobenzene & 330 UG/KG & $u$ & NP & 330 UG/KG & 1 \\
\hline 2-Methylphenol & 330 UG/KG & $u$ & NP & 330 UG/KG & 1 \\
\hline bis(2-Chloroisopropyl) ether & 330 UG/KG & $u$ & NP & 330 UG/KG & 1 \\
\hline 4-Methylphenol & 330 UG/KG & $u$ & NP & 330 UG/KG & 1 \\
\hline N-Nitroso-Di-n-dipropylamine & 330 UG/KG & $u$ & NP & 330 UG/KG & 1 \\
\hline Hexachloroethane & 330 UG/KG & $u$ & NP & 330 UG/KG & 1 \\
\hline Nitrobenzene & 330 UG/KG & $u$ & NP & 330 UG/KG & 1 \\
\hline Isophorone & 330 UG/KG & $u$ & NP & 330 UG/KG & 1 \\
\hline 2-Nitrophenol & 330 UG/KG & $u$ & NP & 330 UG/KG & 1 \\
\hline 2,4-Dimethylphenol & 330 UG/KG & $u$ & NP & 330 UG/KG & 1 \\
\hline Benzoic Acid & 1600 UG/KG & $u$ & NP & 1600 UG/KG & 1 \\
\hline bis(2Chloroethoxy)methane & 330 UG/KG & $u$ & NP & 330 UG/KG & 1 \\
\hline 2,4-Dichiorophenol & 330 UG/KG & $u$ & NP & 330 UG/KG & 1 \\
\hline 1,2,4-Trichlorobenzene & 330 UG/KG & $u$ & NP & $330 \cup G / K G$ & 1 \\
\hline Naphthalene & 330 UG/KG & $u$ & NP & 330 UG/KG & 1 \\
\hline 4-Chloroaniline & 330 UG/KG & $u$ & NP & 330 UG/KG & 1 \\
\hline Hexachlorobutadiene & 330 UG/KG & $u$ & NP & 330 UG/KG & 1 \\
\hline 4-Chloro-3-Methlyphenol & 330 UG/KG & $u$ & NP & 330 UंG/KG & 1 \\
\hline 2-Methylnaphthalene & 330 UG/KG & $u$ & NP & 330 UG/KG & 1 \\
\hline Hexachlorocyclopentadiene & 330 UG/KG & $u$ & NP & 330 UG/KG & 1 \\
\hline 2,4,6-Trichlorophenol & 330 UG/KG & $u$ & NP & 330 UG/KG & 1 \\
\hline 2,4,5-Trichlorophenol & 1600 UG/KG & $u$ & NP & 1600 UG/KG & 1 \\
\hline 2-Chloronaphthalene & 330 UG/KG & $u$ & NP & 330 UG/KG & 1 \\
\hline 2-Nitroaniline & 1600 UG/KG & $u$ & NP & 1600 UG/KG & 1 \\
\hline Dimethyl Phthalate & 330 UG/KG & $u$ & NP & 330 UG/KG & 1 \\
\hline Acenaphthylene & 330 UG/KG & $u$ & NP & 330 UG/KG & 1 \\
\hline 2,6-Dinitrotoluene & 330 UG/KG & $u$ & NP & 330 UG/KG & 1 \\
\hline 3-Nitroaniline & 1600 UG/KG & $u$ & NP & 1600 UG/KG & 1 \\
\hline Acenaphthene & 330 UG/KG & $u$ & NP & 330 UG/KG & 1 \\
\hline 2,4-Dinitrophenol & 1600 UG/KG & $u$ & NP & 1600 UG/KG & 1 \\
\hline 4-Nitrophenol & 1600 UG/KG & $u$ & NP & 1600 UG/KG & 1 \\
\hline Dibenzofuran & 330 UG/KG & $u$ & NP & 330 UG/KG & 1 \\
\hline 2,4-Dinitrotoluene & 330 UG/KG & $u$ & NP & 330 UG/KG & 1 \\
\hline Diethylphthalate & 330 UG/KG & $u$ & NP & 330 UG/KG & 1 \\
\hline 4-Chlorophenyl phenyl ether & 330 UG/KG & $u$ & $\mathrm{NP}$ & 330 UG/KG & 1 \\
\hline Fluorene & 330 UG/KG & $u$ & NP & 330 UG/KG & 1 \\
\hline 4-Nitroaniline & 1600 UG/KG & $u$ & NP & 1600 UG/KG & 1 \\
\hline 4,6-Dinitro-2-methylphenol & 1600 UG/KG & $u$ & NP & 1600 UG/KG & 1 \\
\hline N-Nitrosodiphenylamine & 330 UG/KG & $u$ & NP & 330 UG/KG & 1 \\
\hline
\end{tabular}


APPENDIX D.1 Results of Semivolatile Organic Compound Analyses, Soils, Salmon Site

\begin{tabular}{|c|c|c|}
\hline COMPOUND & RESULTS & $\begin{array}{l}\text { DATA } \\
\text { QUAL. }\end{array}$ \\
\hline \multicolumn{3}{|l|}{ C2-24-S (continued) } \\
\hline 4-Bromophenyl phenylether & 330 UG/KG & $u$ \\
\hline Hexachlorobenzene & 330 UG/KG & $u$ \\
\hline Pentachlorophenol & 1600 UG/KG & $u$ \\
\hline Phenanthrene & 330 UG/KG & $u$ \\
\hline Anthracene & 330 UG/KG & $u$ \\
\hline Di-N-Butylphthalate & 330 UG/KG & $u$ \\
\hline Fluoranthene & 330 UG/KG & $u$ \\
\hline Pyrene & 330 UG/KG & $u$ \\
\hline Butyl Benzyl Phthalate & 330 UG/KG & $u$ \\
\hline 3,3'-Dichlorobenzidine & 660 UG/KG & $u$ \\
\hline Benzo(a)Anthracene & 330 UG/KG & $u$ \\
\hline Chrysene & 330 UG/KG & $u$ \\
\hline bis(2-Ethylhexyl)phthalate & 330 UG/KG & $u$ \\
\hline di-N-Octyl Phthalate & 330 UG/KG & $u$ \\
\hline Benzo(b)Fluoranthene & 330 UG/KG & $u$ \\
\hline Benzo(k)Fluoranthene & 330 UG/KG & $u$ \\
\hline Benzo(a)Pyrene & 330 UG/KG & $u$ \\
\hline Indeno(1,2,3-Cd)pyrene & 330 UG/KG & $u$ \\
\hline Dibenzo(a,h)Anthracene & 330 UG/KG & $u$ \\
\hline Benzo(g,h,i)Perylene & 330 UG/KG & $u$ \\
\hline Aldol Condensation & 1100 UG/KG & BJ \\
\hline Unknown-1 & 97 UG/KG & J \\
\hline Unknown-2 & 130 UG/KG & J \\
\hline Nitrobenzene-d5 & $81 \%$ & \\
\hline 2-Fluorobiphenyi & $75 \%$ & \\
\hline Terphenyl-d14 & $65 \%$ & \\
\hline Phenol-d5 & $78 \%$ & \\
\hline 2-Fluorophenol & $30 \%$ & \\
\hline 2,4,6-Tribromophenol & $70 \%$ & \\
\hline
\end{tabular}

\section{DATA}

VALID.

CRDL UNITS DILUTION

$\begin{array}{crr}\text { NP } & 330 \text { UG/KG } & 1 \\ \text { NP } & 330 \text { UG/KG } & 1 \\ \text { NP } & 1600 \text { UG/KG } & 1 \\ \text { NP } & 330 \text { UG/KG } & 1 \\ \text { NP } & 330 \text { UG/KG } & 1 \\ \text { NP } & 330 \text { UG/KG } & 1 \\ \text { NP } & 330 \text { UG/KG } & 1 \\ \text { NP } & 330 \text { UG/KG } & 1 \\ \text { NP } & 330 \text { UG/KG } & 1 \\ \text { NP } & 660 \text { UG/KG } & 1 \\ \text { NP } & 330 \text { UG/KG } & 1 \\ \text { NP } & 330 \text { UG/KG } & 1 \\ \text { NP } & 330 \text { UG/KG } & 1 \\ \text { NP } & 330 \text { UG/KG } & 1 \\ \text { NP } & 330 \text { UG/KG } & 1 \\ \text { NP } & 330 \text { UG/KG } & 1 \\ \text { NP } & 330 \text { UG/KG } & 1 \\ \text { NP } & 330 \text { UG/KG } & 1 \\ \text { NP } & 330 \text { UG/KG } & 1 \\ \text { NP } & 330 \text { UG/KG } & 1 \\ \text { NP } & \text { UG/KG } & 1 \\ \text { NP } & \text { UG/KG } & 1 \\ \text { NP } & \text { UG/KG } & 1 \\ & \% & 1 \\ & \% & 1 \\ & \% & 1 \\ & \% & 1 \\ & \% & 1\end{array}$


APPENDIX D.1 Results of Semivolatile Organic Compound Analyses, Soils, Salmon Site

\begin{tabular}{|c|c|c|c|c|c|}
\hline COMPOUND & RESULTS & $\begin{array}{l}\text { DATA } \\
\text { QUAL. }\end{array}$ & $\begin{array}{l}\text { DATA } \\
\text { VALID. } \\
\end{array}$ & CRDL UNITS & DILUTION \\
\hline \multicolumn{6}{|l|}{ C2-25-S } \\
\hline Phenol & 330 UG/KG & $u$ & NP & 330 UG/KG & 1 \\
\hline bis(2-Chloroethyl) ether & 330 UG/KG & $u$ & NP & 330 UG/KG & 1 \\
\hline 2-Chlorophenol & 330 UG/KG & $u$ & NP & 330 UG/KG & 1 \\
\hline 1,3-Dichlorobenzene & 330 UG/KG & $u$ & NP & 330 UG/KG & 1 \\
\hline 1,4-Dichlorobenzene & 330 UG/KG & $u$ & NP & 330 UG/KG & 1 \\
\hline Benzyl Alcohol & 330 UG/KG & $u$ & NP & $330 \mathrm{UG} / \mathrm{KG}$ & 1 \\
\hline 1,2-Dichlorobenzene & 330 UG/KG & $u$ & NP & 330 UG/KG & 1 \\
\hline 2-Methylphenol & 330 UG/KG & $u$ & NP & 330 UG/KG & 1 \\
\hline bis(2-Chloroisopropyl) ether & 330 UG/KG & $u$ & NP & 330 UG/KG & 1 \\
\hline 4-Methylphenol & 330 UG/KG & $u$ & NP & 330 UG/KG & 1 \\
\hline N-Nitroso-Di-n-dipropylamine & 330 UG/KG & $u$ & NP & 330 UG/KG & 1 \\
\hline Hexachloroethane & 330 UG/KG & $u$ & NP & 330 UG/KG & 1 \\
\hline Nitrobenzene & 330 UG/KG & $u$ & NP & 330 UG/KG & 1 \\
\hline Isophorone & 330 UG/KG & $u$ & NP & 330 UG/KG & 1 \\
\hline 2-Nitrophenol & 330 UG/KG & $u$ & NP & 330 UG/KG & 1 \\
\hline 2,4-Dimethyiphenol & 330 UG/KG & $u$ & NP & 330 UG/KG & 1 \\
\hline Benzoic Acid & 1600 UG/KG & $u$ & NP & 1600 UG/KG & 1 \\
\hline bis(2Chloroethoxy)methane & 330 UG/KG & $u$ & NP & 330 UG/KG & 1 \\
\hline 2,4-Dichlorophenol & 330 UG/KG & $u$ & NP & 330 UG/KG & 1 \\
\hline 1,2,4-Trichlorobenzene & 330 UG/KG & $u$ & NP & 330 UG/KG & 1 \\
\hline Naphthalene & 330 UG/KG & $u$ & NP & 330 UG/KG & 1 \\
\hline 4-Chloroaniline & 330 UG/KG & $u$ & NP & 330 UG/KG & 1 \\
\hline Hexachlorobutadiene & 330 UG/KG & $u$ & NP & 330 UG/KG & 1 \\
\hline 4-Chloro-3-Methlyphenol & 330 UG/KG & $u$ & NP & 330 UG/KG & 1 \\
\hline 2-Methylnaphthalene & 330 UG/KG & $u$ & NP & 330 UG/KG & 1 \\
\hline Hexachlorocyclopentadiene & 330 UG/KG & $u$ & NP & 330 UG/KG & 1 \\
\hline 2,4,6-Trichlorophenol & 330 UG/KG & $u$ & NP & 330 UG/KG & 1 \\
\hline 2,4,5-Trichlorophenol & 1600 UG/KG & $u$ & NP & 1600 UG/KG & 1 \\
\hline 2-Chloronaphthalene & 330 UG/KG & $u$ & NP & 330 UG/KG & 1 \\
\hline 2-Nitroaniline & 1600 UG/KG & $u$ & NP & 1600 UG/KG & 1 \\
\hline Dimethyl Phthalate & 330 UG/KG & $u$ & NP & 330 UG/KG & 1 \\
\hline Acenaphthylene & 330 UG/KG & $u$ & NP & $330 \mathrm{UG} / \mathrm{KG}$ & 1 \\
\hline 2,6-Dinitrotoluene & 330 UG/KG & $u$ & NP & 330 UG/KG & 1 \\
\hline 3-Nitroaniline & 1600 UG/KG & $u$ & NP & 1600 UG/KG & 1 \\
\hline Acenaphthene & 330 UG/KG & $u$ & NP & 330 UG/KG & 1 \\
\hline 2,4-Dinitrophenol & 1600 UG/KG & $u$ & NP & 1600 UG/KG & 1 \\
\hline 4-Nitrophenol & 1600 UG/KG & $u$ & NP & 1600 UG/KG & 1 \\
\hline Dibenzofuran & 330 UG/KG & $u$ & NP & 330 UG/KG & 1 \\
\hline 2,4-Dinitrotoluene & 330 UG/KG & $u$ & NP & 330 UG/KG & 1 \\
\hline Diethyiphthalate & 330 UG/KG & $u$ & NP & 330 UG/KG & 1 \\
\hline 4-Chlorophenyl phenyl ether & 330 UG/KG & $u$ & NP & 330 UG/KG & 1 \\
\hline Fluorene & 330 UG/KG & $u$ & NP & 330 UG/KG & 1 \\
\hline 4-Nitroaniline & 1600 UG/KG & $u$ & NP & 1600 UG/KG & 1 \\
\hline 4,6-Dinitro-2-methylphenol & 1600 UG/KG & $u$ & NP & 1600 UG/KG & 1 \\
\hline N-Nitrosodiphenylamine & 330 UG/KG & $u$ & NP & 330 UG/KG & 1 \\
\hline
\end{tabular}


APPENDIX D.1 Results of Semivolatile Organic Compound Analyseș, Soils, Salmon Site

\begin{tabular}{|c|c|c|c|c|c|c|}
\hline COMPOUND & RESULTS & $\begin{array}{l}\text { DATA } \\
\text { QUAL. }\end{array}$ & $\begin{array}{l}\text { DATA } \\
\text { VALID. }\end{array}$ & CRDL & UNITS & DILUTION \\
\hline \multicolumn{7}{|l|}{ C2-25-S (continued) } \\
\hline 4-Bromophenyl phenylether & 330 UG/KG & $u$ & NP & 330 & JG/KG & 1 \\
\hline Hexachlorobenzene & 330 UG/KG & $u$ & NP & 330 & JG/KG & 1 \\
\hline Pentachlorophenol & 1600 UG/KG & $u$ & NP & 1600 & UG/KG & 1 \\
\hline Phenanthrene & 330 UG/KG & $u$ & NP & 330 & UG/KG & 1 \\
\hline Anthracene & 330 UG/KG & $u$ & NP & 330 & UG/KG & 1 \\
\hline Di-N-Butylphthalate & $330 \mathrm{UG} / \mathrm{KG}$ & $u$ & NP & 330 & UG/KG & 1 \\
\hline Fiuoranthene & 330 UG/KG & $u$ & NP & 330 & UG/KG & 1 \\
\hline Pyrene & 330 UG/KG & $u$ & NP & 330 & UG/KG & 1 \\
\hline Butyl Benzyl Phthalate & 330 UG/KG & $u$ & NP & 330 & UG/KG & 1 \\
\hline 3,3'-Dichlorobenzidine & 660 UG/KG & $u$ & NP & 660 & UG/KG & 1 \\
\hline Benzo(a)Anthracene & 330 UG/KG & $u$ & NP & 330 & UG/KG & 1 \\
\hline Chrysene & 330 UG/KG & $u$ & NP & 330 & UG/KG & 1 \\
\hline bis(2-Ethylhexyl)phthalate , & 330 UG/KG & $u$ & NP & 330 & UG/KG & 1 \\
\hline di-N-Octyl Phthalate & 330 UG/KG & $u$ & NP & 330 & UG/KG & 1 \\
\hline Benzo(b)Fluoranthene & 330 UG/KG & $u$ & NP & 330 & UG/KG & 1 \\
\hline Benzo(k)Fluoranthene & 330 UG/KG & $u$ & NP & 330 & UG/KG & 1 \\
\hline Benzo(a)Pyrene & 330 UG/KG & $u$ & NP & 330 & UG/KG & 1 \\
\hline Indeno(1,2,3-Cd)pyrene & 330 UG/KG & $u$ & NP & 330 & UG/KG & 1 \\
\hline Dibenzo(a,h)Anthracene & 330 UG/KG & $u$ & NP & 330 & UG/KG & 1 \\
\hline Benzo(g,h,i)Perylene & 330 UG/KG & $u$ & NP & 330 & UG/KG & 1 \\
\hline Aldol Condensation & 1000 UG/KG & BJ & NP & & UG/KG & 1 \\
\hline Unknown-1 & 100 UG/KG & $J$ & NP & & UG/KG & 1 \\
\hline Unknown-2 & 120 UG/KG & $J$ & NP & & UG/KG & 1 \\
\hline Nitrobenzene-d5 & $67 \%$ & & & & $\%$ & 1 \\
\hline 2-Fluorobiphenyl & $72 \%$ & & & & $\%$ & 1 \\
\hline Terphenyl-d14 & $62 \%$ & & & & $\%$ & 1 \\
\hline Phenol-d5 & $69 \%$ & & & & $\%$ & 1 \\
\hline 2-Fluorophenol & $18 \%$ & - & & & $\%$ & 1 \\
\hline 2,4,6-Tribromophenol & $63 \%$ & & & & $\%$ & 1 \\
\hline
\end{tabular}


APPENDIX D.1 Results of Semivolatile Organic Compound Analyses, Soils, Salmon Site

\begin{tabular}{|llllll}
\hline COMPOUND & DESULTS & UNITS & DUTA & VALID. CRDL UNITS DILUTION \\
\hline
\end{tabular}

\section{C2-25A-S}

Phenol

bis(2-Chloroethyl) ether

2-Chlorophenol

1,3-Dichlorobenzene

1,4-Dichlorobenzene

Benzyl Alcohol

1,2-Dichlorobenzene

2-Methylphenol

bis(2-Chloroisopropyl) ether

4-Methylphenol

N-Nitroso-Di-n-dipropylamine

Hexachloroethane

Nitrobenzene

Isophorone

2-Nitrophenol

2,4-Dimethylphenol

Benzoic Acid

bis(2Chloroethoxy)methane

2,4-Dichlorophenol

1,2,4-Trichlorobenzene

Naphthalene

4-Chloroaniline

Hexachlorobutadiene

4-Chloro-3-Methlyphenol

2-Methyinaphthalene

Hexachlorocyclopentadiene

2,4,6-Trichlorophenol

2,4,5-Trichlorophenol

2-Chloronaphthalene

2-Nitroaniline

Dimethyl Phthalate

Acenaphthylene

2,6-Dinitrotoluene

3-Nitroaniline

Acenaphthene

2,4-Dinitrophenol

4-Nitrophenol

Dibenzofuran

2,4-Dinitrotoluene

Diethylphthalate

4-Chlorophenyl phenyl ether

Fluorene

4-Nitroaniline

4,6-Dinitro-2-methylphenol

$\mathrm{N}$-Nitrosodiphenylamine

330 UG/KG
330 UG/KG
330 UG/KG
330 UG/KG
330 UG/KG
330 UG/KG
330 UG/KG
330 UG/KG
330 UG/KG
330 UG/KG
330 UG/KG
330 UG/KG
330 UG/KG
330 UG/KG
330 UG/KG
330 UG/KG
1600 UG/KG
330 UG/KG
330 UG/KG
330 UG/KG
330 UG/KG
330 UG/KG
330 UG/KG
330 UG/KG
330 UG/KG
330 UG/KG
330 UG/KG
1600 UG/KG
330 UG/KG
1600 UG/KG
330 UG/KG
330 UG/KG
330 UG/KG
1600 UG/KG
1600 UG/KG
330 UG/KG
1600 UG/KG
1600 UG/KG
330 UG/KG
330 UG/KG
330 UG/KG
330 UG/KG

\section{NP}

NP

NP

NP

NP

NP

NP

NP

NP

NP

NP

NP

NP

NP

NP

NP

NP

NP

NP

NP

NP

NP

NP

NP

NP

NP

NP

NP

NP

NP

NP

NP

NP

NP

NP

NP

NP

NP

NP

NP

NP

NP

NP

NP

NP
330 UG/KG 1

330 UG/KG 1

330 UG/KG 1

330 UG/KG 1

330 UG/KG 1

330 UG/KG 1

330 UG/KG 1

330 UG/KG 1

330 UG/KG 1

330 UG/KE 1

330 UG/KG 1

330 UG/KG 1

330 UG/KG 1

330 UG/KG 1

330 UG/KG 1

330 UG/KG 1

1600 UG/KG 1

330 UG/KG 1

330 UG/KG $\quad 1$

330 UG/KG 1

330 UG/KG

330 UG/KG

330 UG/KG

330 UG/KG

330 UG/KG

330 UG/KG

330 UG/KG

1600 UG/KG

330 UG/KG

1600 UG/KG

330 UG/KG

330 UG/KG

330 UG/KG

1600 UG/KG

330 UG/KG

1600 UG/KG

1600 UG/KG

330 UG/KG

330 UG/KG

330 UG/KG

330 UG/KG

330 UG/KG

1600 UG/KG

1600 UG/KG

330 UG/KG

1


APPENDIX D.1 Results of Semivolatile Organic Compound Analyses, Soils, Salmon Site

\begin{tabular}{|c|c|c|c|c|c|c|}
\hline COMPOUND & RESULTS & $\begin{array}{l}\text { DATA } \\
\text { QUAL. }\end{array}$ & $\begin{array}{l}\text { DATA } \\
\text { VALID. }\end{array}$ & CRDL & UNITS & DILUTION \\
\hline \multicolumn{7}{|l|}{ C2-25A-S (continued) } \\
\hline 4-Bromophenyl phenylether & 330 UG/KG & $u$ & NP & 330 & UG/KG & 1 \\
\hline Hexachlorobenzene & 330 UG/KG & $u$ & NP & 330 & UG/KG & 1 \\
\hline Pentachlorophenol & 1600 UG/KG & $u$ & NP & 1600 & UG/KG & 1 \\
\hline Phenanthrene & 330 UG/KG & $u$ & NP & 330 & UG/KG & 1 \\
\hline Anthracene & 330 UG/KG & $u$ & NP & 330 & UG/KG & 1 \\
\hline Di-N-Butylphthalate & $330 \mathrm{UG} / \mathrm{KG}$ & $u$ & NP & 330 & UG/KG & 1 \\
\hline Fluoranthene & 330 UG/KG & $u$ & NP & 330 & UG/KG & 1 \\
\hline Pyrene & 330 UG/KG & $u$ & NP & 330 & UG/KG & 1 \\
\hline Butyl Benzyl Phthalate & 330 UG/KG & $u$ & NP & 330 & UG/KG & 1 \\
\hline 3,3'-Dichlorobenzidine & 660 UG/KG & $u$ & NP & 660 & UG/KG & 1 \\
\hline Benzo(a)Anthracene & 330 UG/KG & $u$ & NP & 330 & UG/KG & 1 \\
\hline Chrysene & 330 UG/KG & $u$ & NP & 330 & UG/KG & 1 \\
\hline bis(2-Ethylhexyl)phthalate & 330 UG/KG & $u$ & NP & 330 & UG/KG & 1 \\
\hline di-N-Octyl Phthalate & 330 UG/KG & $u$ & NP & 330 & UG/KG & 1 \\
\hline Benzo(b)Fluoranthene & 330 UG/KG & $u$ & NP & 330 & $\mathrm{UG} / \mathrm{KG}$ & 1 \\
\hline Benzo(k)Fluoranthene & 330 UG/KG & $u$ & NP & 330 & UG/KG & 1 \\
\hline Benzo(a)Pyrene & 330 UG/KG & $u$ & NP & 330 & UG/KG & 1 \\
\hline Indeno(1,2,3-Cd)pyrene & 330 UG/KG & $u$ & NP & 330 & UG/KG & 1 \\
\hline Dibenzo(a,h)Anthracene & 330 UG/KG & $u$ & NP & 330 & UG/KG & 1 \\
\hline Benzo $(g, h, i)$ Perylene & 330 UG/KG & $u$ & NP & 330 & UG/KG & 1 \\
\hline Aldol Condensation & 1300 UG/KG & BJ & NP & & UG/KG & 1 \\
\hline Unknown-1 & 110 UG/KG & $J$ & NP & & UG/KG & 1 \\
\hline Unknown-2 & 91 UG/KG & $J$ & NP & & UG/KG & 1 \\
\hline Unknown-3 & 150 UG/KG & $J$ & NP & & UG/KG & 1 \\
\hline Nitrobenzene-d5 & $80 \%$ & & & & $\%$ & 1 \\
\hline 2-Fluorobiphenyl & $78 \%$ & & & & $\%$ & 1 \\
\hline Terphenyl-d14 & $66 \%$ & & & & $\%$ & 1 \\
\hline Phenol-d5 & $80 \%$ & & & & $\%$ & 1 \\
\hline 2-Fluorophenol & $32 \%$ & & & & $\%$ & 1 \\
\hline 2,4,6-Tribromophenol & $75 \%$ & & & & $\%$ & 1 \\
\hline
\end{tabular}


APPENDIX D.1 Results of Semivolatile Organic Compound Analyses, Soils, Salmon Site

\begin{tabular}{|c|c|c|c|c|c|c|}
\hline COMPOUND & RESULTS & $\begin{array}{l}\text { DATA } \\
\text { QUAL. }\end{array}$ & $\begin{array}{l}\text { DATA } \\
\text { VALID. }\end{array}$ & CRDL & UNITS & DILUTION \\
\hline \multicolumn{7}{|l|}{ C2-26-S } \\
\hline Phenol & 330 UG/KG & $u$ & NP & 330 & UG/KG & 1 \\
\hline bis(2-Chloroethyl) ether & 330 UG/KG & $u$ & NP & 330 & UG/KG & 1 \\
\hline 2-Chlorophenol & 330 UG/KG & $u$ & NP & 330 & UG/KG & 1 \\
\hline 1,3-Dichlorobenzene & 330 UG/KG & $u$ & NP & 330 & UG/KG & 1 \\
\hline 1,4-Dichlorobenzene & 330 UG/KG & $u$ & NP & 330 & UG/KG & 1 \\
\hline Benzyl Alcohol & 330 UG/KG & $u$ & NP & 330 & UG/KG & 1 \\
\hline 1,2-Dichlorobenzene & 330 UG/KG & $u$ & NP & 330 & UG/KG & 1 \\
\hline 2-Methyiphenol & 330 UG/KG & $u$ & NP & 330 & UG/KG & 1 \\
\hline bis(2-Chloroisopropyl) ether & 330 UG/KG & $u$ & NP & 330 & UG/KG & 1 \\
\hline 4-Methyiphenol & 330 UG/KG & $u$ & NP & 330 & UG/KG & 1 \\
\hline N-Nitroso-Di-n-dipropylamine & 330 UG/KG & $u$ & NP & 330 & UG/KG & 1 \\
\hline Hexachloroethane & 330 UG/KG & $u$ & NP & 330 & UG/KG & 1 \\
\hline Nitrobenzene & 330 UG/KG & $u$ & NP & 330 & UG/KG & 1 \\
\hline Isophorone & 330 UG/KG & $u$ & NP & 330 & UG/KG & 1 \\
\hline 2-Nitrophenol & 330 UG/KG & $u$ & NP & 330 & UG/KG & 1 \\
\hline 2,4-Dimethylphenol & 330 UG/KG & $u$ & NP & 330 & UG/KG & 1 \\
\hline Benzoic Acid & 1600 UG/KG & $u$ & NP & 1600 & $\mathrm{UG} / \mathrm{KG}$ & 1 \\
\hline bis(2Chloroethoxy)methane & 330 UG/KG & $u$ & NP & 330 & UG/KG & 1 \\
\hline 2,4-Dichlorophenol & 330 UG/KG & $u$ & NP & 330 & UG/KG & 1 \\
\hline 1,2,4-Trichlorobenzene & $330 \mathrm{UG} / \mathrm{KG}$ & $u$ & NP & 330 & UG/KG & 1 \\
\hline Naphthalene & 330 UG/KG & $u$ & NP & 330 & UG/KG & 1 \\
\hline 4-Chloroaniline & 330 UG/KG & $u$ & NP & 330 & UG/KG & 1 \\
\hline Hexachlorobutadiene & 330 UG/KG & $u$ & NP & 330 & UG/KG & 1 \\
\hline 4-Chloro-3-Methlyphenol & 330 UG/KG & $u$ & NP & 330 & UG/KG & 1 \\
\hline 2-Methylnaphthalene & 330 UG/KG & $u$ & NP & 330 & UG/KG & 1 \\
\hline Hexachlorocyclopentadiene & 330 UG/KG & $u$ & NP & 330 & UG/KG & 1 \\
\hline 2,4,6-Trichlorophenol & 330 UG/KG & $u$ & NP & 330 & UG/KG & 1 \\
\hline 2,4,5-Trichlorophenol & 1600 UG/KG & $u$ & NP & 1600 & UG/KG & 1 \\
\hline 2-Chloronaphthalene & 330 UG/KG & $u$ & NP & 330 & UG/KG & 1 \\
\hline 2-Nitroaniline & 1600 UG/KG & $u$ & NP & 1600 & UG/KG & 1 \\
\hline Dimethyl Phthalate & 330 UG/KG & $u$ & NP & 330 & UG/KG & 1 \\
\hline Acenaphthylene & 330 UG/KG & $u$ & NP & 330 & UGKG & 1 \\
\hline 2,6-Dinitrotoluene & 330 UG/KG & $u$ & NP & 330 & UG/KG & 1 \\
\hline 3-Nitroaniline & 1600 UG/KG & $u$ & NP & 1600 & UG/KG & 1 \\
\hline Acenaphthene & 330 UG/KG & $u$ & NP & 330 & UG/KG & 1 \\
\hline 2,4-Dinitrophenol & 1600 UG/KG & $u$ & NP & 1600 & UG/KG & 1 \\
\hline 4-Nitrophenol & 1600 UG/KG & $u$ & NP & 1600 & UG/KG & 1 \\
\hline Dibenzofuran & 330 UG/KG & $u$ & NP & 330 & UG/KG & 1 \\
\hline 2,4-Dinitrotoluene & 330 UG/KG & $u$ & $\mathrm{NP}$ & 330 & UG/KG & 1 \\
\hline Diethylphthalate & 330 UG/KG & $u$ & NP & 330 & UG/KG & 1 \\
\hline 4-Chlorophenyl phenyl ether & 330 UG/KG & $u$ & NP & 330 & UG/KG & 1 \\
\hline Fluorene & 330 UG/KG & $u$ & NP & 330 & UG/KG & 1 \\
\hline 4-Nitroaniline & 1600 UG/KG & $u$ & NP & 1600 & UG/KG & 1 \\
\hline 4,6-Dinitro-2-methylphenol & 1600 UG/KG & $u$ & NP & 1600 & UG/KG & 1 \\
\hline N-Nitrosodiphenylamine & 330 UG/KG & $u$ & NP & 330 & UG/KG & 1 \\
\hline
\end{tabular}


APPENDIX D.1 Results of Semivolatile Organic Compound Analyses, Soils, Salmon Site

\begin{tabular}{|c|c|c|c|c|c|c|}
\hline COMPOUND & RESULTS & $\begin{array}{l}\text { DATA } \\
\text { QUAL. }\end{array}$ & $\begin{array}{l}\text { DATA } \\
\text { VALID. }\end{array}$ & CRDL & UNITS & DILUTION \\
\hline \multicolumn{7}{|l|}{ C2-26-S (continued) } \\
\hline 4-Bromophenyl phenylether & 330 UG/KG & $u$ & NP & 330 & UG/KG & 1 \\
\hline Hexachlorobenzene & 330 UG/KG & $u$ & NP & 330 & UG/KG & 1 \\
\hline Pentachlorophenol & 1600 UG/KG & $u$ & NP & 1600 & UG/KG & 1 \\
\hline Phenanthrene & 330 UG/KG & $u$ & NP & 330 & UG/KG & 1 \\
\hline Anthracene & 330 UG/KG & $u$ & NP & 330 & UG/KG & 1 \\
\hline Di-N-Butylphthalate & 330 UG/KG & $u$ & NP & 330 & UG/KG & 1 \\
\hline Fluoranthene & 330 UG/KG & $u$ & NP & 330 & UG/KG & 1 \\
\hline Pyrene & 330 UG/KG & $u$ & NP & 330 & UG/KG & 1 \\
\hline Butyl Benzyl Phthalate & 330 UG/KG & $u$ & NP & 330 & UG/KG & 1 \\
\hline 3,3'-Dichlorobenzidine & 660 UG/KG & $u$ & NP & 660 & UG/KG & 1 \\
\hline Benzo(a)Anthracene & $330 \mathrm{UG} / \mathrm{KG}$ & $u$ & NP & 330 & UG/KG & 1 \\
\hline Chrysene & $330 \mathrm{UG} / \mathrm{KG}$ & $u$ & NP & 330 & UG/KG & 1 \\
\hline bis(2-Ethylhexyl)phthalate & 330 UG/KG & $u$ & NP & 330 & UG/KG & 1 \\
\hline di-N-Octyl Phthalate & 330 UG/KG & $u$ & NP & 330 & UG/KG & 1 \\
\hline Benzo(b)Fluoranthene & 330 UG/KG & $u$ & NP & 330 & UG/KG & 1 \\
\hline Benzo(k)Fluoranthene & 330 UG/KG & $u$ & NP & 330 & UG/KG & 1 \\
\hline Benzo(a)Pyrene & 330 UG/KG & $u$ & NP & 330 & UG/KG & 1 \\
\hline Indeno(1,2,3-Cd)pyrene & 330 UG/KG & $u$ & NP & 330 & UG/KG & 1 \\
\hline Dibenzo(a,h)Anthracene & $330 \mathrm{UG} / \mathrm{KG}$ & $u$ & NP & 330 & UG/KG & 1 \\
\hline Benzo(g,h,i)Perylene & 330 UG/KG & $u$ & NP & 330 & UG/KG & 1 \\
\hline Aldol Condensation & 1100 UG/KG & BJ & NP & & UG/KG & 1 \\
\hline Unknown-1 & 73 UG/KG & J & NP & & UG/KG & 1 \\
\hline Unknown-2 & 68 UG/KG & $J$ & NP & & UG/KG & 1 \\
\hline Unknown-3 & 110 UG/KG & J & NP & & UG/KG & 1 \\
\hline Nitrobenzene-d5 & $68 \%$ & & & & $\%$ & 1 \\
\hline 2-Fluorobiphenyl & $71 \%$ & & & & $\%$ & 1 \\
\hline Terphenyl-d14 & $59 \%$ & & & & $\%$ & 1 \\
\hline Phenol-d5 & $74 \%$ & & & & $\%$ & 1 \\
\hline 2-Fluorophenol & $29 \%$ & & & & $\%$ & 1 \\
\hline 2,4,6-Tribromophenol & $69 \%$ & & & & $\%$ & 1 \\
\hline
\end{tabular}


APPENDIX D.1 Results of Semivolatile Organic Compound Analyses, Soils, Salmon Site

\begin{tabular}{|c|c|c|c|c|c|c|}
\hline COMPOUND & RESULTS & $\begin{array}{l}\text { DATA } \\
\text { QUAL. }\end{array}$ & $\begin{array}{l}\text { DATA } \\
\text { VALID. } \\
\end{array}$ & CRDL & UNITS & DILUTION \\
\hline \multicolumn{7}{|l|}{ C2-27-S } \\
\hline Phenol & $330 \mathrm{UG} / \mathrm{L}$ & $u$ & NP & 330 & UG/ & 1 \\
\hline bis(2-Chloroethyl) ether & 330 UG $\Omega$ & $u$ & NP & 330 & $U G / L$ & 1 \\
\hline 2-Chlorophenol & $330 \mathrm{UG} / \mathrm{L}$ & $u$ & NP & 330 & UG $/$ & 1 \\
\hline 1,3-Dichlorobenzene & 330 UG $/$ & $u$ & NP & 330 & UGR & 1 \\
\hline 1,4-Dichlorobenzene & 330 UG & $u$ & NP & 330 & $\mathrm{UG} / \mathrm{L}$ & 1 \\
\hline Benzyl Alcohol & 330 UG几 & $u$ & NP & 330 & UG/ & 1 \\
\hline 1,2-Dichlorobenzene & 330 UG/L & $u$ & NP & 330 & UGR & 1 \\
\hline 2-Methylphenol & 330 UG & $u$ & NP & 330 & UG/ & 1 \\
\hline bis(2-Chloroisopropyl) ether & 330 UG & $u$ & NP & 330 & UG $\Omega$ & 1 \\
\hline 4-Methylphenol & $330 \mathrm{UG} / \mathrm{L}$ & $u$ & NP & 330 & UG/ & 1 \\
\hline N-Nitroso-Di-n-dipropylamine & 330 UG/ & $u$ & NP & 330 & UG $\Omega$ & 1 \\
\hline Hexachloroethane & 330 UG $/$ & $u$ & NP & 330 & UG/L & 1 \\
\hline Nitrobenzene & 330 UG $\Omega$ & $u$ & NP & 330 & UG $\Omega$ & 1 \\
\hline Isophorone & 330 UG/L & $u$ & NP & 330 & UGR & 1 \\
\hline 2-Nitrophenol & $330 \mathrm{UG} / \mathrm{L}$ & $u$ & NP & 330 & UG $\Omega$ & 1 \\
\hline 2,4-Dimethylphenol & $330 \mathrm{UG} / \mathrm{L}$ & $u$ & NP & 330 & UG/L & 1 \\
\hline Benzoic Acid & 1600 UG/L & $u$ & NP & 1600 & UG/L & 1 \\
\hline bis(2Chloroethoxy)methane & $330 \mathrm{UG} / \mathrm{L}$ & $u$ & NP & 330 & UG几 & 1 \\
\hline 2,4-Dichlorophenol & 330 UG & $u$ & NP & 330 & UG/L & 1 \\
\hline 1,2,4-Trichlorobenzene & 330 UG/L & $u$ & NP & 330 & UG/L & 1 \\
\hline Naphthalene & 330 UG/ & $u$ & NP & 330 & UG $\Omega$ & 1 \\
\hline 4-Chloroaniline & 330 UG $/$. & $u$ & NP & 330 & UGR & 1 \\
\hline Hexachlorobutadiene & 330 UG/L & $u$ & NP & 330 & UG $\Omega$ & 1 \\
\hline 4-Chloro-3-Methlyphenol & 330 UG/L & $u$ & NP & 330 & UG/L & 1 \\
\hline 2-Methylnaphthalene & 330 UG/L & $u$ & $\mathrm{NP}$ & 330 & UG/L & 1 \\
\hline Hexachlorocyclopentadiene & 330 UG $\Omega$ & $u$ & NP & 330 & UG几 & 1 \\
\hline 2,4,6-Tribromophenol & 330 UG $\Omega$ & $u$ & NP & 330 & UG/L & 1 \\
\hline 2,4,5-Trichlorophenol & 1600 UG $\Omega$ & $u$ & NP & 1600 & UG/L & 1 \\
\hline 2-Chloronaphthalene & 330 UG $\Omega$ & $u$ & NP & 330 & UG几 & 1 \\
\hline 2-Nitroaniline & 1600 UG/L & $u$ & $N P$ & 1600 & $U G /$ & 1 \\
\hline Dimethyl Phthalate & 330 UG $\Omega$ & $u$ & $N P$ & 330 & UG/L & 1 \\
\hline Acenaphthylene & $330 \mathrm{UG} \Omega$ & $u$ & NP & 330 & UG $\Omega$ & 1 \\
\hline 2,6-Dinitrotoluene & 330 UG $\Omega$ & $u$ & NP & 330 & UG $\Omega$ & 1 \\
\hline 3-Nitroaniline & 1600 UG/L & $u$ & NP & 1600 & UG几 & 1 \\
\hline Acenaphthene & 330 UG/L & $u$ & NP & 330 & UG $\Omega$ & 1 \\
\hline 2,4-Dinitrophenol & $1600 \mathrm{UG} / \mathrm{L}$ & $u$ & NP & 1600 & UG $\Omega$ & 1 \\
\hline 4-Nitrophenol & $1600 \mathrm{UG} / \mathrm{L}$ & $u$ & NP & 1600 & UG $\Omega$ & 1 \\
\hline Dibenzofuran & $330 \cup G /$ & $u$ & NP & 330 & UG/ & 1 \\
\hline 2,4-Dinitrotoluene & 330 UG/L & $u$ & NP & 330 & UG/ & 1 \\
\hline Diethylphthalate & $330 \mathrm{UG} / \mathrm{L}$ & $u$ & NP & 330 & UG/ & 1 \\
\hline 4-Chlorophenyl phenyl ether & 330 UG/ & $u$ & NP & 330 & UG几 & 1 \\
\hline Fluorene & 330 UG $\Omega$ & $u$ & NP & 330 & UGR & 1 \\
\hline 4-Nitroaniline & 1600 UG $\Omega$ & $u$ & NP & 1600 & UG $\Omega$ & 1 \\
\hline 4,6-Dinitro-2-methylphenol & 1600 UG $/$ & $u$ & NP & 1600 & UG $\Omega$ & 1 \\
\hline N-Nitrosodiphenylamine & $330 \mathrm{UG} / \mathrm{L}$ & $u$ & NP & 330 & UG/ & 1 \\
\hline
\end{tabular}


APPENDIX D.1 Results of Semivolatile Organic Compound Analyses, Soils, Salmon Site

\begin{tabular}{|c|c|c|c|c|c|c|}
\hline COMPOUND & RESULTS & $\begin{array}{l}\text { DATA } \\
\text { QUAL. }\end{array}$ & $\begin{array}{l}\text { DATA } \\
\text { VALID. }\end{array}$ & CRDL & UNITS & DILUTION \\
\hline \multicolumn{7}{|l|}{ C2-27-S (continued) } \\
\hline 4-Bromophenyl phenylether & $330 \mathrm{UG} / \mathrm{L}$ & $u$ & NP & 330 & UG $\Omega$ & 1 \\
\hline Hexachlorobenzene & 330 UG/ & $u$ & NP & 330 & UG $\Omega$ & 1 \\
\hline Pentachlorophenol & 1600 UG & $u$ & NP & 1600 & UG $\Omega$ & 1 \\
\hline Phenanthrene & 330 UG/L & $u$ & NP & 330 & UG $\Omega$ & 1 \\
\hline Anthracene & 330 UG/L & $u$ & NP & 330 & UG $\Omega$ & 1 \\
\hline Di-N-Butylphthalate & 330 UG/ & $u$ & NP & 330 & UG $\Omega$ & 1 \\
\hline Fluoranthene & 330 UG $\Omega$ & $u$ & NP & 330 & UG $\Omega$ & 1 \\
\hline Pyrene & 330 UG & $u$ & NP & 330 & UG/L & 1 \\
\hline Butyl Benzyl Phthalate & $330 \mathrm{UG} / \mathrm{L}$ & u & NP & 330 & UG/ & 1 \\
\hline 3,3'-Dichlorobenzidine & 660 UG $/ L$ & $u$ & NP & 660 & UG $\Omega$ & 1 \\
\hline Benzo(a)Anthracene & 330 UG/ & $u$ & NP & 330 & UG/L & 1 \\
\hline Chrysene & $330 \mathrm{UG} / \mathrm{L}$ & $u$ & NP & 330 & UG/ & 1 \\
\hline bis(2-Ethylhexyl)phthalate & $330 \mathrm{UG} / \mathrm{L}$ & $u$ & NP & 330 & UG/ & 1 \\
\hline di-N-Octyl Phthalate & 330 UG $\Omega$ & $u$ & NP & 330 & UG/ & 1 \\
\hline Benzo(b)Fluoranthene & 330 UG & $u$ & NP & 330 & UG $/$ & 1 \\
\hline Benzo(k)Fluoranthene & 330 UG/L & $u$ & NP & 330 & UG $\Omega$ & 1 \\
\hline Benzo(a)Pyrene & 330 UG $/$ & $u$ & NP & 330 & UG/L & 1 \\
\hline Indeno(1,2,3-Cd)pyrene & 330 UG/L & $u$ & NP & 330 & $\mathrm{UG} / \mathrm{L}$ & 1 \\
\hline Dibenzo(a,h)Anthracene & 330 UG $\Omega$ & $u$ & NP & 330 & UG/ & 1 \\
\hline Benzo(g,h,i)Perylene & 330 UG/L & $u$ & NP & 330 & UG $\Omega$ & 1 \\
\hline Aldol Condensation & 700 UGL & BJ & NP & & UGR & 1 \\
\hline Unknown & 74 UG/L & J & NP & & UG $/$ & 1 \\
\hline Nitrobenzene-d5 & $71 \%$ & & & & $\%$ & 1 \\
\hline 2-Fluorobiphenyl & $72 \%$ & & & & $\%$ & 1 \\
\hline Terphenyl-d14 & $63 \%$ & & & & $\%$ & 1 \\
\hline Phenol-d5 & $60 \%$ & & & & $\%$ & 1 \\
\hline 2-Fluorophenol & $28 \%$ & & & & $\%$ & 1 \\
\hline 2,4,6-Tribromophenol & $72 \%$ & & & & $\%$ & 1 \\
\hline
\end{tabular}




\section{Appendix D.2}

Results of Semivolatile Organic Compound Analyses, Water, Salmon Site 
APPENDIX D.2 Results of Semivolatile Organic Compound Analyses, Water,

Salmon Site

\begin{tabular}{|c|c|c|c|c|c|}
\hline COMPOUND & RESULTS & $\begin{array}{l}\text { DATA } \\
\text { QUAL. }\end{array}$ & $\begin{array}{l}\text { VALID. } \\
\text { QUAL. }\end{array}$ & CRDL UNITS & DILUTION \\
\hline \multicolumn{6}{|l|}{ C5-1-W } \\
\hline Phenol & $10 \mathrm{UG} / \mathrm{L}$ & $u$ & NP & $10 \mathrm{UG} / \mathrm{L}$ & 0.5 \\
\hline bis(2-Chloroethyl) ether & 10 UG $\Omega$ & $u$ & NP & 10 UG $\Omega$ & 0.5 \\
\hline 2-Chlorophenol & $10 \mathrm{UG} / \mathrm{L}$ & $u$ & NP & $10 \mathrm{UG} \Omega$ & 0.5 \\
\hline 1,3-Dichlorobenzene & 10 UG $\Omega$ & $u$ & NP & 10 UG $\Omega$ & 0.5 \\
\hline 1,4-Dichlorobenzene & $10 \mathrm{UG} \Omega$ & $u$ & NP & $10 \mathrm{UG} / \mathrm{L}$ & 0.5 \\
\hline Benzyl Alcohol & 10 UG/ & $u$ & NP & $10 \mathrm{UG} / \mathrm{L}$ & 0.5 \\
\hline 1,2-Dichlorobenzene & $10 \mathrm{UG} / \mathrm{L}$ & $u$ & NP & $10 \mathrm{UG} / \mathrm{L}$ & 0.5 \\
\hline 2-Methylphenol & 10 UG/ & $u$ & NP & 10 UG $\Omega$ & 0.5 \\
\hline bis(2-Chloroisopropyl) ether & $10 \mathrm{UG} / \mathrm{L}$ & $u$ & NP & $10 \mathrm{UG} \Omega$ & 0.5 \\
\hline 4-Methylphenol & $10 \mathrm{UG} / \mathrm{L}$ & $u$ & NP & 10 UG/L & 0.5 \\
\hline N-Nitroso-Di-n-dipropylamine & $10 \mathrm{UG} / \mathrm{L}$ & $u$ & NP & 10 UG $/$ & 0.5 \\
\hline Hexachloroethane & 10 UG $\Omega$ & $u$ & NP & $10 \mathrm{UG} / \mathrm{L}$ & 0.5 \\
\hline Nitrobenzene & 10 UG $\Omega$ & $u$ & NP & 10 UG/L & 0.5 \\
\hline Isophorone & 10 UG/L & $u$ & NP & $10 \mathrm{UG} / \mathrm{L}$ & 0.5 \\
\hline 2-Nitrophenol & $10 \mathrm{UG} \Omega$ & $u$ & NP & $10 \mathrm{UG} / \mathrm{L}$ & 0.5 \\
\hline 2,4-Dimethylphenol & $10 \mathrm{UG} / \mathrm{L}$ & $u$ & NP & $10 \mathrm{UG} / \mathrm{L}$ & 0.5 \\
\hline Benzoic Acid & $50 \mathrm{UG} / \mathrm{L}$ & $u$ & NP & 50 UG/L & 0.5 \\
\hline bis(2Chloroethoxy)methane & $10 \mathrm{UG} / \mathrm{L}$ & $u$ & NP & 10 UG $\Omega$ & 0.5 \\
\hline 2,4-Dichlorophenol & $10 \mathrm{UG} / \mathrm{L}$ & $u$ & NP & 10 UG/ & 0.5 \\
\hline 1,2,4-Trichlorobenzene & $10 \mathrm{UG} / \mathrm{L}$ & $u$ & NP & 10 UG $\Omega$ & 0.5 \\
\hline Naphthalene & $10 \mathrm{UG} / \mathrm{L}$ & $u$ & NP & $10 \mathrm{UG} / \mathrm{L}$ & 0.5 \\
\hline 4-Chloroaniline & $10 \mathrm{UG} / \mathrm{L}$ & $u$ & NP & 10 UG $/ L$ & 0.5 \\
\hline Hexachlorobutadiene & $10 \mathrm{UG} / \mathrm{L}$ & $u$ & NP & 10 UG/L & 0.5 \\
\hline 4-Chloro-3-Methlyphenol & 10 UG $\Omega$ & $u$ & NP & 10 UG/ & 0.5 \\
\hline 2-Methylnaphthalene & $10 \mathrm{UG} / \mathrm{L}$ & $u$ & NP & 10 UG $\Omega$ & 0.5 \\
\hline Hexachlorocyclopentadiene & $10 \mathrm{UG} / \mathrm{L}$ & $u$ & NP & $10 \mathrm{UG} / \mathrm{L}$ & 0.5 \\
\hline 2,4,6-Trichlorophenol & 10 UG/ & $u$ & NP & 10 UG $\Omega$ & 0.5 \\
\hline 2,4,5-Trichlorophenol & 50 UG/L & $u$ & NP & 50 UG $/$ & 0.5 \\
\hline 2-Chloronaphthalene & $10 \mathrm{UG} / \mathrm{L}$ & $u$ & NP & 10 UG/ & 0.5 \\
\hline 2-Nitroaniline & $50 \mathrm{UG} \Omega$ & $u$ & NP & 50 UG $\Omega$ & 0.5 \\
\hline Dimethyl Phthalate & 10 UG/L & $u$ & NP & 10 UG/L & 0.5 \\
\hline Acenaphthylene & $10 \mathrm{UG} / \mathrm{L}$ & $u$ & NP & $10 \mathrm{UG} / \mathrm{L}$ & 0.5 \\
\hline 2,6-Dinitrotoluene & 10 UG $\Omega$ & $u$ & NP & 10 UG $\Omega$ & 0.5 \\
\hline 3-Nitroaniline & 50 UG $\Omega$ & $u$ & NP & $50 \mathrm{UG} / \mathrm{L}$ & 0.5 \\
\hline Acenaphthene & $10 \mathrm{UG} / \mathrm{L}$ & $u$ & NP & $10 \mathrm{UG} /$ & 0.5 \\
\hline 2,4-Dinitrophenol & 50 UG $\Omega$ & $u$ & NP & 50 UG $\Omega$ & 0.5 \\
\hline 4-Nitrophenol & 50 UG $\Omega$ & $u$ & NP & 50 UG $\Omega$ & 0.5 \\
\hline Dibenzofuran & 10 UG $\Omega$ & $u$ & NP & 10 UG/ & 0.5 \\
\hline 2,4-Dinitrotoluene & $10 \mathrm{UG} / \mathrm{L}$ & $u$ & NP & $10 \mathrm{UG} / \mathrm{L}$ & 0.5 \\
\hline Diethylphthalate & $10 \mathrm{UG} \Omega$ & $u$ & NP & 10 UG/L. & 0.5 \\
\hline 4-Chlorophenyl phenyl ether & 10 UG $\Omega$ & $u$ & NP & 10 UG $\Omega$ & 0.5 \\
\hline Fluorene & $10 \mathrm{UG} / \mathrm{L}$ & $u$ & NP & $10 \mathrm{UG \Omega}$ & 0.5 \\
\hline 4-Nitroaniline & 50 UG/ & $u$ & NP & $50 \mathrm{UG} \Omega$ & 0.5 \\
\hline 4,6-Dinitro-2-methyiphenol & 50 UGL & $u$ & NP & 50 UG $\Omega$ & 0.5 \\
\hline N-Nitrosodiphenylamine & 10 UG $\Omega$ & $u$ & NP & 10 UG $\Omega$ & 0.5 \\
\hline
\end{tabular}


APPENDIX D.2 Results of Semivolatile Organic Compound Analyses, Water, Salmon Site

\begin{tabular}{|c|c|c|c|c|c|c|}
\hline COMPOUND & RESULTS & UNITS & $\begin{array}{l}\text { DATA } \\
\text { QUAL. }\end{array}$ & $\begin{array}{l}\text { VALID. } \\
\text { QUAL. }\end{array}$ & CRDL UNITS & DILUTIC \\
\hline \multicolumn{7}{|l|}{ C5-1-W (continued) } \\
\hline 4-Bromophenyl phenylether & & 10 UG/L & $u$ & NP & $10 \mathrm{UG} / \mathrm{L}$ & 0.5 \\
\hline Hexachlorobenzene & & 10 UG/ & $u$ & NP & $10 \mathrm{UG} / \mathrm{h}$ & 0.5 \\
\hline Pentachlorophenol & & 50 UGR & $u$ & NP & 50 UGA & 0.5 \\
\hline Phenanthrene & & $10 \mathrm{UG} \Omega$ & $u$ & NP & $10 \mathrm{UG} \Omega$ & 0.5 \\
\hline Anthracene & & 10 UG/ & $u$ & NP & 10 UG & 0.5 \\
\hline Di-N-Butylphthalate & & 10 UG $\Omega$ & $u$ & NP & 10 UG/ & 0.5 \\
\hline Fluoranthene & & 10 UG $\Omega$ & $u$ & NP & 10 UG/ & 0.5 \\
\hline Pyrene & & 10 UG/ & $u$ & NP & 10 UG $/$ & 0.5 \\
\hline Butyl Benzyl Phthalate & & $10 \mathrm{UG} /$ & $u$ & NP & $10 \mathrm{UG} / \mathrm{L}$ & 0.5 \\
\hline 3,3'-Dichlorobenzidine & & 20 UG $\Omega$ & $u$ & NP & 20 UG $\Omega$ & 0.5 \\
\hline Benzo(a)Anthracene & & $10 \mathrm{UG \Omega}$ & $u$ & NP & $10 \mathrm{UG} / \mathrm{L}$ & 0.5 \\
\hline Chrysene & & $10 \mathrm{UG} \Omega$ & $u$ & NP & 10 UG $\Omega$ & 0.5 \\
\hline bis(2-Ethylhexyl)phthaiate & & 7 UGR & BJ & NP & 10 UGL & 0.5 \\
\hline di-N-Octyl Phthalate & & 10 UGR & $u$ & NP & $10 \mathrm{UG} /$ & 0.5 \\
\hline Benzo(b)Fluoranthene & & 10 UG/L & $u$ & NP & 10 UG几 & 0.5 \\
\hline Benzo(k)Fluoranthene & & 10 UG/L & $u$ & NP & 10 Ua/ & 0.5 \\
\hline Benzo(a)Pyrene & & 10 UG/L & $u$ & NP & 10 Ua/ & 0.5 \\
\hline Indeno(1,2,3-Cd)pyrene & & 10 UG/L & $u$ & NP & 10 UG & 0.5 \\
\hline Dibenzo(a,h)Anthracene & & 10 UG/ & $u$ & NP & 10 UG $\Omega$ & 0.5 \\
\hline Benzo(g,h,i)perylene & & 10 UG/ & $u$ & NP & 10 UG $\Omega$ & 0.5 \\
\hline Ethanol,2-butoxy- & & 29 UG/ & 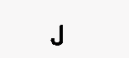 & NP & UG $\Omega$ & 0.5 \\
\hline Unknown & & $8 \mathrm{UG} /$ & J & NP & UG $\Omega$ & 0.5 \\
\hline Unknown Phthalate & & $7 \mathrm{UG} / \mathrm{L}$ & J & NP & UGR & 0.5 \\
\hline Nitrobenzene-d5 & & $35 \%$ & & & $\%$ & 0.5 \\
\hline 2-Fluorobiphenyl & & $31 \%$ & & & $\%$ & 0.5 \\
\hline Terphenyl-d14 & & $35 \%$ & & & $\%$ & 0.5 \\
\hline Phenol-d5 & & $38 \%$ & & & $\%$ & 0.5 \\
\hline 2-Fluorophenol & & $55 \%$ & & & $\%$ & 0.5 \\
\hline 2,4,6-Trichlorophenol & & $79 \%$ & & & $\%$ & 0.5 \\
\hline
\end{tabular}


APPENDIX D.2 Results of Semivolatile Organic Compound Analyses, Water, Salmon Site

\begin{tabular}{|c|c|c|c|c|}
\hline COMPOUND & RESULTS & $\begin{array}{ll}\text { DATA VALID. } \\
\text { QUAL. } \\
\text { QUAL. }\end{array}$ & CRDL UNITS & DILUTION \\
\hline \multicolumn{5}{|l|}{ C5-1-W-MS } \\
\hline Phenol & $36 \%$ & NP & $\%$ & 0.5 \\
\hline 2-Chlorophenol & $72 \%$ & NP & $\%$ & 0.5 \\
\hline 1,4-Dichlorobenzene & $75 \%$ & NP & $\%$ & 0.5 \\
\hline N-Nitroso-Di-n-dipropylamine & $80 \%$ & NP & $\%$ & 0.5 \\
\hline 1,2,4-Trichlorobenzene & $79 \%$ & NP & $\%$ & 0.5 \\
\hline 4-Chloro-3-Methlyphenol- & $82 \%$ & NP & $\%$ & 0.5 \\
\hline Acenaphthene & $74 \%$ & NP & $\%$ & 0.5 \\
\hline 4-Nitrophenol & $42 \%$ & NP & $\%$ & 0.5 \\
\hline 2,4-Dinitrotoluene & $78 \%$ & NP & $\%$ & 0.5 \\
\hline Pentachlorophenol & $96 \%$ & NP & $\%$ & 0.5 \\
\hline Pyrene & $68 \%$ & NP & $\%$ & 0.5 \\
\hline Nitrobenzene-d5 & $81 \%$ & NP & $\%$ & 0.5 \\
\hline 2-Fluorobiphenyl & $75 \%$ & NP & $\%$ & 0.5 \\
\hline Terphenyl-d14 & $67 \%$ & NP & $\%$ & 0.5 \\
\hline Phenol-d5 & $35 \%$ & NP & $\%$ & 0.5 \\
\hline 2-Fluorophenol & $49 \%$ & NP & $\%$ & 0.5 \\
\hline 2,4,6-Trichlorophenol & $79 \%$ & NP & $\%$ & 0.5 \\
\hline \multicolumn{5}{|l|}{ C5-1-W-MSD } \\
\hline Phenol & $36 \%$ & NP & & 0.5 \\
\hline 2-Chlorophenol & $79 \%$ & NP & $\%$ & 0.5 \\
\hline 1,4-Dichlorobenzene & $83 \%$ & NP & $\%$ & 0.5 \\
\hline N-Nitroso-Di-n-dipropylamine & $88 \%$ & NP & $\%$ & 0.5 \\
\hline 1,2,4-Trichlorobenzene & $84 \%$ & NP & $\%$ & 0.5 \\
\hline 4-Chloro-3-Methlyphenol & $89 \%$ & NP & $\%$ & 0.5 \\
\hline Acenaphthene & $81 \%$ & NP & $\%$ & 0.5 \\
\hline 4-Nitrophenol & $45 \%$ & NP & $\%$ & 0.5 \\
\hline 2,4-Dinitrotoluene & $83 \%$ & NP & $\%$ & 0.5 \\
\hline Pentachlorophenol & $99 \%$ & NP & $\%$ & 0.5 \\
\hline Pyrene & $73 \%$ & NP & $\%$ & 0.5 \\
\hline Nitrobenzene-d5 & $91 \%$ & NP & $\%$ & 0.5 \\
\hline 2-Fluorobiphenyl & $84 \%$ & NP & $\%$ & 0.5 \\
\hline Terphenyl-d14 & $71 \%$ & NP & $\%$ & 0.5 \\
\hline Phenol-d5 & $40 \%$ & NP & $\%$ & 0.5 \\
\hline 2-Fluorophenol & $59 \%$ & NP & $\%$ & 0.5 \\
\hline 2,4,6-Trichlorophenol & $84 \%$ & NP & $\%$ & 0.5 \\
\hline
\end{tabular}


APPENDIX D.2 Results of Semivolatile Organic Compound Analyses, Water, Salmon Site

\begin{tabular}{|c|c|c|c|c|c|}
\hline COMPOUND & RESULTS & $\begin{array}{l}\text { DATA } \\
\text { QUAL. }\end{array}$ & $\begin{array}{l}\text { VALID. } \\
\text { QUAL. }\end{array}$ & CRDL UNITS & DILUTION \\
\hline \multicolumn{6}{|l|}{$C 3-8-W$} \\
\hline Phenol & 10 UG/ & $u$ & NP & $10 \mathrm{UG} / \mathrm{L}$ & 0.5 \\
\hline bis(2-Chloroethyl) ether & 10 UG/ & $u$ & NP & $10 \mathrm{UG} \Omega$ & 0.5 \\
\hline 2-Chlorophenol & 10 UG/L & $u$ & NP & 10 UG $\Omega$ & 0.5 \\
\hline 1,3-Dichlorobenzene & $10 \mathrm{UG} / \mathrm{L}$ & $u$ & NP & $10 \mathrm{UG} / \mathrm{L}$ & 0.5 \\
\hline 1,4-Dichlorobenzene & 10 UG/ & $u$ & NP & $10 \mathrm{UG} / \mathrm{L}$ & 0.5 \\
\hline Benzyl Alcohol & 10 UGR & $u$ & NP & 10 UG/ & 0.5 \\
\hline 1,2-Dichlorobenzene & 10 UG & $u$ & NP & 10 UG/ & 0.5 \\
\hline 2-Methyiphenol & 10 UGR & $u$ & NP & $10 \mathrm{UG} / \mathrm{L}$ & 0.5 \\
\hline bis(2-Chloroisopropyl) ether & 10 UG/ & $u$ & NP & $10 \mathrm{UG} / \mathrm{L}$ & 0.5 \\
\hline 4-Methylphenol & 10 UG/L & $u$ & NP & 10 UG/L & 0.5 \\
\hline N-Nitroso-Di-n-dipropylamine & 10 UG/L & u & $\mathrm{NP}$ & $10 \mathrm{UG} / \mathrm{L}$ & 0.5 \\
\hline Hexachloroethane & 10 UG/L & $u$ & NP & 10 UG/L & 0.5 \\
\hline Nitrobenzene & $10 \mathrm{UG} / \mathrm{L}$ & $u$ & $\mathrm{NP}$ & $10 \mathrm{UG} \Omega$ & 0.5 \\
\hline Isophorone & $10 \mathrm{UG} / \mathrm{h}$ & $u$ & NP & 10 UG $\Omega$ & 0.5 \\
\hline 2-Nitrophenol & $10 \mathrm{UG} / \mathrm{L}$ & $u$ & NP & 10 UG/ & 0.5 \\
\hline 2,4-Dimethylphenol & $10 \mathrm{UG} / \mathrm{L}$ & $u$ & NP & $10 \mathrm{UG} / \mathrm{L}$ & 0.5 \\
\hline Benzoic Acid & $50 \mathrm{UG} /$ & $u$ & NP & 50 UG $\Omega$ & 0.5 \\
\hline bis(2Chloroethoxy)methane & 10 UG $\Omega$ & $u$ & NP & 10 Ua凡 & 0.5 \\
\hline 2,4-Dichlorophenol & 10 UG $\Omega$ & $u$ & NP & 10 UG几 & 0.5 \\
\hline 1,2,4-Trichlorobenzene & 10 UG $\Omega$ & $u$ & NP & 10 UG/ & 0.5 \\
\hline Naphthalene & 10 UG $/$ & $u$ & NP & 10 UG $\Omega$ & 0.5 \\
\hline 4-Chloroaniline & 10 UG/L & $u$ & NP & 10 UG $\Omega$ & 0.5 \\
\hline Hexachlorobutadiene & 10 UG $\Omega$ & $u$ & NP & 10 UG $\Omega$ & 0.5 \\
\hline 4-Chloro-3-Methlyphenol & 10 UG $\Omega$ & $u$ & NP & 10 UG $\Omega$ & 0.5 \\
\hline 2-Methyinaphthalene & 10 UG $\Omega$ & $u$ & NP & 10 UGA & 0.5 \\
\hline Hexachlorocyclopentadiene & 10 UG $\Omega$ & $u$ & NP & 10 UG/ & 0.5 \\
\hline 2,4.6-Trichlorophenol & 10 UG $\Omega$ & $u$ & NP & 10 UG/ & 0.5 \\
\hline 2,4,5-Trichlorophenol & 50 UGL & $u$ & NP & 50 UG/L & 0.5 \\
\hline 2-Chloronaphthalene & 10 UG $\Omega$ & $u$ & NP & 10 UG/L & 0.5 \\
\hline 2-Nitroaniline & $50 \mathrm{UG} /$ & $u$ & $\mathrm{NP}$ & 50 UG几 & 0.5 \\
\hline Dimethyl Phthalate & $10 \mathrm{UG} / \mathrm{L}$ & $u$ & NP & 10 UG/L & 0.5 \\
\hline Acenaphthylene & $10 \mathrm{UG} / \mathrm{L}$ & $u$ & NP & 10 UG $\Omega$ & 0.5 \\
\hline 2,6-Dinitrotoluene & 10 UG/ & $u$ & NP & $10 \mathrm{UG} / \mathrm{L}$ & 0.5 \\
\hline 3-Nitroaniline & 50 UG/ & $u$ & NP & 50 UG/ & 0.5 \\
\hline Acenaphthene & 10 UG/L & $u$ & NP & 10 UGL & 0.5 \\
\hline 2,4-Dinitrophenol & 50 Ua/ & $u$ & NP & $50 \mathrm{UG} / \mathrm{L}$ & 0.5 \\
\hline 4-Nitrophenol & 50 UG/ & $u$ & NP & 50 UG $\Omega$ & 0.5 \\
\hline Dibenzofuran & 10 UG/ & $u$ & NP & 10 UG/ & 0.5 \\
\hline 2,4-Dinitrotoluene & 10 UG $\Omega$ & $u$ & NP & 10 UG $\Omega$ & 0.5 \\
\hline Diethylphthalate & 10 UG $\Omega$ & $u$ & NP & 10 UG $\Omega$ & 0.5 \\
\hline 4-Chlorophenyl phenyl ether & 10 UG/ & $u$ & NP & $10 \cup G \Omega$ & 0.5 \\
\hline Fluorene & 10 UG & $u$ & NP & 10 UG $\Omega$ & 0.5 \\
\hline 4-Nitroaniline & 50 UGR & $u$ & NP & 50 UG $\Omega$ & 0.5 \\
\hline 4,6-Dinitro-2-methylphenoi & 50 UG $\Omega$ & $u$ & NP & 50 UG & 0.5 \\
\hline N-Nitrosodiphenylamine & 10 UG $\Omega$ & $u$ & NP & 10 UG $\Omega$ & 0.5 \\
\hline
\end{tabular}


APPENDIX D.2 Results of Semivolatile Organic Compound Analyses, Water, Salmon Site

\begin{tabular}{|c|c|c|c|c|c|}
\hline COMPOUND & RESULTS & $\begin{array}{l}\text { DATA } \\
\text { QUAL. }\end{array}$ & $\begin{array}{l}\text { VALID. } \\
\text { QUAL. }\end{array}$ & CRDL UNITS & DILUTIC \\
\hline \multicolumn{6}{|l|}{ C3-8-W (continued) } \\
\hline 4-Bromophenyl phenylether & $10 \mathrm{UG} \Omega$ & $u$ & NP & 10 UG $\Omega$ & 0.5 \\
\hline Hexachlorobenzene & $10 \mathrm{UG} \Omega$ & $u$ & NP & $10 \mathrm{UG} / \mathrm{L}$ & 0.5 \\
\hline Pentachlorophenol & 50 UG/ & $u$ & NP & $50 \mathrm{UG} /$ & 0.5 \\
\hline Phenanthrene & 10 UG/L & $u$ & NP & 10 UG $\Omega$ & 0.5 \\
\hline Anthracene & $10 \mathrm{UG} / \mathrm{L}$ & $u$ & NP & $10 \mathrm{UG} / \mathrm{L}$ & 0.5 \\
\hline Di-N-Butylphthalate & $10 \mathrm{UG} / \mathrm{L}$ & $u$ & NP & 10 UG $\Omega$ & 0.5 \\
\hline Fluoranthene & $10 \mathrm{UG} \Omega$ & $u$ & NP & 10 UG $\Omega$ & 0.5 \\
\hline Pyrene & $10 \mathrm{UG \Omega}$ & $u$ & NP & $10 \mathrm{UG} / \mathrm{L}$ & 0.5 \\
\hline Butyl Benzyl Phthalate & 10 UG & $u$ & NP & $10 \mathrm{UG} \Omega$ & 0.5 \\
\hline 3,3'-Dichlorobenzidine & 20 UG/L & $u$ & NP & $20 \mathrm{UG} /$ & 0.5 \\
\hline Benzo(a)Anthracene & $10 \mathrm{UG} / \mathrm{L}$ & $u$ & NP & 10 UG $/ 2$ & 0.5 \\
\hline Chrysene & $10 \mathrm{UG} / \mathrm{L}$ & $u$ & NP & $10 \mathrm{UG} /$ & 0.5 \\
\hline bis(2-Ethylhexyl)phthalate & 10 UG/ & $u$ & NP & 10 UG $\Omega$ & 0.5 \\
\hline di-N-Octyl Phthalate & $10 \mathrm{UG} / \mathrm{L}$ & $u$ & NP & 10 UG $\Omega$ & 0.5 \\
\hline Benzo(b)Fluoranthene & $10 \mathrm{UG} / \mathrm{L}$ & $u$ & NP & $10 \mathrm{UG} \Omega$ & 0.5 \\
\hline Benzo(k)Fluoranthene & 10 UG/L & $u$ & NP & $10 \mathrm{UG} / \mathrm{L}$ & 0.5 \\
\hline Benzo(a)Pyrene & 10 UG/L & $u$ & NP & $10 \mathrm{UG} / \mathrm{L}$ & 0.5 \\
\hline Indeno(1,2,3-Cd)pyrene & 10 UGL & $u$ & NP & $10 \mathrm{UG} \Omega$ & 0.5 \\
\hline Dibenzo(a,h)Anthracene & 10 UG/ & $u$ & NP & $10 \mathrm{UG} / \mathrm{L}$ & 0.5 \\
\hline Benzo(g,h,i)Perylene & $10 \mathrm{UG} / \mathrm{L}$ & $u$ & NP & 10 UG $\Omega$ & 0.5 \\
\hline Unknown-1 & 170 UG $/$ & J & NP & UG/ & 0.5 \\
\hline Unknown-2 & 24 UG/ & $J$ & NP & UG $\Omega$ & 0.5 \\
\hline Unknown-3 & 12 UG/L & J & NP & UG $\Omega$ & 0.5 \\
\hline Unknown-4 & $8 \mathrm{UG} \Omega$ & $J$ & NP & UG $\Omega$ & 0.5 \\
\hline Dodecanoic acid & 75 UG/ & $J$ & NP & UG $\Omega$ & 0.5 \\
\hline Unknown-5 & $200 \mathrm{UG} \Omega$ & $J$ & NP & UG $\Omega$ & 0.5 \\
\hline Unknown-6 & 17 UG几 & $\mathrm{J}$ & NP & UG $\Omega$ & 0.5 \\
\hline Unknown-7 & 4 UG/L & $\mathrm{J}$ & NP & UG $/$ L & 0.5 \\
\hline Unknown-8 & $130 \mathrm{UG} / \mathrm{L}$ & $J$ & NP & UG $\Omega$ & 0.5 \\
\hline Unknown-9 & 7 UG/ & $J$ & NP & UG $\Omega$ & 0.5 \\
\hline Unknown-10 & $6 \mathrm{UG} / \mathrm{L}$ & $J$ & NP & UG $\Omega$ & 0.5 \\
\hline Unknown-11 & $4 \mathrm{UG} /$ & $J$ & NP & UG $\Omega$ & 0.5 \\
\hline Unknown-12 & $8 \mathrm{UG} \Omega$ & J & NP & UG $\Omega$ & 0.5 \\
\hline Unknown-13 & $4 \mathrm{UG} /$ & $J$ & NP & UG/L & 0.5 \\
\hline Nitrobenzene-d5 & $72 \%$ & & & $\%$ & 0.5 \\
\hline 2-Fluorobiphenyl & $69 \%$ & & & $\%$ & 0.5 \\
\hline Terphenyl-d14 & $69 \%$ & & & $\%$ & 0.5 \\
\hline Phenol-d5 & $0 \%$ & * & & $\%$ & 0.5 \\
\hline 2-Fluorophenol & $0 \%$ & $\cdot$ & & $\%$ & 0.5 \\
\hline 2,4,6-Tribromophenol & $27 \%$ & & & $\%$ & 0.5 \\
\hline
\end{tabular}


APPENDIX D.2 Results of Semivolatile Organic Compound Analyses, Water, Salmon Site

DATA VALID.

COMPOUND RESULTS UNITS QUAL. QUAL.

CRDL UNITS

DILUTION

C3-9-W

Phenol

bis(2-Chloroethyl) ether

10 UG/

NP

$10 \mathrm{UG/}$

0.5

2-Chlorophenol

10 UG/L

$10 \mathrm{UG} / \mathrm{L}$

0.5

1,3-Dichlorobenzene

$10 \mathrm{UG} / \mathrm{L}$

10 UGR

0.5

1,4-Dichlorobenzene

$10 \mathrm{UG} / \mathrm{L}$

10 UG/

0.5

Benzyl Alcohol

10 UG/L U NP

10 UG

0.5

1,2-Dichlorobenzene

10 UG $\quad U$ NP

10 UG

0.5

10 UGR U NP

10 UG $\Omega$

0.5

2-Methylphenol

bis(2-Chloroisopropyl) ether

10 UGR U NP

$10 \mathrm{UG} / \mathrm{L}$

0.5

4-Methylphenol

N-Nitroso-Di-n-dipropylamine

Hexachloroethane

Nitrobenzene

Isophorone

2-Nitrophenol

2,4-Dimethylphenol

Benzoic Acid

bis(2Chloroethoxy)methane

10 UG/ $\quad U$ NP

$10 \mathrm{UG} / \mathrm{L}$

0.5

10 UGR U NP

$10 \mathrm{UG} / \mathrm{L}$

0.5

$10 \mathrm{UG} / \mathrm{h}$

$10 \mathrm{UG} / \mathrm{L}$

0.5

$10 \mathrm{UG} / \mathrm{L}$

$10 \mathrm{UG} / \mathrm{L}$

0.5

$10 \mathrm{UG} / \mathrm{L}$

$10 \mathrm{UGh}$

0.5

$10 \mathrm{UG} \Omega$

10 UG/

0.5

$10 \mathrm{UG} / \mathrm{L}$

10 UGR

0.5

10 UGR

0.5

50 UGR

50 UG/

0.5

2,4-Dichlorophenol

10 UGR

$10 \mathrm{UG} \Omega$

0.5

10 UGR U NP

10 UG/

0.5

1,2,4-Trichlorobenzene

Naphthalene

4-Chloroaniline

Hexachlorobutadiene

4-Chloro-3-Methlyphenol

2-Methyinaphthalene

Hexachlorocyclopentadiene

10 UG/ U NP

10 UG/

0.5

10 UG/ $U$ NP

10 UG/

0.5

10 UG/ U NP

10 UGR

0.5

$10 \mathrm{UGR} U$ NP

10 UGR

0.5

10 UG $/ \quad U \quad N P$

10 UaR

0.5

10 UG/ U NP

10 UG

0.5

10 UG/

$10 \mathrm{UG} / \mathrm{L}$

0.5

2,4.6-Trichlorophenol

2,4,5-Trichlorophenol

2-Chloronaphthalene

2-Nitroaniline

Dimethyl Phthalate

Acenaphthylene

2,6-Dinitrotoluene

3-Nitroaniline

Acenaphthene

2,4-Dinitrophenol

$10 \mathrm{UG} / \mathrm{L}$

10 UG/

0.5

50 UG/

50 UG/

0.5

10 UG/L

$10 \mathrm{UG} / \mathrm{L}$

0.5

50 UG/

50 UG/

0.5

10 UG/

0.5

10 UG/

$10 \mathrm{UG} / \mathrm{L}$

0.5

10 UGR

10 UGR

0.5

50 UGR

50 UG/

0.5

10 UG/

$10 \mathrm{UG \Omega}$

0.5

50 UGR U NP

50 UG $\Omega$

0.5

4-Nitrophenol

50 UG $\Omega$ U NP

50 UG/

0.5

Dibenzofuran

2,4-Dinitrotoluene

Diethylphthalate

10 UGR U NP

10 UG

0.5

10 UG/

10 UG

0.5

$10 \mathrm{UG} / \mathrm{L}$

$10 \mathrm{UG} \Omega$

0.5

4-Chlorophenyl phenyl ether

$10 \mathrm{UG} /$

$10 \mathrm{UG} \Omega$

0.5

Fluorene

4-Nitroaniline

4,6-Dinitro-2-methylphenol

$\mathrm{N}$-Nitrosodiphenylamine

10 UG/

10 UGR

0.5

50 UG

50 UGR

0.5

50 UGL

50 UGR

0.5

$10 \mathrm{UG} / \mathrm{L}$

10 UG/

0.5 
APPENDIX D.2 Results of Semivolatile Organic Compound Analyses, Water, Salmon Site

DATA VALID.

COMPOUND RESULTS UNITS QUAL. QUAL.

CRDL UNITS

DILUTION

C-3-9-W (continued)

4-Bromophenyl phenylether

\begin{tabular}{|c|c|}
\hline 10 UG & $u$ \\
\hline $10 \mathrm{UG} \Omega$ & $u$ \\
\hline 50 UG $/$ & $u$ \\
\hline 10 UG $\Omega$ & $u$ \\
\hline $10 \mathrm{UG} / \mathrm{L}$ & $u$ \\
\hline $10 \mathrm{UG} / \mathrm{L}$ & $u$ \\
\hline $10 \mathrm{UG} \Omega$ & u \\
\hline $10 \mathrm{UG} / \mathrm{L}$ & $u$ \\
\hline 10 UG/ & $u$ \\
\hline $20 \mathrm{UG} / \mathrm{L}$ & u \\
\hline
\end{tabular}

NP
NP
NP
NP
NP
NP
NP
NP
NP
NP

10 UG $\Omega$

0.5

Hexachlorobenzene

Pentachlorophenol

Phenanthrene

Anthracene

Di-N-Butylphthalate

Fluoranthene

Pyrene

Butyl Benzyl Phthalate

3,3'-Dichlorobenzidine

10 UG/ $U$ NP

10 UG $\Omega$

0.5

Benzo(a)Anthracene

Chrysene

bis(2-Ethylhexyl)phthalate

10 UG/ $U$ NP

50 UG $\Omega$

0.5

10 UGR

0.5

$10 \mathrm{UG} / \mathrm{L}$

0.5

$10 \mathrm{UG} \Omega$

0.5

10 UG

0.5

10 UG/L

0.5

10 UGR

0.5

$20 \mathrm{UG} / \mathrm{L}$

0.5

$10 \mathrm{UG} / \mathrm{L}$

0.5

$10 \mathrm{UG} / \mathrm{U} \quad \mathrm{NP}$

10 UG/L

0.5

$10 \mathrm{UG} / \mathrm{L}$

$10 \mathrm{UG} / \mathrm{L}$

0.5

10 UG/

$10 \mathrm{UG} / \mathrm{L}$

0.5

Benzo(b)Fluoranthene

Benzo(k)Fluoranthene

10 UG/L

$10 \mathrm{UG} / \mathrm{L}$

0.5

Benzo(a)Pyrene

Indeno(1,2,3-Cd)pyrene

Dibenzo(a,h)Anthracene

10 UG/L

10 UG/L

0.5

$10 \mathrm{UG} / \mathrm{L}$

0.5

10 UG/ $U$ NP

10 UG/

0.5

Benzo(g,h,i)Perylene

10 UG/ U NP

Unknown-1

Unknown-2

$28 \mathrm{UGR}$ J NP

10 UG/

0.5

10 UG/

0.5

12 UGR J NP

UGR

0.5

12 UGR J NP

UGR

0.5

Unknown-3

64 UGR

UGR

0.5

12 UGR

UGR

0.5

Unknown-4

Unknown-5

Unknown-6

110 UG/L

$4 \mathrm{UG} /$

6 UG/

Unknown-7

Unknown-8

Unknown-9

Unknown-10

Unknown-11

Unknown-12

Nitrobenzene-d5

2-Fluorobiphenyl

$69 \mathrm{UG} / \mathrm{L}$

$4 \mathrm{UG} \Omega$

6 UG几

13 UG/

56 UG几

UGR

0.5

UG $\Omega$

0.5

UG $L$

0.5

UG/

0.5

UGR

0.5

UGR

0.5

UGR

0.5

UGR

0.5

$69 \%$

$60 \%$

Terphenyl-d14

$60 \%$

Phenol-d5

$22 \%$

2-Fluorophenol

$31 \%$

2,4,6-Tribromophenol

$53 \%$

UG/

0.5

0.5

0.5

0.5

0.5

0.5

0.5 
APPENDIX D.2 Results of Semivolatile Organic Compound Analyses, Water, Salmon Site

\begin{tabular}{|c|c|c|c|c|c|}
\hline COMPOUND & RESULTS & $\begin{array}{l}\text { DATA } \\
\text { QUAL. }\end{array}$ & $\begin{array}{l}\text { VALID. } \\
\text { QUAL. }\end{array}$ & CRDL UNITS & DILUTION \\
\hline \multicolumn{6}{|l|}{$C 4-11-W$} \\
\hline Phenol & $10 \mathrm{UG} / \mathrm{L}$ & $u$ & $N P$ & 10 UG/ & 0.5 \\
\hline bis(2-Chloroethyl) ether & 10 UG/ & $u$ & NP & 10 UG/L & 0.5 \\
\hline 2-Chlorophenol & 10 UG/L & $u$ & NP & $10 \mathrm{UG} / \mathrm{L}$ & 0.5 \\
\hline 1,3-Dichlorobenzene & $10 \mathrm{UG} /$ & $u$ & NP & 10 UG/ & 0.5 \\
\hline 1,4-Dichlorobenzene & $10 \mathrm{UG} / \mathrm{L}$ & $u$ & NP & $10 \mathrm{UG} / \mathrm{L}$ & 0.5 \\
\hline Benzyl Alcohol & 10 UG $\Omega$ & $u$ & NP & 10 UG $/$ & 0.5 \\
\hline 1,2-Dichlorobenzene & $10 \mathrm{UG} \Omega$ & $u$ & NP & $10 \mathrm{UG} \Omega$ & 0.5 \\
\hline 2-Methylphenol & $10 \mathrm{UG} / \mathrm{L}$ & $u$ & NP & $10 \mathrm{UG} /$ & 0.5 \\
\hline bis(2-Chloroisopropyl) ether & $10 \mathrm{UG} / \mathrm{L}$ & $u$ & NP & 10 UG $\Omega$ & 0.5 \\
\hline 4-Methyiphenol & 10 UG/ & $u$ & NP & 10 UG $\Omega$ & 0.5 \\
\hline N-Nitroso-Di-n-dipropylamine & 10 UG/ & $u$ & NP & 10 UG/ & 0.5 \\
\hline Hexachloroethane & $10 \mathrm{UG} / \mathrm{L}$ & $u$ & NP & 10 UG $\Omega$ & 0.5 \\
\hline Nitrobenzene & 10 UG $\Omega$ & $u$ & NP & $10 \mathrm{UG} / \mathrm{L}$ & 0.5 \\
\hline Isophorone & $10 \mathrm{UG} \Omega$ & $u$ & NP & $10 \mathrm{UG} /$ & 0.5 \\
\hline 2-Nitrophenol & $10 \mathrm{UG} / \mathrm{L}$ & $u$ & NP & 10 UG/L & 0.5 \\
\hline 2,4-Dimethylphenol & $10 \mathrm{UG} / \mathrm{L}$ & $u$ & NP & 10 UG/L & 0.5 \\
\hline Benzoic Acid & 50 UG $\Omega$ & $u$ & NP & 50 UG $\Omega$ & 0.5 \\
\hline bis(2Chloroethoxy)methane & 10 UG $\Omega$ & $u$ & NP & 10 UG/ & 0.5 \\
\hline 2,4-Dichlorophenol & 10 UG & $u$ & NP & 10 UG/ & 0.5 \\
\hline 1,2,4-Trichlorobenzene & $10 \mathrm{UG} / \mathrm{L}$ & $u$ & NP & 10 UG/ & 0.5 \\
\hline Naphthalene & 10 UG $\Omega$ & $u$ & NP & 10 UG $\Omega$ & 0.5 \\
\hline 4-Chloroaniline & $10 \mathrm{UG} / \mathrm{L}$ & $u$ & NP & 10 UG & 0.5 \\
\hline Hexachlorobutadiene & $10 \mathrm{UG} / \mathrm{L}$ & $u$ & NP & 10 UG/ & 0.5 \\
\hline 4-Chloro-3-Methlyphenol & $10 \mathrm{UG} / \mathrm{L}$ & $u$ & NP & $10 \mathrm{UG} / \mathrm{\Omega}$ & 0.5 \\
\hline 2-Methylnaphthalene & 10 UGR & $u$ & NP & 10 UG/ & 0.5 \\
\hline Hexachlorocyclopentadiene & $10 \mathrm{UG} / \mathrm{L}$ & $u$ & NP & 10 UG $\Omega$ & 0.5 \\
\hline 2,4.6-Trichlorophenol & 10 UG $\Omega$ & $u$ & NP & $10 \mathrm{UG} / \mathrm{L}$ & 0.5 \\
\hline 2,4,5-Trichlorophenol & 50 UG $\Omega$ & $u$ & NP & 50 UG $\Omega$ & 0.5 \\
\hline 2-Chloronaphthalene & $10 \mathrm{UG} / \mathrm{L}$ & $u$ & NP & 10 UG/L & 0.5 \\
\hline 2-Nitroaniline & 50 UG/L & $u$ & NP & 50 UG $\Omega$ & 0.5 \\
\hline Dimethyl Phthalate & 10 UG $/$ & $u$ & NP & $10 \mathrm{UG} / \mathrm{L}$ & 0.5 \\
\hline Acenaphthylene & 10 UG/ & $u$ & NP & 10 UG/ & 0.5 \\
\hline 2,6-Dinitrotoluene & $10 \mathrm{UG} / \mathrm{L}$ & $u$ & NP & 10 UG/L & 0.5 \\
\hline 3-Nitroaniline & $50 \mathrm{UG} / \mathrm{L}$ & $u$ & NP & 50 UG $\Omega$ & 0.5 \\
\hline Acenaphthene & $10 \mathrm{UG} / \mathrm{L}$ & $u$ & NP & $10 \mathrm{UG} / \mathrm{L}$ & 0.5 \\
\hline 2,4-Dinitrophenol & $50 \mathrm{UG} / \mathrm{L}$ & $u$ & NP & 50 UG/L & 0.5 \\
\hline 4-Nitrophenol & 50 UG/L & $u$ & NP & 50 UG/ & 0.5 \\
\hline Dibenzofuran & $10 \mathrm{UG} / \mathrm{L}$ & $u$ & NP & 10 UG $\Omega$ & 0.5 \\
\hline 2,4-Dinitrotoluene & 10 UG $\Omega$ & $u$ & NP & $10 \mathrm{UG} / \mathrm{L}$ & 0.5 \\
\hline Diethylphthalate & $10 \mathrm{UG} / \mathrm{L}$ & $u$ & NP & 10 UG $\Omega$ & 0.5 \\
\hline 4-Chlorophenyl phenyl ether & $10 \mathrm{UG} / \mathrm{L}$ & $u$ & NP & 10 UG/ & 0.5 \\
\hline Fluorene & 10 UG $\Omega$ & $u$ & NP & 10 UG $/$ & 0.5 \\
\hline 4-Nitroaniline & $50 \mathrm{UG} /$ & $u$ & NP & $50 \mathrm{UG} /$ & 0.5 \\
\hline 4,6-Dinitro-2-methylphenol & $50 \mathrm{UG} \Omega$ & $u$ & NP & 50 UGR & 0.5 \\
\hline N-Nitrosodiphenylamine & $10 \mathrm{UG} / \mathrm{L}$ & $u$ & NP & $10 \mathrm{UG} /$ & 0.5 \\
\hline
\end{tabular}


APPENDIX D.2 Results of Semivolatile Organic Compound Analyses, Water, Salmon Site

\begin{tabular}{|c|c|c|c|c|c|}
\hline COMPOUND & RESULTS & $\begin{array}{l}\text { DATA } \\
\text { QUAL. }\end{array}$ & $\begin{array}{l}\text { VALID. } \\
\text { QUAL. }\end{array}$ & CRDL UNITS & DILUTION \\
\hline \multicolumn{6}{|l|}{ C4-11-W (continued) } \\
\hline 4-Bromophenyl phenylether & $10 \mathrm{UG} / \mathrm{L}$ & $u$ & NP & $10 \mathrm{UG} / \mathrm{L}$ & 0.5 \\
\hline Hexachlorobenzene & 10 UG $\Omega$ & $u$ & NP & 10 UG $\Omega$ & 0.5 \\
\hline Pentachlorophenol & 50 UG $/$ & $u$ & NP & 50 UG/L & 0.5 \\
\hline Phenanthrene & 10 UG $/ 2$ & $u$ & NP & $10 \mathrm{UG} / \mathrm{L}$ & 0.5 \\
\hline Anthracene & 10 UG/ & $u$ & NP & 10 UG $\Omega$ & 0.5 \\
\hline Di-N-Butylphthalate & 10 UG $/$ & $u$ & NP & 10 UG $/$ & 0.5 \\
\hline Fluoranthene & 10 UG/L & $u$ & NP & 10 UG $\Omega$ & 0.5 \\
\hline Pyrene & 10 UGR & $u$ & NP & 10 UG/L & 0.5 \\
\hline Butyl Benzyl Phthalate & 10 UG $\Omega$ & $u$ & NP & $10 \mathrm{UG} / \mathrm{L}$ & 0.5 \\
\hline 3,3'-Dichlorobenzidine & 20 UG $\Omega$ & $u$ & NP & $20 \mathrm{UG} / \mathrm{L}$ & 0.5 \\
\hline Benzo(a)Anthracene & 10 UG $\Omega$ & $u$ & NP & 10 UG $\Omega$ & 0.5 \\
\hline Chrysene & $10 \mathrm{UG} / \mathrm{L}$ & $u$ & NP & 10 UG/L & 0.5 \\
\hline bis(2-Ethylhexyl)phthalate & $7 \mathrm{UG} / \mathrm{L}$ & $J$ & NP & $10 \mathrm{UG} \Omega$ & 0.5 \\
\hline di-N-Octyl Phthalate & 10 UG/L & $u$ & NP & 10 UG/L & 0.5 \\
\hline Benzo(b)Fluoranthene & $10 \mathrm{UG} / \mathrm{L}$ & $u$ & NP & 10 UG/L & 0.5 \\
\hline Benzo(k)Fluoranthene & 10 UG/ & $u$ & NP & $10 \mathrm{UG} / \mathrm{L}$ & 0.5 \\
\hline Benzo(a)Pyrene & 10 UG $/$ & $u$ & NP & 10 UG $/$ & 0.5 \\
\hline Indeno(1,2,3-Cd)pyrene & 10 UG $\Omega$ & $u$ & NP & 10 UG/ & 0.5 \\
\hline Dibenzo(a,h)Anthracene & 10 UG/ & $u$ & NP & 10 UG $/$ & 0.5 \\
\hline Benzo(g,h,i)Perylene & 10 UG/L & $u$ & NP & 10 UG/ & 0.5 \\
\hline Dodecanoic acid & 72 UG/ & $J$ & NP & UG/ & 0.5 \\
\hline Unknown-1 & 140 UGR & J & NP & UG/ & 0.5 \\
\hline Unknown-2 & 8 UGR & $J$ & NP & UG几 & 0.5 \\
\hline Unknown-3 & 110 UG & J & NP & UG/ & 0.5 \\
\hline Unknown-4 & $7 \mathrm{UG} /$ & J & NP & UG $\Omega$ & 0.5 \\
\hline Unknown-5 & 16 UG/L & J & NP & UG $\Omega$ & 0.5 \\
\hline Unknown-6 & 5 UG/ & $\mathrm{J}$ & NP & UG $\Omega$ & 0.5 \\
\hline Unknown-7 & 9 UG/ & $J$ & NP & UG $\Omega$ & 0.5 \\
\hline Unknown-8 & 11 UG/ & J & NP & UGת & 0.5 \\
\hline Unknown-9 & $6 \mathrm{UG} / \mathrm{L}$ & $J$ & NP & UG/L & 0.5 \\
\hline Unknown-10 & $8 \mathrm{UG} \Omega$ & J & NP & UG/ & 0.5 \\
\hline Unknown-11 & $6 \mathrm{UG} /$ & J & NP & UG $\Omega$ & 0.5 \\
\hline Unknown-12 & $16 \mathrm{UG} / \mathrm{L}$ & J & NP & UG/ & 0.5 \\
\hline Unknown-13 & 10 UG $/$ & $J$ & NP & UG $\Omega$ & 0.5 \\
\hline Unknown-14 & $8 \mathrm{UG} / \mathrm{L}$ & $J$ & NP & UG $\Omega$ & 0.5 \\
\hline Unknown-15 & $7 \mathrm{UG} / \mathrm{L}$ & $J$ & NP & UG $\Omega$ & 0.5 \\
\hline Unknown-16 & 20 UG $\Omega$ & J & NP & UG $\Omega$ & 0.5 \\
\hline Unknown-17 & $6 \mathrm{UG} /$ & $J$ & NP & UGR & 0.5 \\
\hline Unknown-18 & 13 UG $\Omega$ & $\mathrm{J}$ & NP & UG/ & 0.5 \\
\hline Unknown-19 & $280 \mathrm{UG} /$ & J & NP & UG/ & 0.5 \\
\hline Unknown-20 & 7.5 UG $\Omega$ & $J$ & NP & UG $\Omega$ & 0.5 \\
\hline Nitrobenzene-d5 & $71 \%$ & & & $\%$ & 0.5 \\
\hline 2-Fluorobiphenyl & $70 \%$ & & & $\%$ & 0.5 \\
\hline Terphenyl-d14 & $71 \%$ & & & $\%$ & 0.5 \\
\hline Phenol-d5 & $25 \%$ & & & $\%$ & 0.5 \\
\hline
\end{tabular}


APPENDIX D.2 Results of Semivolatile Organic Compound Analyses, Water, Salmon Site

\begin{tabular}{|c|c|c|c|c|c|c|}
\hline COMPOUND & RESULTS & UNITS & $\begin{array}{l}\text { DATA } \\
\text { QUAL. }\end{array}$ & $\begin{array}{l}\text { VALID. } \\
\text { QUAL. }\end{array}$ & CRDL UNITS & DILUTION \\
\hline \multicolumn{7}{|l|}{ C4-11-W (continued) } \\
\hline 2-Fluorophenol & & & & & $\%$ & 0.5 \\
\hline 2,4,6-Tribromophenol & & & & & $\%$ & 0.5 \\
\hline
\end{tabular}


APPENDIX D.2 Results of Semivolatile Organic Compound Analyses, Water, Salmon Site

\begin{tabular}{lll}
\hline COMPOUND & & DATA VALID. \\
\hline
\end{tabular}

C4-13-W

Phenol

bis(2-Chloroethyl) ether

2-Chlorophenol

1,3-Dichlorobenzene

1,4-Dichlorobenzene

Benzyl Alcohol

1,2-Dichlorobenzene

2-Methylphenol

bis(2-Chloroisopropyl) ether

4-Methylphenol

N-Nitroso-Di-n-dipropylamine

Hexachloroethane

Nitrobenzene

Isophorone

2-Nitrophenol

2,4-Dimethylphenol

Benzoic Acid

bis(2Chloroethoxy)methane

2,4-Dichlorophenol

1,2,4-Trichlorobenzene

Naphthalene

4-Chloroaniline

Hexachlorobutadiene

4-Chloro-3-Methlyphenol

2-Methylnaphthalene

Hexachlorocyclopentadiene

2,4,6-Trichlorophenol

2,4,5-Trichlorophenol

2-Chloronaphthalene

2-Nitroaniline

Dimethyl Phthalate

Acenaphthylene

2,6-Dinitrotoluene

3-Nitroaniline

Acenaphthene

2,4-Dinitrophenol

4-Nitrophenol

Dibenzofuran

2,4-Dinitrotoluene

Diethylphthalate

4-Chlorophenyl phenyl ether

Fluorene

4-Nitroaniline

4,6-Dinitro-2-methylphenol

$\mathrm{N}$-Nitrosodiphenylamine

\begin{tabular}{|c|c|}
\hline $10 \mathrm{UG} \Omega$ & u \\
\hline $10 \mathrm{UG} / \mathrm{L}$ & $\mathrm{U}$ \\
\hline 10 UG/L & U \\
\hline $10 \mathrm{UG} / \mathrm{L}$ & $u$ \\
\hline $10 \mathrm{UG} / \mathrm{L}$ & $\mathrm{u}$ \\
\hline $10 \mathrm{UG} / \mathrm{L}$ & $u$ \\
\hline $10 \mathrm{UG} /$ & $U$ \\
\hline 10 UG/L & $U$ \\
\hline 10 UG/L & $u$ \\
\hline $10 \mathrm{UG} / \mathrm{L}$ & $u$ \\
\hline $10 \mathrm{UG} / \mathrm{L}$ & $u$ \\
\hline $10 \mathrm{UG} /$ & $u$ \\
\hline $10 \mathrm{UG} /$ & $u$ \\
\hline $10 \mathrm{UG} \Omega$ & $u$ \\
\hline $10 \mathrm{UG} / \mathrm{L}$ & $U$ \\
\hline $10 \mathrm{UG} / \mathrm{L}$ & $u$ \\
\hline 50 UG几 & $\mathrm{U}$ \\
\hline $10 \mathrm{UG} / \mathrm{L}$ & $u$ \\
\hline $10 \mathrm{UG} / \mathrm{L}$ & $\mathrm{u}$ \\
\hline $10 \mathrm{UG} \Omega$ & $\mathrm{U}$ \\
\hline $10 \mathrm{UG} \Omega$ & $u$ \\
\hline $10 \mathrm{UG} / \mathrm{L}$ & $u$ \\
\hline $10 \mathrm{UG} / \mathrm{L}$ & $u$ \\
\hline 10 UG & $U$ \\
\hline 10 UG $/$ & $u$ \\
\hline 10 UG $\Omega$ & $U$ \\
\hline $10 \mathrm{UG} / \mathrm{L}$ & $u$ \\
\hline 50 UG/L & U \\
\hline $10 \mathrm{UG} /$ & U \\
\hline 50 UG/ & $u$ \\
\hline $10 \mathrm{UG} /$ & $u$ \\
\hline $10 \mathrm{UG} / \mathrm{L}$ & $\mathrm{U}$ \\
\hline 10 UG & $U$ \\
\hline $50 \mathrm{UG} \Omega$ & $U$ \\
\hline 10 UG/ & $U$ \\
\hline 50 UG $\Omega$ & $U$ \\
\hline $50 \mathrm{UG} / \mathrm{L}$ & $u$ \\
\hline $10 \mathrm{UG} /$ & $U$ \\
\hline $10 \mathrm{UG} \Omega$ & $U$ \\
\hline 10 UG $\Omega$ & $u$ \\
\hline $10 \mathrm{UG} / \mathrm{L}$ & $\mathrm{U}$ \\
\hline 10 UG & $\mathbf{U}$ \\
\hline $50 \mathrm{UG} \Omega$ & $\mathbf{U}$ \\
\hline 50 UG & $\mathbf{U}$ \\
\hline $10 \mathrm{UG} / \mathrm{L}$ & $u$ \\
\hline
\end{tabular}

CRDL UNITS

DILUTION

NP

$10 \mathrm{UG} / \mathrm{L}$

0.5

$10 \mathrm{UG} /$

0.5

10 UG/

0.5

10 UG/

0.5

10 UG/

0.5

10 UG/

0.5

10 UG/L

0.5

10 UG/L

0.5

10 UG/L

0.5

10 UGL

0.5

10 UG/

0.5

10 UGR

0.5

10 UGR

0.5

10 UG/

0.5

$10 \mathrm{UG} / \mathrm{L}$

0.5

$10 \mathrm{UG} /$

0.5

50 UGR

0.5

10 UG

0.5

10 UG

0.5

10 UG/

0.5

10 UG $/$

0.5

10 UG/

0.5

10 UG/

0.5

10 UG/

0.5

10 UG/

0.5

10 UG/L

0.5

10 UG/L

0.5

50 UGR

0.5

10 UG $\Omega \quad 0.5$

50 UG $\quad 0.5$

10 UG $\quad 0.5$

10 UG/ $\quad 0.5$

10 UG $\Omega \quad 0.5$

$50 \mathrm{UG} / \mathrm{L} \quad 0.5$

$10 \mathrm{UG} \Omega \quad 0.5$

$50 \mathrm{UG} \Omega \quad 0.5$

50 UG $\quad 0.5$

10 UGR $\quad 0.5$

10 UG $\quad 0.5$

10 UG $\quad 0.5$

10 UG $\quad 0.5$

10 UG $\quad 0.5$

50 UG/ $\quad 0.5$

$50 \mathrm{UG} / \mathrm{L} \quad 0.5$

10 UG $\Omega$

0.5 
APPENDIX D.2 Results of Semivolatile Organic Compound Analyses, Water, Salmon Site

\begin{tabular}{|c|c|c|c|c|c|}
\hline COMPOUND & RESULTS & $\begin{array}{l}\text { DATA } \\
\text { QUAL. }\end{array}$ & $\begin{array}{l}\text { VALID. } \\
\text { QUAL. }\end{array}$ & CRDL UNITS & DILUTION \\
\hline \multicolumn{6}{|l|}{ C4-13-W (continued) } \\
\hline 4-Bromophenyl phenylether & 10 UG $\Omega$ & $u$ & NP & $10 \mathrm{UG} / \mathrm{L}$ & 0.5 \\
\hline Hexachlorobenzene & 10 UG $\Omega$ & $u$ & NP & $10 \mathrm{UG} / \mathrm{L}$ & 0.5 \\
\hline Pentachlorophenol & 50 UG $\Omega$ & $u$ & NP & 50 UG/ & 0.5 \\
\hline Phenanthrene & 10 UG $/$ & $u$ & NP & 10 UG & 0.5 \\
\hline Anthracene & $10 \mathrm{UG} / \mathrm{L}$ & $u$ & NP & $10 \mathrm{UG} / \mathrm{L}$ & 0.5 \\
\hline Di-N-Butylphthalate & 10 UG几 & $u$ & NP & $10 \mathrm{UG} / \mathrm{L}$ & 0.5 \\
\hline Fluoranthene & 10 UG $/$ & $u$ & NP & $10 \mathrm{UG} / \mathrm{L}$ & 0.5 \\
\hline Pyrene & $10 \mathrm{UG/L}$ & $u$ & NP & $10 \mathrm{UG} / \mathrm{L}$ & 0.5 \\
\hline Butyl Benzyl Phthalate & 10 UG $/$ & $u$ & NP & 10 UG/ & 0.5 \\
\hline 3,3'-Dichlorobenzidine & 20 UGR & $u$ & NP & 20 UG $\Omega$ & 0.5 \\
\hline Benzo(a)Anthracene & 10 UG $\Omega$ & $u$ & NP & 10 UG $\Omega$ & 0.5 \\
\hline Chrysene & 10 UG/ & $u$ & $\mathrm{NP}$ & 10 UG/ & 0.5 \\
\hline bis(2-Ethylhexyl)phthalate & 12 UG/L & B & NP & 10 UG/L & 0.5 \\
\hline di-N-Octyl Phthalate & $10 \mathrm{UG} / \mathrm{L}$ & $u$ & NP & 10 UG/ & 0.5 \\
\hline Benzo(b)Fluoranthene & 10 UG $\Omega$ & $u$ & NP & 10 UG/L & 0.5 \\
\hline Benzo(k)Fluoranthene & $10 \mathrm{UG} / \mathrm{L}$ & $u$ & NP & $10 \mathrm{UG} / \mathrm{L}$ & 0.5 \\
\hline Benzo(a)Pyrene & 10 UG/ & $u$ & NP & 10 UG/L & 0.5 \\
\hline Indeno(1,2,3-Cd)pyrene & 10 UG $\Omega$ & $u$ & NP & 10 UG $\Omega$ & 0.5 \\
\hline Dibenzo(a,h)Anthracene & $10 \mathrm{UG} / \mathrm{L}$ & $u$ & $\mathrm{NP}$ & 10 UG/ & 0.5 \\
\hline Benzo(g,h,i)perylene & 10 UG $\Omega$ & $u$ & NP & $10 \mathrm{UG} / \mathrm{L}$ & 0.5 \\
\hline Ethanol,2-butoxy- & 240 UG/L & J & NP & UGR & 0.5 \\
\hline Dodecanoic acid & 36 UG/L & J & NP & $U G / L$ & 0.5 \\
\hline Unknown Phthalate & $7 \mathrm{UG} \Omega$ & J & NP & UG/L & 0.5 \\
\hline Hexadecanoic Acid & 21 UG/L & $\mathrm{s}$ & NP & UG/L & 0.5 \\
\hline Unknown-1 & 35 UG/ & J & NP & $U G / L$ & 0.5 \\
\hline Unknown-2 & 17 UG & J & NP & UG $\Omega$ & 0.5 \\
\hline Unknown-3 & $7 \mathrm{UG} \Omega$ & J & NP & UGR & 0.5 \\
\hline Nitrobenzene-d5 & $90 \%$ & & & $\%$ & 0.5 \\
\hline 2-Fluorobiphenyl & $84 \%$ & & & $\%$ & 0.5 \\
\hline Terphenyi-d14 & $65 \%$ & & & $\%$ & 0.5 \\
\hline Phenol-d5 & $42 \%$ & & & $\%$ & 0.5 \\
\hline 2-Fluorophenol & $63 \%$ & & & $\%$ & 0.5 \\
\hline 2,4,6-Trichlorophenol & $87 \%$ & & & $\%$ & 0.5 \\
\hline
\end{tabular}


APPENDIX D.2 Results of Semivolatile Organic Compound Analyses, Water, Salmon Site

\begin{tabular}{|c|c|c|c|c|c|}
\hline COMPOUND & RESULTS & $\begin{array}{l}\text { DATA } \\
\text { QUAL. }\end{array}$ & $\begin{array}{l}\text { VALID. } \\
\text { QUAL. }\end{array}$ & CRDL UNITS & DILUTION \\
\hline \multicolumn{6}{|l|}{ C1-14-W } \\
\hline Phenol & 10 UG $\Omega$ & $u$ & - & $10 \mathrm{UG} \Omega$ & 0.5 \\
\hline bis(2-Chloroethyl) ether & 10 UG/ & $u$ & $\mathbf{R}$ & 10 UG $\Omega$ & 0.5 \\
\hline 2-Chlorophenol & 10 UG $\Omega$ & $u$ & - & 10 UG $/$ & 0.5 \\
\hline 1,3-Dichlorobenzene & $10 \mathrm{UG} / \mathrm{L}$ & $u$ & $\mathbf{R}$ & $10 \mathrm{UG} /$ & 0.5 \\
\hline 1,4-Dichlorobenzene & $10 \mathrm{UG} / \mathrm{L}$ & $u$ & $\mathbf{R}$ & $10 \mathrm{UG} / \mathrm{L}$ & 0.5 \\
\hline Benzyl Alcohol & $10 \mathrm{UG} / \mathrm{L}$ & $u$ & $\mathbf{R}$ & $10 \mathrm{UG} / \mathrm{L}$ & 0.5 \\
\hline 1,2-Dichlorobenzene & $10 \mathrm{UG} / \mathrm{L}$ & $u$ & $\mathbf{R}$ & $10 \mathrm{UG} \Omega$ & 0.5 \\
\hline 2-Methylphenol & 10 UGR & $u$ & - & $10 \mathrm{UG} / \mathrm{L}$ & 0.5 \\
\hline bis(2-Chloroisopropyl) ether & 10 UG $/$ & $u$ & R & 10 UG $\Omega$ & 0.5 \\
\hline 4-Methylphenol & $10 \mathrm{UG} /$ & $u$ & $\ldots$ & $10 \mathrm{UG} \Omega$ & 0.5 \\
\hline N-Nitroso-Di-n-dipropylamine & $10 \mathrm{UG} / \mathrm{L}$ & $u$ & $\mathbf{R}$ & $10 \mathrm{UG} / \mathrm{L}$ & 0.5 \\
\hline Hexachloroethane & 10 UG $\Omega$ & $u$ & $\mathbf{R}$ & $10 \mathrm{UG} /$ & 0.5 \\
\hline Nitrobenzene & 10 UG $\Omega$ & $u$ & $\mathbf{R}$ & 10 UG $\Omega$ & 0.5 \\
\hline Isophorone & $10 \mathrm{UG} / \mathrm{L}$ & $u$ & $\mathbf{R}$ & 10 UG/L & 0.5 \\
\hline 2-Nitrophenol & $10 \mathrm{UG} / \mathrm{L}$ & $u$ & - & $10 \mathrm{UG} / \mathrm{L}$ & 0.5 \\
\hline 2,4-Dimethylphenol & 10 UG/L & $u$ & - & $10 \mathrm{UG} \Omega$ & 0.5 \\
\hline Benzoic Acid & 50 UG/L & $u$ & - & 50 UG $\Omega$ & 0.5 \\
\hline bis(2Chloroethoxy)methane & 10 UG $\Omega$ & $u$ & $\mathbf{R}$ & $10 \mathrm{UG} \Omega$ & 0.5 \\
\hline 2,4-Dichlorophenol & 10 UG $\Omega$ & $u$ & - & 10 UG $\Omega$ & 0.5 \\
\hline 1,2,4-Trichlorobenzene & $10 \mathrm{UG} / \mathrm{L}$ & $u$ & $\mathbf{R}$ & 10 UG $\Omega$ & 0.5 \\
\hline Naphthalene & 10 UG $\Omega$ & $u$ & $\mathbf{R}$ & 10 UG/ & 0.5 \\
\hline 4-Chloroaniline & $10 \mathrm{UG} \Omega$ & $u$ & $\mathbf{R}$ & 10 UG/ & 0.5 \\
\hline Hexachlorobutadiene & $10 \mathrm{UG} /$ & $u$ & R & $10 \mathrm{UG} /$ & 0.5 \\
\hline 4-Chloro-3-Methlyphenol & 10 UG $\Omega$ & $u$ & - & 10 UG $\Omega$ & 0.5 \\
\hline 2-Methylnaphthalene & $10 \mathrm{UG} / \mathrm{L}$ & $u$ & $R$ & $10 \mathrm{UG} / \mathrm{L}$ & 0.5 \\
\hline Hexachlorocyclopentadiene & $10 \mathrm{UG} / \mathrm{L}$ & $u$ & $\mathbf{R}$ & $10 \mathrm{UG} / \mathrm{L}$ & 0.5 \\
\hline 2,4,6-Trichlorophenol & 10 UG $\Omega$ & $u$ & - & $10 \mathrm{UG} \Omega$ & 0.5 \\
\hline 2,4,5-Trichlorophenol & 50 UG/ & $u$ & - & 50 UGR & 0.5 \\
\hline 2-Chloronaphthalene & $10 \mathrm{UG} / \mathrm{L}$ & $u$ & $R$ & $10 \mathrm{UG} /$ & 0.5 \\
\hline 2-Nitroaniline & $50 \mathrm{UG} / \mathrm{L}$ & $u$ & $R$ & 50 UG/ & 0.5 \\
\hline Dimethyl Phthalate & $10 \mathrm{UG} / \mathrm{L}$ & $u$ & R & 10 UG $\Omega$ & 0.5 \\
\hline Acenaphthylene & $10 \mathrm{UG} /$ & $u$ & $\mathbf{R}$ & $10 \mathrm{UG} \Omega$ & 0.5 \\
\hline 2,6-Dinitrotoluene & $10 \mathrm{UG} / \mathrm{L}$ & $u$ & $\mathrm{R}$ & 10 UG & 0.5 \\
\hline 3-Nitroaniline & $50 \mathrm{UG} / \mathrm{L}$ & $u$ & $\mathbf{R}$ & $50 \mathrm{UG} / \mathrm{L}$ & 0.5 \\
\hline Acenaphthene & $10 \mathrm{UG} \Omega$ & $u$ & $R$ & 10 UGR & 0.5 \\
\hline 2,4-Dinitrophenol & 50 UG几 & $u$ & - & 50 UG & 0.5 \\
\hline 4-Nitrophenol & 50 UG $\Omega$ & $u$ & 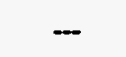 & $50 \mathrm{UG} / \mathrm{L}$ & 0.5 \\
\hline Dibenzofuran & 10 UG $/$ & $u$ & $\mathbf{R}$ & $10 \mathrm{UG} /$ & 0.5 \\
\hline 2,4-Dinitrotoluene & $10 \mathrm{UG} / \mathrm{L}$ & $u$ & R & 10 UG $\Omega$ & 0.5 \\
\hline Diethylphthalate & 10 UG $\Omega$ & $u$ & $\mathbf{R}$ & $10 \mathrm{UG} \Omega$ & 0.5 \\
\hline 4-Chlorophenyl phenyl ether & $10 \mathrm{UG} / \mathrm{L}$ & $u$ & $\mathbf{A}$ & $10 \mathrm{UG} \Omega$ & 0.5 \\
\hline Fluorene & 10 UG $\Omega$ & $u$ & $\mathbf{R}$ & 10 UG $\Omega$ & 0.5 \\
\hline 4-Nitroaniline & $50 \mathrm{UG} / \mathrm{L}$ & $u$ & $\mathbf{R}$ & $50 \mathrm{UG} /$ & 0.5 \\
\hline 4,6-Dinitro-2-methylphenol & 50 UG $\Omega$ & $u$ & - & 50 UG $\Omega$ & 0.5 \\
\hline N-Nitrosodiphenylamine & $10 \mathrm{UG} / \mathrm{L}$ & $u$ & $\mathbf{R}$ & 10 Ua $\Omega$ & 0.5 \\
\hline
\end{tabular}


APPENDIX D.2 Results of Semivolatile Organic Compound Analyses, Water, Salmon Site

\begin{tabular}{|c|c|c|c|c|c|}
\hline COMPOUND & RESULTS & $\begin{array}{l}\text { DATA } \\
\text { QUAL. }\end{array}$ & $\begin{array}{l}\text { VALID. } \\
\text { QUAL. }\end{array}$ & CRDL UNITS & DILUT \\
\hline \multicolumn{6}{|l|}{ C1-14-W (continued) } \\
\hline 4-Bromophenyl phenylether & 10 UG/ & $u$ & $\mathrm{R}$ & 10 UGn & 0. \\
\hline Hexachlorobenzene & 10 UG $/$ & $u$ & $\mathbf{R}$ & 10 UG/ & 0.5 \\
\hline Pentachlorophenol & 50 UG/ & $u$ & -. & 50 UG & 0.5 \\
\hline Phenanthrene & $10 \mathrm{UG} / \mathrm{L}$ & $u$ & R & $10 \mathrm{UG} / \mathrm{L}$ & 0.5 \\
\hline Anthracene & 10 UG/L & $u$ & R & $10 \mathrm{UG} / \mathrm{L}$ & 0.5 \\
\hline Di-N-Butylphthalate & $10 \mathrm{UG} / \mathrm{L}$ & $u$ & R & 10 UGR & 0.5 \\
\hline Fluoranthene & 10 UG $/$ & $u$ & R & 10 UG/L & 0.5 \\
\hline Pyrene & 10 UG/ & $u$ & R & $10 \mathrm{UG} / \mathrm{L}$ & 0.5 \\
\hline Butyl Benzyl Phthalate & 10 UG/ & $u$ & R & $10 \mathrm{UG} / \mathrm{L}$ & 0.5 \\
\hline 3,3'-Dichlorobenzidine & 20 UG/ & $u$ & R & 20 UGL & 0.5 \\
\hline Benzo(a)Anthracene & 10 UG & $u$ & $\mathbf{R}$ & 10 UG/ & 0.5 \\
\hline Chrysene & $10 \mathrm{UG} / \mathrm{L}$ & $u$ & $\mathbf{R}$ & $10 \mathrm{UG} / \mathrm{L}$ & 0.5 \\
\hline bis(2-Ethylhexyl)phthalate & $4 \mathrm{UG} /$ & BJ & UJ & $10 \mathrm{UG} / \mathrm{L}$ & 0.5 \\
\hline di-N-Octyl Phthalate & 10 UG $\Omega$ & $u$ & $R$ & $10 \mathrm{UG} / \mathrm{L}$ & 0. \\
\hline Benzo(b)Fluoranthene & $10 \mathrm{UG} / \mathrm{L}$ & $u$ & $R$ & $10 \mathrm{UG} / \mathrm{L}$ & 0.5 \\
\hline Benzo(k)Fluoranthene & 10 UG/ & $u$ & R & $10 \mathrm{UG} / \mathrm{L}$ & 0.5 \\
\hline Benzo(a)Pyrene & 10 UG/ & $u$ & $R$ & $10 \mathrm{UG} / \mathrm{L}$ & 0.5 \\
\hline Indeno(1,2,3-Cd)pyrene & 10 UG/L & $u$ & $\mathbf{R}$ & $10 \mathrm{UG} \Omega$ & 0.5 \\
\hline Dibenzo(a,h)Anthracene & 10 UG/L & $u$ & R & $10 \mathrm{UG} \Omega$ & 0. \\
\hline Benzo(g,h,i)Perylene & 10 UG/ & $u$ & $R$ & 10 UG $\Omega$ & 0. \\
\hline Unknown-1 & $5 \mathrm{UG} \Omega$ & J & $\ldots$ & UGR & 0.5 \\
\hline Unknown-2 & 35 UG & J & $\ldots$ & UGR & 0.5 \\
\hline Nitrobenzene-d5 & $0 \%$ & * & & $\%$ & 0. \\
\hline 2-Fluorobiphenyl & $52 \%$ & & & $\%$ & 0.5 \\
\hline Terphenyl-d14 & $19 \%$ & $\cdot$ & & $\%$ & 0.5 \\
\hline Phenol-d5 & $20 \%$ & & & $\%$ & 0.5 \\
\hline 2-Fluorophenol & $27 \%$ & & & $\%$ & 0.5 \\
\hline 2,4,6-Tribromophenol & $21 \%$ & & & $\%$ & 0 \\
\hline
\end{tabular}


APPENDIX D.2 Results of Semivolatile Organic Compound Analyses, Water,

Salmon Site

DATA VALID.

COMPOUND

C1-15-W

Phenol

bis(2-Chloroethyl) ether

2-Chlorophenol

1,3-Dichlorobenzene

1,4-Dichlorobenzene

Benzyl Alcohol

1,2-Dichlorobenzene

2-Methylphenol

bis(2-Chloroisopropyl) ether

4-Methylphenol

N-Nitroso-Di-n-dipropylamine

Hexachloroethane

Nitrobenzene

Isophorone

2-Nitrophenol

2,4-Dimethylphenol

Benzoic Acid

bis(2Chloroethoxy)methane

2,4-Dichlorophenol

1,2,4-Trichlorobenzene

Naphthalene

4-Chloroaniline

Hexachlorobutadiene

4-Chloro-3-Methlyphenol

2-Methylnaphthalene

Hexachlorocyclopentadiene

2,4,6-Trichlorophenol

2,4,5-Trichlorophenol

2-Chloronaphthalene

2-Nitroaniline

Dimethyl Phthalate

Acenaphthylene

2,6-Dinitrotoluene

3-Nitroaniline

Acenaphthene

2,4-Dinitrophenol

4-Nitrophenol

Dibenzofuran

2,4-Dinitrotoluene

Diethylphthalate

4-Chlorophenyl phenyl ether

Fluorene

4-Nitroaniline

4,6-Dinitro-2-methylphenol

$\mathrm{N}$-Nitrosodiphenylamine

\begin{tabular}{|c|c|}
\hline 10 UG/ & $U$ \\
\hline $10 \mathrm{UG} / \mathrm{L}$ & $u$ \\
\hline 10 UG $/$ & $u$ \\
\hline $10 \mathrm{UG} / \mathrm{L}$ & $u$ \\
\hline $10 \mathrm{UG} / \mathrm{L}$ & U \\
\hline 10 UG $/$ L & u \\
\hline $10 \mathrm{UG} / \mathrm{L}$ & u \\
\hline 10 UG $/$ & U \\
\hline 10 UG & $u$ \\
\hline $10 \mathrm{UG} \Omega$ & U \\
\hline $10 \mathrm{UG} \Omega$ & $u$ \\
\hline $10 \mathrm{UG} / \mathrm{L}$ & U \\
\hline 10 UG/L & u \\
\hline $10 \mathrm{UG} \Omega$ & $\mathrm{u}$ \\
\hline $10 \mathrm{UG} \Omega$ & $u$ \\
\hline 10 UGR & $u$ \\
\hline $50 \mathrm{UG} / \mathrm{L}$ & U \\
\hline 10 UG/L & U \\
\hline $10 \mathrm{UG} / \mathrm{L}$ & $u$ \\
\hline $10 \mathrm{UG} / \mathrm{L}$ & $u$ \\
\hline 10 UG/L & $\mathrm{u}$ \\
\hline 10 UG & u \\
\hline $10 \mathrm{UG} \Omega$ & $u$ \\
\hline 10 UG $\Omega$ & u \\
\hline 10 UG/ & u \\
\hline $10 \mathrm{UG} \Omega$ & $u$ \\
\hline 10 UG/ & u \\
\hline 50 UG/L & U \\
\hline 10 UG/ & $U$ \\
\hline 50 UG/ & U \\
\hline $10 \mathrm{UG} / \mathrm{L}$ & $u$ \\
\hline $10 \mathrm{UG} /$ & $\mathrm{U}$ \\
\hline $10 \mathrm{UG} \Omega$ & $\mathrm{u}$ \\
\hline $50 \mathrm{UG} \Omega$ & $u$ \\
\hline 10 UG & u \\
\hline 50 UG $\Omega$ & u \\
\hline 50 UG $\Omega$ & $u$ \\
\hline $10 \mathrm{UG} / \mathrm{L}$ & $U$ \\
\hline $10 \mathrm{UG} \Omega$ & $U$ \\
\hline 10 UG几 & $u$ \\
\hline 10 UG $\Omega$ & $U$ \\
\hline $10 \mathrm{UG} \Omega$ & $u$ \\
\hline $50 \mathrm{UG} / \mathrm{L}$ & $U$ \\
\hline 50. UG $\Omega$ & $u$ \\
\hline $10 \mathrm{UG} \Omega$ & $\mathrm{u}$ \\
\hline
\end{tabular}

$-$.

$-$

$\cdots$

$-$

$-$

$--$

$--$

$-$

$-$

$-$

-.

$-$

$-$

$-$

$-$

$-$

$-$

$-$

$-$

$-$

$-$

$-$

$-$

$-$

$-$

$\overline{-}$

$-$

$-$

$-$

$-$

$-$

$-$

$-$

$-$

$-$

$-$

$-$

$-$

$-$

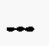

$-$

-

$\overline{-}$

$\overline{-}$

$\overline{-}$

$\overline{-}$

$\overline{-}$
CRDL UNITS

DILUTION

$\begin{array}{ll}10 \mathrm{UG} / \mathrm{L} & 0.5\end{array}$

$10 \mathrm{UG} / \mathrm{L}$

0.5

$10 \mathrm{UG} /$

0.5

10 UG/

0.5

10 UG $/$

0.5

10 UG $\Omega$

0.5

$10 \mathrm{UG} / \mathrm{L}$

0.5

10 UG $\Omega$

0.5

$10 \mathrm{UG} / \mathrm{L}$

0.5

10 UG $\Omega$

0.5

$10 \mathrm{UG} \Omega$

0.5

10 UG/

0.5

10 UG/

0.5

10 UG/

0.5

10 UG $\Omega$

0.5

$10 \mathrm{UG} \Omega$

0.5

50 UG

0.5

10 UG $\Omega$

0.5

10 UG $\Omega$

0.5

10 UG $\Omega$

0.5

10 UGR

0.5

10 UG/

0.5

10 UG $\Omega$

0.5

10 UG $\Omega$

0.5

10 UG/

0.5

10 UG

0.5

$10 \mathrm{UG} \Omega$

0.5

50 UG

0.5

10 UGR

0.5

50 UG/

0.5

$10 \mathrm{UG} / \mathrm{L}$

0.5

$10 \mathrm{UG} / \mathrm{L}$

0.5

$10 \mathrm{UG} / \mathrm{L}$

0.5

50 UG $/$

0.5

$10 \mathrm{UG} /$

0.5

50 UG $/$

0.5

50 UG/L

0.5

10 UGR

0.5

10 UG/

0.5

10 UGL

0.5

10 UG

0.5

$10 \mathrm{UG} /$

0.5

50 UG

0.5

50 UG

0.5

10 UG $\Omega$

0.5 
APPENDIX D.2 Results of Semivolatile Organic Compound Analyses, Water, Salmon Site

\begin{tabular}{|c|c|c|c|c|c|}
\hline COMPOUND & RESULTS & $\begin{array}{l}\text { DATA } \\
\text { QUAL. }\end{array}$ & $\begin{array}{l}\text { VALID. } \\
\text { QUAL. }\end{array}$ & CRDL UNITS & DILUTI \\
\hline \multicolumn{6}{|l|}{ C1-15-W (continued) } \\
\hline 4-Bromophenyl phenylether & 10 UG $\Omega$ & $u$ & $\ldots$ & 10 UG $/ 2$ & 0.5 \\
\hline Hexachlorobenzene & 10 UG $\Omega$ & $u$ & --- & $10 \mathrm{UG} \Omega$ & 0.5 \\
\hline Pentachlorophenol & 50 UG几 & $u$ & $\cdots$ & 50 UG几 & 0.5 \\
\hline Phenanthrene & 10 UG & $u$ & $\cdots$ & $10 \mathrm{UG} / \mathrm{L}$ & 0.5 \\
\hline Anthracene & $10 \mathrm{UG} / \mathrm{L}$ & $u$ & $\cdots$ & $10 \mathrm{UG} / \mathrm{L}$ & 0.5 \\
\hline Di-N-Butyiphthalate & $10 \mathrm{UG} / \mathrm{L}$ & $u$ & - & 10 UG/ & 0.5 \\
\hline Fluoranthene & $10 \mathrm{UG} / \mathrm{L}$ & $u$ & - & $10 \mathrm{UG} / \mathrm{L}$ & 0.5 \\
\hline Pyrene & $10 \mathrm{UG} / \mathrm{L}$ & $\mathrm{u}$ & - & $10 \mathrm{UG} / \mathrm{L}$ & 0.5 \\
\hline Butyl Benzyl Phthalate & $10 \mathrm{UG} / \mathrm{L}$ & $u$ & $\ldots$ & 10 UG/ & 0.5 \\
\hline 3,3'-Dichlorobenzidine & 20 UG & $u$ & $\ldots$ & 20 UG & 0.5 \\
\hline Benzo(a)Anthracene & 10 UG几 & $u$ & $\ldots$ & 10 UG/ & 0.5 \\
\hline Chrysene & 10 UG $\Omega$ & $u$ & $\cdots$ & $10 \mathrm{UG} / \mathrm{L}$ & 0.5 \\
\hline bis(2-Ethylhexyl)phthalate & 11 UG/ & B & - & $10 \mathrm{UG} / \mathrm{L}$ & 0.5 \\
\hline di-N-Octyl Phthalate & 10 UG $\Omega$ & $u$ & $\cdots$ & 10 UG/L & 0.5 \\
\hline Benzo(b)Fluoranthene & 10 UG $\Omega$ & $u$ & -- & $10 \mathrm{UG} / \mathrm{L}$ & 0.5 \\
\hline Benzo(k)Fluoranthene & 10 UG $\Omega$ & $u$ & $\cdots$ & 10 UG $/$ & 0.5 \\
\hline Benzo(a)Pyrene & 10 UG $\Omega$ & $u$ & -. & $10 \mathrm{UG} /$ & 0.5 \\
\hline Indeno(1,2,3-Cd)pyrene & $10 \mathrm{UG \Omega}$ & $u$ & - & $10 \mathrm{UG} \Omega$ & 0.5 \\
\hline Dibenzo(a,h)Anthracene & $10 \mathrm{UG} \Omega$ & $u$ & $\ldots$ & $10 \mathrm{UG} / \mathrm{L}$ & 0.5 \\
\hline Benzo(g,h,i)Perylene & $10 \mathrm{UG} /$ & $u$ & $\ldots$ & $10 \mathrm{UG} \Omega$ & 0.5 \\
\hline Unknown-1 & 30 UG $\Omega$ & $J$ & 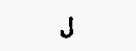 & $U G /$ & 0.5 \\
\hline Unknown-2 & 260 UG几 & $\mathbf{J}$ & J & $U G / L$ & 0.5 \\
\hline Unknown-3 & 5 UG/ & J & J & UG/L & 0.5 \\
\hline Unknown-4 & 14 UG几 & J & R & UG/ & 0.5 \\
\hline Unknown-5 & 5 UGR & J & $R$ & UG $\Omega$ & 0.5 \\
\hline Unknown-6 & 5 UG & J & 8 & UG/ & 0.5 \\
\hline Nitrobenzene-d5 & $0 \%$ & • & & $\%$ & 0.5 \\
\hline 2-Fluorobiphenyl & $75 \%$ & & & $\%$ & 0.5 \\
\hline Terphenyl-d14 & $62 \%$ & & & $\%$ & 0.5 \\
\hline Phenol-d5 & $40 \%$ & & & $\%$ & 0.5 \\
\hline 2-Fluorophenol & $57 \%$ & & & $\%$ & 0.5 \\
\hline 2,4,6-Tribromophenol & $80 \%$ & & & $\%$ & 0.5 \\
\hline
\end{tabular}


APPENDIX D.2 Results of Semivolatile Organic Compound Analyses, Water, Salmon Site

\begin{tabular}{|c|c|c|c|c|c|}
\hline COMPOUND & RESULTS & $\begin{array}{l}\text { DATA } \\
\text { QUAL. }\end{array}$ & $\begin{array}{l}\text { VALID. } \\
\text { QUAL. }\end{array}$ & CRDL UNITS & DILUTION \\
\hline \multicolumn{6}{|l|}{ C1-15-W-MS } \\
\hline Phenol & $32 \%$ & & NP & $\%$ & 0.5 \\
\hline 2-Chlorophenol & $69 \%$ & & NP & $\%$ & 0.5 \\
\hline 1,4-Dichlorobenzene & $83 \%$ & & NP & $\%$ & 0.5 \\
\hline N-Nitroso-Di-n-dipropylamine & $89 \%$ & & NP & $\%$ & 0.5 \\
\hline 1,2,4-Trichlorobenzene & $83 \%$ & & NP & $\%$ & 0.5 \\
\hline 4-Chloro-3-Methlyphenol & $67 \%$ & & NP & $\%$ & 0.5 \\
\hline Acenaphthene & $84 \%$ & & NP & $\%$ & 0.5 \\
\hline 4-Nitrophenol & $17 \%$ & & NP & $\%$ & 0.5 \\
\hline 2,4-Dinitrotoluene & $8 \%$ & * & NP & $\%$ & 0.5 \\
\hline Pentachlorophenol & $88 \%$ & & NP & $\%$ & 0.5 \\
\hline Pyrene & $71 \%$ & & NP & $\%$ & 0.5 \\
\hline Nitrobenzene-d5 & $18 \%$ & • & NP & $\%$ & 0.5 \\
\hline 2-Fluorobiphenyl & $83 \%$ & & NP & $\%$ & 0.5 \\
\hline Terphenyi-d14 & $67 \%$ & & NP & $\%$ & 0.5 \\
\hline Phenol-d5 & $33 \%$ & & NP & $\%$ & 0.5 \\
\hline 2-Fluorophenol & $43 \%$ & & NP & $\%$ & 0.5 \\
\hline 2,4,6-Tribromophenol & $77 \%$ & & NP & $\%$ & 0.5 \\
\hline \multicolumn{6}{|l|}{ C1-15-W-MSD } \\
\hline Phenol & $0 \%$ & * & NP & $\%$ & 0.5 \\
\hline 2-Chlorophenol & $13 \%$ & • & NP & $\%$ & 0.5 \\
\hline 1,4-Dichlorobenzene & $87 \%$ & & NP & $\%$ & 0.5 \\
\hline N-Nitroso-Di-n-dipropylamine & $90 \%$ & & NP & $\%$ & 0.5 \\
\hline 1,2,4-Trichlorobenzene & $86 \%$ & & NP & $\%$ & 0.5 \\
\hline 4-Chloro-3-Methlyphenol & $11 \%$ & * & NP & $\%$ & 0.5 \\
\hline Acenaphthene & $79 \%$ & & NP & $\%$ & 0.5 \\
\hline 4-Nitrophenol & $0 \%$ & * & NP & $\%$ & 0.5 \\
\hline 2,4-Dinitrotoluene & $3 \%$ & * & NP & $\%$ & 0.5 \\
\hline Pentachlorophenol & $67 \%$ & & NP & $\%$ & 0.5 \\
\hline Pyrene & $72 \%$ & & NP & $\%$ & 0.5 \\
\hline Nitrobenzene-d5 & $7 \%$ & * & NP & $\%$ & 0.5 \\
\hline 2-Fluorobiphenyl & $78 \%$ & & NP & $\%$ & 0.5 \\
\hline Terphenyl-d14 & $71 \%$ & & NP & $\%$ & 0.5 \\
\hline Phenol-d5 & $3 \%$ & * & NP & $\%$ & 0.5 \\
\hline 2-Fluorophenol & $5 \%$ & * & NP & $\%$ & 0.5 \\
\hline 2,4,6-Tribromophenol & $40 \%$ & & NP & $\%$ & 0.5 \\
\hline
\end{tabular}


APPENDIX D.2 Results of Semivolatile Organic Compound Analyses, Water, Salmon Site

\begin{tabular}{|c|c|c|c|c|c|}
\hline COMPOUND & RESULTS & $\begin{array}{l}\text { DATA } \\
\text { QUAL. }\end{array}$ & $\begin{array}{l}\text { VALID. } \\
\text { QUAL. }\end{array}$ & CRDL UNITS & DILUTION \\
\hline \multicolumn{6}{|l|}{$C 1-16-W$} \\
\hline Phenol & 12 UG/ & $u$ & ... & 12 UG/L & 0.5 \\
\hline bis(2-Chloroethyl) ether & 12 UG $\Omega$ & $u$ & -.- & 12 UG/ & 0.5 \\
\hline 2-Chlorophenol & 12 UG/L & $u$ & -- & 12 UG/ & 0.5 \\
\hline 1,3-Dichlorobenzene & 12 UG $\Omega$ & $u$ & - & 12 UG/ & 0.5 \\
\hline 1,4-Dichlorobenzene & 12 UG几 & $u$ & -- & 12 UG/ & 0.5 \\
\hline Benzyl Alcohol & 12 UG/ & $u$ & -- & $12 U G / L$ & 0.5 \\
\hline 1,2-Dichlorobenzene & 12 UG/L & $u$ & -. & $12 \mathrm{UG} / \mathrm{L}$ & 0.5 \\
\hline 2-Methylphenol & 12 UG/ & $u$ & $\cdots$ & 12 UG/L & 0.5 \\
\hline bis(2-Chloroisopropyi) ether & 12 UG $\Omega$ & $u$ & -.- & 12 UG $\Omega$ & 0.5 \\
\hline 4-Methylphenol & 12 UGn & $u$ & - & 12 UG/L & 0.5 \\
\hline N-Nitroso-Di-n-dipropylamine & 12 UG/L & $u$ & -- & 12 UG $\Omega$ & 0.5 \\
\hline Hexachloroethane & 12 UG/ & $u$ & - & 12 UG/ & 0.5 \\
\hline Nitrobenzene & $12 \mathrm{UG} / \mathrm{L}$ & $u$ & $\ldots$ & 12 UG/ & 0.5 \\
\hline Isophorone & 12 UG/L & $u$ & $\cdots$ & 12 UG/L & 0.5 \\
\hline 2-Nitrophenol & 12 UG/ & $u$ & $\ldots$ & 12 UG/L & 0.5 \\
\hline 2,4-Dimethylphenol & $12 \mathrm{UG} / \mathrm{L}$ & $u$ & $\cdots$ & 12 UG/L & 0.5 \\
\hline Benzoic Acid & 58 UGR & $u$ & -- & $58 \mathrm{UG} / \mathrm{L}$ & 0.5 \\
\hline bis(2Chloroethoxy)methane & 12 UG/ & $u$ &.- & 12 UG/ & 0.5 \\
\hline 2,4-Dichlorophenol & 12 UG/L & $u$ & $\ldots$ & 12 UG/ & 0.5 \\
\hline 1,2,4-Trichlorobenzene & 12 UG $\Omega$ & $u$ & $\cdots$ & 12 UG $\Omega$ & 0.5 \\
\hline Naphthalene & 12 UGR & $u$ & - & 12 UG $\Omega$ & 0.5 \\
\hline 4-Chloroaniline & 12 UG & $u$ & $\ldots$ & 12 UG $\Omega$ & 0.5 \\
\hline Hexachlorobutadiene & 12 UG/L & $u$ & $\ldots$ & 12 UG/ & 0.5 \\
\hline 4-Chloro-3-Methlyphenol & 12 UG $\Omega$ & $u$ & - & 12 UG $\Omega$ & 0.5 \\
\hline 2-Methyinaphthalene & 12 UG/L & $u$ & $\ldots$ & 12 UG/ & 0.5 \\
\hline Hexachlorocyclopentadiene & 12 UG $/$ & $u$ & $\ldots$ & 12 UG几 & 0.5 \\
\hline 2,4,6-Trichlorophenol & 12 UG $\Omega$ & $u$ & $\ldots$ & 12 UG $\Omega$ & 0.5 \\
\hline 2,4,5-Trichlorophenol & 58 UG $\Omega$ & $u$ & $\ldots$ & 58 UG $\Omega$ & 0.5 \\
\hline 2-Chloronaphthalene & 12 UG $\Omega$ & $u$ & $\ldots$ & 12 UG几 & 0.5 \\
\hline 2-Nitroaniline & 58 UGR & $u$ & $\ldots$ & 58 UG $\Omega$ & 0.5 \\
\hline Dimethyl Phthalate & 12 UG/L & $u$ & $\ldots$ & 12 UG $\Omega$ & 0.5 \\
\hline Acenaphthylene & 12 UG/L & $u$ & - & 12 UG $\Omega$ & 0.5 \\
\hline 2,6-Dinitrotoluene & 12 UG/L & $u$ & $\ldots$ & 12 UG $/$ & 0.5 \\
\hline 3-Nitroaniline & 58 UG几 & $u$ & - & 58 UG几 & 0.5 \\
\hline Acenaphthene & 12 UG几 & $u$ & - & 12 UG几 & 0.5 \\
\hline 2,4-Dinitrophenol & 58 UG/ & $u$ & - & 58 UG/ & 0.5 \\
\hline 4-Nitrophenol & 58 UG $\Omega$ & $u$ & - & 58 UG $\Omega$ & 0.5 \\
\hline Dibenzofuran & 12 UG/ & $u$ & $\ldots$ & 12 UG $\Omega$ & 0.5 \\
\hline 2,4-Dinitrotoluene & 12 UG $\Omega$ & $u$ & $\ldots$ & 12 UG $\Omega$ & 0.5 \\
\hline Diethyiphthalate & 12 UG $\Omega$ & $u$ & $\ldots$ & 12 Uan & 0.5 \\
\hline 4-Chlorophenyl phenyl ether & 12 UG $\Omega$ & $u$ & - & 12 UGn & 0.5 \\
\hline Fluorene & 12 UG $\Omega$ & $u$ & $\ldots$ & 12 UG/L & 0.5 \\
\hline 4-Nitroaniline & 58 UG/ & $u$ & $\ldots$ & 58 UG/ & 0.5 \\
\hline 4,6-Dinitro-2-methylphenol & 58 UG/ & $u$ & - & 58 UG/ & 0.5 \\
\hline N-Nitrosodiphenylamine & 12 UG $\Omega$ & $u$ & $\ldots$ & 12 UG $\Omega$ & 0.5 \\
\hline
\end{tabular}


APPENDIX D.2 Results of Semivolatile Organic Compound Analyses, Water, Salmon Site

\begin{tabular}{|c|c|c|c|}
\hline COMPOUND & RESULTS & UNITS & $\begin{array}{l}\text { DATA } \\
\text { QUAL. }\end{array}$ \\
\hline \multicolumn{4}{|l|}{ C1-16-W (continued) } \\
\hline 4-Bromophenyl phenylether & & 12 UG/L & $u$ \\
\hline Hexachlorobenzene & & 12 UG $\Omega$ & $u$ \\
\hline Pentachlorophenol & & 58 UG几 & $u$ \\
\hline Phenanthrene & & 12 UG $\Omega$ & $u$ \\
\hline Anthracene & & 12 UG & $u$ \\
\hline Di-N-Butylphthalate & & 12 UG/ & $u$ \\
\hline Fluoranthene & & 12 UG $\Omega$ & $u$ \\
\hline Pyrene & & 12 UG & $u$ \\
\hline Butyl Benzyl Phthalate & & 12 UG/L & $u$ \\
\hline 3,3'-Dichlorobenzidine & & 23 UG & $u$ \\
\hline Benzo(a)Anthracene & & 12 UG/ & $u$ \\
\hline Chrysene & & 12 UG/ & $u$ \\
\hline bis(2-Ethylhexyl)phthalate & & 9 UG & BJ \\
\hline di-N-Octyl Phthalate & & 12 UG & $u$ \\
\hline Benzo(b)Fluoranthene & & 12 UG几 & $u$ \\
\hline Benzo(k)Fluoranthene & & 12 UG/ & $u$ \\
\hline Benzo(a)Pyrene & & 12 UG $\Omega$ & $u$ \\
\hline Indeno(1,2,3-Cd)pyrene & & 12 UG/L & $u$ \\
\hline Dibenzo(a,h)Anthracene & & 12 UG/L & $u$ \\
\hline Benzo(g,h,i)Perylene & & 12 UG/L & $u$ \\
\hline Unknown-1 & & 8 UG/ & $J$ \\
\hline Unknown-2 & & $8 \mathrm{UG} / \mathrm{L}$ & J \\
\hline Unknown-3 & & 14 UG/ & J \\
\hline Dodecanoic acid & & 21 UG & J \\
\hline Unknown Phthalate & & 7 UG & $\checkmark$ \\
\hline Unknown-6 & & 7 UG几 & J \\
\hline Unknown-7 & & 7 UG/L & $J$ \\
\hline Unknown-8 & & 7 UG/ & J \\
\hline Unknown-9 & & 9 UG/ & $J$ \\
\hline Unknown-10 & & 12 UG/ & $J$ \\
\hline Unknown-11 & & $5 \mathrm{UG} / \mathrm{L}$ & $J$ \\
\hline Unknown-12 & & 5 UG & 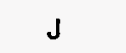 \\
\hline Unknown-13 & & 5 UG & $J$ \\
\hline Unknown-14 & & 5 UG/L & $J$ \\
\hline Unknown-15 & & 5 UG $\Omega$ & J \\
\hline Unknown-16 & & $12 U G \Omega$ & $J$ \\
\hline Sulfur,mol. (S8) & & $31 \mathrm{UG} / \mathrm{L}$ & J \\
\hline Nitrobenzene-d5 & & $88 \%$ & \\
\hline 2-Fluorobiphenyl & & $75 \%$ & \\
\hline Terphenyl-d14 & & $63 \%$ & \\
\hline Phenol-d5 & & $31 \%$ & \\
\hline 2-Fluorophenol & & $40 \%$ & \\
\hline 2,4,6-Tribromophenol & & $69 \%$ & \\
\hline
\end{tabular}


APPENDIX D.2 Results of Semivolatile Organic Compound Analyses, Water, Salmon Site

\begin{tabular}{|c|c|c|c|c|c|}
\hline COMPOUND & RESULTS & $\begin{array}{l}\text { DATA } \\
\text { QUAL. }\end{array}$ & $\begin{array}{l}\text { VALID. } \\
\text { QUAL. }\end{array}$ & CRDL UNITS & DILUTION \\
\hline \multicolumn{6}{|l|}{ C1-17-W } \\
\hline Phenol & $11 \mathrm{UG} /$ & $u$ & -- & $11 \mathrm{UG} / \mathrm{L}$ & 0.5 \\
\hline bis(2-Chloroethyl) ether & $11 \mathrm{UG} /$ & $u$ &.-- & 11 UGR & 0.5 \\
\hline 2-Chlorophenol & $11 \mathrm{UG} \Omega$ & $u$ &.- & 11 UGR & 0.5 \\
\hline 1,3-Dichlorobenzene & 11 UG $/$ & $u$ & -- & $11 \mathrm{UG} / \mathrm{L}$ & 0.5 \\
\hline 1,4-Dichlorobenzene & $11 \mathrm{UG} / \mathrm{L}$ & $u$ & $\ldots$ & 11 UG/ & 0.5 \\
\hline Benzyl Alcohol & 11 UG/ & $u$ & $\ldots$ & $11 \mathrm{UG} / \mathrm{L}$ & 0.5 \\
\hline 1,2-Dichlorobenzene & $11 \mathrm{UG} / \mathrm{L}$ & $u$ & -.. & 11 UG/ & 0.5 \\
\hline 2-Methylphenol & 11 UG $\Omega$ & $u$ & $\ldots$ & $11 \mathrm{UG} /$ & 0.5 \\
\hline bis(2-Chloroisopropyl) ether & 11 UG/ & $u$ & -- & 11 UG/ & 0.5 \\
\hline 4-Methylphenol & 11 UGR & $u$ & $\ldots$ & $11 \mathrm{UG} / \mathrm{L}$ & 0.5 \\
\hline N-Nitroso-Di-n-dipropylamine & 11 UG/ & $u$ & - & 11 UGR & 0.5 \\
\hline Hexachioroethane & 11 UG $\Omega$ & $u$ & - & 11 UG/ & 0.5 \\
\hline Nitrobenzene & 11 UGR & $u$ & - & 11 UG/ & 0.5 \\
\hline Isophorone & $11 \mathrm{UG} / \mathrm{L}$ & $u$ & $-\cdots$ & 11 UG/ & 0.5 \\
\hline 2-Nitrophenol & 11 UG/ & $u$ & $-\cdots$ & $11 \mathrm{UG} / \mathrm{L}$ & 0.5 \\
\hline 2,4-Dimethylphenol & $11 \mathrm{UG} / \mathrm{L}$ & $u$ & $\ldots$ & 11 UGR & 0.5 \\
\hline Benzoic Acid & 54 UG/ & $u$ & - & $54 \mathrm{UG} \Omega$ & 0.5 \\
\hline bis(2Chloroethoxy)methane & 11 UG/L & $u$ & - & 11 UG/ & 0.5 \\
\hline 2,4-Dichlorophenol & 11 UG $\Omega$ & $u$ & $\cdots$ & 11 UG/L & 0.5 \\
\hline 1,2,4-Trichlorobenzene & 11 UG & $u$ & -- & 11 UG/ & 0.5 \\
\hline Naphthalene & 11 UG/ & $u$ & - & $11 \mathrm{UG} / \mathrm{L}$ & 0.5 \\
\hline 4-Chloroaniline & $11 \mathrm{UG} / \mathrm{L}$ & $u$ & $\ldots$ & 11 UG $\Omega$ & 0.5 \\
\hline Hexachlorobutadiene & $11 \mathrm{UG} \Omega$ & $u$ & $\ldots$ & $11 \mathrm{UG} / \mathrm{L}$ & 0.5 \\
\hline 4-Chloro-3-Methlyphenol & 11 UG $\Omega$ & $u$ & $\ldots$ & $11 \mathrm{UG} / \mathrm{L}$ & 0.5 \\
\hline 2-Methylnaphthalene & 11 UG $\Omega$ & $u$ & $\ldots$ & 11 UG $/$ & 0.5 \\
\hline Hexachlorocyclopentadiene & 11 UGR & $u$ & $\ldots$ & 11 UG/ & 0.5 \\
\hline 2,4,6-Trichlorophenol & 11 UG/ & $u$ & $\ldots$ & 11 UG/ & 0.5 \\
\hline 2,4,5-Trichlorophenol & 54 UG/L & $u$ & -- & 54 UG/ & 0.5 \\
\hline 2-Chloronaphthalene & $11 \mathrm{UG} \Omega$ & $u$ & -. & 11 UGL & 0.5 \\
\hline 2-Nitroaniline & 54 UG几 & $u$ & - & 54 UGR & 0.5 \\
\hline Dimethyl Phthalate & 11 UG/ & $u$ & - & 11 UG/ & 0.5 \\
\hline Acenaphthylene & 11 UG $\Omega$ & $u$ & - & 11 UG/L & 0.5 \\
\hline 2,6-Dinitrotoluene & 11 UG $\Omega$ & $u$ & - & 11 UG/ & 0.5 \\
\hline 3-Nitroaniline & 54 UG/ & $u$ & $\ldots$ & 54 UG/ & 0.5 \\
\hline Acenaphthene & $11 \mathrm{UG} \Omega$ & $u$ & - & 11 UG/ & 0.5 \\
\hline 2,4-Dinitrophenol & 54 UG/ & $u$ & - & 54 UG/ & 0.5 \\
\hline 4-Nitrophenol & 54 UG几 & $u$ & -- & 54 UG/L & 0.5 \\
\hline Dibenzofuran & 11 UG $\Omega$ & $u$ & - & $11 \mathrm{UG} / \mathrm{L}$ & 0.5 \\
\hline 2,4-Dinitrotoluene & 11 UG $\Omega$ & $u$ & - & $11 \mathrm{UG} /$ & 0.5 \\
\hline Diethylphthalate & $11 \mathrm{UG} / \mathrm{L}$ & $u$ & - & 11 UG/L & 0.5 \\
\hline 4-Chlorophenyl phenyl ether & $11 \mathrm{UG} / \mathrm{L}$ & $u$ & -- & 11 UG $\Omega$ & 0.5 \\
\hline Fluorene & $11 \mathrm{UG} / \mathrm{L}$ & $u$ & -- & $11 \mathrm{UG} / \mathrm{L}$ & 0.5 \\
\hline 4-Nitroaniline & 54 UG/L & $u$ & - & 54 UG/ & 0.5 \\
\hline 4,6-Dinitro-2-methylphenol & 54 UG $\Omega$ & $u$ & - & 54 UG/L & 0.5 \\
\hline N-Nitrosodiphenylamine & $11 \mathrm{UG} / \mathrm{L}$ & $u$ & - & 11 UG/L & 0.5 \\
\hline
\end{tabular}


APPENDIX D.2 Results of Semivolatile Organic Compound Analyses, Water, Salmon Site

\begin{tabular}{|c|c|c|c|c|c|}
\hline COMPOUND & RESULTS & $\begin{array}{l}\text { DATA } \\
\text { QUAL. }\end{array}$ & $\begin{array}{l}\text { VALID. } \\
\text { QUAL. }\end{array}$ & CRDL UNITS & DILUTION \\
\hline \multicolumn{6}{|l|}{ C1-17-W (continued) } \\
\hline 4-Bromophenyl phenylether & $11 \mathrm{UG} / \mathrm{L}$ & U & $\ldots$ & $11 \mathrm{UG} \Omega$ & 0.5 \\
\hline Hexachlorobenzene & 11 UGL & $u$ & $\ldots$ & $11 \mathrm{UG} / \mathrm{L}$ & 0.5 \\
\hline Pentachlorophenol & 54 UG/L & $u$ & - & 54 UG/L & 0.5 \\
\hline Phenanthrene & 11 UGR & $u$ & - & 11 UG $\Omega$ & 0.5 \\
\hline Anthracene & 11 UGR & $u$ & $\rightarrow$ & 11 UGR & 0.5 \\
\hline Di-N-Butylphthalate & 11 UG/L & $u$ & -- & 11 UG $\Omega$ & 0.5 \\
\hline Fluoranthene & 11 UGR & $u$ & - & 11 UG/ & 0.5 \\
\hline Pyrene & 11 UG/L & $u$ & - & $11 \mathrm{UG} /$ & 0.5 \\
\hline Butyl Benzyl Phthalate & 11 UG/ & $u$ & - & 11 UG $\Omega$ & 0.5 \\
\hline 3,3'-Dichlorobenzidine & 22 UG $\Omega$ & $u$ & $\ldots$ & 22 UG/L & 0.5 \\
\hline Benzo(a)Anthracene & 11 UG/L & $u$ & - & 11 UG $\Omega$ & 0.5 \\
\hline Chrysene & 11 UG/ & $u$ & - & 11 UG $\Omega$ & 0.5 \\
\hline bis(2-Ethylhexyi)phthalate & 12 UG/ & B & - & $11 \mathrm{UG} / \mathrm{L}$ & 0.5 \\
\hline di-N-Octyl Phthalate & $11 \mathrm{UG} / \mathrm{L}$ & $u$ & - & $11 \mathrm{UG} \Omega$ & 0.5 \\
\hline Benzo(b)Fluoranthene & 11 UGR & $u$ & - & 11 UG几 & 0.5 \\
\hline Benzo(k)Fluoranthene & 11 UG $\Omega$ & $u$ & - & 11 UG & 0.5 \\
\hline Benzo(a)Pyrene & 11 UG & $u$ & $\ldots$ & 11 UG/ & 0.5 \\
\hline Indeno(1,2,3-Cd)pyrene & 11 UG $\Omega$ & $u$ & - & 11 UG几 & 0.5 \\
\hline Dibenzo(a,h)Anthracene & $11 \mathrm{UG} \Omega$ & $u$ & - & 11 UG/ & 0.5 \\
\hline Benzo $(g, h, i)$ Perylene & 11 UG $\Omega$ & $u$ & - & 11 UG $\Omega$ & 0.5 \\
\hline Unknown-1 & 380 UG & J & $\mathrm{J}$ & UG/ & 0.5 \\
\hline Unknown-2 & 7 UGR & J & R & UGR & 0.5 \\
\hline Unknown-3 & $6 \mathrm{UG} \Omega$ & $\checkmark$ & $R$ & UG/ & 0.5 \\
\hline Nitrobenzene-d5 & $90 \%$ & & & $\%$ & 0.5 \\
\hline 2-Fluorobiphenyl & $83 \%$ & & & $\%$ & 0.5 \\
\hline Terphenyl-d14 & $66 \%$ & & & $\%$ & 0.5 \\
\hline Phenol-d5 & $36 \%$ & & & $\%$ & 0.5 \\
\hline 2-Fluorophenol & $49 \%$ & & & $\%$ & 0.5 \\
\hline 2,4,6-Tribromophenol & $51 \%$ & & & $\%$ & 0.5 \\
\hline
\end{tabular}


APPENDIX D.2 Results of Semivolatile Organic Compound Analyses, Water, Salmon Site

\begin{tabular}{|c|c|c|c|c|c|}
\hline COMPOUND & RESULTS & $\begin{array}{l}\text { DATA } \\
\text { QUAL. }\end{array}$ & $\begin{array}{l}\text { VALID. } \\
\text { QUAL. }\end{array}$ & CRDL UNITS & DILUTION \\
\hline \multicolumn{6}{|l|}{ C1-18-W } \\
\hline Phenol & $10 \mathrm{UG} / \mathrm{L}$ & $u$ & NP & 10 UG $\Omega$ & 0.5 \\
\hline bis(2-Chloroethyl) ether & $10 \mathrm{UG} / \mathrm{L}$ & $u$ & NP & 10 UG $\Omega$ & 0.5 \\
\hline 2-Chlorophenol & $10 \mathrm{UG} \Omega$ & $u$ & NP & $10 \mathrm{UG} / \mathrm{L}$ & 0.5 \\
\hline 1,3-Dichlorobenzene & $10 \mathrm{UG} / \mathrm{L}$ & $u$ & NP & $10 \mathrm{UG} / \mathrm{L}$ & 0.5 \\
\hline 1,4-Dichlorobenzene & 10 UG $\Omega$ & $u$ & NP & $10 \mathrm{UG} \Omega$ & 0.5 \\
\hline Benzyi Alcohol & $10 \mathrm{UG} / \mathrm{L}$ & $u$ & NP & $10 \mathrm{UG} / \mathrm{L}$ & 0.5 \\
\hline 1,2-Dichlorobenzene & $10 \mathrm{UG} / \mathrm{L}$ & $u$ & NP & $10 \mathrm{UG} / \mathrm{L}$ & 0.5 \\
\hline 2-Methylphenol & $10 \mathrm{UG} / \mathrm{L}$ & $u$ & NP & 10 Ua/ & 0.5 \\
\hline bis(2-Chloroisopropyl) ether & $10 \mathrm{UG} / \mathrm{L}$ & $u$ & NP & $10 \mathrm{UG} / \mathrm{L}$ & 0.5 \\
\hline 4-Methyiphenol & 10 UG/ & $u$ & NP & 10 UG/L & 0.5 \\
\hline N-Nitroso-Di-n-dipropylamine & $10 \mathrm{UG} / \mathrm{L}$ & $u$ & NP & 10 UG/ & 0.5 \\
\hline Hexachloroethane & $10 \mathrm{UG} / \mathrm{L}$ & $u$ & NP & 10 UG/ & 0.5 \\
\hline Nitrobenzene & $10 \mathrm{UG} \Omega$ & $u$ & NP & 10 UGR & 0.5 \\
\hline Isophorone & 10 UG $\Omega$ & $u$ & NP & 10 UG $\Omega$ & 0.5 \\
\hline 2-Nitrophenol & 10 UG $/$ & $u$ & NP & 10 Ua/ & 0.5 \\
\hline 2,4-Dimethylphenol & 10 UG $\Omega$ & $u$ & NP & 10 UG/ & 0.5 \\
\hline Benzoic Acid & 50 UG/ & $u$ & NP & 50 UG/L & 0.5 \\
\hline bis(2Chloroethoxy)methane & $10 \mathrm{UG} / \mathrm{L}$ & $u$ & NP & $10 \mathrm{UG} / \mathrm{L}$ & 0.5 \\
\hline 2,4-Dichlorophenol & 10 UG $\Omega$ & $u$ & NP & 10 UG $\Omega$ & 0.5 \\
\hline 1,2,4-Trichlorobenzene & $10 \mathrm{UG} \Omega$ & u & NP & $10 \mathrm{UG} / \mathrm{L}$ & 0.5 \\
\hline Naphthalene & $10 \mathrm{UG} / \mathrm{L}$ & $u$ & NP & $10 \mathrm{UG} / \mathrm{L}$ & 0.5 \\
\hline 4-Chloroaniline & 10 UG/ & $u$ & NP & 10 UG & 0.5 \\
\hline Hexachlorobutadiene & 10 UG/ & $u$ & NP & 10 UGR & 0.5 \\
\hline 4-Chloro-3-Methlyphenol & 10 UG $\Omega$ & $u$ & NP & $10 \mathrm{UG \Omega}$ & 0.5 \\
\hline 2-Methyinaphthalene & $10 \mathrm{UG} / \mathrm{L}$ & $u$ & NP & $10 \mathrm{UG} \Omega$ & 0.5 \\
\hline Hexachlorocyclopentadiene & $10 \mathrm{UG} / \mathrm{L}$ & $u$ & NP & $10 \mathrm{UG} / \mathrm{L}$ & 0.5 \\
\hline 2,4.6-Trichlorophenol & 10 UG & $u$ & NP & $10 \mathrm{UG} / \mathrm{L}$ & 0.5 \\
\hline 2,4,5-Trichlorophenol & 50 UG/ & $u$ & NP & 50 UG $\Omega$ & 0.5 \\
\hline 2-Chloronaphthalene & $10 \mathrm{UG} / \mathrm{L}$ & $u$ & NP & $10 \mathrm{UG} \Omega$ & 0.5 \\
\hline 2-Nitroaniline & 50 UG/ & $u$ & NP & 50 UG $\Omega$ & 0.5 \\
\hline Dimethyl Phthalate & $10 \mathrm{UG} / \mathrm{L}$ & u & NP & $10 \mathrm{UG} / \mathrm{h}$ & 0.5 \\
\hline Acenaphthylene & 10 UG/ & $u$ & NP & 10 UG $\Omega$ & 0.5 \\
\hline 2,6-Dinitrotoluene & 10 UG/ & $u$ & NP & 10 UG $\Omega$ & 0.5 \\
\hline 3-Nitroaniline & 50 UG/ & $u$ & NP & $50 \mathrm{UG} /$ & 0.5 \\
\hline Acenaphthene & $10 \mathrm{UG} / \mathrm{L}$ & $u$ & NP & 10 UG & 0.5 \\
\hline 2,4-Dinitrophenol & 50 UG/ & $u$ & NP & 50 UG $\Omega$ & 0.5 \\
\hline 4-Nitrophenol & 50 UG/ & $u$ & NP & $50 \mathrm{UG} \Omega$ & 0.5 \\
\hline Dibenzofuran & 10 UG/ & $u$ & NP & $10 \mathrm{UGR}$ & 0.5 \\
\hline 2,4-Dinitrotoluene & $10 \mathrm{UG} / \mathrm{L}$ & $u$ & NP & 10 UG $\Omega$ & 0.5 \\
\hline Diethylphthalate & 10 UG $\Omega$ & $u$ & NP & $10 \mathrm{UG} \Omega$ & 0.5 \\
\hline 4-Chlorophenyl phenyl ether & $10 \mathrm{UG} / \mathrm{L}$ & $u$ & NP & $10 \mathrm{UG} / \mathrm{L}$ & 0.5 \\
\hline Fluorene & $10 \mathrm{UG} / \mathrm{L}$ & $u$ & NP & $10 \mathrm{UG} / \mathrm{L}$ & 0.5 \\
\hline 4-Nitroaniline & 50 UG $/$ & $u$ & NP & 50 UG $\Omega$ & 0.5 \\
\hline 4,6-Dinitro-2-methylphenol & 50 UG $\Omega$ & $u$ & NP & 50 UG $\Omega$ & 0.5 \\
\hline N-Nitrosodiphenylamine & $10 \mathrm{UG \Omega}$ & $u$ & NP & 10 UG $\Omega$ & 0.5 \\
\hline
\end{tabular}


APPENDIX D.2 Results of Semivolatile Organic Compound Analyses, Water, Salmon Site

\begin{tabular}{|c|c|c|c|c|c|}
\hline COMPOUND & RESULTS & $\begin{array}{l}\text { DATA } \\
\text { QUAL. }\end{array}$ & $\begin{array}{l}\text { VALID. } \\
\text { QUAL. }\end{array}$ & CRDL UNITS & DILUTIC \\
\hline \multicolumn{6}{|l|}{ C1-18-W (continued) } \\
\hline 4-Bromophenyl phenylether & $10 \mathrm{UG} / \mathrm{L}$ & $u$ & NP & 10 UG $\Omega$ & 0.5 \\
\hline Hexachlorobenzene & $10 \mathrm{UG} / \mathrm{L}$ & $u$ & NP & $10 \mathrm{UG} \Omega$ & 0.5 \\
\hline Pentachlorophenol & 50 UG $/$ & $u$ & NP & 50 UGR & 0.5 \\
\hline Phenanthrene & $10 \mathrm{UG} / \mathrm{L}$ & $u$ & NP & 10 UG $\Omega$ & 0.5 \\
\hline Anthracene & $10 \mathrm{UG} / \mathrm{L}$ & $u$ & NP & 10 UG/L & 0.5 \\
\hline Di-N-Butylphthalate & 10 UG $/$ & $u$ & NP & $10 \mathrm{UG} / \mathrm{L}$ & 0.5 \\
\hline Fluoranthene & $10 \mathrm{UG} / \mathrm{L}$ & $u$ & NP & 10 UG/ & 0.5 \\
\hline Pyrene & 10 UG/L & $u$ & NP & $10 \mathrm{UG} \Omega$ & 0.5 \\
\hline Butyl Benzyl Phthalate & 10 UG $\Omega$ & $u$ & NP & 10 UG $\Omega$ & 0.5 \\
\hline 3,3'-Dichlorobenzidine & 20 UG/ & $u$ & NP & 20 UG $\Omega$ & 0.5 \\
\hline Benzo(a)Anthracene & 10 UG $\Omega$ & $u$ & NP & 10 UGR & 0.5 \\
\hline Chrysene & $10 \mathrm{UG} / \mathrm{L}$ & $u$ & NP & $10 \mathrm{UG \Omega}$ & 0.5 \\
\hline bis(2-Ethylhexyl)phthalate & 10 UG/L & $u$ & NP & $10 \mathrm{UG} / \mathrm{L}$ & 0.5 \\
\hline di-N-Octyl Phthalate & 10 UG $\Omega$ & $u$ & NP & 10 UGR & 0.5 \\
\hline Benzo(b)Fluoranthene & $10 \mathrm{UG} / \mathrm{L}$ & $u$ & NP & 10 UGR & 0.5 \\
\hline Benzo(k)Fluoranthene & 10 UG/ & $u$ & NP & 10 UG $\Omega$ & 0.5 \\
\hline Benzo(a)Pyrene & $10 \mathrm{UG} / \mathrm{L}$ & $u$ & NP & 10 UGR & 0.5 \\
\hline Indeno(1,2,3-Cd)pyrene & 10 UG/L & $u$ & NP & 10 UG/ & 0.5 \\
\hline Dibenzo(a,h)Anthracene & 10 UG/ & $u$ & NP & $10 \mathrm{UG} / \mathrm{L}$ & 0.5 \\
\hline Benzo $(g, h, i)$ Perylene & 10 UG $\Omega$ & $u$ & NP & $10 \mathrm{UG} /$ & 0.5 \\
\hline Unknown & $6 \mathrm{UG} / \mathrm{L}$ & 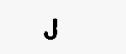 & NP & UGR & 0.5 \\
\hline Nitrobenzene-d5 & $65 \%$ & & & $\%$ & 0.5 \\
\hline 2-Fluorobiphenyl & $59 \%$ & & & $\%$ & 0.5 \\
\hline Terphenyl-d14 & $64 \%$ & & & $\%$ & 0.5 \\
\hline Phenol-d5 & $13 \%$ & & & $\%$ & 0.5 \\
\hline 2-Fluorophenol & $18 \%$ & $*$ & & $\%$ & 0.5 \\
\hline 2,4,6-Tribromophenol & $58 \%$ & & & $\%$ & 0.5 \\
\hline
\end{tabular}


APPENDIX D.2 Results of Semivolatile Organic Compound Analyses, Water, Salmon Site

\begin{tabular}{|c|c|c|c|c|c|}
\hline COMPOUND & RESULTS & $\begin{array}{l}\text { DATA } \\
\text { QUAL. }\end{array}$ & $\begin{array}{l}\text { VALID. } \\
\text { QUAL. }\end{array}$ & CRDL UNITS & DILUTION \\
\hline \multicolumn{6}{|l|}{$C 2-19-W$} \\
\hline Phenol & 10 UG/ & $u$ & NP & $10 \mathrm{UG} / \mathrm{L}$ & 0.5 \\
\hline bis(2-Chloroethyl) ether & $10 \mathrm{UG} / \mathrm{L}$ & $u$ & NP & 10 UG/ & 0.5 \\
\hline 2-Chlorophenol & $10 \mathrm{UG} / \mathrm{L}$ & $u$ & NP & $10 \mathrm{UG} / \mathrm{L}$ & 0.5 \\
\hline 1,3-Dichlorobenzene & 10 UGR & $u$ & NP & 10 UG $\Omega$ & 0.5 \\
\hline 1,4-Dichlorobenzene & 10 UG $\Omega$ & $u$ & NP & $10 \mathrm{UG} \Omega$ & 0.5 \\
\hline Benzyl Alcohol & 10 UG $\Omega$ & $u$ & NP & 10 UG/ & 0.5 \\
\hline 1,2-Dichlorobenzene & 10 UG $\Omega$ & $u$ & NP & 10 UG/L & 0.5 \\
\hline 2-Methylphenol & $10 \mathrm{UG} / \mathrm{L}$ & $u$ & NP & 10 UG/L & 0.5 \\
\hline bis(2-Chloroisopropyl) ether & $10 \mathrm{UG \Omega}$ & $u$ & NP & $10 \mathrm{UG} / \mathrm{L}$ & 0.5 \\
\hline 4-Methylphenol & 10 UGR & $u$ & NP & $10 \mathrm{UG} / \mathrm{L}$ & 0.5 \\
\hline N-Nitroso-Di-n-dipropylamine & $10 \mathrm{UG} / \mathrm{L}$ & $u$ & NP & $10 \mathrm{UG} / \mathrm{L}$ & 0.5 \\
\hline Hexachloroethane & $10 \mathrm{UG} / \mathrm{h}$ & $u$ & NP & $10 \mathrm{UG} / \mathrm{L}$ & 0.5 \\
\hline Nitrobenzene & $10 \mathrm{UG} / \mathrm{L}$ & $u$ & NP & 10 UG $\Omega$ & 0.5 \\
\hline Isophorone & $10 \mathrm{UG} / \mathrm{L}$ & $u$ & NP & 10 UG & 0.5 \\
\hline 2-Nitrophenol & $10 \mathrm{UG} /$ & $u$ & NP & $10 \mathrm{UG} / \mathrm{L}$ & 0.5 \\
\hline 2,4-Dimethylphenol & $10 \mathrm{UG} \Omega$ & $u$ & NP & $10 \mathrm{UG} / \mathrm{L}$ & 0.5 \\
\hline Benzoic Acid & $50 \mathrm{UG} \Omega$ & $u$ & NP & 50 UG/L & 0.5 \\
\hline bis(2Chloroethoxy)methane & $10 \mathrm{UG} \Omega$ & $u$ & NP & 10 UG & 0.5 \\
\hline 2,4-Dichlorophenol & $10 \mathrm{UG} \Omega$ & $u$ & NP & $10 \mathrm{UG} / \mathrm{L}$ & 0.5 \\
\hline 1,2,4-Trichlorobenzene & $10 \mathrm{UG} \Omega$ & $u$ & NP & $10 \mathrm{UG} / \mathrm{L}$ & 0.5 \\
\hline Naphthalene & $10 \mathrm{UG} \Omega$ & $u$ & NP & 10 UG/ & 0.5 \\
\hline 4-Chloroaniline & 10 UG/L & $u$ & NP & $10 \mathrm{UG} / \mathrm{L}$ & 0.5 \\
\hline Hexachlorobutadiene & 10 UG $\Omega$ & $u$ & NP & 10 UG/L & 0.5 \\
\hline 4-Chloro-3-Methlyphenol & $10 \mathrm{UG} \Omega$ & $u$ & NP & 10 UG/ & 0.5 \\
\hline 2-Methylnaphthalene & $10 \mathrm{UG} / \mathrm{L}$ & $u$ & NP & 10 UG/L & 0.5 \\
\hline Hexachlorocyclopentadiene & 10 Ual & $u$ & NP & 10 UG/ & 0.5 \\
\hline 2,4,6-Trichlorophenol & 10 UG/ & $u$ & NP & 10 UG/ & 0.5 \\
\hline 2,4,5-Trichlorophenol & $50 \mathrm{UG} \Omega$ & $u$ & NP & 50 UG/ & 0.5 \\
\hline 2-Chloronaphthalene & $10 \mathrm{UG} / \mathrm{L}$ & $u$ & NP & 10 UG/L & 0.5 \\
\hline 2-Nitroaniline & 50 UG/L & $u$ & NP & 50 UG/L & 0.5 \\
\hline Dimethyl Phthalate & 10 UG $\Omega$ & $u$ & NP & $10 \mathrm{UG} / \mathrm{L}$ & 0.5 \\
\hline Acenaphthylene & 10 UG/ & $u$ & NP & $10 \mathrm{UG} /$ & 0.5 \\
\hline 2,6-Dinitrotoluene & 10 UG & $u$ & NP & 10 UG $\Omega$ & 0.5 \\
\hline 3-Nitroaniline & 50 UG $\Omega$ & $u$ & NP & 50 UG/L & 0.5 \\
\hline Acenaphthene & $10 \mathrm{UG} / \mathrm{L}$ & $u$ & NP & 10 UG/ & 0.5 \\
\hline 2,4-Dinitrophenol & $50 \mathrm{UG} / \mathrm{L}$ & $u$ & NP & $50 \mathrm{UG} /$ & 0.5 \\
\hline 4-Nitrophenol & 50 UG $\Omega$ & $u$ & NP & 50 UG/ & 0.5 \\
\hline Dibenzofuran & 10 UG $\Omega$ & $u$ & NP & 10 UG $\Omega$ & 0.5 \\
\hline 2,4-Dinitrotoluene & $10 \mathrm{UG} / \mathrm{L}$ & $u$ & NP & 10 UG $\Omega$ & 0.5 \\
\hline Diethylphthalate & $34 \mathrm{UG} / \mathrm{L}$ & & NP & 10 UG/ & 0.5 \\
\hline 4-Chlorophenyl phenyl ether & 10 UG/L & $u$ & NP & 10 UG $/$ & 0.5 \\
\hline Fluorene & $10 \mathrm{UG} / \mathrm{L}$ & $u$ & NP & $10 \mathrm{UG} / \mathrm{L}$ & 0.5 \\
\hline 4-Nitroaniline & 50 UG $\Omega$ & $u$ & NP & 50 UG/ & 0.5 \\
\hline 4,6-Dinitro-2-methylphenol & 50 UG/ & $u$ & NP & 50 UG/L & 0.5 \\
\hline N-Nitrosodiphenylamine & 10 Ua $\Omega$ & $u$ & NP & 10 UG/L & 0.5 \\
\hline
\end{tabular}


APPENDIX D.2 Results of Semivolatile Organic Compound Analyses, Water, Salmon Site

\begin{tabular}{|c|c|c|c|c|c|}
\hline COMPOUND & RESULTS & $\begin{array}{l}\text { DATA } \\
\text { QUAL. }\end{array}$ & $\begin{array}{l}\text { VALID. } \\
\text { QUAL. }\end{array}$ & CRDL UNITS & DILUTIC \\
\hline \multicolumn{6}{|l|}{ C2-19-W (continued) } \\
\hline 4-Bromophenyl phenylether & $10 \mathrm{UG} / \mathrm{L}$ & $u$ & NP & $10 \mathrm{UG} \Omega$ & 0.5 \\
\hline Hexachlorobenzene & 10 UG $\Omega$ & $u$ & NP & 10 UG/ & 0.5 \\
\hline Pentachlorophenol & 50 UG & $u$ & NP & 50 UG $/$ & 0.5 \\
\hline Phenanthrene & 10 UG $\Omega$ & $u$ & NP & $10 \mathrm{UG} \Omega$ & 0.5 \\
\hline Anthracene & 10 UG & $u$ & NP & $10 \mathrm{UG} \Omega$ & 0.5 \\
\hline Di-N-Butylphthalate & $10 \mathrm{UG} / \mathrm{L}$ & $u$ & NP & $10 \mathrm{UG} /$ & 0.5 \\
\hline Fluoranthene & 10 UG $\Omega$ & $u$ & NP & $10 \mathrm{UG} / \mathrm{L}$ & 0.5 \\
\hline Pyrene & 10 UG $\Omega$ & $u$ & NP & 10 UG $/$ & 0.5 \\
\hline Butyl Benzyl Phthalate & 10 UG $\Omega$ & $u$ & NP & 10 UG $\Omega$ & 0.5 \\
\hline 3,3'-Dichlorobenzidine & 20 UG $\Omega$ & $u$ & NP & 20 UG $\Omega$ & 0.5 \\
\hline Benzo(a)Anthracene & $10 \mathrm{UG} / \mathrm{L}$ & $u$ & NP & 10 UG $\Omega$ & 0.5 \\
\hline Chrysene & 10 UG/L & $u$ & NP & 10 UG $\Omega$ & 0.5 \\
\hline bis(2-Ethylhexyl)phthalate & $10 \mathrm{UG} / \mathrm{L}$ & $u$ & NP & $10 \mathrm{UG} \Omega$ & 0.5 \\
\hline di-N-Octyl Phthalate & $10 \mathrm{UG} / \mathrm{L}$ & $u$ & NP & 10 UG/ & 0.5 \\
\hline Benzo(b)Fluoranthene & $10 \mathrm{UG} / \mathrm{L}$ & $u$ & NP & $10 \mathrm{UG} /$ & 0.5 \\
\hline Benzo(k)Fluoranthene & 10 UG/ & $u$ & NP & $10 \mathrm{UG} / \mathrm{L}$ & 0.5 \\
\hline Benzo(a)Pyrene & 10 UG/ & $u$ & NP & $10 \mathrm{UG} \Omega$ & 0.5 \\
\hline Indeno(1,2,3-Cd)pyrene & 10 UG $\Omega$ & $u$ & NP & $10 \mathrm{UG} /$ & 0.5 \\
\hline Dibenzo(a,h)Anthracene & $10 \mathrm{UG} / \mathrm{L}$ & $u$ & NP & 10 UG $\Omega$ & 0.5 \\
\hline Benzo(g,h,i)Perylene & $10 \mathrm{UG} \Omega$ & $u$ & NP & $10 \mathrm{UG} / \mathrm{L}$ & 0.5 \\
\hline Unknown Phthalate & $8 \mathrm{UG} \Omega$ & $J$ & NP & UG $/$ L & 0.5 \\
\hline Unknown & 7 UG/ & BJ & NP & UG/L & 0.5 \\
\hline Nitrobenzene-d5 & $52 \%$ & & & $\%$ & 0.5 \\
\hline 2-Fluorobiphenyl & $52 \%$ & & & $\%$ & 0.5 \\
\hline Terphenyl-d14 & $57 \%$ & & & $\%$ & 0.5 \\
\hline Phenol-d5 & $28 \%$ & & & $\%$ & 0.5 \\
\hline 2-Fluorophenol & $39 \%$ & & & $\%$ & 0.5 \\
\hline 2,4,6-Trichlorophenol & $67 \%$ & & & $\%$ & 0.5 \\
\hline
\end{tabular}


APPENDIX D.2 Results of Semivolatile Organic Compound Analyses, Water, Salmon Site

\begin{tabular}{|c|c|c|c|c|c|}
\hline COMPOUND & RESULTS & $\begin{array}{l}\text { DATA } \\
\text { QUAL. }\end{array}$ & $\begin{array}{l}\text { VALID. } \\
\text { QUAL. }\end{array}$ & CRDL UNITS & DILUTION \\
\hline \multicolumn{6}{|l|}{ C2-19-W -MS } \\
\hline Phenol & $34 \%$ & & NP & $\%$ & 0.5 \\
\hline 2-Chlorophenol & $66 \%$ & & NP & $\%$ & 0.5 \\
\hline 1,4-Dichlorobenzene & $60 \%$ & & NP & $\%$ & 0.5 \\
\hline N-Nitroso-Di-n-dipropylamine & $75 \%$ & & NP & $\%$ & 0.5 \\
\hline 1,2,4-Trichlorobenzene & $64 \%$ & & NP & $\%$ & 0.5 \\
\hline 4-Chloro-3-Methlyphenol & $74 \%$ & & NP & $\%$ & 0.5 \\
\hline Acenaphthene & $73 \%$ & & NP & $\%$ & 0.5 \\
\hline 4-Nitrophenol & $40 \%$ & & NP & $\%$ & 0.5 \\
\hline 2,4-Dinitrotoluene & $78 \%$ & & NP & $\%$ & 0.5 \\
\hline Pentachlorophenol & $88 \%$ & & NP & $\%$ & 0.5 \\
\hline Pyrene & $76 \%$ & & NP & $\%$ & 0.5 \\
\hline Nitrobenzene-d5 & $72 \%$ & & NP & $\%$ & 0.5 \\
\hline 2-Fluorobiphenyl & $71 \%$ & & NP & $\%$ & 0.5 \\
\hline Terphenyl-d14 & $68 \%$ & & NP & $\%$ & 0.5 \\
\hline Phenol-d5 & $35 \%$ & & NP & $\%$ & 0.5 \\
\hline 2-Fluorophenol & $49 \%$ & & NP & $\%$ & 0.5 \\
\hline 2,4,6-Trichlorophenol & $77 \%$ & & NP & $\%$ & 0.5 \\
\hline \multicolumn{6}{|l|}{ C2-19-W-MSD } \\
\hline Phenol & $28 \%$ & & NP & $\%$ & 0.5 \\
\hline 2-Chlorophenol & $58 \%$ & & NP & $\%$ & 0.5 \\
\hline 1,4-Dichlorobenzene & $56 \%$ & & NP & $\%$ & 0.5 \\
\hline N-Nitroso-Di-n-dipropylamine & $69 \%$ & & NP & $\%$ & 0.5 \\
\hline 1,2,4-Trichlorobenzene & $60 \%$ & & NP & $\%$ & 0.5 \\
\hline 4-Chloro-3-Methlyphenol & $62 \%$ & & NP & $\%$ & 0.5 \\
\hline Acenaphthene & $64 \%$ & & NP & $\%$ & 0.5 \\
\hline 4-Nitrophenol & $33 \%$ & & NP & $\%$ & 0.5 \\
\hline 2,4-Dinitrotoluene & $68 \%$ & & NP & $\%$ & 0.5 \\
\hline Pentachlorophenol & $66 \%$ & & NP & $\%$ & 0.5 \\
\hline Pyrene & $68 \%$ & & NP & $\%$ & 0.5 \\
\hline Nitrobenzene-d5 & $67 \%$ & & NP & $\%$ & 0.5 \\
\hline 2-Fluorobiphenyl & $62 \%$ & & NP & $\%$ & 0.5 \\
\hline Terphenyl-d14 & $60 \%$ & & NP & $\%$ & 0.5 \\
\hline Phenol-d5 & $28 \%$ & & NP & $\%$ & 0.5 \\
\hline 2-Fluorophenol & $40 \%$ & & NP & $\%$ & 0.5 \\
\hline 2,4,6-Trichlorophenol & $65 \%$ & & NP & $\%$ & 0.5 \\
\hline
\end{tabular}


APPENDIX D.2 Results of Semivolatile Organic Compound Analyses, Water,

Salmon Site

\begin{tabular}{|c|c|c|c|c|c|}
\hline COMPOUND & RESULTS & $\begin{array}{l}\text { DATA } \\
\text { QUAL. }\end{array}$ & $\begin{array}{l}\text { VALID. } \\
\text { QUAL. }\end{array}$ & CRDL UNITS & DILUTION \\
\hline \multicolumn{6}{|l|}{$C-2-27-W$} \\
\hline Phenol & $10 \mathrm{UG} / \mathrm{L}$ & $u$ & NP & $10 \mathrm{UG} / \mathrm{L}$ & 0.5 \\
\hline bis(2-Chloroethyl) ether & $10 \mathrm{UG} / \mathrm{L}$ & $u$ & NP & $10 \mathrm{UG} / \mathrm{L}$ & 0.5 \\
\hline 2-Chlorophenol & $10 \mathrm{UG} / \mathrm{L}$ & $u$ & NP & 10 UG & 0.5 \\
\hline 1,3-Dichlorobenzene & $10 \mathrm{UG} /$ & $u$ & NP & $10 \mathrm{UG} / \mathrm{L}$ & 0.5 \\
\hline 1,4-Dichlorobenzene & 10 UG/ & $u$ & NP & 10 UG/ & 0.5 \\
\hline Benzyl Alcohol & $10 \mathrm{UG} / \mathrm{L}$ & $u$ & NP & 10 UGR & 0.5 \\
\hline 1,2-Dichlorobenzene & $10 \mathrm{UG} / \mathrm{L}$ & $u$ & NP & $10 \mathrm{UG} \Omega$ & 0.5 \\
\hline 2-Methylphenol & $10 \mathrm{UG} /$ & $u$ & NP & 10 UG/L & 0.5 \\
\hline bis(2-Chloroisopropyl) ether & $10 \mathrm{UG} / \mathrm{L}$ & $u$ & NP & $10 \mathrm{UG} / \mathrm{L}$ & 0.5 \\
\hline 4-Methylphenol & $10 \mathrm{UG} / \mathrm{L}$ & $u$ & NP & 10 UG $/$ & 0.5 \\
\hline N-Nitroso-Di-n-dipropylamine & $10 \mathrm{UG} \Omega$ & $u$ & NP & $10 \mathrm{UG} /$ & 0.5 \\
\hline Hexachloroethane & 10 UGR & $u$ & NP & $10 \mathrm{UG} / \mathrm{L}$ & 0.5 \\
\hline Nitrobenzene & 10 UG/L & $u$ & NP & 10 UG/L & 0.5 \\
\hline Isophorone & 10 UG/L & $u$ & NP & 10 UG $\Omega$ & 0.5 \\
\hline 2-Nitrophenol & $10 \mathrm{UG} / \mathrm{L}$ & u & NP & $10 \mathrm{UG} / \mathrm{L}$ & 0.5 \\
\hline 2,4-Dimethylphenol & $10 \mathrm{UG} / \mathrm{L}$ & $u$ & NP & $10 \mathrm{UG} \Omega$ & 0.5 \\
\hline Benzoic Acid & 50 UG $\Omega$ & $u$ & NP & 50 UG $\Omega$ & 0.5 \\
\hline bis(2Chloroethoxy)methane & 10 UG $\Omega$ & $u$ & NP & 10 UG $\Omega$ & 0.5 \\
\hline 2,4-Dichlorophenol & 10 UG $\Omega$ & $u$ & NP & $10 \mathrm{UG} \Omega$ & 0.5 \\
\hline 1,2,4-Trichlorobenzene & $10 \mathrm{UG \Omega}$ & $u$ & NP & 10 UG $\Omega$ & 0.5 \\
\hline Naphthalene & $10 \mathrm{UG} / \mathrm{L}$ & $u$ & NP & $10 \mathrm{UG} \Omega$ & 0.5 \\
\hline 4-Chloroaniline & $10 \mathrm{UG} / \mathrm{L}$ & $u$ & NP & 10 UG $\Omega$ & 0.5 \\
\hline Hexachlorobutadiene & $10 \mathrm{UG} / \mathrm{L}$ & $u$ & NP & 10 UG/ & 0.5 \\
\hline 4-Chloro-3-Methlyphenol & $10 \mathrm{UG} / \mathrm{L}$ & $u$ & NP & 10 UG $\Omega$ & 0.5 \\
\hline 2-Methylnaphthalene & $10 \mathrm{UG} / \mathrm{L}$ & $u$ & NP & 10 UG $\Omega$ & 0.5 \\
\hline Hexachlorocyclopentadiene & $10 \mathrm{UG} / \mathrm{L}$ & $u$ & NP & $10 \mathrm{UG} / \mathrm{L}$ & 0.5 \\
\hline 2,4.6-Trichlorophenol & $10 \mathrm{UG} / \mathrm{L}$ & $u$ & NP & $10 \mathrm{UG} / \mathrm{L}$ & 0.5 \\
\hline 2,4,5-Trichlorophenol & 50 UG $\Omega$ & $u$ & NP & 50 UG $\Omega$ & 0.5 \\
\hline 2-Chloronaphthalene & 10 UG/L & $u$ & NP & 10 UG $\Omega$ & 0.5 \\
\hline 2-Nitroaniline & 50 UG $/$ & $u$ & NP & 50 UG $\Omega$ & 0.5 \\
\hline Dimethyl Phthalate & $10 \mathrm{UG} / \mathrm{L}$ & $u$ & NP & 10 UG $\Omega$ & 0.5 \\
\hline Acenaphthylene & $10 \mathrm{UG} / \mathrm{L}$ & $u$ & NP & 10 UG $/$ & 0.5 \\
\hline 2,6-Dinitrotoluene & 10 UG/ & $u$ & NP & 10 UG $\Omega$ & 0.5 \\
\hline 3-Nitroaniline & 50 UG $\Omega$ & u & NP & 50 UG $\Omega$ & 0.5 \\
\hline Acenaphthene & 10 UG $\Omega$ & $u$ & NP & 10 UG $\Omega$ & 0.5 \\
\hline 2,4-Dinitrophenol & 50 UG $\Omega$ & $u$ & NP & 50 UG几 & 0.5 \\
\hline 4-Nitrophenol & 50 UG & $u$ & NP & 50 UG $\Omega$ & 0.5 \\
\hline Dibenzofuran & $10 \mathrm{UG} /$ & $u$ & NP & $10 \mathrm{UG} \Omega$ & 0.5 \\
\hline 2,4-Dinitrotoluene & 10 UG/ & $u$ & NP & 10 UG $\Omega$ & 0.5 \\
\hline Diethyiphthalate & 10 UG $\Omega$ & $u$ & NP & 10 UG $\Omega$ & 0.5 \\
\hline 4-Chlorophenyl phenyl ether & 10 UG $\Omega$ & $u$ & NP & 10 UG $\Omega$ & 0.5 \\
\hline Fluorene & 10 UG几 & $u$ & NP & 10 UG $\Omega$ & 0.5 \\
\hline 4-Nitroaniline & $50 \mathrm{UG} / \mathrm{L}$ & $u$ & NP & 50 UG $\Omega$ & 0.5 \\
\hline 4,6-Dinitro-2-methylphenol & 50 UG & $u$ & NP & 50 UG $\Omega$ & 0.5 \\
\hline N-Nitrosodiphenylamine & $10 \mathrm{UG} / \mathrm{L}$ & $u$ & NP & 10 UG $/$ & 0.5 \\
\hline
\end{tabular}


APPENDIX D.2 Results of Semivolatile Organic Compound Analyses, Water, Salmon Site

\begin{tabular}{|c|c|c|c|c|c|}
\hline COMPOUND & RESULTS & $\begin{array}{l}\text { DATA } \\
\text { QUAL. }\end{array}$ & $\begin{array}{l}\text { VALID. } \\
\text { QUAL. }\end{array}$ & CRDL UNITS & DILUTION \\
\hline \multicolumn{6}{|l|}{ C-2-27-W (continued) } \\
\hline 4-Bromophenyl phenylether & 10 UG $\Omega$ & $u$ & NP & 10 UG/ & 0.5 \\
\hline Hexachlorobenzene & 10 UG $\Omega$ & $u$ & NP & 10 UG $\Omega$ & 0.5 \\
\hline Pentachlorophenol & 50 UG/ & $u$ & NP & 50 UGЛ & 0.5 \\
\hline Phenanthrene & 10 UG/ & $u$ & NP & 10 UG/L & 0.5 \\
\hline Anthracene & 10 UG $\Omega$ & $u$ & NP & $10 \mathrm{UG} / \mathrm{L}$ & 0.5 \\
\hline Di-N-Butylphthalate & 10 UG/L & $u$ & NP & 10 UG $\Omega$ & 0.5 \\
\hline Fluoranthene & $10 \mathrm{UG} / \mathrm{L}$ & $u$ & NP & $10 \mathrm{UG} \Omega$ & 0.5 \\
\hline Pyrene & $10 \mathrm{UG} / \mathrm{L}$ & $u$ & NP & $10 \mathrm{UG} / \mathrm{R}$ & 0.5 \\
\hline Butyl Benzyl Phthalate & 10 UG & $u$ & NP & $10 \mathrm{UG} / \mathrm{L}$ & 0.5 \\
\hline 3,3'-Dichlorobenzidine & $20 \mathrm{UG} \Omega$ & $u$ & NP & 20 UG $\Omega$ & 0.5 \\
\hline Benzo(a)Anthracene & 10 UG/L & $u$ & NP & $10 \mathrm{UG} / \mathrm{L}$ & 0.5 \\
\hline Chrysene & 10 UG & $u$ & NP & $10 \mathrm{UGl}$ & 0.5 \\
\hline bis(2-Ethylhexyl)phthalate & 10 UG/ & $u$ & NP & 10 UG/L & 0.5 \\
\hline di-N-Octyl Phthalate & $10 \mathrm{UG \Omega}$ & $u$ & NP & 10 UG/L & 0.5 \\
\hline Benzo(b)Fluoranthene & $10 \mathrm{UG} / \mathrm{L}$ & $u$ & NP & $10 \mathrm{UG} / \mathrm{L}$ & 0.5 \\
\hline Benzo(k)Fluoranthene & $10 \mathrm{UG} / \mathrm{L}$ & $u$ & NP & $10 \mathrm{UG} / \mathrm{L}$ & 0.5 \\
\hline Benzo(a)Pyrene & $10 \mathrm{UG} / \mathrm{L}$ & $u$ & NP & $10 \mathrm{UG} / \mathrm{L}$ & 0.5 \\
\hline Indeno(1,2,3-Cd)pyrene & 10 UG & $u$ & NP & $10 \mathrm{UG} / \mathrm{L}$ & 0.5 \\
\hline Dibenzo(a,h)Anthracene & 10 UG $\Omega$ & $u$ & $\mathrm{NP}$ & $10 \mathrm{UG} / \mathrm{L}$ & 0.5 \\
\hline Benzo(g,h,i)Perylene & 10 UGL & $u$ & NP & 10 UG $\Omega$ & 0.5 \\
\hline Unknown-1 & 9 UGR & J & NP & UG几 & 0.5 \\
\hline Unknown-2 & 49 UG $\Omega$ & J & NP & UG/L & 0.5 \\
\hline Unknown-3 & $240 \mathrm{UG} / \mathrm{L}$ & J & NP & UG/L & 0.5 \\
\hline Unknown-4 & $19 \mathrm{UG} / \mathrm{L}$ & $J$ & NP & UG $/$ & 0.5 \\
\hline Unknown-5 & 30 UG/L & J & NP & UG/L & 0.5 \\
\hline Nitrobenzene-d5 & $72 \%$ & & & $\%$ & 0.5 \\
\hline 2-Fluorobiphenyl & $64 \%$ & & & $\%$ & 0.5 \\
\hline Terphenyl-d14 & $68 \%$ & & & $\%$ & 0.5 \\
\hline Phenol-d5 & $28 \%$ & & & $\%$ & 0.5 \\
\hline 2-Fluorophenol & $37 \%$ & & & $\%$ & 0.5 \\
\hline 2,4,6-Tribromophenol & $69 \%$ & & & $\%$ & 0.5 \\
\hline
\end{tabular}


APPENDIX D.2 Results of Semivolatile Organic Compound Analyses, Water, Salmon Site

\begin{tabular}{|c|c|c|c|c|c|}
\hline COMPOUND & RESULTS & $\begin{array}{l}\text { DATA } \\
\text { QUAL. }\end{array}$ & $\begin{array}{l}\text { VALID. } \\
\text { QUAL. }\end{array}$ & CRDL UNITS & DILUTION \\
\hline \multicolumn{6}{|l|}{ C-FB-001-W } \\
\hline Phenol & 12 UG/ & $u$ & - & 12 UG $/$ & 0.5 \\
\hline bis(2-Chloroethyl) ether & 12 UG/ & $u$ & - & 12 UG/L & 0.5 \\
\hline 2-Chlorophenol & 12 UG $\Omega$ & $u$ & - & 12 UG $/ \mathrm{L}$ & 0.5 \\
\hline 1,3-Dichlorobenzene & 12 UG/ & $u$ & - & 12 UG/L & 0.5 \\
\hline 1,4-Dichlorobenzene & 12 UG $\Omega$ & $u$ & - & 12 UG & 0.5 \\
\hline Benzyl Alcohol & 12 UG几 & $u$ & - & 12 UG $\Omega$ & 0.5 \\
\hline 1,2-Dichlorobenzene & $12 \mathrm{UG} / \mathrm{L}$ & $u$ & - & $12 \cup G \Omega$ & 0.5 \\
\hline 2-Methylphenol & 12 UG/L & $u$ & - & 12 UG $\Omega$ & 0.5 \\
\hline bis(2-Chloroisopropyl) ether & 12 UG $\Omega$ & $u$ & $\ldots$ & $12 \mathrm{UG} \Omega$ & 0.5 \\
\hline 4-Methylphenol & 12 UG/ & $U$ & - & 12 UG/L & 0.5 \\
\hline N-Nitroso-Di-n-dipropylamine & 12 UG & $u$ & - & 12 UG/ & 0.5 \\
\hline Hexachloroethane & 12 UG/ & $u$ & - & 12 UG/L & 0.5 \\
\hline Nitrobenzene & 12 UG/ & $u$ & - & 12 UG $\Omega$ & 0.5 \\
\hline Isophorone & 12 UG/ & $u$ & - & 12 UG $\Omega$ & 0.5 \\
\hline 2-Nitrophenol & 12 UG $\Omega$ & $u$ & - & 12 UG $\Omega$ & 0.5 \\
\hline 2,4-Dimethylphenol & 12 UG $\Omega$ & $u$ & - & 12 UG/ & 0.5 \\
\hline Benzoic Acid & 62 UG $\Omega$ & $u$ & - & 62 UG/ & 0.5 \\
\hline bis(2Chloroethoxy)methane & 12 UG $\Omega$ & $u$ & - & 12 UG $\Omega$ & 0.5 \\
\hline 2,4-Dichlorophenol & 12 UG & $u$ & - & 12 UG/ & 0.5 \\
\hline 1,2,4-Trichlorobenzene & 12 UG/ & $U$ & - & 12 UG/L & 0.5 \\
\hline Naphthalene & 12 UG/L & $u$ & - & 12 UG/ & 0.5 \\
\hline 4-Chloroaniline & 12 UG $\Omega$ & $u$ & $\cdots$ & 12 UG/L & 0.5 \\
\hline Hexachlorobutadiene & 12 UG几 & $u$ & $\ldots$ & 12 UG & 0.5 \\
\hline 4-Chloro-3-Methlyphenol & 12 UG/L & $u$ & $\ldots$ & 12 UG $\Omega$ & 0.5 \\
\hline 2-Methylnaphthalene & 12 UG/L & $u$ & - & 12 UG/ & 0.5 \\
\hline Hexachlorocyclopentadiene & 12 UG/L & $u$ & - & 12 UG $\Omega$ & 0.5 \\
\hline 2,4,6-Trichlorophenol & 12 UG $/$ & $u$ & - & 12 UG $\Omega$ & 0.5 \\
\hline 2,4,5-Trichlorophenol & 62 UG/L & $u$ & - & 62 UG/ & 0.5 \\
\hline 2-Chloronaphthalene & 12 UG $\Omega$ & $u$ & -- & 12 UG/ & 0.5 \\
\hline 2-Nitroaniline & 62 UG/L & $u$ & $\ldots$ & 62 UG $\Omega$ & 0.5 \\
\hline Dimethyl Phthalate & 12 UGR & $u$ & $\cdots$ & $12 \mathrm{UG} / \mathrm{L}$ & 0.5 \\
\hline Acenaphthylene & 12 UG/L & $u$ & - & 12 UG/ & 0.5 \\
\hline 2,6-Dinitrotoluene & 12 UGR & $u$ & - & 12 UG/L & 0.5 \\
\hline 3-Nitroaniline & 62 UG/ & $u$ & - & 62 UG/L & 0.5 \\
\hline Acenaphthene & 12 UG $/$ & $u$ & - & $12 \mathrm{UG} / \mathrm{L}$ & 0.5 \\
\hline 2,4-Dinitrophenol & 62 UG $\Omega$ & $u$ & - & 62 UG/ & 0.5 \\
\hline 4-Nitrophenol & 62 UG & $u$ & - & 62 UG/L & 0.5 \\
\hline Dibenzofuran & 12 UG $\Omega$ & $u$ & - & 12 UG/L & 0.5 \\
\hline 2,4-Dinitrotoluene & 12 UG $\Omega$ & $u$ & - & 12 UG/L & 0.5 \\
\hline Diethylphthalate & 12 UG $\Omega$ & $u$ & - & 12 UG $\Omega$ & 0.5 \\
\hline 4-Chlorophenyl phenyl ether & 12 UG/L & $u$ & - & 12 UG/ & 0.5 \\
\hline Fluorene & 12 UG/ & $u$ & - & 12 UG/ & 0.5 \\
\hline 4-Nitroaniline & 62 UG/ & $u$ & - & 62 UGR & 0.5 \\
\hline 4,6-Dinitro-2-methylphenol & 62 UG/L & $u$ & - & 62 UGR & 0.5 \\
\hline N-Nitrosodiphenylamine & 12 UG/ & $u$ & - & 12 UG $\Omega$ & 0.5 \\
\hline
\end{tabular}


APPENDIX D.2 Results of Semivolatile Organic Compound Analyses, Water, Salmon Site

\begin{tabular}{|c|c|c|c|c|c|}
\hline COMPOUND & RESULTS & $\begin{array}{l}\text { DATA } \\
\text { QUAL. }\end{array}$ & $\begin{array}{l}\text { VALID. } \\
\text { QUAL. }\end{array}$ & CRDL UNITS & DILUTIC \\
\hline \multicolumn{6}{|l|}{ C-FB-001-W (continued) } \\
\hline 4-Bromophenyi phenylether & 12 UG/ & $u$ & $\cdots$ & 12 UG/ & 0.5 \\
\hline Hexachlorobenzene & 12 UG/ & $u$ & --- & 12 UG/ & 0.5 \\
\hline Pentachlorophenol & 62 UG/L & $u$ & -- & 62 UG/L & 0.5 \\
\hline Phenanthrene & 12 UG/L & $u$ & $\ldots$ & 12 UGR & 0.5 \\
\hline Anthracene & 12 UGA & $u$ & -- & 12 UGR & 0.5 \\
\hline Di-N-Butylphthalate & 12 UG/ & $u$ & $\cdots$ & 12 UG几 & 0.5 \\
\hline Fluoranthene & 12 UG/ & $\mathrm{u}$ & $\ldots$ & 12 UG $\Omega$ & 0.5 \\
\hline Pyrene & 12 UG/ & $u$ & -- & 12 UG几 & 0.5 \\
\hline Butyl Benzyl Phthalate & 12 UG/L & $u$ & -- & 12 UG $\Omega$ & 0.5 \\
\hline 3,3'-Dichlorobenzidine & 25 UG $\Omega$ & $u$ & $\cdots$ & 25 UG几 & 0.5 \\
\hline Benzo(a)Anthracene & $12 \cup G \Omega$ & $u$ & $\cdots$ & 12 UG几 & 0.5 \\
\hline Chrysene & 12 UG/L & $u$ & -- & 12 UG/ & 0.5 \\
\hline bis(2-Ethylhexyl)phthalate & 9 UG & BJ & $u$ & 12 UG $\Omega$ & 0.5 \\
\hline di-N-Octyl Phthalate & 12 UG/L & $u$ & --- & 12 UG/ & 0.5 \\
\hline Benzo(b)Fluoranthene & 12 UG/L & $u$ & $\ldots$ & 12 UG $\Omega$ & 0.5 \\
\hline Benzo(k)Fluoranthene & 12 UG/L & $u$ & ... & 12 UG/ & 0.5 \\
\hline Benzo(a)Pyrene & 12 UG/ & $u$ & - & 12 UG $\Omega$ & 0.5 \\
\hline Indeno( $1,2,3-C d)$ pyrene & 12 UG/ & $u$ & $\ldots$ & 12 UG $\Omega$ & 0.5 \\
\hline Dibenzo $(a, h)$ Anthracene & 12 UG/ & $u$ & $\ldots$ & 12 UG $\Omega$ & 0.5 \\
\hline Benzo(g,h,i)perylene & 12 UG/ & $u$ & $\cdots$ & 12 UG $\Omega$ & 0.5 \\
\hline Nitrobenzene-d5 & $83 \%$ & & & $\%$ & 0.5 \\
\hline 2-Fluorobiphenyl & $77 \%$ & & & $\%$ & 0.5 \\
\hline Terphenyl-d14 & $65 \%$ & & & $\%$ & 0.5 \\
\hline Phenol-d5 & $43 \%$ & & & $\%$ & 0.5 \\
\hline 2-Fluorophenol & $58 \%$ & & & $\%$ & 0.5 \\
\hline 2,4,6-Trichlorophenol & $79 \%$ & & & $\%$ & 0.5 \\
\hline
\end{tabular}


APPENDIX D.2 Results of Semivolatile Organic Compound Analyses, Water, Salmon Site

\begin{tabular}{|c|c|c|c|c|c|}
\hline COMPOUND & RESULTS & $\begin{array}{l}\text { DATA } \\
\text { QUAL. }\end{array}$ & $\begin{array}{l}\text { VALID. } \\
\text { QUAL. }\end{array}$ & CRDL UNITS & DILUTION \\
\hline \multicolumn{6}{|l|}{ C-RB-001-W } \\
\hline Phenol & 9 UG $\Omega$ & $u$ & $\ldots$ & 9 UGR & 0.5 \\
\hline bis(2-Chloroethyl) ether & 9 UG/ & $u$ & - & 9 UG $\Omega$ & 0.5 \\
\hline 2-Chlorophenol & 9 UG & $u$ & - & 9 UGR & 0.5 \\
\hline 1,3-Dichlorobenzene & 9 UG/ & $u$ & - & 9 UG/ & 0.5 \\
\hline 1,4-Dichlorobenzene & $9 \mathrm{UG} /$ & $u$ & - & $9 \mathrm{UG} \Omega$ & 0.5 \\
\hline Benzyl Alcohol & 9 UG & $u$ & - & 9 UG & 0.5 \\
\hline 1,2-Dichlorobenzene & 9 UG & $u$ & - & 9 UG & 0.5 \\
\hline 2-Methylphenol & 9 UG/ & $u$ & - & 9 UG/L & 0.5 \\
\hline bis(2-Chloroisopropyl) ether & 9 UG/ & $u$ & - & 9 UG/ & 0.5 \\
\hline 4-Methylphenol & 9 UG $\Omega$ & $u$ & $\ldots$ & 9 UG/ & 0.5 \\
\hline N-Nitroso-Di-n-dipropylamine & 9 UG/ & $u$ & - & $9 \mathrm{UG} / \mathrm{L}$ & 0.5 \\
\hline Hexachloroethane & 9 UG/ & $u$ & - & 9 UG/ & 0.5 \\
\hline Nitrobenzene & 9 UG & $u$ & - & 9 UG & 0.5 \\
\hline Isophorone & 9 UG & $u$ & $\ldots$ & 9 UG/ & 0.5 \\
\hline 2-Nitrophenol & 9 UGR & $u$ & -- & 9 UG/ & 0.5 \\
\hline 2,4-Dimethylphenol & 9 UGR & $u$ & - & 9 UG/ & 0.5 \\
\hline Benzoic Acid & $47 \mathrm{UG} \Omega$ & $u$ & - & 47 UG $\Omega$ & 0.5 \\
\hline bis(2Chloroethoxy)methane & 9 UG $/$ & $u$ & - & 9 UG几 & 0.5 \\
\hline 2,4-Dichlorophenol & 9 UG/ & $u$ & - & 9 UG/ & 0.5 \\
\hline 1,2,4-Trichlorobenzene & 9 UGL & $u$ & - & 9 UG & 0.5 \\
\hline Naphthalene & 9 UG $\Omega$ & $u$ & - & $9 \mathrm{UG} /$ & 0.5 \\
\hline 4-Chloroaniline & 9 UGL & $u$ & - & $9 \mathrm{UG} \Omega$ & 0.5 \\
\hline Hexachlorobutadiene & 9 UG/ & $u$ & - & 9 UGR & 0.5 \\
\hline 4-Chloro-3-Methlyphenol & 9 UG/ & $u$ & - & 9 UG & 0.5 \\
\hline 2-Methylnaphthalene & 9 UG & $u$ & - & 9 UG/ & 0.5 \\
\hline Hexachlorocyclopentadiene & 9 UG/ & $u$ & - & 9 UG/ & 0.5 \\
\hline 2,4,6-Trichlorophenol & 9 UG/ & $u$ & - & 9 UG $\Omega$ & 0.5 \\
\hline 2,4,5-Trichlorophenol & 47 UG几 & $u$ & - & $47 \mathrm{UG} / \mathrm{L}$ & 0.5 \\
\hline 2-Chloronaphthalene & 9 UG/ & $u$ & - & 9 UG/ & 0.5 \\
\hline 2-Nitroaniline & 47 UGR & $u$ & - & 47 UG/L & 0.5 \\
\hline Dimethyl Phthalate & 9 UG & $u$ & - & 9 UG/ & 0.5 \\
\hline Acenaphthylene & 9 UGR & $u$ & - & 9 UGL & 0.5 \\
\hline 2,6-Dinitrotoluene & 9 UG/ & $u$ & - & 9 UG & 0.5 \\
\hline 3-Nitroaniline & 47 UG/ & $u$ & -- & 47 UGR & 0.5 \\
\hline Acenaphthene & $9 \mathrm{UG} \Omega$ & $u$ & - & 9 UG & 0.5 \\
\hline 2,4-Dinitrophenol & 47 UG $\Omega$ & $u$ & - & 47 UG/L & 0.5 \\
\hline 4-Nitrophenol & 47 UG $\Omega$ & $u$ & - & 47 UG几 & 0.5 \\
\hline Dibenzofuran & 9 UGR & $u$ & - & 9 UGR & 0.5 \\
\hline 2,4-Dinitrotoluene & 9 UG & $u$ & - & 9 UG/L & 0.5 \\
\hline Diethylphthalate & 9 UGR & $u$ & - & 9 UG/ & 0.5 \\
\hline 4-Chlorophenyl phenyl ether & 9 UG & $u$ & - & 9 UG/L & 0.5 \\
\hline Fluorene & 9 UGR & $u$ & - & 9 UGR & 0.5 \\
\hline 4-Nitroaniline & 47 UGR & $u$ & - & 47 UG/L & 0.5 \\
\hline 4,6-Dinitro-2-methylphenol & 47 UG & $u$ & - & 47 UG $\Omega$ & 0.5 \\
\hline N-Nitrosodiphenylamine & 9 UG/L & $u$ & - & 9 UG & 0.5 \\
\hline
\end{tabular}


APPENDIX D.2 Results of Semivolatile Organic Compound Analyses, Water, Salmon Site

\begin{tabular}{|c|c|c|c|c|c|}
\hline COMPOUND & RESULTS & $\begin{array}{l}\text { DATA } \\
\text { QUAL. }\end{array}$ & $\begin{array}{l}\text { VALID. } \\
\text { QUAL. }\end{array}$ & CRDL UNITS & DILUTION \\
\hline \multicolumn{6}{|l|}{ C-RB-002-W (continued) } \\
\hline 4-Bromophenyl phenylether & 9 UG/ & $u$ & $\cdots$ & 9 UG/ & 0.5 \\
\hline Hexachlorobenzene & 9 UG/ & $u$ & -- & 9 UG $\Omega$ & 0.5 \\
\hline Pentachlorophenol & 47 UG/ & $u$ & $\ldots$ & 47 UG/ & 0.5 \\
\hline Phenanthrene & $9 \mathrm{UG} / \mathrm{h}$ & $u$ & $\cdots$ & 9 UG/L & 0.5 \\
\hline Anthracene & 9 UG/L & $u$ & $\cdots$ & 9 UG/L & 0.5 \\
\hline Di-N-Butylphthalate & 9 UG/ & $u$ & $\ldots$ & 9 UG/ & 0.5 \\
\hline Fluoranthene & 9 UG/ & $u$ & $\ldots$ & 9 UG/L & 0.5 \\
\hline Pyrene & 9 UG/L & $u$ & $\cdots$ & 9 UG/L & 0.5 \\
\hline Butyl Benzyl Phthalate & 9 UG & $u$ & -- & 9 UG & 0.5 \\
\hline 3,3'-Dichlorobenzidine & 19 UG/ & $u$ & -- & 19 UG $\Omega$ & 0.5 \\
\hline Benzo(a)Anthracene & 9 UG/ & $u$ & $\ldots$ & 9 UG/ & 0.5 \\
\hline Chrysene & 9 UG/ & $u$ & -- & 9 UG/L & 0.5 \\
\hline bis(2-Ethylhexyl)phthalate & $10 \mathrm{UG} \Omega$ & 8 & -- & $9 \mathrm{UG} / \mathrm{L}$ & 0.5 \\
\hline di-N-Octyl Phthalate & 9 UG/L & $u$ & $\ldots$ & 9 UG/L & 0.5 \\
\hline Benzo(b)Fiuoranthene & 9 UG/ & $u$ & - & $9 \cup G / L$ & 0.5 \\
\hline Benzo(k)Fluoranthene & 9 UG/ & $u$ & $\ldots$ & 9 UG/L & 0.5 \\
\hline Benzo(a)Pyrene & 9 UG/ & $u$ & $\cdots$ & 9 UG/L & 0.5 \\
\hline Indeno(1,2,3-Cd)pyrene & 9 UG/L & $u$ & $\cdots$ & 9 UG/ & 0.5 \\
\hline Dibenzo(a,h)Anthracene & 9 UG/ & $u$ & - & 9 UG/L & 0.5 \\
\hline Benzo(g,h,i)perylene & 9 UG & $u$ & $\ldots$ & 9 UG/ & 0.5 \\
\hline Dodecanoic acid & 37 UG $\Omega$ & 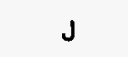 & NJ & & 0.5 \\
\hline Unknown - 1 & 16 UG几 & $\checkmark$ & J & & 0.5 \\
\hline Unknown - 2 & 10 UG/L & 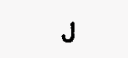 & J & & 0.5 \\
\hline Unknown - 3 & 23 UG/ & J & s & & 0.5 \\
\hline Unknown - 4 & 7 UG/ & J & J & & 0.5 \\
\hline Unknown - 5 & 9 UG/ & J & J & & 0.5 \\
\hline Unknown - 6 & 7 UG/ & $\jmath$ & J & & 0.5 \\
\hline Unknown - 7 & 5 UG & J & J & & 0.5 \\
\hline Unknown - 8 & 5 UG/. & 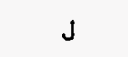 & $J$ & & 0.5 \\
\hline Nitrobenzene-d5 & $84 \%$ & & & $\%$ & 0.5 \\
\hline 2-Fluorobiphenyl & $79 \%$ & & & $\%$ & 0.5 \\
\hline Terphenyi-d14 & $72 \%$ & & & $\%$ & 0.5 \\
\hline Phenol-d5 & $40 \%$ & & & $\%$ & 0.5 \\
\hline 2-Fluorophenol & $56 \%$ & & & $\%$ & 0.5 \\
\hline 2,4,6-Tribromophenol & $82 \%$ & & & $\%$ & 0.5 \\
\hline
\end{tabular}




\section{Appendix E.1}

Results of Pesticide and PCB Analyses, Soils, Salmon Site 
APPENDIX E.1 Results of Pesticide and PCB Anaylses, Soils, Salmon Site

\begin{tabular}{|c|c|c|c|c|c|}
\hline COMPOUND & RESULTS UNITS & $\begin{array}{l}\text { DATA } \\
\text { QUAL. }\end{array}$ & $\begin{array}{l}\text { DATA } \\
\text { VALID. }\end{array}$ & CRDL UNITS & DILUTION \\
\hline \multicolumn{6}{|l|}{ C5-1-S } \\
\hline alpha-BHC & $8 \mathrm{UG} / \mathrm{KG}$ & $u$ & NP & 8 UG/KG & 1 \\
\hline beta-BHC & $8 \mathrm{UG} / \mathrm{KG}$ & $u$ & NP & 8 UG/KG & 1 \\
\hline delta-BHC & 8 UG/KG & $u$ & NP & 8 UG/KG & 1 \\
\hline gamma-BHC (Lindane) & 8 UG/KG & $u$ & NP & 8 UG/KG & 1 \\
\hline Heptachlor & 8 UG/KG & $u$ & NP & 8 UG/KG & 1 \\
\hline Aldrin & 8 UG/KG & $u$ & NP & 8 UG/KG & 1 \\
\hline Heptachlor Epoxide & $8 \mathrm{UG} / \mathrm{KG}$ & $u$ & NP & 8 UG/KG & 1 \\
\hline Endosulfan I & 8 UG/KG & $u$ & NP & 8 UG/KG & 1 \\
\hline Dieldrin & 16 UG/KG & $u$ & NP & 16 UG/KG & 1 \\
\hline 4,4'-DDE & 16 UG/KG & $u$ & NP & 16 UG/KG & 1 \\
\hline Endrin & 16 UG/KG & $u$ & NP & $16 \mathrm{UG} / \mathrm{KG}$ & 1 \\
\hline Endosulfan II & 16 UG/KG & $u$ & NP & 16 UG/KG & 1 \\
\hline 4,4'-DDD & 16 UG/KG & $u$ & NP & 16 UG/KG & 1 \\
\hline Endosulfan Sulfate & 16 UG/KG & $u$ & NP & 16 UG/KG & 1 \\
\hline 4,4'-DDT & 16 UG/KG & $u$ & NP & $16 \mathrm{UG} / \mathrm{KG}$ & 1 \\
\hline Methoxychlor & 80 UG/KG & $u$ & NP & 80 UG/KG & 1 \\
\hline Endrin Ketone & 16 UG/KG & $u$ & NP & 16 UG/KG & 1 \\
\hline alpha-Chlordane & 80 UG/KG & u & NP & 80 UG/KG & 1 \\
\hline gamma-Chlordane & 80 UG/KG & $u$ & NP & 80 UG/KG & 1 \\
\hline Toxaphene & 160 UG/KG & $u$ & NP & 160 UG/KG & 1 \\
\hline Aroclor-1016 & 80 UG/KG & $u$ & NP & 80 UG/KG & 1 \\
\hline Aroclor-1221 & 80 UG/KG & u & NP & 80 UG/KG & 1 \\
\hline Aroclor-1232 & 80 UG/KG & $u$ & NP & 80 UG/KG & 1 \\
\hline Aroclor-1242 & 80 UG/KG & u & NP & 80 UG/KG & 1 \\
\hline Aroclor-1248 & 80 UG/KG & $u$ & NP & 80 UG/KG & 1 \\
\hline Aroclor-1254 & 160 UG/KG & $u$ & NP & 160 UG/KG & 1 \\
\hline Aroclor- 1260 & 160 UG/KG & $u$ & NP & 160 UG/KG & 1 \\
\hline DBC & $84 \%$ & & NP & $\%$ & 1 \\
\hline
\end{tabular}


APPENDIX E.1 Results of Pesticide and PCB Anaylses, Soils, Salmon Site

\begin{tabular}{|c|c|c|c|c|c|}
\hline COMPOUND & RESULTS UNITS & $\begin{array}{l}\text { DATA } \\
\text { QUAL. }\end{array}$ & $\begin{array}{l}\text { DATA } \\
\text { VALID. }\end{array}$ & CRDL UNITS & DILUTION \\
\hline \multicolumn{6}{|l|}{ C5-2-S } \\
\hline alpha-BHC & $8 \mathrm{UG} / \mathrm{KG}$ & $u$ & NP & 8 UG/KG & 1 \\
\hline beta-BHC & 8 UG/KG & $u$ & NP & $8 \mathrm{UG} / \mathrm{KG}$ & 1 \\
\hline delta-BHC & 8 UG/KG & $u$ & NP & 8 UG/KG & 1 \\
\hline gamma-BHC (Lindane) & 8 UG/KG & $u$ & NP & 8 UG/KG & 1 \\
\hline Heptachlor & 8 UG/KG & $u$ & NP & $8 \mathrm{UG} / K G$ & 1 \\
\hline Aldrin & 8 UG/KG & $u$ & NP & $8 \mathrm{UG} / \mathrm{KG}$ & 1 \\
\hline Heptachlor Epoxide & 8 UG/KG & $u$ & NP & 8 UG/KG & 1 \\
\hline Endosulfan 1 & 8 UG/KG & $u$ & NP & $8 \mathrm{UG} / K G$ & 1 \\
\hline Dieldrin & 16 UG/KG & $u$ & NP & 16 UG/KG & 1 \\
\hline $4,4^{\prime}-D D E$ & 16 UG/KG & $u$ & NP & $16 \mathrm{UG} / \mathrm{KG}$ & 1 \\
\hline Endrin & $16 \mathrm{UG} / \mathrm{KG}$ & $u$ & NP & 16 UG/KG & 1 \\
\hline Endosulfan II & 16 UG/KG & $u$ & NP & 16 UG/KG & 1 \\
\hline 4,4'-DDD & 16 UG/KG & $u$ & NP & 16 UG/KG & 1 \\
\hline Endosulfan Sulfate & $16 \mathrm{UG} / \mathrm{KG}$ & $u$ & NP & 16 UG/KG & 1 \\
\hline 4,4'-DDT & 16 UG/KG & $u$ & NP & 16 UG/KG & 1 \\
\hline Methoxychlor & 80 UG/KG & $u$ & NP & 80 UG/KG & 1 \\
\hline Endrin Ketone & 16 UG/KG & $u$ & NP & 16 UG/KG & 1 \\
\hline alpha-Chlordane & 80 UG/KG & $u$ & NP & 80 UG/KG & 1 \\
\hline gamma-Chlordane & 80 UG/KG & $u$ & NP & 80 UG/KG & 1 \\
\hline Toxaphene & 160 UG/KG & $u$ & NP & 160 UG/KG & 1 \\
\hline Aroclor-1016 & 80 UG/KG & $u$ & NP & 80 UG/KG & 1 \\
\hline Aroclor- 1221 & 80 UG/KG & $u$ & NP & 80 UG/KG & 1 \\
\hline Aroclor-1232 & 80 UG/KG & $u$ & NP & 80 UG/KG & 1 \\
\hline Aroclor-1242 & 80 UG/KG & $u$ & NP & 80 UG/KG & 1 \\
\hline Aroclor-1248 & 80 UG/KG & $u$ & NP & 80 UG/KG & 1 \\
\hline Aroclor-1254 & 160 UG/KG & $u$ & NP & $160 \mathrm{UG} / \mathrm{KG}$ & 1 \\
\hline Aroclor- 1260 & 160 UG/KG & $u$ & NP & 160 UG/KG & 1 \\
\hline DBC & $95 \%$ & & & $\%$ & 1 \\
\hline
\end{tabular}


APPENDIX E.1 Results of Pesticide and PCB Anayises, Soils, Salmon Site

\begin{tabular}{|c|c|c|c|c|c|}
\hline COMPOUND & RESULTS UNITS & $\begin{array}{l}\text { DATA } \\
\text { QUAL. }\end{array}$ & $\begin{array}{l}\text { DATA } \\
\text { VALID. }\end{array}$ & CRDL UNITS & DILUTION \\
\hline \multicolumn{6}{|l|}{ C5-2-S-MS } \\
\hline gamma-BHC (Lindane) & $122 \%$ & & NP & $\%$ & 1 \\
\hline Heptachlor & $122 \%$ & & NP & $\%$ & 1 \\
\hline Aldrin & $146 \%$ & * & NP & $\%$ & 1 \\
\hline Dieldrin & $118 \%$ & & NP & $\%$ & 1 \\
\hline Endrin & $124 \%$ & & NP & $\%$ & 1 \\
\hline 4,4'-DDT & $116 \%$ & & NP & $\%$ & 1 \\
\hline DBC & $124 \%$ & & NP & $\%$ & 1 \\
\hline \multicolumn{6}{|l|}{ C5-2-S-MSD } \\
\hline gamma-BHC (Lindane) & $94 \%$ & & NP & $\%$ & 1 \\
\hline Heptachlor & $98 \%$ & & NP & $\%$ & 1 \\
\hline Aldrin & $99 \%$ & & NP & $\%$ & 1 \\
\hline Dieldrin & $94 \%$ & & NP & $\%$ & 1 \\
\hline Endrin & $96 \%$ & & NP & $\%$ & 1 \\
\hline 4,4'-DDT & $96 \%$ & & NP & $\%$ & 1 \\
\hline DBC & $85 \%$ & & NP & $\%$ & 1 \\
\hline
\end{tabular}

E.1-3 
APPENDIX E.1 Results of Pesticide and PCB Anaylses, Soils, Salmon Site

\begin{tabular}{|c|c|c|c|c|c|}
\hline COMPOUND & RESULTS UNITS & $\begin{array}{l}\text { DATA } \\
\text { QUAL. }\end{array}$ & $\begin{array}{l}\text { DATA } \\
\text { VALID. }\end{array}$ & CRDL UNITS & DILUTION \\
\hline \multicolumn{6}{|l|}{ C5-3-S } \\
\hline alpha-BHC & 8 UG/KG & $u$ & NP & $8 \mathrm{UG} / \mathrm{KG}$ & 1 \\
\hline beta-BHC & 8 UG/KG & $u$ & NP & 8 UG/KG & 1 \\
\hline delta-BHC & $8 \mathrm{UG} / \mathrm{KG}$ & $u$ & NP & 8 UG/KG & 1 \\
\hline gamma-BHC (Lindane) & 8 UG/KG & $u$ & NP & 8 UG/KG & 1 \\
\hline Heptachlor & 8 UG/KG & $u$ & NP & 8 UG/KG & 1 \\
\hline Aldrin & 8 UG/KG & $u$ & NP & $8 \mathrm{UG} / K G$ & 1 \\
\hline Heptachlor Epoxide & $8 \mathrm{UG} / \mathrm{KG}$ & $u$ & NP & 8 UG/KG & 1 \\
\hline Endosulfan I & $8 \mathrm{UG} / \mathrm{KG}$ & $u$ & NP & 8 UG/KG & 1 \\
\hline Dieldrin & 16 UG/KG & $u$ & NP & 16 UG/KG & 1 \\
\hline $4,4^{\prime}-\mathrm{DDE}$ & 16 UG/KG & $u$ & NP & 16 UG/KG & 1 \\
\hline Endrin & 16 UG/KG & $u$ & NP & 16 UG/KG & 1 \\
\hline Endosulfan II & 16 UG/KG & $u$ & NP & 16 UG/KG & 1 \\
\hline 4,4'-DDD & 16 UG/KG & $u$ & NP & 16 UG/KG & 1 \\
\hline Endosulfan Sulfate & 16 UG/KG & $u$ & NP & 16 UG/KG & 1 \\
\hline 4,4'-DDT & 16 UG/KG & $u$ & NP & 16 UG/KG & 1 \\
\hline Methoxychlor & 80 UG/KG & $u$ & NP & 80 UG/KG & 1 \\
\hline Endrin Ketone & 16 UG/KG & $u$ & NP & 16 UG/KG & 1 \\
\hline alpha-Chlordane & 80 UG/KG & $u$ & NP & 80 UG/KG & 1 \\
\hline gamma-Chlordane & 80 UG/KG & u & NP & 80 UG/KG & 1 \\
\hline Toxaphene & 160 UG/KG & $u$ & NP & 160 UG/KG & 1 \\
\hline Aroclor-1016 & 80 UG/KG & $u$ & NP & 80 UG/KG & 1 \\
\hline Aroclor-1221 & 80 UG/KG & $u$ & NP & 80 UG/KG & 1 \\
\hline Aroclor-1232 & 80 UG/KG & $u$ & NP & 80 UG/KG & 1 \\
\hline Aroclor-1242 & 80 UG/KG & $u$ & NP & 80 UG/KG & 1 \\
\hline Arocior-1248 & 80 UG/KG & $u$ & NP & 80 UG/KG & 1 \\
\hline Aroclor-1254 & 160 UG/KG & $u$ & NP & 160 UG/KG & 1 \\
\hline Aroclor-1260 & 160 UG/KG & $u$ & NP & 160 UG/KG & 1 \\
\hline DBC & $78 \%$ & & & $\%$ & 1 \\
\hline
\end{tabular}




\section{APPENDIX E.1 Results of Pesticide and PCB Anaylses, Soils, Salmon Site}

\begin{tabular}{|c|c|c|c|c|c|}
\hline COMPOUND & RESULTS UNITS & $\begin{array}{l}\text { DATA } \\
\text { QUAL. }\end{array}$ & $\begin{array}{l}\text { DATA } \\
\text { VALID. }\end{array}$ & CRDL UNITS & DILUTION \\
\hline \multicolumn{6}{|l|}{ C3-4-S } \\
\hline alpha-BHC & 8 UG/KG & $u$ & NP & 8 UG/KG & 1 \\
\hline beta-BHC & $8 \mathrm{UG} / \mathrm{KG}$ & $u$ & NP & 8 UG/KG & 1 \\
\hline delta-BHC & 8 UG/KG & $u$ & NP & 8 UG/KG & 1 \\
\hline gamma-BHC (Lindane) & 8 UG/KG & $u$ & NP & 8 UG/KG & 1 \\
\hline Heptachlor & 8 UG/KG & $u$ & NP & 8 UG/KG & 1 \\
\hline Aldrin & $8 \mathrm{UG} / \mathrm{KG}$ & $u$ & NP & $8 \mathrm{UG} / K G$ & 1 \\
\hline Heptachlor Epoxide & $8 \mathrm{UG} / \mathrm{KG}$ & $u$ & NP & 8 UG/KG & 1 \\
\hline Endosulfan I & 8 UG/KG & $u$ & NP & $8 \mathrm{UG} / \mathrm{KG}$ & 1 \\
\hline Dieldrin & 16 UG/KG & $u$ & NP & 16 UG/KG & 1 \\
\hline 4,4'-DDE & 16 UG/KG & $u$ & NP & $16 \mathrm{UG} / \mathrm{KG}$ & 1 \\
\hline Endrin & 16 UG/KG & $u$ & NP & 16 UG/KG & 1 \\
\hline Endosulfan II & 16 UG/KG & $u$ & NP & 16 UG/KG & 1 \\
\hline 4,4'-DDD & 16 UG/KG & $u$ & NP & 16 UG/KG & 1 \\
\hline Endosulfan Sulfate & 16 UG/KG & $u$ & NP & $16 \mathrm{UG} / \mathrm{KG}$ & 1 \\
\hline 4,4'-DDT & 16 UG/KG & $u$ & NP & 16 UG/KG & 1 \\
\hline Methoxychlor & 80 UG/KG & $u$ & NP & 80 UG/KG & 1 \\
\hline Endrin Ketone & 16 UG/KG & $u$ & NP & $16 \mathrm{UG} / \mathrm{KG}$ & 1 \\
\hline alpha-Chlordane & 80 UG/KG & $u$ & NP & 80 UG/KG & 1 \\
\hline gamma-Chlordane & 80 UG/KG & $u$ & NP & 80 UGKG & 1 \\
\hline Toxaphene & 160 UG/KG & $u$ & NP & 160 UG/KG & 1 \\
\hline Aroclor-1016 & 80 UG/KG & $u$ & NP & 80 UG/KG & 1 \\
\hline Aroclor-1221 & 80 UG/KG & $u$ & NP & 80 UG/KG & 1 \\
\hline Aroclor-1232 & $80 \mathrm{UG} / \mathrm{KG}$ & $u$ & NP & 80 UG/KG & 1 \\
\hline Aroclor-1242 & 80 UG/KG & $u$ & NP & 80 UG/KG & 1 \\
\hline Aroclor-1248 & 80 UG/KG & $u$ & NP & 80 UG/KG & 1 \\
\hline Aroclor-1254 & 160 UG/KG & $u$ & NP & 160 UG/KG & 1 \\
\hline Aroclor-1260 & 160 UG/KG & $u$ & NP & 160 UG/KG & 1 \\
\hline DBC & $65 \%$ & & & $\%$ & 1 \\
\hline
\end{tabular}


APPENDIX E.1 Results of Pesticide and PCB Anaylses, Soils, Salmon Site

\begin{tabular}{|c|c|c|c|c|c|}
\hline COMPOUND & RESULTS UNITS & $\begin{array}{l}\text { DATA } \\
\text { QUAL. }\end{array}$ & $\begin{array}{l}\text { DATA } \\
\text { VALID. }\end{array}$ & CRDL UNITS & DILUTION \\
\hline \multicolumn{6}{|l|}{ C3-5-S } \\
\hline alpha-BHC & 8 UG/KG & $u$ & NP & 8 UG/KG & 1 \\
\hline beta-BHC & 8 UG/KG & $u$ & NP & 8 UG/KG & 1 \\
\hline delta-BHC & 8 UG/KG & $u$ & NP & 8 UG/KG & 1 \\
\hline gamma-BHC (Lindane) & 8 UG/KG & $u$ & NP & 8 UG/KG & 1 \\
\hline Heptachlor & 8 UG/KG & $u$ & NP & 8 UG/KG & 1 \\
\hline Aldrin & 8 UG/KG & $u$ & NP & 8 UG/KG & 1 \\
\hline Heptachlor Epoxide & 8 UG/KG & $u$ & NP & 8 UG/KG & 1 \\
\hline Endosulfan 1 & $8 \mathrm{UG} / \mathrm{KG}$ & $u$ & NP & 8 UG/KG & 1 \\
\hline Dieldrin & 16 UG/KG & $u$ & $\mathrm{NP}$ & 16 UG/KG & 1 \\
\hline 4,4'-DDE & 16 UG/KG & $u$ & NP & 16 UG/KG & 1 \\
\hline Endrin & 16 UG/KG & $u$ & NP & 16 UG/KG & 1 \\
\hline Endosulfan II & 16 UG/KG & $u$ & $\mathrm{NP}$ & 16 UG/KG & 1 \\
\hline 4,4'-DDD & 16 UG/KG & $u$ & NP & 16 UG/KG & 1 \\
\hline Endosulfan Sulfate & 16 UG/KG & $u$ & $\mathrm{NP}$ & 16 UG/KG & 1 \\
\hline 4,4'-DDT & 16 UG/KG & $u$ & NP & 16 UG/KG & 1 \\
\hline Methoxychlor & 80 UG/KG & $u$ & NP & 80 UG/KG & 1 \\
\hline Endrin Ketone & 16 UG/KG & $u$ & NP & 16 UG/KG & 1 \\
\hline alpha-Chlordane & 80 UG/KG & $u$ & NP & 80 UG/KG & 1 \\
\hline gamma-Chlordane & 80 UG/KG & $u$ & NP & 80 UG/KG & 1 \\
\hline Toxaphene & 160 UG/KG & $u$ & NP & $160 \mathrm{UG} / \mathrm{KG}$ & 1 \\
\hline Aroclor-1016 & 80 UG/KG & $u$ & NP & 80 UG/KG & 1 \\
\hline Aroclor-1221 & 80 UG/KG & $u$ & NP & 80 UG/KG & 1 \\
\hline Aroclor-1232 & 80 UG/KG & $u$ & NP & 80 UG/KG & 1 \\
\hline Arocior-1242 & 80 UG/KG & $u$ & NP & 80 UG/KG & 1 \\
\hline Aroclor-1248 & 80 UG/KG & $u$ & NP & 80 UG/KG & 1 \\
\hline Aroclor-1254 & 160 UG/KG & $u$ & NP & 160 UG/KG & 1 \\
\hline Arocior-1260 & 160 UG/KG & $u$ & NP & 160 UG/KG & 1 \\
\hline DBC & $78 \%$ & & & $\%$ & 1 \\
\hline
\end{tabular}




\section{APPENDIX E.1 Results of Pesticide and PCB Anaylses, Soils, Salmon Site}

\begin{tabular}{|c|c|c|c|c|c|}
\hline COMPOUND & RESULTS UNITS & $\begin{array}{l}\text { DATA } \\
\text { QUAL. }\end{array}$ & $\begin{array}{l}\text { DATA } \\
\text { VALID. }\end{array}$ & CRDL UNITS & DILUTION \\
\hline \multicolumn{6}{|l|}{ C3-6-S } \\
\hline alpha-BHC & 8 UG/KG & $u$ & NP & 8 UG/KG & 1 \\
\hline beta-BHC & 8 UG/KG & $u$ & NP & 8 UG/KG & 1 \\
\hline delta-BHC & 8 UG/KG & $u$ & NP & $8 \mathrm{UG} / \mathrm{KG}$ & 1 \\
\hline gamma-BHC (Lindane) & $8 \mathrm{UG} / K \mathrm{G}$ & $u$ & NP & 8 UG/KG & 1 \\
\hline Heptachlor & 8 UG/KG & $u$ & NP & $8 \mathrm{UG} / \mathrm{KG}$ & 1 \\
\hline Aldrin & $8 \mathrm{UG} / \mathrm{KG}$ & $u$ & NP & 8 UG/KG & 1 \\
\hline Heptachlor Epoxide & $8 \mathrm{UG} / \mathrm{KG}$ & $u$ & NP & 8 UG/KG & 1 \\
\hline Endosulfan $\mid$ & 8 UG/KG & $u$ & NP & 8 UG/KG & 1 \\
\hline Dieldrin & 16 UG/KG & $u$ & NP & 16 UG/KG & 1 \\
\hline 4,4'-DDE & 16 UG/KG & $u$ & NP & 16 UG/KG & 1 \\
\hline Endrin & 16 UG/KG & $u$ & NP & 16 UG/KG & 1 \\
\hline Endosulfan II & $16 \mathrm{UG} / \mathrm{KG}$ & $u$ & NP & 16 UG/KG & 1 \\
\hline 4,4'-DDD & 16 UG/KG & $u$ & NP & 16 UG/KG & 1 \\
\hline Endosulfan Sulfate & 16 UG/KG & $u$ & NP & 16 UG/KG & 1 \\
\hline 4,4'-DDT & 16 UG/KG & $u$ & NP & 16 UG/KG & 1 \\
\hline Methoxychlor & 80 UG/KG & $u$ & NP & 80 UG/KG & 1 \\
\hline Endrin Ketone & 16 UG/KG & $u$ & NP & $16 \mathrm{UG} / \mathrm{KG}$ & 1 \\
\hline alpha-Chlordane & 80 UG/KG & $u$ & NP & 80 UG/KG & 1 \\
\hline gamma-Chlordane & 80 UG/KG & $u$ & NP & 80 UG/KG & 1 \\
\hline Toxaphene & 160 UG/KG & $u$ & NP & 160 UG/KG & 1 \\
\hline Aroclor-1016 & 80 UG/KG & $u$ & NP & 80 UG/KG & 1 \\
\hline Aroclor-1221 & 80 UG/KG & $u$ & NP & 80 UG/KG & 1 \\
\hline Aroclor-1232 & 80 UG/KG & $u$ & NP & 80 UG/KG & 1 \\
\hline Aroclor-1242 & 80 UG/KG & $u$ & NP & 80 UG/KG & 1 \\
\hline Aroclor-1248 & 80 UG/KG & $u$ & NP & 80 UG/KG & 1 \\
\hline Aroclor-1254 & 160 UG/KG & $u$ & NP & 160 UG/KG & 1 \\
\hline Aroclor-1260 & 160 UG/KG & $u$ & NP & 160 UG/KG & 1 \\
\hline DBC & $83 \%$ & & & $\%$ & 1 \\
\hline
\end{tabular}


APPENDIX E.1 Results of Pesticide and PCB Anaylses, Soils, Salmon Site

\begin{tabular}{|c|c|c|c|c|c|}
\hline COMPOUND & RESULTS UNITS & $\begin{array}{l}\text { DATA } \\
\text { QUAL. }\end{array}$ & $\begin{array}{l}\text { DATA } \\
\text { VALID. }\end{array}$ & CRDL UNITS & DILUTIC \\
\hline \multicolumn{6}{|l|}{ C3-7-S } \\
\hline alpha-BHC & 8 UG/KG & $u$ & NP & 8 UG/KG & 1 \\
\hline beta-BHC & 8 UG/KG & $u$ & NP & 8 UG/KG & 1 \\
\hline delta-BHC & $8 \mathrm{UG} / \mathrm{KG}$ & $u$ & NP & $8 \mathrm{UG} / \mathrm{KG}$ & 1 \\
\hline gamma-BHC (Lindane) & 8 UG/KG & $u$ & NP & 8 UG/KG & 1 \\
\hline Heptachior & 8 UG/KG & $u$ & NP & $8 \mathrm{UG} / \mathrm{KG}$ & 1 \\
\hline Aldrin & $8 \mathrm{UG} / \mathrm{KG}$ & $u$ & NP & 8 UG/KG & 1 \\
\hline Heptachlor Epoxide & 8 UG/KG & $u$ & NP & 8 UG/KG & 1 \\
\hline Endosulfan I & 8 UG/KG & $u$ & NP & 8 UGKG & 1 \\
\hline Dieldrin & 16 UG/KG & $u$ & NP & 16 UG/KG & 1 \\
\hline 4,4'-DDE & 16 UG/KG & $u$ & NP & 16 UG/KG & 1 \\
\hline Endrin & 16 UG/KG & $u$ & NP & 16 UG/KG & 1 \\
\hline Endosulfan II & $16 \mathrm{UG} / \mathrm{KG}$ & $u$ & NP & 16 UG/KG & 1 \\
\hline 4,4'-DDD & 16 UG/KG & $u$ & $\mathrm{NP}$ & 16 UG/KG & 1 \\
\hline Endosulfan Sulfate & 16 UG/KG & $u$ & NP & 16 UG/KG & 1 \\
\hline 4,4'-DDT & 16 UG/KG & $u$ & $\mathrm{NP}$ & 16 UG/KG & 1 \\
\hline Methoxychlor & 80 UG/KG & $u$ & NP & 80 UGKG & 1 \\
\hline Endrin Ketone & 16 UG/KG & $u$ & NP & 16 UG/KG & 1 \\
\hline alpha-Chlordane & 80 UG/KG & $u$ & NP & 80 UG/KG & 1 \\
\hline gamma-Chlordane & 80 UG/KG & $u$ & NP & 80 UG/KG & 1 \\
\hline Toxaphene & 160 UG/KG & $u$ & NP & 160 UG/KG & 1 \\
\hline Aroclor-1016 & 80 UG/KG & $u$ & NP & 80 UG/KG & 1 \\
\hline Aroclor-1221 & 80 UG/KG & $u$ & NP & 80 UG/KG & 1 \\
\hline Aroclor-1232 & 80 UG/KG & $u$ & NP & 80 UG/KG & 1 \\
\hline Aroclor-1242 & 80 UG/KG & $u$ & NP & 80 UG/KG & 1 \\
\hline Aroclor-1248 & 80 UG/KG & $u$ & NP & 80 UG/KG & 1 \\
\hline Aroclor-1254 & 160 UG/KG & $u$ & NP & 160 UG/KG & 1 \\
\hline Aroclor-1260 & 160 UG/KG & $u$ & NP & 160 UG/KG & 1 \\
\hline $\mathrm{DBC}$ & $85 \%$ & & & $\%$ & 1 \\
\hline
\end{tabular}


APPENDIX E.1 Results of Pesticide and PCB Anaylses, Soils, Salmon Site

\begin{tabular}{|c|c|c|c|c|c|}
\hline COMPOUND & RESULTS UNITS & $\begin{array}{l}\text { DATA } \\
\text { QUAL. }\end{array}$ & $\begin{array}{l}\text { DATA } \\
\text { VALID. }\end{array}$ & CRDL UNITS & DILUTIC \\
\hline \multicolumn{6}{|l|}{ C3-8-S } \\
\hline alpha-BHC & $8 \mathrm{UG} / \mathrm{KG}$ & $u$ & NP & 8 UG/KG & 1 \\
\hline beta-BHC & 8 UG/KG & $u$ & NP & $8 \mathrm{UG} / \mathrm{KG}$ & 1 \\
\hline delta-BHC & 8 UG/KG & $u$ & NP & 8 UG/KG & 1 \\
\hline gamma-BHC (Lindane) & 8 UG/KG & $u$ & NP & 8 UG/KG & 1 \\
\hline Heptachlor & $8 \mathrm{UG} / K G$ & $u$ & NP & $8 \mathrm{UG} / \mathrm{KG}$ & 1 \\
\hline Aldrin & $8 \mathrm{UG} / \mathrm{KG}$ & $u$ & NP & 8 UG/KG & 1 \\
\hline Heptachlor Epoxide & 8 UG/KG & $u$ & NP & 8 UG/KG & 1 \\
\hline Endosulfan I & 8 UG/KG & $u$ & NP & $8 \mathrm{UG} / K G$ & 1 \\
\hline Dieldrin & 16 UG/KG & $u$ & NP & 16 UG/KG & 1 \\
\hline 4,4'-DDE & 16 UG/KG & $u$ & NP & 16 UG/KG & 1 \\
\hline Endrin & 16 UG/KG & $u$ & NP & 16 UG/KG & 1 \\
\hline Endosulfan II & 16 UG/KG & $u$ & NP & 16 UG/KG & 1 \\
\hline 4,4'-DDD & $16 \mathrm{UG} / \mathrm{KG}$ & $u$ & NP & 16 UG/KG & 1 \\
\hline Endosulfan Sulfate & 16 UG/KG & $u$ & $N P$ & 16 UG/KG & 1 \\
\hline 4,4'-DDT & 16 UG/KG & $u$ & NP & 16 UG/KG & 1 \\
\hline Methoxychlor & 80 UG/KG & $u$ & NP & 80 UG/KG & 1 \\
\hline Endrin Ketone & 16 UG/KG & $u$ & NP & 16 UG/KG & 1 \\
\hline alpha-Chlordane & 80 UG/KG & $u$ & NP & 80 UG/KG & 1 \\
\hline gamma-Chlordane & 80 UG/KG & $u$ & NP & 80 UG/KG & 1 \\
\hline Toxaphene & $160 \mathrm{UG} / \mathrm{KG}$ & $u$ & NP & 160 UG/KG & 1 \\
\hline Aroclor-1016 & 80 UG/KG & $u$ & NP & 80 UG/KG & 1 \\
\hline Aroclor-1221 & 80 UG/KG & $u$ & NP & 80 UG/KG & 1 \\
\hline Aroclor-1232 & 80 UG/KG & $u$ & NP & 80 UG/KG & 1 \\
\hline Aroclor-1242 & 80 UG/KG & $u$ & NP & 80 UG/KG & 1 \\
\hline Aroclor-1248 & 80 UG/KG & $u$ & NP & 80 UG/KG & 1 \\
\hline Aroclor-1254 & 160 UG/KG & $u$ & NP & 160 UG/KG & 1 \\
\hline Aroclor-1260 & 160 UG/KG & $u$ & NP & 160 UG/KG & 1 \\
\hline DBC & $88 \%$ & & & $\%$ & 1 \\
\hline
\end{tabular}


APPENDIX E.1 Results of Pesticide and PCB Anaylses, Soils, Salmon Site

\begin{tabular}{|c|c|c|c|c|c|}
\hline COMPOUND & RESULTS UNITS & $\begin{array}{l}\text { DATA } \\
\text { QUAL. }\end{array}$ & $\begin{array}{l}\text { DATA } \\
\text { VALID. }\end{array}$ & CRDL UNITS & DILUTION \\
\hline \multicolumn{6}{|l|}{ C3-9-S } \\
\hline alpha-BHC & $8 \mathrm{UG} / \mathrm{KG}$ & $u$ & NP & 8 UG/KG & 1 \\
\hline beta-BHC & 8 UG/KG & $u$ & NP & 8 UG/KG & 1 \\
\hline delta-BHC & 8 UG/KG & $u$ & NP & 8 UG/KG & 1 \\
\hline gamma-BHC (Lindane) & 8 UG/KG & $u$ & NP & $8 \mathrm{UG} / K G$ & 1 \\
\hline Heptachlor & $8 \mathrm{UG} / \mathrm{KG}$ & $u$ & NP & 8 UG/KG & 1 \\
\hline Aldrin & 8 UG/KG & $u$ & NP & $8 \mathrm{UG} / \mathrm{KG}$ & 1 \\
\hline Heptachlor Epoxide & 8 UG/KG & $\mathrm{u}$ & NP & $8 \mathrm{UG} / \mathrm{KG}$ & 1 \\
\hline Endosulfan I & $8 \mathrm{UG} / \mathrm{KG}$ & $u$ & NP & 8 UG/KG & 1 \\
\hline Dieldrin & $16 \mathrm{UG} / \mathrm{KG}$ & $u$ & NP & 16 UG/KG & 1 \\
\hline 4,4'-DDE & $16 \mathrm{UG} / \mathrm{KG}$ & $u$ & NP & 16 UG/KG & 1 \\
\hline Endrin & 16 UG/KG & $u$ & NP & 16 UG/KG & 1 \\
\hline Endosulfan II & 16 UG/KG & $u$ & NP & 16 UG/KG & 1 \\
\hline $4,4^{\prime}-\mathrm{DDD}$ & 16 UG/KG & $u$ & NP & 16 UG/KG & 1 \\
\hline Endosulfan Sulfate & 16 UG/KG & $u$ & NP & 16 UG/KG & 1 \\
\hline 4,4'-DDT & 16 UG/KG & $u$ & NP & 16 UG/KG & 1 \\
\hline Methoxychlor & 80 UG/KG & $u$ & NP & 80 UG/KG & 1 \\
\hline Endrin Ketone & 16 UG/KG & $u$ & NP & 16 UG/KG & 1 \\
\hline alpha-Chlordane & 80 UG/KG & $u$ & NP & 80 UG/KG & 1 \\
\hline gamma-Chlordane & 80 UG/KG & $u$ & NP & 80 UG/KG & 1 \\
\hline Toxaphene & 160 UG/KG & $u$ & NP & 160 UG/KG & 1 \\
\hline Aroclor-1016 & 80 UG/KG & $u$ & $N P$ & 80 UG/KG & 1 \\
\hline Aroclor-1221 & 80 UG/KG & $u$ & NP & 80 UG/KG & 1 \\
\hline Aroclor-1232 & 80 UG/KG & $u$ & NP & 80 UG/KG & 1 \\
\hline Aroclor-1242 & 80 UG/KG & $u$ & NP & 80 UG/KG & 1 \\
\hline Aroclor-1248 & 80 UG/KG & $u$ & NP & 80 UG/KG & 1 \\
\hline Aroclor-1254 & 160 UG/KG & $u$ & NP & 160 UG/KG & 1 \\
\hline Arocior-1260 & 160 UG/KG & $u$ & NP & 160 UG/KG & 1 \\
\hline DBC & $76 \%$ & & & $\%$ & 1 \\
\hline
\end{tabular}


APPENDIX E.1 Results of Pesticide and PCB Anaylses, Soils, Salmon Site

\begin{tabular}{|c|c|c|c|c|c|}
\hline COMPOUND & RESULTS UNITS & $\begin{array}{l}\text { DATA } \\
\text { QUAL. }\end{array}$ & $\begin{array}{l}\text { DATA } \\
\text { VALID. }\end{array}$ & CRDL UNITS & DILUTION \\
\hline \multicolumn{6}{|l|}{ C4-10-S } \\
\hline alpha-BHC & 8 UG/KG & $u$ & NP & 8 UG/KG & 1 \\
\hline beta-BHC & $8 \mathrm{UG} / \mathrm{KG}$ & $u$ & NP & 8 UG/KG & 1 \\
\hline delta-BHC & 8 UG/KG & $u$ & NP & 8 UG/KG & 1 \\
\hline gamma-BHC (Lindane) & 8 UG/KG & $u$ & NP & 8 UG/KG & 1 \\
\hline Heptachlor & 8 UG/KG & $u$ & NP & 8 UG/KG & 1 \\
\hline Aldrin & 8 UG/KG & $u$ & NP & 8 UG/KG & 1 \\
\hline Heptachlor Epoxide & 8 UG/KG & $u$ & NP & 8 UG/KG & 1 \\
\hline Endosulfan I & $8 \mathrm{UG} / \mathrm{KG}$ & $u$ & NP & 8 UG/KG & 1 \\
\hline Dieldrin & 16 UG/KG & $u$ & NP & 16 UG/KG & 1 \\
\hline 4,4'-DDE & 16 UG/KG & $u$ & NP & $16 \mathrm{UG} / \mathrm{KG}$ & 1 \\
\hline Endrin & 16 UG/KG & $u$ & NP & 16 UG/KG & 1 \\
\hline Endosulfan II & $16 \mathrm{UG} / \mathrm{KG}$ & $u$ & NP & 16 UG/KG & 1 \\
\hline 4,4'-DDD & 16 UG/KG & $u$ & NP & 16 UG/KG & 1 \\
\hline Endosulfan Sulfate & 16 UG/KG & $u$ & NP & 16 UG/KG & 1 \\
\hline 4,4'-DDT & $16 \mathrm{UG} / \mathrm{KG}$ & $u$ & NP & 16 UG/KG & 1 \\
\hline Methoxychlor & 80 UG/KG & $u$ & NP & 80 UG/KG & 1 \\
\hline Endrin Ketone & 16 UG/KG & $u$ & NP & 16 UG/KG & $\cdot 1$ \\
\hline alpha-Chlordane & 80 UG/KG & $u$ & NP & 80 UG/KG & 1 \\
\hline gamma-Chlordane & 80 UG/KG & $u$ & NP & 80 UG/KG & 1 \\
\hline Toxaphene & 160 UG/KG & $u$ & NP & 160 UG/KG & 1 \\
\hline Aroclor-1016 & 80 UG/KG & $u$ & NP & $80 . \mathrm{UG} / \mathrm{KG}$ & 1 \\
\hline Aroclor-1221 & 80 UG/KG & $u$ & NP & 80 UG/KG & 1 \\
\hline Aroclor-1232 & 80 UG/KG & $u$ & NP & 80 UG/KG & 1 \\
\hline Aroclor-1242 & 80 UG/KG & $u$ & NP & 80 UG/KG & 1 \\
\hline Aroclor-1248 & 80 UG/KG & $u$ & NP & 80 UG/KG & 1 \\
\hline Aroclor-1254 & 160 UG/KG & $u$ & NP & 160 UG/KG & 1 \\
\hline Aroclor-1260 & 160 UG/KG & $u$ & NP & $160 \mathrm{UG} / \mathrm{KG}$ & 1 \\
\hline DBC & $81 \%$ & & & $\%$ & 1 \\
\hline
\end{tabular}


APPENDIX E.1 Results of Pesticide and PCB Anaylses, Soils, Salmon Site

\begin{tabular}{|c|c|c|c|c|c|}
\hline COMPOUND & RESULTS UNITS & $\begin{array}{l}\text { DATA } \\
\text { QUAL. }\end{array}$ & $\begin{array}{l}\text { DATA } \\
\text { VALID. }\end{array}$ & CRDL UNITS & DILUTION \\
\hline \multicolumn{6}{|l|}{ C4-11-S } \\
\hline alpha-BHC & 8 UG/KG & $u$ & NP & 8 UG/KG & 1 \\
\hline beta-BHC & $8 \mathrm{UG} / \mathrm{KG}$ & $u$ & NP & $8 \mathrm{UG} / \mathrm{KG}$ & 1 \\
\hline delta-BHC & 8 UG/KG & $u$ & NP & $8 \mathrm{UG} / \mathrm{KG}$ & 1 \\
\hline gamma-BHC (Lindane) & 8 UG/KG & $u$ & NP & $8 \mathrm{UG} / \mathrm{KG}$ & 1 \\
\hline Heptachlor & $8 \mathrm{UG} / \mathrm{KG}$ & $u$ & NP & $8 \mathrm{UG} / K G$ & 1 \\
\hline Aldrin & $8 \mathrm{UG} / K G$ & $u$ & NP & 8 UG/KG & 1 \\
\hline Heptachlor Epoxide & $8 \mathrm{UG} / \mathrm{KG}$ & $u$ & NP & $8 \mathrm{UG} / \mathrm{KG}$ & 1 \\
\hline Endosulfan I & $8 \mathrm{UG} / \mathrm{KG}$ & $u$ & NP & 8 UG/KG & 1 \\
\hline Dieldrin & 16 UG/KG & $u$ & nP & 16 UG/KG & 1 \\
\hline 4,4'-DDE & 16 UG/KG & $u$ & NP & 16 UG/KG & 1 \\
\hline Endrin & 16 UG/KG & $u$ & NP & 16 UG/KG & 1 \\
\hline Endosulfan \| & 16 UG/KG & $u$ & NP & 16 UG/KG & 1 \\
\hline 4, $4^{\prime}-D D D$ & 16 UG/KG & $u$ & NP & 16 UG/KG & 1 \\
\hline Endosulfan Sulfate & 16 UG/KG & $u$ & NP & 16 UG/KG & 1 \\
\hline 4,4'-DDT & 16 UG/KG & $u$ & NP & 16 UG/KG & 1 \\
\hline Methoxychior & 80 UG/KG & $u$ & NP & 80 UG/KG & 1 \\
\hline Endrin Ketone & 16 UG/KG & $u$ & NP & 16 UG/KG & 1 \\
\hline alpha-Chlordane & 80 UG/KG & $u$ & NP & 80 UG/KG & 1 \\
\hline gamma-Chlordane & 80 UG/KG & $u$ & NP & 80 UG/KG & 1 \\
\hline Toxaphene & 160 UG/KG & $u$ & NP & 160 UG/KG & 1 \\
\hline Aroclor-1016 & 80 UG/KG & $u$ & NP & 80 UG/KG & 1 \\
\hline Aroclor-1221 & 80 UG/KG & $u$ & NP & 80 UG/KG & 1 \\
\hline Aroclor-1232 & $80 \mathrm{UG} / \mathrm{KG}$ & $u$ & NP & 80 UG/KG & 1 \\
\hline Aroclor-1242 & 80 UG/KG & $u$ & NP & 80 UG/KG & 1 \\
\hline Aroclor-1248 & 80 UG/KG & $u$ & NP & 80 UG/KG & 1 \\
\hline Aroclor-1254 & 160 UG/KG & $u$ & NP & 160 UG/KG & 1 \\
\hline Aroclor-1260 & 160 UG/KG & $u$ & NP & 160 UG/KG & 1 \\
\hline $\mathrm{DBC}$ & $85 \%$ & & & $\%$ & 1 \\
\hline
\end{tabular}


APPENDIX E.1 Results of Pesticide and PCB Anaylses, Soils, Salmon Site

\begin{tabular}{|c|c|c|c|c|c|}
\hline COMPOUND & RESULTS UNITS & $\begin{array}{l}\text { DATA } \\
\text { QUAL. }\end{array}$ & $\begin{array}{l}\text { DATA } \\
\text { VALID. }\end{array}$ & CRDL UNITS & DILUTION \\
\hline \multicolumn{6}{|l|}{ C4-12-S } \\
\hline alpha-BHC & 8 UG/KG & $u$ & NP & 8 UG/KG & 1 \\
\hline beta-BHC & 8 UG/KG & $u$ & NP & 8 UG/KG & 1 \\
\hline delta-BHC & 8 UG/KG & $u$ & NP & $8 \mathrm{UG} / \mathrm{KG}$ & 1 \\
\hline gamma-BHC (Lindane) & 8 UG/KG & $u$ & NP & 8 UG/KG & 1 \\
\hline Heptachlor & $8 \mathrm{UG} / \mathrm{KG}$ & $u$ & NP & 8 UG/KG & 1 \\
\hline Aldrin & 8 UG/KG & $u$ & NP & 8 UG/KG & 1 \\
\hline Heptachlor Epoxide & 8 UG/KG & $u$ & NP & 8 UG/KG & 1 \\
\hline Endosulfan I & 8 UG/KG & $u$ & NP & $8 \mathrm{UG} / K G$ & 1 \\
\hline Dieldrin & 16 UG/KG & $u$ & NP & 16 UG/KG & 1 \\
\hline 4,4'-DDE & 16 UG/KG & $u$ & NP & 16 UG/KG & 1 \\
\hline Endrin & 16 UG/KG & $u$ & NP & 16 UG/KG & 1 \\
\hline Endosulfan II & 16 UGKG & $u$ & NP & 16 UG/KG & 1 \\
\hline $4,4^{\prime}-\mathrm{DDD}$ & 16 UG/KG & $u$ & NP & 16 UG/KG & 1 \\
\hline Endosulfan Sulfate & 16 UG/KG & $u$ & NP & 16 UG/KG & 1 \\
\hline 4,4'-DDT & 16 UG/KG & $u$ & NP & 16 UG/KG & 1 \\
\hline Methoxychlor & 80 UG/KG & $u$ & NP & 80 UG/KG & 1 \\
\hline Endrin Ketone & 16 UG/KG & $u$ & NP & 16 UG/KG & 1 \\
\hline alpha-Chlordane & 80 UG/KG & $u$ & NP & 80 UG/KG & 1 \\
\hline gamma-Chlordane & 80 UG/KG & $u$ & NP & 80 UG/KG & 1 \\
\hline Toxaphene & 160 UG/KG & $u$ & NP & 160 UG/KG & 1 \\
\hline Aroclor-1016 & 80 UG/KG & $u$ & NP & 80 UG/KG & 1 \\
\hline Aroclor-1221 & 80 UG/KG & $u$ & NP & 80 UG/KG & 1 \\
\hline Aroclor-1232 & 80 UG/KG & $u$ & NP & 80 UG/KG & 1 \\
\hline Aroclor-1242 & 80 UG/KG & $u$ & NP & 80 UG/KG & 1 \\
\hline Aroclor-1248 & 80 UG/KG & $u$ & NP & 80 UG/KG & 1 \\
\hline Aroclor-1254 & 160 UG/KG & $u$ & NP & 160 UG/KG & 1 \\
\hline Aroclor -1260 & 160 UG/KG & $u$ & NP & 160 UG/KG & 1 \\
\hline DBC & $86 \%$ & & & $\%$ & 1 \\
\hline
\end{tabular}


APPENDIX E.1 Results of Pesticide and PCB Anaylses, Soils, Salmon Site

\begin{tabular}{|c|c|c|c|c|c|}
\hline COMPOUND & RESULTS UNITS & $\begin{array}{l}\text { DATA } \\
\text { QUAL. }\end{array}$ & $\begin{array}{l}\text { DATA } \\
\text { VALID. }\end{array}$ & CRDL UNITS & DILUTION \\
\hline \multicolumn{6}{|l|}{ C4-13-S } \\
\hline alpha-BHC & 8 UG/KG & $u$ & NP & 8 UG/KG & 1 \\
\hline beta-BHC & 8 UG/KG & $u$ & NP & $8 \mathrm{UG} / \mathrm{KG}$ & 1 \\
\hline delta-BHC & 8 UG/KG & $u$ & NP & 8 UG/KG & 1 \\
\hline gamma-BHC (Lindane) & $8 \mathrm{UG} / \mathrm{KG}$ & $u$ & NP & 8 UG/KG & 1 \\
\hline Heptachlor & $8 \mathrm{UG} / \mathrm{KG}$ & $u$ & NP & 8 UG/KG & 1 \\
\hline Aldrin & 8 UG/KG & $u$ & NP & $8 \mathrm{UG} / \mathrm{KG}$ & 1 \\
\hline Heptachlor Epoxide & $8 \mathrm{UG} / \mathrm{KG}$ & $u$ & $\mathrm{NP}$ & $8 \mathrm{UG} / \mathrm{KG}$ & 1 \\
\hline Endosulfan I & 8 UG/KG & $u$ & NP & $8 \mathrm{UG} / \mathrm{KG}$ & 1 \\
\hline Dieldrin & 16 UG/KG & $u$ & NP & 16 UG/KG & 1 \\
\hline 4,4'-DDE & 16 UG/KG & $u$ & NP & 16 UG/KG & 1 \\
\hline Endrin & 16 UG/KG & $u$ & NP & 16 UG/KG & 1 \\
\hline Endosulfan II & 16 UG/KG & $u$ & NP & $16 U G / K G$ & 1 \\
\hline $4,4^{\prime}-\mathrm{DDD}$ & 16 UG/KG & u & NP & 16 UG/KG & 1 \\
\hline Endosulfan Sulfate & 16 UG/KG & $u$ & NP & 16 UG/KG & 1 \\
\hline $4,4^{\prime}-\mathrm{DDT}$ & 16 UG/KG & $u$ & NP & 16 UG/KG & 1 \\
\hline Methoxychlor & 80 UG/KG & $u$ & NP & 80 UG/KG & 1 \\
\hline Endrin Ketone & 16 UG/KG & $u$ & NP & $16 \mathrm{UG} / \mathrm{KG}$ & 1 \\
\hline alpha-Chlordane & 80 UG/KG & $u$ & NP & 80 UG/KG & 1 \\
\hline gamma-Chlordane & 80 UG/KG & $u$ & NP & 80 UG/KG & 1 \\
\hline Toxaphene & 160 UG/KG & $u$ & NP & 160 UG/KG & 1 \\
\hline Aroclor-1016 & 80 UG/KG & $u$ & NP & 80 UG/KG & 1 \\
\hline Aroclor-1221 & 80 UG/KG & $u$ & NP & 80 UG/KG & 1 \\
\hline Aroclor-1232 & 80 UG/KG & $u$ & NP & 80 UG/KG & 1 \\
\hline Aroclor-1242 & 80 UG/KG & $u$ & NP & 80 UG/KG & 1 \\
\hline Aroclor-1248 & 80 UG/KG & $u$ & NP & 80 UG/KG & 1 \\
\hline Aroclor-1254 & 160 UG/KG & $u$ & NP & 160 UG/KG & 1 \\
\hline Aroclor-1260 & 160 UG/KG & $u$ & NP & 160 UG/KG & 1 \\
\hline $\mathrm{DBC}$ & $89 \%$ & & & $\%$ & 1 \\
\hline
\end{tabular}




\section{APPENDIX E.1 Results of Pesticide and PCB Anaylses, Soils, Salmon Site}

\begin{tabular}{|c|c|c|c|c|c|}
\hline COMPOUND & RESULTS UNITS & $\begin{array}{l}\text { DATA } \\
\text { QUAL. }\end{array}$ & $\begin{array}{l}\text { DATA } \\
\text { VALID. }\end{array}$ & CRDL UNITS & DILUTION \\
\hline \multicolumn{6}{|l|}{ C1-14-S } \\
\hline alpha-BHC & $8 \mathrm{UG} / \mathrm{KG}$ & $u$ & NP & 8 UG/KG & 1 \\
\hline beta-BHC & 8 UG/KG & $u$ & NP & 8 UG/KG & 1 \\
\hline delta-BHC & $8 \mathrm{UG} / \mathrm{KG}$ & $u$ & NP & $8 \mathrm{UG} / K G$ & 1 \\
\hline gamma-BHC (Lindane) & 8 UG/KG & $u$ & NP & 8 UG/KG & 1 \\
\hline Heptachlor & 8 UG/KG & $u$ & NP & 8 UG/KG & 1 \\
\hline Aldrin & $8 \mathrm{UG} / \mathrm{KG}$ & u & NP & $8 \mathrm{UG} / K G$ & 1 \\
\hline Heptachlor Epoxide & 8 UG/KG & $u$ & NP & $8 \mathrm{UG} / K \mathrm{~K}$ & 1 \\
\hline Endosulfan I & 8 UG/KG & $u$ & NP & 8 UG/KG & 1 \\
\hline Dieldrin & 16 UG/KG & $u$ & NP & 16 UG/KG & 1 \\
\hline 4,4'-DDE & 16 UG/KG & $u$ & NP & 16 UG/KG & 1 \\
\hline Endrin & 16 UG/KG & $u$ & NP & 16 UG/KG & 1 \\
\hline Endosulfan II & 16 UG/KG & $u$ & NP & 16 UG/KG & 1 \\
\hline 4,4'-DDD & 16 UG/KG & $u$ & NP & 16 UG/KG & 1 \\
\hline Endosulfan Sulfate & $16 \mathrm{UG} / \mathrm{KG}$ & $u$ & NP & 16 UG/KG & 1 \\
\hline 4,4'-DDT & 16 UG/KG & $u$ & NP & 16 UG/KG & 1 \\
\hline Methoxychlor & 80 UG/KG & $u$ & NP & 80 UG/KG & 1 \\
\hline Endrin Ketone & 16 UG/KG & $u$ & NP & 16 UG/KG & 1 \\
\hline alpha-Chlordane & 80 UG/KG & $u$ & NP & 80 UG/KG & 1 \\
\hline gamma-Chlordane & 80 UG/KG & $u$ & NP & 80 UG/KG & 1 \\
\hline Toxaphene & 160 UG/KG & $u$ & NP & 160 UG/KG & 1 \\
\hline Aroclor-1016 & 80 UG/KG & $u$ & NP & 80 UG/KG & 1 \\
\hline Aroclor-1221 & 80 UG/KG & $u$ & NP & 80 UG/KG & 1 \\
\hline Aroclor-1232 & 80 UG/KG & $u$ & NP & 80 UG/KG & 1 \\
\hline Aroclor-1242 & 80 UG/KG & $u$ & NP & 80 UG/KG & 1 \\
\hline Aroclor-1248 & 80 UG/KG & $u$ & NP & 80 UG/KG & 1 \\
\hline Aroclor-1254 & 160 UG/KG & $u$ & NP & 160 UG/KG & 1 \\
\hline Aroclor-1260 & $160 \mathrm{UG} / \mathrm{KG}$ & $u$ & NP & 160 UG/KG & 1 \\
\hline DBC & $82 \%$ & & & $\%$ & 1 \\
\hline
\end{tabular}


APPENDIX E.1 Results of Pesticide and PCB Anaylses, Soils, Salmon Site

\begin{tabular}{|c|c|c|c|c|c|}
\hline COMPOUND & RESULTS UNITS & $\begin{array}{l}\text { DATA } \\
\text { QUAL. }\end{array}$ & $\begin{array}{l}\text { DATA } \\
\text { VALID. }\end{array}$ & CRDL UNITS & DILUTION \\
\hline \multicolumn{6}{|l|}{ C1-15-S } \\
\hline alpha-BHC & 7.9 UG/KG & $u$ & NP & 7.9 UG/KG & 1 \\
\hline beta-BHC & 7.9 UG/KG & $u$ & NP & 7.9 UG/KG & 1 \\
\hline delta-BHC & $7.9 \mathrm{UG} / \mathrm{KG}$ & $u$ & NP & 7.9 UG/KG & 1 \\
\hline gamma-BHC (Lindane) & 7.9 UG/KG & $u$ & NP & $7.9 \mathrm{UG} / \mathrm{KG}$ & 1 \\
\hline Heptachlor & 7.9 UG/KG & $u$ & NP & 7.9 UG/KG & 1 \\
\hline Aldrin & 7.9 UG/KG & $u$ & NP & 7.9 UG/KG & 1 \\
\hline Heptachlor Epoxide & 7.9 UG/KG & $u$ & NP & 7.9 UG/KG & 1 \\
\hline Endosulfan I & 7.9 UG/KG & $u$ & NP & 7.9 UG/KG & 1 \\
\hline Dieldrin & 16 UG/KG & $u$ & NP & 16 UG/KG & 1 \\
\hline $4,4^{\prime}-\mathrm{DDE}$ & 16 UG/KG & $u$ & NP & 16 UG/KG & 1 \\
\hline Endrin & $16 \mathrm{UG} / \mathrm{KG}$ & $u$ & NP & 16 UG/KG & 1 \\
\hline Endosulfan II & 16 UG/KG & $u$ & NP & $16 \mathrm{UG} / \mathrm{KG}$ & 1 \\
\hline $4,4^{\prime}-\mathrm{DDD}$ & 16 UG/KG & $u$ & NP & 16 UG/KG & 1 \\
\hline Endosulfan Sulfate & 16 UG/KG & $u$ & NP & 16 UG/KG & 1 \\
\hline 4,4'-DDT & 16 UG/KG & $u$ & NP & 16 UG/KG & 1 \\
\hline Methoxychlor & 79 UG/KG & $u$ & NP & 79 UG/KG & 1 \\
\hline Endrin Ketone & 16 UG/KG & $u$ & NP & 16 UG/KG & 1 \\
\hline alpha-Chlordane & 79 UG/KG & $u$ & NP & 79 UG/KG & 1 \\
\hline gamma-Chlordane & 79 UG/KG & $u$ & NP & 79 UG/KG & 1 \\
\hline Toxaphene & 160 UG/KG & $u$ & NP & 160 UG/KG & 1 \\
\hline Aroclor-1016 & 79 UG/KG & $u$ & NP & 79 UG/KG & 1 \\
\hline Aroclor-1221 & 79 UG/KG & $u$ & NP & 79 UG/KG & 1 \\
\hline Aroclor-1232 & 79 UG/KG & $u$ & NP & 79 UG/KG & 1 \\
\hline Aroclor-1242 & 79 UG/KG & $u$ & NP & 79 UG/KG & 1 \\
\hline Arocior-1248 & 79 UG/KG & $u$ & NP & 79 UG/KG & 1 \\
\hline Aroclor-1254 & $160 \mathrm{UG} / \mathrm{KG}$ & $u$ & NP & 160 UG/KG & 1 \\
\hline Aroclor-1260 & 160 UG/KG & $u$ & & 160 UG/KG & 1 \\
\hline $\mathrm{DBC}$ & $89 \%$ & & & $\%$ & 1 \\
\hline
\end{tabular}




\section{APPENDIX E.1 Results of Pesticide and PCB Anaylses, Soils, Salmon Site}

\begin{tabular}{|c|c|c|c|c|c|}
\hline COMPOUND & RESULTS UNITS & $\begin{array}{l}\text { DATA } \\
\text { QUAL. }\end{array}$ & $\begin{array}{l}\text { DATA } \\
\text { VALID. }\end{array}$ & CRDL UNITS & DILUTION \\
\hline \multicolumn{6}{|l|}{ C1-16-S } \\
\hline alpha-BHC & 8 UG/KG & $u$ & NP & 8 UG/KG & 1 \\
\hline beta-BHC & $8 \mathrm{UG} / K G$ & $\mathrm{u}$ & NP & 8 UG/KG & 1 \\
\hline delta-BHC & 8 UG/KG & $u$ & NP & 8 UG/KG & 1 \\
\hline gamma-BHC (Lindane) & $8 \mathrm{UG} / \mathrm{KG}$ & $u$ & NP & 8 UG/KG & 1 \\
\hline Heptachlor & 8 UG/KG & $u$ & NP & 8 UG/KG & 1 \\
\hline Aldrin & $8 \mathrm{UG} / K G$ & $u$ & NP & 8 UG/KG & 1 \\
\hline Heptachlor Epoxide & 8 UG/KG & $u$ & NP & 8 UG/KG & 1 \\
\hline Endosulfan I & 8 UG/KG & $u$ & NP & 8 UG/KG & 1 \\
\hline Dieldrin & 16 UG/KG & $\mathrm{u}$ & NP & 16 UG/KG & 1 \\
\hline 4,4'-DDE & 16 UG/KG & $u$ & NP & 16 UG/KG & 1 \\
\hline Endrin & 16 UG/KG & $u$ & NP & 16 UG/KG & 1 \\
\hline Endosulfan II & 16 UG/KG & $u$ & NP & 16 UG/KG & 1 \\
\hline $4,4^{\prime}-\mathrm{DDD}$ & 16 UG/KG & $u$ & NP & 16 UG/KG & 1 \\
\hline Endosulfan Sulfate & 16 UG/KG & $u$ & NP & 16 UG/KG & 1 \\
\hline 4,4'-DDT & 16 UG/KG & $u$ & NP & 16 UG/KG & 1 \\
\hline Methoxychlor & 80 UG/KG & $u$ & NP & 80 UG/KG & 1 \\
\hline Endrin Ketone & 16 UG/KG & $u$ & NP & 16 UG/KG & 1 \\
\hline alpha-Chlordane & 80 UG/KG & $u$ & NP & 80 UG/KG & 1 \\
\hline gamma-Chlordane & 80 UG/KG & $u$ & NP & 80 UG/KG & 1 \\
\hline Toxaphene & 160 UG/KG & $u$ & NP & 160 UG/KG & 1 \\
\hline Aroclor-1016 & 80 UG/KG & $u$ & NP & 80 UG/KG & 1 \\
\hline Aroclor-1221 & 80 UG/KG & $u$ & NP & 80 UG/KG & 1 \\
\hline Aroclor-1232 & 80 UG/KG & $u$ & NP & 80 UG/KG & 1 \\
\hline Aroclor-1242 & 80 UG/KG & $u$ & NP & 80 UG/KG & 1 \\
\hline Aroclor-1248 & 80 UG/KG & $u$ & NP & 80 UG/KG & 1 \\
\hline Aroclor-1254 & 160 UG/KG & $u$ & NP & 160 UG/KG & 1 \\
\hline Aroclor-1260 & 160 UG/KG & $u$ & NP & 160 UG/KG & 1 \\
\hline$D B C$ & $88 \%$ & & & $\%$ & 1 \\
\hline
\end{tabular}


APPENDIX E.1 Results of Pesticide and PCB Anaylses, Soils, Salmon Site

\begin{tabular}{|c|c|c|c|c|c|}
\hline COMPOUND & RESULTS UNITS & $\begin{array}{l}\text { DATA } \\
\text { QUAL. }\end{array}$ & $\begin{array}{l}\text { DATA } \\
\text { VALID. }\end{array}$ & CRDL UNITS & DILUTION \\
\hline \multicolumn{6}{|l|}{ C1-17-S } \\
\hline alpha-BHC & $8 \mathrm{UG} / \mathrm{KG}$ & $u$ & NP & 8 UG/KG & 1 \\
\hline beta-BHC & 8 UG/KG & $u$ & NP & 8 UG/KG & 1 \\
\hline delta-BHC & 8 UG/KG & $u$ & NP & 8 UG/KG & 1 \\
\hline gamma-BHC (Lindane) & $8 \mathrm{UG} / \mathrm{KG}$ & $u$ & NP & $8 \mathrm{UG} / \mathrm{KG}$ & 1 \\
\hline Heptachlor & 8 UG/KG & $u$ & NP & 8 UG/KG & 1 \\
\hline Aldrin & $8 \mathrm{UG} / \mathrm{KG}$ & $u$ & NP & 8 UG/KG & 1 \\
\hline Heptachlor Epoxide & 8 UG/KG & $u$ & NP & 8 UG/KG & 1 \\
\hline Endosulfan I & 8 UG/KG & $u$ & NP & $8 \mathrm{UG} / \mathrm{KG}$ & 1 \\
\hline Dieldrin & 16 UG/KG & $u$ & NP & 16 UG/KG & 1 \\
\hline 4,4'-DDE & 16 UG/KG & $u$ & NP & 16 UG/KG & 1 \\
\hline Endrin & 16 UG/KG & $u$ & NP & 16 UG/KG & 1 \\
\hline Endosulfan $\|$ & 16 UG/KG & $u$ & NP & 16 UG/KG & 1 \\
\hline $4,4^{\prime}-D D D$ & 16 UG/KG & $u$ & NP & 16 UG/KG & 1 \\
\hline Endosulfan Sulfate & 16 UG/KG & $u$ & NP & 16 UG/KG & 1 \\
\hline 4,4'-DDT & 16 UG/KG & $u$ & NP & 16 UG/KG & 1 \\
\hline Methoxychlor & 80 UG/KG & $u$ & NP & 80 UG/KG & 1 \\
\hline Endrin Ketone & 16 UG/KG & $u$ & NP & 16 UG/KG & 1 \\
\hline alpha-Chlordane & 80 UG/KG & $u$ & NP & 80 UG/KG & 1 \\
\hline gamma-Chlordane & 80 UG/KG & $u$ & NP & 80 UG/KG & 1 \\
\hline Toxaphene & 160 UG/KG & $u$ & NP & 160 UG/KG & 1 \\
\hline Aroclor-1016 & 80 UG/KG & $u$ & NP & 80 UG/KG & 1 \\
\hline Aroclor-1221 & 80 UG/KG & $u$ & NP & 80 UG/KG & 1 \\
\hline Aroclor-1232 & 80 UG/KG & $u$ & NP & 80 UG/KG & 1 \\
\hline Aroclor-1242 & 80 UG/KG & $u$ & NP & 80 UG/KG & 1 \\
\hline Aroclor-1248 & 80 UG/KG & $u$ & NP & 80 UG/KG & 1 \\
\hline Aroclor-1254 & 160 UG/KG & $u$ & NP & 160 UG/KG & 1 \\
\hline Arocior-1260 & 160 UG/KG & $u$ & NP & 160 UG/KG & 1 \\
\hline DBC & $86 \%$ & & & $\%$ & 1 \\
\hline
\end{tabular}


APPENDIX E.1 Results of Pesticide and PCB Anaylses, Soils, Salmon Site

\begin{tabular}{lcccc|}
\hline COMPOUND & RESULTS UNITS & $\begin{array}{c}\text { DATA } \\
\text { QUAL. }\end{array}$ & DATA & \\
VALID. & CRDL UNITS & DILUTION \\
\hline C1-17-S-MS & & & & \\
gamma-BHC (Lindane) & $113 \%$ & NP & $\%$ & 1 \\
Heptachlor & $114 \%$ & NP & $\%$ & 1 \\
Aldrin & $125 \%$ & NP & $\%$ & 1 \\
Dieldrin & $110 \%$ & NP & $\%$ & 1 \\
Endrin & $106 \%$ & NP & $\%$ & 1 \\
$4,4 '-D D T$ & $129 \%$ & NP & $\%$ & 1 \\
DBC & $92 \%$ & NP & $\%$ & 1 \\
& & & & \\
C1-17-S-MSD & & & & \\
gamma-BHC (Lindane) & $87 \%$ & NP & $\%$ & 1 \\
Heptachlor & $89 \%$ & NP & $\%$ & 1 \\
Aldrin & $97 \%$ & NP & $\%$ & 1 \\
Dieldrin & $86 \%$ & NP & $\%$ & 1 \\
Endrin & $84 \%$ & NP & $\%$ & 1 \\
4,4 -DDT & $100 \%$ & NP & $\%$ & 1 \\
DBC & $71 \%$ & NP & $\%$ & 1
\end{tabular}


APPENDIX E.1 Results of Pesticide and PCB Anaylses, Soils, Salmon Site

\begin{tabular}{|c|c|c|c|c|c|}
\hline COMPOUND & RESULTS UNITS & $\begin{array}{l}\text { DATA } \\
\text { QUAL. }\end{array}$ & $\begin{array}{l}\text { DATA } \\
\text { VALID. }\end{array}$ & CRDL UNITS & DILUTION \\
\hline \multicolumn{6}{|l|}{ C1-18-S } \\
\hline alpha-BHC & 8 UG/KG & $u$ & NP & $8 \mathrm{UG} / \mathrm{KG}$ & 1 \\
\hline beta-BHC & 8 UG/KG & $u$ & NP & 8 UG/KG & 1 \\
\hline delta-BHC & $8 \mathrm{UG} / \mathrm{KG}$ & $u$ & NP & 8 UG/KG & 1 \\
\hline gamma-BHC (Lindane) & 8 UG/KG & $u$ & NP & 8 UG/KG & 1 \\
\hline Heptachlor & 8 UG/KG & $u$ & NP & 8 UG/KG & 1 \\
\hline Aldrin & 8 UG/KG & $u$ & NP & 8 UG/KG & 1 \\
\hline Heptachlor Epoxide & 8 UG/KG & $u$ & NP & 8 UG/KG & 1 \\
\hline Endosulfan I & 8 UG/KG & $u$ & NP & 8 UG/KG & 1 \\
\hline Dieldrin & 16 UG/KG & $u$ & NP & 16 UG/KG & 1 \\
\hline 4,4'-DDE & 16 UG/KG & $u$ & NP & 16 UG/KG & 1 \\
\hline Endrin & 16 UG/KG & $u$ & NP & 16 UG/KG & 1 \\
\hline Endosulfan II & 16 UG/KG & $u$ & NP & 16 UGKG & 1 \\
\hline $4,4^{\prime}-\mathrm{DDD}$ & 16 UG/KG & $u$ & NP & 16 UG/KG & 1 \\
\hline Endosulfan Sulfate & 16 UG/KG & $u$ & NP & 16 UG/KG & 1 \\
\hline 4,4'-DDT & 16 UG/KG & $u$ & NP & 16 UG/KG & 1 \\
\hline Methoxychlor & 80 UG/KG & $u$ & NP & 80 UG/KG & 1 \\
\hline Endrin Ketone & 16 UG/KG & $u$ & NP & 16 UG/KG & 1 \\
\hline aipha-Chlordane & 80 UG/KG & $u$ & NP & 80 UG/KG & 1 \\
\hline gamma-Chlordane & 80 UG/KG & $u$ & NP & 80 UG/KG & 1 \\
\hline Toxaphene & $160 \mathrm{UG} / \mathrm{KG}$ & $u$ & NP & 160 UG/KG & 1 \\
\hline Aroclor-1016 & 80 UG/KG & $u$ & NP & 80 UG/KG & 1 \\
\hline Arocior-1221 & 80 UG/KG & $u$ & NP & 80 UG/KG & 1 \\
\hline Aroclor- 1232 & 80 UG/KG & $u$ & NP & 80 UG/KG & 1 \\
\hline Aroclor-1242 & 80 UG/KG & $u$ & NP & 80 UG/KG & 1 \\
\hline Aroclor-1248 & 80 UG/KG & $u$ & NP & 80 UG/KG & 1 \\
\hline Arocior-1254 & 160 UG/KG & $u$ & NP & 160 UG/KG & 1 \\
\hline Aroclor-1260 & $160 \mathrm{UG} / \mathrm{KG}$ & $u$ & NP & 160 UG/KG & 1 \\
\hline $\mathrm{DBC}$ & $86 \%$ & & & $\%$ & 1 \\
\hline
\end{tabular}


APPENDIX E.1 Results of Pesticide and PCB Anaylses, Soils, Salmon Site

\begin{tabular}{|c|c|c|c|c|c|}
\hline COMPOUND & RESULTS UNITS & $\begin{array}{l}\text { DATA } \\
\text { QUAL. }\end{array}$ & $\begin{array}{l}\text { DATA } \\
\text { VALID. }\end{array}$ & CRDL UNITS & DILUTION \\
\hline \multicolumn{6}{|l|}{ C2-19-S } \\
\hline alpha-BHC & 8 UG/KG & $u$ & NP & 8 UG/KG & 1 \\
\hline beta-BHC & 8 UG/KG & $u$ & NP & 8 UG/KG & 1 \\
\hline delta-BHC & 8 UG/KG & $u$ & NP & $8 \mathrm{UG} / \mathrm{KG}$ & 1 \\
\hline gamma-BHC (Lindane) & 8 UG/KG & $u$ & NP & 8 UG/KG & 1 \\
\hline Heptachlor & 8 UG/KG & $u$ & NP & 8 UG/KG & 1 \\
\hline Aldrin & $8 \mathrm{UG} / \mathrm{KG}$ & $u$ & NP & 8 UG/KG & 1 \\
\hline Heptachlor Epoxide & 8 UG/KG & $u$ & NP & $8 \mathrm{UG} / \mathrm{KG}$ & 1 \\
\hline Endosulfan l & $8 \mathrm{UG} / \mathrm{KG}$ & $u$ & NP & 8 UG/KG & 1 \\
\hline Dieldrin & 16 UG/KG & $u$ & NP & 16 UG/KG & 1 \\
\hline $4,4^{\prime}-\mathrm{DDE}$ & 16 UG/KG & $u$ & NP & 16 UG/KG & 1 \\
\hline Endrin & 16 UG/KG & $u$ & NP & 16 UG/KG & 1 \\
\hline Endosulfan II & 16 UG/KG & $u$ & NP & 16 UG/KG & 1 \\
\hline 4,4'-DDD & 16 UG/KG & $u$ & NP & 16 UG/KG & 1 \\
\hline Endosulfan Sulfate & 16 UG/KG & $u$ & NP & 16 UG/KG & 1 \\
\hline 4,4'-DDT & $16 \mathrm{UG} / \mathrm{KG}$ & $u$ & NP & 16 UG/KG & 1 \\
\hline Methoxychlor & 80 UG/KG & $u$ & NP & 80 UG/KG & 1 \\
\hline Endrin Ketone & 16 UG/KG & $u$ & NP & 16 UG/KG & 1 \\
\hline alpha-Chlordane & 80 UG/KG & $u$ & NP & 80 UG/KG & 1 \\
\hline gamma-Chlordane & 80 UG/KG & $u$ & NP & 80 UG/KG & 1 \\
\hline Toxaphene & 160 UG/KG & $u$ & NP & 160 UG/KG & 1 \\
\hline Aroclor-1016 & 80 UG/KG & $u$ & NP & 80 UG/KG & 1 \\
\hline Aroclor-1221 & 80 UG/KG & $u$ & NP & 80 UG/KG & 1 \\
\hline Aroclor-1232 & 80 UG/KG & $u$ & NP & 80 UG/KG & 1 \\
\hline Aroclor-1242 & 80 UG/KG & $u$ & NP & 80 UG/KG & 1 \\
\hline Aroclor-1248 & 80 UG/KG & $u$ & NP & 80 UG/KG & 1 \\
\hline Aroclor-1254 & 160 UG/KG & $u$ & NP & 160 UG/KG & 1 \\
\hline Aroclor-1260 & 160 UG/KG & $u$ & NP & 160 UG/KG & 1 \\
\hline DBC & $71 \%$ & & & $\%$ & 1 \\
\hline
\end{tabular}


APPENDIX E.1 Results of Pesticide and PCB Anaylses, Soils, Salmon Site

\begin{tabular}{|c|c|c|c|c|c|}
\hline COMPOUND & RESULTS UNITS & $\begin{array}{l}\text { DATA } \\
\text { QUAL. }\end{array}$ & $\begin{array}{l}\text { DATA } \\
\text { VALID. }\end{array}$ & CRDL UNITS & DILUTION \\
\hline \multicolumn{6}{|l|}{ C2-20-S } \\
\hline alpha-BHC & 8 UG/KG & $u$ & NP & $8 \mathrm{UG} / K G$ & 1 \\
\hline beta-BHC & 8 UG/KG & $u$ & NP & 8 UG/KG & 1 \\
\hline delta-BHC & 8 UG/KG & $u$ & NP & $8 \mathrm{UG} / \mathrm{KG}$ & 1 \\
\hline gamma-BHC (Lindane) & 8 UG/KG & $u$ & NP & 8 UG/KG & 1 \\
\hline Heptachlor & 8 UG/KG & $u$ & NP & 8 UG/KG & 1 \\
\hline Aldrin & 8 UG/KG & $u$ & NP & $8 \mathrm{UG} / K \mathrm{~K}$ & 1 \\
\hline Heptachlor Epoxide & $8 \mathrm{UG} / \mathrm{KG}$ & $u$ & NP & 8 UG/KG & 1 \\
\hline Endosulfan I & $8 \mathrm{UG} / K G$ & $u$ & NP & 8 UG/KG & 1 \\
\hline Dieldrin & 16 UG/KG & $u$ & NP & 16 UG/KG & 1 \\
\hline 4,4'-DDE & 16 UG/KG & $u$ & NP & 16 UG/KG & 1 \\
\hline Endrin & 16 UG/KG & $u$ & NP & 16 UG/KG & 1 \\
\hline Endosulfan II & 16 UG/KG & $u$ & NP & $16 \mathrm{UG} / \mathrm{KG}$ & 1 \\
\hline $4,4^{\prime}-\mathrm{DDD}$ & 16 UG/KG & $u$ & NP & 16 UG/KG & 1 \\
\hline Endosulfan Sulfate & 16 UG/KG & $u$ & NP & 16 UG/KG & 1 \\
\hline $4,4^{\prime}-\mathrm{DDT}$ & 16 UG/KG & $u$ & NP & 16 UG/KG & 1 \\
\hline Methoxychlor & 80 UG/KG & $u$ & NP & 80 UG/KG & 1 \\
\hline Endrin Ketone & 16 UG/KG & $u$ & NP & $16 \mathrm{UG} / \mathrm{KG}$ & 1 \\
\hline alpha-Chlordane & 80 UG/KG & $u$ & NP & 80 UG/KG & 1 \\
\hline gamma-Chlordane & 80 UG/KG & $u$ & NP & 80 UG/KG & 1 \\
\hline Toxaphene & 160 UG/KG & $u$ & NP & 160 UG/KG & 1 \\
\hline Aroclor-1016 & 80 UG/KG & $u$ & NP & 80 UG/KG & 1 \\
\hline Aroclor-1221 & 80 UG/KG & $u$ & NP & 80 UG/KG & 1 \\
\hline Aroclor-1232 & 80 UG/KG & $u$ & NP & 80 UG/KG & 1 \\
\hline Aroclor-1242 & 80 UG/KG & u & NP & 80 UG/KG & 1 \\
\hline Aroclor-1248 & 80 UG/KG & $u$ & NP & 80 UG/KG & 1 \\
\hline Aroclor-1254 & 160 UG/KG & $u$ & NP & 160 UG/KG & 1 \\
\hline Aroclor-1260 & 160 UG/KG & $u$ & NP & 160 UG/KG & 1 \\
\hline DBC & $74 \%$ & & & $\%$ & 1 \\
\hline
\end{tabular}


APPENDIX E.1 Results of Pesticide and PCB Anaylses, Soils, Salmon Site

\begin{tabular}{|c|c|c|c|c|c|}
\hline COMPOUND & RESULTS UNITS & $\begin{array}{l}\text { DATA } \\
\text { QUAL. }\end{array}$ & $\begin{array}{l}\text { DATA } \\
\text { VALID. }\end{array}$ & CRDL UNITS & DILUTION \\
\hline \multicolumn{6}{|l|}{ C2-21-S } \\
\hline alpha-BHC & 8 UG/KG & $u$ & NP & 3 UG/KG & 1 \\
\hline beta-BHC & $8 \mathrm{UG} / K G$ & $u$ & NP & 8 UG/KG & 1 \\
\hline delta-BHC & $8 \mathrm{UG} / K G$ & $u$ & NP & 8 UG/KG & 1 \\
\hline gamma-BHC (Lindane) & 8 UG/KG & $u$ & NP & $8 \mathrm{UG} / K G$ & 1 \\
\hline Heptachlor & $8 \mathrm{UG} / \mathrm{KG}$ & $u$ & NP & ३ UG/KG & 1 \\
\hline Aldrin & 8 UG/KG & u & NP & 8 UG/KG & 1 \\
\hline Heptachlor Epoxide & 8 UG/KG & $u$ & NP & 8 UG/KG & 1 \\
\hline Endosulfan I & 8 UG/KG & $u$ & NP & 8 UG/KG & 1 \\
\hline Dieldrin & 16 UG/KG & $u$ & NP & 16 UG/KG & 1 \\
\hline 4,4'-DDE & 16 UG/KG & $u$ & NP & 16 UG/KG & 1 \\
\hline Endrin & 16 UG/KG & $u$ & NP & 16 UG/KG & 1 \\
\hline Endosulfan II & 16 UG/KG & $u$ & NP & 16 UG/KG & 1 \\
\hline 4,4'-DDD & 16 UG/KG & $u$ & NP & 16 UG/KG & 1 \\
\hline Endosulfan Sulfate & 16 UG/KG & $u$ & NP & 16 UG/KG & 1 \\
\hline 4,4'-DDT & 16 UG/KG & $u$ & NP & 16 UG/KG & 1 \\
\hline Methoxychlor & 80 UG/KG & $u$ & NP & 80 UG/KG & 1 \\
\hline Endrin Ketone & 16 UG/KG & $u$ & NP & 16 UG/KG & 1 \\
\hline alpha-Chlordane & 80 UG/KG & $u$ & NP & 80 UG/KG & 1 \\
\hline gamma-Chlordane & 80 UG/KG & $u$ & NP & 80 UG/KG & 1 \\
\hline Toxaphene & $160 \mathrm{UG} / \mathrm{KG}$ & $u$ & NP & 160 UG/KG & 1 \\
\hline Aroclor-1016 & 80 UG/KG & $u$ & NP & 80 UG/KG & 1 \\
\hline Aroclor-1221 & 80 UG/KG & $u$ & NP & 80 UG/KG & 1 \\
\hline Aroclor-1232 & 80 UG/KG & $u$ & NP & 80 UG/KG & 1 \\
\hline Aroclor-1242 & 80 UG/KG & $u$ & NP & 80 UG/KG & 1 \\
\hline Aroclor-1248 & 80 UG/KG & $u$ & NP & 80 UG/KG & 1 \\
\hline Aroclor-1254 & $160 \mathrm{UG} / \mathrm{KG}$ & $u$ & NP & 160 UG/KG & 1 \\
\hline Aroclor-1260 & $160 \mathrm{UG} / \mathrm{KG}$ & $u$ & NP & 160 UG/KG & 1 \\
\hline $\mathrm{DBC}$ & $71 \%$ & & & $\%$ & 1 \\
\hline
\end{tabular}


APPENDIX E.1 Results of Pesticide and PCB Anaylses, Soils, Salmon Site

\begin{tabular}{|c|c|c|c|c|c|}
\hline COMPOUND & RESULTS UNITS & $\begin{array}{l}\text { DATA } \\
\text { QUAL. }\end{array}$ & $\begin{array}{l}\text { DATA } \\
\text { VALID. }\end{array}$ & CRDL UNITS & DILUTION \\
\hline \multicolumn{6}{|l|}{ C2-21-S-MS } \\
\hline gamma-BHC (Lindane) & $86 \%$ & & NP & $\%$ & 1 \\
\hline Heptachlor & $90 \%$ & & NP & $\%$ & 1 \\
\hline Aldrin & $100 \%$ & & NP & $\%$ & 1 \\
\hline Dieldrin & $82 \%$ & & NP & $\%$ & 1 \\
\hline Endrin & $82 \%$ & & NP & $\%$ & 1 \\
\hline 4,4'-DDT & $97 \%$ & & NP & $\%$ & 1 \\
\hline DBC & $65 \%$ & & NP & $\%$ & 1 \\
\hline \multicolumn{6}{|l|}{ C2-21-S-MSD } \\
\hline gamma-BHC (Lindane) & $94 \%$ & & NP & $\%$ & 1 \\
\hline Heptachlor & $98 \%$ & & NP & $\%$ & 1 \\
\hline Aldrin & $109 \%$ & & NP & $\%$ & 1 \\
\hline Dieldrin & $91 \%$ & & NP & $\%$ & 1 \\
\hline Endrin & $92 \%$ & & NP & $\%$ & 1 \\
\hline 4,4'-DDT & $107 \%$ & & NP & $\%$ & 1 \\
\hline $\mathrm{DBC}$ & $76 \%$ & & NP & $\%$ & 1 \\
\hline
\end{tabular}


APPENDIX E.1 Results of Pesticide and PCB Anaylses, Soils, Salmon Site

\begin{tabular}{|c|c|c|c|c|c|}
\hline COMPOUND & RESULTS UNITS & $\begin{array}{l}\text { DATA } \\
\text { QUAL. }\end{array}$ & $\begin{array}{l}\text { DATA } \\
\text { VALID. }\end{array}$ & CRDL UNITS & DILUTION \\
\hline \multicolumn{6}{|l|}{ C2-22-S } \\
\hline alpha-BHC & 8 UG/KG & $u$ & NP & 8 UG/KG & 1 \\
\hline beta-BHC & 8 UG/KG & $u$ & NP & 8 UG/KG & 1 \\
\hline delta-BHC & $8 \mathrm{UG} / \mathrm{KG}$ & $u$ & NP & $8 \mathrm{UG} / \mathrm{KG}$ & 1 \\
\hline gamma-BHC (Lindane) & $8 \mathrm{UG} / \mathrm{KG}$ & $u$ & NP & 8 UG/KG & 1 \\
\hline Heptachlor & $8 \mathrm{UG} / K G$ & $u$ & NP & 8 UG/KG & 1 \\
\hline Aldrin & $8 \mathrm{UG} / \mathrm{KG}$ & $u$ & NP & 8 UG/KG & 1 \\
\hline Heptachlor Epoxide & 8 UG/KG & $u$ & NP & $8 \mathrm{UG} / \mathrm{KG}$ & 1 \\
\hline Endosulfan I & $8 \mathrm{UG} / \mathrm{KG}$ & $u$ & $\mathrm{NP}$ & 8 UG/KG & 1 \\
\hline Dieldrin & 16 UG/KG & $u$ & NP & 16 UG/KG & 1 \\
\hline 4,4'-DDE & $16 \mathrm{UG} / \mathrm{KG}$ & $u$ & NP & 16 UG/KG & 1 \\
\hline Endrin & 16 UG/KG & $u$ & NP & 16 UG/KG & 1 \\
\hline Endosulfan II & 16 UG/KG & $u$ & NP & 16 UG/KG & 1 \\
\hline 4,4'-DDD & 16 UG/KG & $u$ & NP & 16 UG/KG & 1 \\
\hline Endosulfan Sulfate & 16 UG/KG & $u$ & $\mathrm{NP}$ & 16 UG/KG & 1 \\
\hline 4,4'-DDT & 16 UG/KG & $u$ & NP & 16 UG/KG & 1 \\
\hline Methoxychlor & 80 UG/KG & $u$ & NP & 80 UG/KG & 1 \\
\hline Endrin Ketone & 16 UG/KG & $u$ & NP & 16 UG/KG & 1 \\
\hline alpha-Chlordane & 80 UG/KG & $u$ & $\mathrm{NP}$ & 80 UG/KG & 1 \\
\hline gamma-Chlordane & 80 UG/KG & $u$ & NP & 80 UG/KG & 1 \\
\hline Toxaphene & 160 UG/KG & $u$ & NP & 160 UG/KG & 1 \\
\hline Aroclor-1016 & 80 UG/KG & $u$ & NP & 80 UG/KG & 1 \\
\hline Aroclor-1221 & 80 UG/KG & $u$ & $\mathrm{NP}$ & 80 UG/KG & 1 \\
\hline Aroclor-1232 & 80 UG/KG & $u$ & NP & 80 UG/KG & 1 \\
\hline Aroclor-1242 & 80 UG/KG & $u$ & NP & 80 UG/KG & 1 \\
\hline Aroclor-1248 & 80 UG/KG & $u$ & NP & 80 UG/KG & 1 \\
\hline Aroclor-1254 & 160 UG/KG & $u$ & NP & 160 UG/KG & 1 \\
\hline Aroclor- 1260 & 160 UG/KG & $u$ & NP & 160 UG/KG & 1 \\
\hline DBC & $61 \%$ & & & $\%$ & 1 \\
\hline
\end{tabular}


APPENDIX E.1 Results of Pesticide and PCB Anaylses, Soils, Salmon Site

\begin{tabular}{|c|c|c|c|c|c|}
\hline COMPOUND & RESULTS UNITS & $\begin{array}{l}\text { DATA } \\
\text { QUAL. }\end{array}$ & $\begin{array}{l}\text { DATA } \\
\text { VALID. }\end{array}$ & CRDL UNITS & DILUTION \\
\hline \multicolumn{6}{|l|}{ C2-23-S } \\
\hline alpha-BHC & 8 UG/KG & $\mathrm{u}$ & NP & $8 \mathrm{UG} / \mathrm{KG}$ & 1 \\
\hline beta-BHC & 8 UG/KG & $u$ & NP & 8 UG/KG & 1 \\
\hline delta-BHC & 8 UG/KG & $u$ & NP & 8 UG/KG & 1 \\
\hline gamma-BHC (Lindane) & $8 \mathrm{UG} / \mathrm{KG}$ & $u$ & NP & 8 UG/KG & 1 \\
\hline Heptachlor & $8 \mathrm{UG} / \mathrm{KG}$ & $u$ & NP & 8 UG/KG & 1 \\
\hline Aldrin & 8 UG/KG & $u$ & NP & $8 \mathrm{UG} / \mathrm{KG}$ & 1 \\
\hline Heptachlor Epoxide & $8 \mathrm{UG} / \mathrm{KG}$ & $u$ & NP & 8 UG/KG & 1 \\
\hline Endosulfan I & 8 UG/KG & $u$ & NP & 8 UG/KG & 1 \\
\hline Dieldrin & $16 \mathrm{UG} / \mathrm{KG}$ & $u$ & NP & 16 UG/KG & 1 \\
\hline 4,4'-DDE & 16 UG/KG & $u$ & $N P$ & 16 UG/KG & 1 \\
\hline Endrin & 16 UG/KG & $u$ & NP & 16 UG/KG & 1 \\
\hline Endosulfan II & 16 UG/KG & $u$ & NP & 16 UG/KG & 1 \\
\hline 4,4'-DDD & $16 \mathrm{UG} / \mathrm{KG}$ & $u$ & NP & 16 UG/KG & 1 \\
\hline Endosulfan Sulfate & 16 UG/KG & $u$ & NP & 16 UG/KG & 1 \\
\hline $4,4^{\prime}$-DDT & 16 UG/KG & $u$ & NP & 16 UG/KG & 1 \\
\hline Methoxychlor & 80 UG/KG & $u$ & NP & 80 UG/KG & 1 \\
\hline Endrin Ketone & 16 UG/KG & $u$ & NP & 16 UG/KG & 1 \\
\hline alpha-Chlordane & 80 UG/KG & $u$ & NP & 80 UG/KG & 1 \\
\hline gamma-Chlordane & 80 UG/KG & $u$ & NP & 80 UG/KG & 1 \\
\hline Toxaphene & 160 UG/KG & $u$ & NP & 160 UG/KG & 1 \\
\hline Aroclor-1016 & 80 UG/KG & $u$ & NP & 80 UG/KG & 1 \\
\hline Arocior-1221 & 80 UG/KG & $u$ & NP & 80 UG/KG & 1 \\
\hline Aroclor-1232 & 80 UG/KG & $u$ & NP & 80 UG/KG & 1 \\
\hline Aroclor-1242 & 80 UG/KG & $u$ & NP & 80 UG/KG & 1 \\
\hline Aroclor-1248 & 80 UG/KG & $u$ & NP & 80 UG/KG & 1 \\
\hline Aroclor-1254 & $160 \mathrm{UG} / \mathrm{KG}$ & $u$ & NP & $160 \mathrm{UG} / \mathrm{KG}$ & 1 \\
\hline Aroclor-1260 & $160 \mathrm{UG} / \mathrm{KG}$ & $u$ & NP & $160 \mathrm{UG} / \mathrm{KG}$ & 1 \\
\hline DBC & $70 \%$ & & & $\%$ & 1 \\
\hline
\end{tabular}


APPENDIX E.1 Results of Pesticide and PCB Anaylses, Soils, Salmon Site

\begin{tabular}{|c|c|c|c|c|c|}
\hline COMPOUND & RESULTS UNITS & $\begin{array}{l}\text { DATA } \\
\text { QUAL. }\end{array}$ & $\begin{array}{l}\text { DATA } \\
\text { VALID. }\end{array}$ & CRDL UNITS & DILUTION \\
\hline \multicolumn{6}{|l|}{ C2-24-S } \\
\hline alpha-BHC & 8 UG/KG & $u$ & NP & 8 UG/KG & 1 \\
\hline beta-BHC & $8 \mathrm{UG} / \mathrm{KG}$ & $u$ & NP & 8 UG/KG & 1 \\
\hline delta-BHC & $8 \mathrm{UG} / \mathrm{KG}$ & $u$ & NP & $3 \mathrm{UG} / \mathrm{KG}$ & 1 \\
\hline gamma-BHC (Lindane) & 8 UG/KG & $u$ & NP & 3 UG/KG & 1 \\
\hline Heptachlor & 8 UG/KG & $u$ & NP & $8 \mathrm{UG} / \mathrm{KG}$ & 1 \\
\hline Aldrin & $8 \mathrm{UG} / \mathrm{KG}$ & $u$ & NP & 8 UG/KG & 1 \\
\hline Heptachlor Epoxide & 8 UG/KG & $u$ & NP & 8 UG/KG & 1 \\
\hline Endosulfan I & $8 \mathrm{UG} / \mathrm{KG}$ & $u$ & NP & $8 \mathrm{UG} / K G$ & 1 \\
\hline Dieldrin & 16 UG/KG & $u$ & NP & 16 UG/KG & 1 \\
\hline 4,4'-DDE & 16 UG/KG & $u$ & NP & $16 \mathrm{UG} / \mathrm{KG}$ & 1 \\
\hline Endrin & 16 UG/KG & $u$ & NP & 16 UG/KG & 1 \\
\hline Endosulfan II & 16 UG/KG & $u$ & NP & 16 UG/KG & 1 \\
\hline 4,4'-DDD & 16 UG/KG & $u$ & NP & $16 \mathrm{UG} / \mathrm{KG}$ & 1 \\
\hline Endosulfan Sulfate & 16 UG/KG & $u$ & NP & 16 UG/KG & 1 \\
\hline 4,4'-DDT & 16 UG/KG & $u$ & NP & 16 UG/KG & 1 \\
\hline Methoxychlor & 80 UG/KG & $u$ & NP & 80 UG/KG & 1 \\
\hline Endrin Ketone & 16 UG/KG & $u$ & NP & 16 UG/KG & 1 \\
\hline alpha-Chlordane & 80 UG/KG & $u$ & NP & 80 UG/KG & 1 \\
\hline gamma-Chlordane & 80 UG/KG & $u$ & NP & 80 UG/KG & 1 \\
\hline Toxaphene & 160 UG/KG & $u$ & NP & 160 UG/KG & 1 \\
\hline Aroclor-1016 & 80 UG/KG & $u$ & NP & 80 UG/KG & 1 \\
\hline Aroclor-1221 & 80 UG/KG & $u$ & NP & 80 UG/KG & 1 \\
\hline Aroclor-1232 & 80 UG/KG & $u$ & NP & 80 UG/KG & 1 \\
\hline Aroclor-1242 & 80 UG/KG & $u$ & NP & 80 UG/KG & 1 \\
\hline Aroclor-1248 & 80 UG/KG & $u$ & NP & 80 UG/KG & 1 \\
\hline Arocior-1254 & 160 UG/KG & $u$ & NP & 160 UG/KG & 1 \\
\hline Aroclor-1260 & 160 UG/KG & $u$ & NP & 160 UG/KG & 1 \\
\hline DBC & $75 \%$ & & & $\%$ & 1 \\
\hline
\end{tabular}


APPENDIX E.1 Results of Pesticide and PCB Anaylses, Soils, Salmon Site

\begin{tabular}{|c|c|c|c|c|c|}
\hline COMPOUND & RESULTS UNITS & $\begin{array}{l}\text { DATA } \\
\text { QUAL. }\end{array}$ & $\begin{array}{l}\text { DATA } \\
\text { VALID. }\end{array}$ & CRDL UNITS & DILUTION \\
\hline \multicolumn{6}{|l|}{ C2-25-S } \\
\hline alpha-BHC & $8 \mathrm{UG} / \mathrm{KG}$ & $u$ & NP & 8 UG/KG & 1 \\
\hline beta-BHC & $8 \mathrm{UG} / \mathrm{KG}$ & $u$ & NP & $8 \mathrm{UG} / K G$ & 1 \\
\hline delta-BHC & 8 UG/KG & $u$ & NP & 8 UG/KG & 1 \\
\hline gamma-BHC (Lindane) & $8 \mathrm{UG} / \mathrm{KG}$ & $u$ & NP & $8 U G / K G$ & 1 \\
\hline Heptachlor & 8 UG/KG & $u$ & NP & $8 \mathrm{UG} / K G$ & 1 \\
\hline Aldrin & 8 UG/KG & $u$ & NP & $8 \mathrm{UG} / \mathrm{KG}$ & 1 \\
\hline Heptachlor Epoxide & 8 UG/KG & $u$ & NP & 8 UG/KG & 1 \\
\hline Endosulfan I & 8 UG/KG & $u$ & NP & 8 UG/KG & 1 \\
\hline Dieldrin & 16 UG/KG & $u$ & NP & 16 UG/KG & 1 \\
\hline 4,4'-DDE & 16 UG/KG & $u$ & NP & 16 UG/KG & 1 \\
\hline Endrin & $16 \mathrm{UG} / \mathrm{KG}$ & $u$ & NP & 16 UG/KG & 1 \\
\hline Endosulfan II & 16 UG/KG & $u$ & NP & 16 UG/KG & 1 \\
\hline 4,4'-DDD & $16 \mathrm{UG} / \mathrm{KG}$ & $u$ & NP & 16 UG/KG & 1 \\
\hline Endosulfan Sulfate & 16 UG/KG & $u$ & NP & 16 UG/KG & 1 \\
\hline $4,4^{\prime}-$ DDT & 16 UG/KG & $u$ & NP & $16 \mathrm{UG} / \mathrm{KG}$ & 1 \\
\hline Methoxychior & 80 UG/KG & $u$ & NP & 80 UG/KG & 1 \\
\hline Endrin Ketone & 16 UG/KG & $u$ & NP & 16 UG/KG & 1 \\
\hline alpha-Chlordane & 80 UG/KG & $u$ & NP & 80 UG/KG & 1 \\
\hline gamma-Chlordane & 80 UG/KG & $u$ & NP & 80 UG/KG & 1 \\
\hline Toxaphene & 160 UG/KG & $u$ & NP & 160 UGMG & 1 \\
\hline Aroclor-1016 & 80 UG/KG & $u$ & NP & 80 UG/KG & 1 \\
\hline Aroclor-1221 & 80 UG/KG & $u$ & NP & 80 UG/KG & 1 \\
\hline Arocior-1232 & 80 UG/KG & $u$ & NP & 80 UG/KG & 1 \\
\hline Aroclor-1242 & 80 UG/KG & $u$ & NP & 80 UG/KG & 1 \\
\hline Aroclor- 1248 & 80 UG/KG & $u$ & NP & 80 UG/KG & 1 \\
\hline Arocior-1254 & 160 UG/KG & $u$ & NP & 160 UG/KG & 1 \\
\hline Aroclor- 1260 & 160 UG/KG & $u$ & NP & 160 UG/KG & 1 \\
\hline DBC & $76 \%$ & & & $\%$ & 1 \\
\hline
\end{tabular}


APPENDIX E.1 Results of Pesticide and PCB Anaylses, Soils, Salmon Site

\begin{tabular}{|c|c|c|c|c|c|}
\hline COMPOUND & RESULTS UNITS & $\begin{array}{l}\text { DATA } \\
\text { QUAL. }\end{array}$ & $\begin{array}{l}\text { DATA } \\
\text { VALID. }\end{array}$ & CRDL UNITS & DILUTION \\
\hline \multicolumn{6}{|l|}{ C2-25A-S } \\
\hline alpha-BHC & $8 \mathrm{UG} / \mathrm{KG}$ & $u$ & NP & 8 UG/KG & 1 \\
\hline beta-BHC & 8 UG/KG & $u$ & NP & $8 \mathrm{UG} / \mathrm{KG}$ & 1 \\
\hline delta-BHC & 8 UG/KG & $u$ & NP & $8 \mathrm{UG} / \mathrm{KG}$ & 1 \\
\hline gamma-BHC (Lindane) & 8 UG/KG & $u$ & NP & $8 \mathrm{UG} / \mathrm{KG}$ & 1 \\
\hline Heptachlor & $8 \mathrm{UG} / K G$ & $u$ & NP & $8 \mathrm{UG} / \mathrm{KG}$ & 1 \\
\hline Aldrin & 8 UG/KG & $u$ & NP & 8 UG/KG & 1 \\
\hline Heptachlor Epoxide & 8 UG/KG & $u$ & NP & 8 UG/KG & 1 \\
\hline Endosulfan I & 8 UG/KG & $u$ & NP & $8 \mathrm{UG} / \mathrm{KG}$ & 1 \\
\hline Dieldrin & 16 UG/KG & $u$ & NP & 16 UG/KG & 1 \\
\hline 4,4'-DDE & 16 UG/KG & $u$ & NP & 16 UG/KG & 1 \\
\hline Endrin & 16 UG/KG & $u$ & NP & 16 UG/KG & 1 \\
\hline Endosulfan II & 16 UG/KG & $u$ & NP & 16 UG/KG & 1 \\
\hline 4,4'-DDD & 16 UG/KG & $u$ & NP & 16 UG/KG & 1 \\
\hline Endosulfan Sulfate & 16 UG/KG & $u$ & NP & 16 UG/KG & 1 \\
\hline 4,4'-DDT & 16 UG/KG & $u$ & NP & $16 \mathrm{UG} / \mathrm{KG}$ & 1 \\
\hline Methoxychlor & 80 UG/KG & $u$ & NP & 80 UG/KG & 1 \\
\hline Endrin Ketone & 16 UG/KG & $u$ & $\mathrm{NP}$ & 16 UG/KG & 1 \\
\hline alpha-Chlordane & 80 UG/KG & $u$ & NP & 80 UG/KG & 1 \\
\hline gamma-Chlordane & 80 UG/KG & $u$ & NP & 80 UG/KG & 1 \\
\hline Toxaphene & 160 UG/KG & $u$ & NP & 160 UG/KG & 1 \\
\hline Aroclor-1016 & 80 UG/KG & $u$ & NP & 80 UG/KG & 1 \\
\hline Aroclor-1221 & 80 UG/KG & $u$ & NP & 80 UG/KG & 1 \\
\hline Aroclor-1232 & 80 UG/KG & $u$ & NP & 80 UG/KG & 1 \\
\hline Aroclor-1242 & 80 UG/KG & $u$ & NP & 80 UG/KG & 1 \\
\hline Aroclor-1248 & 80 UG/KG & $u$ & NP & 80 UG/KG & 1 \\
\hline Aroclor-1254 & $160 \mathrm{UG} / \mathrm{KG}$ & $u$ & NP & 160 UG/KG & 1 \\
\hline Aroclor-1260 & 160 UG/KG & $u$ & NP & 160 UG/KG & 1 \\
\hline DBC & $75 \%$ & & & $\%$ & 1 \\
\hline
\end{tabular}


APPENDIX E.1 Results of Pesticide and PCB Anaylses, Soils, Salmon Site

\begin{tabular}{|c|c|c|c|c|c|}
\hline COMPOUND & RESULTS UNITS & $\begin{array}{l}\text { DATA } \\
\text { QUAL. }\end{array}$ & $\begin{array}{l}\text { DATA } \\
\text { VALID. } \\
\end{array}$ & CRDL UNITS & DILUTION \\
\hline \multicolumn{6}{|l|}{ C2-26-S } \\
\hline alpha-BHC & 8 UG/KG & $u$ & NP & 8 UG/KG & 1 \\
\hline beta-BHC & 8 UG/KG & $u$ & NP & $8 \mathrm{UG} / \mathrm{KG}$ & 1 \\
\hline delta-BHC & $8 \mathrm{UG} / \mathrm{KG}$ & $u$ & $\mathrm{NP}$ & 8 UG/KG & 1 \\
\hline gamma-BHC (Lindane) & $8 \mathrm{UG} / \mathrm{KG}$ & $u$ & NP & 8 UG/KG & 1 \\
\hline Heptachlor & $8 \mathrm{UG} / \mathrm{KG}$ & $u$ & NP & 8 UG/KG & 1 \\
\hline Aldrin & 8 UG/KG & $u$ & NP & $8 \mathrm{UG} / \mathrm{KG}$ & 1 \\
\hline Heptachlor Epoxide & 8 UG/KG & $u$ & NP & $8 \mathrm{UG} / \mathrm{KG}$ & 1 \\
\hline Endosulfan I & 8 UG/KG & $u$ & NP & $8 \mathrm{UG} / \mathrm{KG}$ & 1 \\
\hline Dieldrin & 16 UG/KG & $u$ & NP & $16 \mathrm{UG} / \mathrm{KG}$ & 1 \\
\hline 4,4'-DDE & 16 UG/KG & $u$ & NP & 16 UG/KG & 1 \\
\hline Endrin & 16 UG/KG & $u$ & NP & $16 \mathrm{UG} / \mathrm{KG}$ & 1 \\
\hline Endosulfan II & 16 UG/KG & $u$ & NP & 16 UG/KG & 1 \\
\hline 4,4'-DDD & 16 UG/KG & $u$ & NP & 16 UG/KG & 1 \\
\hline Endosulfan Sulfate & 16 UG/KG & $u$ & NP & 16 UG/KG & 1 \\
\hline $4,4^{\prime}-\mathrm{DDT}$ & 16 UG/KG & $u$ & NP & 16 UG/KG & 1 \\
\hline Methoxychlor & 80 UG/KG & $u$ & NP & 80 UG/KG & 1 \\
\hline Endrin Ketone & 16 UGKG & $u$ & NP & 16 UG/KG & 1 \\
\hline alpha-Chlordane & 80 UG/KG & $u$ & NP & 80 UG/KG & 1 \\
\hline gamma-Chlordane & 80 UG/KG & $u$ & NP & 80 UG/KG & 1 \\
\hline Toxaphene & $160 \mathrm{UG} / \mathrm{KG}$ & $u$ & NP & 160 UG/KG & 1 \\
\hline Aroclor-1016 & 80 UG/KG & $u$ & NP & 80 UG/KG & 1 \\
\hline Aroclor-1221 & 80 UG/KG & $u$ & NP & 80 UG/KG & 1 \\
\hline Aroclor-1232 & 80 UG/KG & $u$ & NP & 80 UG/KG & 1 \\
\hline Aroclor-1242 & 80 UG/KG & $u$ & NP & 80 UG/KG & 1 \\
\hline Arocior-1248 & 80 UG/KG & $u$ & NP & 80 UG/KG & 1 \\
\hline Aroclor-1254 & 160 UG/KG & $u$ & NP & 160 UG/KG & 1 \\
\hline Arocior-1260 & 160 UG/KG & $u$ & NP & 160 UG/KG & 1 \\
\hline DBC & $76 \%$ & & & $\%$ & 1 \\
\hline
\end{tabular}


APPENDIX E.1 Results of Pesticide and PCB Anaylses, Soils, Salmon Site

\begin{tabular}{|c|c|c|c|c|c|}
\hline COMPOUND & RESULTS UNITS & $\begin{array}{l}\text { DATA } \\
\text { QUAL. }\end{array}$ & $\begin{array}{l}\text { DATA } \\
\text { VALID. }\end{array}$ & CRDL UNITS & DILUTION \\
\hline \multicolumn{6}{|l|}{ C2-27-S } \\
\hline alpha-BHC & 8 UG/KG & $u$ & NP & 8 UG/KG & 1 \\
\hline beta-BHC & 8 UG/KG & $u$ & NP & 8 UG/KG & 1 \\
\hline delta-BHC & 8 UG/KG & $u$ & NP & 8 UG/KG & 1 \\
\hline gamma-BHC (Lindane) & 8 UG/KG & $u$ & NP & $8 \mathrm{UG} / \mathrm{KG}$ & 1 \\
\hline Heptachlor & $8 \mathrm{UG} / \mathrm{KG}$ & $u$ & NP & $8 \mathrm{UG} / \mathrm{KG}$ & 1 \\
\hline Aldrin & 8 UG/KG & $u$ & NP & 8 UG/KG & 1 \\
\hline Heptachlor Epoxide & 8 UG/KG & $u$ & NP & 8 UG/KG & 1 \\
\hline Endosulfan I & 8 UG/KG & $u$ & NP & $8 \mathrm{UG} / \mathrm{KG}$ & 1 \\
\hline Dieldrin & 16 UGRG & $u$ & NP & 16 UG/KG & 1 \\
\hline 4,4'-DDE & 16 UG/KG & $u$ & NP & 16 UG/KG & 1 \\
\hline Endrin & 16 UG/KG & $u$ & NP & 16 UG/KG & 1 \\
\hline Endosulfan II & 16 UG/KG & $u$ & NP & 16 UG/KG & 1 \\
\hline 4,4'-DDD & 16 UG/KG & $u$ & NP & 16 UG/KG & 1 \\
\hline Endosulfan Sulfate & 16 UG/KG & $u$ & NP & 16 UG/KG & 1 \\
\hline $4,4^{\prime}-\mathrm{DDT}$ & 16 UG/KG & $u$ & NP & 16 UG/KG & 1 \\
\hline Methoxychlor & 80 UG/KG & $u$ & NP & 80 UG/KG & 1 \\
\hline Endrin Ketone & 16 UG/KG & $u$ & NP & 16 UG/KG & 1 \\
\hline alpha-Chlordane & 80 UG/KG & $u$ & NP & 80 UG/KG & 1 \\
\hline gamma-Chlordane & 80 UG/KG & $u$ & NP & 80 UG/KG & 1 \\
\hline Toxaphene & 160 UG/KG & $u$ & NP & 160 UG/KG & 1 \\
\hline Aroclor-1016 & 80 UG/KG & $u$ & NP & 80 UG/KG & 1 \\
\hline Aroclor-1221 & 80 UG/KG & $u$ & NP & 80 UG/KG & 1 \\
\hline Aroclor-1232 & 80 UG/KG & $u$ & NP & 80 UG/KG & 1 \\
\hline Aroclor-1242 & 80 UG/KG & $u$ & NP & 80 UG/KG & 1 \\
\hline Aroclor-1248 & 80 UG/KG & $u$ & NP & 80 UG/KG & 1 \\
\hline Aroclor-1254 & 160 UG/KG & $u$ & NP & 160 UG/KG & 1 \\
\hline Aroclor-1260 & 160 UG/KG & $u$ & NP & 160 UG/KG & 1 \\
\hline DBC & $63 \%$ & & & $\%$ & 1 \\
\hline
\end{tabular}




\section{Appendix E.2}

Results of Pesticide and PCB Analyses, Water, Salmon Site 
APPENDIX E.2 Results of Pesticide and PCB Analyses, Water,

Salmon Site

\begin{tabular}{|c|c|c|c|c|c|}
\hline COMPOUND & RESULTS UNITS & $\begin{array}{l}\text { DATA } \\
\text { QUAL. }\end{array}$ & $\begin{array}{l}\text { DATA } \\
\text { VALID. }\end{array}$ & CRDL UNITS & DILUTION \\
\hline \multicolumn{6}{|l|}{$C 5-1-W$} \\
\hline alpha-BHC & 0.05 UG/L & $u$ & NP & 0.05 UG/L & 1 \\
\hline beta-BHC & 0.05 UG/L & $u$ & NP & 0.05 UG $/$ & 1 \\
\hline delta-BHC & $0.05 \mathrm{UG} / \mathrm{L}$ & $u$ & NP & 0.05 UG/L & 1 \\
\hline gamma-BHC (Lindane) & $0.05 \mathrm{UG} /$ & $u$ & NP & 0.05 UG $\Omega$ & 1 \\
\hline Heptachlor & 0.05 UG $/$ & $u$ & NP & 0.05 UG/ & 1 \\
\hline Aldrin & 0.05 UG/L & $u$ & NP & 0.05 UG $\Omega$ & 1 \\
\hline Heptachlor Epoxide & 0.05 UG/ & $u$ & NP & 0.05 UG/L & 1 \\
\hline Endosulfan I & 0.05 UG $\Omega$ & $u$ & NP & 0.05 UG $\Omega$ & 1 \\
\hline Dieldrin & 0.1 UGR & $u$ & NP & $0.1 \mathrm{UG} \Omega$ & 1 \\
\hline 4,4'-DDE & $0.1 \mathrm{UG} /$ & $u$ & NP & 0.1 UG $\Omega$ & 1 \\
\hline Endrin & 0.1 UG $\Omega$ & $u$ & NP & 0.1 UG $\Omega$ & 1 \\
\hline Endosulfan II & $0.1 \mathrm{UG} / \mathrm{L}$ & $u$ & NP & 0.1 UG $\Omega$ & 1 \\
\hline 4,4'-DDD & 0.1 UG $\Omega$ & $u$ & NP & 0.1 UG $\Omega$ & 1 \\
\hline Endosulfan Sulfate & 0.1 UG $\Omega$ & $u$ & NP & $0.1 \mathrm{UG} \Omega$ & 1 \\
\hline 4,4'-DDT & $0.1 \mathrm{UG} \Omega$ & $u$ & NP & 0.1 UG $\Omega$ & 1 \\
\hline Methoxychlor & $0.5 \mathrm{UG} / \mathrm{L}$ & $u$ & NP & 0.5 UG $\Omega$ & 1 \\
\hline Endrin Ketone & $0.1 \mathrm{UG} \Omega$ & $u$ & NP & $0.1 \mathrm{UG} \Omega$ & 1 \\
\hline alpha-Chlordane & $0.5 \mathrm{UG} / \mathrm{L}$ & $u$ & NP & $0.5 \mathrm{UG} \Omega$ & 1 \\
\hline gamma-Chlordane & 0.5 UG $\Omega$ & $u$ & NP & 0.5 UG $\Omega$ & 1 \\
\hline Toxaphene & 1 UG $\Omega$ & $u$ & NP & 1 UG $\Omega$ & 1 \\
\hline Aroclor-1016 & $0.5 \mathrm{UG} / \mathrm{L}$ & $u$ & NP & $0.5 \mathrm{UG} /$ & 1 \\
\hline Aroclor-1221 & 0.5 UG $\Omega$ & $u$ & NP & 0.5 UG/L & 1 \\
\hline Aroclor-1232 & $0.5 \mathrm{UG} / \mathrm{L}$ & $u$ & NP & 0.5 UG $\Omega$ & 1 \\
\hline Aroclor-1242 & 0.5 UG & $u$ & NP & $0.5 \mathrm{UG} \Omega$ & 1 \\
\hline Aroclor-1248 & 0.5 UG $\Omega$ & $u$ & NP & 0.5 UG $\Omega$ & 1 \\
\hline Aroclor-1254 & $1 \mathrm{UG} /$ & $u$ & NP & 1 UG $\Omega$ & 1 \\
\hline Aroclor-1260 & $1 \mathrm{UG} /$ & $u$ & NP & 1 UG $\Omega$ & 1 \\
\hline DBC & $73 \%$ & & & $\%$ & 1 \\
\hline
\end{tabular}


APPENDIX E.2 Results of Pesticide and PCB Analyses, Water, Salmon Site

\begin{tabular}{lcccc|}
\hline COMPOUND & RESULTS UNITS & DATA & DATA & \\
QUAL. & VALID. & CRDL UNITS & DILUTION \\
\hline C5-1-W-MS & & & & \\
gamma-BHC (Lindane) & $98 \%$ & NP & $\%$ & 1 \\
Heptachlor & $106 \%$ & NP & $\%$ & 1 \\
Aldrin & $101 \%$ & NP & $\%$ & 1 \\
Dieldrin & $116 \%$ & NP & $\%$ & 1 \\
Endrin & $103 \%$ & NP & $\%$ & 1 \\
4,4 -DDT & $86 \%$ & NP & $\%$ & 1 \\
DBC & $58 \%$ & NP & $\%$ & 1 \\
& & & & \\
C5-1-W-MSD & & & & \\
gamma-BHC (Lindane) & $83 \%$ & NP & $\%$ & 1 \\
Heptachlor & $96 \%$ & NP & $\%$ & 1 \\
Aldrin & $84 \%$ & NP & $\%$ & 1 \\
Dieldrin & $106 \%$ & NP & $\%$ & 1 \\
Endrin & $85 \%$ & NP & $\%$ & 1 \\
$4,4-D D T$ & $66 \%$ & NP & $\%$ & 1 \\
DBC & $43 \%$ & NP & $\%$ & 1
\end{tabular}


APPENDIX E.2 Results of Pesticide and PCB Analyses, Water,

Salmon Site

\begin{tabular}{|c|c|c|c|c|c|}
\hline COMPOUND & RESULTS UNITS & $\begin{array}{l}\text { DATA } \\
\text { QUAL. }\end{array}$ & $\begin{array}{l}\text { DATA } \\
\text { VALID. }\end{array}$ & CRDL UNITS & DILUTION \\
\hline \multicolumn{6}{|l|}{ C3-8-W } \\
\hline alpha-BHC & 0.062 UG/ & $u$ & NP & 0.062 UG $/$ & 1 \\
\hline beta-BHC & 0.062 UG/L & $u$ & NP & 0.062 UG $\Omega$ & 1 \\
\hline delta-BHC & $0.062 \mathrm{UG} \Omega$ & $u$ & NP & 0.062 UG $\Omega$ & 1 \\
\hline gamma-BHC (Lindane) & 0.062 UG $\Omega$ & $u$ & NP & 0.062 UG $\Omega$ & 1 \\
\hline Heptachlor & 0.062 UG $\Omega$ & $u$ & NP & 0.062 UG/ & 1 \\
\hline Aldrin & $0.062 \mathrm{UG} / \mathrm{L}$ & $u$ & NP & 0.062 UG $\Omega$ & 1 \\
\hline Heptachlor Epoxide & 0.062 UG $/$ & $u$ & NP & 0.062 UG/ & 1 \\
\hline Endosulfan I & 0.062 UGR & $u$ & NP & 0.062 UG $\Omega$ & 1 \\
\hline Dieldrin & 0.12 UG/L & $u$ & NP & 0.12 UG $\Omega$ & 1 \\
\hline 4,4'-DDE & $0.12 \mathrm{UG} / \mathrm{L}$ & $u$ & NP & 0.12 UG $\Omega$ & 1 \\
\hline Endrin & 0.12 UG/L & $u$ & NP & 0.12 UG/L & 1 \\
\hline Endosulfan II & 0.12 UG/L & $u$ & NP & 0.12 UG/L & 1 \\
\hline 4,4'-DDD & $0.12 \mathrm{UG} / \mathrm{L}$ & $u$ & NP & 0.12 UG/ & 1 \\
\hline Endosulfan Sulfate & 0.12 UG/L & $u$ & NP & 0.12 UG/ & 1 \\
\hline 4,4'-DDT & 0.12 UG/L & $u$ & NP & 0.12 UG/L & 1 \\
\hline Methoxychlor & $0.62 \mathrm{UG} / \mathrm{L}$ & $u$ & NP & 0.62 UG/L & 1 \\
\hline Endrin Ketone & $0.12 \mathrm{UG} / \mathrm{L}$ & $u$ & NP & 0.12 UG $\Omega$ & 1 \\
\hline alpha-Chlordane & 0.62 UG/L & $u$ & NP & 0.62 UG & 1 \\
\hline gamma-Chlordane & 0.62 UG/ & $u$ & NP & 0.62 UG/ & 1 \\
\hline Toxaphene & 1.2 UG/L & $u$ & NP & $1.2 \mathrm{UG} /$ & 1 \\
\hline Aroclor-1016 & 0.62 UG/L & $u$ & NP & 0.62 UG/L & 1 \\
\hline Aroclor-1221 & 0.62 UG/L & $u$ & NP & 0.62 UG/ & 1 \\
\hline Aroclor-1232 & $0.62 \mathrm{UG} \Omega$ & $u$ & NP & 0.62 UG/ & 1 \\
\hline Aroclor-1242 & $0.62 \mathrm{UG} /$ & u & NP & 0.62 UG/ & 1 \\
\hline Aroclor-1248 & 0.62 UG $\Omega$ & $u$ & NP & 0.62 UG/ & 1 \\
\hline Aroclor-1254 & $1.2 \mathrm{UG} / \mathrm{L}$ & $u$ & NP & $1.2 \mathrm{UG} / \mathrm{L}$ & 1 \\
\hline Aroclor-1260 & $1.2 \mathrm{UG} / \mathrm{L}$ & $u$ & NP & 1.2 UG/L & 1 \\
\hline DBC & $38 \%$ & & & $\%$ & 1 \\
\hline
\end{tabular}


APPENDIX E.2 Results of Pesticide and PCB Analyses, Water, Salmon Site

\begin{tabular}{|c|c|c|c|c|c|c|}
\hline COMPOUND & RESULTS & UNITS & $\begin{array}{l}\text { DATA } \\
\text { QUAL. }\end{array}$ & $\begin{array}{l}\text { DATA } \\
\text { VALID. }\end{array}$ & CRDL UNITS & DILUTION \\
\hline \multicolumn{7}{|l|}{ C3-9-W } \\
\hline alpha-BHC & 0.07 & UG/ & $u$ & NP & 0.074 UG $\Omega$ & 1 \\
\hline beta-BHC & $0.07<$ & UG & $u$ & NP & $0.074 \mathrm{UG} \Omega$ & 1 \\
\hline delta-BHC & 0.07 & UG/L & $u$ & NP & 0.074 UG/ & 1 \\
\hline gamma-BHC (Lindane) & 0.07 & UG/ & $u$ & NP & 0.074 UG/L & 1 \\
\hline Heptachlor & 0.07 & UGR & $u$ & NP & 0.074 UG/L & 1 \\
\hline Aldrin & 0.07 & UGR & $u$ & NP & 0.074 UG/L & 1 \\
\hline Heptachlor Epoxide & 0.07 & UGR & $u$ & NP & 0.074 UG几 & 1 \\
\hline Endosulfan I & 0.07 & UG $\Omega$ & $u$ & NP & 0.074 UGL & 1 \\
\hline Dieldrin & 0.15 & UG/L & $u$ & NP & $0.15 \mathrm{UG} / \mathrm{L}$ & 1 \\
\hline $4,4^{\prime}-\mathrm{DDE}$ & 0.15 & UG & $u$ & NP & 0.15 UGR & 1 \\
\hline Endrin & 0.15 & UGR & $u$ & NP & 0.15 UG/L & 1 \\
\hline Endosulfan II & 0.15 & UG $\Omega$ & $u$ & NP & 0.15 UG几 & 1 \\
\hline 4,4'-DDD & 0.15 & UG/L & $u$ & NP & 0.15 UG/ & 1 \\
\hline Endosulfan Sulfate & 0.3 & UG $/$ & $u x$ & NP & 0.15 UG/ & 1 \\
\hline $4,4^{\prime}-D D T$ & 0.1 & UG/L & $u$ & NP & 0.15 UG/L & 1 \\
\hline Methoxychlor & 0.7 & UG $\Omega$ & $u$ & NP & 0.74 UG/ & 1 \\
\hline Endrin Ketone & 0.1 & UG $\Omega$ & $u$ & NP & 0.15 UG/L & 1 \\
\hline alpha-Chlordane & 0.7 & $U G /$ & $u$ & NP & $0.74 \mathrm{UG} / \mathrm{L}$ & 1 \\
\hline gamma-Chlordane & 0.7 & UG几 & $u$ & NP & 0.74 UG $\Omega$ & 1 \\
\hline Toxaphene & & UG/ & $u$ & NP & $1.5 \mathrm{UG} /$ & 1 \\
\hline Aroclor-1016 & 0.7 & UG $\Omega$ & $u$ & NP & $0.74 \mathrm{UG} / \mathrm{L}$ & 1 \\
\hline Aroclor-1221 & 0.7 & UG/L & $u$ & NP & $0.74 \mathrm{UG} \Omega$ & 1 \\
\hline Aroclor-1232 & 0.7 & UG/ & $u$ & NP & 0.74 UG/L & 1 \\
\hline Aroclor-1242 & 0.7 & UG/ & $u$ & NP & $0.74 \mathrm{UG} \Omega$ & 1 \\
\hline Aroclor- 1248 & 0.7 & $U G /$ & $u$ & NP & 0.74 UG/L & 1 \\
\hline Aroclor-1254 & & UG/ & $u$ & NP & $1.5 \mathrm{UG} / \mathrm{L}$ & 1 \\
\hline Aroclor-1260 & & UG & $u$ & NP & 1.5 UG $\Omega$ & 1 \\
\hline DBC & & $\%$ & & & $\%$ & 1 \\
\hline
\end{tabular}


APPENDIX E.2 Results of Pesticide and PCB Analyses, Water, Salmon Site

\begin{tabular}{|c|c|c|c|c|c|}
\hline COMPOUND & RESULTS UNITS & $\begin{array}{l}\text { DATA } \\
\text { QUAL. }\end{array}$ & $\begin{array}{l}\text { DATA } \\
\text { VALID. }\end{array}$ & CRDL UNITS & DILUTION \\
\hline \multicolumn{6}{|l|}{ C4-11-W } \\
\hline alpha-BHC & 0.084 UGR & $u$ & NP & 0.084 UG $\Omega$ & 1 \\
\hline beta-BHC & 0.084 UG & $u$ & NP & 0.084 UG $/$ & 1 \\
\hline delta-BHC & 0.084 UG $\Omega$ & $u$ & NP & 0.084 UG $\Omega$ & 1 \\
\hline gamma-BHC (Lindane) & 0.084 UG/ & $u$ & NP & $0.084 \mathrm{UG} / \mathrm{L}$ & 1 \\
\hline Heptachlor & 0.084 UG/L & $u$ & NP & 0.084 UG $\Omega$ & 1 \\
\hline Aldrin & 0.084 UG/L & $u$ & NP & 0.084 UG/L & 1 \\
\hline Heptachlor Epoxide & 0.084 UGR & $u$ & NP & 0.084 UG/ & 1 \\
\hline Endosulfan I & 0.084 UGR & $u$ & NP & $0.084 \mathrm{UG} /$ & 1 \\
\hline Dieldrin & 0.17 UG几 & $u$ & NP & 0.17 UG $\Omega$ & 1 \\
\hline 4,4'-DDE & 0.17 UG $\Omega$ & $u$ & NP & 0.17 UG/L & 1 \\
\hline Endrin & 0.17 UG/ & $u$ & NP & 0.17 UG/ & 1 \\
\hline Endosulfan II & 0.17 UG $\Omega$ & $u$ & NP & 0.17 UG/ & 1 \\
\hline 4,4'-DDD & 0.17 UG/ & $u$ & NP & 0.17 UG/ & 1 \\
\hline Endosulfan Sulfate & 0.17 UG/ & $u$ & NP & 0.17 UG $\Omega$ & 1 \\
\hline 4,4'-DDT & 0.17 UG/L & $u$ & NP & 0.17 UG/ & 1 \\
\hline Methoxychlor & $0.84 \mathrm{UG} \Omega$ & $u$ & NP & 0.84 UG/ & 1 \\
\hline Endrin Ketone & 0.17 UG几 & $u$ & NP & 0.17 UG/L & 1 \\
\hline alpha-Chlordane & $0.84 \mathrm{UG} /$ & $u$ & NP & 0.84 UG $\Omega$ & 1 \\
\hline gamma-Chlordane & $0.84 \mathrm{UG} / \mathrm{L}$ & $u$ & NP & 0.84 UG/ & 1 \\
\hline Toxaphene & 1.7 UGR & $u$ & NP & $1.7 \mathrm{UG} / \mathrm{L}$ & 1 \\
\hline Aroclor-1016 & 0.84 UG/ & $u$ & NP & 0.84 UG $\Omega$ & 1 \\
\hline Aroclor-1221 & 0.84 UGL & $u$ & NP & 0.84 UG $\Omega$ & 1 \\
\hline Aroclor-1232 & 0.84 UG $\Omega$ & $u$ & NP & $0.84 \mathrm{UG} \Omega$ & 1 \\
\hline Aroclor-1242 & 0.84 UG/ & $u$ & NP & 0.84 UG/ & 1 \\
\hline Aroclor-1248 & 0.84 UG/ & $u$ & NP & $0.84 \mathrm{UG} / \mathrm{L}$ & 1 \\
\hline Aroclor- 1254 & $1.7 \mathrm{UG} / \mathrm{L}$ & $u$ & NP & $1.7 \mathrm{UG} \Omega$ & 1 \\
\hline Aroclor- 1260 & $1.7 \mathrm{UG} / \mathrm{L}$ & $u$ & NP & $1.7 \mathrm{UG} /$ & 1 \\
\hline DBC & $29 \%$ & & & $\%$ & 1 \\
\hline
\end{tabular}


APPENDIX E.2 Results of Pesticide and PCB Analyses, Water,

Salmon Site

\begin{tabular}{|c|c|c|c|c|c|}
\hline COMPOUND & RESULTS UNITS & $\begin{array}{l}\text { DATA } \\
\text { QUAL. }\end{array}$ & $\begin{array}{l}\text { DATA } \\
\text { VALID. }\end{array}$ & CRDL UNITS & DILUTION \\
\hline \multicolumn{6}{|l|}{ C4-13-W } \\
\hline alpha-BHC & 0.05 UG/ & $\mathrm{u}$ & NP & 0.05 UG/ & 1 \\
\hline beta-BHC & 0.05 UG $\Omega$ & $u$ & NP & 0.05 UG $\Omega$ & 1 \\
\hline delta-BHC & 0.05 UG $\Omega$ & $u$ & NP & 0.05 UG/ & 1 \\
\hline gamma-BHC (Lindane) & 0.05 UG/L & $u$ & NP & 0.05 UG/L & 1 \\
\hline Heptachlor & 0.05 UG/L & $u$ & NP & 0.05 UG/L & 1 \\
\hline Aldrin & 0.05 UG/ & $u$ & NP & $0.05 \mathrm{UG} /$ & 1 \\
\hline Heptachlor Epoxide & $0.05 \mathrm{UG} / \mathrm{L}$ & $u$ & NP & $0.05 \mathrm{UG} / \mathrm{L}$ & 1 \\
\hline Endosulfan 1 & 0.05 UG/L & $u$ & NP & 0.05 UG/ & 1 \\
\hline Dieldrin & $0.1 \mathrm{UG} \Omega$ & $u$ & NP & $0.1 \mathrm{UG} \Omega$ & 1 \\
\hline 4,4'-DDE & $0.1 \mathrm{UG} \Omega$ & $u$ & NP & $0.1 \mathrm{UG} \Omega$ & 1 \\
\hline Endrin & 0.1 UG $/$ & $u$ & NP & 0.1 UG $\Omega$ & 1 \\
\hline Endosulfan II & 0.1 UG/ & $u$ & NP & 0.1 UG/ & 1 \\
\hline $4,4^{\prime}-\mathrm{DDD}$ & 0.1 UG/L & $u$ & NP & 0.1 UG/ & 1 \\
\hline Endosulfan Sulfate & 0.1 UG & $u$ & NP & 0.1 UG/ & 1 \\
\hline 4,4'-DDT & 0.1 UG/ & $u$ & NP & 0.1 UG/L & 1 \\
\hline Methoxychlor & 0.5 UG/ & $u$ & NP & $0.5 \mathrm{UG} / \mathrm{L}$ & 1 \\
\hline Endrin Ketone & $0.1 \mathrm{UG} /$ & $u$ & NP & $0.1 \mathrm{UG} / \mathrm{L}$ & 1 \\
\hline alpha-Chlordane & 0.5 UG & $u$ & NP & 0.5 UG/ & 1 \\
\hline gamma-Chlordane & 0.5 UG $\Omega$ & $u$ & NP & 0.5 UG/L & 1 \\
\hline Toxaphene & 1 UGR & $u$ & NP & 1 UG/ & 1 \\
\hline Aroclor-1016 & $0.5 \mathrm{UG} / \mathrm{L}$ & $u$ & NP & 0.5 UG/ & 1 \\
\hline Aroclor-1221 & 0.5 UG $\Omega$ & $u$ & NP & 0.5 UG/L & 1 \\
\hline Aroclor-1232 & $0.5 \mathrm{UG} / \mathrm{L}$ & $u$ & NP & 0.5 UG & 1 \\
\hline Aroclor-1242 & 0.5 UG $\Omega$ & $u$ & NP & $0.5 \mathrm{UG} \Omega$ & 1 \\
\hline Aroclor-1248 & 0.5 UG $\Omega$ & $u$ & NP & 0.5 UG & 1 \\
\hline Aroclor-1254 & $1 \mathrm{UG} / \mathrm{L}$ & $u$ & NP & $1 \mathrm{UG} / \mathrm{L}$ & 1 \\
\hline Aroclor-1260 & 1 UG $\Omega$ & $u$ & NP & $1 \mathrm{UG} / \mathrm{L}$ & 1 \\
\hline $\mathrm{DBC}$ & $77 \%$ & & & $\%$ & 1 \\
\hline
\end{tabular}


APPENDIX E.2 Results of Pesticide and PCB Analyses, Water, Salmon Site

\begin{tabular}{|c|c|c|c|c|c|}
\hline COMPOUND & RESULTS UNITS & $\begin{array}{l}\text { DATA } \\
\text { QUAL. }\end{array}$ & $\begin{array}{l}\text { DATA } \\
\text { VALID. }\end{array}$ & CRDL UNITS & DILUTION \\
\hline \multicolumn{6}{|l|}{ C1-14-W } \\
\hline alpha-BHC & 0.05 UG $\Omega$ & $u$ & -. & 0.05 UG $\Omega$ & 1 \\
\hline beta-BHC & 0.05 UG/L & $u$ & $\cdots$ & 0.05 UG/ & 1 \\
\hline delta-BHC & 0.05 UGR & $u$ & - & 0.05 UG $\Omega$ & 1 \\
\hline gamma-BHC (Lindane) & 0.05 UG $\Omega$ & $u$ & - & 0.05 UG/ & 1 \\
\hline Heptachlor & 0.05 UG/L & $u$ & - & 0.05 UG/ & 1 \\
\hline Aldrin & 0.05 UGR & $u$ & - & 0.05 UG $\Omega$ & 1 \\
\hline Heptachlor Epoxide & 0.05 UG/ & $u$ & - & 0.05 UG $\Omega$ & 1 \\
\hline Endosulfan I & 0.05 UG $\Omega$ & $u$ & - & 0.05 UG $\Omega$ & 1 \\
\hline Dieldrin & $0.1 \mathrm{UG} /$ & $u$ & - & $0.1 \mathrm{UG} / \mathrm{L}$ & 1 \\
\hline 4,4'-DDE & 0.1 UG/L & $u$ & $\cdots$ & 0.1 UG/ & 1 \\
\hline Endrin & 0.1 UG $/$ & $u$ & - & $0.1 \mathrm{UG} / \mathrm{L}$ & 1 \\
\hline Endosulfan II & $0.1 \mathrm{UG} \Omega$ & $u$ & - & $0.1 \mathrm{UG} / \mathrm{L}$ & 1 \\
\hline 4,4'-DDD & $0.1 \mathrm{UG} \Omega$ & $u$ & - & 0.1 UG/L & 1 \\
\hline Endosulfan Sulfate & 0.1 UG/ & $u$ & $\cdots$ & $0.1 \mathrm{UG} / \mathrm{L}$ & 1 \\
\hline 4,4'-DDT & $0.1 \mathrm{UG} /$ & $u$ & $\ldots$ & $0.1 \mathrm{UG} \Omega$ & 1 \\
\hline Methoxychlor & $0.5 \mathrm{UG} / \mathrm{L}$ & $u$ & $\ldots$ & 0.5 UG $\Omega$ & 1 \\
\hline Endrin Ketone & $0.1 \mathrm{UG} /$ & $u$ & $\ldots$ & $0.1 \mathrm{UG} / \mathrm{L}$ & 1 \\
\hline alpha-Chlordane & $0.5 \cup G \Omega$ & $u$ & - & 0.5 UG/L & 1 \\
\hline gamma-Chlordane & $0.5 \mathrm{UG} / \mathrm{L}$ & $u$ & - & 0.5 UG $\Omega$ & 1 \\
\hline Toxaphene & 1 UG & $u$ & - & 1 UG/ & 1 \\
\hline Aroclor-1016 & 0.5 UG $\Omega$ & $u$ & - & $0.5 \cup G \Omega$ & 1 \\
\hline Aroclor-1221 & $0.5 \mathrm{UG} \Omega$ & $u$ & - & $0.5 \mathrm{UG} \Omega$ & 1 \\
\hline Aroclor-1232 & 0.5 UG $\Omega$ & $U$ & - & $0.5 \mathrm{UG} /$ & 1 \\
\hline Aroclor-1242 & 0.5 UG $\Omega$ & $u$ & - & 0.5 UGR & 1 \\
\hline Aroclor-1248 & 0.5 UG $/$ & $u$ & -- & $0.5 \mathrm{UG} \Omega$ & 1 \\
\hline Aroclor-1254 & $1 \mathrm{UG} / \mathrm{L}$ & $u$ & - & 1 UG $\Omega$ & 1 \\
\hline Aroclor-1260 & $1 \mathrm{UG} /$ & $u$ & - & 1 UG/L & 1 \\
\hline DBC & $72 \%$ & & & $\%$ & 1 \\
\hline
\end{tabular}


APPENDIX E.2 Results of Pesticide and PCB Analyses, Water,

Salmon Site

\begin{tabular}{|c|c|c|c|c|c|c|}
\hline COMPOUND & RESULTS & UNITS & $\begin{array}{l}\text { DATA } \\
\text { QUAL. }\end{array}$ & $\begin{array}{l}\text { DATA } \\
\text { VALID. }\end{array}$ & CRDL UNITS & DILUTION \\
\hline \multicolumn{7}{|l|}{ C1-15-W } \\
\hline alpha-BHC & 0.05 & UG/ & $u$ & -- & 0.05 UG/L & 1 \\
\hline beta-BHC & 0.05 & UG $\Omega$ & $u$ & -- & 0.05 UG/ & 1 \\
\hline delta-BHC & 0.05 & UG & $u$ & $\cdots$ & 0.05 UG $\Omega$ & 1 \\
\hline gamma-BHC (Lindane) & 0.05 & $U G /$ & $u$ & -- & 0.05 UG/L & 1 \\
\hline Heptachlor & 0.05 & UGR & $u$ & -. & 0.05 UGL & 1 \\
\hline Aldrin & 0.05 & UGR & $u$ & -.- & 0.05 UG/ & 1 \\
\hline Heptachlor Epoxide & 0.05 & UGR & $u$ & --- & 0.05 UGR & 1 \\
\hline Endosulfan I & 0.05 & UG/L & $u$ & --- & 0.05 UG/ & 1 \\
\hline Dieldrin & & UG/L & $u$ & $\ldots$ & 0.1 UG/L & 1 \\
\hline $4,4^{\prime}-D D E$ & & UG/L & $u$ & $\ldots$ & 0.1 UG & 1 \\
\hline Endrin & & UGR & $u$ & -.- & 0.1 UG $\Omega$ & 1 \\
\hline Endosulfan II & & UGR & $u$ & $\ldots$ & $0.1 \mathrm{UG} / \mathrm{L}$ & 1 \\
\hline 4,4'-DDD & & UGR & $u$ & -- & $0.1 \mathrm{UG} /$ & 1 \\
\hline Endosulfan Sulfate & & UG $\Omega$ & u & $\ldots$ & $0.1 \mathrm{UG} /$ & 1 \\
\hline 4,4'-DDT & & UG $\Omega$ & $u$ & -.. & $0.1 \mathrm{UG} / \mathrm{L}$ & 1 \\
\hline Methoxychlor & & UG $\Omega$ & $u$ & -- & 0.5 UG $\Omega$ & 1 \\
\hline Endrin Ketone & & $U G \Omega$ & $u$ & ... & $0.1 \mathrm{UG} /$ & 1 \\
\hline alpha-Chlordane & & UG $\Omega$ & $u$ & $\ldots$ & $0.5 \mathrm{UG} / \mathrm{L}$ & 1 \\
\hline gamma-Chlordane & & UG $\Omega$ & $u$ & -- & 0.5 UGR & 1 \\
\hline Toxaphene & & UG $\Omega$ & $u$ & -- & $1 \mathrm{UG} / \mathrm{L}$ & 1 \\
\hline Aroclor-1016 & & UG $\Omega$ & $u$ & - & 0.5 UG几 & 1 \\
\hline Aroclor-1221 & 0. & UG $/$ & $u$ & $\ldots$ & 0.5 UG $\Omega$ & 1 \\
\hline Aroclor-1232 & 0. & UG/L & $u$ & $\ldots$ & $0.5 \cup G / L$ & 1 \\
\hline Aroclor-1242 & 0. & UG $\Omega$ & $u$ & $\ldots$ & $0.5 \mathrm{UG} / \mathrm{L}$ & 1 \\
\hline Aroclor-1248 & & UG $\Omega$ & $u$ & $\ldots$ & 0.5 UGR & 1 \\
\hline Arocior-1254 & & UG & $u$ & $\ldots$ & 1 UG $\Omega$ & 1 \\
\hline Aroclor- 1260 & & UG/ & $u$ & -- & 1 UG & 1 \\
\hline DBC & 9 & $\%$ & & & $\%$ & 1 \\
\hline
\end{tabular}


APPENDIX E.2 Results of Pesticide and PCB Analyses, Water, Salmon Site

\begin{tabular}{|c|c|c|c|c|}
\hline COMPOUND & RESULTS UNITS & $\begin{array}{l}\text { DATA DATA } \\
\text { QUAL. VALID. }\end{array}$ & CRDL UNITS & DILUTION \\
\hline \multicolumn{5}{|l|}{ C1-15-W-MS } \\
\hline gamma-BHC (Lindane) & $100 \%$ & & $\%$ & 1 \\
\hline Heptachlor & $105 \%$ & & $\%$ & 1 \\
\hline Aldrin & $92 \%$ & & $\%$ & 1 \\
\hline Dieldrin & $99 \%$ & & $\%$ & 1 \\
\hline Endrin & $100 \%$ & & $\%$ & 1 \\
\hline 4,4'-DDT & $79 \%$ & & $\%$ & 1 \\
\hline DBC & $69 \%$ & & $\%$ & 1 \\
\hline \multicolumn{5}{|l|}{ C1-15-W -MSD } \\
\hline gamma-BHC (Lindane) & $110 \%$ & & $\%$ & 1 \\
\hline Heptachlor & $128 \%$ & & $\%$ & 1 \\
\hline Aldrin & $106 \%$ & & $\%$ & 1 \\
\hline Dieldrin & $101 \%$ & & $\%$ & 1 \\
\hline Endrin & $98 \%$ & & $\%$ & 1 \\
\hline 4,4'-DDT & $112 \%$ & & $\%$ & 1 \\
\hline $\mathrm{DBC}$ & $93 \%$ & & $\%$ & 1 \\
\hline
\end{tabular}


APPENDIX E.2 Results of Pesticide and PCB Analyses, Water, Salmon Site

\section{DATA DATA}

COMPOUND RESULTS UNITS QUAL. VALID.

CRDL UNITS DILUTION

C1-16-W

alpha-BHC

beta-BHC

delta-BHC

gamma-BHC (Lindane)

Heptachlor

Aldrin

Heptachlor Epoxide

Endosulfan I

Dieldrin

4,4'-DDE

Endrin

Endosulfan II

4,4'-DDD

Endosulfan Sulfate

4,4'-DDT

Methoxychior

Endrin Ketone

alpha-Chlordane

gamma-Chlordane

Toxaphene

Aroclor-1016

Aroclor-1221

Aroclor-1232

Aroclor-1242

Aroclor-1248

Aroclor-1254

Arocior-1260

DBC

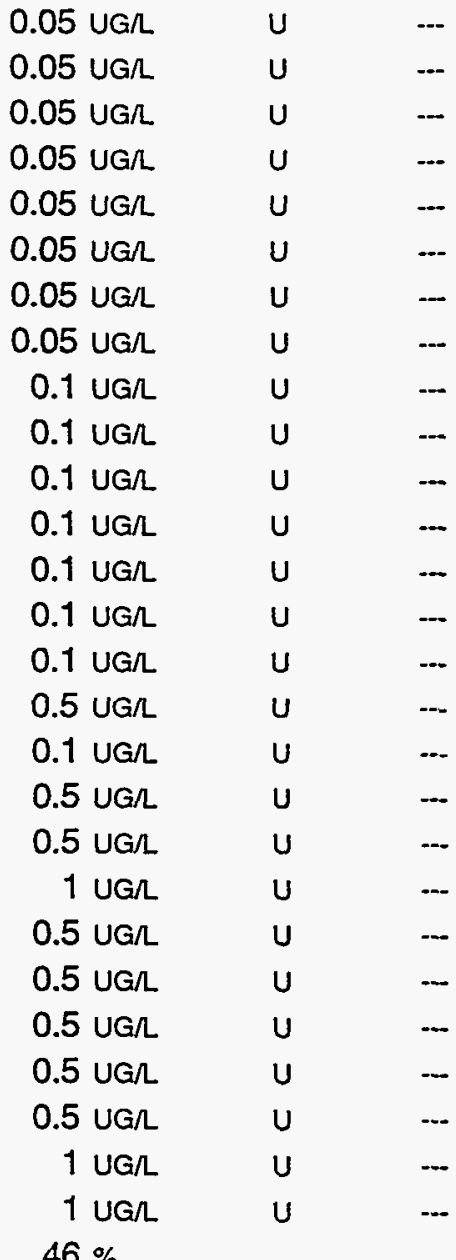

0.05 UG/

0.05 UG/

0.05 UGR

0.05 UGA

0.05 UG/

0.05 UG/L

0.05 UG/

0.05 UG/

0.1 UGR

0.1 UG/

0.1 UGR

0.1 UGR

0.1 UG/

0.1 UGR

0.1 UGR

$0.5 \mathrm{UG} / \mathrm{L}$

$0.1 \mathrm{UG} / \mathrm{L}$

$0.5 \mathrm{UG} / \mathrm{L}$

0.5 UG/

$1 \mathrm{UG \Omega}$

$0.5 \mathrm{UGR}$

$0.5 \mathrm{UG} \Omega$

$0.5 \mathrm{UG} / \mathrm{L}$

0.5 UG/

0.5 UG/L

1 UGR

1 UGR

$\%$

1 
APPENDIX E.2 Results of Pesticide and PCB Analyses, Water,

Salmon Site

\begin{tabular}{|c|c|c|c|c|c|}
\hline COMPOUND & RESULTS UNITS & $\begin{array}{l}\text { DATA } \\
\text { QUAL. }\end{array}$ & $\begin{array}{l}\text { DATA } \\
\text { VALID. }\end{array}$ & CRDL UNITS & DILUTION \\
\hline \multicolumn{6}{|l|}{ C1-17-W } \\
\hline alpha-BHC & 0.05 UG/L & $u$ & - & 0.05 UG/ & 1 \\
\hline beta-BHC & 0.05 UG/L & $u$ & - & 0.05 UG/ & 1 \\
\hline delta-BHC & 0.05 UG $\Omega$ & $u$ & - & 0.05 UG/L & 1 \\
\hline gamma-BHC (Lindane) & 0.05 UG $\Omega$ & $u$ & - & 0.05 UG/L & 1 \\
\hline Heptachlor & 0.05 UG $\Omega$ & $u$ & - & 0.05 UG $\Omega$ & 1 \\
\hline Aldrin & 0.05 UG $\Omega$ & $u$ & $\ldots$ & 0.05 UG $\Omega$ & 1 \\
\hline Heptachlor Epoxide & 0.05 UGL & $u$ & - & 0.05 UGL & 1 \\
\hline Endosulfan I & 0.05 UG/L & $u$ & - & $0.05 \mathrm{UG} \Omega$ & 1 \\
\hline Dieldrin & 0.1 UG & $u$ & - & 0.1 UGR & 1 \\
\hline 4,4'-DDE & 0.1 UG $\Omega$ & $u$ & $\ldots$ & 0.1 UG $\Omega$ & 1 \\
\hline Endrin & 0.1 UG $\Omega$ & $u$ & - & 0.1 UG $\Omega$ & 1 \\
\hline Endosulfan II & $0.1 \mathrm{UG} \Omega$ & $u$ & $\ldots$ & 0.1 UG/L & 1 \\
\hline 4,4'-DDD & 0.1 UG & $u$ & - & $0.1 \mathrm{UG} / \mathrm{L}$ & 1 \\
\hline Endosulfan Sulfate & $0.1 \mathrm{UG} / \mathrm{L}$ & $u$ & - & $0.1 \mathrm{UG} / \mathrm{L}$ & 1 \\
\hline 4,4'-DDT & $0.1 \mathrm{UG} / \mathrm{L}$ & $u$ & - & 0.1 UG/ & 1 \\
\hline Methoxychlor & $0.5 \mathrm{UG} \Omega$ & $u$ & - & 0.5 UG $\Omega$ & 1 \\
\hline Endrin Ketone & $0.1 \mathrm{UG} \Omega$ & $u$ & $\ldots$ & $0.1 \mathrm{UG} \Omega$ & 1 \\
\hline alpha-Chlordane & 0.5 UGR & $u$ & $\ldots$ & 0.5 UG $\Omega$ & 1 \\
\hline gamma-Chlordane & 0.5 UGR & $u$ & - & 0.5 UG $\Omega$ & 1 \\
\hline Toxaphene & 1 UG & $u$ & $\ldots$ & 1 UG $\Omega$ & 1 \\
\hline Aroclor-1016 & $0.5 \mathrm{UG} / \mathrm{L}$ & u & $\ldots$ & 0.5 UG $\Omega$ & 1 \\
\hline Aroclor-1221 & 0.5 UG/ & $u$ & - & $0.5 \mathrm{UG} \Omega$ & 1 \\
\hline Arocior-1232 & 0.5 UG & $u$ & - & 0.5 UG & 1 \\
\hline Aroclor-1242 & $0.5 \mathrm{UG} / \mathrm{L}$ & $u$ & - & 0.5 UG/ & 1 \\
\hline Aroclor-1248 & 0.5 UG/L & $u$ & - & 0.5 UG/L & 1 \\
\hline Aroclor-1254 & $1 \mathrm{UG} /$ & $u$ & - & $1 \mathrm{UG} /$ & 1 \\
\hline Aroclor-1260 & $1 \mathrm{UG} /$ & $u$ & $\ldots$ & 1 UG/ & 1 \\
\hline $\mathrm{DBC}$ & $86 \%$ & & & $\%$ & 1 \\
\hline
\end{tabular}


APPENDIX E.2 Results of Pesticide and PCB Analyses, Water,

Salmon Site

\begin{tabular}{|c|c|c|c|c|c|c|}
\hline COMPOUND & RESULTS & UNITS & $\begin{array}{l}\text { DATA } \\
\text { QUAL. }\end{array}$ & $\begin{array}{r}\text { DATA } \\
\text { VALID. } \\
\end{array}$ & CRDL UNITS & DILUTION \\
\hline \multicolumn{7}{|l|}{ C1-18-W } \\
\hline alpha-BHC & 0.052 & UG/ & $u$ & --- & 0.052 UG/ & 1 \\
\hline beta-BHC & 0.052 & UG/L & $u$ & -- & 0.052 UG/ & 1 \\
\hline delta-BHC & 0.052 & $U G \Omega$ & $u$ & -- & 0.052 UG/L & 1 \\
\hline gamma-BHC (Lindane) & 0.052 & UG/L & $u$ & -- & 0.052 UG/L & 1 \\
\hline Heptachlor & 0.052 & UGR & $u$ & --- & 0.052 UG/ & 1 \\
\hline Aldrin & 0.052 & UG $\Omega$ & $u$ & -- & $0.052 \mathrm{UG} / \mathrm{L}$ & 1 \\
\hline Heptachlor Epoxide & 0.052 & UG $/$ & $u$ & -- & 0.052 UG/ & 1 \\
\hline Endosulfan l & 0.05 & UG $/$ & $u$ & - & 0.052 UGR & 1 \\
\hline Dieldrin & & UG/L & $u$ & -- & 0.1 UG & 1 \\
\hline $4,4^{\prime}-\mathrm{DDE}$ & & UG/L & $u$ & - & 0.1 UGR & 1 \\
\hline Endrin & & UG $\Omega$ & $u$ & $\cdots$ & 0.1 UG/L & 1 \\
\hline Endosulfan II & & UG/L & $u$ & - & $0.1 \mathrm{UG} /$ & 1 \\
\hline 4,4'-DDD & & UG/L & $u$ & - & 0.1 UG/ & 1 \\
\hline Endosulfan Sulfate & & UG/ & $u$ & $\ldots$ & $0.1 \mathrm{UG} / \mathrm{L}$ & 1 \\
\hline $4,4^{\prime}-\mathrm{DDT}$ & & UG/L & $u$ & -- & 0.1 UG/ & 1 \\
\hline Methoxychlor & 0.5 & UG/L & $u$ & -- & 0.52 UG/ & 1 \\
\hline Endrin Ketone & & $U G / L$ & $u$ & -- & 0.1 UG & 1 \\
\hline alpha-Chlordane & 0.5 & $U G /$ & $u$ & - & 0.52 UG/ & 1 \\
\hline gamma-Chlordane & 0.5 & UG & $u$ & $\cdots$ & 0.52 UG几 & 1 \\
\hline Toxaphene & & UG $\Omega$ & $u$ & -- & 1 UG几 & 1 \\
\hline Aroclor-1016 & 0.5 & UG $/$ & $u$ & $\ldots$ & 0.52 UG $\Omega$ & 1 \\
\hline Aroclor-1221 & 0.5 & UGR & $u$ & $\ldots$ & 0.52 UG/ & 1 \\
\hline Aroclor-1232 & 0.5 & $U G /$ & $u$ & - & 0.52 UG/ & 1 \\
\hline Aroclor-1242 & 0.5 & UG/L & $u$ & - & 0.52 UG/ & 1 \\
\hline Arocior-1248 & 0.5 & UG/L & $u$ & --- & 0.52 UG/L & 1 \\
\hline Aroclor-1254 & & $U G / 2$ & $u$ & -- & 1 UG/ & 1 \\
\hline Aroclor-1260 & & $U G / L$ & $u$ & -- & $1 \mathrm{UG} \Omega$ & 1 \\
\hline $\mathrm{DBC}$ & 3 & $\%$ & & & $\%$ & 1 \\
\hline
\end{tabular}




\section{APPENDIX E.2 Results of Pesticide and PCB Analyses, Water,}

Salmon Site

\begin{tabular}{|c|c|c|c|c|c|}
\hline COMPOUND & RESULTS UNITS & $\begin{array}{l}\text { DATA } \\
\text { QUAL. }\end{array}$ & $\begin{array}{r}\text { DATA } \\
\text { VALID. }\end{array}$ & CRDL UNITS & DILUTION \\
\hline \multicolumn{6}{|l|}{ C2-19-W } \\
\hline alpha-BHC & $0.052 \mathrm{UG} / \mathrm{L}$ & $u$ & NP & 0.052 UGR & 1 \\
\hline beta-BHC & 0.12 UG/L & & NP & $0.052 \mathrm{UG} / \mathrm{L}$ & 1 \\
\hline delta-BHC & 0.052 UG/L & $u$ & NP & 0.052 UG/L & 1 \\
\hline gamma-BHC (Lindane) & 0.052 UGR & $u$ & NP & 0.052 UG $\Omega$ & 1 \\
\hline Heptachlor & 0.052 UG/ & $u$ & NP & 0.052 UG $\Omega$ & 1 \\
\hline Aldrin & $0.067 \mathrm{UG} / \mathrm{L}$ & & NP & 0.052 UG/ & 1 \\
\hline Heptachlor Epoxide & 0.12 UG/ & & NP & 0.052 UG $\Omega$ & 1 \\
\hline Endosulfan I & $0.052 \mathrm{UG} \Omega$ & $u$ & NP & 0.052 UG/ & 1 \\
\hline Dieldrin & 0.1 UG & $u$ & NP & 0.1 UG/ & 1 \\
\hline 4,4'-DDE & 0.1 UG & $u$ & NP & 0.1 UG/ & 1 \\
\hline Endrin & 0.1 UG & $u$ & NP & $0.1 \mathrm{UG} \Omega$ & 1 \\
\hline Endosulfan II & 0.1 UG & $u$ & NP & $0.1 \mathrm{UG} / \mathrm{L}$ & 1 \\
\hline $4,4^{\prime}-\mathrm{DDD}$ & $0.1 \mathrm{UG} /$ & $u$ & NP & 0.1 UG/L & 1 \\
\hline Endosulfan Sulfate & 0.1 UG/ & $u$ & NP & $0.1 \mathrm{UG} / \mathrm{L}$ & 1 \\
\hline 4,4'-DDT & 0.1 UG $\Omega$ & $u$ & NP & 0.1 UG & 1 \\
\hline Methoxychlor & 0.52 UG $\Omega$ & $u$ & NP & $0.52 \mathrm{UG} / \mathrm{L}$ & 1 \\
\hline Endrin Ketone & 0.1 UG/ & $u$ & NP & 0.1 UG $\Omega$ & 1 \\
\hline alpha-Chlordane & 0.52 UGL & $u$ & NP & 0.52 UG $\Omega$ & 1 \\
\hline gamma-Chlordane & 0.52 UG/L & $u$ & $\mathrm{NP}$ & 0.52 UG $\Omega$ & 1 \\
\hline Toxaphene & 1 UGR & $u$ & NP & 1 UG & 1 \\
\hline Aroclor-1016 & 0.52 UGR & $u$ & NP & 0.52 UG $\Omega$ & 1 \\
\hline Aroclor-1221 & 0.52 UGL & $u$ & NP & 0.52 UG $/$ & 1 \\
\hline Aroclor-1232 & 0.52 UG $\Omega$ & $u$ & NP & 0.52 UG $\Omega$ & 1 \\
\hline Arocior-1242 & 0.52 UG $\Omega$ & $u$ & NP & 0.52 UG/ & 1 \\
\hline Aroclor-1248 & 0.52 UG $\Omega$ & $u$ & NP & $0.52 \mathrm{UG} / \mathrm{L}$ & 1 \\
\hline Aroclor-1254 & 1 UG/ & $u$ & NP & $1 \mathrm{UG} \Omega$ & 1 \\
\hline Aroclor-1260 & 1 UG $\Omega$ & $u$ & NP & 1 UG $\Omega$ & 1 \\
\hline $\mathrm{DBC}$ & $97 \%$ & & & $\%$ & 1 \\
\hline
\end{tabular}


APPENDIX E.2 Results of Pesticide and PCB Analyses, Water, Salmon Site

\begin{tabular}{|c|c|c|c|c|}
\hline COMPOUND & RESULTS UNITS & $\begin{array}{l}\text { DATA DATA } \\
\text { QUAL. VALID. }\end{array}$ & CRDL UNITS & DILUTION \\
\hline \multicolumn{5}{|l|}{ C2-19-W-MS } \\
\hline gamma-BHC (Lindane) & $165 \%$ & * & $\%$ & 1 \\
\hline Heptachlor & $138 \%$ & * & $\%$ & 1 \\
\hline Aldrin & $97 \%$ & & $\%$ & 1 \\
\hline Dieldrin & $141 \%$ & * & $\%$ & 1 \\
\hline Endrin & $166 \%$ & * & $\%$ & 1 \\
\hline 4,4'-DDT & $123 \%$ & & $\%$ & 1 \\
\hline DBC & $136 \%$ & & $\%$ & 1 \\
\hline \multicolumn{5}{|l|}{ C2-19-W-MSD } \\
\hline gamma-BHC (Lindane) & $180 \%$ & - & $\%$ & 1 \\
\hline Heptachlor & $146 \%$ & * & $\%$ & 1 \\
\hline Aldrin & $112 \%$ & & $\%$ & 1 \\
\hline Dieldrin & $142 \%$ & * & $\%$ & 1 \\
\hline Endrin & $157 \%$ & * & $\%$ & 1 \\
\hline 4,4'-DDT & $99 \%$ & & $\%$ & 1 \\
\hline $\mathrm{DBC}$ & $120 \%$ & & $\%$ & 1 \\
\hline
\end{tabular}


APPENDIX E.2 Results of Pesticide and PCB Analyses, Water, Salmon Site

\begin{tabular}{|c|c|c|c|c|c|}
\hline COMPOUND & RESULTS UNITS & $\begin{array}{l}\text { DATA } \\
\text { QUAL. }\end{array}$ & $\begin{array}{l}\text { DATA } \\
\text { VALID. }\end{array}$ & CRDL UNITS & DILUTION \\
\hline \multicolumn{6}{|l|}{ C2-27-W } \\
\hline alpha-BHC & 0.062 UG/ & $u$ & NP & 0.062 UG/ & 1 \\
\hline beta-BHC & 0.062 UG/ & $u$ & NP & 0.062 UG/ & 1 \\
\hline delta-BHC & 0.062 UG $\Omega$ & $u$ & NP & 0.062 UGL & 1 \\
\hline gamma-BHC (Lindane) & 0.062 UG & $u$ & NP & 0.062 UG/L & 1 \\
\hline Heptachlor & 0.062 UG/ & $u$ & NP & 0.062 UG/L & 1 \\
\hline Aldrin & 0.062 UG/ & $u$ & NP & 0.062 UG/L & 1 \\
\hline Heptachlor Epoxide & 0.062 UG/L & $u$ & NP & 0.062 UG/L & 1 \\
\hline Endosulfan I & 0.062 UG/ & $u$ & NP & 0.062 UG/ & 1 \\
\hline Dieldrin & 0.12 UG/ & $u$ & NP & $0.12 \mathrm{UG} / \mathrm{L}$ & 1 \\
\hline 4,4'-DDE & 0.12 UG $\Omega$ & $u$ & NP & 0.12 UG/ & 1 \\
\hline Endrin & 0.12 UG/ & $u$ & NP & 0.12 UG $\Omega$ & 1 \\
\hline Endosulfan II & 0.12 UG $\Omega$ & $u$ & NP & $0.12 \mathrm{UG} / \mathrm{L}$ & 1 \\
\hline 4,4'-DDD & 0.12 UG & $u$ & NP & 0.12 UG/ & 1 \\
\hline Endosulfan Sulfate & 0.12 UG/ & $u$ & NP & 0.12 UG/L & 1 \\
\hline 4,4'-DDT & 0.12 UG/L & $u$ & NP & 0.12 UG & 1 \\
\hline Methoxychlor & 0.62 UG $\Omega$ & $u$ & NP & 0.62 UGR & 1 \\
\hline Endrin Ketone & 0.12 UG/ & $u$ & NP & 0.12 UG/L & 1 \\
\hline alpha-Chlordane & 0.62 UG/ & $u$ & NP & 0.62 UG/L & 1 \\
\hline gamma-Chlordane & 0.62 UG/L & $u$ & NP & 0.62 UG/ & 1 \\
\hline Toxaphene & $1.2 \mathrm{UG} \Omega$ & $u$ & NP & $1.2 \mathrm{UG} / \mathrm{L}$ & 1 \\
\hline Aroclor-1016 & 0.62 UG/ & $u$ & NP & $0.62 \mathrm{UG} \Omega$ & 1 \\
\hline Aroclor-1221 & 0.62 UG & $u$ & NP & $0.62 \mathrm{UG} / \mathrm{L}$ & 1 \\
\hline Aroclor-1232 & $0.62 \mathrm{UG} \Omega$ & $u$ & NP & 0.62 UG/L & 1 \\
\hline Aroclor-1242 & 0.62 UG $\Omega$ & $u$ & NP & 0.62 UG $/$ & 1 \\
\hline Aroclor-1248 & 0.62 UG & $u$ & NP & 0.62 UG/L & 1 \\
\hline Aroclor-1254 & 1.2 UG/ & $u$ & NP & $1.2 \mathrm{UG} / \mathrm{L}$ & 1 \\
\hline Aroclor-1260 & 1.2 UG $\Omega$ & $u$ & NP & 1.2 UGR & 1 \\
\hline $\mathrm{DBC}$ & $23 \%$ & $*$ & & $\%$ & 1 \\
\hline
\end{tabular}


APPENDIX E.2 Results of Pesticide and PCB Analyses, Water,

Salmon Site

\begin{tabular}{|c|c|c|c|c|c|}
\hline COMPOUND & RESULTS UNITS & $\begin{array}{l}\text { DATA } \\
\text { QUAL. }\end{array}$ & $\begin{array}{l}\text { DATA } \\
\text { VALID. } \\
\end{array}$ & CRDL UNITS & DILUTION \\
\hline \multicolumn{6}{|l|}{ C-FB-001-W } \\
\hline alpha-BHC & 0.05 UG $\Omega$ & $u$ & $\ldots$ & 0.05 UG/ & 1 \\
\hline beta-BHC & 0.05 UG $\Omega$ & $u$ & -.. & 0.05 UG/ & 1 \\
\hline delta-BHC & 0.05 UG $\Omega$ & $u$ & --- & 0.05 UG $\Omega$ & 1 \\
\hline gamma-BHC (Lindane) & 0.05 UG/L & $u$ & -- & 0.05 UG $\Omega$ & 1 \\
\hline Heptachlor & 0.05 UG/ & $u$ & -- & 0.05 UGR & 1 \\
\hline Aldrin & 0.05 UG $\Omega$ & $u$ & -- & 0.05 UG/ & 1 \\
\hline Heptachlor Epoxide & $0.05 \mathrm{UG} / \mathrm{L}$ & $u$ & $\ldots$ & 0.05 UG $\Omega$ & 1 \\
\hline Endosulfan I & 0.05 UGR & $u$ & - & 0.05 UG & 1 \\
\hline Dieldrin & 0.1 UGR & $u$ & -- & $0.1 \mathrm{UG} /$ & 1 \\
\hline $4,4^{\prime}-\mathrm{DDE}$ & $0.1 \mathrm{UG} / \mathrm{L}$ & $u$ & $\ldots$ & 0.1 UGR & 1 \\
\hline Endrin & 0.1 UG/L & $u$ & -- & 0.1 UG $\Omega$ & 1 \\
\hline Endosulfan II & 0.1 UG/ & $u$ & -- & 0.1 UGR & 1 \\
\hline 4,4'-DDD & 0.1 UG几 & $u$ & -.- & 0.1 UG/ & 1 \\
\hline Endosulfan Sulfate & 0.1 UG/ & $u$ & $\ldots$ & 0.1 UG & 1 \\
\hline 4,4'-DDT & 0.1 UG & $u$ & -- & $0.1 \mathrm{UG} \Omega$ & 1 \\
\hline Methoxychlor & 0.5 UGん & $u$ & $\ldots$ & 0.5 UG/ & 1 \\
\hline Endrin Ketone & 0.1 UG & $u$ & -- & 0.1 UG $\Omega$ & 1 \\
\hline alpha-Chlordane & 0.5 UG & $u$ & $\ldots$ & 0.5 UG & 1 \\
\hline gamma-Chlordane & $0.5 \mathrm{UG} \Omega$ & $u$ & $\cdots$ & $0.5 \mathrm{UG} / \mathrm{L}$ & 1 \\
\hline Toxaphene & 1 UGR & $u$ & $\ldots$ & 1 UG/ & 1 \\
\hline Aroclor-1016 & 0.5 UG/ & $u$ & $\ldots$ & 0.5 Uan & 1 \\
\hline Aroclor-1221 & 0.5 UGR & $u$ & $\ldots$ & 0.5 UG/ & 1 \\
\hline Aroclor-1232 & 0.5 UG/L & $u$ & -- & 0.5 UG/ & 1 \\
\hline Aroclor-1242 & $0.5 \mathrm{UG} / \mathrm{L}$ & $u$ & $\cdots$ & 0.5 UG/L & 1 \\
\hline Aroclor-1248 & 0.5 UG/ & $u$ & -. & 0.5 UG/L & 1 \\
\hline Aroclor-1254 & 1 UG/ & $u$ & - & 1 UG/ & 1 \\
\hline Aroclor-1260 & 1 UG/L & $u$ & - & 1 UG/ & 1 \\
\hline DBC & $90 \%$ & & & $\%$ & 1 \\
\hline
\end{tabular}




\section{APPENDIX E.2 Results of Pesticide and PCB Analyses, Water, Salmon Site}

\begin{tabular}{|c|c|c|c|c|c|}
\hline COMPOUND & RESULTS UNITS & $\begin{array}{l}\text { DATA } \\
\text { QUAL. }\end{array}$ & $\begin{array}{l}\text { DATA } \\
\text { VALID. }\end{array}$ & CRDL UNITS & DILUTION \\
\hline \multicolumn{6}{|l|}{ C-FB-002-W } \\
\hline alpha-BHC & 0.05 UG/ & $u$ & NP & 0.05 UG/L & 1 \\
\hline beta-BHC & 0.05 UG/L & $u$ & NP & 0.05 UG $/$ & 1 \\
\hline delta-BHC & 0.05 UG/L & $u$ & NP & 0.05 UG $\Omega$ & 1 \\
\hline gamma-BHC (Lindane) & 0.05 UG $\Omega$ & $u$ & NP & 0.05 UG $\Omega$ & 1 \\
\hline Heptachlor & 0.05 UG $\Omega$ & $u$ & NP & $0.05 \mathrm{UG} / \mathrm{L}$ & 1 \\
\hline Aldrin & 0.05 UGR & $u$ & NP & 0.05 UG/L & 1 \\
\hline Heptachlor Epoxide & $0.05 \mathrm{UG} / \mathrm{L}$ & $u$ & NP & $0.05 \mathrm{UG} / \mathrm{L}$ & 1 \\
\hline Endosulfan I & 0.05 UG/L & $u$ & NP & 0.05 UG $\Omega$ & 1 \\
\hline Dieldrin & $0.1 \mathrm{UG} / \mathrm{L}$ & $u$ & NP & $0.1 \mathrm{UG} \Omega$ & 1 \\
\hline 4,4'-DDE & $0.1 \mathrm{UG} \Omega$ & $u$ & NP & $0.1 \mathrm{UG} / \mathrm{L}$ & 1 \\
\hline Endrin & 0.1 UGR & $u$ & NP & 0.1 UG/ & 1 \\
\hline Endosulfan II & $0.1 \mathrm{UG} / \mathrm{L}$ & $u$ & NP & $0.1 \mathrm{UG} / \mathrm{L}$ & 1 \\
\hline 4,4'-DDD & $0.1 \mathrm{UG} / \mathrm{L}$ & $u$ & NP & $0.1 \mathrm{UG} \Omega$ & 1 \\
\hline Endosulfan Sulfate & $0.1 \mathrm{UG} / \mathrm{L}$ & $u$ & NP & 0.1 UGR & 1 \\
\hline 4,4'-DDT & $0.1 \mathrm{UG} / \mathrm{L}$ & $u$ & NP & 0.1 UG $\Omega$ & 1 \\
\hline Methoxychlor & $0.5 \mathrm{UG} \Omega$ & $u$ & NP & 0.5 UG几 & 1 \\
\hline Endrin Ketone & $0.1 \mathrm{UG} / \mathrm{L}$ & $u$ & NP & 0.1 UGR & 1 \\
\hline alpha-Chlordane & 0.5 UG/ & $u$ & NP & $0.5 \mathrm{UG} / \mathrm{L}$ & 1 \\
\hline gamma-Chlordane & $0.5 \mathrm{UG} / \mathrm{L}$ & $u$ & NP & 0.5 UG $\Omega$ & 1 \\
\hline Toxaphene & 1 UG/ & $u$ & NP & 1 UG & 1 \\
\hline Aroclor-1016 & 0.5 UG/ & $u$ & NP & $0.5 \mathrm{UG} \Omega$ & 1 \\
\hline Aroclor-1221 & $0.5 \mathrm{UG} / \mathrm{L}$ & $u$ & NP & $0.5 \mathrm{UG} / \mathrm{L}$ & 1 \\
\hline Aroclor-1232 & $0.5 \mathrm{UG} \Omega$ & $u$ & NP & $0.5 \mathrm{UG} /$ & 1 \\
\hline Aroclor-1242 & $0.5 \mathrm{UG} / \mathrm{L}$ & $u$ & NP & 0.5 UG $\Omega$ & 1 \\
\hline Aroclor-1248 & 0.5 UG几 & $u$ & NP & 0.5 UG & 1 \\
\hline Aroclor-1254 & 1 UG/ & $u$ & NP & $1 \mathrm{UG} \Omega$ & 1 \\
\hline Aroclor-1260 & $1 \mathrm{UG} \Omega$ & $u$ & NP & $1 \mathrm{UG} / \mathrm{L}$ & 1 \\
\hline DBC & $99 \%$ & & & $\%$ & 1 \\
\hline
\end{tabular}


APPENDIX E.2 Results of Pesticide and PCB Analyses, Water, Salmon Site

\begin{tabular}{|c|c|c|c|c|c|}
\hline COMPOUND & RESULTS UNITS & $\begin{array}{l}\text { DATA } \\
\text { QUAL. }\end{array}$ & $\begin{array}{l}\text { DATA } \\
\text { VALID. }\end{array}$ & CRDL UNITS & DILUTION \\
\hline \multicolumn{6}{|l|}{ C-FB-003-W } \\
\hline alpha-BHC & 0.05 UG/ & $u$ & NP & 0.05 UG/ & 1 \\
\hline beta-BHC & 0.05 UG $\Omega$ & $u$ & NP & 0.05 UG/L & 1 \\
\hline delta-BHC & 0.05 UG $\Omega$ & $u$ & NP & 0.05 UG/ & 1 \\
\hline gamma-BHC (Lindane) & 0.05 UG/L & $u$ & NP & $0.05 \mathrm{UG} / \mathrm{L}$ & 1 \\
\hline Heptachlor & 0.05 UGR & $u$ & NP & 0.05 UG/L & 1 \\
\hline Aldrin & 0.05 UGA & $u$ & NP & 0.05 UG $\Omega$ & 1 \\
\hline Heptachlor Epoxide & $0.05 \mathrm{UG} / \mathrm{L}$ & $u$ & NP & 0.05 UG $\Omega$ & 1 \\
\hline Endosulfan I & $0.05 \mathrm{UG} / \mathrm{L}$ & $u$ & NP & 0.05 UG/ & 1 \\
\hline Dieldrin & $0.1 \mathrm{UG} /$ & $u$ & NP & 0.1 UG/ & 1 \\
\hline $4,4^{\prime}-\mathrm{DDE}$ & $0.1 \mathrm{UG} /$ & $u$ & NP & $0.1 \mathrm{UG} / \mathrm{L}$ & 1 \\
\hline Endrin & $0.1 \mathrm{UG} / \mathrm{L}$ & $u$ & NP & 0.1 UGR & 1 \\
\hline Endosulfan II & $0.1 \mathrm{UG} /$ & $u$ & NP & 0.1 UG/L & 1 \\
\hline $4,4^{\prime}-\mathrm{DDD}$ & 0.1 UG/ & $u$ & NP & $0.1 \mathrm{UG} / \mathrm{L}$ & 1 \\
\hline Endosulfan Sulfate & $0.1 \mathrm{UG} / \mathrm{L}$ & $u$ & $\mathrm{NP}$ & $0.1 \mathrm{UG} / \mathrm{L}$ & 1 \\
\hline $4,4^{\prime}-\mathrm{DDT}$ & 0.1 UG & $u$ & NP & $0.1 \mathrm{UG} / \mathrm{L}$ & 1 \\
\hline Methoxychlor & $0.5 \mathrm{UG} / \mathrm{L}$ & $u$ & NP & 0.5 UG & 1 \\
\hline Endrin Ketone & $0.1 \mathrm{UG} / \mathrm{L}$ & $u$ & NP & 0.1 UG/L & 1 \\
\hline alpha-Chlordane & 0.5 UG/ & $u$ & $N P$ & 0.5 UG/ & 1 \\
\hline gamma-Chlordane & 0.5 UG/L & $u$ & NP & 0.5 UG & 1 \\
\hline Toxaphene & $1 \mathrm{UG} / \mathrm{L}$ & $u$ & NP & $1 \mathrm{UG} / \mathrm{L}$ & 1 \\
\hline Aroclor-1016 & $0.5 \mathrm{UG} / \mathrm{L}$ & $u$ & NP & 0.5 UG/ & 1 \\
\hline Aroclor-1221 & $0.5 \mathrm{UG} / \mathrm{L}$ & $u$ & NP & 0.5 UGR & 1 \\
\hline Aroclor-1232 & 0.5 UG & $u$ & NP & 0.5 UG/L & 1 \\
\hline Aroclor-1242 & 0.5 UG & $u$ & NP & 0.5 UG $\Omega$ & 1 \\
\hline Arocior-1248 & 0.5 UG $\Omega$ & $u$ & NP & 0.5 UG & 1 \\
\hline Arocior-1254 & $1 \mathrm{UG} /$ & $u$ & NP & $1 \mathrm{UG} \Omega$ & 1 \\
\hline Arocior-1260 & 1.05 UG/L & $u$ & NP & $1 \mathrm{UG} / \mathrm{L}$ & 1 \\
\hline $\mathrm{DBC}$ & $93 \%$ & & & $\%$ & 1 \\
\hline
\end{tabular}




\section{APPENDIX E.2 Results of Pesticide and PCB Analyses, Water,}

Salmon Site

\begin{tabular}{|c|c|c|c|c|c|}
\hline COMPOUND & RESULTS UNITS & $\begin{array}{l}\text { DATA } \\
\text { QUAL. }\end{array}$ & $\begin{array}{l}\text { DATA } \\
\text { VALID. }\end{array}$ & CRDL UNITS & DILUTION \\
\hline \multicolumn{6}{|l|}{ C-RB-001-W } \\
\hline alpha-BHC & 0.05 UG $\Omega$ & $u$ & $\ldots$ & $0.05 \mathrm{UG} / \mathrm{L}$ & 1 \\
\hline beta-BHC & 0.05 UGL & $u$ & - & $0.05 \mathrm{UG} / \mathrm{L}$ & 1 \\
\hline delta-BHC & 0.05 UG/ & $u$ & - & 0.05 UG $\Omega$ & 1 \\
\hline gamma-BHC (Lindane) & $0.05 \mathrm{UG} / \mathrm{L}$ & $u$ & - & 0.05 UG/ & 1 \\
\hline Heptachlor & 0.05 UG $\Omega$ & $u$ & - & 0.05 UG/L & 1 \\
\hline Aldrin & 0.05 UGR. & $u$ & - & 0.05 UG $\Omega$ & 1 \\
\hline Heptachlor Epoxide & 0.05 UGR & $u$ & - & 0.05 UGR & 1 \\
\hline Endosulfan I & 0.05 UGL & $u$ & - & 0.05 UG $\Omega$ & 1 \\
\hline Dieldrin & $0.1 \mathrm{UG} / \mathrm{L}$ & $u$ & - & 0.1 UGL & 1 \\
\hline 4,4'-DDE & $0.1 \mathrm{UG} / \mathrm{L}$ & $u$ & - & 0.1 UG $\Omega$ & 1 \\
\hline Endrin & 0.1 UG & $u$ & - & 0.1 UG $\Omega$ & 1 \\
\hline Endosulfan II & $0.1 \mathrm{UG} \Omega$ & $u$ & - & 0.1 UG $\Omega$ & 1 \\
\hline 4,4'-DDD & 0.1 UG $\Omega$ & $u$ & - & 0.1 UGR & 1 \\
\hline Endosulfan Sulfate & $0.1 \mathrm{UG} / \mathrm{L}$ & $u$ & - & $0.1 \mathrm{UG} /$ & 1 \\
\hline $4,4^{4}-\mathrm{DDT}$ & $0.1 \mathrm{UG} \Omega$ & $u$ & $\ldots$ & $0.1 \mathrm{UG} /$ & 1 \\
\hline Methoxychlor & 0.5 UG $/$ & $u$ & $\ldots$ & 0.5 UG & 1 \\
\hline Endrin Ketone & 0.1 UG/ & $u$ & - & $0.1 \mathrm{UG} \Omega$ & 1 \\
\hline alpha-Chlordane & 0.5 UG/ & $u$ & $\ldots$ & 0.5 UG $\Omega$ & 1 \\
\hline gamma-Chlordane & $0.5 \mathrm{UG} \Omega$ & $u$ & - & 0.5 UG $\Omega$ & 1 \\
\hline Toxaphene & 1 UG/ & $u$ & - & 1 UG $\Omega$ & 1 \\
\hline Aroclor-1016 & $0.5 \mathrm{UG} \Omega$ & $u$ & - & 0.5 UG $/$ & 1 \\
\hline Aroclor-1221 & $0.5 \mathrm{UG} \Omega$ & $u$ & -- & $0.5 \mathrm{UG} \Omega$ & 1 \\
\hline Aroclor-1232 & $0.5 \mathrm{UG} / \mathrm{L}$ & $u$ & - & 0.5 UG几 & 1 \\
\hline Aroclor-1242 & $0.5 \mathrm{UG} / \mathrm{L}$ & $u$ & - & 0.5 UG $\Omega$ & 1 \\
\hline Aroclor-1248 & $0.5 \mathrm{UG} /$ & $u$ & - & $0.5 \mathrm{UG} / \mathrm{L}$ & 1 \\
\hline Aroclor-1254 & 1 UG $\Omega$ & $u$ & $\ldots$ & $1 \mathrm{UG} \Omega$ & 1 \\
\hline Aroclor-1260 & $1 \mathrm{UG} \Omega$ & $u$ & - & 1 UG & 1 \\
\hline $\mathrm{DBC}$ & $46 \%$ & & & $\%$ & 1 \\
\hline
\end{tabular}


APPENDIX E.2 Results of Pesticide and PCB Analyses, Water, Salmon Site

\begin{tabular}{|c|c|c|c|c|c|c|}
\hline COMPOUND & RESULTS & UNITS & $\begin{array}{l}\text { DATA } \\
\text { QUAL. }\end{array}$ & $\begin{array}{l}\text { DATA } \\
\text { VALID. }\end{array}$ & CRDL UNITS & DILUTION \\
\hline \multicolumn{7}{|l|}{ C-RB-002-W } \\
\hline alpha-BHC & 0.05 & 5 UG/ & $u$ & NP & 0.05 UGA & 1 \\
\hline beta-BHC & 0.05 & 5 UG/ & $u$ & NP & 0.05 UG/L & 1 \\
\hline delta-BHC & 0.05 & 5 UG/ & $u$ & NP & 0.05 UG $\Omega$ & 1 \\
\hline gamma-BHC (Lindane) & 0.05 & 5 UG/ & $u$ & NP & 0.05 UG & 1 \\
\hline Heptachlor & 0.0 & 5 UG/ & $u$ & NP & 0.05 UG $\Omega$ & 1 \\
\hline Aldrin & 0.0 & 5 UG/ & $u$ & NP & 0.05 UG $\Omega$ & 1 \\
\hline Heptachlor Epoxide & 0.0 & 5 UG/ & $u$ & NP & 0.05 UG $\Omega$ & 1 \\
\hline Endosulfan l & 0.0 & 5 UG/L & $u$ & NP & $0.05 \mathrm{UG} / \mathrm{L}$ & 1 \\
\hline Dieldrin & & 1 UG/ & $u$ & NP & 0.1 UG/ & 1 \\
\hline 4,4'-DDE & & 1 UG/ & $u$ & NP & $0.1 \mathrm{UG} / \mathrm{L}$ & 1 \\
\hline Endrin & & 1 UG/ & $u$ & NP & $0.1 \mathrm{UG} / \mathrm{L}$ & 1 \\
\hline Endosulfan II & & 1 UG/L & $u$ & NP & $0.1 \mathrm{UG} /$ & 1 \\
\hline 4,4'-DDD & & $1 \mathrm{UG} / \mathrm{L}$ & $u$ & NP & 0.1 UG & 1 \\
\hline Endosulfan Sulfate & & 1 UG/L & $u$ & NP & 0.1 UG $/$ & 1 \\
\hline 4,4'-DDT & & 1 UG/ & $u$ & NP & 0.1 UG & 1 \\
\hline Methoxychlor & & 5 UG/ & $u$ & NP & 0.5 UG & 1 \\
\hline Endrin Ketone & & 1 UG/L & $u$ & NP & 0.1 UG/ & 1 \\
\hline alpha-Chlordane & & 5 UG/L & $u$ & NP & 0.5 UGR & 1 \\
\hline gamma-Chlordane & & 5 UG $/$ & $u$ - & NP & 0.5 UG/L & 1 \\
\hline Toxaphene & & 1 UG $\Omega$ & $u$ & NP & $1 \mathrm{UG} \Omega$ & 1 \\
\hline Aroclor-1016 & & 5 UG $\Omega$ & $u$ & NP & $0.5 \mathrm{UG} \Omega$ & 1 \\
\hline Aroclor-1221 & & 5 UG & $u$ & NP & $0.5 \mathrm{UG} \Omega$ & 1 \\
\hline Aroclor-1232 & & 5 UG $\Omega$ & $u$ & NP & $0.5 \mathrm{UG} /$ & 1 \\
\hline Arocior-1242 & & 5 UG $\Omega$ & $u$ & NP & 0.5 UG $\Omega$ & 1 \\
\hline Aroclor-1248 & & 5 UG/ & $u$ & NP & 0.5 UGA & 1 \\
\hline Aroclor-1254 & & 1 UGR & $u$ & NP & 1 UG $\Omega$ & 1 \\
\hline Aroclor-1260 & & 1 UG/ & $u$ & NP & $1 \mathrm{UG} / \mathrm{L}$ & 1 \\
\hline $\mathrm{DBC}$ & & $1 \%$ & & & $\%$ & 1 \\
\hline
\end{tabular}


APPENDIX E.2 Results of Pesticide and PCB Analyses, Water,

Salmon Site

\begin{tabular}{|c|c|c|c|c|c|}
\hline COMPOUND & RESULTS UNITS & $\begin{array}{l}\text { DATA } \\
\text { QUAL. }\end{array}$ & $\begin{array}{l}\text { DATA } \\
\text { VALID. }\end{array}$ & CRDL UNITS & DILUTION \\
\hline \multicolumn{6}{|l|}{ C-RB-003-W } \\
\hline alpha-BHC & 0.054 UGR & $u$ & NP & $0.054 \mathrm{UG} \Omega$ & 1 \\
\hline beta-BHC & $0.054 \mathrm{UG} \Omega$ & $u$ & NP & $0.054 \mathrm{UG} \Omega$ & 1 \\
\hline delta-BHC & 0.054 UG $\Omega$ & $u$ & NP & $0.054 \mathrm{UG} /$ & 1 \\
\hline gamma-BHC (Lindane) & 0.054 UG/L & $u$ & NP & 0.054 UG/ & 1 \\
\hline Heptachlor & 0.054 UG/L & $u$ & NP & 0.054 UG $\Omega$ & 1 \\
\hline Aldrin & 0.054 UG $/$ & $u$ & NP & $0.054 \mathrm{UG} \Omega$ & 1 \\
\hline Heptachlor Epoxide & $0.054 \mathrm{UG} \Omega$ & $u$ & NP & 0.054 UG $\Omega$ & 1 \\
\hline Endosulfan I & $0.054 \mathrm{UG} / \mathrm{L}$ & $u$ & NP & 0.054 UG/L & 1 \\
\hline Dieldrin & $0.11 \mathrm{UG} / \mathrm{L}$ & $u$ & NP & 0.11 UGL & 1 \\
\hline 4,4'-DDE & 0.11 UG/ & $u$ & NP & $0.11 \mathrm{UG} \Omega$ & 1 \\
\hline Endrin & $0.11 \mathrm{UG} / \mathrm{L}$ & $u$ & NP & $0.11 \mathrm{UG} \Omega$ & 1 \\
\hline Endosulfan II & $0.11 \mathrm{UG} /$ & $u$ & NP & 0.11 UG & 1 \\
\hline 4,4'-DDD & $0.11 \mathrm{UG \Omega}$ & $u$ & NP & $0.11 \mathrm{UG} / \mathrm{L}$ & 1 \\
\hline Endosulfan Sulfate & $0.11 \mathrm{UG} /$ & $u$ & NP & 0.11 UG/ & 1 \\
\hline 4,4'-DDT & 0.11 UG $\Omega$ & $u$ & NP & 0.11 UG/L & 1 \\
\hline Methoxychlor & 0.54 UG $\Omega$ & $u$ & NP & $0.54 \mathrm{UG} / \mathrm{L}$ & 1 \\
\hline Endrin Ketone & 0.11 UG $\Omega$ & $u$ & NP & $0.11 \mathrm{UG} / \mathrm{L}$ & 1 \\
\hline alpha-Chlordane & $0.54 \mathrm{UG} /$ & $u$ & NP & $0.54 \mathrm{UG} / \mathrm{L}$ & 1 \\
\hline gamma-Chlordane & 0.54 UG $\Omega$ & $u$ & NP & 0.54 UG $\Omega$ & 1 \\
\hline Toxaphene & $1.1 \mathrm{UG} \Omega$ & $u$ & NP & $1.1 \mathrm{UG} \Omega$ & 1 \\
\hline Aroclor-1016 & $0.54 \mathrm{UG} /$ & $u$ & NP & 0.54 UGR & 1 \\
\hline Aroclor-1221 & 0.54 UG $\Omega$ & $u$ & NP & 0.54 UG $\Omega$ & 1 \\
\hline Aroclor-1232 & 0.54 UG & $u$ & NP & 0.54 UG $\Omega$ & 1 \\
\hline Aroclor-1242 & $0.54 \mathrm{UG} /$ & $u$ & NP & 0.54 UGR & 1 \\
\hline Aroclor-1248 & $0.54 \mathrm{UG} \Omega$ & $u$ & NP & 0.54 UG $\Omega$ & 1 \\
\hline Aroclor-1254 & 1.5 UG/ & & NP & $1.1 \mathrm{UG} \Omega$ & 1 \\
\hline Aroclor-1260 & 1.1 UG $\Omega$ & $u$ & NP & $1.1 \mathrm{UG} \Omega$ & 1 \\
\hline $\mathrm{DBC}$ & $83 \%$ & & & $\%$ & 1 \\
\hline
\end{tabular}




\section{Appendix F.1 \\ Results of Metals Analyses, Soils, Salmon Site}


APPENDIX F.1 Results of Metals Analyses, Soils, Salmon Site

\begin{tabular}{lrrrrr}
\hline COMPOUND & RESULTS UNITS & DUTA & DATA & & \\
C5-1-S & & & & & \\
Arsenic & $1.4 \mathrm{MG} / \mathrm{KG}$ & $\mathrm{B}$ & $\mathrm{NP}$ & $2 \mathrm{MG} / \mathrm{KG}$ & 1 \\
Cyanide & $0.05 \mathrm{MG} / \mathrm{KG}$ & $\mathrm{U}$ & $\mathrm{NP}$ & $0.49 \mathrm{MG} / \mathrm{KG}$ & 1 \\
Mercury & $0.05 \mathrm{MG} / \mathrm{KG}$ & $\mathrm{U}$ & $\mathrm{NP}$ & $0.1 \mathrm{MG} / \mathrm{KG}$ & 1 \\
Aluminum & $1420 \mathrm{MG} / \mathrm{KG}$ & & $\mathrm{NP}$ & $40 \mathrm{MG} / \mathrm{KG}$ & 1 \\
Antimony & $4.5 \mathrm{MG} / \mathrm{KG}$ & $\mathrm{U}$ & $\mathrm{NP}$ & $12 \mathrm{MG} / \mathrm{KG}$ & 1 \\
Barium & $19 \mathrm{MG} / \mathrm{KG}$ & $\mathrm{B}$ & $\mathrm{NP}$ & $40 \mathrm{MG} / \mathrm{KG}$ & 1 \\
Beryllium & $0.34 \mathrm{MG} / \mathrm{KG}$ & $\mathrm{U}$ & $\mathrm{NP}$ & $1 \mathrm{MG} / \mathrm{KG}$ & 1 \\
Cadmium & $0.58 \mathrm{MG} / \mathrm{KG}$ & $\mathrm{U}$ & $\mathrm{NP}$ & $1 \mathrm{MG} / \mathrm{KG}$ & 1 \\
Calcium & $319 \mathrm{MG} / \mathrm{KG}$ & $\mathrm{B}$ & $\mathrm{NP}$ & $1000 \mathrm{MG} / \mathrm{KG}$ & 1 \\
Chromium & $73.5 \mathrm{MG} / \mathrm{KG}$ & & $\mathrm{NP}$ & $2 \mathrm{MG} / \mathrm{KG}$ & 1 \\
Cobalt & $2.5 \mathrm{MG} / \mathrm{KG}$ & $\mathrm{B}$ & $\mathrm{NP}$ & $10 \mathrm{MG} / \mathrm{KG}$ & 1 \\
Copper & $16.5 \mathrm{MG} / \mathrm{KG}$ & & $\mathrm{NP}$ & $5 \mathrm{MG} / \mathrm{KG}$ & 1 \\
Iron & $10200 \mathrm{MG} / \mathrm{KG}$ & & $\mathrm{NP}$ & $20 \mathrm{MG} / \mathrm{KG}$ & 1 \\
Magnesium & $111 \mathrm{MG} / \mathrm{KG}$ & $\mathrm{B}$ & $\mathrm{NP}$ & $1000 \mathrm{MG} / \mathrm{KG}$ & 1 \\
Manganese & $99.4 \mathrm{MG} / \mathrm{KG}$ & & $\mathrm{NP}$ & $3 \mathrm{MG} / \mathrm{KG}$ & 1 \\
Nickel & $20.2 \mathrm{MG} / \mathrm{KG}$ & & $\mathrm{NP}$ & $8 \mathrm{MG} / \mathrm{KG}$ & 1 \\
Potassium & $139 \mathrm{MG} / \mathrm{KG}$ & $\mathrm{U}$ & $\mathrm{NP}$ & $1000 \mathrm{MG} / \mathrm{KG}$ & 1 \\
Silver & $0.82 \mathrm{MG} / \mathrm{KG}$ & $\mathrm{U}$ & $\mathrm{NP}$ & $2 \mathrm{MG} / \mathrm{KG}$ & 1 \\
Sodium & $43.4 \mathrm{MG} / \mathrm{KG}$ & $\mathrm{B}$ & $\mathrm{NP}$ & $1000 \mathrm{MG} / \mathrm{KG}$ & 1 \\
Vanadium & $13.4 \mathrm{MG} / \mathrm{KG}$ & & $\mathrm{NP}$ & $10 \mathrm{MG} / \mathrm{KG}$ & 1 \\
Zinc & $4.1 \mathrm{MG} / \mathrm{KG}$ & & $\mathrm{NP}$ & $4 \mathrm{MG} / \mathrm{KG}$ & 1 \\
Lead & $4.7 \mathrm{MG} / \mathrm{KG}$ & & $\mathrm{NP}$ & $0.6 \mathrm{MG} / \mathrm{KG}$ & 1 \\
Selenium & $0.16 \mathrm{MG} / \mathrm{KG}$ & $\mathrm{U}$ & $\mathrm{NP}$ & $1 \mathrm{MG} / \mathrm{KG}$ & 1 \\
Thallium & $0.2 \mathrm{MG} / \mathrm{KG}$ & $\mathrm{U}$ & $\mathrm{NP}$ & $2 \mathrm{MG} / \mathrm{KG}$ & 1 \\
& & & &
\end{tabular}


APPENDIX F.1 Results of Metals Analyses, Soils, Salmon Site

\begin{tabular}{|c|c|c|c|c|c|}
\hline COMPOUND & RESULTS UNITS & $\begin{array}{l}\text { DATA } \\
\text { QUAL. }\end{array}$ & $\begin{array}{l}\text { DATA } \\
\text { VALID. }\end{array}$ & CRDL UNITS & DILUTION \\
\hline \multicolumn{6}{|l|}{ C5-2-S } \\
\hline Arsenic & $0.3 \mathrm{MG} / \mathrm{KG}$ & $B$ & NP & $2 M G / K G$ & 1 \\
\hline Cyanide & $0.04 \mathrm{MG} / \mathrm{KG}$ & $u$ & NP & $0.44 \mathrm{MG} / \mathrm{KG}$ & 1 \\
\hline Mercury & $0.05 \mathrm{MG} / \mathrm{KG}$ & $u$ & $\mathrm{NP}$ & $0.1 \mathrm{MG} / \mathrm{KG}$ & 1 \\
\hline Aluminum & $1470 \mathrm{MG} / \mathrm{KG}$ & & NP & $40 \mathrm{MG} / \mathrm{KG}$ & 1 \\
\hline Antimony & $4.5 \mathrm{MG} / \mathrm{KG}$ & $u$ & $\mathrm{NP}$ & $12 M G / K G$ & 1 \\
\hline Barium & $4.8 \mathrm{MG} / \mathrm{KG}$ & B & $\mathrm{NP}$ & $40 \mathrm{MG} / \mathrm{KG}$ & 1 \\
\hline Beryllium & $0.34 \mathrm{MG} / \mathrm{KG}$ & $u$ & NP & $1 \mathrm{MG} / \mathrm{KG}$ & 1 \\
\hline Cadmium & $0.58 \mathrm{MG} / \mathrm{KG}$ & $u$ & $\mathrm{NP}$ & $1 \mathrm{MG} / \mathrm{KG}$ & 1 \\
\hline Calcium & 205 MG/KG & B & NP & $1000 \mathrm{MG} / \mathrm{KG}$ & 1 \\
\hline Chromium & $2.5 \mathrm{MG} / \mathrm{KG}$ & & $\mathrm{NP}$ & $2 \mathrm{MG} / \mathrm{KG}$ & 1 \\
\hline Cobalt & $1.1 \mathrm{MG} / \mathrm{KG}$ & $u$ & NP & $10 \mathrm{MG} / \mathrm{KG}$ & 1 \\
\hline Copper & $3.1 \mathrm{MG} / \mathrm{KG}$ & $B$ & NP & $5 \mathrm{MG} / K \mathrm{G}$ & 1 \\
\hline Iron & $3840 \mathrm{MG} / \mathrm{KG}$ & & $\mathrm{NP}$ & $20 \mathrm{MG} / \mathrm{KG}$ & 1 \\
\hline Magnesium & $91.5 \mathrm{MG} / \mathrm{KG}$ & B & NP & $1000 \mathrm{MG} / \mathrm{KG}$ & 1 \\
\hline Manganese & $2.9 \mathrm{MG} / \mathrm{KG}$ & $B$ & $\mathrm{NP}$ & $3 \mathrm{MG} / \mathrm{KG}$ & 1 \\
\hline Nickel & $2.3 \mathrm{MG} / \mathrm{KG}$ & $B$ & NP & $8 \mathrm{MG} / \mathrm{KG}$ & 1 \\
\hline Potassium & $139 \mathrm{MG} / \mathrm{KG}$ & u & NP & $1000 \mathrm{MG} / \mathrm{KG}$ & 1 \\
\hline Silver & $0.82 \mathrm{MG} / \mathrm{KG}$ & $u$ & NP & $2 M G / K G$ & 1 \\
\hline Sodium & $39.9 \mathrm{MG} / \mathrm{KG}$ & B & NP & $1000 \mathrm{MG} / \mathrm{KG}$ & 1 \\
\hline Vanadium & $8.5 \mathrm{MG} / \mathrm{KG}$ & B & $\mathrm{NP}$ & $10 \mathrm{MG} / \mathrm{KG}$ & 1 \\
\hline Zinc & $6.9 \mathrm{MG} / \mathrm{KG}$ & & NP & $4 M G / K G$ & 1 \\
\hline Lead & $1.3 \mathrm{MG} / \mathrm{KG}$ & & NP & $0.6 \mathrm{MG} / \mathrm{KG}$ & 1 \\
\hline Selenium & $0.16 \mathrm{MG} / \mathrm{KG}$ & $u$ & $\mathrm{NP}$ & $1 \mathrm{MG} / \mathrm{KG}$ & 1 \\
\hline Thallium & $0.2 \mathrm{MG} / \mathrm{KG}$ & $u$ & $\mathrm{NP}$ & $2 M G / K G$ & 1 \\
\hline
\end{tabular}




\section{APPENDIX F.1 Results of Metals Analyses, Soils, Salmon Site}

\begin{tabular}{|c|c|c|c|c|c|c|}
\hline COMPOUND & RESULTS & UNITS & $\begin{array}{l}\text { DATA } \\
\text { QUAL. }\end{array}$ & $\begin{array}{l}\text { DATA } \\
\text { VALID. }\end{array}$ & CRDL UNITS & DILUTION \\
\hline \multicolumn{7}{|l|}{ C5-2-S-DUP } \\
\hline Arsenic & 0.36 & $M G / K G$ & $B$ & NP & $2 M C: K G$ & 1 \\
\hline Cyanide & 0.05 & $M G / K G$ & $u$ & NP & $0.49 \mathrm{MG} / \mathrm{KG}$ & 1 \\
\hline Mercury & 0.05 & $M G / K G$ & $u$ & NP & $0.1 k:=: K G$ & 1 \\
\hline Aluminum & 1300 & $M G / K G$ & & NP & $40 \mathrm{Mr} \cdot K G$ & 1 \\
\hline Antimony & 4.5 & $M G / K G$ & $u$ & NP & $12 \mathrm{MG} / \mathrm{KG}$ & 1 \\
\hline Barium & 4.4 & $M G / K G$ & B & NP & 40 MG/KG & 1 \\
\hline Beryllium & 0.34 & MG/KG & $u$ & NP & $1 \mathrm{MG/KG}$ & 1 \\
\hline Cadmium & 0.58 & $M G / K G$ & $u$ & NP & $1 \mathrm{MG} / K G$ & 1 \\
\hline Calcium & 215 & $M G / K G$ & B & NP & $1000 \mathrm{MG} / \mathrm{KG}$ & 1 \\
\hline Chromium & & MG/KG & & NP & $2 M G / K G$ & 1 \\
\hline Cobalt & 1.1 & MG/KG & $u$ & NP & $10 \mathrm{MG} / \mathrm{KG}$ & 1 \\
\hline Copper & 0.88 & $M G / K G$ & B & $\mathrm{NP}$ & $5 \mathrm{MG} / \mathrm{KG}$ & 1 \\
\hline Iron & 3340 & $M G / K G$ & & NP & 20 MG/KG & 1 \\
\hline Magnesium & 88.3 & $M G / K G$ & 8 & NP & $1000 \mathrm{MG} / \mathrm{KG}$ & 1 \\
\hline Manganese & 3.6 & $M G / K G$ & & NP & $3 \mathrm{MG} / K \mathrm{~K}$ & 1 \\
\hline Nickel & 1.7 & $M G / K G$ & $u$ & NP & $8 M G / K G$ & 1 \\
\hline Potassium & 139 & $M G / K G$ & $u$ & NP & $1000 \mathrm{MG} / \mathrm{KG}$ & 1 \\
\hline Silver & 0.82 & MG/KG & $u$ & NP & $2 \mathrm{MG} / \mathrm{KG}$ & 1 \\
\hline Sodium & 32.1 & $M G / K G$ & B & NP & $1000 \mathrm{MG} / \mathrm{KG}$ & 1 \\
\hline Vanadium & 8.2 & $M G / K G$ & B & NP & $10 \mathrm{MG} / \mathrm{KG}$ & 1 \\
\hline Zinc & 2.8 & $M G / K G$ & B & NP & $4 \mathrm{MG} / \mathrm{KG}$ & 1 \\
\hline Lead & & MG/KG & & NP & $0.6 \mathrm{MG} / \mathrm{KG}$ & 1 \\
\hline Selenium & 0.16 & $M G / K G$ & $u$ & NP & $1 \mathrm{MG} / \mathrm{KG}$ & 1 \\
\hline Thallium & 0.2 & $M G / K G$ & u & $\mathrm{NP}$ & $2 M G / K G$ & 1 \\
\hline
\end{tabular}


APPENDIX F.1 Results of Metals Analyses, Soils, Salmon Site

\begin{tabular}{lcccc}
\hline COMPOUND & RESULTS UNITS & $\begin{array}{c}\text { DATA } \\
\text { QUAL. }\end{array}$ & DATA & \\
VALID. & CRDL UNITS & DILUTION \\
\hline C5-2-S-MS & $79 \%$ & NP & $\%$ & 1 \\
Arsenic & $94 \%$ & NP & $\%$ & 1 \\
Cyanide & $92 \%$ & NP & $\%$ & 1 \\
Mercury & $65 \%$ & NP & $\%$ & 1 \\
Antimony & $100 \%$ & NP & $\%$ & 1 \\
Barium & $103 \%$ & NP & $\%$ & 1 \\
Beryllium & $95 \%$ & NP & $\%$ & 1 \\
Cadmium & $100 \%$ & NP & $\%$ & 1 \\
Chromium & $103 \%$ & NP & $\%$ & 1 \\
Cobalt & $99 \%$ & NP & $\%$ & 1 \\
Copper & $102 \%$ & NP & $\%$ & 1 \\
Manganese & $101 \%$ & NP & $\%$ & 1 \\
Nickel & $107 \%$ & NP & $\%$ & 1 \\
Silver & $104 \%$ & NP & $\%$ & 1 \\
Vanadium & $96 \%$ & NP & $\%$ & 1 \\
Zinc & $114 \%$ & NP & $\%$ & 1 \\
Lead & $43 \%$ & NP & $\%$ & 1 \\
Selenium & $105 \%$ & NP & $\%$ & 1 \\
Thallium & & & &
\end{tabular}




\section{APPENDIX F.1 Results of Metals Analyses, Soils, Salmon Site}

\begin{tabular}{|c|c|c|c|c|c|}
\hline COMPOUND & RESULTS UNITS & $\begin{array}{l}\text { DATA } \\
\text { QUAL. }\end{array}$ & $\begin{array}{l}\text { DATA } \\
\text { VALID. }\end{array}$ & CRDL UNITS & DILUTION \\
\hline \multicolumn{6}{|l|}{ C5-3-S } \\
\hline Arsenic & $0.97 \mathrm{MG} / \mathrm{KG}$ & B & NP & $2 M G / K G$ & 1 \\
\hline Cyanide & $0.04 \mathrm{MG} / \mathrm{KG}$ & $u$ & NP & $0.44 \mathrm{MG} / \mathrm{KG}$ & 1 \\
\hline Mercury & $0.05 \mathrm{MG} / \mathrm{KG}$ & $u$ & NP & $0.1 \mathrm{MG} / \mathrm{KG}$ & 1 \\
\hline Aluminum & 4200 MG/KG & & NP & $40 \mathrm{MG} / \mathrm{KG}$ & 1 \\
\hline Antimony & $4.5 \mathrm{MG} / K \mathrm{KG}$ & $u$ & NP & $12 \mathrm{MG} / \mathrm{KG}$ & 1 \\
\hline Barium & $57.2 \mathrm{MG} / \mathrm{KG}$ & & $\mathrm{NP}$ & $40 \mathrm{MG} / \mathrm{KG}$ & 1 \\
\hline Beryllium & $0.43 \mathrm{MG} / \mathrm{KG}$ & B & NP & $1 \mathrm{MG} / \mathrm{KG}$ & 1 \\
\hline Cadmium & $0.58 \mathrm{MG} / \mathrm{KG}$ & u & NP & $1 \mathrm{MG} / \mathrm{KG}$ & 1 \\
\hline Calcium & $2140 \mathrm{MG} / \mathrm{KG}$ & & NP & $1000 \mathrm{MG} / \mathrm{KG}$ & 1 \\
\hline Chromium & $16.2 \mathrm{MG} / \mathrm{KG}$ & & NP & $2 M G / K G$ & 1 \\
\hline Cobalt & $2.2 \mathrm{MG} / \mathrm{KG}$ & B & NP & $10 \mathrm{MG} / \mathrm{KG}$ & 1 \\
\hline Copper & $6.7 \mathrm{MG} / \mathrm{KG}$ & & NP & $5 \mathrm{MG/KG}$ & 1 \\
\hline Iron & $10300 \mathrm{MG} / \mathrm{KG}$ & & NP & $20 \mathrm{MG} / \mathrm{KG}$ & 1 \\
\hline Magnesium & 1010 MG/KG & & NP & $1000 \mathrm{MG} / \mathrm{KG}$ & 1 \\
\hline Manganese & $40.5 \mathrm{MG} / \mathrm{KG}$ & & NP & $3 \mathrm{MG} / K G$ & 1 \\
\hline Nickel & $6 \mathrm{MG} / \mathrm{KG}$ & B & $\mathrm{NP}$ & $8 \mathrm{MG} / \mathrm{KG}$ & 1 \\
\hline Potassium & $501 \mathrm{MG} / \mathrm{KG}$ & B & NP & $1000 \mathrm{MG} / \mathrm{KG}$ & 1 \\
\hline Silver & $0.82 \mathrm{MG} / \mathrm{KG}$ & $u$ & NP & $2 \mathrm{MG} / \mathrm{KG}$ & 1 \\
\hline Sodium & $54.1 \mathrm{MG} / \mathrm{KG}$ & B & NP & $1000 \mathrm{MG} / \mathrm{KG}$ & 1 \\
\hline Vanadium & $17 \mathrm{MG} / \mathrm{KG}$ & & $\mathrm{NP}$ & $10 \mathrm{MG} / \mathrm{KG}$ & 1 \\
\hline Zinc & $11.4 \mathrm{MG} / \mathrm{KG}$ & & NP & $4 \mathrm{MG} / \mathrm{KG}$ & 1 \\
\hline Lead & $3.2 \mathrm{MG} / \mathrm{KG}$ & & NP & $0.6 \mathrm{MG} / \mathrm{KG}$ & 1 \\
\hline Selenium & $0.16 \mathrm{MG} / \mathrm{KG}$ & $u$ & NP & $1 \mathrm{MG} / \mathrm{KG}$ & 1 \\
\hline Thallium & $0.2 \mathrm{MG} / \mathrm{KG}$ & $u$ & NP & $2 M G / K G$ & 1 \\
\hline
\end{tabular}


APPENDIX F.1 Results of Metals Analyses, Soils, Salmon Site

\begin{tabular}{|c|c|c|c|c|c|}
\hline COMPOUND & RESULTS UNITS & $\begin{array}{l}\text { DATA } \\
\text { QUAL. }\end{array}$ & $\begin{array}{l}\text { DATA } \\
\text { VALID. }\end{array}$ & CRDL UNITS & DILUTION \\
\hline \multicolumn{6}{|l|}{ C3-4-S } \\
\hline Arsenic & $4.1 \mathrm{MG} / \mathrm{KG}$ & & NP & $2 \mathrm{MG} / \mathrm{KG}$ & 1 \\
\hline Cyanide & $0.05 \mathrm{MG} / \mathrm{KG}$ & $u$ & $N P$ & $0.49 \mathrm{MG} / \mathrm{KG}$ & 1 \\
\hline Mercury & $0.05 \mathrm{MG} / \mathrm{KG}$ & $u$ & NP & $0.1 \mathrm{MG} / \mathrm{KG}$ & 1 \\
\hline Aluminum & $4330 \mathrm{MG} / \mathrm{KG}$ & & NP & $40 M G / K G$ & 1 \\
\hline Antimony & $4.5 \mathrm{MG} / \mathrm{KG}$ & $u$ & NP & $12 M G / K G$ & 1 \\
\hline Barium & $32.6 \mathrm{MG} / \mathrm{KG}$ & B & NP & $40 \mathrm{MG} / \mathrm{KG}$ & 1 \\
\hline Beryllium & $0.69 \mathrm{MG} / \mathrm{KG}$ & B & NP & $1 \mathrm{MG} / \mathrm{KG}$ & 1 \\
\hline Cadmium & $0.58 \mathrm{MG} / \mathrm{KG}$ & $u$ & NP & $1 \mathrm{MG} / \mathrm{KG}$ & 1 \\
\hline Calcium & $6100 \mathrm{MG} / \mathrm{KG}$ & & NP & $1000 \mathrm{MG} / \mathrm{KG}$ & 1 \\
\hline Chromium & $4.9 \mathrm{MG} / \mathrm{KG}$ & & $\mathrm{NP}$ & $2 \mathrm{MG} / \mathrm{KG}$ & 1 \\
\hline Cobalt & $11.4 \mathrm{MG} / \mathrm{KG}$ & & NP & $10 \mathrm{MG} / \mathrm{KG}$ & 1 \\
\hline Copper & $17.1 \mathrm{MG} / \mathrm{KG}$ & & NP & $5 \mathrm{MG} / \mathrm{KG}$ & 1 \\
\hline Iron & $9520 \mathrm{MG} / \mathrm{KG}$ & & NP & $20 \mathrm{MG} / K \mathrm{G}$ & 1 \\
\hline Magnesium & $3100 \mathrm{MG} / \mathrm{KG}$ & & NP & $1000 \mathrm{MG} / \mathrm{KG}$ & 1 \\
\hline Manganese & $1250 \mathrm{MG} / \mathrm{KG}$ & & NP & $3 \mathrm{MG} / \mathrm{KG}$ & 1 \\
\hline Nickel & $20.6 \mathrm{MG} / \mathrm{KG}$ & & NP & $8 \mathrm{MG} / \mathrm{KG}$ & 1 \\
\hline Potassium & $957 \mathrm{MG} / \mathrm{KG}$ & B & NP & $1000 \mathrm{MG} / \mathrm{KG}$ & 1 \\
\hline Silver & $0.82 \mathrm{MG} / \mathrm{KG}$ & $u$ & NP & $2 M G / K G$ & 1 \\
\hline Sodium & $147 \mathrm{MG} / \mathrm{KG}$ & B & NP & $1000 \mathrm{MG} / \mathrm{KG}$ & 1 \\
\hline Vanadium & $8.8 \mathrm{MG} / \mathrm{KG}$ & $B$ & NP & $10 \mathrm{MG} / \mathrm{KG}$ & 1 \\
\hline Zinc & $35 \mathrm{MG} / \mathrm{KG}$ & & NP & $4 \mathrm{MG} / \mathrm{KG}$ & 1 \\
\hline Lead & $18.8 \mathrm{MG} / \mathrm{KG}$ & & NP & $1.2 \mathrm{MG} / \mathrm{KG}$ & 2 \\
\hline Selenium & $0.2 \mathrm{MG} / \mathrm{KG}$ & u & NP & $1 \mathrm{MG} / \mathrm{KG}$ & 1 \\
\hline Thallium & $0.2 \mathrm{MG} / \mathrm{KG}$ & u & NP & $2 M G / K G$ & 1 \\
\hline
\end{tabular}


APPENDIX F.1 Results of Metals Analy'ses, Soils, Salmon Site

\begin{tabular}{|c|c|c|c|c|c|}
\hline COMPOUND & RESULTS UNITS & $\begin{array}{l}\text { DATA } \\
\text { QUAL. }\end{array}$ & $\begin{array}{l}\text { DATA } \\
\text { VALID. }\end{array}$ & CRDL UNITS & DILUTION \\
\hline \multicolumn{6}{|l|}{ C3-5-S } \\
\hline Arsenic & $18.9 \mathrm{MG} / \mathrm{KG}$ & & NP & $6 \mathrm{MCKG}$ & 3 \\
\hline Cyanide & $0.05 \mathrm{MG} / \mathrm{KG}$ & $u$ & NP & $0.5 \mathrm{MG} / \mathrm{KG}$ & 1 \\
\hline Mercury & $0.18 \mathrm{MG} / \mathrm{KG}$ & & NP & $0.1 i^{\prime} \mathrm{GKG}$ & 1 \\
\hline Aluminum & $1460 \mathrm{MG} / \mathrm{KG}$ & & NP & $40 \mathrm{M} \cdot \mathrm{KG}$ & 1 \\
\hline Antimony & $4.5 \mathrm{MG} / \mathrm{KG}$ & $u$ & NP & 12 MG/KG & 1 \\
\hline Barium & $39.6 \mathrm{MG} / \mathrm{KG}$ & B & NP & 40 MG/KG & 1 \\
\hline Beryllium & $0.82 \mathrm{MG} / \mathrm{KG}$ & B & NP & 1 MGKG & 1 \\
\hline Cadmium & $0.58 \mathrm{MG} / \mathrm{KG}$ & $u$ & NP & $1 \mathrm{MG} / \mathrm{KG}$ & 1 \\
\hline Calcium & $1870 \mathrm{MG} / \mathrm{KG}$ & & NP & $1000 \mathrm{MG} / \mathrm{KG}$ & 1 \\
\hline Chromium & $0.96 \mathrm{MG} / \mathrm{KG}$ & $u$ & NP & $2 \mathrm{MG} / K \mathrm{~K}$ & 1 \\
\hline Cobalt & $56.9 \mathrm{MG} / \mathrm{KG}$ & & NP & $10 \mathrm{MG} / \mathrm{KG}$ & 1 \\
\hline Copper & $21.9 \mathrm{MG} / \mathrm{KG}$ & & NP & $5 \mathrm{MG} / \mathrm{KG}$ & 1 \\
\hline Iron & $3640 \mathrm{MG} / \mathrm{KG}$ & & NP & $20 \mathrm{MG} / \mathrm{KG}$ & 1 \\
\hline Magnesium & $841 \mathrm{MG} / \mathrm{KG}$ & B & NP & $1000 \mathrm{MG} / \mathrm{KG}$ & 1 \\
\hline Manganese & $19.1 \mathrm{MG} / \mathrm{KG}$ & & NP & $3 \mathrm{MG} / \mathrm{KG}$ & 1 \\
\hline Nickel & 41.2 MG/KG & & NP & $8 \mathrm{MG} / \mathrm{KG}$ & 1 \\
\hline Potassium & 354 MG/KG & B & NP & $1000 \mathrm{MG} / \mathrm{KG}$ & 1 \\
\hline Silver & $0.82 \mathrm{MG} / \mathrm{KG}$ & $u$ & NP & $2 \mathrm{MG} / K \mathrm{~K}$ & 1 \\
\hline Sodium & 119 MG/KG & B & NP & $1000 \mathrm{MG} / \mathrm{KG}$ & 1 \\
\hline Vanadium & $1.7 \mathrm{MG} / \mathrm{KG}$ & B & NP & $10 \mathrm{MG} / \mathrm{KG}$ & 1 \\
\hline Zinc & $11.6 \mathrm{MG} / \mathrm{KG}$ & & NP & $4 \mathrm{MG} / \mathrm{KG}$ & 1 \\
\hline Lead & $15.6 \mathrm{MG} / \mathrm{KG}$ & & NP & $1.2 \mathrm{MG} / \mathrm{KG}$ & 2 \\
\hline Selenium & $0.2 \mathrm{MG} / \mathrm{KG}$ & $u$ & NP & $1 \mathrm{MG} / \mathrm{KG}$ & 1 \\
\hline Thallium & 0.22 MG/KG & $B$ & NP & $2 M G / K G$ & 1 \\
\hline
\end{tabular}


APPENDIX F.1 Results of Metals Analyses, Soils, Salmon Site

\begin{tabular}{lrrrrr}
\hline COMPOUND & RESULTS UNITS & $\begin{array}{r}\text { DATA } \\
\text { QUAL. }\end{array}$ VATA & VALID. & CRDL UNITS & DILUTION \\
\hline C3-6-S & $11.5 \mathrm{MG} / \mathrm{KG}$ & & $\mathrm{NP}$ & $2 \mathrm{MG} / \mathrm{KG}$ & 1 \\
Arsenic & $0.05 \mathrm{MG} / \mathrm{KG}$ & $\mathrm{U}$ & $\mathrm{NP}$ & $0.48 \mathrm{MG} / \mathrm{KG}$ & 1 \\
Cyanide & $0.05 \mathrm{MG} / \mathrm{KG}$ & $\mathrm{U}$ & $\mathrm{NP}$ & $0.1 \mathrm{MG} / \mathrm{KG}$ & 1 \\
Mercury & $1750 \mathrm{MG} / \mathrm{KG}$ & & $\mathrm{NP}$ & $40 \mathrm{MG} / \mathrm{KG}$ & 1 \\
Aluminum & $4.5 \mathrm{MG} / \mathrm{KG}$ & $\mathrm{U}$ & $\mathrm{NP}$ & $12 \mathrm{MG} / \mathrm{KG}$ & 1 \\
Antimony & $104 \mathrm{MG} / \mathrm{KG}$ & & $\mathrm{NP}$ & $40 \mathrm{MG} / \mathrm{KG}$ & 1 \\
Barium & $0.43 \mathrm{MG} / \mathrm{KG}$ & $\mathrm{B}$ & $\mathrm{NP}$ & $1 \mathrm{MG} / \mathrm{KG}$ & 1 \\
Beryllium & $0.58 \mathrm{MG} / \mathrm{KG}$ & $\mathrm{U}$ & $\mathrm{NP}$ & $1 \mathrm{MG} / \mathrm{KG}$ & 1 \\
Cadmium & $3380 \mathrm{MG} / \mathrm{KG}$ & & $\mathrm{NP}$ & $1000 \mathrm{MG} / \mathrm{KG}$ & 1 \\
Calcium & $0.96 \mathrm{MG} / \mathrm{KG}$ & $\mathrm{U}$ & $\mathrm{NP}$ & $2 \mathrm{MG} / \mathrm{KG}$ & 1 \\
Chromium & $5.3 \mathrm{MG} / \mathrm{KG}$ & $\mathrm{B}$ & $\mathrm{NP}$ & $10 \mathrm{MG} / \mathrm{KG}$ & 1 \\
Cobalt & $6 \mathrm{MG} / \mathrm{KG}$ & & $\mathrm{NP}$ & $5 \mathrm{MG} / \mathrm{KG}$ & 1 \\
Copper & $1830 \mathrm{MG} / \mathrm{KG}$ & & $\mathrm{NP}$ & $20 \mathrm{MG} / \mathrm{KG}$ & 1 \\
Iron & $1200 \mathrm{MG} / \mathrm{KG}$ & & $\mathrm{NP}$ & $1000 \mathrm{MG} / \mathrm{KG}$ & 1 \\
Magnesium & $310 \mathrm{MG} / \mathrm{KG}$ & & $\mathrm{NP}$ & $3 \mathrm{MG} / \mathrm{KG}$ & 1 \\
Manganese & $9.2 \mathrm{MG} / \mathrm{KG}$ & & $\mathrm{NP}$ & $8 \mathrm{MG} / \mathrm{KG}$ & 1 \\
Nickel & $240 \mathrm{MG} / \mathrm{KG}$ & $\mathrm{B}$ & $\mathrm{NP}$ & $1000 \mathrm{MG} / \mathrm{KG}$ & 1 \\
Potassium & $0.89 \mathrm{MG} / \mathrm{KG}$ & $\mathrm{B}$ & $\mathrm{NP}$ & $2 \mathrm{MG} / \mathrm{KG}$ & 1 \\
Silver & $108 \mathrm{MG} / \mathrm{KG}$ & $\mathrm{B}$ & $\mathrm{NP}$ & $1000 \mathrm{MG} / \mathrm{KG}$ & 1 \\
Sodium & $2.5 \mathrm{MG} / \mathrm{KG}$ & $\mathrm{B}$ & $\mathrm{NP}$ & $10 \mathrm{MG} / \mathrm{KG}$ & 1 \\
Vanadium & $7.3 \mathrm{MG} / \mathrm{KG}$ & & $\mathrm{NP}$ & $4 \mathrm{MG} / \mathrm{KG}$ & 1 \\
Zinc & $8.3 \mathrm{MG} / \mathrm{KG}$ & & $\mathrm{NP}$ & $0.6 \mathrm{MG} / \mathrm{KG}$ & 1 \\
Lead & $0.16 \mathrm{MG} / \mathrm{KG}$ & $\mathrm{U}$ & $\mathrm{NP}$ & $1 \mathrm{MG} / \mathrm{KG}$ & 1 \\
Selenium & $0.2 \mathrm{MG} / \mathrm{KG}$ & $\mathrm{U}$ & $\mathrm{NP}$ & $2 \mathrm{MG} / \mathrm{KG}$ & 1 \\
Thallium & & & & & \\
& & & & &
\end{tabular}


APPENDIX F.1 Results of Metals Analyses, Soils, Salmon Site

\begin{tabular}{lrrrrr}
\hline COMPOUND & RESULTS UNITS & $\begin{array}{r}\text { DATA } \\
\text { QUAL. }\end{array}$ & DATA & \\
C3-7-S & & & & & \\
Arsenic & $10 \mathrm{MG} / \mathrm{KG}$ & & $\mathrm{NP}$ & $2 \mathrm{MG} / \mathrm{KG}$ & 1 \\
Cyanide & $0.05 \mathrm{MG} / \mathrm{KG}$ & $\mathrm{U}$ & $\mathrm{NP}$ & $0.5 \mathrm{MG} / \mathrm{KG}$ & 1 \\
Mercury & $0.05 \mathrm{MG} / \mathrm{KG}$ & $\mathrm{U}$ & $\mathrm{NP}$ & $0.1 \mathrm{MG} / \mathrm{KG}$ & 1 \\
Aluminum & $5740 \mathrm{MG} / \mathrm{KG}$ & & $\mathrm{NP}$ & $40 \mathrm{MG} / \mathrm{KG}$ & 1 \\
Antimony & $4.5 \mathrm{MG} / \mathrm{KG}$ & $\mathrm{U}$ & $\mathrm{NP}$ & $12 \mathrm{MG} / \mathrm{KG}$ & 1 \\
Barium & $109 \mathrm{MG} / \mathrm{KG}$ & & $\mathrm{NP}$ & $40 \mathrm{MG} / \mathrm{KG}$ & 1 \\
Beryllium & $0.98 \mathrm{MG} / \mathrm{KG}$ & $\mathrm{B}$ & $\mathrm{NP}$ & $1 \mathrm{MG} / \mathrm{KG}$ & 1 \\
Cadmium & $0.58 \mathrm{MG} / \mathrm{KG}$ & $\mathrm{U}$ & $\mathrm{NP}$ & $1 \mathrm{MG} / \mathrm{KG}$ & 1 \\
Calcium & $6890 \mathrm{MG} / \mathrm{KG}$ & & $\mathrm{NP}$ & $1000 \mathrm{MG} / \mathrm{KG}$ & 1 \\
Chromium & $6 \mathrm{MG} / \mathrm{KG}$ & & $\mathrm{NP}$ & $2 \mathrm{MG} / \mathrm{KG}$ & 1 \\
Cobalt & $26.8 \mathrm{MG} / \mathrm{KG}$ & & $\mathrm{NP}$ & $10 \mathrm{MG} / \mathrm{KG}$ & 1 \\
Copper & $12.9 \mathrm{MG} / \mathrm{KG}$ & & $\mathrm{NP}$ & $5 \mathrm{MG} / \mathrm{KG}$ & 1 \\
Iron & $13900 \mathrm{MG} / \mathrm{KG}$ & & $\mathrm{NP}$ & $20 \mathrm{MG} / \mathrm{KG}$ & 1 \\
Magnesium & $3890 \mathrm{MG} / \mathrm{KG}$ & & $\mathrm{NP}$ & $1000 \mathrm{MG} / \mathrm{KG}$ & 1 \\
Manganese & $2690 \mathrm{MG} / \mathrm{KG}$ & & $\mathrm{NP}$ & $3 \mathrm{MG} / \mathrm{KG}$ & 1 \\
Nickel & $29.2 \mathrm{MG} / \mathrm{KG}$ & & $\mathrm{NP}$ & $8 \mathrm{MG} / \mathrm{KG}$ & 1 \\
Potassium & $1380 \mathrm{MG} / \mathrm{KG}$ & & $\mathrm{NP}$ & $1000 \mathrm{MG} / \mathrm{KG}$ & 1 \\
Silver & $0.82 \mathrm{MG} / \mathrm{KG}$ & $\mathrm{U}$ & $\mathrm{NP}$ & $2 \mathrm{MG} / \mathrm{KG}$ & 1 \\
Sodium & $198 \mathrm{MG} / \mathrm{KG}$ & $\mathrm{B}$ & $\mathrm{NP}$ & $1000 \mathrm{MG} / \mathrm{KG}$ & 1 \\
Vanadium & $8.2 \mathrm{MG} / \mathrm{KG}$ & $\mathrm{B}$ & $\mathrm{NP}$ & $10 \mathrm{MG} / \mathrm{KG}$ & 1 \\
Zinc & $47.6 \mathrm{MG} / \mathrm{KG}$ & & $\mathrm{NP}$ & $4 \mathrm{MG} / \mathrm{KG}$ & 1 \\
Lead & $15.1 \mathrm{MG} / \mathrm{KG}$ & & $\mathrm{NP}$ & $1.2 \mathrm{MG} / \mathrm{KG}$ & 2 \\
Selenium & $0.16 \mathrm{MG} / \mathrm{KG}$ & $\mathrm{U}$ & $\mathrm{NP}$ & $1 \mathrm{MG} / \mathrm{KG}$ & 1 \\
Thallium & $0.46 \mathrm{MG} / \mathrm{KG}$ & $\mathrm{B}$ & $\mathrm{NP}$ & $2 \mathrm{MG} / \mathrm{KG}$ & 1 \\
& & & & & \\
& & & & &
\end{tabular}


APPENDIX F.1 Results of Metals Analyses, Soils, Salmon Site

\begin{tabular}{|c|c|c|c|c|c|}
\hline COMPOUND & RESULTS UNITS & $\begin{array}{l}\text { DATA } \\
\text { QUAL. }\end{array}$ & $\begin{array}{l}\text { DATA } \\
\text { VALID. } \\
\end{array}$ & CRDL UNITS & DILUTION \\
\hline \multicolumn{6}{|l|}{ C3-8-S } \\
\hline Arsenic & $9.8 \mathrm{MG} / \mathrm{KG}$ & & NP & $2 M G / K G$ & 1 \\
\hline Cyanide & $0.05 \mathrm{MG} / \mathrm{KG}$ & $u$ & $\mathrm{NP}$ & $0.49 \mathrm{MG} / \mathrm{KG}$ & 1 \\
\hline Mercury & $0.05 \mathrm{MG} / \mathrm{KG}$ & $u$ & NP & $0.1 \mathrm{MG} / \mathrm{KG}$ & 1 \\
\hline Aluminum & 2250 MG/KG & & $\mathrm{NP}$ & $40 \mathrm{MG} / \mathrm{KG}$ & 1 \\
\hline Antimony & $4.5 \mathrm{MG} / K G$ & $u$ & NP & $12 \mathrm{MG} / \mathrm{KG}$ & 1 \\
\hline Barium & $55.8 \mathrm{MG} / \mathrm{KG}$ & & NP & $40 \mathrm{MG} / \mathrm{KG}$ & 1 \\
\hline Beryllium & $0.34 \mathrm{MG} / \mathrm{KG}$ & $u$ & NP & $1 \mathrm{MG} / \mathrm{KG}$ & 1 \\
\hline Cadmium & $0.58 \mathrm{MG} / \mathrm{KG}$ & $u$ & NP & $1 \mathrm{MG} / K G$ & 1 \\
\hline Calcium & $3960 \mathrm{MG} / \mathrm{KG}$ & & NP & $1000 \mathrm{MG} / \mathrm{KG}$ & 1 \\
\hline Chromium & $1.2 \mathrm{MG} / \mathrm{KG}$ & B & NP & $2 M G / K G$ & 1 \\
\hline Cobalt & $1.1 \mathrm{MG} / \mathrm{KG}$ & $u$ & $N P$ & $10 \mathrm{MG} / \mathrm{KG}$ & 1 \\
\hline Copper & $4.8 \mathrm{MG} / \mathrm{KG}$ & B & NP & $5 \mathrm{MG} / K \mathrm{~K}$ & 1 \\
\hline Iron & $8160 \mathrm{MG} / \mathrm{KG}$ & & NP & $20 \mathrm{MG} / \mathrm{KG}$ & 1 \\
\hline Magnesium & 924 MG/KG & B & NP & $1000 \mathrm{MG} / \mathrm{KG}$ & 1 \\
\hline Manganese & $9.1 \mathrm{MG} / \mathrm{KG}$ & & NP & 3 MG/KG & 1 \\
\hline Nickel & $2 \mathrm{MG} / \mathrm{KG}$ & B & $\mathrm{NP}$ & $8 \mathrm{MG} / \mathrm{KG}$ & 1 \\
\hline Potassium & 317 MG/KG & B & NP & $1000 \mathrm{MG} / \mathrm{KG}$ & 1 \\
\hline Silver & $0.82 \mathrm{MG} / \mathrm{KG}$ & $u$ & NP & $2 \mathrm{MG} / \mathrm{KG}$ & 1 \\
\hline Sodium & $60.8 \mathrm{MG} / \mathrm{KG}$ & B & $\mathrm{NP}$ & $1000 \mathrm{MG} / \mathrm{KG}$ & 1 \\
\hline Vanadium & $8 \mathrm{MG} / \mathrm{KG}$ & B & NP & $10 \mathrm{MG} / \mathrm{KG}$ & 1 \\
\hline Zinc & $5.3 \mathrm{MG} / \mathrm{KG}$ & & NP & 4 MG/KG & 1 \\
\hline Lead & $2.9 \mathrm{MG} / \mathrm{KG}$ & & NP & $0.6 \mathrm{MG} / \mathrm{KG}$ & 1 \\
\hline Selenium & $0.2 \mathrm{MG} / \mathrm{KG}$ & $u$ & NP & 1 MG/KG & 1 \\
\hline Thallium & $0.2 \mathrm{MG} / \mathrm{KG}$ & $u$ & NP & $2 M G / K G$ & 1 \\
\hline
\end{tabular}


APPENDIX F.1 Results of Metals Analyses, Soils, Salmon Site

\begin{tabular}{|c|c|c|c|c|c|c|}
\hline COMPOUND & RESULTS & UNITS & $\begin{array}{l}\text { DATA } \\
\text { QUAL. }\end{array}$ & $\begin{array}{l}\text { DATA } \\
\text { VALID. }\end{array}$ & CRDL LNITS & DILUTION \\
\hline \multicolumn{7}{|l|}{ C3-9-S } \\
\hline Arsenic & 2.1 & $M G / K G$ & & NP & 2 MG.KG & 1 \\
\hline Cyanide & 0.05 & MG/KG & $u$ & NP & $0.49 \mathrm{MG} / \mathrm{KG}$ & 1 \\
\hline Mercury & 0.05 & MG/KG & $u$ & NP & 0.1 MG"KG & 1 \\
\hline Aluminum & 548 & MG/KG & & NP & $40 M E:-G$ & 1 \\
\hline Antimony & 4.5 & MG/KG & u & NP & $12 M G K G$ & 1 \\
\hline Barium & 17.2 & MG/KG & B & NP & $40 \mathrm{MG} / \mathrm{KG}$ & 1 \\
\hline Beryllium & 0.34 & MG/KG & u & NP & 1 MG:KG & 1 \\
\hline Cadmium & 0.58 & MG/KG & $u$ & NP & $1 \mathrm{MG} / \mathrm{KG}$ & 1 \\
\hline Calcium & 863 & 3 MG/KG & B & NP & $1000 \mathrm{MG} / \mathrm{KG}$ & 1 \\
\hline Chromium & 0.96 & $M G / K G$ & $u$ & NP & $2 M G / K G$ & 1 \\
\hline Cobalt & 1.1 & $M G / K G$ & $u$ & NP & $10 \mathrm{MG} / \mathrm{KG}$ & 1 \\
\hline Copper & 0.88 & MG/KG & u & NP & $5 \mathrm{MG} / \mathrm{KG}$ & 1 \\
\hline Iron & 1040 & $M G / K G$ & & NP & $20 \mathrm{MG} / K G$ & 1 \\
\hline Magnesium & 179 & $M G / K G$ & $B$ & NP & 1000 MG/KG & 1 \\
\hline Manganese & 44.7 & $M G / K G$ & & NP & $3 M G / K G$ & 1 \\
\hline Nickel & 1.7 & MG/KG & $u$ & NP & $8 \mathrm{MG} / \mathrm{KG}$ & 1 \\
\hline Potassium & 139 & MG/KG & $u$ & NP & 1000 MG/KG & 1 \\
\hline Silver & 0.82 & $M G / K G$ & $u$ & NP & $2 M G / K G$ & 1 \\
\hline Sodium & 44.4 & $M G / K G$ & B & NP & $1000 \mathrm{MG} / \mathrm{KG}$ & 1 \\
\hline Vanadium & 1.3 & $M G / K G$ & B & NP & $10 \mathrm{MG} / \mathrm{KG}$ & 1 \\
\hline Zinc & 1.7 & $M G / K G$ & B & NP & 4 MG/KG & 1 \\
\hline Lead & 1.8 & 3 MG/KG & & NP & $0.6 \mathrm{MG} / \mathrm{KG}$ & 1 \\
\hline Selenium & 0.2 & MG/KG & $u$ & NP & $1 M G / K G$ & 1 \\
\hline Thallium & 0.2 & MG/KG & $u$ & NP & $2 \mathrm{MG} / \mathrm{KG}$ & 1 \\
\hline
\end{tabular}




\section{APPENDIX F.1 Results of Metals Analyses, Soils, Salmon Site}

\begin{tabular}{lrrrrr}
\hline COMPOUND & RESULTS UNITS & DATA & DATA & \\
QUAL. & VALID. & CRDL UNITS & DILUTION \\
\hline C4-10-S & $0.68 \mathrm{MG} / \mathrm{KG}$ & $\mathrm{B}$ & $\mathrm{NP}$ & $2 \mathrm{MG} / \mathrm{KG}$ & 1 \\
Arsenic & $0.05 \mathrm{MG} / \mathrm{KG}$ & $\mathrm{U}$ & $\mathrm{NP}$ & $0.49 \mathrm{MG} / \mathrm{KG}$ & 1 \\
Cyanide & $0.05 \mathrm{MG} / \mathrm{KG}$ & $\mathrm{U}$ & $\mathrm{NP}$ & $0.1 \mathrm{MG} / \mathrm{KG}$ & 1 \\
Mercury & $1330 \mathrm{MG} / \mathrm{KG}$ & & $\mathrm{NP}$ & $40 \mathrm{MG} / \mathrm{KG}$ & 1 \\
Aluminum & $4.5 \mathrm{MG} / \mathrm{KG}$ & $\mathrm{U}$ & $\mathrm{NP}$ & $12 \mathrm{MG} / \mathrm{KG}$ & 1 \\
Antimony & $13.6 \mathrm{MG} / \mathrm{KG}$ & $\mathrm{B}$ & $\mathrm{NP}$ & $40 \mathrm{MG} / \mathrm{KG}$ & 1 \\
Barium & $0.34 \mathrm{MG} / \mathrm{KG}$ & $\mathrm{U}$ & $\mathrm{NP}$ & $1 \mathrm{MG} / \mathrm{KG}$ & 1 \\
Beryllium & $0.58 \mathrm{MG} / \mathrm{KG}$ & $\mathrm{U}$ & $\mathrm{NP}$ & $1 \mathrm{MG} / \mathrm{KG}$ & 1 \\
Cadmium & $2430 \mathrm{MG} / \mathrm{KG}$ & & $\mathrm{NP}$ & $1000 \mathrm{MG} / \mathrm{KG}$ & 1 \\
Calcium & $1.3 \mathrm{MG} / \mathrm{KG}$ & $\mathrm{B}$ & $\mathrm{NP}$ & $2 \mathrm{MG} / \mathrm{KG}$ & 1 \\
Chromium & $1.1 \mathrm{MG} / \mathrm{KG}$ & $\mathrm{U}$ & $\mathrm{NP}$ & $10 \mathrm{MG} / \mathrm{KG}$ & 1 \\
Cobalt & $3.6 \mathrm{MG} / \mathrm{KG}$ & $\mathrm{B}$ & $\mathrm{NP}$ & $5 \mathrm{MG} / \mathrm{KG}$ & 1 \\
Copper & $2740 \mathrm{MG} / \mathrm{KG}$ & & $\mathrm{NP}$ & $20 \mathrm{MG} / \mathrm{KG}$ & 1 \\
Iron & $1020 \mathrm{MG} / \mathrm{KG}$ & & $\mathrm{NP}$ & $1000 \mathrm{MG} / \mathrm{KG}$ & 1 \\
Magnesium & $581 \mathrm{MG} / \mathrm{KG}$ & & $\mathrm{NP}$ & $3 \mathrm{MG} / \mathrm{KG}$ & 1 \\
Manganese & $2.2 \mathrm{MG} / \mathrm{KG}$ & $\mathrm{B}$ & $\mathrm{NP}$ & $8 \mathrm{MG} / \mathrm{KG}$ & 1 \\
Nickel & $213 \mathrm{MG} / \mathrm{KG}$ & $\mathrm{B}$ & $\mathrm{NP}$ & $1000 \mathrm{MG} / \mathrm{KG}$ & 1 \\
Potassium & $0.82 \mathrm{MG} / \mathrm{KG}$ & $\mathrm{U}$ & $\mathrm{NP}$ & $2 \mathrm{MG} / \mathrm{KG}$ & 1 \\
Silver & $109 \mathrm{MG} / \mathrm{KG}$ & $\mathrm{B}$ & $\mathrm{NP}$ & $1000 \mathrm{MG} / \mathrm{KG}$ & 1 \\
Sodium & $2.2 \mathrm{MG} / \mathrm{KG}$ & $\mathrm{B}$ & $\mathrm{NP}$ & $10 \mathrm{MG} / \mathrm{KG}$ & 1 \\
Vanadium & $2.2 \mathrm{MG} / \mathrm{KG}$ & $\mathrm{B}$ & $\mathrm{NP}$ & $4 \mathrm{MG} / \mathrm{KG}$ & 1 \\
Zinc & $6.1 \mathrm{MG} / \mathrm{KG}$ & & $\mathrm{NP}$ & $0.6 \mathrm{MG} / \mathrm{KG}$ & 1 \\
Lead & $0.2 \mathrm{MG} / \mathrm{KG}$ & $\mathrm{U}$ & $\mathrm{NP}$ & $1 \mathrm{MG} / \mathrm{KG}$ & 1 \\
Selenium & $0.2 \mathrm{MG} / \mathrm{KG}$ & $\mathrm{U}$ & $\mathrm{NP}$ & $2 \mathrm{MG} / \mathrm{KG}$ & 1 \\
Thallium & & & & &
\end{tabular}


APPENDIX F.1 Results of Metals Analyses, Soils, Salmon Site

\begin{tabular}{lrrrrr}
\hline COMPOUND & RESULTS UNITS & DATA & DATA & & \\
C4-11-S & & & & & \\
Arsenic & $3 \mathrm{MG} / \mathrm{KG}$ & & $\mathrm{NP}$ & $2 \mathrm{MG} / \mathrm{KG}$ & 1 \\
Cyanide & $0.05 \mathrm{MG} / \mathrm{KG}$ & $\mathrm{U}$ & $\mathrm{NP}$ & $0.5 \mathrm{MG} / \mathrm{KG}$ & 1 \\
Mercury & $0.05 \mathrm{MG} / \mathrm{KG}$ & $\mathrm{U}$ & $\mathrm{NP}$ & $0.1 \mathrm{MG} / \mathrm{KG}$ & 1 \\
Aluminum & $1180 \mathrm{MG} / \mathrm{KG}$ & & $\mathrm{NP}$ & $40 \mathrm{MG} / \mathrm{KG}$ & 1 \\
Antimony & $4.5 \mathrm{MG} / \mathrm{KG}$ & $\mathrm{U}$ & $\mathrm{NP}$ & $12 \mathrm{MG} / \mathrm{KG}$ & 1 \\
Barium & $18.1 \mathrm{MG} / \mathrm{KG}$ & $\mathrm{B}$ & $\mathrm{NP}$ & $40 \mathrm{MG} / \mathrm{KG}$ & 1 \\
Beryllium & $0.46 \mathrm{MG} / \mathrm{KG}$ & $\mathrm{B}$ & $\mathrm{NP}$ & $1 \mathrm{MG} / \mathrm{KG}$ & 1 \\
Cadmium & $0.58 \mathrm{MG} / \mathrm{KG}$ & $\mathrm{U}$ & $\mathrm{NP}$ & $1 \mathrm{MG} / \mathrm{KG}$ & 1 \\
Calcium & $1890 \mathrm{MG} / \mathrm{KG}$ & & $\mathrm{NP}$ & $1000 \mathrm{MG} / \mathrm{KG}$ & 1 \\
Chromium & $0.96 \mathrm{MG} / \mathrm{KG}$ & $\mathrm{U}$ & $\mathrm{NP}$ & $2 \mathrm{MG} / \mathrm{KG}$ & 1 \\
Cobalt & $11.1 \mathrm{MG} / \mathrm{KG}$ & & $\mathrm{NP}$ & $10 \mathrm{MG} / \mathrm{KG}$ & 1 \\
Copper & $5.1 \mathrm{MG} / \mathrm{KG}$ & & $\mathrm{NP}$ & $5 \mathrm{MG} / \mathrm{KG}$ & 1 \\
Iron & $4210 \mathrm{MG} / \mathrm{KG}$ & & $\mathrm{NP}$ & $20 \mathrm{MG} / \mathrm{KG}$ & 1 \\
Magnesium & $602 \mathrm{MG} / \mathrm{KG}$ & $\mathrm{B}$ & $\mathrm{NP}$ & $1000 \mathrm{MG} / \mathrm{KG}$ & 1 \\
Manganese & $55.2 \mathrm{MG} / \mathrm{KG}$ & & $\mathrm{NP}$ & $3 \mathrm{MG} / \mathrm{KG}$ & 1 \\
Nickel & $9.5 \mathrm{MG} / \mathrm{KG}$ & & $\mathrm{NP}$ & $8 \mathrm{MG} / \mathrm{KG}$ & 1 \\
Potassium & $167 \mathrm{MG} / \mathrm{KG}$ & $\mathrm{B}$ & $\mathrm{NP}$ & $1000 \mathrm{MG} / \mathrm{KG}$ & 1 \\
Silver & $0.82 \mathrm{MG} / \mathrm{KG}$ & $\mathrm{U}$ & $\mathrm{NP}$ & $2 \mathrm{MG} / \mathrm{KG}$ & 1 \\
Sodium & $54.3 \mathrm{MG} / \mathrm{KG}$ & $\mathrm{B}$ & $\mathrm{NP}$ & $1000 \mathrm{MG} / \mathrm{KG}$ & 1 \\
Vanadium & $1.6 \mathrm{MG} / \mathrm{KG}$ & $\mathrm{B}$ & $\mathrm{NP}$ & $10 \mathrm{MG} / \mathrm{KG}$ & 1 \\
Zinc & $8.9 \mathrm{MG} / \mathrm{KG}$ & & $\mathrm{NP}$ & $4 \mathrm{MG} / \mathrm{KG}$ & 1 \\
Lead & $6.1 \mathrm{MG} / \mathrm{KG}$ & & $\mathrm{NP}$ & $0.6 \mathrm{MG} / \mathrm{KG}$ & 1 \\
Selenium & $0.16 \mathrm{MG} / \mathrm{KG}$ & $\mathrm{U}$ & $\mathrm{NP}$ & $1 \mathrm{MG} / \mathrm{KG}$ & 1 \\
Thallium & $0.2 \mathrm{MG} / \mathrm{KG}$ & $\mathrm{U}$ & $\mathrm{NP}$ & $2 \mathrm{MG} / \mathrm{KG}$ & 1 \\
& & & & &
\end{tabular}


APPENDIX F.1 Results of Metals Analyses, Soils, Salmon Site

\begin{tabular}{|c|c|c|c|c|c|}
\hline COMPOUND & RESULTS UNITS & $\begin{array}{l}\text { DATA } \\
\text { QUAL. }\end{array}$ & $\begin{array}{l}\text { DATA } \\
\text { VALID. }\end{array}$ & CRDL UNITS & DILUTION \\
\hline \multicolumn{6}{|l|}{ C4-12-S } \\
\hline Arsenic & $1 \mathrm{MG} / \mathrm{KG}$ & B & NP & $2 \mathrm{MG} / \mathrm{KG}$ & 1 \\
\hline Cyanide & $0.05 \mathrm{MG} / \mathrm{KG}$ & $u$ & NP & $0.49 \mathrm{MG} / \mathrm{KG}$ & 1 \\
\hline Mercury & $0.05 \mathrm{MG} / \mathrm{KG}$ & $u$ & NP & $0.1 \mathrm{MG} / \mathrm{KG}$ & 1 \\
\hline Aluminum & $1710 \mathrm{MG} / \mathrm{KG}$ & & NP & $40 \mathrm{MG} / \mathrm{KG}$ & 1 \\
\hline Antimony & $4.5 \mathrm{MG} / \mathrm{KG}$ & $u$ & NP & $12 \mathrm{MG} / \mathrm{KG}$ & 1 \\
\hline Barium & 25.2 MG/KG & B & NP & $40 \mathrm{MG} / \mathrm{KG}$ & 1 \\
\hline Beryllium & 0.34 MG/KG & $u$ & NP & $1 \mathrm{MG} / \mathrm{KG}$ & 1 \\
\hline Cadmium & $0.58 \mathrm{MG} / \mathrm{KG}$ & $u$ & NP & $1 \mathrm{MG} / \mathrm{KG}$ & 1 \\
\hline Calcium & 409 MG/KG & B & NP & $1000 \mathrm{MG} / \mathrm{KG}$ & 1 \\
\hline Chromium & 99.6 MG/KG & & NP & $2 M G / K G$ & 1 \\
\hline Cobalt & $1.7 \mathrm{MG} / \mathrm{KG}$ & B & NP & $10 \mathrm{MG} / \mathrm{KG}$ & 1 \\
\hline Copper & $21.4 \mathrm{MG} / \mathrm{KG}$ & & NP & $5 \mathrm{MG} / K \mathrm{~K}$ & 1 \\
\hline Iron & $3660 \mathrm{MG} / \mathrm{KG}$ & & NP & $20 \mathrm{MG} / \mathrm{KG}$ & 1 \\
\hline Magnesium & $99.5 \mathrm{MG} / \mathrm{KG}$ & B & NP & $1000 \mathrm{MG} / \mathrm{KG}$ & 1 \\
\hline Manganese & $43.2 \mathrm{MG} / \mathrm{KG}$ & & $\mathrm{NP}$ & $3 \mathrm{MG} / \mathrm{KG}$ & 1 \\
\hline Nickel & $27 \mathrm{MG} / \mathrm{KG}$ & & NP & $8 \mathrm{MG} / \mathrm{KG}$ & 1 \\
\hline Potassium & $139 \mathrm{MG} / \mathrm{KG}$ & $u$ & $\mathrm{NP}$ & $1000 \mathrm{MG} / \mathrm{KG}$ & 1 \\
\hline Silver & $0.82 \mathrm{MG} / \mathrm{KG}$ & $u$ & NP & $2 M G / K G$ & 1 \\
\hline Sodium & $93.9 \mathrm{MG} / \mathrm{KG}$ & B & NP & $1000 \mathrm{MG} / \mathrm{KG}$ & 1 \\
\hline Vanadium & $6.1 \mathrm{MG} / \mathrm{KG}$ & $B$ & $N P$ & $10 \mathrm{MG} / \mathrm{KG}$ & 1 \\
\hline Zinc & $8.5 \mathrm{MG} / \mathrm{KG}$ & & NP & $4 \mathrm{MG} / \mathrm{KG}$ & 1 \\
\hline Lead & $3.5 \mathrm{MG} / \mathrm{KG}$ & & $\mathrm{NP}$ & $0.6 \mathrm{MG} / \mathrm{KG}$ & 1 \\
\hline Selenium & $0.16 \mathrm{MG} / \mathrm{KG}$ & $u$ & NP & $1 \mathrm{MG} / \mathrm{KG}$ & 1 \\
\hline Thallium & $0.2 \mathrm{MG} / \mathrm{KG}$ & u & $\mathrm{NP}$ & $2 M G / K G$ & 1 \\
\hline
\end{tabular}


APPENDIX F.1 Results of Metals Analyses, Soils, Salmon Site

\begin{tabular}{|c|c|c|c|c|c|}
\hline COMPOUND & RESULTS UNITS & $\begin{array}{l}\text { DATA } \\
\text { QUAL. }\end{array}$ & $\begin{array}{l}\text { DATA } \\
\text { VALID. }\end{array}$ & CRDL UNITS & DILUTION \\
\hline \multicolumn{6}{|l|}{ C4-13-s } \\
\hline Arsenic & $0.48 \mathrm{MG} / \mathrm{KG}$ & B & NP & $2 M C: / K G$ & 1 \\
\hline Cyanide & $0.05 \mathrm{MG} / \mathrm{KG}$ & $u$ & NP & $0.49 \mathrm{MG} / \mathrm{KG}$ & 1 \\
\hline Mercury & $0.05 \mathrm{MG} / \mathrm{KG}$ & $u$ & NP & $0.1 \mathrm{Mr.KG}$ & 1 \\
\hline Aluminum & $1820 \mathrm{MG} / \mathrm{KG}$ & & NP & $40 \mathrm{M}=\mathrm{KG}$ & 1 \\
\hline Antimony & $4.5 \mathrm{MG} / \mathrm{KG}$ & $u$ & NP & $12 \mathrm{MG} / \mathrm{KG}$ & 1 \\
\hline Barium & $31.2 \mathrm{MG} / \mathrm{KG}$ & B & NP & $40 \mathrm{MG} / \mathrm{KG}$ & 1 \\
\hline Beryllium & $2.4 \mathrm{MG} / \mathrm{KG}$ & & NP & 1 MOKG & 1 \\
\hline Cadmium & $0.58 \mathrm{MG} / \mathrm{KG}$ & $u$ & NP & 1 MG/KG & 1 \\
\hline Calcium & $4420 \mathrm{MG} / \mathrm{KG}$ & & NP & $1000 \mathrm{MG} / \mathrm{KG}$ & 1 \\
\hline Chromium & $1.6 \mathrm{MG} / \mathrm{KG}$ & B & NP & $2 M G / K G$ & 1 \\
\hline Cobalt & $43.1 \mathrm{MG} / \mathrm{KG}$ & & NP & $10 \mathrm{MG} / \mathrm{KG}$ & 1 \\
\hline Copper & $12.8 \mathrm{MG} / \mathrm{KG}$ & & NP & 5 MG/KG & 1 \\
\hline Iron & 425 MG/KG & & NP & $20 \mathrm{MG} / \mathrm{KG}$ & 1 \\
\hline Magnesium & $1250 \mathrm{MG} / \mathrm{KG}$ & & NP & $1000 \mathrm{MG} / \mathrm{KG}$ & 1 \\
\hline Manganese & $30.5 \mathrm{MG} / K G$ & & NP & $3 M G / K G$ & 1 \\
\hline Nickel . & $29.8 \mathrm{MG} / \mathrm{KG}$ & & NP & $8 \mathrm{MG} / \mathrm{KG}$ & 1 \\
\hline Potassium & $447 \mathrm{MG} / \mathrm{KG}$ & B & NP & $1000 \mathrm{MG} / \mathrm{KG}$ & 1 \\
\hline Silver & $0.82 \mathrm{MG} / \mathrm{KG}$ & u & NP & $2 M G / K G$ & 1 \\
\hline Sodium & $87.8 \mathrm{MG} / \mathrm{KG}$ & B & NP & $1000 \mathrm{MG} / \mathrm{KG}$ & 1 \\
\hline Vanadium & $4.1 \mathrm{MG} / \mathrm{KG}$ & B & NP & $10 \mathrm{MG} / \mathrm{KG}$ & 1 \\
\hline Zinc & $26.4 \mathrm{MG} / \mathrm{KG}$ & & NP & 4 MG/KG & 1 \\
\hline Lead & $14.6 \mathrm{MG} / \mathrm{KG}$ & & NP & $1.2 \mathrm{MG} / \mathrm{KG}$ & 2 \\
\hline Selenium & $0.16 \mathrm{MG} / \mathrm{KG}$ & u & NP & $1 \mathrm{MG} / K G$ & 1 \\
\hline Thallium & $0.2 \mathrm{MG} / \mathrm{KG}$ & $u$ & NP & $2 \mathrm{MG} / \mathrm{KG}$ & 1 \\
\hline
\end{tabular}


APPENDIX F.1 Results of Metals Analyses, Soils, Salmon Site

\begin{tabular}{lrrrrr}
\hline COMPOUND & RESULTS UNITS & DATA & DATA & \\
Q & QUAL. & VALID. & CRDL UNITS & DILUTION \\
Arsenic & $0.26 \mathrm{MG} / \mathrm{KG}$ & $\mathrm{U}$ & $\mathrm{NP}$ & $2 \mathrm{MG} / \mathrm{KG}$ & 1 \\
Cyanide & $0.05 \mathrm{MG} / \mathrm{KG}$ & $\mathrm{U}$ & $\mathrm{NP}$ & $0.5 \mathrm{MG} / \mathrm{KG}$ & 1 \\
Mercury & $0.05 \mathrm{MG} / \mathrm{KG}$ & $\mathrm{U}$ & $\mathrm{NP}$ & $0.1 \mathrm{MG} / \mathrm{KG}$ & 1 \\
Aluminum & $495 \mathrm{MG} / \mathrm{KG}$ & & $\mathrm{NP}$ & $40 \mathrm{MG} / \mathrm{KG}$ & 1 \\
Antimony & $4.5 \mathrm{MG} / \mathrm{KG}$ & $\mathrm{U}$ & $\mathrm{NP}$ & $12 \mathrm{MG} / \mathrm{KG}$ & 1 \\
Barium & $8.2 \mathrm{MG} / \mathrm{KG}$ & $\mathrm{B}$ & $\mathrm{NP}$ & $40 \mathrm{MG} / \mathrm{KG}$ & 1 \\
Beryllium & $0.34 \mathrm{MG} / \mathrm{KG}$ & $\mathrm{U}$ & $\mathrm{NP}$ & $1 \mathrm{MG} / \mathrm{KG}$ & 1 \\
Cadmium & $0.58 \mathrm{MG} / \mathrm{KG}$ & $\mathrm{U}$ & $\mathrm{NP}$ & $1 \mathrm{MG} / \mathrm{KG}$ & 1 \\
Calcium & $355 \mathrm{MG} / \mathrm{KG}$ & $\mathrm{B}$ & $\mathrm{NP}$ & $1000 \mathrm{MG} / \mathrm{KG}$ & 1 \\
Chromium & $5.7 \mathrm{MG} / \mathrm{KG}$ & & $\mathrm{NP}$ & $2 \mathrm{MG} / \mathrm{KG}$ & 1 \\
Cobalt & $1.1 \mathrm{MG} / \mathrm{KG}$ & $\mathrm{U}$ & $\mathrm{NP}$ & $10 \mathrm{MG} / \mathrm{KG}$ & 1 \\
Copper & $2.2 \mathrm{MG} / \mathrm{KG}$ & $\mathrm{B}$ & $\mathrm{NP}$ & $5 \mathrm{MG} / \mathrm{KG}$ & 1 \\
Iron & $814 \mathrm{MG} / \mathrm{KG}$ & & $\mathrm{NP}$ & $20 \mathrm{MG} / \mathrm{KG}$ & 1 \\
Magnesium & $96.1 \mathrm{MG} / \mathrm{KG}$ & $\mathrm{B}$ & $\mathrm{NP}$ & $1000 \mathrm{MG} / \mathrm{KG}$ & 1 \\
Manganese & $5.3 \mathrm{MG} / \mathrm{KG}$ & & $\mathrm{NP}$ & $3 \mathrm{MG} / \mathrm{KG}$ & 1 \\
Nickel & $1.7 \mathrm{MG} / \mathrm{KG}$ & $\mathrm{U}$ & $\mathrm{NP}$ & $8 \mathrm{MG} / \mathrm{KG}$ & 1 \\
Potassium & $139 \mathrm{MG} / \mathrm{KG}$ & $\mathrm{U}$ & $\mathrm{NP}$ & $1000 \mathrm{MG} / \mathrm{KG}$ & 1 \\
Silver & $0.82 \mathrm{MG} / \mathrm{KG}$ & $\mathrm{U}$ & $\mathrm{NP}$ & $2 \mathrm{MG} / \mathrm{KG}$ & 1 \\
Sodium & $166 \mathrm{MG} / \mathrm{KG}$ & $\mathrm{B}$ & $\mathrm{NP}$ & $1000 \mathrm{MG} / \mathrm{KG}$ & 1 \\
Vanadium & $3.6 \mathrm{MG} / \mathrm{KG}$ & $\mathrm{B}$ & $\mathrm{NP}$ & $10 \mathrm{MG} / \mathrm{KG}$ & 1 \\
Zinc & $4.2 \mathrm{MG} / \mathrm{KG}$ & & $\mathrm{NP}$ & $4 \mathrm{MG} / \mathrm{KG}$ & 1 \\
Lead & $1.2 \mathrm{MG} / \mathrm{KG}$ & & $\mathrm{NP}$ & $0.6 \mathrm{MG} / \mathrm{KG}$ & 1 \\
Selenium & $0.16 \mathrm{MG} / \mathrm{KG}$ & $\mathrm{U}$ & $\mathrm{NP}$ & $1 \mathrm{MG} / \mathrm{KG}$ & 1 \\
Thallium & $0.2 \mathrm{MG} / \mathrm{KG}$ & $\mathrm{U}$ & $\mathrm{NP}$ & $2 \mathrm{MG} / \mathrm{KG}$ & 1
\end{tabular}


APPENDIX F.1 Results of Metals Analyses, Soils, Salmon Site

\begin{tabular}{|c|c|c|c|c|c|}
\hline COMPOUND & RESULTS UNITS & $\begin{array}{l}\text { DATA } \\
\text { QUAL. }\end{array}$ & $\begin{array}{l}\text { DATA } \\
\text { VALID. }\end{array}$ & CRDL UNITS & DILUTION \\
\hline \multicolumn{6}{|l|}{ C1-15-S } \\
\hline Arsenic & $0.26 \mathrm{MG} / \mathrm{KG}$ & $u$ & NP & $2 \mathrm{MG} / \mathrm{KG}$ & 1 \\
\hline Cyanide & 0.05 MG/KG & $u$ & NP & $0.48 \mathrm{MG} / \mathrm{KG}$ & 1 \\
\hline Mercury & 0.05 MG/KG & $u$ & NP & $0.1 \mathrm{MG} / \mathrm{KG}$ & 1 \\
\hline Aluminum & 394 MG/KG & & NP & $40 \mathrm{MG} / \mathrm{KG}$ & 1 \\
\hline Antimony & $4.5 \mathrm{MG} / \mathrm{KG}$ & $u$ & NP & $12 \mathrm{MG} / \mathrm{KG}$ & 1 \\
\hline Barium & $7.5 \mathrm{MG} / \mathrm{KG}$ & B & NP & $40 \mathrm{MG} / \mathrm{KG}$ & 1 \\
\hline Beryllium & $0.34 \mathrm{MG} / K G$ & $u$ & NP & $1 \mathrm{MG} / K \mathrm{~K}$ & 1 \\
\hline Cadmium & $0.58 \mathrm{MG} / \mathrm{KG}$ & $u$ & NP & 1 MG/KG & 1 \\
\hline Calcium & 286 MG/KG & 8 & NP & 1000 MG/KG & 1 \\
\hline Chromium & $2.5 \mathrm{MG} / \mathrm{KG}$ & & NP & $2 M G / K G$ & 1 \\
\hline Cobalt & $1.1 \mathrm{MG} / \mathrm{KG}$ & $u$ & NP & $10 \mathrm{MG} / \mathrm{KG}$ & 1 \\
\hline Copper & $1.4 \mathrm{MG} / \mathrm{KG}$ & B & NP & 5 MG/KG & 1 \\
\hline Iron & 785 MG/KG & & NP & $20 \mathrm{MG} / K \mathrm{~K}$ & 1 \\
\hline Magnesium & 82 MG/KG & B & NP & $1000 \mathrm{MG} / \mathrm{KG}$ & 1 \\
\hline Manganese & $4.1 \mathrm{MG} / \mathrm{KG}$ & & NP & 3 MG/KG & 1 \\
\hline Nickel & $1.7 \mathrm{MG} / \mathrm{KG}$ & $u$ & NP & $8 \mathrm{MG} / \mathrm{KG}$ & 1 \\
\hline Potassium & $139 \mathrm{MG} / \mathrm{KG}$ & $u$ & NP & $1000 \mathrm{MG} / \mathrm{KG}$ & 1 \\
\hline Silver & $0.82 \mathrm{MG} / \mathrm{KG}$ & $u$ & NP & $2 \mathrm{MG} / \mathrm{KG}$ & 1 \\
\hline Sodium & 79.4 MG/KG & B & NP & $1000 \mathrm{MG} / \mathrm{KG}$ & 1 \\
\hline Vanadium & 4.2 MG/KG & B & NP & $10 \mathrm{MG} / \mathrm{KG}$ & 1 \\
\hline Zinc & $4.4 \mathrm{MG} / \mathrm{KG}$ & & NP & $4 \mathrm{MG} / \mathrm{KG}$ & 1 \\
\hline Lead & $1.2 \mathrm{MG} / \mathrm{KG}$ & & NP & $0.6 \mathrm{MG} / \mathrm{KG}$ & 1 \\
\hline Selenium & $0.16 \mathrm{MG} / \mathrm{KG}$ & $u$ & NP & $1 \mathrm{MG} / \mathrm{KG}$ & 1 \\
\hline Thallium & $0.2 \mathrm{MG} / \mathrm{KG}$ & $u$ & NP & $2 \mathrm{MG} / \mathrm{KG}$ & 1 \\
\hline
\end{tabular}


APPENDIX F.1 Results of Metals Analyses, Soils, Salmon Site

\begin{tabular}{lrrrrr}
\hline COMPOUND & RESULTS UNITS & DATA & DATA & \\
C1-16-S & VALID. & CRDL UNITS & DILUTION \\
Arsenic & $0.26 \mathrm{MG} / \mathrm{KG}$ & $\mathrm{U}$ & $\mathrm{NP}$ & $2 \mathrm{MG} / \mathrm{KG}$ & 1 \\
Cyanide & $0.05 \mathrm{MG} / \mathrm{KG}$ & $\mathrm{U}$ & $\mathrm{NP}$ & $0.5 \mathrm{MG} / \mathrm{KG}$ & 1 \\
Mercury & $0.05 \mathrm{MG} / \mathrm{KG}$ & $\mathrm{U}$ & $\mathrm{NP}$ & $0.1 \mathrm{MG} / \mathrm{KG}$ & 1 \\
Aluminum & $551 \mathrm{MG} / \mathrm{KG}$ & & $\mathrm{NP}$ & $40 \mathrm{MG} / \mathrm{KG}$ & 1 \\
Antimony & $4.5 \mathrm{MG} / \mathrm{KG}$ & $\mathrm{U}$ & $\mathrm{NP}$ & $12 \mathrm{MG} / \mathrm{KG}$ & 1 \\
Barium & $6.9 \mathrm{MG} / \mathrm{KG}$ & $\mathrm{B}$ & $\mathrm{NP}$ & $40 \mathrm{MG} / \mathrm{KG}$ & 1 \\
Beryllium & $0.34 \mathrm{MG} / \mathrm{KG}$ & $\mathrm{U}$ & $\mathrm{NP}$ & $1 \mathrm{MG} / \mathrm{KG}$ & 1 \\
Cadmium & $0.58 \mathrm{MG} / \mathrm{KG}$ & $\mathrm{U}$ & $\mathrm{NP}$ & $1 \mathrm{MG} / \mathrm{KG}$ & 1 \\
Calcium & $304 \mathrm{MG} / \mathrm{KG}$ & $\mathrm{B}$ & $\mathrm{NP}$ & $1000 \mathrm{MG} / \mathrm{KG}$ & 1 \\
Chromium & $1.6 \mathrm{MG} / \mathrm{KG}$ & $\mathrm{B}$ & $\mathrm{NP}$ & $2 \mathrm{MG} / \mathrm{KG}$ & 1 \\
Cobalt & $1.1 \mathrm{MG} / \mathrm{KG}$ & $\mathrm{U}$ & $\mathrm{NP}$ & $10 \mathrm{MG} / \mathrm{KG}$ & 1 \\
Copper & $0.88 \mathrm{MG} / \mathrm{KG}$ & $\mathrm{B}$ & $\mathrm{NP}$ & $5 \mathrm{MG} / \mathrm{KG}$ & 1 \\
Iron & $187 \mathrm{MG} / \mathrm{KG}$ & & $\mathrm{NP}$ & $20 \mathrm{MG} / \mathrm{KG}$ & 1 \\
Magnesium & $50.3 \mathrm{MG} / \mathrm{KG}$ & $\mathrm{B}$ & $\mathrm{NP}$ & $1000 \mathrm{MG} / \mathrm{KG}$ & 1 \\
Manganese & $2.1 \mathrm{MG} / \mathrm{KG}$ & $\mathrm{B}$ & $\mathrm{NP}$ & $3 \mathrm{MG} / \mathrm{KG}$ & 1 \\
Nickel & $1.7 \mathrm{MG} / \mathrm{KG}$ & $\mathrm{U}$ & $\mathrm{NP}$ & $8 \mathrm{MG} / \mathrm{KG}$ & 1 \\
Potassium & $139 \mathrm{MG} / \mathrm{KG}$ & $\mathrm{U}$ & $\mathrm{NP}$ & $1000 \mathrm{MG} / \mathrm{KG}$ & 1 \\
Silver & $0.82 \mathrm{MG} / \mathrm{KG}$ & $\mathrm{U}$ & $\mathrm{NP}$ & $2 \mathrm{MG} / \mathrm{KG}$ & 1 \\
Sodium & $53.3 \mathrm{MG} / \mathrm{KG}$ & $\mathrm{B}$ & $\mathrm{NP}$ & $1000 \mathrm{MG} / \mathrm{KG}$ & 1 \\
Vanadium & $0.66 \mathrm{MG} / \mathrm{KG}$ & $\mathrm{U}$ & $\mathrm{NP}$ & $10 \mathrm{MG} / \mathrm{KG}$ & 1 \\
Zinc & $4 \mathrm{MG} / \mathrm{KG}$ & & $\mathrm{NP}$ & $4 \mathrm{MG} / \mathrm{KG}$ & 1 \\
Lead & $1.1 \mathrm{MG} / \mathrm{KG}$ & & $\mathrm{NP}$ & $0.6 \mathrm{MG} / \mathrm{KG}$ & 1 \\
Selenium & $0.16 \mathrm{MG} / \mathrm{KG}$ & $\mathrm{U}$ & $\mathrm{NP}$ & $1 \mathrm{MG} / \mathrm{KG}$ & 1 \\
Thallium & $0.2 \mathrm{MG} / \mathrm{KG}$ & $\mathrm{U}$ & $\mathrm{NP}$ & $2 \mathrm{MG} / \mathrm{KG}$ & 1 \\
& & & & &
\end{tabular}




\section{APPENDIX F.1 Results of Metals Analyses, Soils, Salmon Site}

\begin{tabular}{|c|c|c|c|c|c|}
\hline COMPOUND & RESULTS UNITS & $\begin{array}{l}\text { DATA } \\
\text { QUAL. }\end{array}$ & $\begin{array}{l}\text { DATA } \\
\text { VALID. }\end{array}$ & CRDL UNITS & DILUTION \\
\hline \multicolumn{6}{|l|}{ C1-17-s } \\
\hline Arsenic & $0.26 \mathrm{MG} / \mathrm{KG}$ & $u$ & NP & $2 M G / K G$ & 1 \\
\hline Cyanide & $0.05 \mathrm{MG} / \mathrm{KG}$ & $u$ & NP & $0.49 \mathrm{MG} / \mathrm{KG}$ & 1 \\
\hline Mercury & $0.05 \mathrm{MG} / \mathrm{KG}$ & $u$ & NP & $0.1 \mathrm{Mr}=\mathrm{KG}$ & 1 \\
\hline Aluminum & $313 \mathrm{MG} / \mathrm{KG}$ & & NP & $40 M: G$ & 1 \\
\hline Antimony & $4.5 \mathrm{MG} / \mathrm{KG}$ & $u$ & NP & $12 \mathrm{MüKG}$ & 1 \\
\hline Barium & $5.6 \mathrm{MG} / \mathrm{KG}$ & B & NP & $40 \mathrm{MG} / \mathrm{KG}$ & 1 \\
\hline Beryllium & $0.34 \mathrm{MG} / \mathrm{KG}$ & $u$ & NP & 1 MGiKG & 1 \\
\hline Cadmium & $0.58 \mathrm{MG} / \mathrm{KG}$ & $u$ & NP & 1 MG/KG & 1 \\
\hline Calcium & $229 \mathrm{MG} / \mathrm{KG}$ & B & NP & $1000 \mathrm{MG} / \mathrm{KG}$ & 1 \\
\hline Chromium & $2 M G / K G$ & & NP & $2 \mathrm{MG} / \mathrm{KG}$ & 1 \\
\hline Cobalt & $1.2 \mathrm{MG} / \mathrm{KG}$ & B & NP & $10 \mathrm{MG} / \mathrm{KG}$ & 1 \\
\hline Copper & $1.8 \mathrm{MG} / \mathrm{KG}$ & B & NP & $5 \mathrm{MG} / \mathrm{KG}$ & 1 \\
\hline Iron & $239 \mathrm{MG} / \mathrm{KG}$ & & NP & 20 MG/KG & 1 \\
\hline Magnesium & $69.6 \mathrm{MG} / \mathrm{KG}$ & 8 & NP & $1000 \mathrm{MG} / \mathrm{KG}$ & 1 \\
\hline Manganese & $4.9 \mathrm{MG} / \mathrm{KG}$ & & NP & $3 \mathrm{MG} / \mathrm{KG}$ & 1 \\
\hline Nickel & $1.7 \mathrm{MG} / \mathrm{KG}$ & $u$ & NP & $8 \mathrm{MG} / \mathrm{KG}$ & 1 \\
\hline Potassium & $139 \mathrm{MG} / \mathrm{KG}$ & $u$ & NP & $1000 \mathrm{MG} / \mathrm{KG}$ & 1 \\
\hline Silver & $0.82 \mathrm{MG} / \mathrm{KG}$ & $u$ & NP & $2 M G / K G$ & 1 \\
\hline Sodium & $221 \mathrm{MG} / \mathrm{KG}$ & B & NP & $1000 \mathrm{MG} / \mathrm{KG}$ & 1 \\
\hline Vanadium & $2.1 \mathrm{MG} / \mathrm{KG}$ & B & NP & $10 \mathrm{MG} / \mathrm{KG}$ & 1 \\
\hline Zinc & 4.8 MG/KG & & NP & $4 M G / K G$ & 1 \\
\hline Lead & $1.4 \mathrm{MG} / \mathrm{KG}$ & & NP & $0.6 \mathrm{MG} / \mathrm{KG}$ & 1 \\
\hline Selenium & $0.16 \mathrm{MG} / \mathrm{KG}$ & $u$ & NP & $1 \mathrm{MG} / \mathrm{KG}$ & 1 \\
\hline Thallium & $0.2 \mathrm{MG} / \mathrm{KG}$ & $u$ & NP & $2 M G / K G$ & 1 \\
\hline
\end{tabular}


APPENDIX F.1 Results of Metals Analyses, Soils, Salmon Site

\begin{tabular}{|c|c|c|c|c|c|c|}
\hline COMPOUND & RESULTS & UNITS & $\begin{array}{l}\text { DATA } \\
\text { QUAL. }\end{array}$ & $\begin{array}{r}\text { DATA } \\
\text { VALID. } \\
\end{array}$ & CRDL UNITS & DILUTION \\
\hline \multicolumn{7}{|l|}{ C1-17-S-DUP } \\
\hline Arsenic & 0.26 & $M G / K G$ & $u$ & NP & $2 M G / K G$ & 1 \\
\hline Cyanide & 0.05 & $M G / K G$ & $u$ & NP & $0.49 \mathrm{MG} / \mathrm{KG}$ & 1 \\
\hline Mercury & 0.05 & $M G / K G$ & $u$ & NP & $0.1 \mathrm{MG} / \mathrm{KG}$ & 1 \\
\hline Aluminum & 353 & $\mathrm{MG} / \mathrm{KG}$ & & NP & $40 \mathrm{MG} / \mathrm{KG}$ & 1 \\
\hline Antimony & 4.5 & $\mathrm{MG} / \mathrm{KG}$ & $u$ & NP & $12 \mathrm{MG} / \mathrm{KG}$ & 1 \\
\hline Barium & 5.3 & $\mathrm{MG} / \mathrm{KG}$ & B & NP & $40 \mathrm{MG} / \mathrm{KG}$ & 1 \\
\hline Beryilium & 0.34 & $M G / K G$ & $u$ & NP & $1 \mathrm{MG} / \mathrm{KG}$ & 1 \\
\hline Cadmium & 0.58 & $3 \mathrm{MG} / K \mathrm{G}$ & $u$ & NP & $1 \mathrm{MG} / \mathrm{KG}$ & 1 \\
\hline Calcium & 236 & MG/KG & B & NP & 1000 MG/KG & 1 \\
\hline Chromium & 2.6 & $M G / K G$ & & NP & $2 M G / K G$ & 1 \\
\hline Cobalt & & MG/KG & $u$ & NP & $10 \mathrm{MG} / \mathrm{KG}$ & 1 \\
\hline Copper & & MG/KG & 8 & NP & $5 \mathrm{MG} / \mathrm{KG}$ & 1 \\
\hline Iron & 259 & $M G / K G$ & & NP & $20 \mathrm{MG} / \mathrm{KG}$ & 1 \\
\hline Magnesium & 76.8 & $3 \mathrm{MG} / \mathrm{KG}$ & B & NP & $1000 \mathrm{MG} / \mathrm{KG}$ & 1 \\
\hline Manganese & & $3 \mathrm{MG} / K \mathrm{~K}$ & & NP & $3 \mathrm{MG} / \mathrm{KG}$ & 1 \\
\hline Nickel & & MG/KG & $u$ & NP & $8 \mathrm{MG} / \mathrm{KG}$ & 1 \\
\hline Potassium & 139 & MG/KG & $u$ & $\mathrm{NP}$ & $1000 \mathrm{MG} / \mathrm{KG}$ & 1 \\
\hline Silver & 0.82 & MG/KG & $u$ & NP & $2 \mathrm{MG} / \mathrm{KG}$ & 1 \\
\hline Sodium & 234 & I MG/KG & $B$ & $\mathrm{NP}$ & $1000 \mathrm{MG} / \mathrm{KG}$ & 1 \\
\hline Vanadium & & $M G / K G$ & $B$ & $\mathrm{NP}$ & $10 \mathrm{MG} / \mathrm{KG}$ & 1 \\
\hline Zinc & & 3 MG/KG & B & NP & $4 M G / K G$ & 1 \\
\hline Lead & & 5 MG/KG & & NP & $0.6 \mathrm{MG} / \mathrm{KG}$ & 1 \\
\hline Selenium & 0.16 & $M G / K G$ & $u$ & NP & $1 \mathrm{MG} / \mathrm{KG}$ & 1 \\
\hline Thallium & & MG/KG & $u$ & NP & $2 M G / K G$ & 1 \\
\hline
\end{tabular}




\section{APPENDIX F.1 Results of Metals Analyses, Soils, Salmon Site}

\begin{tabular}{lccccc}
\hline COMPOUND & RESULTS UNITS & $\begin{array}{c}\text { DATA } \\
\text { QUAL. }\end{array}$ & DATA & & \\
C1-17-S-MS & & & & \\
Arsenic & $117 \%$ & NP & CRDL UNITS & DILUTION \\
Cyanide & $95 \%$ & NP & $\%$ & 1 \\
Mercury & $92 \%$ & NP & $\%$ & 1 \\
Antimony & $90 \%$ & NP & $\%$ & 1 \\
Barium & $96 \%$ & NP & $\%$ & 1 \\
Beryllium & $100 \%$ & NP & $\%$ & 1 \\
Cadmium & $100 \%$ & NP & $\%$ & 1 \\
Chromium & $95 \%$ & NP & $\%$ & 1 \\
Cobalt & $99 \%$ & NP & $\%$ & 1 \\
Copper & $94 \%$ & NP & $\%$ & 1 \\
Maniganese & $98 \%$ & NP & $\%$ & 1 \\
Nickel & $102 \%$ & NP & $\%$ & 1 \\
Silver & $106 \%$ & NP & $\%$ & 1 \\
Vanadium & $100 \%$ & NP & $\%$ & 1 \\
Zinc & $96 \%$ & NP & $\%$ & 1 \\
Lead & $89 \%$ & NP & $\%$ & 1 \\
Selenium & $98 \%$ & NP & $\%$ & 1 \\
Thallium & $114 \%$ & NP & $\%$ & 1
\end{tabular}


APPENDIX F.1 Results of Metals Analyses, Soils, Salmon Site

\begin{tabular}{lrrrrr}
\hline COMPOUND & RESULTS UNITS & DATA & DATA & \\
C1-18-S & & & & & \\
Arsenic & $1.2 \mathrm{MG} / \mathrm{KG}$ & $\mathrm{B}$ & $\mathrm{NP}$ & $2 \mathrm{MG} / \mathrm{KG}$ & 1 \\
Cyanide & $0.08 \mathrm{MG} / \mathrm{KG}$ & $\mathrm{B}$ & $\mathrm{NP}$ & $0.5 \mathrm{MG} / \mathrm{KG}$ & 1 \\
Mercury & $0.05 \mathrm{MG} / \mathrm{KG}$ & $\mathrm{U}$ & $\mathrm{NP}$ & $0.1 \mathrm{MG} / \mathrm{KG}$ & 1 \\
Aluminum & $2200 \mathrm{MG} / \mathrm{KG}$ & & $\mathrm{NP}$ & $40 \mathrm{MG} / \mathrm{KG}$ & 1 \\
Antimony & $4.5 \mathrm{MG} / \mathrm{KG}$ & $\mathrm{U}$ & $\mathrm{NP}$ & $12 \mathrm{MG} / \mathrm{KG}$ & 1 \\
Barium & $8.4 \mathrm{MG} / \mathrm{KG}$ & $\mathrm{B}$ & $\mathrm{NP}$ & $40 \mathrm{MG} / \mathrm{KG}$ & 1 \\
Beryilium & $0.34 \mathrm{MG} / \mathrm{KG}$ & $\mathrm{U}$ & $\mathrm{NP}$ & $1 \mathrm{MG} / \mathrm{KG}$ & 1 \\
Cadmium & $0.58 \mathrm{MG} / \mathrm{KG}$ & $\mathrm{U}$ & $\mathrm{NP}$ & $1 \mathrm{MG} / \mathrm{KG}$ & 1 \\
Calcium & $823 \mathrm{MG} / \mathrm{KG}$ & $\mathrm{B}$ & $\mathrm{NP}$ & $1000 \mathrm{MG} / \mathrm{KG}$ & 1 \\
Chromium & $14.5 \mathrm{MG} / \mathrm{KG}$ & & $\mathrm{NP}$ & $2 \mathrm{MG} / \mathrm{KG}$ & 1 \\
Cobalt & $1.4 \mathrm{MG} / \mathrm{KG}$ & $\mathrm{B}$ & $\mathrm{NP}$ & $10 \mathrm{MG} / \mathrm{KG}$ & 1 \\
Copper & $4.9 \mathrm{MG} / \mathrm{KG}$ & $\mathrm{B}$ & $\mathrm{NP}$ & $5 \mathrm{MG} / \mathrm{KG}$ & 1 \\
Iron & $5310 \mathrm{MG} / \mathrm{KG}$ & & $\mathrm{NP}$ & $20 \mathrm{MG} / \mathrm{KG}$ & 1 \\
Magnesium & $92.2 \mathrm{MG} / \mathrm{KG}$ & $\mathrm{B}$ & $\mathrm{NP}$ & $1000 \mathrm{MG} / \mathrm{KG}$ & 1 \\
Manganese & $14 \mathrm{MG} / \mathrm{KG}$ & & $\mathrm{NP}$ & $3 \mathrm{MG} / \mathrm{KG}$ & 1 \\
Nickel & $1.7 \mathrm{MG} / \mathrm{KG}$ & $\mathrm{U}$ & $\mathrm{NP}$ & $8 \mathrm{MG} / \mathrm{KG}$ & 1 \\
Potassium & $139 \mathrm{MG} / \mathrm{KG}$ & $\mathrm{U}$ & $\mathrm{NP}$ & $1000 \mathrm{MG} / \mathrm{KG}$ & 1 \\
Silver & $0.82 \mathrm{MG} / \mathrm{KG}$ & $\mathrm{U}$ & $\mathrm{NP}$ & $2 \mathrm{MG} / \mathrm{KG}$ & 1 \\
Sodium & $293 \mathrm{MG} / \mathrm{KG}$ & $\mathrm{B}$ & $\mathrm{NP}$ & $1000 \mathrm{MG} / \mathrm{KG}$ & 1 \\
Vanadium & $13.9 \mathrm{MG} / \mathrm{KG}$ & & $\mathrm{NP}$ & $10 \mathrm{MG} / \mathrm{KG}$ & 1 \\
Zinc & $4 \mathrm{MG} / \mathrm{KG}$ & & $\mathrm{NP}$ & $4 \mathrm{MG} / \mathrm{KG}$ & 1 \\
Lead & $2.4 \mathrm{MG} / \mathrm{KG}$ & & $\mathrm{NP}$ & $0.6 \mathrm{MG} / \mathrm{KG}$ & 1 \\
Selenium & $0.16 \mathrm{MG} / \mathrm{KG}$ & $\mathrm{U}$ & $\mathrm{NP}$ & $1 \mathrm{MG} / \mathrm{KG}$ & 1 \\
Thallium & $0.2 \mathrm{MG} / \mathrm{KG}$ & $\mathrm{U}$ & $\mathrm{NP}$ & $2 \mathrm{MG} / \mathrm{KG}$ & 1
\end{tabular}




\section{APPENDIX F.1 Results of Metals Analyses, Soils, Salmon Site}

\begin{tabular}{|c|c|c|c|c|c|}
\hline COMPOUND & RESULTS UNITS & $\begin{array}{l}\text { DATA } \\
\text { QUAL. }\end{array}$ & $\begin{array}{l}\text { DATA } \\
\text { VALID. }\end{array}$ & CRDL UNITS & DILUTION \\
\hline \multicolumn{6}{|l|}{ C2-19-S } \\
\hline Arsenic & $1.5 \mathrm{MG} / \mathrm{KG}$ & B & NP & 2 MG.KG & 1 \\
\hline Cyanide & $0.05 \mathrm{MG} / \mathrm{KG}$ & $u$ & NP & $0.49 \mathrm{MG} / \mathrm{KG}$ & 1 \\
\hline Mercury & $0.05 \mathrm{MG} / \mathrm{KG}$ & $u$ & NP & $0.1 \mathrm{MG} " \mathrm{KG}$ & 1 \\
\hline Aluminum & 1420 MG/KG & & NP & 40 ME:KG & 1 \\
\hline Antimony & $4.5 \mathrm{MG} / \mathrm{KG}$ & $u$ & NP & $12 \mathrm{MG} / \mathrm{KG}$ & 1 \\
\hline Barium & $67.4 \mathrm{MG} / \mathrm{KG}$ & & NP & 40 MG/KG & 1 \\
\hline Beryllium & $0.34 M G / K G$ & $u$ & NP & $1 \mathrm{M}: \mathrm{GKG}$ & 1 \\
\hline Cadmium & $0.58 \mathrm{MG} / \mathrm{KG}$ & $u$ & NP & $1 \mathrm{MG} / \mathrm{KG}$ & 1 \\
\hline Calcium & $2120 \mathrm{MG} / \mathrm{KG}$ & & NP & $1000 \mathrm{MG} / \mathrm{KG}$ & 1 \\
\hline Chromium & $0.96 \mathrm{MG} / \mathrm{KG}$ & $u$ & NP & $2 \mathrm{MG} / \mathrm{KG}$ & 1 \\
\hline Cobalt & $1.1 \mathrm{MG} / \mathrm{KG}$ & $u$ & NP & $10 \mathrm{MG} / \mathrm{KG}$ & 1 \\
\hline Copper & $0.93 \mathrm{MG} / \mathrm{KG}$ & B & NP & $5 \mathrm{MG} / \mathrm{KG}$ & 1 \\
\hline Iron & $10300 \mathrm{MG} / \mathrm{KG}$ & & NP & $20 \mathrm{MG} / \mathrm{KG}$ & 1 \\
\hline Magnesium & $437 \mathrm{MG} / \mathrm{KG}$ & B & NP & $1000 \mathrm{MG} / \mathrm{KG}$ & 1 \\
\hline Manganese & $451 \mathrm{MG} / \mathrm{KG}$ & & NP & $3 \mathrm{MG} / \mathrm{KG}$ & 1 \\
\hline Nickel & $1.7 \mathrm{MG} / \mathrm{KG}$ & $u$ & NP & $8 \mathrm{MG} / \mathrm{KG}$ & 1 \\
\hline Potassium & $139 \mathrm{MG} / \mathrm{KG}$ & $u$ & NP & $1000 \mathrm{MG} / \mathrm{KG}$ & 1 \\
\hline Silver & $0.82 \mathrm{MG} / \mathrm{KG}$ & $u$ & NP & $2 \mathrm{MG} / \mathrm{KG}$ & 1 \\
\hline Sodium & $39.9 \mathrm{MG} / \mathrm{KG}$ & B & NP & $1000 \mathrm{MG} / \mathrm{KG}$ & 1 \\
\hline Vanadium & $6.3 \mathrm{MG} / \mathrm{KG}$ & B & NP & $10 \mathrm{MG} / \mathrm{KG}$ & 1 \\
\hline Zinc & $3.2 \mathrm{MG} / \mathrm{KG}$ & B & NP & $4 \mathrm{MG} / \mathrm{KG}$ & 1 \\
\hline Lead & $0.26 \mathrm{MG} / \mathrm{KG}$ & B & NP & $0.6 \mathrm{MG} / \mathrm{KG}$ & 1 \\
\hline Selenium & $0.2 \mathrm{MG} / \mathrm{KG}$ & $u$ & NP & $1 \mathrm{MG} / \mathrm{KG}$ & 1 \\
\hline Thallium & $0.2 \mathrm{MG} / \mathrm{KG}$ & $u$ & NP & $2 M G / K G$ & 1 \\
\hline
\end{tabular}


APPENDIX F.1 Results of Metals Analyses, Soils, Salmon Site

\begin{tabular}{lrrrrr}
\hline COMPOUND & RESULTS UNITS & DATA & DATA & & \\
C2-20-S & & & & & \\
Arsenic & $1.1 \mathrm{MG} / \mathrm{KG}$ & $\mathrm{B}$ & $\mathrm{NP}$ & $2 \mathrm{MG} / \mathrm{KG}$ & 1 \\
Cyanide & $0.05 \mathrm{MG} / \mathrm{KG}$ & $\mathrm{U}$ & $\mathrm{NP}$ & $0.48 \mathrm{MG} / \mathrm{KG}$ & 1 \\
Mercury & $0.05 \mathrm{MG} / \mathrm{KG}$ & $\mathrm{U}$ & $\mathrm{NP}$ & $0.1 \mathrm{MG} / \mathrm{KG}$ & 1 \\
Aluminum & $1230 \mathrm{MG} / \mathrm{KG}$ & & $\mathrm{NP}$ & $40 \mathrm{MG} / \mathrm{KG}$ & 1 \\
Antimony & $4.5 \mathrm{MG} / \mathrm{KG}$ & $\mathrm{U}$ & $\mathrm{NP}$ & $12 \mathrm{MG} / \mathrm{KG}$ & 1 \\
Barium & $23.1 \mathrm{MG} / \mathrm{KG}$ & $\mathrm{B}$ & $\mathrm{NP}$ & $40 \mathrm{MG} / \mathrm{KG}$ & 1 \\
Beryllium & $0.34 \mathrm{MG} / \mathrm{KG}$ & $\mathrm{U}$ & $\mathrm{NP}$ & $1 \mathrm{MG} / \mathrm{KG}$ & 1 \\
Cadmium & $0.58 \mathrm{MG} / \mathrm{KG}$ & $\mathrm{U}$ & $\mathrm{NP}$ & $1 \mathrm{MG} / \mathrm{KG}$ & 1 \\
Calcium & $3760 \mathrm{MG} / \mathrm{KG}$ & & $\mathrm{NP}$ & $1000 \mathrm{MG} / \mathrm{KG}$ & 1 \\
Chromium & $1 \mathrm{MG} / \mathrm{KG}$ & $\mathrm{B}$ & $\mathrm{NP}$ & $2 \mathrm{MG} / \mathrm{KG}$ & 1 \\
Cobalt & $2.5 \mathrm{MG} / \mathrm{KG}$ & $\mathrm{B}$ & $\mathrm{NP}$ & $10 \mathrm{MG} / \mathrm{KG}$ & 1 \\
Copper & $3.9 \mathrm{MG} / \mathrm{KG}$ & $\mathrm{B}$ & $\mathrm{NP}$ & $5 \mathrm{MG} / \mathrm{KG}$ & 1 \\
Iron & $1240 \mathrm{MG} / \mathrm{KG}$ & & $\mathrm{NP}$ & $20 \mathrm{MG} / \mathrm{KG}$ & 1 \\
Magnesium & $428 \mathrm{MG} / \mathrm{KG}$ & $\mathrm{B}$ & $\mathrm{NP}$ & $1000 \mathrm{MG} / \mathrm{KG}$ & 1 \\
Manganese & $78.9 \mathrm{MG} / \mathrm{KG}$ & & $\mathrm{NP}$ & $3 \mathrm{MG} / \mathrm{KG}$ & 1 \\
Nickel & $3.2 \mathrm{MG} / \mathrm{KG}$ & $\mathrm{B}$ & $\mathrm{NP}$ & $8 \mathrm{MG} / \mathrm{KG}$ & 1 \\
Potassium & $230 \mathrm{MG} / \mathrm{KG}$ & $\mathrm{B}$ & $\mathrm{NP}$ & $1000 \mathrm{MG} / \mathrm{KG}$ & 1 \\
Silver & $0.82 \mathrm{MG} / \mathrm{KG}$ & $\mathrm{U}$ & $\mathrm{NP}$ & $2 \mathrm{MG} / \mathrm{KG}$ & 1 \\
Sodium & $52.6 \mathrm{MG} / \mathrm{KG}$ & $\mathrm{B}$ & $\mathrm{NP}$ & $1000 \mathrm{MG} / \mathrm{KG}$ & 1 \\
Vanadium & $0.94 \mathrm{MG} / \mathrm{KG}$ & $\mathrm{B}$ & $\mathrm{NP}$ & $10 \mathrm{MG} / \mathrm{KG}$ & 1 \\
Zinc & $3.3 \mathrm{MG} / \mathrm{KG}$ & $\mathrm{B}$ & $\mathrm{NP}$ & $4 \mathrm{MG} / \mathrm{KG}$ & 1 \\
Lead & $4.5 \mathrm{MG} / \mathrm{KG}$ & & $\mathrm{NP}$ & $0.6 \mathrm{MG} / \mathrm{KG}$ & 1 \\
Selenium & $0.2 \mathrm{MG} / \mathrm{KG}$ & $\mathrm{U}$ & $\mathrm{NP}$ & $1 \mathrm{MG} / \mathrm{KG}$ & 1 \\
Thallium & $0.2 \mathrm{MG} / \mathrm{KG}$ & $\mathrm{U}$ & $\mathrm{NP}$ & $2 \mathrm{MG} / \mathrm{KG}$ & 1 \\
& & & & &
\end{tabular}




\section{APPENDIX F.1 Results of Metals Analyses, Soils, Salmon Site}

\begin{tabular}{lrrrrr}
\hline COMPOUND & RESULTS UNITS & $\begin{array}{l}\text { DATA } \\
\text { QUAL. }\end{array}$ & VATA & \\
C2-21-S & & & & & \\
Arsenic & $0.54 \mathrm{MG} / \mathrm{KG}$ & $\mathrm{B}$ & $\mathrm{NP}$ & $2 \mathrm{MG} / \mathrm{KG}$ & 1 \\
Cyanide & $0.05 \mathrm{MG} / \mathrm{KG}$ & $\mathrm{U}$ & $\mathrm{NP}$ & $0.5 \mathrm{MG} / \mathrm{KG}$ & 1 \\
Mercury & $0.05 \mathrm{MG} / \mathrm{KG}$ & $\mathrm{U}$ & $\mathrm{NP}$ & $0.1 \mathrm{MG} / \mathrm{KG}$ & 1 \\
Aluminum & $1960 \mathrm{MG} / \mathrm{KG}$ & & $\mathrm{NP}$ & $40 \mathrm{MG} / \mathrm{KG}$ & 1 \\
Antimony & $4.5 \mathrm{MG} / \mathrm{KG}$ & $\mathrm{U}$ & $\mathrm{NP}$ & $12 \mathrm{MG} / \mathrm{KG}$ & 1 \\
Barium & $10.8 \mathrm{MG} / \mathrm{KG}$ & $\mathrm{B}$ & $\mathrm{NP}$ & $40 \mathrm{MG} / \mathrm{KG}$ & 1 \\
Beryllium & $0.34 \mathrm{MG} / \mathrm{KG}$ & $\mathrm{U}$ & $\mathrm{NP}$ & $1 \mathrm{MG} / \mathrm{KG}$ & 1 \\
Cadmium & $0.58 \mathrm{MG} / \mathrm{KG}$ & $\mathrm{U}$ & $\mathrm{NP}$ & $1 \mathrm{MG} / \mathrm{KG}$ & 1 \\
Calcium & $624 \mathrm{MG} / \mathrm{KG}$ & $\mathrm{B}$ & $\mathrm{NP}$ & $1000 \mathrm{MG} / \mathrm{KG}$ & 1 \\
Chromium & $1.8 \mathrm{MG} / \mathrm{KG}$ & $\mathrm{B}$ & $\mathrm{NP}$ & $2 \mathrm{MG} / \mathrm{KG}$ & 1 \\
Cobalt & $1.1 \mathrm{MG} / \mathrm{KG}$ & $\mathrm{U}$ & $\mathrm{NP}$ & $10 \mathrm{MG} / \mathrm{KG}$ & 1 \\
Copper & $1 \mathrm{MG} / \mathrm{KG}$ & $\mathrm{B}$ & $\mathrm{NP}$ & $5 \mathrm{MG} / \mathrm{KG}$ & 1 \\
Iron & $1720 \mathrm{MG} / \mathrm{KG}$ & & $\mathrm{NP}$ & $20 \mathrm{MG} / \mathrm{KG}$ & 1 \\
Magnesium & $274 \mathrm{MG} / \mathrm{KG}$ & $\mathrm{B}$ & $\mathrm{NP}$ & $1000 \mathrm{MG} / \mathrm{KG}$ & 1 \\
Manganese & $15.8 \mathrm{MG} / \mathrm{KG}$ & & $\mathrm{NP}$ & $3 \mathrm{MG} / \mathrm{KG}$ & 1 \\
Nickel & $1.7 \mathrm{MG} / \mathrm{KG}$ & $\mathrm{U}$ & $\mathrm{NP}$ & $8 \mathrm{MG} / \mathrm{KG}$ & 1 \\
Potassium & $139 \mathrm{MG} / \mathrm{KG}$ & $\mathrm{U}$ & $\mathrm{NP}$ & $1000 \mathrm{MG} / \mathrm{KG}$ & 1 \\
Silver & $0.82 \mathrm{MG} / \mathrm{KG}$ & $\mathrm{U}$ & $\mathrm{NP}$ & $2 \mathrm{MG} / \mathrm{KG}$ & 1 \\
Sodium & $447 \mathrm{MG} / \mathrm{KG}$ & $\mathrm{B}$ & $\mathrm{NP}$ & $1000 \mathrm{MG} / \mathrm{KG}$ & 1 \\
Vanadium & $4.7 \mathrm{MG} / \mathrm{KG}$ & $\mathrm{B}$ & $\mathrm{NP}$ & $10 \mathrm{MG} / \mathrm{KG}$ & 1 \\
Zinc & $3.7 \mathrm{MG} / \mathrm{KG}$ & $\mathrm{B}$ & $\mathrm{NP}$ & $4 \mathrm{MG} / \mathrm{KG}$ & 1 \\
Lead & $1.8 \mathrm{MG} / \mathrm{KG}$ & & $\mathrm{NP}$ & $0.6 \mathrm{MG} / \mathrm{KG}$ & 1 \\
Selenium & $0.2 \mathrm{MG} / \mathrm{KG}$ & $\mathrm{U}$ & $\mathrm{NP}$ & $1 \mathrm{MG} / \mathrm{KG}$ & 1 \\
Thallium & $0.2 \mathrm{MG} / \mathrm{KG}$ & $\mathrm{U}$ & $\mathrm{NP}$ & $2 \mathrm{MG} / \mathrm{KG}$ & 1 \\
& & & & & \\
& & & & &
\end{tabular}


APPENDIX F.1 Results of Metals Analyses, Soils, Salmon Site

\begin{tabular}{lrrrrr}
\hline COMPOUND & RESULTS UNITS & DATA & DATA & \\
QUAL. & VALID. & CRDL UNITS & DILUTION \\
\hline C2-21-S-DUP & $0.69 \mathrm{MG} / \mathrm{KG}$ & $\mathrm{B}$ & $\mathrm{NP}$ & $2 \mathrm{MG} / \mathrm{KG}$ & 1 \\
Arsenic & $0.05 \mathrm{MG} / \mathrm{KG}$ & $\mathrm{U}$ & $\mathrm{NP}$ & $0.49 \mathrm{MG} / \mathrm{KG}$ & 1 \\
Cyanide & $0.05 \mathrm{MG} / \mathrm{KG}$ & $\mathrm{U}$ & $\mathrm{NP}$ & $0.1 \mathrm{MG} / \mathrm{KG}$ & 1 \\
Mercury & $867 \mathrm{MG} / \mathrm{KG}$ & & $\mathrm{NP}$ & $40 \mathrm{MG} / \mathrm{KG}$ & 1 \\
Aluminum & $4.5 \mathrm{MG} / \mathrm{KG}$ & $\mathrm{U}$ & $\mathrm{NP}$ & $12 \mathrm{MG} / \mathrm{KG}$ & 1 \\
Antimony & $7.8 \mathrm{MG} / \mathrm{KG}$ & $\mathrm{B}$ & $\mathrm{NP}$ & $40 \mathrm{MG} / \mathrm{KG}$ & 1 \\
Barium & $0.34 \mathrm{MG} / \mathrm{KG}$ & $\mathrm{U}$ & $\mathrm{NP}$ & $1 \mathrm{MG} / \mathrm{KG}$ & 1 \\
Beryllium & $0.58 \mathrm{MG} / \mathrm{KG}$ & $\mathrm{U}$ & $\mathrm{NP}$ & $1 \mathrm{MG} / \mathrm{KG}$ & 1 \\
Cadmium & $254 \mathrm{MG} / \mathrm{KG}$ & $\mathrm{B}$ & $\mathrm{NP}$ & $1000 \mathrm{MG} / \mathrm{KG}$ & 1 \\
Calcium & $3.7 \mathrm{MG} / \mathrm{KG}$ & & $\mathrm{NP}$ & $2 \mathrm{MG} / \mathrm{KG}$ & 1 \\
Chromium & $1.1 \mathrm{MG} / \mathrm{KG}$ & $\mathrm{U}$ & $\mathrm{NP}$ & $10 \mathrm{MG} / \mathrm{KG}$ & 1 \\
Cobalt & $0.88 \mathrm{MG} / \mathrm{KG}$ & $\mathrm{U}$ & $\mathrm{NP}$ & $5 \mathrm{MG} / \mathrm{KG}$ & 1 \\
Copper & $2600 \mathrm{MG} / \mathrm{KG}$ & & $\mathrm{NP}$ & $20 \mathrm{MG} / \mathrm{KG}$ & 1 \\
Iron & $78.5 \mathrm{MG} / \mathrm{KG}$ & $\mathrm{B}$ & $\mathrm{NP}$ & $1000 \mathrm{MG} / \mathrm{KG}$ & 1 \\
Magnesium & $16.3 \mathrm{MG} / \mathrm{KG}$ & & $\mathrm{NP}$ & $3 \mathrm{MG} / \mathrm{KG}$ & 1 \\
Manganese & $1.7 \mathrm{MG} / \mathrm{KG}$ & $\mathrm{U}$ & $\mathrm{NP}$ & $8 \mathrm{MG} / \mathrm{KG}$ & 1 \\
Nickel & $139 \mathrm{MG} / \mathrm{KG}$ & $\mathrm{U}$ & $\mathrm{NP}$ & $1000 \mathrm{MG} / \mathrm{KG}$ & 1 \\
Potassium & $0.82 \mathrm{MG} / \mathrm{KG}$ & $\mathrm{U}$ & $\mathrm{NP}$ & $2 \mathrm{MG} / \mathrm{KG}$ & 1 \\
Silver & $148 \mathrm{MG} / \mathrm{KG}$ & $\mathrm{B}$ & $\mathrm{NP}$ & $1000 \mathrm{MG} / \mathrm{KG}$ & 1 \\
Sodium & $6 \mathrm{MG} / \mathrm{KG}$ & $\mathrm{B}$ & $\mathrm{NP}$ & $10 \mathrm{MG} / \mathrm{KG}$ & 1 \\
Vanadium & $2.5 \mathrm{MG} / \mathrm{KG}$ & $\mathrm{B}$ & $\mathrm{NP}$ & $4 \mathrm{MG} / \mathrm{KG}$ & 1 \\
Zinc & $3.9 \mathrm{MG} / \mathrm{KG}$ & & $\mathrm{NP}$ & $0.6 \mathrm{MG} / \mathrm{KG}$ & 1 \\
Lead & $0.2 \mathrm{MG} / \mathrm{KG}$ & $\mathrm{U}$ & $\mathrm{NP}$ & $1 \mathrm{MG} / \mathrm{KG}$ & 1 \\
Selenium & $0.2 \mathrm{MG} / \mathrm{KG}$ & $\mathrm{U}$ & $\mathrm{NP}$ & $2 \mathrm{MG} / \mathrm{KG}$ & 1 \\
Thallium & & & & & \\
& & & &
\end{tabular}


APPENDIX F.1 Results of Metals Analyses, Soils, Salmon Site

\begin{tabular}{lcccc}
\hline COMPOUND & RESULTS UNITS & $\begin{array}{c}\text { DATA } \\
\text { QUAL. }\end{array}$ & DATA & \\
C2-21-S-MS & & & & \\
Arsenic & $113 \%$ & NP & CRDL UNITS & DILUTION \\
Cyanide & $86 \%$ & NP & $\%$ & 1 \\
Mercury & $119 \%$ & NP & $\%$ & 1 \\
Antimony & $101 \%$ & NP & $\%$ & 1 \\
Barium & $104 \%$ & NP & $\%$ & 1 \\
Beryilium & $108 \%$ & NP & $\%$ & 1 \\
Cadmium & $106 \%$ & NP & $\%$ & 1 \\
Chromium & $109 \%$ & NP & $\%$ & 1 \\
Cobalt & $108 \%$ & NP & $\%$ & 1 \\
Copper & $111 \%$ & NP & $\%$ & 1 \\
Manganese & $117 \%$ & NP & $\%$ & 1 \\
Nickel & $111 \%$ & NP & $\%$ & 1 \\
Silver & $104 \%$ & NP & $\%$ & 1 \\
Vanadium & $112 \%$ & NP & $\%$ & 1 \\
Zinc & $108 \%$ & NP & $\%$ & 1 \\
Lead & $113 \%$ & NP & $\%$ & 1 \\
Selenium & $75 \%$ & NP & $\%$ & 1 \\
Thallium & $89 \%$ & NP & $\%$ & 1
\end{tabular}


APPENDIX F.1 Results of Metals Analyses, Soils, Salmon Site

\begin{tabular}{|c|c|c|c|c|c|c|}
\hline COMPOUND & RESULTS & UNITS & $\begin{array}{l}\text { DATA } \\
\text { QUAL. }\end{array}$ & $\begin{array}{l}\text { DATA } \\
\text { VALID. }\end{array}$ & CRDL UNITS & DILUTION \\
\hline \multicolumn{7}{|l|}{ C2-22-S } \\
\hline Arsenic & & $M G / K G$ & $B$ & $N P$ & $2 M G / K G$ & 1 \\
\hline Cyanide & 0.05 & $M G / K G$ & $u$ & $N P$ & $0.5 M G / K G$ & 1 \\
\hline Mercury & 0.05 & $M G / K G$ & $u$ & NP & $0.1 \mathrm{MG} / \mathrm{KG}$ & 1 \\
\hline Aluminum & 856 & $M G / K G$ & & NP & $40 \mathrm{MG} / \mathrm{KG}$ & 1 \\
\hline Antimony & 4.5 & $M G / K G$ & $u$ & NP & $12 \mathrm{MG} / \mathrm{KG}$ & 1 \\
\hline Barium & 7.4 & $M G / K G$ & B & NP & $40 \mathrm{MG} / \mathrm{KG}$ & 1 \\
\hline Beryllium & 0.34 & $M G / K G$ & $u$ & NP & $1 \mathrm{MG} / \mathrm{KG}$ & 1 \\
\hline Cadmium & 0.58 & $M G / K G$ & $u$ & NP & $1 \mathrm{MG} / \mathrm{KG}$ & 1 \\
\hline Calcium & 307 & $M G / K G$ & B & NP & $1000 \mathrm{MG} / \mathrm{KG}$ & 1 \\
\hline Chromium & & $M G / K G$ & & NP & $2 M G / K G$ & 1 \\
\hline Cobalt & 1.1 & $M G / K G$ & $u$ & NP & $10 \mathrm{MG} / \mathrm{KG}$ & 1 \\
\hline Copper & $0.8 \varepsilon$ & $M G / K G$ & $u$ & NP & $5 \mathrm{MG} / \mathrm{KG}$ & 1 \\
\hline Iron & 3410 & $M G / K G$ & & NP & $20 \mathrm{MG} / \mathrm{KG}$ & 1 \\
\hline Magnesium & 41.8 & $M G / K G$ & B & NP & $1000 \mathrm{MG} / \mathrm{KG}$ & 1 \\
\hline Manganese & 24.7 & $M G / K G$ & & NP & $3 \mathrm{MG} / \mathrm{KG}$ & 1 \\
\hline Nickel & 1.7 & $M G / K G$ & $u$ & NP & $8 \mathrm{MG} / \mathrm{KG}$ & 1 \\
\hline Potassium & 139 & $M G / K G$ & $u$ & NP & $1000 \mathrm{MG} / \mathrm{KG}$ & 1 \\
\hline Silver & 0.82 & $M G / K G$ & $u$ & NP & $2 \mathrm{MG} / \mathrm{KG}$ & 1 \\
\hline Sodium & 104 & $M G / K G$ & $B$ & $N P$ & $1000 \mathrm{MG} / \mathrm{KG}$ & 1 \\
\hline Vanadium & 7.8 & $M G / K G$ & B & NP & $10 \mathrm{MG} / \mathrm{KG}$ & 1 \\
\hline Zine & 1.8 & $M G / K G$ & 8 & NP & $4 \mathrm{MG} / \mathrm{KG}$ & 1 \\
\hline Lead & 2.8 & $M G / K G$ & & NP & $0.6 \mathrm{MG} / \mathrm{KG}$ & 1 \\
\hline Selenium & 0.2 & $M G / K G$ & $u$ & NP & $1 M G / K G$ & 1 \\
\hline Thallium & 0.2 & $M G / K G$ & $u$ & NP & $2 M G / K G$ & 1 \\
\hline
\end{tabular}


APPENDIX F.1 Results of Metals Analyses, Soils, Salmon Site

\begin{tabular}{lrrrrr}
\hline COMPOUND & RESULTS UNITS & $\begin{array}{r}\text { DATA } \\
\text { QUAL. }\end{array}$ & DATA & \\
C2-23-S & & & & & \\
Arsenic & $0.76 \mathrm{MG} / \mathrm{KG}$ & $\mathrm{B}$ & $\mathrm{NP}$ & $2 \mathrm{MG} / \mathrm{KG}$ & 1 \\
Cyanide & $0.35 \mathrm{MG} / \mathrm{KG}$ & $\mathrm{B}$ & $\mathrm{NP}$ & $0.49 \mathrm{MG} / \mathrm{KG}$ & 1 \\
Mercury & $0.05 \mathrm{MG} / \mathrm{KG}$ & $\mathrm{U}$ & $\mathrm{NP}$ & $0.1 \mathrm{MG} / \mathrm{KG}$ & 1 \\
Aluminum & $782 \mathrm{MG} / \mathrm{KG}$ & & $\mathrm{NP}$ & $40 \mathrm{MG} / \mathrm{KG}$ & 1 \\
Antimony & $4.5 \mathrm{MG} / \mathrm{KG}$ & $\mathrm{U}$ & $\mathrm{NP}$ & $12 \mathrm{MG} / \mathrm{KG}$ & 1 \\
Barium & $11.6 \mathrm{MG} / \mathrm{KG}$ & $\mathrm{B}$ & $\mathrm{NP}$ & $40 \mathrm{MG} / \mathrm{KG}$ & 1 \\
Beryllium & $0.34 \mathrm{MG} / \mathrm{KG}$ & $\mathrm{U}$ & $\mathrm{NP}$ & $1 \mathrm{MG} / \mathrm{KG}$ & 1 \\
Cadmium & $0.58 \mathrm{MG} / \mathrm{KG}$ & $\mathrm{U}$ & $\mathrm{NP}$ & $1 \mathrm{MG} / \mathrm{KG}$ & 1 \\
Calcium & $158 \mathrm{MG} / \mathrm{KG}$ & $\mathrm{B}$ & $\mathrm{NP}$ & $1000 \mathrm{MG} / \mathrm{KG}$ & 1 \\
Chromium & $11.7 \mathrm{MG} / \mathrm{KG}$ & & $\mathrm{NP}$ & $2 \mathrm{MG} / \mathrm{KG}$ & 1 \\
Cobalt & $1.1 \mathrm{MG} / \mathrm{KG}$ & $\mathrm{U}$ & $\mathrm{NP}$ & $10 \mathrm{MG} / \mathrm{KG}$ & 1 \\
Copper & $2.1 \mathrm{MG} / \mathrm{KG}$ & $\mathrm{B}$ & $\mathrm{NP}$ & $5 \mathrm{MG} / \mathrm{KG}$ & 1 \\
Iron & $8640 \mathrm{MG} / \mathrm{KG}$ & & $\mathrm{NP}$ & $20 \mathrm{MG} / \mathrm{KG}$ & 1 \\
Magnesium & $43.8 \mathrm{MG} / \mathrm{KG}$ & $\mathrm{B}$ & $\mathrm{NP}$ & $1000 \mathrm{MG} / \mathrm{KG}$ & 1 \\
Manganese & $50.2 \mathrm{MG} / \mathrm{KG}$ & & $\mathrm{NP}$ & $3 \mathrm{MG} / \mathrm{KG}$ & 1 \\
Nickel & $1.7 \mathrm{MG} / \mathrm{KG}$ & $\mathrm{U}$ & $\mathrm{NP}$ & $8 \mathrm{MG} / \mathrm{KG}$ & 1 \\
Potassium & $139 \mathrm{MG} / \mathrm{KG}$ & $\mathrm{U}$ & $\mathrm{NP}$ & $1000 \mathrm{MG} / \mathrm{KG}$ & 1 \\
Silver & $0.82 \mathrm{MG} / \mathrm{KG}$ & $\mathrm{U}$ & $\mathrm{NP}$ & $2 \mathrm{MG} / \mathrm{KG}$ & 1 \\
Sodium & $39 \mathrm{MG} / \mathrm{KG}$ & $\mathrm{B}$ & $\mathrm{NP}$ & $1000 \mathrm{MG} / \mathrm{KG}$ & 1 \\
Vanadium & $11.9 \mathrm{MG} / \mathrm{KG}$ & & $\mathrm{NP}$ & $10 \mathrm{MG} / \mathrm{KG}$ & 1 \\
Zinc & $3.4 \mathrm{MG} / \mathrm{KG}$ & $\mathrm{B}$ & $\mathrm{NP}$ & $4 \mathrm{MG} / \mathrm{KG}$ & 1 \\
Lead & $1.5 \mathrm{MG} / \mathrm{KG}$ & & $\mathrm{NP}$ & $0.6 \mathrm{MG} / \mathrm{KG}$ & 1 \\
Selenium & $0.2 \mathrm{MG} / \mathrm{KG}$ & $\mathrm{U}$ & $\mathrm{NP}$ & $1 \mathrm{MG} / \mathrm{KG}$ & 1 \\
Thallium & $0.2 \mathrm{MG} / \mathrm{KG}$ & $\mathrm{U}$ & $\mathrm{NP}$ & $2 \mathrm{MG} / \mathrm{KG}$ & 1 \\
& & & & &
\end{tabular}


APPENDIX F.1 Results of Metals Analyses, Soils, Salmon Site

\begin{tabular}{lrrrrr}
\hline COMPOUND & RESULTS UNITS & DATA & DATA & \\
C2-24-S & & & & & \\
Arsenic & $1.7 \mathrm{MG} / \mathrm{KG}$ & $\mathrm{B}$ & $\mathrm{NP}$ & $2 \mathrm{MG} / \mathrm{KG}$ & 1 \\
Cyanide & $0.05 \mathrm{MG} / \mathrm{KG}$ & $\mathrm{U}$ & $\mathrm{NP}$ & $0.49 \mathrm{MG} / \mathrm{KG}$ & 1 \\
Mercury & $0.05 \mathrm{MG} / \mathrm{KG}$ & $\mathrm{U}$ & $\mathrm{NP}$ & $0.1 \mathrm{MG} / \mathrm{KG}$ & 1 \\
Aluminum & $695 \mathrm{MG} / \mathrm{KG}$ & & $\mathrm{NP}$ & $40 \mathrm{MG} / \mathrm{KG}$ & 1 \\
Antimony & $4.5 \mathrm{MG} / \mathrm{KG}$ & $\mathrm{U}$ & $\mathrm{NP}$ & $12 \mathrm{MG} / \mathrm{KG}$ & 1 \\
Barium & $277 \mathrm{MG} / \mathrm{KG}$ & & $\mathrm{NP}$ & $40 \mathrm{MG} / \mathrm{KG}$ & 1 \\
Beryllium & $0.34 \mathrm{MG} / \mathrm{KG}$ & $\mathrm{U}$ & $\mathrm{NP}$ & $1 \mathrm{MG} / \mathrm{KG}$ & 1 \\
Cadmium & $0.58 \mathrm{MG} / \mathrm{KG}$ & $\mathrm{U}$ & $\mathrm{NP}$ & $1 \mathrm{MG} / \mathrm{KG}$ & 1 \\
Calcium & $2780 \mathrm{MG} / \mathrm{KG}$ & & $\mathrm{NP}$ & $1000 \mathrm{MG} / \mathrm{KG}$ & 1 \\
Chromium & $1.9 \mathrm{MG} / \mathrm{KG}$ & $\mathrm{B}$ & $\mathrm{NP}$ & $2 \mathrm{MG} / \mathrm{KG}$ & 1 \\
Cobalt & $1.1 \mathrm{MG} / \mathrm{KG}$ & $\mathrm{U}$ & $\mathrm{NP}$ & $10 \mathrm{MG} / \mathrm{KG}$ & 1 \\
Copper & $2.4 \mathrm{MG} / \mathrm{KG}$ & $\mathrm{B}$ & $\mathrm{NP}$ & $5 \mathrm{MG} / \mathrm{KG}$ & 1 \\
Iron & $1640 \mathrm{MG} / \mathrm{KG}$ & & $\mathrm{NP}$ & $20 \mathrm{MG} / \mathrm{KG}$ & 1 \\
Magnesium & $454 \mathrm{MG} / \mathrm{KG}$ & $\mathrm{B}$ & $\mathrm{NP}$ & $1000 \mathrm{MG} / \mathrm{KG}$ & 1 \\
Manganese & $1250 \mathrm{MG} / \mathrm{KG}$ & & $\mathrm{NP}$ & $3 \mathrm{MG} / \mathrm{KG}$ & 1 \\
Nickel & $1.7 \mathrm{MG} / \mathrm{KG}$ & $\mathrm{B}$ & $\mathrm{NP}$ & $8 \mathrm{MG} / \mathrm{KG}$ & 1 \\
Potassium & $151 \mathrm{MG} / \mathrm{KG}$ & $\mathrm{B}$ & $\mathrm{NP}$ & $1000 \mathrm{MG} / \mathrm{KG}$ & 1 \\
Silver & $0.82 \mathrm{MG} / \mathrm{KG}$ & $\mathrm{U}$ & $\mathrm{NP}$ & $2 \mathrm{MG} / \mathrm{KG}$ & 1 \\
Sodium & $41.4 \mathrm{MG} / \mathrm{KG}$ & $\mathrm{B}$ & $\mathrm{NP}$ & $1000 \mathrm{MG} / \mathrm{KG}$ & 1 \\
Vanadium & $1.7 \mathrm{MG} / \mathrm{KG}$ & $\mathrm{B}$ & $\mathrm{NP}$ & $10 \mathrm{MG} / \mathrm{KG}$ & 1 \\
Zinc & $3.6 \mathrm{MG} / \mathrm{KG}$ & $\mathrm{B}$ & $\mathrm{NP}$ & $4 \mathrm{MG} / \mathrm{KG}$ & 1 \\
Lead & $3.8 \mathrm{MG} / \mathrm{KG}$ & & $\mathrm{NP}$ & $0.6 \mathrm{MG} / \mathrm{KG}$ & 1 \\
Selenium & $0.23 \mathrm{MG} / \mathrm{KG}$ & $\mathrm{B}$ & $\mathrm{NP}$ & $1 \mathrm{MG} / \mathrm{KG}$ & 1 \\
Thallium & $0.2 \mathrm{MG} / \mathrm{KG}$ & $\mathrm{U}$ & $\mathrm{NP}$ & $2 \mathrm{MG} / \mathrm{KG}$ & 1 \\
& & & & &
\end{tabular}


APPENDIX F.1 Results of Metals Analyses, Soils, Salmon Site

\begin{tabular}{lrrrrr}
\hline COMPOUND & RESULTS UNITS & QATA & DATA & & \\
C2-25-S & & & & & \\
Arsenic & $0.66 \mathrm{MG} / \mathrm{KG}$ & $\mathrm{B}$ & $\mathrm{NP}$ & $2 \mathrm{MG} / \mathrm{KG}$ & 1 \\
Cyanide & $0.13 \mathrm{MG} / \mathrm{KG}$ & $\mathrm{B}$ & $\mathrm{NP}$ & $0.49 \mathrm{MG} / \mathrm{KG}$ & 1 \\
Mercury & $0.05 \mathrm{MG} / \mathrm{KG}$ & $\mathrm{U}$ & $\mathrm{NP}$ & $0.1 \mathrm{MG} / \mathrm{KG}$ & 1 \\
Aluminum & $3220 \mathrm{MG} / \mathrm{KG}$ & & $\mathrm{NP}$ & $40 \mathrm{MG} / \mathrm{KG}$ & 1 \\
Antimony & $4.5 \mathrm{MG} / \mathrm{KG}$ & $\mathrm{U}$ & $\mathrm{NP}$ & $12 \mathrm{MG} / \mathrm{KG}$ & 1 \\
Barium & $17.5 \mathrm{MG} / \mathrm{KG}$ & $\mathrm{B}$ & $\mathrm{NP}$ & $40 \mathrm{MG} / \mathrm{KG}$ & 1 \\
Beryllium & $0.34 \mathrm{MG} / \mathrm{KG}$ & $\mathrm{U}$ & $\mathrm{NP}$ & $1 \mathrm{MG} / \mathrm{KG}$ & 1 \\
Cadmium & $0.58 \mathrm{MG} / \mathrm{KG}$ & $\mathrm{U}$ & $\mathrm{NP}$ & $1 \mathrm{MG} / \mathrm{KG}$ & 1 \\
Calcium & $244 \mathrm{MG} / \mathrm{KG}$ & $\mathrm{B}$ & $\mathrm{NP}$ & $1000 \mathrm{MG} / \mathrm{KG}$ & 1 \\
Chromium & $6.5 \mathrm{MG} / \mathrm{KG}$ & & $\mathrm{NP}$ & $2 \mathrm{MG} / \mathrm{KG}$ & 1 \\
Cobalt & $1.1 \mathrm{MG} / \mathrm{KG}$ & $\mathrm{U}$ & $\mathrm{NP}$ & $10 \mathrm{MG} / \mathrm{KG}$ & 1 \\
Copper & $2.8 \mathrm{MG} / \mathrm{KG}$ & $\mathrm{B}$ & $\mathrm{NP}$ & $5 \mathrm{MG} / \mathrm{KG}$ & 1 \\
Iron & $6780 \mathrm{MG} / \mathrm{KG}$ & & $\mathrm{NP}$ & $20 \mathrm{MG} / \mathrm{KG}$ & 1 \\
Magnesium & $171 \mathrm{MG} / \mathrm{KG}$ & $\mathrm{B}$ & $\mathrm{NP}$ & $1000 \mathrm{MG} / \mathrm{KG}$ & 1 \\
Manganese & $3.5 \mathrm{MG} / \mathrm{KG}$ & & $\mathrm{NP}$ & $3 \mathrm{MG} / \mathrm{KG}$ & 1 \\
Nickel & $1.9 \mathrm{MG} / \mathrm{KG}$ & $\mathrm{B}$ & $\mathrm{NP}$ & $8 \mathrm{MG} / \mathrm{KG}$ & 1 \\
Potassium & $139 \mathrm{MG} / \mathrm{KG}$ & $\mathrm{U}$ & $\mathrm{NP}$ & $1000 \mathrm{MG} / \mathrm{KG}$ & 1 \\
Silver & $0.82 \mathrm{MG} / \mathrm{KG}$ & $\mathrm{U}$ & $\mathrm{NP}$ & $2 \mathrm{MG} / \mathrm{KG}$ & 1 \\
Sodium & $74.7 \mathrm{MG} / \mathrm{KG}$ & $\mathrm{B}$ & $\mathrm{NP}$ & $1000 \mathrm{MG} / \mathrm{KG}$ & 1 \\
Vanadium & $13.3 \mathrm{MG} / \mathrm{KG}$ & & $\mathrm{NP}$ & $10 \mathrm{MG} / \mathrm{KG}$ & 1 \\
Zinc & $3.3 \mathrm{MG} / \mathrm{KG}$ & $\mathrm{B}$ & $\mathrm{NP}$ & $4 \mathrm{MG} / \mathrm{KG}$ & 1 \\
Lead & $3.7 \mathrm{MG} / \mathrm{KG}$ & & $\mathrm{NP}$ & $0.6 \mathrm{MG} / \mathrm{KG}$ & 1 \\
Selenium & $0.2 \mathrm{MG} / \mathrm{KG}$ & $\mathrm{U}$ & $\mathrm{NP}$ & $1 \mathrm{MG} / \mathrm{KG}$ & 1 \\
Thallium & $0.2 \mathrm{MG} / \mathrm{KG}$ & $\mathrm{U}$ & $\mathrm{NP}$ & $2 \mathrm{MG} / \mathrm{KG}$ & 1 \\
& & & &
\end{tabular}


APPENDIX F.1 Results of Metals Analyses, Soils, Salmon Site

\begin{tabular}{|c|c|c|c|c|c|}
\hline COMPOUND & RESULTS UNITS & $\begin{array}{l}\text { DATA } \\
\text { QUAL. }\end{array}$ & $\begin{array}{l}\text { DATA } \\
\text { VALID. }\end{array}$ & CRDL UNITS & DILUTION \\
\hline \multicolumn{6}{|l|}{ C2-25A-S } \\
\hline Arsenic & $0.26 \mathrm{MG} / \mathrm{KG}$ & $u$ & NP & $2 \mathrm{MG} / \mathrm{KG}$ & 1 \\
\hline Cyanide & $0.05 \mathrm{MG} / \mathrm{KG}$ & $u$ & $\mathrm{NP}$ & $0.49 \mathrm{MG} / \mathrm{KG}$ & 1 \\
\hline Mercury & $0.05 \mathrm{MG} / \mathrm{KG}$ & $u$ & NP & $0.1 \mathrm{MG} / \mathrm{KG}$ & 1 \\
\hline Aluminum & 3690 MG/KG & & NP & $40 \mathrm{MG} / \mathrm{KG}$ & 1 \\
\hline Antimony & $4.5 \mathrm{MG} / \mathrm{KG}$ & $u$ & NP & $12 \mathrm{MG} / \mathrm{KG}$ & 1 \\
\hline Barium & $100 \mathrm{MG} / \mathrm{KG}$ & & NP & $40 \mathrm{MG} / \mathrm{KG}$ & 1 \\
\hline Beryllium & $1.7 \mathrm{MG} / \mathrm{KG}$ & & NP & $1 \mathrm{MG} / \mathrm{KG}$ & 1 \\
\hline Cadmium & $0.58 \mathrm{MG} / \mathrm{KG}$ & $u$ & NP & $1 \mathrm{MG} / \mathrm{KG}$ & 1 \\
\hline Calcium & $3740 \mathrm{MG} / \mathrm{KG}$ & & NP & $1000 \mathrm{MG} / \mathrm{KG}$ & 1 \\
\hline Chromium & 4.8 MG/KG & & NP & $2 \mathrm{MG} / \mathrm{KG}$ & 1 \\
\hline Cobalt & $7.4 \mathrm{MG} / \mathrm{KG}$ & B & $\mathrm{NP}$ & $10 \mathrm{MG} / \mathrm{KG}$ & 1 \\
\hline Copper & $3.7 \mathrm{MG} / \mathrm{KG}$ & B & NP & $5 \mathrm{MG} / \mathrm{KG}$ & 1 \\
\hline Iron & $4500 \mathrm{MG} / \mathrm{KG}$ & & NP & $20 \mathrm{MG} / \mathrm{KG}$ & 1 \\
\hline Magnesium & $1720 \mathrm{MG} / \mathrm{KG}$ & & NP & $1000 \mathrm{MG} / \mathrm{KG}$ & 1 \\
\hline Manganese & $77.4 \mathrm{MG} / \mathrm{KG}$ & & NP & $3 \mathrm{MG} / \mathrm{KG}$ & 1 \\
\hline Nickel & $12.8 \mathrm{MG} / \mathrm{KG}$ & & $\mathrm{NP}$ & $8 \mathrm{MG} / \mathrm{KG}$ & 1 \\
\hline Potassium & 622 MG/KG & B & NP & $1000 \mathrm{MG} / \mathrm{KG}$ & 1 \\
\hline Silver & $0.82 \mathrm{MG} / \mathrm{KG}$ & $u$ & NP & $2 M G / K G$ & 1 \\
\hline Sodium & $74.6 \mathrm{MG} / \mathrm{KG}$ & B & $N P$ & $1000 \mathrm{MG} / \mathrm{KG}$ & 1 \\
\hline Vanadium & $7.4 \mathrm{MG} / \mathrm{KG}$ & $B$ & NP & $10 \mathrm{MG} / \mathrm{KG}$ & 1 \\
\hline Zinc & $26.8 \mathrm{MG} / \mathrm{KG}$ & & $\mathrm{NP}$ & $4 M G / K G$ & 1 \\
\hline Lead & $8.9 \mathrm{MG} / \mathrm{KG}$ & & $N P$ & $0.6 \mathrm{MG} / \mathrm{KG}$ & 1 \\
\hline Selenium & $0.2 \mathrm{MG} / K \mathrm{~K}$ & $u$ & $N P$ & $1 \mathrm{MG} / \mathrm{KG}$ & 1 \\
\hline Thallium & 0.2 MG/KG & $u$ & $N P$ & $2 M G / K G$ & 1 \\
\hline
\end{tabular}




\section{APPENDIX F.1 Results of Metals Analyses, Soils, Salmon Site}

\begin{tabular}{|c|c|c|c|c|c|}
\hline COMPOUND & RESULTS UNITS & $\begin{array}{l}\text { DATA } \\
\text { QUAL. }\end{array}$ & $\begin{array}{l}\text { DATA } \\
\text { VALID. }\end{array}$ & CRDL UNITS & DILUTION \\
\hline \multicolumn{6}{|l|}{ C2-26-S } \\
\hline Arsenic & $0.72 \mathrm{MG} / \mathrm{KG}$ & B & NP & $2 M G / K G$ & 1 \\
\hline Cyanide & $0.05 \mathrm{MG} / \mathrm{KG}$ & $u$ & NP & $0.49 \mathrm{MG} / \mathrm{KG}$ & 1 \\
\hline Mercury & $0.05 \mathrm{MG} / \mathrm{KG}$ & $u$ & NP & $0.1 \mathrm{MG} / \mathrm{KG}$ & 1 \\
\hline Aluminum & 762 MG/KG & & NP & $40 \mathrm{MG} / \mathrm{KG}$ & 1 \\
\hline Antimony & $4.5 \mathrm{MG} / \mathrm{KG}$ & $u$ & NP & 12 MG/KG & 1 \\
\hline Barium & $3.4 \mathrm{MG} / \mathrm{KG}$ & B & NP & $40 \mathrm{MG} / \mathrm{KG}$ & 1 \\
\hline Beryllium & $0.34 \mathrm{MG} / \mathrm{KG}$ & $u$ & NP & $1 \mathrm{MG} / \mathrm{KG}$ & 1 \\
\hline Cadmium & $0.58 \mathrm{MG} / \mathrm{KG}$ & $u$ & NP & $1 \mathrm{MG} / K \mathrm{~K}$ & 1 \\
\hline Calcium & 142 MG/KG & B & NP & $1000 \mathrm{MG} / \mathrm{KG}$ & 1 \\
\hline Chromium & $5.9 \mathrm{MG} / \mathrm{KG}$ & & NP & $2 \mathrm{MG} / K \mathrm{G}$ & 1 \\
\hline Cobalt & $1.1 \mathrm{MG} / \mathrm{KG}$ & $u$ & NP & $10 \mathrm{MG} / \mathrm{KG}$ & 1 \\
\hline Copper & $1.1 \mathrm{MG} / \mathrm{KG}$ & B & NP & $5 \mathrm{MG} / \mathrm{KG}$ & 1 \\
\hline Iron & $3600 \mathrm{MG} / \mathrm{KG}$ & & NP & 20 MG/KG & 1 \\
\hline Magnesium & $41.7 \mathrm{MG} / \mathrm{KG}$ & B & NP & $1000 \mathrm{MG} / \mathrm{KG}$ & 1 \\
\hline Manganese & $9.4 \mathrm{MG} / \mathrm{KG}$ & & NP & $3 \mathrm{MG} / \mathrm{KG}$ & 1 \\
\hline Nickel & $1.7 \mathrm{MG} / \mathrm{KG}$ & $u$ & NP & $8 \mathrm{MG} / \mathrm{KG}$ & 1 \\
\hline Potassium & 139 MG/KG & $u$ & NP & $1000 \mathrm{MG} / \mathrm{KG}$ & 1 \\
\hline Silver & $0.82 \mathrm{MG} / \mathrm{KG}$ & $u$ & NP & $2 \mathrm{MG} / \mathrm{KG}$ & 1 \\
\hline Sodium & $46.4 \mathrm{MG} / \mathrm{KG}$ & $B$ & NP & $1000 \mathrm{MG} / \mathrm{KG}$ & 1 \\
\hline Vanadium & $8.6 \mathrm{MG} / \mathrm{KG}$ & B & NP & $10 \mathrm{MG} / \mathrm{KG}$ & 1 \\
\hline Zinc & $2.8 \mathrm{MG} / \mathrm{KG}$ & B & NP & $4 \mathrm{MG} / \mathrm{KG}$ & 1 \\
\hline Lead & $1.7 \mathrm{MG} / \mathrm{KG}$ & & NP & $0.6 \mathrm{MG} / \mathrm{KG}$ & 1 \\
\hline Selenium & $0.2 \mathrm{MG} / \mathrm{KG}$ & $u$ & NP & $1 \mathrm{MG} / \mathrm{KG}$ & 1 \\
\hline Thallium & $0.2 \mathrm{MG} / \mathrm{KG}$ & $u$ & NP & $2 M G / K G$ & 1 \\
\hline
\end{tabular}


APPENDIX F.1 Results of Metals Analyses, Soils, Salmon Site

\begin{tabular}{|c|c|c|c|c|c|c|}
\hline COMPOUND & RESULTS & UNITS & $\begin{array}{l}\text { DATA } \\
\text { QUAL. }\end{array}$ & $\begin{array}{l}\text { DATA } \\
\text { VALID. }\end{array}$ & CRDL UNITS & DILUTION \\
\hline \multicolumn{7}{|l|}{ C2-27-S } \\
\hline Arsenic & 3.2 & $M G / K G$ & & NP & $2 \mathrm{MG} / \mathrm{KG}$ & 1 \\
\hline Cyanide & 0.05 & $M G / K G$ & $\mathrm{u}$ & NP & $0.48 \mathrm{MG} / \mathrm{KG}$ & 1 \\
\hline Mercury & 0.05 & $M G / K G$ & u & NP & $0.1 \mathrm{MG} / \mathrm{KG}$ & 1 \\
\hline Aluminum & 1810 & $M G / K G$ & & NP & $40 \mathrm{MG} / \mathrm{KG}$ & 1 \\
\hline Antimony & 4.5 & $M G / K G$ & $u$ & NP & $12 \mathrm{MG} / \mathrm{KG}$ & 1 \\
\hline Barium & 31.6 & $M G / K G$ & B & NP & $40 \mathrm{MG} / \mathrm{KG}$ & 1 \\
\hline Beryllium & 0.34 & $M G / K G$ & $u$ & NP & $1 \mathrm{MG} / \mathrm{KG}$ & 1 \\
\hline Cadmium & 0.58 & $M G / K G$ & $u$ & NP & $1 \mathrm{MG} / \mathrm{KG}$ & 1 \\
\hline Calcium & 1730 & $M G / K G$ & & NP & $1000 \mathrm{MG} / \mathrm{KG}$ & 1 \\
\hline Chromium & 1.5 & $M G / K G$ & B & NP & 2 MG/KG & 1 \\
\hline Cobalt & 1.1 & $M G / K G$ & $u$ & NP & $10 \mathrm{MG} / \mathrm{KG}$ & 1 \\
\hline Copper & 1.9 & $M G / K G$ & B & NP & $5 \mathrm{MG} / \mathrm{KG}$ & 1 \\
\hline Iron & 5210 & $M G / K G$ & & NP & $20 \mathrm{MG} / \mathrm{KG}$ & 1 \\
\hline Magnesium & 681 & $M G / K G$ & B & NP & $1000 \mathrm{MG} / \mathrm{KG}$ & 1 \\
\hline Manganese & 10.1 & $M G / K G$ & & NP & $3 \mathrm{MG} / \mathrm{KG}$ & 1 \\
\hline Nickel & 1.7 & $M G / K G$ & $u$ & NP & $8 \mathrm{MG} / \mathrm{KG}$ & 1 \\
\hline Potassium & 213 & $M G / K G$ & B & NP & 1000 MGKKG & 1 \\
\hline Silver & 0.82 & $M G / K G$ & $u$ & NP & $2 \mathrm{MG} / \mathrm{KG}$ & 1 \\
\hline Sodium & 99.9 & $M G / K G$ & B & NP & $1000 \mathrm{MG} / \mathrm{KG}$ & 1 \\
\hline Vanadium & 8.7 & $M G / K G$ & B & NP & $10 \mathrm{MG} / \mathrm{KG}$ & 1 \\
\hline Zinc & & $M G / K G$ & & $N P$ & $4 M G / K G$ & 1 \\
\hline Lead & 1.7 & $M G / K G$ & & $N P$ & $0.6 \mathrm{MG} / \mathrm{KG}$ & 1 \\
\hline Selenium & 0.2 & $M G / K G$ & $u$ & NP & $1 \mathrm{MG} / \mathrm{KG}$ & 1 \\
\hline Thallium & 0.2 & $M G / K G$ & $u$ & NP & $2 \mathrm{MG} / \mathrm{KG}$ & 1 \\
\hline
\end{tabular}


Appendix F.2

Results of Metals Analyses, Water, Salmon Site 
APPENDIX F.2 Results of Metals Analyses, Water, Salmon Site

\begin{tabular}{|c|c|c|c|c|c|}
\hline COMPOUND & RESULTS UNITS & $\begin{array}{l}\text { DATA } \\
\text { QUAL. }\end{array}$ & $\begin{array}{l}\text { DATA } \\
\text { VALID. }\end{array}$ & CRDL UNITS & DILUTION \\
\hline \multicolumn{6}{|l|}{$C 5-1-W$} \\
\hline Arsenic & 1.8 UG/L & B & NP & $10 \mathrm{UG} / \mathrm{L}$ & 1 \\
\hline Aluminum & 2430 UG $\Omega$ & & NP & $200 \mathrm{UG} / \mathrm{L}$ & 1 \\
\hline Antimony & 22.7 UG & $u$ & NP & 60 UGIL & 1 \\
\hline Barium & 39.2 UG/ & B & NP & 200 UG/L & 1 \\
\hline Beryllium & 1.7 UG $\Omega$ & $u$ & NP & 5 UG/L & 1 \\
\hline Cadmium & 2.9 UG/ & $u$ & NP & $5 \mathrm{UG} / \mathrm{L}$ & 1 \\
\hline Calcium & 3710 UG/L & $B$ & NP & $5000 \mathrm{UG} / \mathrm{L}$ & 1 \\
\hline Chromium & 42.7 UG $\Omega$ & & NP & $10 \mathrm{UG} / \mathrm{L}$ & 1 \\
\hline Cobalt & 5.6 UGL & $u$ & NP & $50 \mathrm{UG} / \mathrm{L}$ & 1 \\
\hline Copper & 40 UG/L & & NP & 25 UG/L & 1 \\
\hline Iron & 35300 UG & & NP & 100 UG/L & 1 \\
\hline Lead & 4.4 UG/ & & NP & $3 \mathrm{UG} / \mathrm{L}$ & 1 \\
\hline Magnesium & 1250 UG $/$ & B & NP & 5000 UG/L & 1 \\
\hline Manganese & 222 UG/ & & NP & 15 UG/L & 1 \\
\hline Mercury & 0.1 UGR & $u$ & NP & $0.2 \mathrm{UG} / \mathrm{L}$ & 1 \\
\hline Nickel & $21.4 \mathrm{UG} \Omega$ & B & NP & 40 UG/L & 1 \\
\hline Potassium & 5840 UG/L & & NP & 5000 UG/L & 1 \\
\hline Selenium & $1 \mathrm{UG} / \mathrm{L}$ & $u$ & NP & 5 UG/L & 1 \\
\hline Silver & 4.1 UG & $u$ & $\mathrm{NP}$ & $10 \mathrm{UG} / \mathrm{L}$ & 1 \\
\hline Sodium & 3210 UG/ & B & $N P$ & 5000 UG/L & 1 \\
\hline Thallium & $1 \mathrm{UG} \Omega$ & $u$ & NP & $10 \mathrm{UG} / \mathrm{L}$ & 1 \\
\hline Vanadium & 23.3 UG $\Omega$ & B & NP & $50 \mathrm{UG} / \mathrm{L}$ & 1 \\
\hline Zinc & 221 UG & & NP & $20 \mathrm{UG} / \mathrm{L}$ & 1 \\
\hline Cyanide & 2.1 UG/ & B & NP & $10 \mathrm{UG} / \mathrm{L}$ & 1 \\
\hline
\end{tabular}


APPENDIX F.2 Results of Metals Analyses, Water, Salmon Site

\begin{tabular}{|c|c|c|c|c|c|}
\hline COMPOUND & RESULTS UNITS & $\begin{array}{l}\text { DATA } \\
\text { QUAL. }\end{array}$ & $\begin{array}{l}\text { DATA } \\
\text { VALID. }\end{array}$ & CRDL UNITS & DILUTION \\
\hline \multicolumn{6}{|l|}{ C5-1-W-DUP } \\
\hline Arsenic & $1.4 \mathrm{UG} /$ & B & NP & $10 \mathrm{UG} / \mathrm{L}$ & 1 \\
\hline Aluminum & 4380 UG/ & & NP & $200 \mathrm{UG} / \mathrm{L}$ & 1 \\
\hline Antimony & 22.7 UG $\Omega$ & $u$ & $\mathrm{NP}$ & 60 UG/L & 1 \\
\hline Barium & 41.5 UG/ & B & NP & 200 UG/L & 1 \\
\hline Beryilium & 1.7 UG/ & u & NP & 5 UG/L & 1 \\
\hline Cadmium & $2.9 \mathrm{UG} / \mathrm{L}$ & $u$ & NP & 5 UG/L & 1 \\
\hline Calcium & 3760 UG/L & $B$ & $\mathrm{NP}$ & 5000 UG/L & 1 \\
\hline Chromium & 93.5 UaR & & $\mathrm{NP}:$ & $10 \mathrm{UG} / \mathrm{L}$ & 1 \\
\hline Cobalt & 5.8 UG $\Omega$ & B & NP & $50 \mathrm{UG} / \mathrm{L}$ & 1 \\
\hline Copper & $38.6 \mathrm{UG} / \mathrm{L}$ & & NP & $25 \mathrm{UG} / \mathrm{L}$ & 1 \\
\hline Iron & 37400 UG $\Omega$ & & NP & $100 \mathrm{UG} / \mathrm{L}$ & 1 \\
\hline Lead & 3 UG $\Omega$ & & NP & 3 UG/L & 1 \\
\hline Magnesium & 1360 UG & B & NP & 5000 UG/L & 1 \\
\hline Manganese & 229 UG/ & & NP & 15 UG/L & 1 \\
\hline Mercury & 0.1 UG/L & $u$ & NP & 0.2 UG/L & 1 \\
\hline Nickel & $43.8 \mathrm{UG} / \mathrm{L}$ & & $\mathrm{NP}$ & $40 \mathrm{UG} / \mathrm{L}$ & 1 \\
\hline Potassium & $5890 \mathrm{UG} / \mathrm{L}$ & & NP & 5000 UG/L & 1 \\
\hline Selenium & $1 \mathrm{UG \Omega}$ & $u$ & $\mathrm{NP}$ & $5 \mathrm{UG} / \mathrm{L}$ & 1 \\
\hline Silver & 4.1 UG $\Omega$ & $u$ & NP & 10 UG/L & 1 \\
\hline Sodium & 3260 UG $\Omega$ & B & NP & $5000 \mathrm{UG} / \mathrm{L}$ & 1 \\
\hline Thallium & 1 UGR & $u$ & NP & $10 \mathrm{UG} / \mathrm{L}$ & 1 \\
\hline Vanadium & 27.3 UGR & B & NP & 50 UG/L & 1 \\
\hline Zinc & $223 \mathrm{UG/L}$ & & $N P$ & $20 \mathrm{UG} / \mathrm{L}$ & 1 \\
\hline Cyanide & 2 UG/ & B & $N P$ & $10 \mathrm{UG} / \mathrm{L}$ & 1 \\
\hline
\end{tabular}


APPENDIX F.2 Results of Metals Analyses, Water, Salmon Site

\begin{tabular}{|c|c|c|c|c|}
\hline COMPOUND & RESULTS UNITS & $\begin{array}{l}\text { DATA DATA } \\
\text { QUAL. VALID. }\end{array}$ & CRDL UNITS & DILUTION \\
\hline \multicolumn{5}{|l|}{ C5-1-W-MS } \\
\hline Arsenic & $70 \%$ & NP & $\%$ & 1 \\
\hline Aluminum & $280 \%$ & NP & $\%$ & 1 \\
\hline Antimony & $94 \%$ & NP & $\%$ & 1 \\
\hline Barium & $96 \%$ & NP & $\%$ & 1 \\
\hline Beryllium & $98 \%$ & NP & $\%$ & 1 \\
\hline Cadmium & $78 \%$ & NP & $\%$ & 1 \\
\hline Chromium & $118 \%$ & NP & $\%$ & 1 \\
\hline Cobalt & $99 \%$ & NP & $\%$ & 1 \\
\hline Copper & $102 \%$ & NP & $\%$ & 1 \\
\hline Iron & $905 \%$ & NP & $\%$ & 1 \\
\hline Lead & $90 \%$ & NP & $\%$ & 1 \\
\hline Manganese & $104 \%$ & NP & $\%$ & 1 \\
\hline Mercury & $111 \%$ & NP & $\%$ & 1 \\
\hline Nickel & $103 \%$ & NP & $\%$ & 1 \\
\hline Selenium & $57 \%$ & NP & $\%$ & 1 \\
\hline Silver & $93 \%$ & NP & $\%$ & 1 \\
\hline Thallium & $97 \%$ & NP & $\%$ & 1 \\
\hline Vanadium & $101 \%$ & NP & $\%$ & 1 \\
\hline Zinc & $101 \%$ & NP & $\%$ & 1 \\
\hline Cyanide & $94 \%$ & NP & $\%$ & 1 \\
\hline
\end{tabular}

F.2 - 3 
APPENDIX F.2 Results of Metals Analyses, Water, Salmon Site

\begin{tabular}{|c|c|c|c|c|c|c|}
\hline COMPOUND & RESULTS & UNITS & $\begin{array}{l}\text { DATA } \\
\text { QUAL. }\end{array}$ & $\begin{array}{l}\text { DATA } \\
\text { VALID. }\end{array}$ & CRDL UNITS & DILUTION \\
\hline \multicolumn{7}{|l|}{ C3-8-W } \\
\hline Arsenic & 15.2 & UG/L & & NP & $10 \cup G / L$ & 1 \\
\hline Aluminum & 61200 & UG/L & & NP & 200 UG/L & 1 \\
\hline Antimony & 227 & UGR & $u$ & NP & $60 \mathrm{UG} / \mathrm{L}$ & 1 \\
\hline Barium & 2690 & UGR & & $\mathrm{NP}$ & 200 UG/L & 1 \\
\hline Beryllium & 10.1 & $U G /$ & & NP & 5 UG/L & 1 \\
\hline Cadmium & & UGR & $u$ & NP & 5 UG/L & 1 \\
\hline Calcium & 189000 & UG/L & & NP & 5000 UG/L & 1 \\
\hline Ctiromium & 353 & UGR & & NP & $10 \mathrm{UG} / \mathrm{L}$ & 1 \\
\hline Cobalt & 265 & UG/ & & NP & 50 UG/L & 1 \\
\hline Copper & 140 & UG/L & & NP & 25 UG/L & 1 \\
\hline Iron & 84400 & UG $\Omega$ & & NP & $100 \mathrm{UG} / \mathrm{L}$ & 1 \\
\hline Lead & 68.4 & UG & & NP. & $6 \mathrm{UG} /$ & 2 \\
\hline Magnesium & 61200 & UG/L & & NP & 5000 UG/L & 1 \\
\hline Manganese & 3500 & UGR & & NP & $15 \mathrm{UG} / \mathrm{L}$ & 1 \\
\hline Mercury & 0.14 & UGR & B & NP & 0.2 UG/L & 1 \\
\hline Nickel & 235 & UGR & & $\mathrm{NP}$ & 40 UG/L & 1 \\
\hline Potassium & 20800 & UG/ & & NP & 5000 UG/L & 1 \\
\hline Selenium & & UGR & u & NP & 50 UG/L & 10 \\
\hline Silver & & UG/L & $u$ & NP & $10 \mathrm{UG} / \mathrm{L}$ & 1 \\
\hline Sodium & 96900 & UG/L & & NP & 5000 UG/L & 1 \\
\hline Thallium & & Uar & $u$ & NP: & $50 \mathrm{UG} / \mathrm{L}$ & 5 \\
\hline Vanadium & 82.8 & UG $\Omega$ & & $N P$ & 50 UG/L & 1 \\
\hline Zinc & 606 & UG/ & & $\mathrm{NP}$ & $20 \mathrm{UG} / \mathrm{L}$ & 1 \\
\hline Cyanide & 3.6 & UG/L & B & NP & 10 UG/L & 1 \\
\hline
\end{tabular}


APPENDIX F.2 Results of Metals Analyses, Water, Salmon Site

\begin{tabular}{|c|c|c|c|c|c|c|c|}
\hline COMPOUND & & RESULTS & UNITS & $\begin{array}{l}\text { DATA } \\
\text { QUAL. }\end{array}$ & $\begin{array}{l}\text { DATA } \\
\text { VALID. }\end{array}$ & CRDL UNITS & DILUTION \\
\hline \multicolumn{8}{|l|}{ C-3-9-W } \\
\hline Arsenic & & 12.4 & UG/ & & NP & $10 \mathrm{UG} / \mathrm{L}$ & 1 \\
\hline Aluminum & & 227000 & UGR & & NP & 200 UG/L & 1 \\
\hline Antimony & & 22.7 & UGR: & $u$ & NP & 60 UGR & 1 \\
\hline Barium & & 4530 & UGR & & $\mathrm{NP}$ & $200 \mathrm{UG} /$ & 1 \\
\hline Beryllium & & 60.2 & UGR & & $N P$ & 5. UG/L & 1 \\
\hline Cadmium & & 2.9 & UG $\Omega$ & $u$ & NP & 5 UG/L & 1 \\
\hline Calcium & & 189000 & UG/ & & NP & 5000 UG/L & 1 \\
\hline Chromium & & 966 & UGR & . & NP. & $10 \mathrm{UG} / \mathrm{L}$ & 1 \\
\hline Cobalt & & 385 & UGR & & NP & $50 \mathrm{UG} / \mathrm{L}$ & 1 \\
\hline Copper & & 385 & UG/L & & NP & 25 UG/L & 1 \\
\hline Iron & & 401000 & UG $/$ & & NP & $100 \mathrm{UG} / \mathrm{L}$ & 1 \\
\hline Lead & & 258 & UGR & & NP: & 30 UG $\Omega$ & 10 \\
\hline Magnesium & & 52900 & UGL & & NP & $5000 \mathrm{UG} / \mathrm{L}$ & 1 \\
\hline Manganese & & 11600 & UG $/$ & & NP & 15 UG/L & 1 \\
\hline Mercury & & 0.15 & UG $\Omega$ & $B$ & $\mathrm{NP}$ & 0.2 UG/L & 1 \\
\hline Nickel & & 882 & UG & & NP & $40 \mathrm{UG/L}$ & 1 \\
\hline Potassium & & 26600 & UG/L & & NP & 5000 UG/L & 1 \\
\hline Selenium & & & UG/L & $u$ & NP & $50 \mathrm{UG} / \mathrm{L}$ & 10 \\
\hline Silver & & 4.1 & UG $\Omega$ & $u$ & NP & $10 \mathrm{UG} / \mathrm{L}$ & 1 \\
\hline Sodium & & 19400 & UG & & NP & 5000 UG/L & 1 \\
\hline Thallium & $\ldots \ldots$ & & UGR & $u$ & NP & 50 UG/L. & 5 \\
\hline Vanadium & & 334 & $U G \Omega$ & & NP & 50 UG/L & 1 \\
\hline Zinc & & 769 & UG/ & & NP & 20 UG/L & 1 \\
\hline Cyanide & & 4.8 & UG $\Omega$ & B & NP & $10 \mathrm{UG} / \mathrm{L}$ & 1 \\
\hline
\end{tabular}


APPENDIX F.2 Results of Metals Analyses, Water, Salmon Site

\begin{tabular}{|c|c|c|c|c|c|c|}
\hline COMPOUND & RESULTS & UNITS & $\begin{array}{l}\text { DATA } \\
\text { QUAL. }\end{array}$ & $\begin{array}{l}\text { DATA } \\
\text { VALID. }\end{array}$ & CRDL UNITS & DILUTION \\
\hline \multicolumn{7}{|l|}{ C4-11-W } \\
\hline Arsenic & 1.3 & UGR & $u$ & NP & $10 \mathrm{UG} / \mathrm{L}$ & 1 \\
\hline Aluminum & 136000 & UG/L & & NP & 200 UG/L & 1 \\
\hline Antimony & 22.7 & UG/L & $u$ & NP & $60 \mathrm{UG} /$ & 1 \\
\hline Barium & 1430 & UG/L & & NP & $200 \mathrm{UG} / \mathrm{L}$ & 1 \\
\hline Beryllium & 8.8 & UGR & & NP. & $5 \mathrm{UG} / \mathrm{L}$ & 1 \\
\hline Cadmium & 2.9 & UG/L & $u$ & NP & 5 UG/L & 1 \\
\hline Calcium & 10300 & UG/L & & NP & 5000 UG/L & 1 \\
\hline Chromium & 496 & UGR & & NP. & 10 UG/ & 1 \\
\hline Cobalt & 204 & UG/ & & NP & $50 \mathrm{UG} / \mathrm{L}$ & 1 \\
\hline Copper & 152 & UG/ & & NP & 25 UG/L & 1 \\
\hline Iron & 284000 & UG/ & & NP & 100 UG/L & 1 \\
\hline Lead & 155 & UGR & & $\stackrel{N P}{ }$ & $15 \mathrm{UG} \Omega$ & 5 \\
\hline Magnesium & 7470 & UG/ & & $\mathrm{NP}$ & $5000 \mathrm{UG} / \mathrm{L}$ & 1 \\
\hline Manganese & 4680 & UG/L & & NP & $15 \mathrm{UG} / \mathrm{L}$ & 1 \\
\hline Mercury & 0.98 & $U G / L$ & & NP & 0.2 UG/L & 1 \\
\hline Nickel & 114 & UGR & & NP & 40 UG/L & 1 \\
\hline Potassium & 6230 & $U G \Omega$ & & NP & 5000 UG/L & 1 \\
\hline Selenium & & UG $\Omega$ & $u$ & NP & 25 UG/L & 5 \\
\hline Silver & 4.1 & UGR & $u$ & NP & 10 UG/L & 1 \\
\hline Sodium & 6320 & UG/ & & NP & 5000 UG/L & 1 \\
\hline Thallium & 1.5 & UG/ & B & NP & $10 \mathrm{UG} / \mathrm{L}$ & 1 \\
\hline Vanadium & 703 & UGR & & NP & $50 \mathrm{UG} / \mathrm{L}$ & 1 \\
\hline Zinc & 360 & UG/ & & NP & $20 \mathrm{UG} / \mathrm{L}$ & 1 \\
\hline Cyanide & 3.3 & UGR & B & NP & $10 \mathrm{UG} / \mathrm{L}$ & 1 \\
\hline
\end{tabular}


APPENDIX F.2 Results of Metals Analyses, Water, Salmon Site

\section{DATA DATA}

COMPOUND

C4-13-W

Arsenic

Aluminum

Antimony

Barium

Beryllium

Cadmium

Calcium

Chromium

Cobalt

Copper

Iron

Lead

Magnesium

Manganese

Mercury

Nickel

Potassium

Selenium

Silver

Sodium

Thallium

Vanadium

Zinc

Cyanide
B

NP

$U \quad N P$

B NP

$U \quad N P$

NP

NP

NP

NP

NP

NP

NP

NP

B

NP

NP

NP

NP

u

u

u

NP

NP

NP

NP

NP

B

NP
RESULTS UNITS QUAL. VALID.

CRDL UNITS DILUTION

2.5 UG
16900 UG
22.7 UG $\Omega$
704 UG/
4 UG
2.9 UG $\Omega$
16900 UG $\Omega$
393. UG
18.5 UG
192 UG $/$
340000 UG

92.4 UGR

5640 UG/

1660 UG/

0.11 UGL

115 UGR

14600 UGR

1 UGR

4.1 UG/L

$14500 \mathrm{UG} / \mathrm{L}$

5 UGR

387 UGR

$810 \mathrm{UG} / \mathrm{L}$

$2.1 \mathrm{UGR}$
$10 \mathrm{UG} / \mathrm{L}$ 200 UG/L

60 UGR. 200 UG/L

$5 \mathrm{UG} / \mathrm{L}$

5 UG/L

5000 UG/L

10 UGR

$50 \mathrm{UG} / \mathrm{L}$

25 UG/L

$100 \mathrm{UG} / \mathrm{L}$

6 UG/

5000 UG/L

15 UG/L

$0.2 \mathrm{UG} / \mathrm{L}$

40 UG/

5000 UG/L

5 UG/L

10 UG/L

5000 UG/L

50 UGR

50 UG/L

20 UG/L

$10 \mathrm{UG} / \mathrm{L}$
1

1

1

1

1

1

1

1

1

1

2

1

1

1

1 .

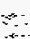


APPENDIX F.2 Results of Metals Analyses, Water, Salmon Site

\begin{tabular}{|c|c|c|c|c|c|c|c|}
\hline COMPOUND & & RESULTS & UNITS & $\begin{array}{l}\text { DATA } \\
\text { QUAL. }\end{array}$ & $\begin{array}{l}\text { DATA } \\
\text { VALID. }\end{array}$ & CRDL UNITS & DILUTION \\
\hline \multicolumn{8}{|l|}{ C1-14-W } \\
\hline Arsenic & & 3.6 & UG/ & B & $s$ & $10 \mathrm{UG} / \mathrm{L}$ & 1 \\
\hline Aluminum & & 331000 & UG $\Omega$ & & -- & 200 UG/L & 1 \\
\hline Antimony & & & UG $\Omega$ & $u$ & UJ: & 60 UG/L & 1 \\
\hline Barium & & 8650 & UGR & & - & $200 \mathrm{UG} / \mathrm{L}$ & 1 \\
\hline Beryllium & & 30.5 & UG/ & & - & 5 UG/L & 1 \\
\hline Cadmium & & 6.9 & UGR & & - & $5 \mathrm{UG} / \mathrm{L}$ & 1 \\
\hline Calcium & & 500000 & UGR & & - & $100000 \mathrm{UG} / \mathrm{L}$ & 20 \\
\hline Chromium & & 2050 & UGR & & - & $10 \mathrm{UG} / \mathrm{L}$ & 1 \\
\hline Cobalt & & 291 & UG/ & B & $J$ & 1000 UG/L & 20 \\
\hline Copper & & 983 & UG/L & & $\mathrm{J}$ & 25 UG/L & 1 \\
\hline Iron & & 1190000 & UG/ & & - & 2000 UG/L & 20 \\
\hline Lead & & 800 & UGR & & - & 60 UG/ & 20 \\
\hline Magnesium & & 140000 & UG/L & & - & $100000 \mathrm{UG} / \mathrm{L}$ & 20 \\
\hline Manganese & & 15700 & $U G / L$ & & - & $300 \mathrm{UG} / \mathrm{L}$ & 20 \\
\hline Mercury & & 0.9 & UG/L & & $\ldots$ & 0.2 UG/L & 1 \\
\hline Nickel & & 720 & UG $\Omega$ & & - & $40 \mathrm{UG} / \mathrm{L}$ & 1 \\
\hline Potassium & & 28600 & UG $\Omega$ & & - & $5000 \mathrm{UG} / \mathrm{L}$ & 1 \\
\hline Selenium & & & UG/ & $u$ & $R$ & $25 \mathrm{UG} / \mathrm{L}$ & 5 \\
\hline Silver & & 3.4 & UG/L & $u$ & -- & $10 \mathrm{UG} / \mathrm{L}$ & 1 \\
\hline Sodium & & 571000 & UG/ & & $\ldots$ & $100000 \mathrm{UG} / \mathrm{L}$ & 20 \\
\hline Thallium & $\therefore$ & & UGR & $u$ & $\mathrm{R}$ & $\therefore \quad 100 \mathrm{UG} / \mathrm{L}$ & 10 \\
\hline Vanadium & & 1380 & UG/L & & - & $1000 \mathrm{UG} / \mathrm{L}$ & 20 \\
\hline Zinc & & 1500 & UG/L & & J & 400 UG/L & 20 \\
\hline Cyanide & & 0.99 & UG/L & B & UJ & $10 \mathrm{UG} / \mathrm{L}$ & 1 \\
\hline
\end{tabular}




\section{APPENDIX F.2 Results of Metals Analyses, Water, Salmon Site}

\begin{tabular}{|c|c|c|c|c|c|c|c|}
\hline COMPOUND & & RESULTS & UNITS & $\begin{array}{l}\text { DATA } \\
\text { QUAL. }\end{array}$ & $\begin{array}{l}\text { DATA } \\
\text { VALID. }\end{array}$ & CRDL UNITS & DILUTION \\
\hline \multicolumn{8}{|l|}{ C1-15-W } \\
\hline Arsenic & & 20.2 & UG/L & & $\mathrm{J}$ & $10 \mathrm{UG} / \mathrm{L}$ & 1 \\
\hline Aluminum & & 121000 & UG $\Omega$ & & J & $200 \mathrm{UG} / \mathrm{L}$ & 1 \\
\hline Antimony & & 35.2 & UGL & $u=$ & US & $60 \mathrm{UG}$ & 1 \\
\hline Barium & & 2590 & UGR. & & J & 200.UGL & 1 . \\
\hline Beryllium & & 15.2 & UGR & & $J$ & $5 \mathrm{UG} /$ & 1 \\
\hline Cadmium & - & 5.7 & UGL & $\because$ & $\mathrm{J}$ & $5 \mathrm{UG}$ & 1 \\
\hline Calcium & & 104000 & UG $\Omega$ & - & $J$ & $25000 \mathrm{UG} / \mathrm{L}$ & 5 \\
\hline Chromium & & 1310 & Uar. & & $\mathrm{j}$ & 10 Uan & 1 \\
\hline Cobalt & & 75.9 & $U G \Omega$ & & J & $50 \mathrm{UG} / \mathrm{L}$ & 1 \\
\hline Copper & & 412 & UG/L & & J & $25 \mathrm{UG} / \mathrm{L}$ & 1 \\
\hline Iron & & 426000 & UG $/$ & & $J$ & 500 UG/L & 5 \\
\hline Lead & & 338 & UGR. & & $\mathrm{j}$ & 30 UG/L & 10 \\
\hline Magnesium & & 36600 & UG $\Omega$ & & $J$ & 5000 UG/L & 1 \\
\hline Manganese & & 3510 & UG/L & & $\mathrm{J}$ & 15 UG/L & 1 \\
\hline Mercury & & 0.61 & UG & & J & 0.2 UG/L & 1 \\
\hline Nickel & & 437 & UGL & & $\checkmark$ & $40 \mathrm{UG} / \mathrm{L}$ & 1 \\
\hline Potassium & & 17100 & UG/ & & UJ & 5000 UG/L & 1 \\
\hline Selenium & & & + UG $\Omega$ & $u$ & R & 25 UG/L & 5 \\
\hline Silver & & 3.4 & $+U G \Omega$ & $u$ & Us & $10 \mathrm{UG} / \mathrm{L}$ & 1 \\
\hline Sodium & & 117000 & UGR & & $J$ & $5000 \mathrm{UG} / \mathrm{L}$ & 1 \\
\hline Thallium & .. & & YUGL & $\mathrm{U}$ & $\mathrm{R}$ & 100 UG $/$ & 10 \\
\hline Vanadium & & 883 & 3 UGR & & $J$ & 50 UG/L & 1 \\
\hline Zinc & & 734 & 4 UG & & $\mathrm{J}$ & $20 \mathrm{UG} / \mathrm{L}$ & 1 \\
\hline Cyanide & & 0.92 & 2 UG/L & B & UJ & $10 \mathrm{UG} / \mathrm{L}$ & 1 \\
\hline
\end{tabular}


APPENDIX F.2 Results of Metals Analyses, Water, Salmon Site

\begin{tabular}{|c|c|c|c|c|c|}
\hline COMPOUND & RESULTS UNITS & $\begin{array}{l}\text { DATA } \\
\text { QUAL. }\end{array}$ & $\begin{array}{l}\text { DATA } \\
\text { VALID. }\end{array}$ & CRDL UNITS & DILUTION \\
\hline \multicolumn{6}{|l|}{ C1-15-W -DUP } \\
\hline Arsenic & 19 UGR & & $\mathrm{NP}$ & $10 \mathrm{UG} / \mathrm{L}$ & 1 \\
\hline Aluminum & 116000 UGR & & NP & 200 UG/L & 1 \\
\hline Antimony & $35: 2$ UG & $u$ & NP. & $60 \mathrm{UG} / \mathrm{L}$ & 1 \\
\hline Barium & 2660 UG/L & & NP & 200 UGR & 1 \\
\hline Beryllium & 15.2 UGR & & $\mathrm{NP}$ & 5 UG/L & 1 \\
\hline Cadmium & 6:UG & & NP & 5 UG/L & 1 \\
\hline Calcium & 97400 UG/L & & $\mathrm{NP}$ & 25000 UG/L & 5 \\
\hline Chromium & 1350 UGR & & $\mathrm{NP}$ & $10 \mathrm{UG} / \mathrm{L}$ & 1 \\
\hline Cobalt & 76 UG $\Omega$ & & $\mathrm{NP}$ & $50 \mathrm{UG} / \mathrm{L}$ & 1 \\
\hline Copper & 416 UG/ & & NP & 25 UG/L & 1 \\
\hline Iron & $395000 \mathrm{UG} /$ & & $\mathrm{NP}$ & 500 UG/L & 5 \\
\hline Lead & 351 UG & & $N P$ & $15 \cdot \mathrm{UG} / \mathrm{L}$ & 5 \\
\hline Magnesium & 36600 UG & & NP & 5000 UG/L & 1 \\
\hline Manganese & 3540 UG/L & & NP & 15 UG/L & 1 \\
\hline Mercury & 0.64 UG几 & & NP & 0.2 UG/L & 1 \\
\hline Nickel & 433 UGR & & $\mathrm{NP}$ & $40 \mathrm{UG} /$ & 1 \\
\hline Potassium & $17400 \mathrm{UG} / \mathrm{L}$ & & NP & 5000 UG/L & 1 \\
\hline Selenium & 4 UG $\Omega$ & $u$ & NP & 25 UG/L & 5 \\
\hline Silver & $3.4 \mathrm{UG} / \mathrm{L}$ & $u$ & NP & $10 \mathrm{UG} / \mathrm{L}$ & 1 \\
\hline Sodium & $117000 \mathrm{UG} / \mathrm{L}$ & & NP & 5000 UG/L & 1 \\
\hline Thallium & 9UGR & $u$ & $\mathrm{NP}$ & $100 \mathrm{UGL}$ & $10^{\circ}$ \\
\hline Vanadium & 893 UG/L & & NP & 50 UG/L & 1 \\
\hline Zinc & 723 UG/L & & NP & 20 UG/L & 1 \\
\hline Cyanide & $1.3 \mathrm{UG} / \mathrm{L}$ & $B$ & $\mathrm{NP}$ & $10 \mathrm{UG} / \mathrm{L}$ & 1 \\
\hline
\end{tabular}


APPENDIX F.2 Results of Metals Analyses, Water, Salmon Site

\begin{tabular}{lrccc|}
\hline COMPOUND & RESULTS UNITS & DATA & DATA & \\
QUA. & VALID. & CRDL UNITS & DILUTION \\
\hline C1-15-W -MS & & & & \\
Arsenic & $37 \%$ & NP & $\%$ & 1 \\
Aluminum & $1540 \%$ & NP & $\%$ & 1 \\
Antimony & $40 \%$ & NP & $\%$ & 1 \\
Barium & $104 \%$ & NP & $\%$ & 1 \\
Beryllium & $110 \%$ & NP & $\%$ & 1 \\
Cadmium & $112 \%$ & NP & $\%$ & 1 \\
Chromium & $6 \%$ & NP & $\%$ & 1 \\
Cobalt & $103 \%$ & NP & $\%$ & 1 \\
Copper & $95 \%$ & NP & $\%$ & 1 \\
Iron & $-1240 \%$ & NP & $\%$ & 5 \\
Lead & $-222 \%$ & NP & $\%$ & 5 \\
Manganese & $106 \%$ & NP & $\%$ & 1 \\
Mercury & $105 \%$ & NP & $\%$ & 1 \\
Nickel & $84 \%$ & NP & $\%$ & 1 \\
Selenium & $11 \%$ & NP & $\%$ & 1 \\
Silver & $93 \%$ & NP & $\%$ & 1 \\
Thallium & $0 \%$ & NP & $\%$ & 1 \\
Vanadium & $114 \%$ & NP & $\%$ & 1 \\
Zinc & $105 \%$ & NP & $\%$ & 1 \\
Cyanide & $94 \%$ & NP & $\%$ & 1
\end{tabular}


APPENDIX F.2 Results of Metals Analyses, Water, Salmon Site

\begin{tabular}{|c|c|c|c|c|c|c|}
\hline COMPOUND & RESULTS & UNITS & $\begin{array}{l}\text { DATA } \\
\text { QUAL. }\end{array}$ & $\begin{array}{c}\text { DATA } \\
\text { VALID. }\end{array}$ & CRDL UNITS & DILUTION \\
\hline \multicolumn{7}{|l|}{ C1-16-W } \\
\hline Arsenic & 28.6 & UG/ & & J & $10 \mathrm{UG} / \mathrm{L}$ & 1 \\
\hline Aluminum & 109000 & UGR & & $J$ & 200 UG/L & 1 \\
\hline Antimony & 35.2 & $U G \Omega$ & $u$ & US & $60 \mathrm{UG} / \mathrm{L}$ & 1 \\
\hline Barium & 246 & UGR & & J & $200 \mathrm{UG} / \mathrm{L}$ & 1 \\
\hline Beryilium & & UGR & & J & 5 UG/ & 1 \\
\hline Cadmium & 2.7 & UG/L & $u$ & UJ & $5 \mathrm{UG/L}$ & 1 \\
\hline Calcium & 165000 & UGR & & $J$ & $100000 \mathrm{UG} / \mathrm{L}$ & 20 \\
\hline Chromium & 333 & UGR & & 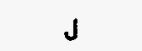 & $10 \mathrm{UG} / \mathrm{L}$ & 1 \\
\hline Cobalt & 123 & UGR & & $J$ & $50 \mathrm{UG} / \mathrm{L}$ & 1 \\
\hline Copper & 205 & UGR & & US & 25 UG/L & 1 \\
\hline Iron & 125000 & UG/L & & J & $100 \mathrm{UG} / \mathrm{L}$ & 1 \\
\hline Lead & 196 & Uar & & J & 30. UG/L & 10 \\
\hline Magnesium & 71290 & UG/L & & J & 5000 UG/L & 1 \\
\hline Manganese & 3120 & UG/L & & J & 15 UG/L & 1 \\
\hline Mercury & 1.6 & UG/L & & J & 0.2 UG/L & 1 \\
\hline Nickel & 83.9 & UG/ & & J & $40 \mathrm{UG} / \mathrm{L}$ & 1 \\
\hline Potassium & 14900 & UGR & & J & 5000 UG/L & 1 \\
\hline Selenium & 4.9 & UG $\Omega$ & B & UJ & 25 UG/L & 5 \\
\hline Silver & 3.4 & UG $\Omega$ & u & us & 10 UG/L & 1 \\
\hline Sodium & 94500 & UG $\Omega$ & & $J$ & 5000 UG/L & 1 \\
\hline Thallium & & UGR. & $u$ & $R$ & $100 \mathrm{UG} / \mathrm{L}$ & 10 \\
\hline Vanadium & 358 & $U G \Omega$ & & J & $50 \mathrm{UG} / \mathrm{L}$ & 1 \\
\hline Zinc & 228 & UGR & & UJ & $20 \mathrm{UG} / \mathrm{L}$ & 1 \\
\hline Cyanide & 2.1 & $U G / L$ & B & J & $10 \mathrm{UG} / \mathrm{L}$ & 1 \\
\hline
\end{tabular}


APPENDIX F.2 Results of Metals Analyses, Water, Salmon Site

\begin{tabular}{|c|c|c|c|c|c|c|c|}
\hline COMPOUND & & RESULTS & UNITS & $\begin{array}{l}\text { DATA } \\
\text { QUAL. }\end{array}$ & $\begin{array}{l}\text { DATA } \\
\text { VALID. }\end{array}$ & CRDL UNITS & DILUTION \\
\hline \multicolumn{8}{|l|}{ C1-17-W } \\
\hline Arsenic & & & UG/L & & J & $10 \mathrm{UG} / \mathrm{L}$ & 1 \\
\hline Aluminum & & 95200 & UG $/$. & & J & 200 UG/L & 1 \\
\hline Antimony & & $35: 2$ & UGR & $u$ & us. & 60 UGL & 1 \\
\hline Barium & & 1120 & UG/L & & $\mathrm{J}$ & 200 UG/L & 1 \\
\hline Beryllium & & 13.6 & UGR & & $d$ & $5 \mathrm{UG} /$ & 1 \\
\hline Cadmium & & & UGR & B & $J$ & $5 \mathrm{UG} / \mathrm{L}$ & 1 \\
\hline Calcium & & 30800 & UGR & & $J$ & 5000 UG/L & 1 \\
\hline Chromium & & 2490 & UGR & & $\mathrm{J}$ & $10 U G \Omega$ & $t$ \\
\hline Cobalt & & 40.8 & UG/L & B & J & $50 \mathrm{UG} / \mathrm{L}$ & 1 \\
\hline Copper & & 544 & UG $\Omega$ & & 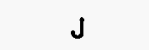 & 25 UG/L & 1 \\
\hline Iron & & 133000 & UGR & & $J$ & $100 \mathrm{UG} / \mathrm{L}$ & 1 \\
\hline Lead & & 133 & UGR & & J & 30 UGI & $10=$ \\
\hline Magnesium & & 13400 & UG/ & & J & 5000 UG/L & 1 \\
\hline Manganese & & 2480 & UG/L & & $\checkmark$ & 15 UG/L & 1 \\
\hline Mercury & & 0.38 & UG/L & & $\mathrm{J}$ & $0.2 \mathrm{UG} / \mathrm{L}$ & 1 \\
\hline Nickel & & 861 & UGR. & & J & 40 UG/L & 1 \\
\hline Potassium & & 14100 & UG $\Omega$ & & us & 5000 UG/L & 1 \\
\hline Selenium & & & UG $\Omega$ & $u$ & $\mathrm{R}$ & 25 UG/L & 5 \\
\hline Silver & & 3.4 & UG $\Omega$ & $u$ & Us & $10 \mathrm{UG} / \mathrm{L}$ & 1 \\
\hline Sodium & & 157000 & UG & & $J$ & 5000 UG/L & 1 \\
\hline Thallium & & & UG $\Omega$ & $u$ & $A$ & 100 UG $\Omega$ & 10 \\
\hline Vanadium & & 277 & UGR. & & $J$ & $50 \mathrm{UG} / \mathrm{L}$ & 1 \\
\hline Zinc & & 560 & UG $\Omega$ & & Us & $20 \mathrm{UG} / \mathrm{L}$ & 1 \\
\hline Cyanide & & 1.1 & UG $/ \mathrm{L}$ & B & UJ & $10 \mathrm{UG} / \mathrm{L}$ & 1 \\
\hline
\end{tabular}


APPENDIX F.2 Results of Metals Analyses, Water, Salmon Site

\begin{tabular}{|c|c|c|c|c|c|}
\hline COMPOUND & RESULTS UNITS & $\begin{array}{l}\text { DATA } \\
\text { QUAL. }\end{array}$ & $\begin{array}{l}\text { DATA } \\
\text { VALID. }\end{array}$ & CRDL UNITS & DILUTION \\
\hline \multicolumn{6}{|l|}{$C 1-18-W$} \\
\hline Arsenic & 12.2 UG/L & & J & $10 \mathrm{UG} / \mathrm{L}$ & 1 \\
\hline Aluminum & 17800 UG/L & & $\ldots$ & 200 UG/L & 1 \\
\hline Antimony & 40.3 UG $\Omega$ & $B$ & J & $60 \mathrm{UG} / \mathrm{L}$ & 1 \\
\hline Barium & 59.2 UG/L & B & J & $200 \mathrm{UG} / \mathrm{L}$ & 1 \\
\hline Beryllium & 2.5 UG/L & B & J & 5 UG/L & 1 \\
\hline Cadmium & 2.7 UG/ & $u$ & $\ldots$ & 5 UG/L & 1 \\
\hline Calcium & 456000 UG/L & & -- & 100000 UG/L & 20 \\
\hline Chromium & 241 UG $\Omega$ & & - & 10 UG/L & 1 \\
\hline Cobalt & 19.7 UG/ & B & $J$ & $50 \mathrm{UG} / \mathrm{L}$ & 1 \\
\hline Copper & 90.2 UG/L & & J & 25 UG/L & 1 \\
\hline Iron & 99700 UGR & & -- & $100 \mathrm{UG} / \mathrm{L}$ & 1 \\
\hline Lead & 102 UG & & - & 6 UG/L & 2 \\
\hline Magnesium & 11400 UG/L & & - & 5000 UG/L & 1 \\
\hline Manganese & 2840 UG/ & & -- & 15 UG/L & 1 \\
\hline Mercury & 0.22 UG $\Omega$ & & -- & $0.2 \mathrm{UG} / \mathrm{L}$ & 1 \\
\hline Nickel & 79.9 UG/L & & -- & $40 \mathrm{UG} / \mathrm{L}$ & 1 \\
\hline Potassium & 12600 UG/ & & $u$ & $5000 \mathrm{UG} / \mathrm{L}$ & 1 \\
\hline Selenium & $1 \mathrm{UG} \Omega$ & B & UJ & $5 \mathrm{UG} / \mathrm{L}$ & 1 \\
\hline Silver & 3.4 UG/L & $u$ & $u$ & 10 UG/L & 1 \\
\hline Sodium & 92400 UG/L & & $\ldots$ & 5000 UG/L & 1 \\
\hline Thallium & 0.9 UG $\Omega$ & $u$ & $R$ & $10 \mathrm{UG} / \mathrm{L}$ & 1 \\
\hline Vanadium & 86 UG几 & & $\ldots$ & 50 UG/L & 1 \\
\hline Zinc & 750 UG & & $J$ & $20 \mathrm{UG} / \mathrm{L}$ & 1 \\
\hline Cyanide & 3.3 UG & $B$ & UJ & $10 \mathrm{UG} / \mathrm{L}$ & 1 \\
\hline
\end{tabular}


APPENDIX F.2 Results of Metals Analyses, Water, Salmon Site

\begin{tabular}{|c|c|c|c|c|c|}
\hline COMPOUND & RESULTS UNITS & $\begin{array}{l}\text { DATA } \\
\text { QUAL. }\end{array}$ & $\begin{array}{l}\text { DATA } \\
\text { VALID. }\end{array}$ & CRDL UNITS & DILUTION \\
\hline \multicolumn{6}{|l|}{$C 2-19-W$} \\
\hline Arsenic & $22.7 \mathrm{UG} \Omega$ & & NP & $10 \mathrm{UG} / \mathrm{L}$ & 1 \\
\hline Aluminum & 4790 UG/L & & $N P$ & 200 UG/L & 1 \\
\hline Antimony & $227 \mathrm{UG} \Omega$ & $u$ & $\mathrm{NP}$ & $60 \mathrm{UG} / \mathrm{L}$ & 1. \\
\hline Barium & $135 \mathrm{UG} / \mathrm{L}$ & B & NP & 200 UG/L & 1 \\
\hline Beryllium & 1.7 UG/ & $u$ & NP & $5 \mathrm{UG} / \mathrm{L}$ & 1 \\
\hline Cadmium & $2.9 \mathrm{UG} / \mathrm{L}$ & $u$ & NP & 5 UG/L & 1 \\
\hline Calcium & $17100 \mathrm{UG} / \mathrm{L}$ & & NP & 5000 UG/L & 1 \\
\hline Chromium & 63.9 UG/ & & NP & $10 \mathrm{UG} / \mathrm{L}$ & 1 \\
\hline Cobalt & 29.5 UG/ & B & NP & $50 \mathrm{UG} / \mathrm{L}$ & 1 \\
\hline Copper & 41.5 UG $\Omega$ & & NP & 25 UG/L & 1 \\
\hline Iron & 80900 UG $\Omega$ & & NP & $100 \mathrm{UG} / \mathrm{L}$ & 1 \\
\hline Lead & 47.T UGR & & NP & 3 UG/L. & 1 \\
\hline Magnesium & 4950 UG/L & B & NP & $5000 \mathrm{UG} / \mathrm{L}$ & 1 \\
\hline Manganese & 1530 UG/ & & NP & $15 \mathrm{UG} / \mathrm{L}$ & 1 \\
\hline Mercury & 0.11 UG $/$ & B & NP & $0.2 \mathrm{UG} / \mathrm{L}$ & 1 \\
\hline Nickel & 56.7 UGR & & NP & 40 UG/L & 1 \\
\hline Potassium & $6220 \mathrm{UG} \Omega$ & & NP & 5000 UG/L & 1 \\
\hline Selenium & 4.6 UG/L & B & NP & $5 \mathrm{UG} / \mathrm{L}$ & 1 \\
\hline Silver & 4.1 UG & $u$ & NP & $10 \mathrm{UG} / \mathrm{L}$ & 1 \\
\hline Sodium & 6780 UG & & $N P$ & 5000 UG/L & 1 \\
\hline Thallium & 1 UG $\Omega$ & $u$ & NP & $10 \mathrm{UG} / \mathrm{L}$ & 1 \\
\hline Vanadium & 44.5 UG $\Omega$ & B & NP & 50 UG/L & 1 \\
\hline Zinc & 122 UG/ & & NP & $20 \mathrm{UG} / \mathrm{L}$ & 1 \\
\hline Cyanide & $11.8 \mathrm{UG} /$ & & NP & $10 \mathrm{UG} / \mathrm{L}$ & 1 \\
\hline
\end{tabular}


APPENDIX F.2 Results of Metals Analyses, Water, Salmon Site

\begin{tabular}{|c|c|c|c|c|c|}
\hline COMPOUND & RESULTS UNITS & $\begin{array}{l}\text { DATA } \\
\text { QUAL. }\end{array}$ & $\begin{array}{l}\text { DATA } \\
\text { VALID. }\end{array}$ & CRDL UNITS & DILUTIC \\
\hline \multicolumn{6}{|l|}{ C2-19-W -DUP } \\
\hline Arsenic & 9.9 UG/ & B & NP & $10 \mathrm{UG} / \mathrm{L}$ & 1 \\
\hline Aluminum & 16800 UG/ & & NP & 200 UG/L & 1 \\
\hline Antimony & 22.7 UG几 & $u$ & NP. & $60 \mathrm{UG} / \mathrm{L}$ & 1 \\
\hline Barium & 174 UG/L & B & NP & 200 UG/L & 1 \\
\hline Beryllium & 1.7 UGL & $u$ & NP & $5 \mathrm{UG} / \mathrm{L}$ & 1 \\
\hline Cadmium & 2.9 UG & $u$ & NP & $5 \mathrm{UG} / \mathrm{L}$ & 1 \\
\hline Calcium & 16900 UG $\Omega$ & & NP & 5000 UG/L & 1 \\
\hline Chromium & 196 UG & & & $10 \mathrm{UG} / \mathrm{L}$ & 1 \\
\hline Cobalt & 34.5 UG/L & B & NP & $50 \mathrm{UG} / \mathrm{L}$ & 1 \\
\hline Copper & 74.2 UG/L & & NP & 25 UG/L & 1 \\
\hline Iron & $137000 \mathrm{UG} /$ & & NP & $100 \mathrm{UG} / \mathrm{L}$ & 1 \\
\hline Lead & 79 UG $\Omega$ & & NP & $3 \mathrm{UG} / \mathrm{L}$ & 1 \\
\hline Magnesium & 5700 UG/L & & NP & 5000 UG/L & 1 \\
\hline Manganese & 2120 UG $\Omega$ & & NP & 15 UG/L & 1 \\
\hline Mercury & 0.1 UG $\Omega$ & $u$ & NP & 0.2 UG/L & 1 \\
\hline Nickel & 129 UG & & $N P$ & 40 UG/L & 1 \\
\hline Potassium & 7140 UG/ & & NP & 5000 UG/L & 1 \\
\hline Selenium & 1 UG $\Omega$ & $u$ & NP & $5 \mathrm{UG} / \mathrm{L}$ & 1 \\
\hline Silver & 4.1 UG/L & $u$ & NP & $10 \mathrm{UG} / \mathrm{L}$ & 1 \\
\hline Sodium & $6390 \mathrm{UG} / \mathrm{L}$ & & NP & 5000 UG/L & 1 \\
\hline Thallium & 1 UG & $u$ & NP & $10 \mathrm{UG} / \mathrm{L}$ & 1 \\
\hline Vanadium & $104 \mathrm{UG} /$ & & NP & 50 UG/L & 1 \\
\hline Zinc & $163 \mathrm{UG} / \mathrm{L}$ & & NP & $20 \mathrm{UG} / \mathrm{L}$ & 1 \\
\hline Cyanide & $18.8 \mathrm{UG} / \mathrm{L}$ & B & NP & 20 UG/L & 1 \\
\hline
\end{tabular}




\section{APPENDIX F.2 Results of Metals Analyses, Water, Salmon Site}

\begin{tabular}{lcccc|}
\hline COMPOUND & RESULTS UNITS & DATA & DATA & \\
QUA. & VALID. & CRDL UNITS & DILUTION \\
\hline C2-19-W -MS & & & & \\
Arsenic & $-34 \%$ & NP & $\%$ & 1 \\
Aluminum & $784 \%$ & NP & $\%$ & 1 \\
Antimony & $62 \%$ & NP & $\%$ & 1 \\
Barium & $93 \%$ & NP & $\%$ & 1 \\
Beryllium & $97 \%$ & NP & $\%$ & 1 \\
Cadmium & $52 \%$ & NP & $\%$ & 1 \\
Chromium & $157 \%$ & NP & $\%$ & 1 \\
Cobalt & $95 \%$ & NP & $\%$ & 1 \\
Copper & $108 \%$ & NP & $\%$ & 1 \\
Iron & $5240 \%$ & NP & $\%$ & 1 \\
Lead & $94 \%$ & NP & $\%$ & 1 \\
Manganese & NP & $\%$ & 1 \\
Mercury & $205 \%$ & NP & $\%$ & 1 \\
Nickel & $95 \%$ & NP & $\%$ & 1 \\
Selenium & $108 \%$ & NP & $\%$ & 1 \\
Silver & $-46 \%$ & NP & $\%$ & 1 \\
Thallium & $90 \%$ & NP & $\%$ & 1 \\
Vanadium & $98 \%$ & NP & $\%$ & 1 \\
Zinc & $104 \%$ & NP & $\%$ & 1 \\
Cyanide & $102 \%$ & NP & $\%$ & 1
\end{tabular}


APPENDIX F.2 Results of Metals Analyses, Water, Salmon Site

\begin{tabular}{|c|c|c|c|c|c|c|}
\hline COMPOUND & RESULTS & UNITS & $\begin{array}{l}\text { DATA } \\
\text { QUAL. }\end{array}$ & $\begin{array}{c}\text { DATA } \\
\text { VALID. }\end{array}$ & CRDL UNITS & DILUTION \\
\hline \multicolumn{7}{|l|}{$C-2-27-W$} \\
\hline Arsenic & 1.3 & UG/L & $u$ & NP & $10 \mathrm{UG} / \mathrm{L}$ & 1 \\
\hline Aluminum & 77300 & $U G / L$ & & $\mathrm{NP}$ & 200 UG/L & 1 \\
\hline Antimony & 22.7 & UGR & $u$ & $\mathrm{NP}$ & $60 \mathrm{UG} / \mathrm{L}$ & 1 \\
\hline Barium & 1260 & UGR & & $N P$ & 200 UG/L & 1 \\
\hline Beryllium & 11.9 & UGR & & NP & $5 \mathrm{UG} / \mathrm{L}$ & 1 \\
\hline Cadmium & 2.9 & $U G / L$ & $u$ & NP & $5 \mathrm{UG} / \mathrm{L}$ & 1 \\
\hline Calcium & 17100 & UG/L & & NP & 5000 UG/L & 1 \\
\hline Chromium & 4250 & UGR & & NP & $10 \mathrm{UG} / \mathrm{L}$ & 1 \\
\hline Cobalt & 137 & $U G / L$ & & $N P$ & 50 UG/L & 1 \\
\hline Copper & 515 & UG/L & & NP & 25 UG/L & 1 \\
\hline Iron & 353000 & UG/L & & NP & $100 \mathrm{UG} / \mathrm{L}$ & 1 \\
\hline Lead & 442 & UGR & & NP & 15 UG/L & 5 \\
\hline Magnesium & 4340 & UG/L & B & $N P$ & 5000 UG/L & 1 \\
\hline Manganese & 4560 & UG/ & & NP & 15 UG/L & 1 \\
\hline Mercury & 3.1 & UGR & & NP & $0.2 \mathrm{UG} \Omega$ & 1 \\
\hline Nickel & 2060 & UGR & & NP & 40 UG/L & 1 \\
\hline Potassium & 7410 & UG/L & & NP & 5000 UG/L & 1 \\
\hline Selenium & & UG/ & $u$ & NP & 25 UG/L & 5 \\
\hline Silver & 4.1 & UG/L & $u$ & NP & $10 \mathrm{UG} / \mathrm{L}$ & 1 \\
\hline Sodium & 9180 & UG/L & & $N P$ & $5000 \mathrm{UG} / \mathrm{L}$ & 1 \\
\hline Thallium & & UG/L & B & $N P$ & $10 \mathrm{UG} / \mathrm{L}$ & 1 \\
\hline Vanadium & 781 & UG $\Omega$ & & $N P$ & 50 UG/L & 1 \\
\hline Zinc & 395 & UG/L & & NP & $20 \mathrm{UG} / \mathrm{L}$ & 1 \\
\hline Cyanide & 6.9 & UGA & & $\mathrm{NP}$ & $10 \mathrm{UG} / \mathrm{L}$ & 1 \\
\hline
\end{tabular}


APPENDIX F.2 Results of Metals Analyses, Water, Salmon Site

\begin{tabular}{|c|c|c|c|c|c|}
\hline COMPOUND & RESULTS UNITS & $\begin{array}{l}\text { DATA } \\
\text { QUAL. }\end{array}$ & $\begin{array}{l}\text { DATA } \\
\text { VALID. }\end{array}$ & CRDL UNITS & DILUTION \\
\hline \multicolumn{6}{|l|}{ C-FB-001-W } \\
\hline Arsenic & $1.3 \mathrm{UG} \Omega$ & $u$ & Us & $10 \mathrm{UG} / \mathrm{L}$ & 1 \\
\hline Aluminum & 151 UG/L & B & UJ & 200 UG/L & 1 \\
\hline Antimony & 35.2 UGi & $u$ & UJ & 60 UGA & 1 \\
\hline Barium & $3.2 \mathrm{UG} / \mathrm{L}$ & B & Us & 200 UG/L & 1 \\
\hline Beryllium & 2.4 UG/ & $u$ & us & 5 UG/L & 1 \\
\hline Cadmium & $2.7 \mathrm{UG} \Omega$ & $u$ & Us & 5 UG/L & 1 \\
\hline Calcium & 127 UG/ & B & UJ & 5000 UG/L & 1 \\
\hline Chromium & 3 UG/L & $u$ & UJ & $10 \mathrm{UG} / \mathrm{L}$ & 1 \\
\hline Cobalt & $3.8 \mathrm{UG} / \mathrm{L}$ & $u$ & us & 50 UG/L & 1 \\
\hline Copper & $81.4 \mathrm{UG} / \mathrm{L}$ & & UJ & 25 UG/L & 1 \\
\hline Iron & $128 \mathrm{UG} /$ & & UJ & 100 UG/L & 1 \\
\hline Lead & 3.5 UGR & & J & 3 UG/L & 1 \\
\hline Magnesium & 32 UG/L & $B$ & J & 5000 UG/L & 1 \\
\hline Manganese & 5 UG/ & $B$ & US & 15 UG/L & 1 \\
\hline Mercury & 0.1 UG & $u$ & us & 0.2 UG/L & 1 \\
\hline Nickel & 17.8 UGR & $u$ & Us & 40 UG/L & 1 \\
\hline Potassium & 5020 UG/ & & $J$ & 5000 UG/L & 1 \\
\hline Selenium & 0.95 UG $\Omega$ & B & J & $5 \mathrm{UG} / \mathrm{L}$ & 1 \\
\hline Silver & 3.4 UG/ & B & J & $10 \mathrm{UG} / \mathrm{L}$ & 1 \\
\hline Sodium & 216 UG/ & $B$ & Us & 5000 UG/L & 1 \\
\hline Thallium & 0.9 UGR & $u$ & $R$ & 10 UG/L & 1 \\
\hline Vanadium & 13 UG/L & B & UJ & 50 UG/L & 1 \\
\hline Zinc & 19.6 UG/ & B & UJ & 20 UG/L & 1 \\
\hline Cyanide & 0.99 UG/ & B & us & 10 UG/L & 1 \\
\hline
\end{tabular}


APPENDIX F.2 Results of Metals Analyses, Water, Salmon Site

\begin{tabular}{|c|c|c|c|c|c|c|}
\hline COMPOUND & RESULTS & UNITS & $\begin{array}{l}\text { DATA } \\
\text { QUAL. }\end{array}$ & $\begin{array}{l}\text { DATA } \\
\text { VALID. }\end{array}$ & CRDL UNITS & DILUTIC \\
\hline \multicolumn{7}{|l|}{ C-FB-002-W } \\
\hline Arsenic & 1.3 & UG/ & $u$ & NP & $10 \mathrm{UG} / \mathrm{L}$ & 1 \\
\hline Aluminum & 22.5 & UG/L & $u$ & NP & 200 UG/L & 1 \\
\hline Antimony & 22.7 & UGR & $u$ & NP: & 60 UG/L & 1 \\
\hline Barium & 2.1 & UGR & $u$ & NP & 200 UG/L & 1 \\
\hline Beryllium & 1.7 & UGR & $u$ & NP & 5 UG/L & 1 \\
\hline Cadmium & 2.9 & UG/L & $u$ & NP & $5 \mathrm{UG} / \mathrm{L}$ & 1 \\
\hline Calcium & 245 & UG/ & B & NP & $5000 \mathrm{UG} / \mathrm{L}$ & 1 \\
\hline Chromium & 4.8 & UG $\Omega$ & $u$ & NP & $10 \mathrm{UG} / \mathrm{L}$ & 1 \\
\hline Cobalt & 5.6 & UG/L & $u$ & NP & $50 \mathrm{UG} / \mathrm{L}$ & 1 \\
\hline Copper & 4.4 & UGR & $u$ & NP & 25 UG/L & 1 \\
\hline Iron & 45.6 & UGR & $B$ & NP & 100 UG/L & 1 \\
\hline Lead & 0.7 & UGR & $u$ & NP & $3 U G / L$ & 1 \\
\hline Magnesium & 29.9 & UG $\Omega$ & B & NP & 5000 UG/L & 1 \\
\hline Manganese & 1.6 & UG/L & $u$ & NP & 15 UG/L & 1 \\
\hline Mercury & 0.1 & UG/L & $u$ & NP & 0.2 UG/L & 1 \\
\hline Nickel & 8.6 & UG/ & $u$ & NP & 40 UG/L & 1 \\
\hline Potassium & 694 & $U G / L$ & $u$ & NP & $5000 \mathrm{UG} / \mathrm{L}$ & 1 \\
\hline Selenium & & UG/L & $u$ & NP & 5 UG/L & 1 \\
\hline Silver & 4.1 & UG/L & u & NP & $10 \mathrm{UG} / \mathrm{L}$ & 1 \\
\hline Sodium & 555 & UG $\Omega$ & B & NP & 5000 UG/L & 1 \\
\hline Thallium & & UG/L & $u$ & NP & 10 UG/L & 1 \\
\hline Vanadium & 3.3 & UG $\Omega$ & $u$ & NP & 50 UG/L & 1 \\
\hline Zinc & 19.5 & UGR & $B$ & NP & $20 \mathrm{UG} / \mathrm{L}$ & 1 \\
\hline Cyanide & 0.99 & UG & B & $\mathrm{NP}$ & $10 \mathrm{UG} / \mathrm{L}$ & 1 \\
\hline
\end{tabular}


APPENDIX F.2 Results of Metals Analyses, Water, Salmon Site

\begin{tabular}{|c|c|c|c|c|c|}
\hline COMPOUND & RESULTS UNITS & $\begin{array}{l}\text { DATA } \\
\text { QUAL. }\end{array}$ & $\begin{array}{l}\text { DATA } \\
\text { VALID. }\end{array}$ & CRDL UNITS & DILUTION \\
\hline \multicolumn{6}{|l|}{ C-FB-003-W } \\
\hline Arsenic & $1.3 \mathrm{UG} /$ & $u$ & $\mathrm{NP}$ & $10 \mathrm{UG} / \mathrm{L}$ & 1 \\
\hline Aluminum & $22.5 \mathrm{UG} / \mathrm{L}$ & $u$ & $\mathrm{NP}$ & 200. UG/L & 1 \\
\hline Antimony & 22.7 UG $\Omega$ & $u$ & NP. & 60.UG几 & 1 \\
\hline Barium & $2.1 \mathrm{UG} \Omega$ & $u$ & NP & 200 UG/L & 1 \\
\hline Beryllium & 1.7 UG几 & $u$ & NP & 5 UG/L & 1 \\
\hline Cadmium & 2.9 UG & $u$ & NP & $5 \mathrm{UG} / \mathrm{L}$ & 1 \\
\hline Calcium & 330 UG & 8 & NP & 5000 UG/L & 1 \\
\hline Chromium & $4.8 \mathrm{UG} /$ & $u$ & $\mathrm{NP}$ & 10 UG/L & 1 \\
\hline Cobalt & 5.6 UG $\Omega$ & $u$ & NP & 50 UG/L & 1 \\
\hline Copper & $4.4 \mathrm{UG} \Omega$ & $u$ & $\mathrm{NP}$ & 25 UG/L & 1 \\
\hline Iron & 83.2 UG $\Omega$ & B & NP & $100 \mathrm{UG} / \mathrm{L}$ & 1 \\
\hline Lead & 0.7 UGR & $u$ & NP & 3 UG/L & 1 \\
\hline Magnesium & $28.6 \mathrm{UG} \Omega$ & $u$ & $\mathrm{NP}$ & 5000 UG/L & 1 \\
\hline Manganese & $2.4 \mathrm{UG} / \mathrm{L}$ & B & NP & 15 UG/L & 1 \\
\hline Mercury & $0.1 \mathrm{UGR}$ & u & NP & $0.2 \mathrm{UG} / \mathrm{L}$ & 1 \\
\hline Nickel & 8.6 UG & $u$ & $\mathrm{NP}$ & 40 UG/L & 1 \\
\hline Potassium & 694 UG/ & u & $\mathrm{NP}$ & 5000 UG/L & 1 \\
\hline Selenium & $1 \mathrm{UG} / \mathrm{L}$ & u & $\mathrm{NP}$ & 5 UG/L & 1 \\
\hline Silver & $4.1 \mathrm{UG} /$ & $u$ & $\mathrm{NP}$ & $10 \mathrm{UG} / \mathrm{L}$ & 1 \\
\hline Sodium & 810 UGR & B & NP & 5000 UG/L & 1 \\
\hline Thallium & $1 \mathrm{UG} /$ & u & $\mathrm{NP}$ & $10 \mathrm{UG} / \mathrm{L}$ & 1 \\
\hline Vanadium & $3.3 \mathrm{UG} / \mathrm{L}$ & $u$ & $N P$ & 50 UG/L & 1 \\
\hline Zinc & $15.8 \mathrm{UG} / \mathrm{L}$ & B & NP & $20 \mathrm{UG} / \mathrm{L}$ & 1 \\
\hline Cyanide & $3.4 \mathrm{UG} / \mathrm{L}$ & B & NP & 10 UG/L & 1 \\
\hline
\end{tabular}


APPENDIX F.2 Results of Metals Analyses, Water, Salmon Site

\begin{tabular}{lccccc}
\hline COMPOUND & RESULTS UNITS & QUAL. & DATA & \\
C-RB-001-W & & & & \\
Crsenic & $1.3 \mathrm{UG} / \mathrm{L}$ & $\mathrm{U}$ & $\mathrm{UJ}$ & $10 \mathrm{UG} / \mathrm{L}$ & 1 \\
Aluminum & $160 \mathrm{UG} / \mathrm{L}$ & $\mathrm{B}$ & $\mathrm{UJ}$ & $200 \mathrm{UG} / \mathrm{L}$ & 1 \\
Antimony & $35.2 \mathrm{UG} / \mathrm{L}$ & $\mathrm{U}$ & $\mathrm{UJ}$ & $60 \mathrm{UG} / \mathrm{L}$ & 1 \\
Barium & $3.5 \mathrm{UG} / \mathrm{L}$ & $\mathrm{B}$ & $\mathrm{UJ}$ & $200 \mathrm{UG} / \mathrm{L}$ & 1 \\
Beryllium & $2.4 \mathrm{UG} / \mathrm{L}$ & $\mathrm{U}$ & $\mathrm{UJ}$ & $5 \mathrm{UG} / \mathrm{L}$ & 1 \\
Cadmium & $2.7 \mathrm{UG} / \mathrm{L}$ & $\mathrm{U}$ & $\mathrm{UJ}$ & $5 \mathrm{UG} / \mathrm{L}$ & 1 \\
Calcium & $396 \mathrm{UG} / \mathrm{L}$ & $\mathrm{B}$ & $\mathrm{UJ}$ & $5000 \mathrm{UG} / \mathrm{L}$ & 1 \\
Chromium & $31.9 \mathrm{UG} / \mathrm{L}$ & & $\mathrm{J}$ & $10 \mathrm{UG} / \mathrm{L}$ & 1 \\
Cobalt & $3.8 \mathrm{UG} / \mathrm{L}$ & $\mathrm{U}$ & $\mathrm{UJ}$ & $50 \mathrm{UG} / \mathrm{L}$ & 1 \\
Copper & $75.8 \mathrm{UG} / \mathrm{L}$ & & $\mathrm{UJ}$ & $25 \mathrm{UG} / \mathrm{L}$ & 1 \\
Iron & $401 \mathrm{UG} / \mathrm{L}$ & & $\mathrm{J}$ & $100 \mathrm{UG} / \mathrm{L}$ & 1 \\
Lead & $0.7 \mathrm{UG} / \mathrm{L}$ & $\mathrm{U}$ & $\mathrm{UJ}$ & $3 \mathrm{UG} / \mathrm{L}$ & 1 \\
Magnesium & $102 \mathrm{UG} / \mathrm{L}$ & $\mathrm{B}$ & $\mathrm{J}$ & $5000 \mathrm{UG} / \mathrm{L}$ & 1 \\
Manganese & $6.8 \mathrm{UG} / \mathrm{L}$ & $\mathrm{B}$ & $\mathrm{UJ}$ & $15 \mathrm{UG} / \mathrm{L}$ & 1 \\
Mercury & $0.1 \mathrm{UG} / \mathrm{L}$ & $\mathrm{U}$ & $\mathrm{UJ}$ & $0.2 \mathrm{UG} / \mathrm{L}$ & 1 \\
Nickel & $17.8 \mathrm{UG} / \mathrm{L}$ & $\mathrm{U}$ & $\mathrm{UJ}$ & $40 \mathrm{UG} / \mathrm{L}$ & 1 \\
Potassium & $4130 \mathrm{UG} / \mathrm{L}$ & $\mathrm{B}$ & $\mathrm{UJ}$ & $5000 \mathrm{UG} / \mathrm{L}$ & 1 \\
Selenium & $0.8 \mathrm{UG} / \mathrm{L}$ & $\mathrm{U}$ & $\mathrm{R}$ & $5 \mathrm{UG} / \mathrm{L}$ & 1 \\
Silver & $3.4 \mathrm{UG} / \mathrm{L}$ & $\mathrm{U}$ & $\mathrm{UJ}$ & $10 \mathrm{UG} / \mathrm{L}$ & 1 \\
Sodium & $725 \mathrm{UG} / \mathrm{L}$ & $\mathrm{B}$ & $\mathrm{J}$ & $5000 \mathrm{UG} / \mathrm{L}$ & 1 \\
Thallium & $0.9 \mathrm{UG} / \mathrm{L}$ & $\mathrm{U}$ & $\mathrm{R}$ & $10 \mathrm{UG} / \mathrm{L}$ & 1 \\
Vanadium & $9 \mathrm{UG} / \mathrm{L}$ & $\mathrm{B}$ & $\mathrm{UJ}$ & $50 \mathrm{UG} / \mathrm{L}$ & 1 \\
Zinc & $122 \mathrm{UG} / \mathrm{L}$ & & $\mathrm{J}$ & $20 \mathrm{UG} / \mathrm{L}$ & 1 \\
Cyanide & $0.7 \mathrm{UG} / \mathrm{L}$ & $\mathrm{B}$ & $\mathrm{UJ}$ & $10 \mathrm{UG} / \mathrm{L}$ & 1 \\
& & & & & \\
& & & & &
\end{tabular}




\section{APPENDIX F.2 Results of Metals Analyses, Water, Salmon Site}

\begin{tabular}{|c|c|c|c|c|c|}
\hline COMPOUND & RESULTS UNITS & $\begin{array}{l}\text { DATA } \\
\text { QUAL. }\end{array}$ & $\begin{array}{l}\text { DATA } \\
\text { VALID. }\end{array}$ & CRDL UNITS & DILUTION \\
\hline \multicolumn{6}{|l|}{ C-RB-002-W } \\
\hline Arsenic & $1.3 \mathrm{UG} / \mathrm{L}$ & $u$ & NP & $10 \mathrm{UG} / \mathrm{L}$ & 1 \\
\hline Aluminum & 22.5 UG $\Omega$ & $u$ & NP & 200 UG/L & 1 \\
\hline Antimony & 22.7 UG & $u$ & $N P:=$ & 60 UG/L & 1 \\
\hline Barium & $2.1 \mathrm{UG} \Omega$ & $u$ & NP & $200 \mathrm{UG} / \mathrm{L}$ & 1 \\
\hline Beryllium & 1.7 UGR & $u$ & NP & 5 UG/L & 1 \\
\hline Cadmium & 2.9 UG/L & $u$ & NP & $5 \mathrm{UG} / \mathrm{L}$ & 1 \\
\hline Calcium & 339 UG/L & B & NP & 5000 UG/L & 1 \\
\hline Chromium & 13.9 UG/L & & NP & $10 \mathrm{UG} / \mathrm{L}$ & 1 \\
\hline Cobalt & $5.6 \mathrm{UG} / \mathrm{L}$ & $u$ & NP & $50 \mathrm{UG} / \mathrm{L}$ & 1 \\
\hline Copper & $4.4 \mathrm{UG} / \mathrm{L}$ & $u$ & NP & 25 UG/L & 1 \\
\hline Iron & 154 UG/ & & NP & 100 UG/L & 1 \\
\hline Lead & 0.7 UG $\Omega$ & $u$ & NP & $3 \mathrm{UG} / \mathrm{L}$ & 1 \\
\hline Magnesium & 61.9 UG/ & B & NP & 5000 UG/L & 1 \\
\hline Manganese & $2.4 \mathrm{UG} / \mathrm{L}$ & B & NP & 15 UG/L & 1 \\
\hline Mercury & $0.1 \mathrm{UG} / \mathrm{L}$ & $u$ & NP & 0.2 UG/L & 1 \\
\hline Nickel & 8.6 UG/ & $u$ & NP & $40 \mathrm{UG} / \mathrm{L}$ & 1 \\
\hline Potassium & 694 UG/L & u & NP & 5000 UG/L & 1 \\
\hline Selenium & $1 \mathrm{UG} \Omega$ & $u$ & NP & 5 UG/L & 1 \\
\hline Silver & $4.1 \mathrm{UG} / \mathrm{L}$ & $u$ & NP & $10 \mathrm{UG} / \mathrm{L}$ & 1 \\
\hline Sodium & 683 UG & B & NP & $5000 \mathrm{UG} / \mathrm{L}$ & 1 \\
\hline Thallium & 1 UGR & $u$ & NP & $10 \mathrm{UG} / \mathrm{L}$ & 1 \\
\hline Vanadium & 3.3 UG $\Omega$ & $u$ & NP & $50 \mathrm{UG} / \mathrm{L}$ & 1 \\
\hline Zinc & 29 UG几 & & NP & $20 \mathrm{UG} / \mathrm{L}$ & 1 \\
\hline Cyanide & 0.99 UG $\Omega$ & B & NP & $10 \mathrm{UG} / \mathrm{L}$ & 1 \\
\hline
\end{tabular}


APPENDIX F.2 Results of Metals Analyses, Water, Salmon Site

\begin{tabular}{|c|c|c|c|c|c|c|}
\hline COMPOUND & RESULTS & UNITS & $\begin{array}{l}\text { DATA } \\
\text { QUAL. }\end{array}$ & $\begin{array}{l}\text { DATA } \\
\text { VALID. }\end{array}$ & CRDL UNITS & DILUTION \\
\hline \multicolumn{7}{|l|}{ C-RB-003-W } \\
\hline Arsenic & 1.3 & UG & $u$ & NP & $10 \mathrm{UG} / \mathrm{L}$ & 1 \\
\hline Aluminum & 46.3 & UGR & B & NP & $200 \mathrm{UG} / \mathrm{L}$ & 1 \\
\hline Antimony & 22.7 & UGR & $u$ & NP & $60 \mathrm{UG} / \mathrm{L}$ & 1 \\
\hline Barium & 2.1 & UG $\Omega$ & $u$ & NP & $200 \mathrm{UG} / \mathrm{L}$ & 1 \\
\hline Beryllium & 1.7 & UGR & $u$ & NP & 5 UG/L & 1 \\
\hline Cadmium & 2.9 & UG/L & $u$ & NP & $5 \mathrm{UG} / \mathrm{L}$ & 1 \\
\hline Calcium & 366 & $U G \Omega$ & B & NP & 5000 UG/L & 1 \\
\hline Chromium & 4.8 & UG/ & $u$ & NP & $10 \mathrm{UG} / \mathrm{L}$ & 1 \\
\hline Cobalt & 5.6 & UG & $u$ & NP & $50 \mathrm{UG} / \mathrm{L}$ & 1 \\
\hline Copper & 6.8 & UG & B & NP & 25 UG/L & 1 \\
\hline Iron & 151 & UG $\Omega$ & & NP & $100 \mathrm{UG} / \mathrm{L}$ & 1 \\
\hline Lead & 0.7 & UG $\Omega$ & $u$ & NP & $3 U G / L$ & 1 \\
\hline Magnesium & 68.6 & UG $\Omega$ & B & NP & 5000 UG/L & 1 \\
\hline Manganese & & UG/L & B & NP & 15 UG/L & 1 \\
\hline Mercury & 0.1 & UG/ & $u$ & NP & 0.2 UG/L & 1 \\
\hline Nickel & 8.6 & UG几 & $u$ & NP & 40 UG/L & 1 \\
\hline Potassium & 694 & UG/L & $u$ & NP & 5000 UG/L & 1 \\
\hline Selenium & & $U G \Omega$ & $u$ & NP & 5 UG/L & 1 \\
\hline Silver & 4.1 & UG/L & u & NP & $10 \mathrm{UG} / \mathrm{L}$ & 1 \\
\hline Sodium & 703 & UG $\Omega$ & 8 & NP & 5000 UG/L & 1 \\
\hline Thallium & & UG $\Omega$ & $u$ & NP & $10 \mathrm{UG} / \mathrm{L}$ & 1 \\
\hline Vanadium & 3.3 & $U G \Omega$ & $u$ & NP & 50 UG/L & 1 \\
\hline Zinc & 36.7 & UG $\Omega$ & & $N P$ & $20 \mathrm{UG} / \mathrm{L}$ & 1 \\
\hline Cyanide & 3.3 & UG $\Omega$ & B & NP & $10 \mathrm{UG} / \mathrm{L}$ & 1 \\
\hline
\end{tabular}




\section{Appendix G.1 \\ Results of Gamma Spectroscopy Analyses, Soils, Salmon Site}




\begin{tabular}{|c|c|c|c|c|c|c|c|}
\hline SAMPLE & PARAMETER & RESULT & UNITS & $\begin{array}{l}\text { DATA } \\
\text { VALID. }\end{array}$ & $\begin{array}{l}2 \text { SIGMA } \\
\text { ERROR }(+/-)\end{array}$ & UNITS & MDA UNITS \\
\hline C5-1-S & Cesium 137 & ND & $\mathrm{PCl} / \mathrm{G}$ & $\mathrm{NP}$ & & $P C I / G$ & $0.19 \mathrm{PCl} / \mathrm{G}$ \\
\hline C5-1-S & Potassium 40 & $6.05^{\circ}$ & PCI/G & $\mathrm{NP}$ & 1.36 & $P C I / G$ & $2.40 \mathrm{PCl} / \mathrm{G}$ \\
\hline C5-1-S & Lead 210. & 1.66: & PCUG & $N P$ & 0.98 & PCUG & $1.30 \mathrm{PCl} / \mathrm{G}$ \\
\hline C5-1-S & Lead 212 & 0.92 & PCUG & NP & 0.11 & PCI/G: & 0.16 PCI/G \\
\hline C5-1-S & Radium-226 & 0.51 & PCI/G & NP. & 0.10 & PCUG: & $0.25 \mathrm{PCI} / \mathrm{G}$ \\
\hline C5-1-S & Thorium-228 & ND & $\mathrm{PCI} / \mathrm{G}$ & NP & & $\mathrm{PCl} / \mathrm{G}$ & $3.91 \mathrm{PCI} / \mathrm{G}$ \\
\hline C5-1-S & Thorium 234 & ND & $\mathrm{PCl} / \mathrm{G}$ & NP & & PCI/G & $1.35 \mathrm{PCl} / \mathrm{G}$ \\
\hline C5-2-S & Cesium 137 & ND & $P C I / G$ & NP & & PCI/G & $0.25 \mathrm{PCl} / \mathrm{G}$ \\
\hline C5-2-S & Lead 212 & 0.37 & PCVG & NP & 0.08 & PCVG & $0.17 \mathrm{PCI} / \mathrm{G}$ \\
\hline C5-2-S & Thorium 234 & ND & $\mathrm{PCl} / \mathrm{G}$ & NP & & PCI/G & $2.11 \mathrm{PCl} / \mathrm{G}$ \\
\hline C5-2-S-DUP & Cesium 137 & ND & $\mathrm{PCl} / \mathrm{G}$ & NP & & $\mathrm{PCl} / \mathrm{G}$ & $0.16 \mathrm{PCl} / \mathrm{G}$ \\
\hline C5-2-S-DUP & Potassium 40 & ND & $\mathrm{PCI} / \mathrm{G}$ & NP & & PCI/G & 2.37 PCI/G \\
\hline C5-2-S-DUP & Lead 212 & 0.29 & $\mathrm{PCl} / \mathrm{G}$ & NP & 0.08 & PCI/G & $0.14 \mathrm{PCI} / \mathrm{G}$ \\
\hline C5-3-S & Cesium 137 & ND & PCl/G & NP & & PCI/G & $0.19 \mathrm{PCI} / \mathrm{G}$ \\
\hline C5-3-S & Potassium 40 & ND & $\mathrm{PCI} / \mathrm{G}$ & NP & & $\mathrm{PCl} / \mathrm{G}$ & $3.25 \mathrm{PCl} / \mathrm{G}$ \\
\hline C5-3-S & Thallium 208 & ND & $\mathrm{PCl} / \mathrm{G}$ & NP & & PCI/G & $0.15 \mathrm{PCl} / \mathrm{G}$ \\
\hline C5-3-S & Lead 212 & $0: 40^{\circ}$ & PCUG & NP: & 0.09 & PCUG: & $0.18 \mathrm{PCI} / \mathrm{G}$. \\
\hline C5-3-S & Radium-226 & 0.50 & PCUG & NP. & 0.10 & PCUG & $0.22 \mathrm{PCI} / \mathrm{G}$ \\
\hline C3-4-S & Cesium 137 & ND & PCI/G & NP & & $\mathrm{PCl} / \mathrm{G}$ & 0.16 PCUG \\
\hline C3-4-S: & Potassium $40^{\circ}$ & 16.80 & PCIG & NP. & $3: 00$ & PCIG & 3.30 PCI/G \\
\hline C3-4-S & : Thallium 208 & 0.40 & PCIG & NP & 0.08 & PCWIG & $0.13 \mathrm{PCI} / \mathrm{G}$ \\
\hline C3-4-S & : Lead 210 & 1.78 & PCIG & NP & 0.61 & PCIG & 1:43 PCI/G: \\
\hline C3-4-S & - Lead 212 & 1.59 & PCVG & NP & 0.16 & PCUG: & $0.20 \mathrm{PCI} / \mathrm{G}$ \\
\hline C3-4-S & Radium-226 & 1.12 & PCI/G & NP: & 0.14 & PCI/G. & $0.24 \mathrm{PCl} / \mathrm{G}=$ \\
\hline C3-4-S & Radium-228 & 1.63 & PCI/G & $\mathrm{NP}$ & 0.28 & PCUG & $0.47 \mathrm{PCl} / \mathrm{G}$ \\
\hline C3-5-S & Cesium 137 & ND & $\mathrm{PCl} / \mathrm{G}$ & NP & & $P C I / G$ & $0.30 \mathrm{PCl} / \mathrm{G}$ \\
\hline C3-5-S & Potassium 40 & ND & $\mathrm{PCI} / \mathrm{G}$ & NP & & PCI/G & $7.58 \mathrm{PCl} / \mathrm{G}$ \\
\hline C3-5-S & Thallium 208 & 0.45 & PCI/G & NP $=$ & 0.11 & PCIIG & $0.18 \mathrm{PCI} / \mathrm{G}$ \\
\hline C3-5-S & Lead 212 & 1.27 & PCVG & NP: & 0.21 & PCIG & $0.24 \mathrm{PCI} / \mathrm{G}$ \\
\hline C3-5-S. & Radium-226 & 1.86 & PCIG & $N P$ & 0.17 & PCUG: & $0.34 \mathrm{PCI} / \mathrm{G}$ \\
\hline C3-6-S & Cesium 137 & ND & PCI/G & NP & & $\mathrm{PCI} / \mathrm{G}$ & $0.28 \mathrm{PCl} / \mathrm{G}$ \\
\hline C3-6-S & Thallium 208 & 0.44 & PCVG & NP & 0.09 & PCIG & $0.17 \mathrm{PCl} / \mathrm{G}$ \\
\hline C3-6-S & Lead 212 & 1.10 & PCIG & $\mathbf{N P}$ & 0.19 & PCIG & $0.22 \mathrm{PCI} / \mathrm{G}$ \\
\hline C3:6-S & Radium-226 & 0.75 & PCIG & $\mathrm{NP}$ & 0.13 & PCI/G & $0.35 \mathrm{PCI} / \mathrm{G}$ \\
\hline C3-7-s & Cesium 137 & ND & $P C I / G$ & NP & & PCI/G & $0.16 \mathrm{PCI} / \mathrm{G}$ \\
\hline C3-7-S & Potassium 40 & 17.50 & PCIG: & NP & 1.60 & PCI/G & $7.80 \mathrm{PCI} / \mathrm{G}$ \\
\hline C3-7-S & Thallium 208 & 0.50 & PCUG & $\mathrm{NP}$ & 0.09 & PCVG & 0.14 PCI/G \\
\hline C3-7-S & Lead 212 & 1.58 & PCIG & $N P$ & 0.12 & PCIG & $0.21 \mathrm{PCl} / \mathrm{G}$ \\
\hline C3-7-S, & Radium-228 & 1.46 & PCIG & $\mathrm{NP}$ & 0.20 & PCVG & $0.39 \mathrm{PCI} / \mathrm{G}$ \\
\hline C3-7-S & Thorium 234 & 1.41 & $\mathrm{PCl} / \mathrm{G}$ & NP & 0.45 & PCIG & $1.23 \mathrm{PCl} / \mathrm{G}$ \\
\hline
\end{tabular}




\begin{tabular}{|c|c|c|c|c|c|c|c|}
\hline SAMPLE & PARAMETER & RESULT & UNITS & $\begin{array}{l}\text { DATA } \\
\text { VALID. } \\
\end{array}$ & $\begin{array}{l}2 \text { SIGMA } \\
\text { ERROR }(+/-)\end{array}$ & UNITS & MDA UNITS \\
\hline C3-8-S & Cesium 137 & ND & $\mathrm{PCl} / \mathrm{G}$ & $\mathrm{NP}$ & & $\mathrm{PCl} / \mathrm{G}$ & $0.17 \mathrm{PCl} / \mathrm{G}$ \\
\hline C3-8-S & Pötassium 40 & 8.23 & PCI/G & NP & $1.17 \mathrm{~F}$ & $\mathrm{PCI/G}$ & $2.11 \mathrm{PCl} / \mathrm{G}$ \\
\hline C3-8-S & Thallium 208 & 0.57 & PCUG & NP & $0.08 \mathrm{~F}$ & PCU/G & $0.13 \mathrm{PCl} / \mathrm{G}$ \\
\hline C3-8-S & Lead 212 & 1.54 & $\mathrm{PCI} / \mathrm{G}$ & NP & $0.14 F$ & PCI/G & $0.22 \mathrm{PCl} / \mathrm{G}$ \\
\hline C3-8-S & Radium-226 & 1.66 & PCI/G & NP & $0.13 F$ & PCI/G & $0.20 \mathrm{PCl} / \mathrm{G}$ \\
\hline C3-8-5 & Radium-228 & 1.51 & PCI/G & NP: & $0.20 \mathrm{~F}$ & PCI/G & $0.43 \mathrm{PCl} / \mathrm{G}$ \\
\hline C3-8-S & Thorium 234 & ND & $\mathrm{PCl} / \mathrm{G}$ & NP & & $\mathrm{PCl} / \mathrm{G}$ & $1.39 \mathrm{PCl} / \mathrm{G}$ \\
\hline C3-9-S & Cesium 137 & ND & $\mathrm{PCl} / \mathrm{G}$ & NP & & PCl/G & $0.26 \mathrm{PCl} / \mathrm{G}$ \\
\hline C3-9-S & Potassium 40 & ND & $\mathrm{PCI} / \mathrm{G}$ & NP & & $\mathrm{PCl} / \mathrm{G}$ & $3.15 \mathrm{PCl} / \mathrm{G}$ \\
\hline C3-9-5 & Lead 212 & 0.43 & PCIIG & NP: & 0.15 & PCI/G & $0.20 \mathrm{PCI} / \mathrm{G}$ \\
\hline C3-9-S & Bismuth 214 & ND & $\mathrm{PCI} / \mathrm{G}$ & NP & & PCI/G & $0.34 \mathrm{PCl} / \mathrm{G}$ \\
\hline C4-10-S & Cesium 137 & ND & $\mathrm{PCl} / \mathrm{G}$ & NP & & $\mathrm{PCl} / \mathrm{G}$ & $0.18 \mathrm{PCl} / \mathrm{G}$ \\
\hline C4-10-S & Potassium 40 & 6.41 & PCI/G & NP & 1.14 & PCI/G & $2.30 \mathrm{PCI} / \mathrm{G}$ \\
\hline$C 4-10-5$ & Thallium 208 & 0.34 & PCI/G & NP & 0.09 & PCl/G & $0.14 \mathrm{PCI} / \mathrm{G}$ \\
\hline C4-10-S & Lead 212 & 1.62 & $P C I / G$ & NP & 0.14 & PCI/G & $0.18 \mathrm{PCl} / \mathrm{G}$ \\
\hline C4-10-S & Radium-226 & 1.36 & PCI/G & NP & 0.11 & PCI/G & $0.25 \mathrm{PCI} / \mathrm{G}$ \\
\hline C4-10-S & Thorium 234 & ND & $\mathrm{PCl} / \mathrm{G}$ & NP & & $\mathrm{PCl} / \mathrm{G}$ & $1.41 \mathrm{PCl} / \mathrm{G}$ \\
\hline C4-11-S & Cesium 137 & ND & $\mathrm{PCl} / \mathrm{G}$ & NP & & $\mathrm{PCl} / \mathrm{G}$ & $0.16 \mathrm{PCl} / \mathrm{G}$ \\
\hline C4-11-S & Potassium 40 & 8.04 & PCIVG & $N P:$ & 2.28 & PCI/G & $3.40 \mathrm{PCl} / \mathrm{G}$ \\
\hline C4-11-S & Thallium 208 & 0.48 & POUG & NP: & 0.08 & PCI/G & $0.12 \mathrm{PCI} / \mathrm{G}$ \\
\hline$C 4-11-s$ & Lead 210 & 2.84 & PCUG & NP. & $1.16 f$ & PCUG & $1.58 \mathrm{PCl} / \mathrm{G}$ \\
\hline C4-11-S & Lead 212 & 1.19 & PCUG & NP. & 0.13 & PCUG & $0.20 \mathrm{PCl} / \mathrm{G}$ \\
\hline C4-11-S & Radium 224 & ND & $\mathrm{PCl} / \mathrm{G}$ & NP & & PCI/G & $1.87 \mathrm{PCl} / \mathrm{G}$ \\
\hline C4-11-S & Radium-226 & 2.25 & PCV/G & NP & 0.20 & PCI/G & $0.25 \mathrm{PCl} / \mathrm{G}$ \\
\hline C4-11-S & Thorium 234 & 2.56 & $P C I / G$ & NP & 0.48 & PCI/G & $1.48 \mathrm{PCl} / \mathrm{G}$ \\
\hline C4-12-S & Cesium 137 & ND & $P C l / G$ & NP & & PCI/G & $0.26 \mathrm{PCI} / \mathrm{G}$ \\
\hline C4-13-S & Cesium 137 & ND & $\mathrm{PCl} / \mathrm{G}$ & NP & & $\mathrm{PCI} / \mathrm{G}$ & $0.18 \mathrm{PCI} / \mathrm{G}$ \\
\hline$C 4-13-5$ & Potassium 40 & 14.40 & $\mathrm{PCI} / \mathrm{G}$ & NP & 2.20 & PCUG & $2.40 \mathrm{PCl} / \mathrm{G}$ \\
\hline C4-13-S & Thallium 208 & 0.42 & PCVG & NP. & 0.11 & PCUG & $0.13 \mathrm{PCl} / \mathrm{G}$ \\
\hline C4-13-S & Lead 212 & 1.61 & PCUG & NP & 0.14 & PCIVG. & $0.16 \mathrm{PCI} / \mathrm{G}$ \\
\hline C4-13-S & Radium-226 & 0.95 & PCIVG & NP: & 0.13 & PCI/G & $0.23 \mathrm{PCl} / \mathrm{G}$ \\
\hline C1-14-S & Cesium 137 & ND & PCI/G & NP & & PCI/G & $0.15 \mathrm{PCl} / \mathrm{G}$ \\
\hline C1-14-S & Lead 212 & 0.18 & PCI/G & NP & 0.06 & PCI/G & $0.18 \mathrm{PCl} / \mathrm{G}$ \\
\hline C1-14-S & Bismuth 214 & 0.36 & $P C I / G$ & NP & 0.12 & PCI/G & $0.24 \mathrm{PCl} / \mathrm{G}$ \\
\hline C1-14-S & Thorium 234 & ND & $P C l / G$ & NP & & $\mathrm{PCl} / \mathrm{G}$ & $1.19 \mathrm{PCl} / \mathrm{G}$ \\
\hline C1-15-S & Cesium 137 & ND & $\mathrm{PCl} / \mathrm{G}$ & NP & & $\mathrm{PCl} / \mathrm{G}$ & $0.25 \mathrm{PCl} / \mathrm{G}$ \\
\hline C1-16-S & Cesium 137 & ND & $\mathrm{PCI} / \mathrm{G}$ & NP & & $\mathrm{PCl} / \mathrm{G}$ & $0.16 \mathrm{PCl} / \mathrm{G}$ \\
\hline C.1-16-s & Potassium 40 & 6.59 & PCIIG & $N P$ & 123 & PCl/G. & $1.33 \mathrm{PCl} / \mathrm{G}$ \\
\hline C1-16-S & Lead 212 & ND & PCI/G & NP & & PCI/G & $0.17 \mathrm{PCl} / \mathrm{G}$ \\
\hline
\end{tabular}




\begin{tabular}{|c|c|c|c|c|c|c|c|}
\hline SAMPLE & PARAMETER & RESULT & UNITS & $\begin{array}{l}\text { DATA } \\
\text { VALID. }\end{array}$ & $\begin{array}{l}2 \text { SIGMA } \\
\text { ERROR }(+/-)\end{array}$ & UNITS & MDA UNITS \\
\hline C1-17-s & Cesium 137 & ND & $\mathrm{PCl} / \mathrm{G}$ & $\mathrm{NP}$ & & $\mathrm{PCl} / \mathrm{G}$ & $0.22 \mathrm{PCl} / \mathrm{G}$ \\
\hline C1-17-S-DUP & Cesium 137 & ND & $P C I / G$ & NP & & $P C I / G$ & $0.10 \mathrm{PCI} / \mathrm{G}$ \\
\hline C1-17-S-DUP & Lead 212 & 0.29 & $\mathrm{PCl} / \mathrm{G}$ & NP & 0.10 & $P C I / G$ & $0.17 \mathrm{PCl} / \mathrm{G}$ \\
\hline C1-17-S-DUP & Bismuth 214 & 0.48 & PCI/G & NP & 0.22 & $P C I / G$ & $0.20 \mathrm{PCl} / \mathrm{G}$ \\
\hline C1-17-S-DUP & Radium-226 & 0.53 & PCCI/G & $N P$ : & 0.14 & PCUG & $0.20 \mathrm{PCl} / \mathrm{G}$ \\
\hline C1-17-S-DUP & Thorium 234 & ND & PCl/G & NP & & PCI/G & $1.12 \mathrm{PCl} / \mathrm{G}$ \\
\hline C1-18-S & Cesium 137 & ND & $\mathrm{PCl} / \mathrm{G}$ & NP & & $\mathrm{PCl} / \mathrm{G}$ & $0.20 \mathrm{PCl} / \mathrm{G}$ \\
\hline C1-18-S & Lead 212 & 0.24 & PCI/G & NP & 0.10 & $\mathrm{PCl} / \mathrm{G}$ & $0.15 \mathrm{PCl} / \mathrm{G}$ \\
\hline C1-18-S & Bismuth 214 & ND & $\mathrm{PCI} / \mathrm{G}$ & NP & & $\mathrm{PCl} / \mathrm{G}$ & $0.31 \mathrm{PCl} / \mathrm{G}$ \\
\hline C1-18-S & Radium-226 & 0.32 & $\mathrm{PCl} / \mathrm{G}$ & NP & 0.17 & $\mathrm{PCl} / \mathrm{G}$ & $0.23 \mathrm{PCl} / \mathrm{G}$ \\
\hline$C 1-18-5$ & Thorium 234 & ND & PCl/G & NP & & PCI/G & $1.35 \mathrm{PCl} / \mathrm{G}$ \\
\hline C2-19-S & Cesium 137 & ND & PCI/G & NP & & PCI/G & $0.17 \mathrm{PCVG}$ \\
\hline C2-19-S & Potassium 40 & 8.50 & PCVG & $\mathbf{N P}$ & 1.39 & PCI/G & $2.10 \mathrm{PCI} / \mathrm{G}$ \\
\hline C2-19-S & Thallium 208 & 0.34 & $P C I / G$ & NP & 0.07 & $\mathrm{PCl} / \mathrm{G}$ & $0.12 \mathrm{PCl} / \mathrm{G}$ \\
\hline C2-19-S & Lead 210 & 1.73 & $P C I / G$ & NP & 0.68 & PCUG & $1.47 \mathrm{PCl} / \mathrm{G}$ \\
\hline C2-19-S. & Lead 212 . & 1.46 & PGI/G & NP & 0.10 & PCUG: & $0.19 \mathrm{PCI} / \mathrm{G}$ \\
\hline C2-19-S & Radium-226: & 1.64 & PCIUG & $\mathrm{NP}=$ & 0.14 & PCI/G. & $0.23 \mathrm{PCI} / \mathrm{G}$ \\
\hline C2-19-S & Thorium 234 & 2.25 & PCI/G & NP & 0.50 & PCI/G & 1.43 PCI/G \\
\hline C2-20-S & Cesium 137 & ND & $P C V / G$ & NP & & $P C I / G$ & $0.15 \mathrm{PCI} / \mathrm{G}$ \\
\hline C2-20-S & Potassium 40 & 9.16 & PCIIG & NP & 2.13 & PCIG & $3.31 \mathrm{PCl} / \mathrm{G}$ \\
\hline C2-2O-S & Thallium 208 & 0.38 & PCVG & $\mathbf{N P}$ & 0.09 & PCIG & $0.15 . P C I / G$ \\
\hline $\mathrm{C} 2-20-\mathrm{S}$ & Lead 210 & 2.38 & PCVG & $N P$ & 0.71 & PGUG & $1.50 \mathrm{PCI} / \mathrm{G}$ \\
\hline C2-20-S & Lead 212 & 1.70 & PCUG & NP & 0.18 & PCI/G: & $0.20 \mathrm{PCI} / \mathrm{G}$ \\
\hline $\mathrm{C} 2-20-\mathrm{S}$ & Rädium-226 & 1.28 & PCI/G & $\mathrm{NP}$ & 0.12 & PCUG & $0.23 \mathrm{PCI} / \mathrm{G}$ \\
\hline C2-20-S & Radium-228 & 1.22 & PCI/G: & $N P$ & 0.37 & PCIG & $0.52 \mathrm{PCl} / \mathrm{G}$ \\
\hline C2-20-S & Thorium 234 & 2.41 & PCI/G & NP & 0.84 & PCI/G & $1.34 \mathrm{PCl} / \mathrm{G}$ \\
\hline C2-21-S & Cesium 137 & ND & PCI/G & NP & & PCI/G & $0.17 \mathrm{PCI} / \mathrm{G}$ \\
\hline C2-21-S & Potassium 40 & ND & PCI/G & $N P$ & & PCI/G & $2.20 \mathrm{PCI} / \mathrm{G}$ \\
\hline C2-21-S & Lead 212 & 0.35 & PCIG & NP & 0.07 & PCIG & $0.15 \mathrm{PCl} / \mathrm{G}$ \\
\hline C2-21-S & Thorium 234 & ND & PCI/G & NP & & PCI/G & $1.27 \mathrm{PCI} / \mathrm{G}$ \\
\hline C2-21-S & Cesium 137 & ND & $\mathrm{PCI} / \mathrm{G}$ & NP & & $\mathrm{PCl} / \mathrm{G}$ & $0.14 \mathrm{PCI} / \mathrm{G}$ \\
\hline C2-21-S & Lead 212 & 0.50 & $P C I / G$ & NP & 0.10 & $P C I / G$ & $0.18 \mathrm{PCl} / \mathrm{G}$ \\
\hline C2-21-S & Radium-226 & 0.48 & PCI/G & NP & 0.07 & PCI/G & $0.16 \mathrm{PCl} / \mathrm{G}$ \\
\hline C2-22-S & Cesium 137 & ND & $P C I / G$ & NP & & PCI/G & $0.18 \mathrm{PCl} / \mathrm{G}$ \\
\hline C2-22-S & Bismuth 214 & 0.33 & $\mathrm{PCI} / \mathrm{G}$ & NP & 0.16 & PCI/G & 0.33 PCI/G \\
\hline C2-23-S & Cesium 137 & ND & $\mathrm{PCl} / \mathrm{G}$ & NP & & $\mathrm{PCl} / \mathrm{G}$ & $0.16 \mathrm{PCl} / \mathrm{G}$ \\
\hline C2-23-S & Potassium 40 & ND & $P C I / G$ & $\mathrm{NP}$ & & PCI/G & $2.45 \mathrm{PCI} / \mathrm{G}$ \\
\hline C2-23-S & Thallium 208 & 0.15 & PCIG & NP & 0.05 & PCLG & $0.10 \mathrm{PCI} / \mathrm{G}$ \\
\hline C2-23-S & Lead 210 & ND & PCI/G & NP & & PCUG & $1.45 \mathrm{PCI} / \mathrm{G}$ \\
\hline$c 2-23-5$ & Lead 212 & 0.48 & PCIV & NP. & 0.07 & PCUG & $0.16 \mathrm{PCl} / \mathrm{G}$ \\
\hline C2-23-S & Radium-226 & 0.83 & PCUG & $\mathrm{NP}$ & 0.09 & PCUG & $0.23 \mathrm{PCI} / \mathrm{G}$ \\
\hline C2-23-S & Thorium 234 & ND & $\mathrm{PCl} / \mathrm{G}$ & NP & & PCI/G & $1.31 \mathrm{PCl} / \mathrm{G}$ \\
\hline
\end{tabular}


APPENDIX G.1 Results of Gamma Spectroscopy Analyses, Soils, Salmon Site

\begin{tabular}{|c|c|c|c|c|c|c|c|}
\hline SAMPLE & PARAMETER & RESULT & UNITS & $\begin{array}{l}\text { DATA } \\
\text { VALID. }\end{array}$ & $\begin{array}{l}2 \text { SIGMA } \\
\text { ERROR }(t /-)\end{array}$ & UNITS & MDA UNITS \\
\hline C2-24-S & Cesium 137 & ND & $\mathrm{PCl} / \mathrm{G}$ & NP & & $\mathrm{PCl} / \mathrm{G}$ & $0.12 \mathrm{PCl} / \mathrm{G}$ \\
\hline C2-24-S & Lead 212 & 0.58 & PCI/G & NP & 0.09 & PCI/G & $0.15 \mathrm{PCl} / \mathrm{G}$ \\
\hline$C 2-24-5$ & Radium-226 & 0.54 & PCUG & NP. & 0.09 & PCVG & $0.26 \mathrm{PCl} / \mathrm{G}$ \\
\hline C2-24-S & Thorium 234 & ND & $\mathrm{PCl} / \mathrm{G}$ & NP & & PCI/G & $1.25 \mathrm{PCl} / \mathrm{G}$ \\
\hline C2-25-S & Cesium 137 & ND & $\mathrm{PCI} / \mathrm{G}$ & NP & & PCI/G & $0.18 \mathrm{PCI} / \mathrm{G}$ \\
\hline C2-25-S & Potassium 40 & 2.51 & $\mathrm{PCl} / \mathrm{G}$ & NP & 0.58 & PCI/G & $2.33 \mathrm{PCl} / \mathrm{G}$ \\
\hline C2-25-S & Thallium 208 & 0.30 & $\mathrm{PCI} / \mathrm{G}$ & $\mathrm{NP}$ & 0.08 & PCI/G & $0.12 \mathrm{PCl} / \mathrm{G}$ \\
\hline$C 2-25-S$ & Lead 212 & 0.88 & PCI/G & NP & 0.09 & PCUG & $0.17 \mathrm{PCl} / \mathrm{G}$ \\
\hline C2-25-S & Bismuth 214 & 0.34 & $\mathrm{PCI} / \mathrm{G}$ & NP & 0.25 & PCI/G & $0.25 \mathrm{PCl} / \mathrm{G}$ \\
\hline C2-25-S & Radium 224 & 1.71 & PCUG & NP. & 1.12 & $\mathrm{PCl} / \mathrm{G}$ & $1.58 \mathrm{PCl} / \mathrm{G}$ \\
\hline$C 2-25-S$ & Radium-226 & 1.02 & PCI/G & NP & 0.13 & PCI/G & $0.21 \mathrm{PCI} / \mathrm{G}$ \\
\hline C2-25A-S & Cesium 137 & ND & PCI/G & NP & & PCI/G & $0.28 \mathrm{PCl} / \mathrm{G}$ \\
\hline C2-25A-S & Potassium 40 & 13.50 & $P C l / G$ & NP & 1.80 & PCI/G & $3.10 \mathrm{PCl} / \mathrm{G}$ \\
\hline C2-25A-S & Thallium 208 & 0.36 & $\mathrm{PCI} / \mathrm{G}$ & NP & 0.08 & PCI/G & $0.19 \mathrm{PCl} / \mathrm{G}$ \\
\hline C2-25A-S & Lead 212 & 1.29 & PCVG & NP & 0.19 & PCI/G & $0.25 \mathrm{PCl} / \mathrm{G}$ \\
\hline C2-25A-S & Bismuth 214 & 0.70 & $\mathrm{PCl} / \mathrm{G}$ & NP & 0.17 & PCI/G & $0.40 \mathrm{PCl} / \mathrm{G}$ \\
\hline$C 2-25 A-S$ & Radium-228 & 1.49 & PCUG & NP & 0.25 & PCI/G & $0.61 \mathrm{PCI} / \mathrm{G}$ \\
\hline C2-26-S & Cesium 137 & ND & $\mathrm{PCl} / \mathrm{G}$ & NP & & PCI/G & $0.14 \mathrm{PCl} / \mathrm{G}$ \\
\hline C2-26-S & Lead 212 & 0.42 & PCUG & NP. & 0.11 & PCIVG & $0.14 \mathrm{PCl} / \mathrm{G}$ \\
\hline$C 2-26-S$ & Thorium 234 & ND & $\mathrm{PCl} / \mathrm{G}$ & NP & & PCI/G & $1.26 \mathrm{PCl} / \mathrm{G}$ \\
\hline C2-27-S & Cesium 137 & ND & $P C l / G$ & NP & & PCI/G & $0.16 \mathrm{PCI} / \mathrm{G}$ \\
\hline C2-27-S & Potassium 40 & 12.40 & PCVG & $\mathrm{NP}$ & 1.40 & PCUG & $6.70 \mathrm{PCl} / \mathrm{G}$ \\
\hline $\mathrm{C} 2-27-\mathrm{S}$ & Thallium 208 & 0.52 & PCIG & NP & 0.09 & PCVG & $0.12 \mathrm{PCl} / \mathrm{G}$ \\
\hline C2-27-S & Lead 210 & 1.80 & PCUG & NP & 0.7 .4 & PCUG & $1.33 \mathrm{PCl} / \mathrm{G}$ \\
\hline$C 2-27-S$ & Lead 212 & 1.94 & PCI/G & NP & 0.12 & PCIVG & $0.16 \mathrm{PCl} / \mathrm{G}$ \\
\hline$C 2-27-5$ & Radium-226 & 0.96 & PCI/G & NP & 0.12 & PCIVG & $0.20 \mathrm{PCl} / \mathrm{G}$ \\
\hline C2-27-S & Radium-228 & 1.73 & PCI/G & NP & 0.28 & PCUG & $0.46 \mathrm{PCl} / \mathrm{G}$ \\
\hline C2-27-S & Thorium-228 & ND & $\mathrm{PCI} / \mathrm{G}$ & NP & & $\mathrm{PCl} / \mathrm{G}$ & $4.49 \mathrm{PCl} / \mathrm{G}$ \\
\hline C2-27-S & Thorium 234 & 2.34 & $\mathrm{PCl} / \mathrm{G}$ & NP & 0.60 & $P C l / G$ & $1.26 \mathrm{PCl} / \mathrm{G}$ \\
\hline
\end{tabular}


Appendix G.2

Results of Gamma Spectroscopy Analyses, Water, Salmon Site 


\begin{tabular}{|c|c|c|c|c|c|c|c|}
\hline SAMPLE & PARAMETER & RESULT & UNITS & $\begin{array}{l}\text { DATA } \\
\text { VALID. } \\
\end{array}$ & $\begin{array}{l}2 \text { SIGMA } \\
\text { ERROR }(+/-)\end{array}$ & UNITS & MDA UNITS \\
\hline C5-1-W & Cesium 137 & ND & $\mathrm{PCI} /$ & NP & & PCI/L & $9.64 \mathrm{PCI} /$ \\
\hline C5-1-W-DUP & Cesium 137 & ND & $\mathrm{PCI} / \mathrm{L}$ & NP & & PCI/ & $9.87 \mathrm{PCl} / \mathrm{L}$ \\
\hline C5-1-W-DUP & Potassium 40 & 452 & PCIn & NP & & $\mathrm{PCI} / \mathrm{L}$ & $248 \mathrm{PCI} / \mathrm{L}$ \\
\hline C3-8-W & Cesium 137 & ND & PCIn & NP & & $\mathrm{PCl} / \mathrm{L}$ & $11.6 \mathrm{PCI} /$ \\
\hline C3-8-W & Potassium 40 & 461 & $\mathrm{PCI} / \mathrm{L}$ & NP & & $\mathrm{PCI} /$ & $162 \mathrm{PCI} / \mathrm{L}$ \\
\hline C3-8-W & Lead 212 & 29.6 & $\mathrm{PCl} / \mathrm{L}$ & NP & & $\mathrm{PCI} / \mathrm{L}$ & $13.3 \mathrm{PCI} / \mathrm{L}$ \\
\hline C-3-9-W & Cesium 137 & ND & $\mathrm{PCI} / \mathrm{L}$ & NP & & PCIR & $20.5 \mathrm{PCl} / \mathrm{L}$ \\
\hline$C-3-9-W$ & Potassium 40 & 1930 & PCIR & NP & 199 & $\mathrm{PCI} / \mathrm{L}$ & $160 \mathrm{PCI} / \mathrm{L}$ \\
\hline$c-3-9-w$ & Lead 212 & 87 & $\mathrm{PCI} / \mathrm{L}$ & NP & 10.9 & $\mathrm{PCl} / \mathrm{L}$ & $22.5 \mathrm{PCl} / \mathrm{L}$ \\
\hline C-3-9-W & Radium-226 & 80.8 & $\mathrm{PCI} / \mathrm{L}$ & NP & 13.5 & PCIR & $24.1 \mathrm{PCI} / \mathrm{L}$ \\
\hline$C 4-11-W$ & Cesium 137 & ND & $\mathrm{PCI} / \mathrm{L}$ & NP & & $\mathrm{PCI} / \mathrm{L}$ & $9.66 \mathrm{PCl} / \Omega$ \\
\hline$C 4-11-W$ & Potassium 40 & ND & $\mathrm{PCI} / \mathrm{L}$ & NP & & PCIL & $196 \mathrm{PCI}$ \\
\hline$C 4-11-W$ & Lead 212 & 23.9 & PCIL & NP & & $\mathrm{PCI} / \mathrm{L}$ & $14.2 \mathrm{PCl} / \mathrm{L}$ \\
\hline$C 4-11-W$ & Radium-226 & 57.6 & PCI/L & NP & 6.6 & $\mathrm{PCI} / \mathrm{L}$ & $16.3 \mathrm{PCl} / \mathrm{L}$ \\
\hline C4-13-W & Cesium 137 & ND & PCI/ & NP & & $\mathrm{PCl} / \mathrm{L}$ & $9.86 \mathrm{PCI} / \mathrm{L}$ \\
\hline C4-13-W & Potassium 40 & 564 & $\mathrm{PCI} / \mathrm{L}$ & NP & & $\mathrm{PCI} / \mathrm{L}$ & $46 \mathrm{PCI} / \mathrm{L}$ \\
\hline C4-13-W & Lead 212 & ND & PCI/ & NP & & PCI & $13.5 \mathrm{PCI}$ \\
\hline C4-13-W & Radium-226 & ND & $\mathrm{PCI} / \mathrm{L}$ & NP & & PCI/ & $17.5 \mathrm{PCl} / \mathrm{L}$ \\
\hline C1-14-W & Cesium 137 & ND & $\mathrm{PCI} / \mathrm{L}$ & - & & PCI/L & $9.52 \mathrm{PCI} / \mathrm{L}$ \\
\hline C1-14-W & Potassium 40 & ND & $\mathrm{PCI} / \mathrm{L}$ & -- & & PCI/L & 175 PCIn \\
\hline$C 1-14-W$ & Thallium 208 & 29.6 & $\mathrm{PCI} / \mathrm{L}$ & - & & $3 \mathrm{PCI} / \mathrm{L}$ & $10.9 \mathrm{PCI} /$ \\
\hline$C 1-14-W$ & Lead 212 & 73.7 & $\mathrm{PCl} / \mathrm{L}$ & - & 14.7 & $7 \mathrm{PCI} / \mathrm{L}$ & $16.8 \mathrm{PCl} /$ \\
\hline C1-14-W & Bismuth 214 & 15.8 & PCIR & -- & 14.1 & $\mathrm{PCI}$ & $14.6 \mathrm{PCl} / \mathrm{L}$ \\
\hline C1-14-W & Radium-226 & 69.9 & PCI/L & - & 11.1 & $1 \mathrm{PCI}$ & $14.6 \mathrm{PCI} / \mathrm{L}$ \\
\hline C1-14-W & Radium-228 & 96.3 & $\mathrm{PCI} /$ & - & 14.5 & $5 \mathrm{PCI} /$ & $34.2 \mathrm{PCI}$ \\
\hline$C 1-15-W$ & Cesium 137 & ND & PCIn & - & & $\mathrm{PCI} / \mathrm{L}$ & $9.62 \mathrm{PCl} / \mathrm{L}$ \\
\hline C1-15-W & Potassium 40 & 533 & PCIn & J & & $4 \mathrm{PCI} /$ & $76 \mathrm{PCl} \Omega$ \\
\hline$C 1-15-W$ & Thallium 208 & ND & PCI/L & - & & $\mathrm{PCI} / \mathrm{L}$ & $10.0 \mathrm{PCl} / \mathrm{L}$ \\
\hline$C 1-15-W$ & Lead 212 & 39.6 & PCIn & $J$ & 8.3 & $3 \mathrm{PCl} / \mathrm{L}$ & $14.1 \mathrm{PCI} / \mathrm{L}$ \\
\hline$C 1-15-W$ & Radium-226 & 19.4 & PCIn & $\mathrm{J}$ & 4.8 & $3 \mathrm{PCI} / \mathrm{L}$ & $17.9 \mathrm{PCl} / \mathrm{L}$ \\
\hline C1-15-W & Radium-228 & 54.8 & PCI/ & J & 18.5 & $5 \mathrm{PCl} / \mathrm{L}$ & $36.9 \mathrm{PCl} / \mathrm{L}$ \\
\hline
\end{tabular}


APPENDIX G.2 Results of Gamma Spectroscopy Analyses, Water, Salmon Site

\begin{tabular}{|c|c|c|c|c|c|c|c|c|}
\hline SAMPLE & PARAMETER & RESULT & UNITS & $\begin{array}{l}\text { DATA } \\
\text { VALID. }\end{array}$ & $\begin{array}{l}2 \text { SIGMA } \\
\text { ERROR }(+/-)\end{array}$ & UNITS & MDA & UNITS \\
\hline$C 1-16-W$ & Cesium 137 & ND & $\mathrm{PCI}$ & UJ & & PCIR & 16.6 & $\mathrm{PCI} / \mathrm{L}$ \\
\hline C1-16-W & Potassium 40 & ND & $\mathrm{PCI} /$ & UJ & & $\mathrm{PCI} / \mathrm{L}$ & 450 & PCI/L \\
\hline C1-16-W & Lead 212 & ND & $\mathrm{PCI} / \mathrm{L}$ & UJ & & $\mathrm{PCI} /$ & 20.2 & PCIR \\
\hline C1-16-W & Bismuth 214 & ND & PCI/L & us & & $\mathrm{PCI} / \mathrm{L}$ & 27.3 & PCIR \\
\hline C1-16-W & Thorium 234 & ND & PCI/ & UJ & & PCI/ & 153 & PCIR \\
\hline C1-17-W & Cesium 137 & ND & PCIR & $\ldots$ & & $\mathrm{PCI} / \mathrm{L}$ & 14.0 & $\mathrm{PCI} / \mathrm{L}$ \\
\hline C1-17-W & Lead 212 & 14.6 & $\mathrm{PCI} / \mathrm{L}$ & J & 5.5 & PCI/L & 14.3 & PCI/L \\
\hline C1-17-W & Bismuth 214 & ND & PCIR & - & & PCI/ & 21.0 & PCIR \\
\hline$C 1-17-W$ & Radium-226 & ND & PCI/L & -- & & PCIR & 20.2 & $\mathrm{PCI} / \mathrm{L}$ \\
\hline$C 1-18-W$ & Cesium 137 & ND & PCI/ & $\ldots$ & & PCIn & 13.2 & $\mathrm{PCI} / \mathrm{L}$ \\
\hline C1-18-W -DUP & Cesium 137 & ND & $\mathrm{PCI} / \mathrm{L}$ & - & & $\mathrm{PCI} / \mathrm{L}$ & 13.4 & PCIR \\
\hline$C 2-19-W$ & Cesium 137 & ND & PCI/ & NP & & PCI/L & 7.34 & PCI/L \\
\hline C2-19-W & Radium-226 & 45.5 & PCIR & $\mathrm{NP}$ & 5.8 & PCI/L & 15.5 & $\mathrm{PCI} / \mathrm{L}$ \\
\hline C2-19-W & Thorium 234 & 176 & $\mathrm{PCl} / \mathrm{L}$ & NP & & PCIR. & 126 & $\mathrm{PCI} / \mathrm{L}$ \\
\hline C2-19-W-DUP & Cesium 137 & ND & $\mathrm{PCI} / \mathrm{L}$ & $N P$ & & $\mathrm{PCI} / \mathrm{L}$ & 18.5 & $\mathrm{PCI} / \mathrm{L}$ \\
\hline C2-19-W-DUP & Radium-226 & 54.2 & PCIR & NP & 7.6 & $\mathrm{PCI}$ & 19.5 & $\mathrm{PCI} / \mathrm{L}$ \\
\hline$C 2-27-W$ & Cesium 137 & ND & PCIR & NP & & $\mathrm{PCI} / \Omega$ & 9.93 & $\mathrm{PCI} / \mathrm{L}$ \\
\hline$C 2-27-W$ & Potassium 40 & 387 & $\mathrm{PCI} / \mathrm{L}$ & NP & 82 & $\mathrm{PCI} / \mathrm{L}$ & 170 & PCI/L \\
\hline$C 2-27-W$ & Thallium 208 & 17.7 & $\mathrm{PCI} / \mathrm{L}$ & NP & 7.1 & $\mathrm{PCI}$ & 9.3 & $\mathrm{PCI} / \mathrm{L}$ \\
\hline$C 2-27-W$ & Lead 212 & 89 & PCIL & NP & 10.1 & PCI/ & 16.8 & $\mathrm{PCI} / \mathrm{L}$ \\
\hline$C 2-27-W$ & Radium-226 & 89.5 & $\mathrm{PCI} / \mathrm{L}$ & NP & 8.4 & $\mathrm{PCI} / \mathrm{L}$ & 19.7 & PCI/L \\
\hline$C 2-27-W$ & Thorium 234 & ND & PCI & NP & & PCI/L & 155 & $\mathrm{PCI} / \mathrm{L}$ \\
\hline C-FB-001-W & Cesium 137 & ND & PCI/ & -. & & $\mathrm{PCI} / \mathrm{L}$ & 14.4 & PCI/L \\
\hline C-FB-002-W & Cesium 137 & ND & $\mathrm{PCI} / \mathrm{L}$ & NP & & PCIR & 18.4 & $\mathrm{PCI} / \mathrm{L}$ \\
\hline C-FB-003-W & Cesium 137 & ND & $\mathrm{PCI} /$ & NP & & $\mathrm{PCl} / \mathrm{L}$ & 8.86 & $\mathrm{PCl} / \mathrm{L}$ \\
\hline C-RB-001-W & Cesium 137 & ND & PCIL & - & & $\mathrm{PCl} / \mathrm{L}$ & 14.9 & $\mathrm{PCI} / \mathrm{L}$ \\
\hline C-RB-002-W & Cesium 137 & ND & PCIR & NP & & $\mathrm{PCI} / \mathrm{L}$ & 14.3 & $\mathrm{PCI} / \mathrm{L}$ \\
\hline C-RB-003-W & Cesium 137 & ND & $\mathrm{PCI} / 2$ & NP & & $\mathrm{PCI} / \mathrm{L}$ & 18.6 & $\mathrm{PCI} / \mathrm{L}$ \\
\hline C-RB-003-W & Potassium 40 & 253 & PCIR & NP & 44 & $P C I / L$ & 78 & PCI/L \\
\hline
\end{tabular}




\section{Appendix $\mathrm{H} .1$ \\ Results of Gross Alpha and Beta Analyses, Soils, Salmon Site}


APPENDIX H.1 Results of Gross Alpha and Beta Analyses, Soils, Salmon Site

\begin{tabular}{|c|c|c|c|c|c|c|}
\hline SAMPLE & & RESULT UNITS & $\begin{array}{l}\text { DATA } \\
\text { VALID. }\end{array}$ & $\begin{array}{l}2 \text { SIGMA } \\
\text { ERROR (+l-; UNITS }\end{array}$ & MDA UNITS & DILUTION \\
\hline C5-1-S & Alpha (total) & $11.4 \mathrm{PCl} / \mathrm{G}$ & $N P$ & $2.6 \mathrm{PCl} / \mathrm{G}$ & 2.8 PCI/G & 1 \\
\hline C5-1-S & Beta (total) & 13.1 PCI/G & NP & 1.8 PCI/G & 1.8.PCI/G & 1 \\
\hline C5-2-S & Alpha (total) & $5.36 \mathrm{PCl} / \mathrm{G}$ & NP & $1.91 \mathrm{PCI} / \mathrm{G}$ & $2.5 \mathrm{PCl} / \mathrm{G}$ & 1 \\
\hline C5-2-S & Beta (total) & $11.1 \mathrm{PCl} / \mathrm{G}$ & NP & $1.7 \mathrm{PCl} / \mathrm{G}$ & $1.8 \mathrm{PCl} / \mathrm{G}$ & 1 \\
\hline C5-2-S-DUP & Alpha (total) & $6.4 \mathrm{PCl} / \mathrm{G}$ & NP & $2.09 \mathrm{PCl} / \mathrm{G}$ & $2.63 \mathrm{PCl} / \mathrm{G}$ & 1 \\
\hline C5-2-S-DUP & Beta (total) & $9.35 \mathrm{PCl} / \mathrm{G}$ & NP & $1.59 \mathrm{PCl} / \mathrm{G}$ & 1.92 PCI/G & 1 \\
\hline C5-3-S & Alpha (total) & $8.55 \mathrm{PCl} / \mathrm{G}$ & NP: & $2.35 \mathrm{PCUG}$ & 2.79 PCUG & 1 \\
\hline$C 5-3-5$ & Beta (total) & 14.5 PCI/G & NP: & 1.9 PCUG & $1.8 \mathrm{PCI} / \mathrm{G}$ & 1 \\
\hline C3-4-S & Alpha (total) & 12.9 PCI/G & $N P$ & 2.9 PCUG & 3 PCI/G: & 1 \\
\hline C3-4-S & Beta (total). & $28: 9$ PCI/G & NP: : & 3.3.PCUG & 1.8. PCl/G & 1 \\
\hline C3-5-S & Alpha (total) & $12.3 \mathrm{PCl} / \mathrm{G}$ & NP & 2.6 PCI/G & $2.5 \mathrm{PCI} / \mathrm{G}$ & 1 \\
\hline C3-5-S & Beta (total) & 16.6 PCI/G & NP & 2.PCUG & $1.5 \mathrm{PCl} / \mathrm{G}$ & 1 \\
\hline C3-6-S & Alpha (total) & $12 \mathrm{PCl} / \mathrm{G}$ & NP: & 2.7 PCUG & $2.8 \mathrm{PCI} / \mathrm{G}$ & $1=$ \\
\hline C3-6-S & Beta:(total) & $17: 2$ PCI/G. & NP & 2.2 PCIG & 1.9.PCI/G & 1 \\
\hline C3-7-S & Alpha (total) & $16.7 \mathrm{PCI} / \mathrm{G}$ & $\mathrm{NP}$ & $3.6 \mathrm{PCIG}$ & $3.9 \mathrm{PCI} / \mathrm{G}$ & 1 \\
\hline C3-7-S & Beta (total) & 33,4 PCI/G & $\mathrm{NP}$ & 3.7 PCUG & 2PCI/G & $1:$ \\
\hline C3-8-S & Alpha (total) & $15.6 \mathrm{PCl} / \mathrm{G}$ & NP. & 2.9 PCIG & $2.3 \mathrm{PCI} / \mathrm{G}$ & 1 \\
\hline C3-8-S & Beta (total) & $23.2 \mathrm{PCl} / \mathrm{G}$ & NP. & 2.7 PCUG: & $1.6 \mathrm{PCl} / \mathrm{G}$ & 1 \\
\hline C3-9-S & Alpha (total) & $6.15 \mathrm{PCl} / \mathrm{G}$ & NP & $2.12 \mathrm{PCI} / \mathrm{G}$ & $2.74 \mathrm{PCl} / \mathrm{G}$ & 1 \\
\hline C3-9-S & Beta (total) & 15.1 PCI/G & NP & 1.9 PCI/G & $1.6 \mathrm{PCI} / \mathrm{G}$ & 1 \\
\hline C4-10-S & Alpha (total) & $11.7 \mathrm{PCl} / \mathrm{G}$ & $N P$ & 2.8.PCUG & $3 \mathrm{PCI} / \mathrm{G}$ & 1 \\
\hline$C 4-10-5$ & Beta (total) & $18.1 \mathrm{PCl} / \mathrm{G}$ & NP: & 2.3 PCVG & $1.7 \mathrm{PCl} / \mathrm{G}$ & 1 \\
\hline C4-11-s & Alpha (total) & 35.9 pCI/G & NP & 5.7 PCUG & 5.2 PCI/G & 1 \\
\hline$C 4-11-S$ & Beta (total) & $34.4 \mathrm{PCI} / \mathrm{G}$ & $\mathrm{NP}$ & 3.9 PCUG & 2.1 PCI/G & 1 \\
\hline C4-12-S & Alpha (total) & $4.69 \mathrm{PCl} / \mathrm{G}$ & NP & $2.48 \mathrm{PCI} / \mathrm{G}$ & $3.78 \mathrm{PCl} / \mathrm{G}$ & 1 \\
\hline C4-12-S & Beta (total) & $8.52 \mathrm{PCI} / \mathrm{G}$ & NP & $1.76 \mathrm{PCl} / \mathrm{G}$ & 2.37 PCU/G & 1 \\
\hline C4-13-s & Alpha (total) & $19.4 \mathrm{PCI} / \mathrm{G}$ & $\mathrm{NP}$ & 3.7 PCUG & $3.4 \mathrm{PCI} / \mathrm{G}$ & 1 \\
\hline$c 4-13-s$ & Beta (total) & 25.9 PCI/G & $\mathrm{NP}$ & 3PCUG & $1.9 \mathrm{PCI} / \mathrm{G}$ & 1 \\
\hline C1-14-S & Alpha (total) & 3.27 PCVG & NP & $1.69 \mathrm{PCl} / \mathrm{G}$ & $2.49 \mathrm{PCl} / \mathrm{G}$ & 1 \\
\hline C1-14-s & Beta (total) & $7.96 \mathrm{PCI} / \mathrm{G}$ & NP & $1.35 \mathrm{PCl} / \mathrm{G}$ & 1.59 PCI/G & 1 \\
\hline C1-15-s & Alpha (total) & $3.59 \mathrm{PCl} / \mathrm{G}$ & NP & $1.79 \mathrm{PCI} / \mathrm{G}$ & $2.61 \mathrm{PCl} / \mathrm{G}$ & 1 \\
\hline
\end{tabular}


APPENDIX H.1 Results of Gross Alpha and Beta Analyses, Soils, Salmon Site

\begin{tabular}{|c|c|c|c|c|c|c|}
\hline SAMPLE & & RESULT UNITS & $\begin{array}{l}\text { DATA } \\
\text { VALID. }\end{array}$ & $\begin{array}{l}2 \text { SIGMA } \\
\text { ERROR (+/:-: UNITS }\end{array}$ & MDA UNITS & DILUTION \\
\hline C1-15-s & Beta (total) & $9.26 \mathrm{PCl} / \mathrm{G}$ & $\overline{N P}$ & $1.44 \mathrm{PCI} / \mathrm{G}$ & $1.56 \mathrm{PCl} / \mathrm{G}$ & 1 \\
\hline C1-16-S & Alpha (total) & $3.53 \mathrm{PCI} / \mathrm{G}$ & NP & $1.72 \mathrm{PCl} / \mathrm{G}$ & $2.46 \mathrm{PCl} / \mathrm{G}$ & 1 \\
\hline C1-16-S & Beta (total) & $8.46 \mathrm{PCl} / \mathrm{G}$ & NP & $1.43 \mathrm{PCl} / \mathrm{G}$ & $1.66 \mathrm{PCl} / \mathrm{G}$ & 1 \\
\hline C1-17-s & Alpha (total) & $2.09 \mathrm{PCl} / \mathrm{G}$ & NP & $1.75 \mathrm{PCI} / \mathrm{G}$ & $2.8 \mathrm{PCl} / \mathrm{G}$ & 1 \\
\hline C1-17-S & Beta (total) & $8.38 \mathrm{PCl} / \mathrm{G}$ & NP & $1.5 \mathrm{PCI} / \mathrm{G}$ & $1.86 \mathrm{PCl} / \mathrm{G}$ & 1 \\
\hline C1-17-S-DUP & Alpha (total) & $1.73 \mathrm{PCI} / \mathrm{G}$ & NP & $1.25 \mathrm{PCI} / \mathrm{G}$ & $1.96 \mathrm{PCl} / \mathrm{G}$ & 1 \\
\hline C1-17-S-DUP & Beta (total) & $2.63 \mathrm{PCl} / \mathrm{G}$ & NP & 0.87 PCI/G & $1.32 \mathrm{PCl} / \mathrm{G}$ & 1 \\
\hline C1-18-S & Alpha (total) & $5.41 \mathrm{PCl} / \mathrm{G}$ & NP & $2.27 \mathrm{PCI} / \mathrm{G}$ & $3.22 \mathrm{PCI} / \mathrm{G}$ & 1 \\
\hline C1-18-S & Beta (total) & $8.73 \mathrm{PCl} / \mathrm{G}$ & NP & $1.5 \mathrm{PCI} / \mathrm{G}$ & $1.79 \mathrm{PCl} / \mathrm{G}$ & 1 \\
\hline $\mathrm{C} 2-19-\mathrm{S}$ & Alpha (total) & 19.2 PCI/G & NP & 4 PCVG & $3.8 \mathrm{PCl} / \mathrm{G}$ & 1 \\
\hline C2-19-S & Beta (total) & 22.7 PCI/G & NP & $2.8 \mathrm{PCI} / \mathrm{G}$ & $1.9 \mathrm{PCl} / \mathrm{G}$ & 1 \\
\hline C2-20-S & Alpha (total) & $11.6 \mathrm{PCI} / \mathrm{G}$ & NP & $3.9 \mathrm{PC} / \mathrm{G}$ & $5.4 \mathrm{PCl} / \mathrm{G}$ & 1 \\
\hline $\mathrm{C} 2-20-\mathrm{S}$ & Beta (total) & $23.4 \mathrm{PCI} / \mathrm{G}$ & NP: & 2.9 PCUG & $2 \mathrm{PCl} / \mathrm{G}$ & 1 \\
\hline C2-21-S & Alpha (total) & $5.97 \mathrm{PCl} / \mathrm{G}$ & NP & $2.76 \mathrm{PCl} / \mathrm{G}$ & $3.99 \mathrm{PCI} / \mathrm{G}$ & 1 \\
\hline C2-21-S & Beta (total) & $7.05 \mathrm{PCl} / \mathrm{G}$ & NP & $1.62 \mathrm{PCl} / \mathrm{G}$ & $2.21 \mathrm{PCl} / \mathrm{G}$ & 1 \\
\hline C2-21-S-DUP & Alpha (total) & $9.81 \mathrm{PCl} / \mathrm{G}$ & NP & $2.87 \mathrm{PCVG}$ & $3.39 \mathrm{PCI} / \mathrm{G}$ & 1 \\
\hline C2-21-S-DUP & Beta (total) & $7.72 \mathrm{PCl} / \mathrm{G}$ & NP & $1.53 \mathrm{PCl} / \mathrm{G}$ & $1.94 \mathrm{PCl} / \mathrm{G}$ & 1 \\
\hline C2-22-S & Alpha (total) & $7.61 \mathrm{PCI} / \mathrm{G}$ & NP & $2.44 \mathrm{PCl} / \mathrm{G}$ & $2.95 \mathrm{PCI} / \mathrm{G}$ & 1 \\
\hline C2-22-S & Beta (total) & $10.2 \mathrm{PCI} / \mathrm{G}$ & NP & $1.7 \mathrm{PCl} / \mathrm{G}$ & $1.8 \mathrm{PCl} / \mathrm{G}$ & 1 \\
\hline C2-23-S & Alpha (total) & $9.43 \mathrm{PCl} / \mathrm{G}$ & NP & $2.27 \mathrm{PCVG}$ & $2.27 \mathrm{PCl} / \mathrm{G}$ & 1 \\
\hline C2-23-S & Beta (total) & $9.61 \mathrm{PCl} / \mathrm{G}$ & NP & $1.48 \mathrm{PCl} / \mathrm{G}$ & $1.58 \mathrm{PCl} / \mathrm{G}$ & 1 \\
\hline C2-24-S & Alpha (total) & $8.33 \mathrm{PCl} / \mathrm{G}$ & NP & $2.17 \mathrm{PCl} / \mathrm{G}$ & $2.31 \mathrm{PCl} / \mathrm{G}$ & 1 \\
\hline C2-24-S & Beta (total) & $12.9 \mathrm{PCI} / \mathrm{G}$ & NP. & $1.8 \mathrm{PCl} / \mathrm{G}$ & $1.6 \mathrm{PCl} / \mathrm{G}$ & 1 \\
\hline C2-25-S & Alpha (total) & 8.2 PCl/G & NP & $2.28 \mathrm{PCI} / \mathrm{G}$ & $2.66 \mathrm{PCl} / \mathrm{G}$ & 1 \\
\hline C2-25-S & Beta (total) & $11.4 \mathrm{PCl} / \mathrm{G}$ & NP & $1.6 \mathrm{PCl} / \mathrm{G}$ & 1.6 PCI/G & 1 \\
\hline C2-25A-S & Alpha (total) & $14.6 \mathrm{PCI} / \mathrm{G}$ & NP & 2.8 PCVG & $2.4 \mathrm{PCl} / \mathrm{G}$ & 1 \\
\hline C2-25A-S & Beta (total) & $276 \mathrm{PCl} / \mathrm{G}$ & NP: & 3.1. PCI/G & $1.5 \mathrm{PCI} / \mathrm{G}$ & 1 \\
\hline C2-26-S & Alpha (total) & $5.61 \mathrm{PCl} / \mathrm{G}$ & NP & $1.85 \mathrm{PCl} / \mathrm{G}$ & $2.19 \mathrm{PCl} / \mathrm{G}$ & 1 \\
\hline C2-26-S & Beta (total) & $7.37 \mathrm{PCI} / \mathrm{G}$ & NP & $1.37 \mathrm{PCl} / \mathrm{G}$ & $1.72 \mathrm{PCl} / \mathrm{G}$ & 1 \\
\hline $\mathrm{C} 2-27-\mathrm{S}$ & Alpha (total) & $18.9 \mathrm{PCl} / \mathrm{G}$ & NP. & 4. PCUG: & $3.1 \mathrm{PCI} / \mathrm{G}$ & 1 \\
\hline C2-27-S & Böta (total) & $28.8 \mathrm{PCl} / \mathrm{G}$ & NP: & 3.4 PCIG. & 2.1 $\mathrm{PCl} / \mathrm{G}$ & 1 \\
\hline
\end{tabular}




\section{Appendix H.2 \\ Results of Gross Alpha and Beta Analyses, Water, Salmon Site}




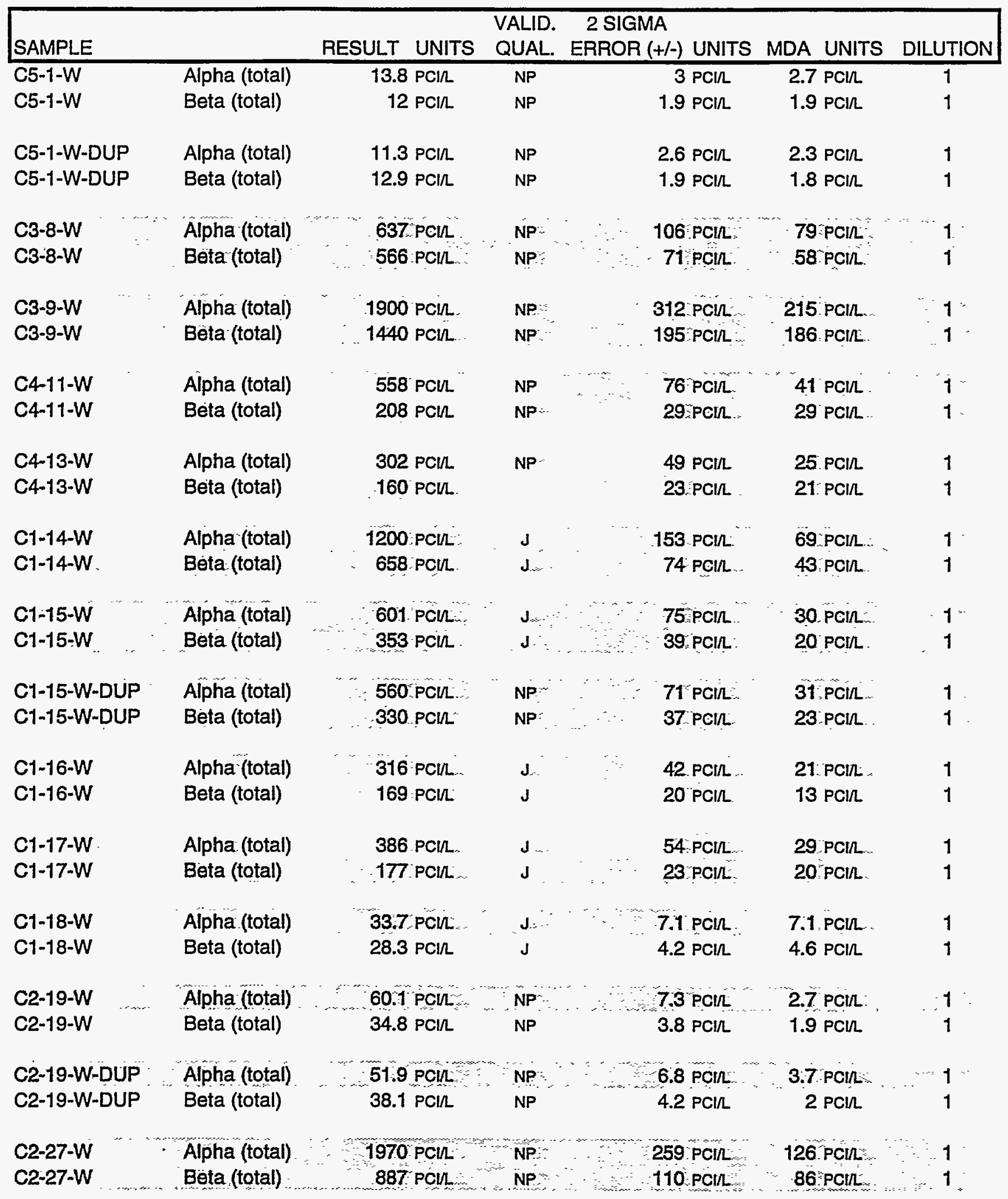


APPENDIX H.2 - Results of Gross Alpha and Beta Analyses, Water, Salmon Site

\begin{tabular}{|c|c|c|c|c|c|c|c|c|}
\hline SAMPLE & & RESULT & UNITS & $\begin{array}{l}\text { VALID. } \\
\text { QUAL. }\end{array}$ & $\begin{array}{c}2 \text { SIGMA } \\
\text { ERROR }(+/-)\end{array}$ & UNITS & MDA UNITS & DILUTION \\
\hline C-FB-001-W & Alpha (total) & 0.26 & $\mathrm{PCI} / \mathrm{L}$ & $J$ & 0.16 & $P C I / L$ & $0.24 \mathrm{PCl} / \mathrm{L}$ & 1 \\
\hline C-FB-001-W & Beta (total) & 0.01 & $\mathrm{PCI} / \mathrm{L}$ & $u$ & 0.43 & $\mathrm{PCI} / \mathrm{L}$ & $0.73 \mathrm{PCl} / \mathrm{L}$ & 1 \\
\hline C-FB-002-W & Alpha (total) & -0.13 & $\mathrm{PCI} / \mathrm{L}$ & NP & 1.2 & $\mathrm{PCl} / \mathrm{L}$ & $2.31 \mathrm{PCI} / \mathrm{L}$ & 1 \\
\hline C-FB-002-W & Beta (total) & 0.07 & $\mathrm{PCI} / \mathrm{L}$ & NP & 1.12 & PCI/ & $1.93 \mathrm{PCI} / \mathrm{L}$ & 1 \\
\hline C-FB-003-W & Alpha (total) & -0.03 & $\mathrm{PCI} / \mathrm{L}$ & NP & 0.15 & $\mathrm{PCI} / \mathrm{L}$ & $0.27 \mathrm{PCI} /$ & 1 \\
\hline C-FB-003-W & Beta (total) & -0.2 & $\mathrm{PCI} / \mathrm{L}$ & NP & 0.45 & $\mathrm{PCl} / \mathrm{L}$ & $0.78 \mathrm{PCI} / \mathrm{L}$ & 1 \\
\hline C-RB-001-W & Alpha (total) & -0.07 & PCI/ & $u$ & 0.14 & $\mathrm{PCI} / \mathrm{L}$ & $0.27 \mathrm{PCl} / \mathrm{L}$ & 1 \\
\hline C-RB-001-W & Beta (total) & 0.11 & $\mathrm{PCI}$ & $u$ & 0.4 & $\mathrm{PCI} / \mathrm{L}$ & $0.68 \mathrm{PCI}$ & 1 \\
\hline C-RB-002-W & Alpha (total) & -0.41 & $\mathrm{PCI} / \mathrm{L}$ & NP & 1.27 & $\mathrm{PCI} / \mathrm{L}$ & $2.51 \mathrm{PCI} /$ & 1 \\
\hline C-RB-002-W & Beta (total) & 0.19 & PCI/L & NP & 1.05 & $\mathrm{PCI}$ & $1.8 \mathrm{PCI} /$ & 1 \\
\hline C-RB-003-W & Alpha (total) & 0.23 & $\mathrm{PCl} / \mathrm{L}$ & NP & 0.18 & $\mathrm{PCI} / \mathrm{L}$ & $0.29 \mathrm{PCI} / \mathrm{L}$ & 1 \\
\hline C-RB-003-W & Beta (total) & 0.16 & $\mathrm{PCI}$ & NP & 0.43 & PCI/L & $0.72 \mathrm{PCI} / \mathrm{L}$ & 1 \\
\hline
\end{tabular}




\section{Appendix I}

Results of Tritium Analyses, Water, Salmon Site 
APPENDIX I - Results of Tritium Analyses, Water, Salmon Site

\begin{tabular}{|c|c|c|c|c|}
\hline SAMPLE & PARAMETER & RESULT UNITS & $\begin{array}{ll}2 \text { SIGMA } & \text { VALID. } \\
\text { ERROR (+/-) } & \text { QUAL. }\end{array}$ & MDA UNITS \\
\hline C5-1-W & Hydrogen 3 (Tritium) & $-91 \mathrm{PCI} / \mathrm{L}$ & 182 & $324 \mathrm{PCI} / \mathrm{L}$ \\
\hline C5-1-W-DUP & Hydrogen 3 (Tritium) & $-247 \mathrm{PCI} / \mathrm{L}$ & 177 & $324 \mathrm{PCI} / \mathrm{L}$ \\
\hline C3-8-W & Hydrogen 3 (Tritium) & $1540 \mathrm{PCI} / \mathrm{L}$ & 218 & $199 \mathrm{PCl} / \mathrm{L}$ \\
\hline C3-9-W & Hydrogen 3 (Tritium) & $66 \mathrm{PCl} / \mathrm{L}$ & 114 & $192 \mathrm{PCI} /$ \\
\hline$C 4-11-W$ & Hydrogen 3 (Tritium) & $-4 \mathrm{PCl} / 2$ & 115 & 199 PCI/ \\
\hline C4-13-W & Hydrogen 3 (Tritium) & $233 \mathrm{PCI} / \mathrm{L}$ & 185 & $301 \mathrm{PCI}$ \\
\hline C1-14-W & Hydrogen 3 (Tritium) & $13 \mathrm{PCl}$ & $114196 U$ & $196 \mathrm{PCI} / \mathrm{L}$ \\
\hline C1-15-W & Hydrogen 3 (Tritium) & $13 \mathrm{PCI} /$ & $114196 \mathrm{U}$ & $196 \mathrm{PCI} /$ \\
\hline C1-16-W & Hydrogen 3 (Tritium) & $-66 \mathrm{PCI} /$ & $199351 \mathrm{U}$ & $351 \mathrm{PCI} / \mathrm{L}$ \\
\hline $\mathrm{C} 1-17-\mathrm{W}$ & Hydrogen 3 (Tritium) & $51300 \mathrm{PCI} /$ & 5440 & $864 \mathrm{PCl}$ \\
\hline C1-18-W & Hydrogen 3 (Tritium) & $348 \mathrm{PCI} / \mathrm{L}$ & $228367 U$ & $367 \mathrm{PCI} /$ \\
\hline$C 2-19-W$ & Hydrogen 3 (Tritium) & $-117 \mathrm{PCI} / \mathrm{L}$ & 121 & $213 \mathrm{PCI} /$ \\
\hline C2-19-W & Hydrogen 3 (Tritium) & $-129 \mathrm{PCI} / \mathrm{L}$ & 120 & $213 \mathrm{PCI} /$ \\
\hline$C 2-24-W$ & Hydrogen 3 (Tritium) & $-66 \mathrm{PCI} / \mathrm{L}$ & 112 & $196 \mathrm{PCI} / \mathrm{L}$ \\
\hline$C 2-27-W$ & Hydrogen 3 (Tritium) & -62 PCI/ & 113 & 199 PCI/ \\
\hline C-FB-001-W & Hydrogen 3 (Tritium) & $38 \mathrm{PCI} / \mathrm{n}$ & $163281 U$ & $281 \mathrm{PCI} / \mathrm{L}$ \\
\hline C-FB-002-W & Hydrogen 3 (Tritium) & $-60 \mathrm{PCI} / \mathrm{L}$ & 159 & $281 \mathrm{PCl} / \mathrm{L}$ \\
\hline C-FB-003-W & Hydrogen 3 (Tritium) & $39 \mathrm{PCI} / \mathrm{L}$ & 113 & 192 PCI \\
\hline C-RB-001-W & Hydrogen 3 (Tritium) & $105 \mathrm{PCl} / \mathrm{L}$ & $172291 \mathrm{U}$ & $291 \mathrm{PCI} /$ \\
\hline C-RB-002-W & Hydrogen 3 (Tritium) & $16 \mathrm{PCI}$ & 168 & $291 \mathrm{PCI}$ \\
\hline C-RB-003-W & Hydrogen 3 (Tritium) & $79 \mathrm{PCI}$ & 114 & 192 PCI/ \\
\hline
\end{tabular}




\section{Distribution List}

\section{Copies}

Robert Bell

Mississippi Department of Health

Division of Radiological Health

P.O. Box 1700

Jackson, Mississippi 39215-1700

Roxanne Danz

DOE/Nevada Operations Office

P.O. Box 98518

Las Vegas, Nevada $89193-8518$

Philip Weathersby, Chief

CERCLA Section

Office of Pollution Control

Department of Environmental Quality

P.O. Box 10385

Jackson, Mississippi 39289-0385

IT Corporation

4330 South Valley View, Suite 114

Las Vegas, Nevada 89023-0345

Ethan Merril

U.S. Department of Energy Headquarters

12800 Littlebrook Road

Germantown, Maryland 20874

Anita Mullen

U.S. Environmental Protection Agency

Environmental Monitoring Systems Laboratory

P.O. Box 93478

Las Vegas, Nevada 89193-3478

Purvis Library

Purvis, Mississippi 39475

Paul Tatum

Tatum Lumber Company

P.O. Box 15547

Hattiesburg, Mississippi 39404

U.S. Department of Energy

Office of Scientific and Technical Information

175 Oak Ridge Turnpike

P.O. Box 62

Oak Ridge, Tennessee 37831

U.S. Department of Energy 